\title{
APPENDICES
}

for

\section{Framework for Identifying Key Environmental Concerns in Marine Renewable Energy Projects}

Appendix A - Potential generic effects of wave and tidal energy conversion devices

Appendix B - Raptools methodology

Appendix C - Attributes and scoring ranges for trial run of the Raptools analysis

Appendix D - Effects of a Small Commercial OPT Power Buoy Project at Humboldt on physical and biological indicators

Appendix E - Effects of Small Commercial Pelamis at Makapu’u Appendix F - Effects of Pilot Scale MCT SeaGen project at Tacoma Narrows 


\section{Appendix A. Potential generic effects of wave and tidal energy conversion devices.}

Potential generic effects of wave and tidal energy conversion devices on marine species.

\begin{tabular}{|c|c|c|c|c|c|c|c|}
\hline \multirow[b]{2}{*}{ Action } & \multicolumn{7}{|c|}{ Organisms affected and description of potential effect } \\
\hline & Marine birds & Cetaceans & Pinnipeds & Fish & Invertebrates & Mustelids & Sea turtles \\
\hline $\begin{array}{l}\text { Noise and } \\
\text { vibration }\end{array}$ & $\begin{array}{l}\text { Disrupt } \\
\text { movement, } \\
\text { behavior }\end{array}$ & $\begin{array}{l}\text { Disrupt } \\
\text { movement, } \\
\text { behavior }\end{array}$ & $\begin{array}{l}\text { Disrupt } \\
\text { movement, } \\
\text { behavior }\end{array}$ & $\begin{array}{l}\text { Disrupt } \\
\text { movement, } \\
\text { behavior }\end{array}$ & Effects unlikely & $\begin{array}{l}\text { Disrupt } \\
\text { movement, } \\
\text { behavior }\end{array}$ & $?$ \\
\hline $\begin{array}{l}\text { Seabed } \\
\text { disturbance }\end{array}$ & $\begin{array}{l}\text { Affect food } \\
\text { resources }\end{array}$ & $\begin{array}{l}\text { Affect food } \\
\text { resources }\end{array}$ & $\begin{array}{l}\text { Affect food } \\
\text { resources }\end{array}$ & $\begin{array}{l}\text { Affect food and } \\
\text { habitat resources }\end{array}$ & Affect habitat & $\begin{array}{l}\text { Affect food } \\
\text { resources }\end{array}$ & Effects unlikely \\
\hline Structure & $\begin{array}{l}\text { Provide rest } \\
\text { sites, foraging, } \\
\text { collision and } \\
\text { entanglement }\end{array}$ & $\begin{array}{l}\text { Collision and } \\
\text { entanglement }\end{array}$ & $\begin{array}{l}\text { Provide rest } \\
\text { sites, foraging, } \\
\text { entanglement }\end{array}$ & $\begin{array}{c}\text { Acts as } \\
\text { attractant, } \\
\text { provides habitat, } \\
\text { foraging }\end{array}$ & Provide habitat & $\begin{array}{l}\text { Provide rest } \\
\text { sites, foraging }\end{array}$ & Effects unlikely \\
\hline Moving parts & $\begin{array}{l}\text { Strike or } \\
\text { impact }\end{array}$ & $\begin{array}{l}\text { Strike or } \\
\text { impact }\end{array}$ & Strike or impact & Strike or impact & Effects unlikely & Strike or impact & Strike or impact \\
\hline $\begin{array}{l}\text { Water } \\
\text { circulation } \\
\text { changes }\end{array}$ & $\begin{array}{l}\text { Affect food } \\
\text { resources }\end{array}$ & $\begin{array}{l}\text { Affect food } \\
\text { resources }\end{array}$ & $\begin{array}{l}\text { Affect food } \\
\text { resources, } \\
\text { behavior }\end{array}$ & $\begin{array}{l}\text { Affect food } \\
\text { resources, } \\
\text { behavior }\end{array}$ & $\begin{array}{c}\text { Change } \\
\text { community } \\
\text { composition }\end{array}$ & $\begin{array}{l}\text { Affect food } \\
\text { resources, } \\
\text { behavior }\end{array}$ & Effects unlikely \\
\hline $\begin{array}{l}\text { Electromag- } \\
\text { netic field } \\
\text { (EMF) } \\
\end{array}$ & $?$ & $\begin{array}{c}\text { Disrupt } \\
\text { movement, } \\
\text { behavior }\end{array}$ & $\begin{array}{c}\text { Disrupt } \\
\text { movement, } \\
\text { behavior }\end{array}$ & $\begin{array}{c}\text { Disrupt } \\
\text { movement, } \\
\text { behavior }\end{array}$ & $\begin{array}{c}\text { Disrupt } \\
\text { movement, } \\
\text { behavior }\end{array}$ & $?$ & $\begin{array}{c}\text { Disrupt } \\
\text { movement, } \\
\text { behavior }\end{array}$ \\
\hline Lights & $\begin{array}{l}\text { Disorientation, } \\
\text { collision }\end{array}$ & $?$ & $?$ & $\begin{array}{l}\text { Affect behavior } \\
\text { for some fish } \\
\text { species }\end{array}$ & $\begin{array}{l}\text { Attract some } \\
\text { species of pelagic } \\
\text { invertebrates }\end{array}$ & $?$ & Effects unlikely \\
\hline $\begin{array}{l}\text { Chemical } \\
\text { releases }\end{array}$ & $\begin{array}{l}\text { Hypothermia, } \\
\text { toxicity to } \\
\text { individuals } \\
\text { and prey }\end{array}$ & $\begin{array}{l}\text { Toxicity to } \\
\text { individuals } \\
\text { and prey }\end{array}$ & $\begin{array}{c}\text { Toxicity to } \\
\text { individuals and } \\
\text { prey }\end{array}$ & $\begin{array}{c}\text { Toxicity to } \\
\text { individuals and } \\
\text { prey }\end{array}$ & $\begin{array}{l}\text { Toxicity to } \\
\text { individuals }\end{array}$ & $\begin{array}{c}\text { Toxicity to } \\
\text { individuals and } \\
\text { prey }\end{array}$ & $\begin{array}{c}\text { Toxicity to } \\
\text { individuals and } \\
\text { prey }\end{array}$ \\
\hline
\end{tabular}

Environmental Assessment for Siting Wave \& Tidal Energy Projects
A-1
H. T. Harvey \& Associates

24 November 2009 
Potential generic effects of wave and tidal energy conversion devices on site physical characteristics $(\mathrm{NA}=$ not affected)

\begin{tabular}{|c|c|c|c|c|c|c|c|}
\hline Action & $\begin{array}{l}\text { Substrate } \\
\text { Dynamics }\end{array}$ & $\begin{array}{l}\text { Hydrodynamic } \\
\text { regime (tidal only) }\end{array}$ & $\begin{array}{l}\text { Sediment } \\
\text { chemistry }\end{array}$ & $\begin{array}{c}\text { Water } \\
\text { chemistry }\end{array}$ & $\begin{array}{c}\text { Acoustic } \\
\text { environment }\end{array}$ & $\begin{array}{c}\text { Visual } \\
\text { environment }\end{array}$ & $\begin{array}{l}\text { Electromagnetic } \\
\text { environment }\end{array}$ \\
\hline $\begin{array}{l}\text { Seabed } \\
\text { disturbance }\end{array}$ & $\begin{array}{c}\text { Increases in } \\
\text { substrate size if } \\
\text { fine-grained or } \\
\text { unconsolidated } \\
\text { substrates } \\
\text { disturbed/ eroded } \\
\end{array}$ & NA & $\begin{array}{c}\text { Releases } \\
\text { pollutants from } \\
\text { contaminated } \\
\text { sediments or } \\
\text { causes reduction- } \\
\text { oxidation } \\
\end{array}$ & $\begin{array}{l}\text { Increased } \\
\text { suspended } \\
\text { sediment }\end{array}$ & NA & NA & NA \\
\hline Structure & $\begin{array}{l}\text { Localized current } \\
\text { velocity reduction; } \\
\text { contribution to } \\
\text { sediments from } \\
\text { biofouling } \\
\text { organisms on } \\
\text { structures } \\
\end{array}$ & $\begin{array}{l}\text { Localized current } \\
\text { velocity reduction }\end{array}$ & $\begin{array}{l}\text { Biological } \\
\text { contribution to } \\
\text { sediments from } \\
\text { biofouling } \\
\text { organisms on } \\
\text { structures }\end{array}$ & NA & $\begin{array}{c}\text { Increased noise, } \\
\text { vibrations }\end{array}$ & $\begin{array}{l}\text { Introduces } \\
\text { visual } \\
\text { stimuli }\end{array}$ & Effects unlikely \\
\hline Moving parts & Effects unlikely & Effects unlikely & Effects unlikely & Effects unlikely & $\begin{array}{c}\text { Increased noise, } \\
\text { vibrations }\end{array}$ & NA & Effects unlikely \\
\hline $\begin{array}{l}\text { Water } \\
\text { circulation } \\
\text { changes }\end{array}$ & $\begin{array}{l}\text { Altered substrate } \\
\text { size, sediment } \\
\text { transport, and/or } \\
\text { littoral cells }\end{array}$ & $\begin{array}{c}\text { Reduced } \\
\text { downstream } \\
\text { current velocities, } \\
\text { may affect } \\
\text { sediment transport, } \\
\text { water quality in } \\
\text { inlets and estuaries }\end{array}$ & $\begin{array}{l}\text { Sediment } \\
\text { transport and } \\
\text { deposition } \\
\text { changes alters } \\
\text { chemistry }\end{array}$ & $\begin{array}{l}\text { Changes in } \\
\text { mixing and } \\
\text { stratification }\end{array}$ & NA & NA & NA \\
\hline $\begin{array}{l}\text { Electromagnetic } \\
\text { field (EMF) }\end{array}$ & Effects unlikely & Effects unlikely & Effects unlikely & Effects unlikely & NA & NA & $\begin{array}{l}\text { Alters local } \\
\text { geomagnetic } \\
\text { fields }\end{array}$ \\
\hline Lights & NA & NA & NA & NA & NA & $\begin{array}{l}\text { Introduces } \\
\text { artificial } \\
\text { light sources }\end{array}$ & NA \\
\hline $\begin{array}{l}\text { Chemical } \\
\text { releases }\end{array}$ & NA & NA & $\begin{array}{c}\text { Addition of } \\
\text { contaminants to } \\
\text { sediment }\end{array}$ & $\begin{array}{l}\text { Addition of } \\
\text { contaminants to } \\
\text { water column }\end{array}$ & NA & NA & NA \\
\hline
\end{tabular}




\section{Appendix B. Raptools Methodology}

\section{RAPTOOLS DESCRIPTION}

Raptools is a spreadsheet application that uses multidimensional scaling (MDS), leveraging analyses, and graphical representations so that one can evaluate the interactions and effects of numerous attributes of many scenarios. These statistical techniques are readily accepted and well-vetted but complex, and a simplified and conceptual description is given here.

\subsection{MDS MECHANICS}

MDS is a set of statistical techniques that has recently been applied to aid in the relatively new field of information visualization (Kruskal and Wish 1978). MDS enables objective comparison between complex entities - in this case the "entities" are wave and tidal energy development scenarios; MDS allows identification of clusters or "more similar" entities. MDS was selected because we needed a way to evaluate and visualize the relationships between many site attributes (e.g., beach slope, numbers of special status species, or cost of energy per kilowatt), three potential project locations (Makapu'u, Hawaii; Humboldt, California, and Tacoma, Washington), and three project scales (pilot, small commercial, and large commercial).

Most people can easily evaluate and visualize relationships between 2 or 3 attributes or dimensions. An example of analysis in 2 dimensions would be evaluating whether the project generates any noise or vibrations in frequencies that affect whale behavior; the first dimension is noise, the second is whale behavior. A third dimension could be project size. We can visualize 3 dimensions as an $x-y-z$ graph, but more than 3 dimensions are difficult to visualize.

"The purpose of multidimensional scaling (MDS) is to provide a visual representation of the pattern of proximities (i.e., similarities or distances) among a set of objects" (Kruskal and Wish 1978). For MDS to be applied to resource management and evaluation, Kruskal and Wish's "similarities or distances" are the distances between two attributes if they were measured, scored, and compared. The scoring of the attributes is standardized to simple valuations of "few effects" to "most effects" so that the attributes may be compared against each other. In our 2 dimensional example above, whales could be scored 1 for very disturbed or affected, to a 3 for no behavioral changes observed; the noise generated during project construction could be scored 1 for that noise associated with a pilot project, to a 3 for power associated with a large commercial scale project.

If the relationship between whales and noise during construction is weak, then no behavioral changes (that is a "good" effect, 3 on the x axis) would be observed during construction of a large commercial project (a large commercial project would create the most construction noise, 3 on the $y$ axis), and one can imagine a line or vector on an $x-y$ graph, from the origin $(0,0)$ to a point $(3,3)$ (Figure B-1). 


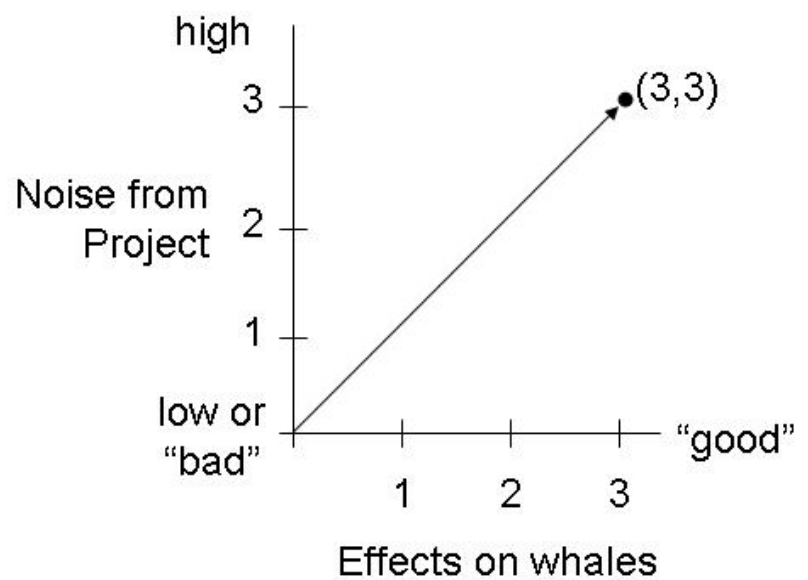

Figure B-1. High levels of noise that do not affect whales are represented by a vector from the origin $(\mathbf{0 , 0 )}$ to $(3,3)$.

If the relationship is very strong (whales are highly affected by noise from a pilot project), then the vector would be from $(0,0)$ to $(1,1)$; this second vector would be shorter than the first one (Figure B-2). In this way, relationships between attributes can be represented by distances; the MDS algorithms are performed on the distances between scored attributes.

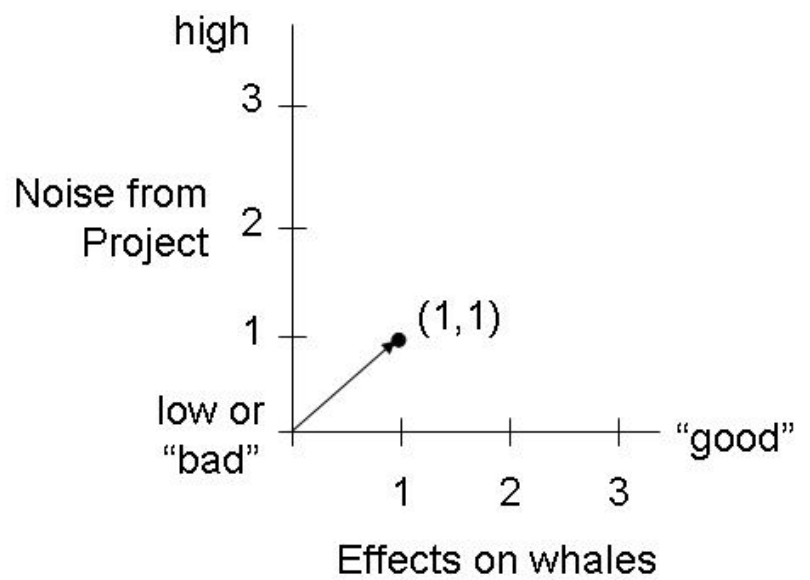

Figure B-2. Low levels of noise that do affect whales are represented by a vector from the origin $(0,0)$ to $(1,1)$.

We have identified 4 sets of attributes and 8 attributes within each set. (The statistics literature calls these sets of attributes "evaluation fields".) When distances between the many standardized attributes are obtained, the attributes can be put in order. MDS is considered an "ordination method" by statisticians.

MDS techniques have been applied to a variety of complex, multivariate problems from a variety of disciplines (Schiffman et al. 1981; Jongman et al. 1995; McCune and Grace 2002). This MDS methodology has been used as the center piece for a suite of analyses to examine the 
multidisciplinary task of evaluating the relative sustainability of commercial fisheries; this approach is called "Rapfish" (Pitcher et al. 1998; Pitcher 1999; Pitcher and Preikshot 2001). With Raptools, we have adapted the Rapfish software with the expectation that this approach offers suitable means for the objective assessment of diverse attributes (personal communication, $\mathrm{T}$. Pitcher, 12 February 2009).

\subsection{RUNNING RAPTOOLS}

In Raptools, we selected these evaluation fields:

1. Siting attributes, which are measurable site characteristics

2. Technology attributes, which are characteristics of a project's technology

3. Ecological attributes, which are environmental attributes that could be affected

4. Human environment attributes, which are attributes that are defined by society

In applying MDS to a resource management evaluation, the exercise depends on 1) the selection of the individual attributes, and 2) their scorings.

Within each attribute set, we selected 8 attributes. The ideal number of attributes is between 8 to 12 (Kavanagh and Pitcher 2004), which are enough to capture the complexity of an evaluation field, but not so many that the ordinations become too difficult to interpret visually. Kavanagh (2004) found that the resultant level of detail was a good match between the diversity of factors at play in complex scenarios, and the amount of information available to inform the evaluation of each attribute.)

The scoring of each attribute, on a coarse "few effects" to "more effects" scale, was assigned based on the literature and professional judgment. The range of scores is based on how well we can discern potential effects. For example, if our understanding of an effect on an attribute can only be judged to be "high", "medium," or "low", the scores would be 0,1 , or 2 and the range would be 0 to 2; however if we have a better understanding and can discern the effects better, the score range would be greater, say from 0 to 4, representing "no effect", "minor", "moderate", "moderately high" and "very high."

However, another approach--one that is sometimes used in the Rapfish protocol--is to have stakeholders and experts come to a consensus on the attributes and their scorings. Rapfish uses expert opinion to evaluate the probable importance of a broad range of physical, biological and sociological attributes. Rapfish creates 'best case' and 'worst case' scenarios, using the same attributes; these extreme scenarios allow multiple ordinations ${ }^{1}$ to be oriented consistently for direct comparison. Rapfish is unusual, however, in its capacity to compare multiple situations and compares favorably to other less inclusive (and often less quantitative) methods for evaluating complex systems (Leadbitter and Ward 2007). By adopting the Rapfish approach and methods in Raptools, we have also adopted its capabilities and advantages.

In this application of Raptools, we generated scores for wave energy and tidal energy development projects. The scores were entered into a spreadsheet. We downloaded the Rapfish spreadsheet from the University of British Columbia website ${ }^{2}$ and followed the instructions for

\footnotetext{
${ }^{1}$ Ordination is a technique of multivariate statistics that maps multiple measures from multiple factors, enabling the user to compare the relative importance of these factors.

${ }^{2} \mathrm{http}: / / \mathrm{www} 2$.fisheries.com/archive/projects/rapfish.php
} 
performing the calculations with our new attributes. After obtaining the $\mathrm{x}$, $\mathrm{y}$ ordinations, we used graphing software to produce the $\mathrm{X}-\mathrm{Y}$ ordination plots, the leveraging bar graphs, and the kite diagrams.

Jongman RHG, Ter Braak CJF, van Tongeren OFR (1995) Data Analysis in Community and Landscape Ecology. Cambridge University Press $321 \mathrm{pp.}$

Kavanagh P, Pitcher TJ (2004) Implementing Microsoft Excel software for Rapfish: A technique for the rapid appraisal of fisheries status. Fisheries Centre Research Reports 12: 1-75

Kruskal JB, Wish M (1978) Multidimensional Scaling. Sage University 96 pp.

Leadbitter D, Ward TJ (2007) An evaluation of systems for the integrated assessment of capture fisheries. Marine Policy 31: 458-469

McCune B, Grace JB (2002) Analysis of Ecological Communities. MjM Software Design, Gleneden Beach, Oregon, 300 pp.

Pitcher T, Bundy A, Preikshot D, Hutton T, Pauly D (1998) Measuring the unmeasurable: a multivariate and interdisciplinary method for rapid appraisal of the health of fisheries. In: Pitcher T, Hart PJB, Pauly D (eds) Reinventing Fisheries Management. Kluwer Academic Publishers, London, pp 31-54

Pitcher TJ (1999) Rapfish, a rapid appraisal technique for fisheries, and its application to the code of conduct for responsible fisheries. Food and Agricultural Organization of the United Nations, Rome, pp 52

Pitcher TJ, Preikshot D (2001) RAPFISH: A rapid appraisal technique to evaluate the sustainability status of fisheries. Fisheries Research 49: 255-270

Schiffman SS, Reynolds ML, Young FW (1981) Introduction to Multidimensional Scaling, Theory, Methods and Applications. Elsevier 440 pp. 
Attributes and scoring ranges for trial run of the Raptools analysis, as applied to evaluating effects of siting wave energy technology

\begin{tabular}{|c|c|c|c|}
\hline Attributes & $\begin{array}{l}\text { Fewest } \\
\text { adverse } \\
\text { effects }\end{array}$ & $\begin{array}{c}\text { Most } \\
\text { adverse } \\
\text { effects }\end{array}$ & Explanation \\
\hline \multicolumn{4}{|r|}{ SITING } \\
\hline distance from shore & 0 & 3 & $\begin{array}{l}\text { Although shorter distance reduces installation and maintenance costs, greater distances present } \\
\text { fewer effects due to greater wave attenuation and lower biodiversity. }\end{array}$ \\
\hline water depth & 4 & 0 & Greater depth presents fewer effects; higher biodiversity in shallow water. \\
\hline estuarine distance & 0 & 2 & $\begin{array}{l}\text { The farther away an estuary (or rivermouth) is, the fewer effects because change in sediment } \\
\text { transport will be less, with less effect on estuary closure and opening. }\end{array}$ \\
\hline proximity to natural reef & 2 & 0 & $\begin{array}{l}\text { The farther away a natural reef is, the fewer adverse effects because reefs are associated with } \\
\text { high diversity and species attracted to artificial habitat presented by devices. }\end{array}$ \\
\hline beach slope & 2 & 0 & $\begin{array}{l}\text { The steeper the slope, the fewer adverse effects expected, because the width of the intertidal } \\
\text { zone would be narrower, so less of this important zone would be affected. }\end{array}$ \\
\hline biological hot spot & 0 & 2 & $\begin{array}{l}\text { Ecologically important areas ideally would be avoided; increasing number or magnitude is } \\
\text { assumed to be detrimental. }\end{array}$ \\
\hline $\begin{array}{r}\text { Sea surface temperature (SST) } \\
\text { predictability }\end{array}$ & 0 & 3 & $\begin{array}{l}\text { SST is associated with biological predictability; greater predictability would create fewer } \\
\text { effects because ecological effects could be avoided or minimized if they can be predicted. }\end{array}$ \\
\hline distance to port & 0 & 2 & $\begin{array}{l}\text { The shorter the distance to port, the fewer effects because vessel transit distances are reduced; } \\
\text { accident probabilities would be lower. }\end{array}$ \\
\hline \multicolumn{4}{|r|}{ PROJECT/TECHNOLOGY } \\
\hline Project size (MW generated) & 0 & 2 & $\begin{array}{l}\text { Smaller projects translate to fewer environmental effects, however, the relationship is almost } \\
\text { certainly nonlinear. }\end{array}$ \\
\hline energy extraction & & & $\begin{array}{l}\text { Less energy extraction would create the fewest adverse effects. Technologies' extraction } \\
\text { efficiencies are a function of device design and siting, so this attribute was scored primarily on } \\
\text { scale. }\end{array}$ \\
\hline $\begin{array}{r}\text { impingement/entrainment/moving } \\
\text { parts }\end{array}$ & & & The fewer moving parts, the fewer effects created. \\
\hline \# of vertical cables & 0 & 3 & The fewer cables, the smaller the risk of seabird or marine mammal strike. \\
\hline anchoring system & 0 & 2 & $\begin{array}{l}\text { Anchoring/foundation system designs vary, as would their potential effects through } \\
\text { displacement or adding substrate. }\end{array}$ \\
\hline operations and maintenance & 0 & 2 & $\begin{array}{l}\text { The less O\&M activity, the fewer effects because risk of accidents through vessel traffic is also } \\
\text { reduced. }\end{array}$ \\
\hline noise and vibration generated & 0 & 2 & $\begin{array}{l}\text { Device noise and vibration is generally unknown, but many taxa are potentially affected; must } \\
\text { consider frequency (Hz), amplitude, duration as well as longer temporal patterns }\end{array}$ \\
\hline
\end{tabular}




\begin{tabular}{|c|c|c|c|}
\hline Attributes & $\begin{array}{l}\text { Fewest } \\
\text { adverse } \\
\text { effects }\end{array}$ & $\begin{array}{l}\text { Most } \\
\text { adverse } \\
\text { effects }\end{array}$ & Explanation \\
\hline hydraulic fluid & 0 & 3 & Least volumes used create the fewest effects. \\
\hline \multirow[t]{2}{*}{ operational/navigational lighting } & 0 & 2 & $\begin{array}{l}\text { Lighting strength and patterns create effects. Efforts or projects with designs explicitly } \\
\text { intended to mitigate effects on seabird behavior recognized in the scoring. }\end{array}$ \\
\hline & \multicolumn{3}{|r|}{ ECOLOGICAL } \\
\hline de facto marine reserve & 2 & 0 & $\begin{array}{l}\text { Protection results in fewer effects. Fishing access restrictions likely to result in protected } \\
\text { subpopulation; scale effects are likely; assumes some degree of compliance. }\end{array}$ \\
\hline artificial reef effect & 2 & 0 & $\begin{array}{l}\text { Attraction is detrimental and production is beneficial. This attribute combines distance to } \\
\text { nearest existing reefs with presence and swimming ability of local fishes. A lower } \\
\text { redistribution is preferable for causing the fewest adverse effects. Artificial reef effect + } \\
\text { project scale may combine to result in local production (increased regional biomass through } \\
\text { larval/juvenile recruitment). Smaller projects may result in attraction and migration from } \\
\text { existing reefs. }\end{array}$ \\
\hline local concentration of piscivores & 0 & 1 & $\begin{array}{l}\text { Fewer piscivores is preferable because concentrating potential predators may affect species of } \\
\text { special concern (e.g., salmon smolts, sea turtle hatchlings) near known/suspected migration } \\
\text { routes. }\end{array}$ \\
\hline species of special concern & 0 & 2 & Fewer species of special concern is better. \\
\hline EMF-responsive species & 0 & 3 & Fewer EMF-responsive species is better. \\
\hline Marine mammal strike & 0 & 3 & $\begin{array}{l}\text { Fewer vulnerable species is better. Presence determined by siting of project with a migration } \\
\text { corridor, and prior experience with local strike or entanglement (e.g., crab pot lines). }\end{array}$ \\
\hline noise-responsive species & 0 & 2 & The fewer species known to be noise-responsive is better. \\
\hline light-responsive birds & 0 & 2 & The fewer species known to be light-responsive is better. \\
\hline & & 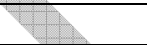 & HUMAN ENVIRONMENT \\
\hline local population size & & 0 & $\begin{array}{l}\text { The greater the population size, the fewer the adverse effects because any negative impact } \\
\text { would be spread across a greater population size (ecological footprint concept). }\end{array}$ \\
\hline \# of temporary jobs & & 0 & $\begin{array}{l}\text { The greater number of temporary jobs created, the fewer adverse (greater beneficial) impacts to } \\
\text { the human environment. }\end{array}$ \\
\hline \# of permanent jobs & 3 & 0 & $\begin{array}{l}\text { The greater number of permanent jobs created, the fewer adverse (greater beneficial) impacts } \\
\text { to the human environment. }\end{array}$ \\
\hline lost fishing grounds & 0 & 2 & The lower the area of lost fishing grounds, the fewer effects. \\
\hline existing vessel traffic & 0 & 2 & $\begin{array}{l}\text { The lower the existing traffic, the fewer effects because the probability of vessel collision is } \\
\text { lower. }\end{array}$ \\
\hline cost of energy $(\$ / \mathrm{kwhr})$ & 2 & 0 & The higher the local cost of energy, the greater the positive effect of the project. \\
\hline energy output (MWh/year) & 2 & 0 & The greater the energy output, the greater the contribution to reduction in greenhouse gas \\
\hline
\end{tabular}

Page C-2 


\begin{tabular}{c|c|c|l}
\hline Attributes & $\begin{array}{c}\text { Fewest } \\
\text { adverse } \\
\text { effects }\end{array}$ & $\begin{array}{c}\text { Most } \\
\text { adverse } \\
\text { effects }\end{array}$ & \multicolumn{1}{|c}{ Explanation } \\
\hline & & & emissions. \\
\hline indirect economic effects & 2 & 0 & Indirect economic development assumed to occur with energy development. \\
\hline
\end{tabular}

\section{Page C-3}




\begin{tabular}{|c|c|c|c|c|c|c|c|c|c|c|c|c|c|c|c|c|c|}
\hline $\begin{array}{l}\text { Attributes > } \\
\text { Ocean Energy } \\
\text { Projects } \\
\text { V }\end{array}$ & 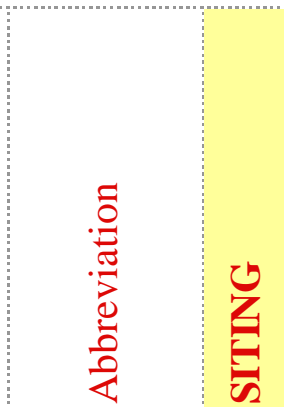 & 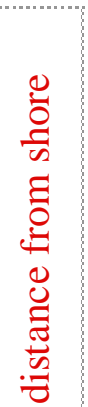 & 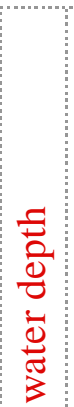 & 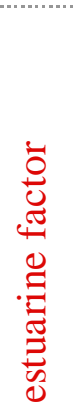 & 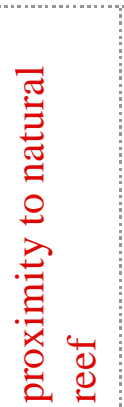 & 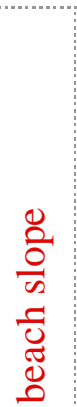 & 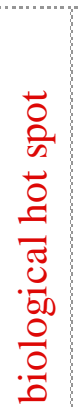 & 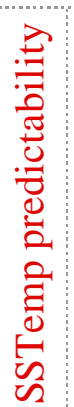 & 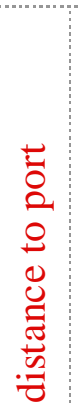 & 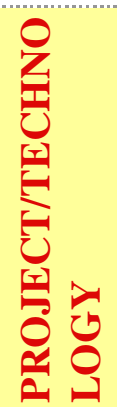 & 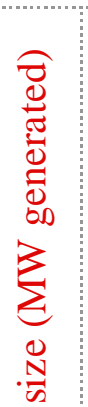 & 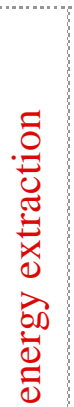 & 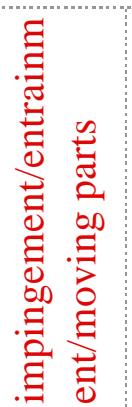 & 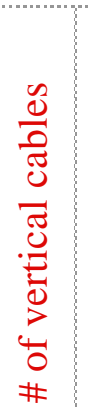 & 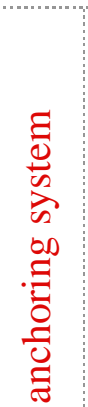 & 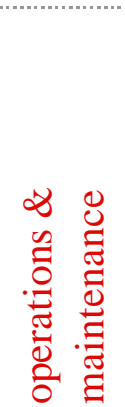 & 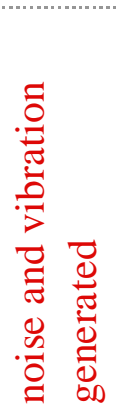 \\
\hline Hawaii Pelamis mec & HI_PeI_med & 2 & 3 & 0 & 0 & 2 & 2 & 0 & 2 & & 1 & 1 & 0 & 1 & 0 & 1 & 1 \\
\hline Hawaii Pelamis Irg & HI_Pel_Irg & 2 & 3 & 0 & 0 & 2 & 2 & 0 & 2 & & 2 & 2 & 0 & 2 & 0 & 1 & 1 \\
\hline Hawaii OPT sml & HI_OPT_sml & 2 & 3 & 0 & 0 & 2 & 2 & 0 & 2 & & 0 & 0 & 0 & 2 & 3 & 1 & 1 \\
\hline Hawaii OPT med & HI_OPT_med & 2 & 3 & 0 & 0 & 2 & 2 & 0 & 2 & & 1 & 1 & 0 & 2 & 3 & 1 & 1 \\
\hline Hawaii OPT Irg & HI_OPT_Irg & 2 & 3 & 0 & 0 & 2 & 2 & 0 & 2 & & 2 & 2 & 0 & 3 & 3 & 1 & 1 \\
\hline Hawaii Wdragon sml & HI_WD_sml & 2 & 3 & 0 & 0 & 2 & 2 & 0 & 2 & & 0 & 0 & 2 & 1 & 2 & 1 & 2 \\
\hline Hawaii Wdragon med & HI_WD_med & 2 & 3 & 0 & 0 & 2 & 2 & 0 & 2 & & 1 & 1 & 2 & 1 & 2 & 1 & 2 \\
\hline California Pelamis sml & CA_Pel_sml & 2 & 2 & 1 & 2 & 1 & 1 & 2 & 0 & & 0 & 0 & 0 & 1 & 0 & 1 & 1 \\
\hline California Pelamis med & CA_Pel_med & 2 & 2 & 1 & 2 & 1 & 1 & 2 & 0 & & 1 & 1 & 0 & 1 & 0 & 1 & 1 \\
\hline California Pelamis Irg & CA_Pel_Irg & 2 & 2 & 2 & 2 & 1 & 1 & 2 & 0 & & 2 & 2 & 0 & 2 & 0 & 1 & 1 \\
\hline California OPT sml & CA_OPT_sml & 2 & 2 & 1 & 2 & 1 & 1 & 2 & 0 & & 0 & 0 & 0 & 2 & 3 & 1 & 1 \\
\hline California OPT med & CA_OPT_med & 2 & 2 & 1 & 2 & 1 & 1 & 2 & 0 & & 1 & 1 & 0 & 2 & 3 & 1 & 1 \\
\hline California OPT Irg & CA_OPT_Irg & 2 & 2 & 2 & 2 & 1 & 1 & 2 & 0 & & 2 & 2 & 0 & 3 & 3 & 1 & 1 \\
\hline California Wdragon sml & CA_WD_sml & 2 & 2 & 1 & 2 & 1 & 1 & 2 & 0 & & 0 & 0 & 2 & 1 & 2 & 1 & 2 \\
\hline California Wdragon med & CA WD med & 2 & 2 & 1 & 2 & 1 & 1 & 2 & 0 & & 1 & 1 & 2 & 1 & 2 & 1 & 2 \\
\hline California Wdragon Irg & CA_WD_Irg & 2 & 2 & 2 & 2 & 1 & 1 & 2 & 0 & & 2 & 2 & 2 & 2 & 2 & 1 & 2 \\
\hline California Oyster sml & CA_Oys_sml & 0 & 1 & 1 & 2 & 1 & 1 & 2 & 0 & & 0 & 0 & 1 & 0 & 1 & 1 & 0 \\
\hline California Oyster med & CA_Oys_med & 0 & 1 & 1 & 2 & 1 & 1 & 2 & 0 & & 1 & 1 & 1 & 0 & 1 & 1 & 0 \\
\hline California Oyster Irg & CA_Oys_Irg & 0 & 1 & 2 & 2 & 1 & 1 & 2 & 0 & & 2 & 2 & 1 & 0 & 1 & 1 & 0 \\
\hline Washington SMD Irg & WA_SMD_Irg & 1 & 3 & 2 & 1 & 2 & 1 & 1 & 0 & & 2 & 2 & 2 & 1 & 1 & 1 & 2 \\
\hline
\end{tabular}


Appendix C -- Raptools Trial Run

\begin{tabular}{|c|c|c|c|c|c|c|c|c|c|c|c|c|c|c|c|c|c|c|c|c|}
\hline 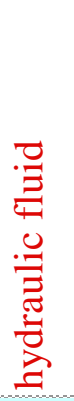 & 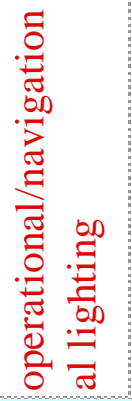 & $\begin{array}{l}\qquad \\
0 \\
0 \\
0 \\
0 \\
0 \\
0 \\
0\end{array}$ & 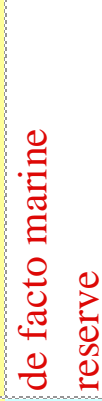 & 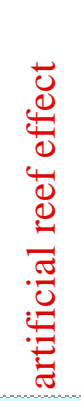 & 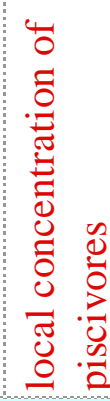 & 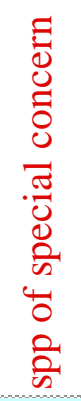 & 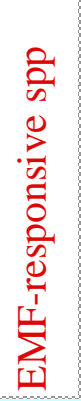 & 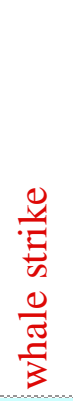 & 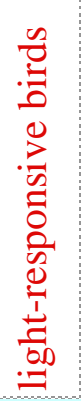 & 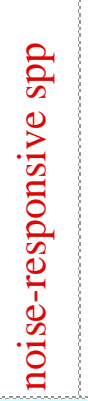 & 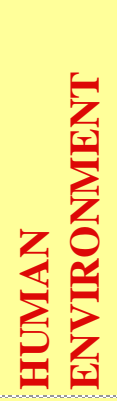 & 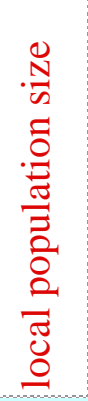 & 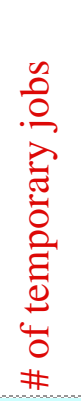 & 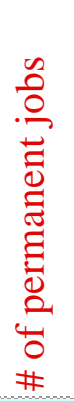 & 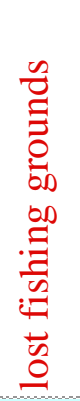 & 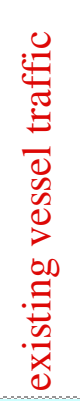 & 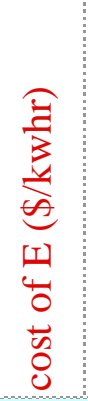 & 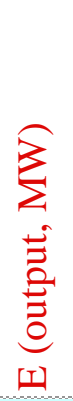 & 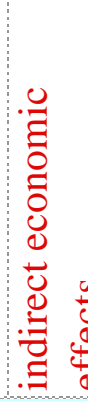 & 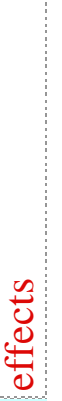 \\
\hline 3 & 1 & & 1 & 0 & & 1 & 1 & 1 & 1 & 2 & & 3 & 1 & 0 & 0 & 0 & 2 & 0 & & 0 \\
\hline 3 & 1 & & 1 & 0 & & 1 & 1 & 1 & 1 & 2 & & 3 & 1 & 0 & 0 & 0 & 2 & 1 & & 0 \\
\hline 4 & 2 & & 2 & 0 & & 1 & 2 & 2 & 2 & 2 & & 2 & 1 & 1 & 0 & 0 & 2 & 2 & & 1 \\
\hline 2 & 1 & & 1 & 1 & & 1 & 1 & 1 & 1 & 2 & & 3 & 2 & 0 & 0 & 0 & 2 & 0 & & 0 \\
\hline 2 & 1 & & 1 & 1 & & 1 & 1 & 2 & 1 & 2 & & 3 & 2 & 0 & 0 & 0 & 2 & 1 & & 0 \\
\hline 3 & 2 & & 2 & 1 & & 1 & 2 & 2 & 2 & 2 & & 2 & 2 & 1 & 0 & 0 & 2 & 2 & & 1 \\
\hline 3 & 1 & & 1 & 1 & 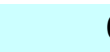 & 1 & 1 & 0 & 1 & 2 & & 3 & 1 & 0 & 0 & 0 & 2 & 0 & & 0 \\
\hline 3 & 1 & & 1 & 1 & ( & 1 & 1 & 1 & 1 & 2 & & 3 & 1 & 0 & 0 & 0 & 2 & 1 & & 0 \\
\hline 4 & 2 & & 2 & 1 & & 1 & 2 & 2 & 2 & 2 & & 2 & 1 & 1 & 1 & 0 & 2 & 2 & & 1 \\
\hline 0 & 0 & & 0 & 1 & 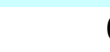 & 0 & 1 & 1 & 0 & 1 & & 3 & 1 & 0 & 0 & 0 & 2 & 0 & & 0 \\
\hline 0 & 0 & & 0 & 1 & 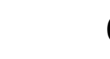 & 0 & 1 & 1 & 0 & 1 & & 3 & 1 & 0 & 0 & 0 & 2 & 1 & & 0 \\
\hline 0 & 0 & & 1 & 1 & 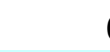 & 0 & 2 & 1 & 0 & 2 & & 2 & 1 & 1 & 0 & 0 & 2 & 2 & & 1 \\
\hline 3 & 1 & & 1 & 0 & & 2 & 2 & 2 & 1 & 1 & & 1 & 1 & 0 & 0 & 0 & 1 & 0 & & 0 \\
\hline 3 & 1 & & 1 & 0 & & 2 & 2 & 2 & 1 & 1 & & 1 & 1 & 0 & 1 & 1 & 1 & 1 & & 0 \\
\hline 4 & 2 & & 2 & 0 & & 2 & 3 & 3 & 2 & 2 & & 0 & 1 & 1 & 2 & 1 & 1 & 2 & & 1 \\
\hline 2 & 1 & & 1 & 1 & & 2 & 2 & 2 & 1 & 1 & & 1 & 2 & 0 & 0 & 0 & 1 & 0 & & 0 \\
\hline 2 & 1 & & 1 & 1 & & 2 & 2 & 3 & 1 & 1 & & 1 & 2 & 1 & 1 & 1 & 1 & 1 & & 0 \\
\hline 3 & 2 & & 2 & 1 & & 2 & 3 & 3 & 2 & 2 & & 0 & 2 & 1 & 2 & 1 & 1 & 2 & & 1 \\
\hline 3 & 1 & & 1 & 1 & 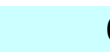 & 2 & 2 & 0 & 1 & 1 & & 1 & 1 & 0 & 0 & 0 & 1 & 0 & & 0 \\
\hline 3 & 1 & & 1 & 1 & 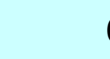 & 2 & 2 & 1 & 1 & 1 & & 1 & 1 & 0 & 1 & 1 & 1 & 1 & & 0 \\
\hline 4 & 2 & & 2 & 1 & & 2 & 3 & 2 & 2 & 2 & & 0 & 1 & 0 & 2 & 1 & 1 & 2 & & 1 \\
\hline 0 & 0 & & 0 & 1 & & 1 & 2 & 0 & 0 & 0 & & 1 & 1 & 0 & 0 & 0 & 1 & 0 & & 0 \\
\hline 0 & 0 & & 0 & 1 & & 1 & 2 & 0 & 0 & 0 & & 1 & 1 & 0 & 0 & 0 & 1 & 1 & & 0 \\
\hline 0 & 0 & & 1 & 1 & & 1 & 3 & 0 & 0 & 1 & & 0 & 1 & 1 & 0 & 0 & 1 & 2 & & 1 \\
\hline 0 & 0 & & 0 & 1 & & 1 & 2 & 1 & 0 & 0 & & 4 & 1 & 0 & 0 & 0 & 0 & 0 & & 0 \\
\hline 0 & 0 & & 0 & 1 & 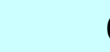 & 1 & 2 & 1 & 0 & 0 & & 4 & 1 & 0 & 0 & 0 & 0 & 1 & & 1 \\
\hline 1 & 0 & & 1 & 1 & & 1 & 3 & 2 & 0 & 1 & & 3 & 1 & 1 & 1 & 1 & 0 & 2 & & 1 \\
\hline 1 & 0 & & 0 & 1 & & 1 & 2 & 1 & 0 & 0 & & 4 & 1 & 0 & 0 & 0 & 0 & 0 & & 0 \\
\hline 1 & 0 & & 0 & 1 & & 1 & 2 & 1 & 0 & 0 & & 4 & 1 & 0 & 0 & 0 & 0 & 1 & & 1 \\
\hline 2 & 0 & & 1 & 1 & & 1 & 3 & 2 & 0 & 1 & & 3 & 1 & 1 & 1 & 1 & 0 & 2 & & 1 \\
\hline 2 & 0 & & 0 & 1 & & 1 & 2 & 1 & 0 & 0 & & 4 & 1 & 0 & 0 & 0 & 0 & 0 & & 0 \\
\hline 2 & 0 & & 0 & 1 & 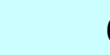 & 1 & 2 & 1 & 0 & 0 & & 4 & 1 & 0 & 0 & 0 & 0 & 1 & & 1 \\
\hline 3 & 0 & & 1 & 1 & & 1 & 3 & 2 & 0 & 1 & & 3 & 1 & 1 & 1 & 1 & 0 & 2 & & 1 \\
\hline
\end{tabular}




\section{Appendix D. Effects of a Small Commercial OPT Power Buoy Project at Humboldt on physical and biological indicators}

Included in Appendix D are 1) a project description of the small commercial OPT Power Buoy project at the Humboldt project site; 2) the marine indicators selection process based on species' distribution, behavior, and biology; 3) an effects analysis of the project on site physical and biological indicators in tabular format; and 4) a list of references used to complete the effects analysis.

\section{Project description for construction, operations and maintenance, and decommissioning phases for Small Commercial OPT Power Buoy project at the Humboldt project site.}

\begin{tabular}{|c|c|c|}
\hline Project phase & Project activity or characteristic & Technology and site specific information \\
\hline \multirow{14}{*}{ Construction } & Location and deployment depths & Off Humboldt Bay, 50m water depth, 3 NM from shore \\
\hline & Footprint & 67 devices, $3.3 \mathrm{~km} \mathrm{x} 0.8$ km (2.6 km² $)$. \\
\hline & Loading ports and dock locations & Humboldt Bay \\
\hline & $\begin{array}{l}\text { Shipping routes for delivery and } \\
\text { installation }\end{array}$ & Assumed in and out of Humboldt Bay docks \\
\hline & Ship types and sizes & 2 tug boats, 1 barge \\
\hline & $\begin{array}{l}\text { Installation and assembly } \\
\text { procedures }\end{array}$ & $\begin{array}{l}\text { Cable directionally drilled from land to subsea opening, } \\
\text { the subsea cable installation is from the subsea opening } \\
\text { to turbine site. Tug boats bring barge, anchor from } \\
\text { barge lowered via strand jacks, and mooring buoys } \\
\text { deployed. Repeat until each OPT buoy gets } 3 \text { mooring } \\
\text { buoys, but some share buoys. OPT buoy is towed to } \\
\text { mooring sites with a tug boat, then connected to mooring } \\
\text { buoys. }\end{array}$ \\
\hline & Installation equipment & Barge, tug boats, strand jacks, directional drilling rig \\
\hline & Temporary structures & N/A \\
\hline & $\begin{array}{l}\text { Types, composition, locations, and } \\
\text { numbers of anchoring and mooring } \\
\text { systems }\end{array}$ & $\begin{array}{l}3 \text { anchors and subsurface mooring buoys for each } \\
\text { device, } 67 \text { devices. Anchors are } 6 \text { × } 6 \text { × } 3.1 \text { m concrete } \\
\text { blocks. }\end{array}$ \\
\hline & Installation schedule and phasing & $\begin{array}{l}\sim 4 \text { months, could be over } 2 \text { summers. Construction } \\
\text { assumed to occur } 24 \text { hrs/day. }\end{array}$ \\
\hline & Chemicals and fuels used & Hydraulic fluids, boat fuel, paints, anti-fouling agents \\
\hline & Sources and levels of noise & Ship engines, drilling rig for directional drilling \\
\hline & $\begin{array}{l}\text { Sources, levels, and characteristics } \\
\text { of light }\end{array}$ & $\begin{array}{l}\text { Navigational lights on boats and on devices as they are } \\
\text { installed. Construction and deck lights will be brighter } \\
\text { than navigation lights }\end{array}$ \\
\hline & Number of vessel trips & Unknown \\
\hline \multirow[t]{4}{*}{$\begin{array}{l}\text { Operations and } \\
\text { Maintenance }\end{array}$} & General description of technology & $\begin{array}{l}\text { The OPT Buoy is a heaving point absorber, reacting } \\
\text { against a subsea reaction plate. The relative movement } \\
\text { between the absorber buoy and the reaction mass is } \\
\text { converted into electricity using a hydraulic power } \\
\text { conversion system. The device is "slack-moored" to the } \\
\text { seabed; the wires are under tension. }\end{array}$ \\
\hline & O\&M procedures and schedule & $\begin{array}{l}\text { Routine O\&M is assumed to be annually; devices will } \\
\text { likely be towed to pier. }\end{array}$ \\
\hline & $\begin{array}{l}\text { Operating equipment other than } \\
\text { wave device(s) }\end{array}$ & $\begin{array}{l}\text { Mooring lines and anchors, maintenance boats, electrical } \\
\text { collector system, subsea cable }\end{array}$ \\
\hline & Listing of all moving parts & OPT buoys \\
\hline
\end{tabular}


Appendix D - Effects of Small Commercial OPT Power Buoy Project Humboldt

\begin{tabular}{|c|c|c|}
\hline Project phase & Project activity or characteristic & Technology and site specific information \\
\hline & $\begin{array}{l}\text { Listing of all structures on surface } \\
\text { and below }\end{array}$ & $\begin{array}{l}67 \text { OPT buoys. Each OPT buoy extends } 8 \mathrm{~m} \text { above } \\
\text { surface and } 34 \mathrm{~m} \text { below surface, and is } 11 \mathrm{~m} \text { diameter. } 3 \\
\text { subsurface mooring buoys (at depths } 9 \text { to } 15 \text { m below } \\
\text { surface) are moored to anchor on bottom. }\end{array}$ \\
\hline & $\begin{array}{l}\text { Vessel routes and schedule for } \\
\text { operation and maintenance }\end{array}$ & Assumed from Humboldt Bay docks to project site \\
\hline & Ship types and sizes & Tug boat if device towed to pier \\
\hline & $\begin{array}{l}\text { Potential emergency conditions } \\
\text { and procedures }\end{array}$ & Unknown \\
\hline & $\begin{array}{l}\text { Chemicals used by devices, e.g., } \\
\text { hydraulic fluids, antifouling paints. }\end{array}$ & Antifouling paint, hydraulic fluid \\
\hline & Sources and levels of noise & $\begin{array}{l}\text { Unknown. Possibly splash sound of waves on devices. } \\
\text { Assume no audio navigational warnings on devices. } \\
\text { Maintenance vessel noise. }\end{array}$ \\
\hline & Sources and levels of light & $\begin{array}{l}\text { Navigational lights on devices, surface piercing mooring } \\
\text { buoys, and on maintenance vessels. }\end{array}$ \\
\hline \multirow{13}{*}{ Decommissioning } & $\begin{array}{l}\text { Description of equipment or } \\
\text { structures removed }\end{array}$ & $\begin{array}{l}\text { Assumed all buoys, devices, mooring lines, anchors, } \\
\text { subsea cable, and other electrical collection removed } \\
\text { with custom vessel, tug boats, and barges }\end{array}$ \\
\hline & $\begin{array}{l}\text { Description of equipment or } \\
\text { structures to be left in place }\end{array}$ & None. \\
\hline & $\begin{array}{l}\text { Monitoring procedure and schedule } \\
\text { for equipment left in place }\end{array}$ & None. \\
\hline & $\begin{array}{l}\text { Shipping routes for equipment } \\
\text { removed }\end{array}$ & Assumed project site to Humboldt dock \\
\hline & Ship types and size & $\begin{array}{l}\text { Assumed same as construction, } 2 \text { tug boats, } 1 \text { barge plus } \\
\text { a cable handling vessel }\end{array}$ \\
\hline & $\begin{array}{l}\text { Decommissioning and disassembly } \\
\text { procedures }\end{array}$ & Assumed similar but reverse of construction procedures \\
\hline & Decommissioning equipment & Barges, tug boats, supply boat, cable handling vessel \\
\hline & Temporary structures & None \\
\hline & $\begin{array}{l}\text { Decommissioning schedule and } \\
\text { phasing }\end{array}$ & Assumed over 1 summer season \\
\hline & Chemicals and fuels used & Boat fuels, hydraulic fluids \\
\hline & Sources and levels of noise & $\begin{array}{l}\text { Shipping noise, subsea cable removal, dismantling of } \\
\text { mooring cables and device }\end{array}$ \\
\hline & Vessels required, number of trips & Unknown \\
\hline & $\begin{array}{l}\text { Best management practices } \\
\text { planned }\end{array}$ & $\begin{array}{l}\text { Assumed to follow BMPs for marine construction and } \\
\text { decommissioning }\end{array}$ \\
\hline
\end{tabular}




\section{Selection of biological indicators for the Humboldt project effects analysis}

\section{Selection of pinnipeds and cetacean indicators for Humboldt project}

Cetacean and pinniped indicator species were selected for the Humboldt wave energy project effects analysis by considering their temporal distribution, habitat and abundance, for all pinnipeds and cetacean species that could occur in the project area.

Cetaceans. Many baleen whales (Order Mysticeti) and toothed whales (Order Odontoceti) could occur in the continental shelf waters off the Humboldt coast (Table 1). However, the only two baleen whale species that regularly occur within the Humboldt wave energy project area are the humpback whale and the gray whale. The remaining baleen whales either occur further offshore along the continental slope and beyond, and/or are very rare in the area. Although not selected as indicator species, blue whales and minke whales also occasionally occur in the project area. If they do occur in the project area, the effects on the blue whales are likely similar to effects on the humpback whales, and effects on the minke whales are likely similar to effects on the gray whales.

The toothed whale species that regularly occur within the project area are the killer whale, harbor porpoise, and three smaller species: Pacific white-sided dolphin, Dall's porpoise, and Risso's dolphin. The remaining odontocetes occur farther offshore, along the continental slope and beyond. The harbor porpoise is the most common toothed whale species in the project area with its preference for nearshore sandy bottom habitats. Killer whales are infrequently sighted but are known to appear closer to shore, including inside Humboldt Bay. Dall’s porpoise, Risso’s dolphin, and Pacific white-sided dolphin regularly occur in the area, but tend to prefer more offshore waters.

The following cetaceans were selected as indicator species to assess the effects of a Humboldt wave energy project: 1) humpback whale; 2) gray whale; 3) killer whale; 4) harbor porpoise; and 5) "small odontocetes" (Pacific white-sided dolphin, Dall's porpoise, and Risso's dolphin). All cetaceans have highly developed acoustic abilities capable of detecting sounds at great distances; therefore, the acoustic zone of influence of project activities (i.e., noise emitted from construction equipment or device structures) extends far beyond the project area. Types of potential impacts on these species include collision and injury with boats; toxicity from oil or chemical spills/ releases, noise disturbance from turbines, moorings, boats or construction/ decommissioning activities; and entanglement or collision with moorings and wave structures.

Table 1. Cetaceans species that could occur in the Humboldt wave energy project area

\begin{tabular}{|c|c|c|c|c|c|c|}
\hline Common Name & Scientific Name & Habitat & $\begin{array}{l}\text { Temporal } \\
\text { Distribution }\end{array}$ & Life Stage & $\begin{array}{l}\text { Federal/ } \\
\text { State } \\
\text { Status }\end{array}$ & References \\
\hline \multicolumn{7}{|l|}{ Baleen whales } \\
\hline Blue whale & Balaenoptera musculus & $\begin{array}{l}\text { Offshore, } \\
\text { occasionally } \\
\text { nearshore }\end{array}$ & Spring to fall & Adult, calf & FE 1970 & 1 \\
\hline Humpback whale & $\begin{array}{l}\text { Megaptera } \\
\text { novaeangliae }\end{array}$ & Nearshore & Spring to fall & Adult, calf & FE 1970 & 1 \\
\hline Fin whale & Balaenoptera physalus & Nearshore & Spring to fall & Adult, calf & FE 1970 & 1 \\
\hline Gray whale & Eschrichtius robustus & Nearshore & Spring to fall & Adult, calf & Recovered & 2 \\
\hline
\end{tabular}


Table 1. Cetaceans species that could occur in the Humboldt wave energy project area

\begin{tabular}{|c|c|c|c|c|c|c|}
\hline Common Name & Scientific Name & Habitat & $\begin{array}{l}\text { Temporal } \\
\text { Distribution }\end{array}$ & Life Stage & $\begin{array}{l}\text { Federal/ } \\
\text { State } \\
\text { Status }\end{array}$ & References \\
\hline Sei Whale & Balaenoptera borealis & $\begin{array}{l}\text { Nearshore } \\
\text { and offshore }\end{array}$ & Rare & Adult, calf & FE 1970 & 1 \\
\hline Minke Whale & $\begin{array}{l}\text { Balaenoptera } \\
\text { acutorostrata }\end{array}$ & $\begin{array}{l}\text { Nearshore } \\
\text { and offshore }\end{array}$ & Rare & Adult, calf & MMPA & 5,6 \\
\hline Bryde's Whale & Balaenoptera edeni & $\begin{array}{l}\text { Tropical } \\
\text { offshore }\end{array}$ & Rare & Adult, calf & MMPA & 5,6 \\
\hline $\begin{array}{l}\text { Northern Right } \\
\text { Whale }\end{array}$ & Eubalaena japonica & $\begin{array}{l}\text { Nearshore } \\
\text { and offshore }\end{array}$ & Rare & Adult, calf? & $\begin{array}{l}\text { FE, } \\
\text { MMPA }\end{array}$ & 3 \\
\hline \multicolumn{7}{|l|}{ Toothed whales } \\
\hline Sperm Whale & $\begin{array}{l}\text { Physeter } \\
\text { macrocephalus }\end{array}$ & $\begin{array}{l}\text { Shelf, slope, } \\
\text { offshore }\end{array}$ & Year-round & Adult, calf & FE, MMPA & 1 \\
\hline $\begin{array}{l}\text { Killer whale } \\
\text { Southern Resident }\end{array}$ & Orcinus orca & Nearshore & Winter & Adult, calf & FE, MMPA & 4 \\
\hline $\begin{array}{l}\text { Killer whale } \\
\text { Offshore }\end{array}$ & Orcinus orca & $\begin{array}{l}\text { Nearshore } \\
\text { and offshore }\end{array}$ & Year-round & Adult, calf & MMPA & 5,6 \\
\hline $\begin{array}{l}\text { Killer whale } \\
\text { Transient }\end{array}$ & Orcinus orca & $\begin{array}{l}\text { Nearshore } \\
\text { and offshore }\end{array}$ & Year-round & Adult, calf & MMPA & 5,6 \\
\hline Harbor Porpoise & Phocoena phocoena & Nearshore & Year-round & All & MMPA & 5,6 \\
\hline Dall's Porpoise & Phocoenoides dalli & $\begin{array}{l}\text { Nearshore } \\
\text { and offshore }\end{array}$ & Year-round & All & MMPA & 5,6 \\
\hline $\begin{array}{l}\text { Pacific White- } \\
\text { Sided Dolphin }\end{array}$ & $\begin{array}{l}\text { Lagenorhynchus } \\
\text { obliquidens }\end{array}$ & $\begin{array}{l}\text { Nearshore } \\
\text { and offshore }\end{array}$ & Year-round & All & MMPA & 5,6 \\
\hline Risso's Dolphin & Grampus griseus & $\begin{array}{l}\text { Nearshore } \\
\text { and offshore }\end{array}$ & Year-round & All & MMPA & 5,6 \\
\hline $\begin{array}{l}\text { Short-beaked } \\
\text { Common Dolphin }\end{array}$ & Delphinus delphis & Offshore & Rare & All & MMPA & 5,6 \\
\hline $\begin{array}{l}\text { Long-beaked } \\
\text { Common Dolphin }\end{array}$ & Delphinus capensis & Offshore & Rare & All & MMPA & 5,6 \\
\hline Striped Dolphin & Stenella coeruleoalba & Offshore & Rare & All & MMPA & 5,6 \\
\hline $\begin{array}{l}\text { Northern Right } \\
\text { Whale Dolphin }\end{array}$ & Lissodelphis borealis & Offshore & Rare & All & MMPA & 5,6 \\
\hline $\begin{array}{l}\text { Bottlenose } \\
\text { Dolphin (offshore) }\end{array}$ & Tursiops truncatus & Offshore & Rare & All & MMPA & 5,6 \\
\hline $\begin{array}{l}\text { Short-finned Pilot } \\
\text { Whale }\end{array}$ & $\begin{array}{l}\text { Globicephala } \\
\text { macrorhynchus }\end{array}$ & Offshore & Rare & All & MMPA & 5,6 \\
\hline $\begin{array}{l}\text { Baird's Beaked } \\
\text { Whale }\end{array}$ & Berardius bairdii & Offshore & Rare & All & MMPA & 5,6 \\
\hline $\begin{array}{l}\text { Cuvier's Beaked } \\
\text { Whale }\end{array}$ & Ziphius cavirostris & Offshore & Rare & All & MMPA & 5,6 \\
\hline
\end{tabular}


Table 1. Cetaceans species that could occur in the Humboldt wave energy project area

\begin{tabular}{|c|c|c|c|c|c|c|}
\hline Common Name & Scientific Name & Habitat & $\begin{array}{l}\text { Temporal } \\
\text { Distribution }\end{array}$ & Life Stage & $\begin{array}{l}\text { Federal/ } \\
\text { State } \\
\text { Status }\end{array}$ & References \\
\hline $\begin{array}{l}\text { Blainville's } \\
\text { Beaked Whale }\end{array}$ & $\begin{array}{l}\text { Mesoplodon } \\
\text { densirostris }\end{array}$ & Offshore & Rare & All & MMPA & 5,6 \\
\hline $\begin{array}{l}\text { Perrin's Beaked } \\
\text { Whale }\end{array}$ & Mesoplodon perrini & Offshore & Rare & All & MMPA & 5,6 \\
\hline $\begin{array}{l}\text { Lesser Beaked } \\
\text { Whale }\end{array}$ & Mesoplodon peruvianus & Offshore & Rare & All & MMPA & 5,6 \\
\hline $\begin{array}{l}\text { Gingko-toothed } \\
\text { Beaked Whale }\end{array}$ & Mesoplodon gingkodens & Offshore & Rare & All & MMPA & 5,6 \\
\hline $\begin{array}{l}\text { Hubbs' Beaked } \\
\text { Whale }\end{array}$ & Mesoplodon carlhubbsi & Offshore & Rare & All & MMPA & 5,6 \\
\hline $\begin{array}{l}\text { Stejneger's Beaked } \\
\text { Whale }\end{array}$ & Mesoplodon stejnergeri & Offshore & Rare & All & MMPA & 5,6 \\
\hline $\begin{array}{l}\text { Pygmy Sperm } \\
\text { Whale }\end{array}$ & Kogia breviceps & Offshore & Rare & All & MMPA & 5,6 \\
\hline $\begin{array}{l}\text { Dwarf Sperm } \\
\text { Whale }\end{array}$ & Kogia sima & Offshore & Rare & All & MMPA & 5,6 \\
\hline
\end{tabular}

MMPA = Marine Mammal Protection Act; FE = Federally endangered

References: ${ }^{1}$ USFWS 1970, ${ }^{2}$ USFWS $1994,{ }^{3}$ NMFS 2008, ${ }^{4}$ NMFS 2005c, ${ }^{5}$ Carretta et al. 2009, ${ }^{6}$ NOAA 2009a

Pinnipeds. Six species of pinnipeds could occur along the Humboldt coast (Table 2). However, harbor seals and Steller sea lions are the only pinnipeds that breed along the Humboldt coast and inhabit the area throughout the year. The other species of pinnipeds breed on beaches along the central and southern California mainland, on central and southern California islands, or on islands off of Alaska, and their presence in the project area would be rare and infrequent. Therefore, harbor seals and Steller sea lions were selected as indicator pinniped species for the Humboldt wave energy project effects analysis. Steller sea lions are listed as federally endangered. Potential impacts of the project on these species include collision and injury with boats; toxicity from oil or chemical spills/releases; noise disturbance from turbines, moorings, boats or construction and decommissioning activities; attraction to the wave energy structures because they aggregate prey or provide haul-out structures; and disorientation from construction lights. If any other pinniped species occur in the project area, the effects of the project are likely similar to effects on harbor seals and Steller sea lions. 
Table 2. Pinniped species that could occur in the Humboldt wave energy project area

\begin{tabular}{|c|c|c|c|c|c|c|}
\hline $\begin{array}{l}\text { Common } \\
\text { Name }\end{array}$ & Scientific Name & Habitat & $\begin{array}{l}\text { Temporal } \\
\text { Distribution }\end{array}$ & Life Stage & $\begin{array}{l}\text { Federal/ } \\
\text { State } \\
\text { Status }\end{array}$ & References \\
\hline $\begin{array}{l}\text { California sea } \\
\text { lion }\end{array}$ & $\begin{array}{l}\text { Zalophus } \\
\text { californianus }\end{array}$ & $\begin{array}{l}\text { Coastal, Cont. } \\
\text { shelf }\end{array}$ & $\begin{array}{l}\text { Fall and Spring } \\
\text { peaks }\end{array}$ & Adult, juvenile & MMPA & 1,2 \\
\hline Harbor seal & Phoca vitulina & $\begin{array}{l}\text { Coastal, Cont. } \\
\text { shelf }\end{array}$ & Year-round & Adult, pup & MMPA & 1,2 \\
\hline $\begin{array}{l}\text { Steller sea } \\
\text { lion }\end{array}$ & Eumetopias jubatus & $\begin{array}{l}\text { Coastal, Cont. } \\
\text { shelf }\end{array}$ & $\begin{array}{l}\text { April-October } \\
\text { Few in winter }\end{array}$ & Adult, pup & $\begin{array}{l}\text { FT, } \\
\text { MMPA }\end{array}$ & $3,4,5$ \\
\hline Elephant seal & $\begin{array}{l}\text { Mirounga } \\
\text { angustirostris }\end{array}$ & $\begin{array}{l}\text { Oceanic, Cont. } \\
\text { slope }\end{array}$ & Year-round & Adult, juvenile & MMPA & 1,2 \\
\hline $\begin{array}{l}\text { Northern fur } \\
\text { seal }\end{array}$ & Callorhinus ursinus & $\begin{array}{l}\text { Oceanic, Cont. } \\
\text { slope }\end{array}$ & Year-round & Adult, juvenile & MMPA & 6,7 \\
\hline $\begin{array}{l}\text { Guadalupe fur } \\
\text { seal }\end{array}$ & $\begin{array}{l}\text { Arctocephalus } \\
\text { townsendi }\end{array}$ & $\begin{array}{l}\text { Oceanic, Cont. } \\
\text { slope }\end{array}$ & Summer, rare & Adult, juvenile & $\begin{array}{l}\text { FT, ST, } \\
\text { MMPA }\end{array}$ & 6,7 \\
\hline
\end{tabular}

MMPA = Marine Mammal Protection Act; FE = Federally endangered; FT = Federally threatened; ST = California State threatened

References: ${ }^{1}$ Carretta et al. 2009, ${ }^{2}$ NOAA 2009b, ${ }^{3}$ NMFS 1990, ${ }^{4}$ NMFS 1993, ${ }^{5}$ Angliss and Allen $2009,{ }^{6}$ NMFS $1988,{ }^{7}$ NMFS 1985

\section{Selection of fish indicators for Humboldt wave energy project}

Fish indicator species were selected for the Humboldt wave energy project effects analysis if they are known to occur in northern California coastal waters, suitable habitat either occurs in the project area or would be created by the project, and aspects of their behavior and/or biology makes it likely that they would be affected by the project. Fish species were also selected if the project area is located within designated Essential Fish Habitat (EFH), and/or if they are designated as federally threatened or endangered and could occur in the project area. The following indicator species groups were selected for the Humboldt wave energy project effects analysis: 1) sharks, skates, and rays (elasmobranchs), 2) flatfishes, 3) rockfishes, 4) pelagic schooling fishes, 5) juvenile salmonids, and 6) adult salmonids.

Sharks, skates and rays - There are eight sharks, skates and ray species that could occur in the project area (Table 3). Other elasmobranchs could be in the vicinity of the project site; however, these eight species were selected as indicators because they are present in significant numbers year-round or seasonally, they have special conservation or management status, their behavioral ecology lends itself to a high probability of interaction with electromagnetic fields associated with the wave energy project, or some combination of these factors. Some other locally abundant species were not included (i.e., blue sharks (Prionace glauca) and salmon sharks (Lamna ditropis), because their behavioral ecology is such that the likelihood of interaction is very low.

Most of these species prey on benthic invertebrates and fishes; however, larger, more open water sharks are frequent visitors or transients and feed on pelagic organisms (Klimley et al. 2001). The wave structures could affect sharks by aggregating fishes, which could then attract sharks to the area. Marine

Environmental Assessment for Siting Wave \& Tidal Energy Projects
D-6

H. T. Harvey \& Associates 24 November 2009 
mammals are also important prey for white sharks (Klimley et al. 2001), and sea lions could haul out on the wave buoys and attract white sharks. These are not necessarily negative effects; however, they could change the community composition of the project area and result in changes in shark behavior. The more concerning potential effect to these species is from electromagnetic fields (EMF) associated with the wave energy project. Sharks, rays, and skates have highly sensitive electroreceptive sense organs for predation, mate detection and, possibly orientation and navigation (Bodznick et al. 2003).

Electromagnetic fields (EMF) could result in disorientation and behavioral changes (Nelson 2008).

Table 3. Elasmobranchs associated with Nearshore, Soft-Bottom Habitat Off Humboldt County, California

\begin{tabular}{|c|c|c|c|c|c|}
\hline Common Name & Species & Life Stage & $\begin{array}{l}\text { Seasonal } \\
\text { Presence }\end{array}$ & $\begin{array}{c}\text { Locally } \\
\text { Abundant }\end{array}$ & Reference \\
\hline Sevengill shark & Notorynchus cepedianus & juvenile \& adult & $?^{*}$ & & $1,2,3,4$ \\
\hline Spiny dogfish & Squalus acanthias & juvenile \& adult & $?$ & $\mathrm{X}$ & 2,3 \\
\hline White shark & Carcharodon carcharias & adult & Aug-Jan & & 2,3 \\
\hline $\begin{array}{l}\text { Brown } \\
\text { smoothhound shark }\end{array}$ & Mustelus henlei & juvenile \& adult & $?$ & $\mathrm{X}$ & $1,2,3,4$ \\
\hline Leopard shark & Triakis semifasciata & adult & All year* & $\mathrm{X}$ & $1,2,3$ \\
\hline Soupfin shark & Galeorhinus zyopterus & adult & $\begin{array}{l}\text { Annual N/S } \\
\text { migrations }\end{array}$ & $?$ & $3,4,5$ \\
\hline Big skate & Raja binoculata & juvenile \& adult & All year & $\mathrm{X}$ & $1,2,6$ \\
\hline Bat ray & Myliobatis californica & juvenile \& adult & All year* & $\mathrm{X}$ & $1,2,3$ \\
\hline
\end{tabular}

* Seasonally abundant in Humboldt Bay. References: ${ }^{1}$ Allen et al. 2006, ${ }^{2}$ Fritzsche and Cavanagh $1995,{ }^{3}$ Love 1996, ${ }^{4}$ Miller and Lea 1976, ${ }^{5}$ Ebert 2001, ${ }^{6}$ Pequegnat et al. 1995

Flatfishes- Species most likely to occur in the project area include sand sole (Psettichthys melanostictus), starry flounder (Platichthys stellatus), English sole (Parophrys vetulus), Pacific sanddab (Citharichthys sordidas), Dover sole (Microstomus pacificus), Pacific halibut (Hippoglossus stenolepis), and Rex sole (Errex zachirus) (Allen 2006, Pequegnat et al. 1990). Most of these flatfishes spawn in the winter, with juveniles settling to demersal habitats in the summer (Brodeur et al. 2004). Flatfishes are associated with the sand/mud bottom habitats that occur within the project area (Allen 2006). They feed on or near the bottom on crustaceans, copepods, polychaetes, squid, octopus and small fishes, and are preyed upon by larger marine fishes, diving birds and marine mammals (Allen 2006, Allen and Leos 2001). Underwater structures associated with the wave energy project could attract new species (i.e., rockfish, sharks) to the project area, which could affect flatfishes through increased predation or other species interactions (Nelson 2008).

Rockfish, lingcod, cabezon- The typical habitat for rockfishes (Sebastes spp.), cabezon (Scorpaenichthys marmoratus), and lingcod (Ophiodon elongatus) in the adult life stage is rocky substrate with some relief, although some species (e.g., lingcod) make extensive use of other habitats, including sand bottom habitats (Allen 2006, Lea et al. 1999, Love et al. 1991). These species are pelagic during their early life history stages (larval through pre-settlement; Allen and Cross 2006), therefore, these species could occur in the project area during their early life history prior to construction of the wave energy project. After construction of the wave energy project, adults of these species could be attracted to the underwater wave energy structures (i.e., anchors, mooring devices, and chains) that become an artificial reef, resulting in changes to species composition and distribution in the project area (Nelson 2008). 
Pelagic schooling fishes- Species that could occur in the project area include small- to medium-sized species that occur in coastal waters, including Pacific sardine (Sardinops sagax), northern anchovy (Engraulis mordax), jack mackerel (Trachurus symmetricus), Pacific mackerel (Scomber japonicus), and true smelts such as eulachon (Thaleichthys pacificus), whitebait smelt (Allosmerus elngates), longfin smelt (Spirinchus thaleichthys), and surf smelt (Hypomesus pretiosus), and night smelt (Spirinchus starksi). Collectively, these species are the largest marine fisheries in California in terms of biomass (California Department of Fish and Game 2008). Populations tend to be very dynamic; for example, sardines tend to be more abundant during "warm" regimes and anchovies more abundant during "cool" regimes (Horn and Stephens Jr. 2006). They are also highly mobile and migratory in coastal waters. Schooling in these species occurs can vary from well-defined compact aggregations to widespread, scattering layers, and some species are in deeper waters during the day and closer to the surface and more scattered at night (Allen and Cross 2006). Because of their mobility and patchy distribution, there may be a low likelihood of effects on these species from the wave energy project. However, some species are known to be attracted to lights; therefore, they could be attracted to bright construction lights, making them more vulnerable to predation. They could also be attracted to underwater or surface structures associated with the project, resulting in increased predation, or other changes in community composition.

Salmonids - Salmonid species that could occur in the project area include coho salmon (Oncorhynchus kisutch), Chinook salmon (O. tshawytscha), steelhead (O. mykiss), chum salmon (O. keta), pink salmon (O. gorbuscha), and cutthroat trout (O. clarki). Pacific salmonids spawn in streams and tributaries along the Pacific coast and occur in northern California ocean waters during their oceanic stage. They migrate to the ocean mostly in spring and early summer, coinciding with the greatest availability of prey, and grow rapidly by feeding on small fishes, crustaceans, and squid. They occur in the epipelagic zone in offshore and coastal nearshore waters, and are more abundant in the subarctic and northern Pacific waters, decreasing in abundance towards subtropic waters. They are known to migrate long distances in oceanic waters, although some species and individuals remain in coastal waters near their natal rivers.

Salmonids as a group were selected as indicator species because there are several Evolutionarily Significant Units (ESU) and Distinct Population Segments (DPS) that are federally listed as threatened or endangered that could occur in the project area (Table 4). The effects of the Humboldt wave energy project would be different for juvenile and adult salmonids and were analyzed separately. Bright construction lights or lights on the wave structures could result in behavioral changes (i.e., attraction or reduced movements) of juvenile salmonids, making them more vulnerable to predation. Changes in community composition around the underwater wave structures (i.e., aggregating fish and fish predators) could make juvenile or adult salmonids more vulnerable to predation. Both juvenile and adult salmonids are not likely to be affected by noise and vibration, seabed disturbance, and oil/chemical releases associated with the project because they are highly motile.

Table 4. Salmonids that could occur in the Humboldt wave energy project area.

\begin{tabular}{llll}
\multicolumn{1}{c}{ Common Name } & Scientific Name & $\begin{array}{c}\text { Federal/ } \\
\text { State Status }\end{array}$ & References \\
\hline Central California Coast coho salmon ESU & Oncorhynchus kisutch & FE, ST & NMFS 2005b \\
$\begin{array}{l}\text { S. OR/N. CA Coast Coho salmon ESU } \\
\begin{array}{l}\text { Sacramento River winter-run Chinook } \\
\text { salmon ESU }\end{array}\end{array}$ & Oncorhynchus kisutch & FT, ST & $\begin{array}{l}\text { NMFS 1997, } \\
1999\end{array}$ \\
\end{tabular}


Appendix D - Effects of Small Commercial OPT Power Buoy Project Humboldt

Table 4. Salmonids that could occur in the Humboldt wave energy project area.

\begin{tabular}{llll}
\hline \multicolumn{1}{c}{ Common Name } & \multicolumn{1}{c}{ Scientific Name } & \multicolumn{1}{c}{$\begin{array}{c}\text { Federal/ } \\
\text { State Status }\end{array}$} & \multicolumn{1}{c}{ References } \\
\hline $\begin{array}{l}\text { California Coast Chinook salmon ESU } \\
\text { Central Valley spring-run Chinook salmon } \\
\text { ESU }\end{array}$ & Oncorhynchus tshawytscha & FT & NMFS 2005b \\
Northern CA Steelhead DPS & Oncorhynchus tshawytscha & FT, ST & NMFS 2005b \\
Central Valley steelhead DPS & Oncorhynchus mykiss & FT & $\begin{array}{l}\text { NMFS 2000, } \\
\text { Central California Coast steelhead DPS }\end{array}$ \\
\hline
\end{tabular}

$\mathrm{FE}=$ federally endangered; FT= federally threatened; SE= California State endangered; ST=California State threatened

Green Sturgeon- Green sturgeon (Acipenser medirostris) spend the majority of their lives in coastal marine waters, coastal bays, and estuaries along the Pacific coast, and Humboldt Bay provides habitat for large numbers of adult and subadult green sturgeon. Juveniles inhabit bays and estuaries for 1 to 4 years before traveling to the ocean. They spend about 15 years at sea before returning to spawn in their natal habitat, and spawn every two to four years thereafter (Moyle 2002). They spend summers in coastal waters up to $110 \mathrm{~m}$ deep along California, Oregon, and Washington, migrate north in the fall to as far as southeast Alaska, and then return in the spring (Erickson and Hightower 2007, Lindley et al. 2008). They occur on the bottom and feed on benthic invertebrates and small fishes.

There are two DPSs of the North American green sturgeon that could occur in the project area, the Northern DPS and Southern DPS. The Northern and Southern DPS populations are distinguished only by their spawning locations; otherwise they are identical and their ranges overlap (Adams et al. 2002, NMFS 2005d, USFWS 2006, USFWS 2009). The Northern DPS breeds north of the Eel River and is not listed as threatened or endangered, and the Southern DPS breeds only in the Sacramento River and is listed as federally threatened (USFWS 2006). Critical habitat for the Southern DPS was proposed in 2008, and all of Humboldt Bay, its coastal estuaries and river mouths, and coastal waters along the Pacific coast outside of Humboldt Bay are included in proposed critical habitat (USFWS 2008). Sturgeon have highly sensitive electroreceptive sense organs for predation, mate detection and, orientation and navigation (Bodznick et al. 2003). Electromagnetic fields (EMF) could result in disorientation and behavioral changes (Nelson 2008). Sturgeon could also be attracted to the wave energy structures if they aggregate prey, and they could be impacted by seabed disturbance because they occur in benthic habitats. Sturgeons are not likely to be affected by noise and vibration and oil/chemical releases associated with the project because they are highly migratory and motile.

\section{Selection of marine bird indicators for Humboldt wave energy project}

Marine bird indicators were selected for the Humboldt wave energy project effects analysis by determining the foraging mode, foraging habitat and diurnal rhythms of all marine bird species that could occur in the project area (Table 5). This information was used to evaluate whether the species could be affected by the project. Of the below groups of marine birds, the following species or species groups were selected as indicators for the Humboldt wave energy project effects analysis: 1) diving ducks, loons and

Environmental Assessment for Siting Wave \& Tidal Energy Projects
D-9

H. T. Harvey \& Associates 24 November 2009 
grebes; 2) shearwaters and storm-petrels; 3) pelicans and gulls; 4) cormorants; and 5) alcids. These species were selected because they fit one or more of the following criteria: 1) marine birds that exhibit nocturnal behaviors and are known to be attracted to or disoriented by lights, 2) marine birds that are known to roost on buoys or other at-sea structures, and 3) diving marine birds that could strike underwater structures. All surface-feeding and plunge-diving diurnal marine birds were excluded as indicators, unless they exhibit nocturnal behaviors or are known to roost on at-sea structures. Occurrence of the selected marine bird indicators in the project area was used for effects analysis (Table 6).

Table 5. Marine birds that are known to, or are likely to, regularly occur in the project area and the area between the Humboldt project site and shore.

\begin{tabular}{|c|c|c|c|c|c|c|}
\hline Group & Common name & Scientific name & $\begin{array}{l}\text { Foraging } \\
\text { mode }^{1}\end{array}$ & $\begin{array}{l}\text { Foraging } \\
\text { habitat }^{2}\end{array}$ & $\begin{array}{l}\text { Diurnal } \\
\text { rhythm }^{3}\end{array}$ & $\begin{array}{l}\text { Federal } \\
\text { /state } \\
\text { status }\end{array}$ \\
\hline \multirow{15}{*}{$\begin{array}{l}\text { Ducks, } \\
\text { geese, swans }\end{array}$} & Brant & Branta bernicla & $\mathrm{S}$ & $\mathrm{N}$ & $\mathrm{D}$ & None \\
\hline & Canada goose & Branta canadensis & $\mathrm{S}$ & $\mathrm{N}$ & $\mathrm{D}$ & None \\
\hline & Snow goose & Chen caerulescens & $\mathrm{S}$ & $\mathrm{N}$ & $\mathrm{D}$ & None \\
\hline & Cackling goose & Branta hutchinsii & $\mathrm{S}$ & $\mathrm{N}$ & $\mathrm{D}$ & None \\
\hline & Greater scaup & Aythya marila & $\mathrm{D}$ & $\mathrm{N}$ & $\mathrm{D}$ & None \\
\hline & Lesser scaup & Aythya affinis & $\mathrm{D}$ & $\mathrm{N}$ & $\mathrm{D}$ & None \\
\hline & Harlequin duck & Histrionicus histrionicus & $\mathrm{D}$ & $\mathrm{N}$ & $\mathrm{D}$ & None \\
\hline & Surf scoter & Melanitta perspicillata & $\mathrm{D}$ & $\mathrm{N}$ & $\mathrm{D}$ & None \\
\hline & White-winged scoter & Melanitta fusca & $\mathrm{D}$ & $\mathrm{N}$ & $\mathrm{D}$ & None \\
\hline & Black scoter & Melanitta nigra & $\mathrm{D}$ & $\mathrm{N}$ & $\mathrm{D}$ & None \\
\hline & Long-tailed duck & Clangula hyemalis & $\mathrm{D}$ & $\mathrm{N}$ & $\mathrm{D}$ & None \\
\hline & Bufflehead & Bucephala albeola & $\mathrm{D}$ & $\mathrm{N}$ & $\mathrm{D}$ & None \\
\hline & Common goldeneye & Bucephala clangula & $\mathrm{D}$ & $\mathrm{N}$ & $\mathrm{D}$ & None \\
\hline & $\begin{array}{l}\text { Red-breasted } \\
\text { merganser }\end{array}$ & Mergus serrator & $\mathrm{D}$ & $\mathrm{N}$ & $\mathrm{D}$ & None \\
\hline & Ruddy duck & Oxyura jamaicensis & $\mathrm{D}$ & $\mathrm{N}$ & $\mathrm{D}$ & None \\
\hline \multirow{8}{*}{$\begin{array}{l}\text { Loons and } \\
\text { grebes }\end{array}$} & Red-throated loon & Gavia stellata & $\mathrm{D}$ & $\mathrm{N}$ & $\mathrm{D}$ & None \\
\hline & Pacific loon & Gavia pacifica & $\mathrm{D}$ & $\mathrm{N}$ & $\mathrm{D}$ & None \\
\hline & Common loon & Gavia immer & $\mathrm{D}$ & $\mathrm{N}$ & $\mathrm{D}$ & None \\
\hline & Horned grebe & Podiceps auritus & $\mathrm{D}$ & $\mathrm{N}$ & $\mathrm{D}$ & None \\
\hline & Red-necked grebe & Podiceps grisegena & $\mathrm{D}$ & $\mathrm{N}$ & $\mathrm{D}$ & None \\
\hline & Eared grebe & Podiceps nigricollis & $\mathrm{D}$ & $\mathrm{N}$ & $\mathrm{D}$ & None \\
\hline & Western grebe & Aechmophorus occidentalis & $\mathrm{D}$ & $\mathrm{N}$ & $\mathrm{D}$ & None \\
\hline & Clark's grebe & Aechmophorus clarkii & $\mathrm{D}$ & $\mathrm{N}$ & $\mathrm{D}$ & None \\
\hline \multirow[t]{2}{*}{ Albatrosses } & Laysan albatross & Phoebastria immutabilis & $\mathrm{S}$ & $\mathrm{P}$ & $\mathrm{D}, \mathrm{N}$ & None \\
\hline & $\begin{array}{l}\text { Black-footed } \\
\text { albatross }\end{array}$ & Phoebastria nigripes & S & $\mathrm{P}$ & $\mathrm{D}, \mathrm{N}$ & None \\
\hline \multirow[t]{6}{*}{ Shearwaters } & Northern fulmar & Fulmarus glacialis & $\mathrm{S}$ & $\mathrm{P}$ & $\mathrm{D}, \mathrm{N}$ & None \\
\hline & $\begin{array}{l}\text { Pink-footed } \\
\text { shearwater }\end{array}$ & Puffinus creatopus & $\mathrm{S}$ & $\mathrm{P}$ & $\mathrm{D}, \mathrm{N}$ & None \\
\hline & $\begin{array}{l}\text { Flesh-footed } \\
\text { shearwater }\end{array}$ & Puffinus carneipes & $\mathrm{S}$ & $\mathrm{P}$ & $\mathrm{D}, \mathrm{N}$ & None \\
\hline & Buller's shearwater & Puffinus bulleri & $\mathrm{S}$ & $\mathrm{P}$ & $\mathrm{D}, \mathrm{N}$ & None \\
\hline & Sooty shearwater & Puffinus griseus & $\mathrm{S}$ & $\mathrm{P}$ & $\mathrm{D}, \mathrm{N}$ & None \\
\hline & $\begin{array}{l}\text { Short-tailed } \\
\text { shearwater }\end{array}$ & Puffinus tenuirostris & $\mathrm{S}$ & $\mathrm{P}$ & $\mathrm{D}, \mathrm{N}$ & None \\
\hline \multirow[t]{2}{*}{$\begin{array}{l}\text { Storm- } \\
\text { petrels }\end{array}$} & $\begin{array}{l}\text { Fork-tailed storm- } \\
\text { petrel }\end{array}$ & Oceanodroma furcata & $\mathrm{S}$ & $P$ & $\mathrm{D}, \mathrm{N}$ & None \\
\hline & Leach’s storm-petrel & Oceanodroma leucorhoa & $\mathrm{S}$ & $\mathrm{P}$ & $\mathrm{D}, \mathrm{N}$ & None \\
\hline
\end{tabular}


Appendix D - Effects of Small Commercial OPT Power Buoy Project Humboldt

\begin{tabular}{|c|c|c|c|c|c|c|}
\hline & Ashy storm-petrel & Oceanodroma homochroa & $\mathrm{S}$ & $\mathrm{P}$ & $\mathrm{D}, \mathrm{N}$ & None \\
\hline & Black storm-petrel & Oceanodroma melania & $\mathrm{S}$ & $\mathrm{P}$ & $\mathrm{D}, \mathrm{N}$ & None \\
\hline Pelicans & Brown pelican & Pelecanus occidentalis & $\mathrm{P}$ & $\mathrm{N}$ & $\mathrm{D}$ & FE \\
\hline \multirow[t]{3}{*}{ Cormorants } & Brandt's cormorant & Phalacrocorax penicillatus & $\mathrm{D}$ & $\mathrm{N}$ & $\mathrm{D}$ & None \\
\hline & $\begin{array}{l}\text { Double-crested } \\
\text { cormorant }\end{array}$ & Phalacrocorax auritus & $\mathrm{D}$ & $\mathrm{N}$ & $\mathrm{D}$ & None \\
\hline & Pelagic cormorant & Phalacrocorax pelagicus & $\mathrm{D}$ & $\mathrm{N}$ & $\mathrm{D}$ & None \\
\hline \multirow[t]{2}{*}{ Birds of prey } & Osprey & Pandion haliaetus & $\mathrm{S}$ & $\mathrm{N}$ & $\mathrm{D}$ & None \\
\hline & Peregrine falcon & Falco peregrinus & $\mathrm{S}$ & $\mathrm{N}$ & $\mathrm{D}$ & SE \\
\hline \multirow[t]{2}{*}{ Phalaropes } & $\begin{array}{l}\text { Red-necked } \\
\text { phalarope }\end{array}$ & Phalaropus lobatus & S & $\mathrm{P}$ & $\mathrm{D}, \mathrm{N}$ & None \\
\hline & Red phalarope & Phalaropus fulicarius & $\mathrm{S}$ & $\mathrm{P}$ & $\mathrm{D}, \mathrm{N}$ & None \\
\hline \multirow[t]{12}{*}{ Gulls } & $\begin{array}{l}\text { Black-legged } \\
\text { kittiwake }\end{array}$ & Rissa tridactyla & S, P & $\mathrm{N}$ & $\mathrm{D}$ & None \\
\hline & Sabine’s gull & Xema sabini & $\mathrm{S}, \mathrm{P}$ & $\mathrm{N}$ & $\mathrm{D}$ & None \\
\hline & Bonaparte's gull & Larus philadephia & S, P & $\mathrm{N}$ & $\mathrm{D}$ & None \\
\hline & Heerman's gull & Larus heermanni & $\mathrm{S}, \mathrm{P}$ & $\mathrm{N}$ & $\mathrm{D}$ & None \\
\hline & Mew gull & Larus canus & S, P & $\mathrm{N}$ & $\mathrm{D}$ & None \\
\hline & Ring-billed gull & Larus delawarensis & $\mathrm{S}, \mathrm{P}$ & $\mathrm{N}$ & $\mathrm{D}$ & None \\
\hline & California gull & Larus californicus & S, P & $\mathrm{N}$ & $\mathrm{D}$ & None \\
\hline & Herring gull & Larus argentatus & S, P & $\mathrm{N}$ & $\mathrm{D}$ & None \\
\hline & Thayer's gull & Larus thayeri & S, P & $\mathrm{N}$ & $\mathrm{D}$ & None \\
\hline & Western gull & Larus occidentalis & S, P & $\mathrm{N}$ & $\mathrm{D}$ & None \\
\hline & $\begin{array}{l}\text { Glaucous-winged } \\
\text { gull }\end{array}$ & Larus glaucescens & S, P & $\mathrm{N}$ & $\mathrm{D}$ & None \\
\hline & Glaucous gull & Larus hyperboreus & S, P & $\mathrm{N}$ & $\mathrm{D}$ & None \\
\hline \multirow[t]{5}{*}{ Terns } & Caspian tern & Hydroprogne caspia & $\mathrm{P}$ & $\mathrm{N}$ & $\mathrm{D}$ & None \\
\hline & Common tern & Sterna hirundo & $\mathrm{P}$ & $\mathrm{N}$ & $\mathrm{D}$ & None \\
\hline & Arctic tern & Sterna paradisaea & $\mathrm{P}$ & $\mathrm{N}$ & $\mathrm{D}$ & None \\
\hline & Forster's tern & Sterna forsteri & $\mathrm{P}$ & $\mathrm{N}$ & $\mathrm{D}$ & None \\
\hline & Elegant tern & Thalasseus elegans & $P$ & $\mathrm{~N}$ & $\mathrm{D}$ & None \\
\hline \multirow[t]{4}{*}{ Jaegers } & South polar skua & Stercorarius maccormicki & S & $\mathrm{N}$ & $\mathrm{D}$ & None \\
\hline & Pomarine jaeger & Stercorarius pomarinus & $\mathrm{S}$ & $\mathrm{N}$ & $\mathrm{D}$ & None \\
\hline & Parasitic jaeger & Stercorarius parasiticus & $\mathrm{S}$ & $\mathrm{N}$ & $\mathrm{D}$ & None \\
\hline & Long-tailed jaeger & Stercorarius longicaudus & $\mathrm{S}$ & $\mathrm{N}$ & $\mathrm{D}$ & None \\
\hline \multirow[t]{10}{*}{ Alcids } & Common murre & Uria aalge & $\mathrm{D}$ & $\mathrm{P}$ & $\mathrm{D}$ & None \\
\hline & Thick-billed murre & Uria lomvia & $\mathrm{D}$ & $\mathrm{P}$ & $\mathrm{D}$ & None \\
\hline & Pigeon guillemot & Cepphus columba & $\mathrm{D}$ & $\mathrm{P}$ & $\mathrm{D}$ & None \\
\hline & Marbled murrelet & Brachyramphus marmoratus & $\mathrm{D}$ & $\mathrm{P}$ & $\mathrm{D}, \mathrm{N}$ & FT, SE \\
\hline & Xantus's murrelet & $\begin{array}{l}\text { Synthliboramphus } \\
\text { hypoleucus }\end{array}$ & $\mathrm{D}$ & $\mathrm{P}$ & $\mathrm{D}, \mathrm{N}$ & FC, ST \\
\hline & Ancient murrelet & Synthliboramphus antiquus & $\mathrm{D}$ & $\mathrm{P}$ & $\mathrm{D}, \mathrm{N}$ & None \\
\hline & Rhinoceros auklet & Cerorhinca monocerata & $\mathrm{D}$ & $\mathrm{P}$ & $\mathrm{D}, \mathrm{N}$ & None \\
\hline & Cassin's auklet & Ptychoramphus aleuticus & $\mathrm{D}$ & $\mathrm{P}$ & $\mathrm{D}, \mathrm{N}$ & None \\
\hline & Horned puffin & Fratercula corniculata & $\mathrm{D}$ & $\mathrm{P}$ & $\mathrm{D}, \mathrm{N}$ & None \\
\hline & Tufted puffin & Fratercula cirrhata & $\mathrm{D}$ & $\mathrm{P}$ & $\mathrm{D}, \mathrm{N}$ & None \\
\hline Kingfishers & Belted kingfisher & Ceryle alcylon & $\mathrm{S}$ & $\mathrm{N}$ & $\mathrm{D}$ & None \\
\hline \multicolumn{7}{|c|}{$\begin{array}{l}{ }^{1} \mathrm{~S}=\text { surface feeder, dabbler; } \mathrm{P}=\text { shallon } \\
{ }^{2} \mathrm{~N}=\text { nearshore; } \mathrm{P}=\text { pelagic } \\
{ }^{3} \mathrm{D}=\text { foraging or return to nests diurnal } \\
{ }^{4} \mathrm{FE}=\text { Federally endangered; } \mathrm{FT}=\text { Fede } \\
\text { California State threatened }\end{array}$} \\
\hline
\end{tabular}

Environmental Assessment for Siting Wave \& Tidal

D-11

H. T. Harvey \& Associates

Energy Projects

24 November 2009 
There are a large number of marine bird species that could occur in the project area, however, not all of them are likely to be affected by the Humboldt wave energy project. The sensitivity of a group of marine birds to the project is dependent on factors such as foraging mode, habitat, and timing of activity (diurnal or nocturnal) and is examined below:

Ducks, geese, and swans - Members of this group are either foot-propelled surface feeders or divers that feed in a variety of nearshore habitats including above tideflats, reefs, eelgrass beds, and kelp forests over a variety of substrates (sand, rock, and mud). They feed on small mollusks, crustaceans, benthic invertebrates, small fish and roe, vegetable matter such as sea lettuce and eelgrass, and some species are known to pry mussels from reefs and ledges (Angell 1982). Surface feeding ducks, and geese and swans are unlikely to be affected by the project. However, diving ducks could be affected by the project if they strike underwater structures while diving, especially if these structures serve as prey habitat and become an attractant to ducks. If present in the project area, they could also be affected by chemical releases or oil spills. Ducks, geese, and swans are not known to roost on at-sea structures, or be attracted to lights at night.

Loons and Grebes — Loons and grebes are wing-propelled pursuit divers that feed on small fishes and crustaceans in a variety of habitats, including in open water or nearshore, and over mudflats, estuaries, reefs, and eelgrass beds (Angell 1982). Loons are known to dive up to $60 \mathrm{~m}$ to capture prey (Angell 1982). Diving depths for grebes are not well-known; however, one study found that great crested grebes in the Netherlands dove up to $10 \mathrm{~m}$ (Wiersma et al. 1995). Loons and grebes could be affected by the project if they strike underwater structures while diving, especially if these structures aggregate prey and become an attractant to loons and grebes. If present in the project area, they could also be affected by chemical releases or oil spills. Loons and grebes are not known to roost on at-sea structures, or be attracted to lights at night.

Albatrosses - Members of this group are nocturnal and diurnal surface-feeders and feed on shrimp, squid, fish, crustaceans, and zooplankton that can be picked off the surface of the water. Many species follow fishing boats and scavenge ship offal (USFWS 2005). Albatrosses are unlikely to be affected by the project because they are highly pelagic and would not be expected to occur in the project area (Harris 2006).

Shearwaters - Members of this group are nocturnal and diurnal surface-feeders and feed on shrimp, squid, fish, crustaceans, and zooplankton that can be picked off the surface of the water. Many species follow fishing boats and scavenge ship offal (USFWS 2005). They are known to be attracted by lights and could become disoriented and/or vulnerable to predation by construction lights or by lights on the wave energy structures. If present in the project area, they could also be affected by chemical releases or oil spills. Shearwaters are not known to roost on at-sea structures.

Storm-petrels - Storm-petrel are surface-feeders and feed on crustaceans, squid, jellyfish, zooplankton, fish, and animal detritus picked off the surface of the water. They often feed near ocean fronts, tide-rips, eddies, and large floating objects where prey is available near the water's surface. They are generally diurnal feeders, although some species also forage at night (USFWS 2005). Storm-petrels are known to be attracted by lights and could become disoriented and/or vulnerable to predation by construction lights or by lights on the wave energy structures. If present in the project area, they could also be affected by chemical releases or oil spills. Storm-petrels are not known to roost on at-sea structures.

Pelicans - Pelicans feed by plunge-diving on small schooling fishes (USFWS 2005). Pelicans are known to roost on at-sea structures and could roost on the wave energy structures. If present in the project area, 
they could also be affected by chemical releases or oil spills. They are not known to be attracted to lights at night.

Cormorants - Cormorants are foot-propelled pursuit divers known to dive up to $100 \mathrm{~m}$ to capture prey. They feed on small fish and crustaceans at or near the bottom in nearshore waters and in intertidal waters over rocky substrates (USFWS 2005). Cormorants could be affected by the project if they strike underwater structures while diving, especially if these structures aggregate prey and become an attractant to cormorants. If present in the project area, they could also be affected by chemical releases or oil spills. Cormorants are also known to roost on at-sea structures and could roost on the wave energy structures. They are not known to be attracted to lights at night.

Birds of prey - Birds of prey feed on birds, small mammals, and fish, capturing prey items from the surface of the water, or from the ground with their talons (Poole et al. 2002, White et al. 2002). They are unlikely to be affected by the project because they are not known to roost on at-sea structures, are not known to be attracted to lights at night, and do not dive under the water's surface for prey.

Phalaropes - Phalaropes are pelagic surface-feeders, and forage by picking prey items off the surface of the water. They feed on aquatic insects, crustaceans, and zooplankton, often at upwelling and convergence zones where prey is available near the surface of the water (Colwell and Jehl Jr. 1994, Rubega et al. 2000). When swimming, they will spin in tight circles and create upwellings of food, and pick up small bits of food from the water's surface. They are generally diurnal feeders, although they are also known to forage at night (Colwell and Jehl Jr. 1994, Rubega et al. 2000). They are unlikely to be affected by the project because they do not roost on at-sea structures, are not known to be attracted to lights at night, and do not dive under the water's surface for prey.

Small gulls - Members of this group include kittiwakes and small gulls such as Bonaparte's gulls. They often feed in mixed flocks with other seabird species in offshore waters. They feed opportunistically on small schooling fishes, euphausids, amphipods, and insects using a variety of foraging methods including plunge-diving, surface-dipping, surface-seizing, and jump-plunging (Burger and Gochfeld 2002, Hatch et al. 2009). They are unlikely to be affected by the project because they do not roost on at-sea structures, are not known to be attracted to lights at night, and do not dive under the water's surface for prey.

Gulls - Gulls are generalist predators and scavengers, feeding on pelagic and intertidal marine invertebrates and fish, eggs, chicks, and adults of other seabirds, and human refuse, carrion, and ship offal. They join multi-species feeding aggregations and use a variety of foraging methods including surface-dipping, shallow plunge-diving, surface-seizing, walking or swimming along shore at low tide, or by kleptoparasitism (Hayward and Verbeek 2008, Pierotti and Annett 1995). Gulls could be affected by the project because they are known to roost on at-sea structures and could roost on the wave energy structures. If present in the project area, they could also be affected by chemical releases or oil spills. They are not known to be attracted to lights at night.

Terns - Terns generally feed close to shore or close to their island nesting colonies, mostly on small fishes, although arthropods, crustaceans, and insects are sometimes taken (Cuthbert and Wires 1999, Mcnicholl et al. 2001, Nisbet 2002). They capture prey by plunge-diving but will also steal prey items from conspecifics. They are unlikely to be affected by the project because they do not roost on at-sea structures, are not known to be attracted to lights at night, and do not dive under the water's surface for prey.

Jaegers - Jaegers feed on birds, eggs, rodents, insects, and berries, and also often forage by chasing other seabirds and forcing them to drop their prey. They are unlikely to be affected by the project because they 
do not roost on at-sea structures, are not known to be attracted to lights at night, and do not dive under the water's surface for prey.

Alcids - Alcids are wing-propelled pursuit divers that feed on small schooling fishes, crustaceans, squid, zooplankton, and other benthic and pelagic invertebrates in a variety of nearshore and offshore habitats. Diving depths vary widely by species; common murres dive up to $180 \mathrm{~m}$, other smaller alcids have maximum dive depths of 30-60 m (USFWS 2005). Alcids could be affected by the project if they strike underwater structures while diving, especially if these structures serve as prey habitat and become an attractant to alcids. If present in the project area, they could also be affected by chemical releases or oil spills. Some alcids are also known to be attracted by lights and could become disoriented and/or vulnerable to predation by construction lights or by lights on the wave energy structures. Alcids are not known to roost on at-sea structures. The marbled murrelet is listed as federally threatened; this alcid species could be affected by the project and effects were analyzed separately.

Kingfishers - Kingfishers feed on small fish, crayfish, frogs, and tadpoles. They will hover or perch over open water to locate prey, and then take prey items near the surface of the water. They are unlikely to be affected by the project because they do not roost on at-sea structures, are not known to be attracted to lights at night, and do not dive under the water's surface for prey. 
Appendix D - Effects of Small Commercial OPT Power Buoy Project Humboldt

Table 6. Occurrence of marine birds that may be affected by the Humboldt wave energy project.

\begin{tabular}{|c|c|c|c|c|c|c|c|c|c|c|c|c|c|c|c|}
\hline \multirow[b]{2}{*}{ Group } & \multirow[b]{2}{*}{ Common name } & \multirow[b]{2}{*}{ Foraging mode } & \multirow[b]{2}{*}{ Diurnal rhythm } & \multicolumn{12}{|c|}{ Temporal distribution } \\
\hline & & & & Jan & Feb & Mar & April & May & June & July & Aug & Sept & Oct & Nov & Dec \\
\hline \multirow[t]{10}{*}{ Diving ducks } & Greater scaup & $\mathrm{D}$ & $\mathrm{D}$ & & & & & & & & & & & & \\
\hline & Harlequin duck & $\mathrm{D}$ & $\mathrm{D}$ & & - & & & & & & & & & & \\
\hline & Surf scoter & $\mathrm{D}$ & $\mathrm{D}$ & & & & & & & & & & & & \\
\hline & White-winged scoter & $\mathrm{D}$ & $\mathrm{D}$ & & & & & & & & & & & & \\
\hline & Black scoter & $\mathrm{D}$ & $\mathrm{D}$ & & & & & & & & & & & & \\
\hline & Long-tailed duck & $\mathrm{D}$ & $\mathrm{D}$ & & & & & & & & & & & & \\
\hline & Bufflehead & $\mathrm{D}$ & $\mathrm{D}$ & - & - & & & & & & & & & - & \\
\hline & Common goldeneye & $\mathrm{D}$ & $\mathrm{D}$ & & & & & & & & & & & & \\
\hline & Red-breasted merganser & $\mathrm{D}$ & $\mathrm{D}$ & & & & & & & & & & & & \\
\hline & Ruddy duck & $\mathrm{D}$ & $\mathrm{D}$ & & & & & & & & & & & & \\
\hline \multirow{8}{*}{$\begin{array}{l}\text { Loons and } \\
\text { grebes }\end{array}$} & Red-throated loon & $\mathrm{D}$ & $\mathrm{D}$ & & & & & & & & & & & & \\
\hline & Pacific loon & $\mathrm{D}$ & $\mathrm{D}$ & & & & & & & & & & & & \\
\hline & Common loon & $\mathrm{D}$ & $\mathrm{D}$ & & & & & & & - & & & & & \\
\hline & Horned grebe & $\mathrm{D}$ & $\mathrm{D}$ & & & & & & & & & & & & \\
\hline & Red-necked grebe & $\mathrm{D}$ & $\mathrm{D}$ & & & & & & & & & & & & \\
\hline & Eared grebe & $\mathrm{D}$ & $\mathrm{D}$ & & & & & & & & & & & & \\
\hline & Western grebe & $\mathrm{D}$ & $\mathrm{D}$ & & & & & & & & & & & & \\
\hline & Clark’s grebe & $\mathrm{D}$ & $\mathrm{D}$ & & & & & & & & & & & & \\
\hline \multirow[t]{4}{*}{ Shearwaters } & Northern fulmar & $\mathrm{P}$ & $\mathrm{D}, \mathrm{N}$ & & & & & & & & & & & & \\
\hline & Pink-footed shearwater & $\mathrm{P}$ & $\mathrm{D}, \mathrm{N}$ & & & & & & & & & & & & \\
\hline & Sooty shearwater & $\mathrm{P}$ & $\mathrm{D}, \mathrm{N}$ & & & & F & & & & - & - & & & \\
\hline & Short-tailed shearwater & $\mathrm{P}$ & $\mathrm{D}, \mathrm{N}$ & & & & & & & & & & & & \\
\hline Storm-petrels & Fork-tailed storm petrel & $\mathrm{S}$ & $\mathrm{D}, \mathrm{N}$ & & & & & & & & & & & & \\
\hline Pelicans & Brown Pelican & $\mathrm{P}$ & $\mathrm{D}$ & & & & & & & & & & & & \\
\hline Cormorants & Brandt's cormorant & $\mathrm{D}$ & $\mathrm{D}$ & & & & & & & & & & & & \\
\hline
\end{tabular}

\begin{tabular}{lrr}
\hline $\begin{array}{l}\text { Environmental Assessment for Siting Wave \& Tidal } \\
\text { Energy Projects }\end{array}$ & D-15 & H. T. Harvey \& Associates \\
24 November 2009
\end{tabular}


Appendix D - Effects of Small Commercial OPT Power Buoy Project Humboldt

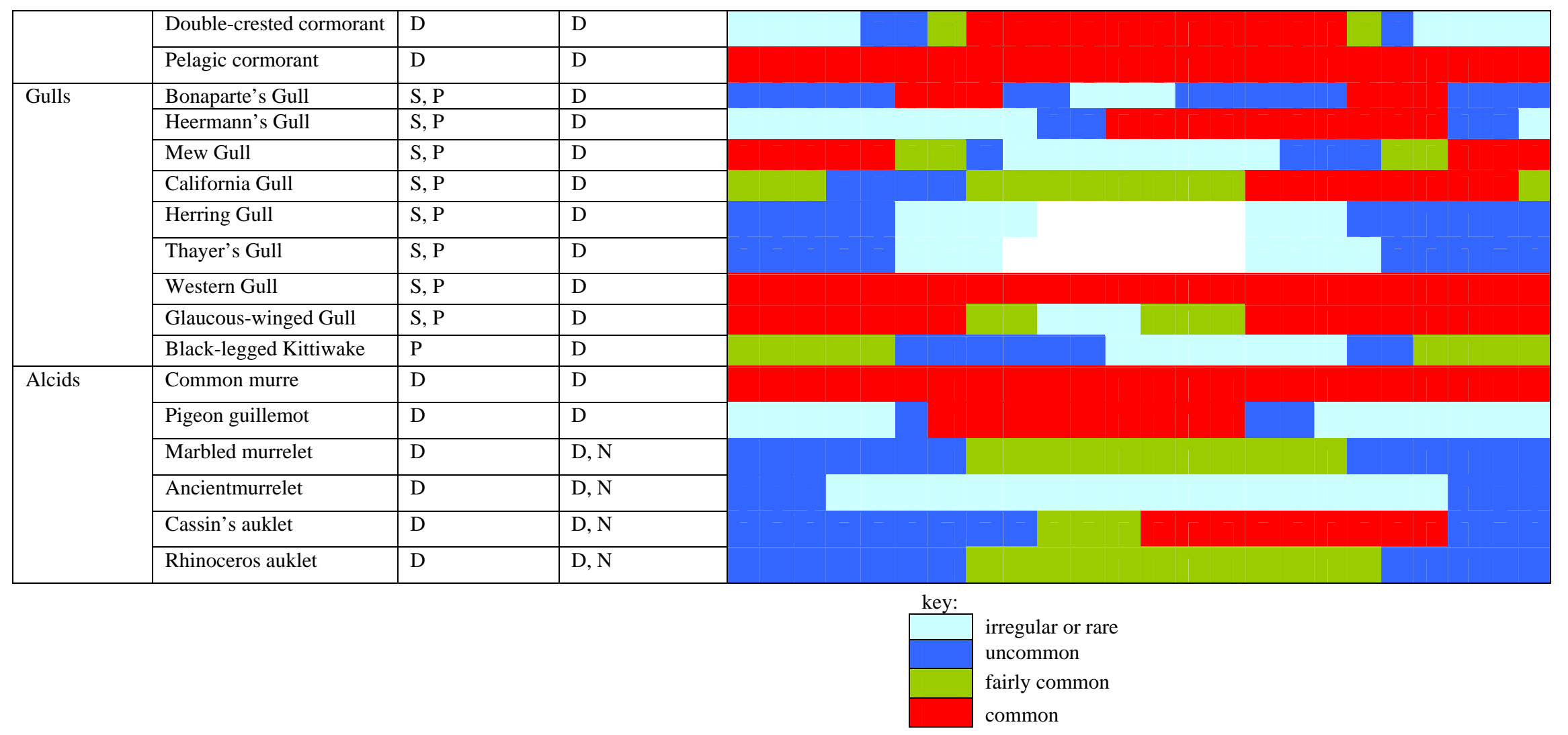

\begin{tabular}{lrr}
\hline Environmental Assessment for Siting Wave \& Tidal & D-16 & H. T. Harvey \& Associates \\
Energy Projects & & 24 November 2009
\end{tabular}


Potential effects on acoustic environment due to Small Commercial OPT Power Buoy Project at Humboldt site

\begin{tabular}{|c|c|c|c|c|c|c|}
\hline Project activity & $\begin{array}{l}\text { Project } \\
\text { action }\end{array}$ & $\begin{array}{l}\text { Description of } \\
\text { action's effect on site } \\
\text { physical attribute }\end{array}$ & $\begin{array}{l}\text { Spatial exposure of } \\
\text { attribute (low, med, high) }\end{array}$ & $\begin{array}{l}\text { Temporal exposure of } \\
\text { attribute (low, med, } \\
\text { high) }\end{array}$ & $\begin{array}{l}\text { Overall risk to } \\
\text { attribute (low, med, } \\
\text { high or unknown) }\end{array}$ & Source(s) \\
\hline \multicolumn{7}{|c|}{ Construction } \\
\hline Boat traffic & $\begin{array}{l}\text { Noise and } \\
\text { vibration }\end{array}$ & $\begin{array}{l}\text { Propellers cavitate, } \\
\text { causing pressure } \\
\text { differences }\end{array}$ & $\begin{array}{l}\text { High, modeled noise of } 120 \\
\mathrm{~dB} \text { extended approx } 20 \mathrm{~km} \\
\text { in ocean wind turbine } \\
\text { project }\end{array}$ & $\begin{array}{l}\text { Low, vessel traffic will } \\
\text { occur over } 1 \text { to } 2 \text { years } \\
\text { during the summer }\end{array}$ & $\begin{array}{l}\text { Med, noise could be } \\
\text { elevated but would be } \\
\text { short-term }\end{array}$ & $\begin{array}{l}\text { Austin et } \\
\text { al. } 2009\end{array}$ \\
\hline $\begin{array}{l}\text { Construction and installation of } \\
\text { electrical collector system, mooring } \\
\text { cables, anchors or footings, devices }\end{array}$ & $\begin{array}{l}\text { Noise and } \\
\text { vibration }\end{array}$ & $\begin{array}{l}\text { Adds to existing } \\
\text { natural and man-made } \\
\text { noise in project area }\end{array}$ & $\begin{array}{l}\text { High, modeled noise of } 120 \\
\mathrm{~dB} \text { extended approx } 20 \mathrm{~km} \\
\text { in ocean wind turbine } \\
\text { project }\end{array}$ & $\begin{array}{l}\text { Low, construction noise } \\
\text { will occur over } 1 \text { to } 2 \\
\text { years during the summer }\end{array}$ & $\begin{array}{l}\text { Med, noise could be } \\
\text { elevated but would be } \\
\text { short-term }\end{array}$ & $\begin{array}{l}\text { Austin et } \\
\text { al. } 2009\end{array}$ \\
\hline $\begin{array}{l}\text { Directional drilling, and laying cable } \\
\text { under/on seabed }\end{array}$ & $\begin{array}{l}\text { Noise and } \\
\text { vibration }\end{array}$ & $\begin{array}{l}\text { Vibration of immediate } \\
\text { area being drilled }\end{array}$ & $\begin{array}{l}\text { Low, vibration could be } \\
\text { localized, assuming similar } \\
\text { to directional drilling on } \\
\text { land }\end{array}$ & $\begin{array}{l}\text { Low, drilling will occur } \\
\text { for 1-2 weeks }\end{array}$ & $\begin{array}{l}\text { Low, effect expected } \\
\text { to be localized and } \\
\text { short-term }\end{array}$ & $\begin{array}{l}\text { CPUC } \\
2009\end{array}$ \\
\hline \multicolumn{7}{|c|}{ Operation and maintenance } \\
\hline Boat traffic & $\begin{array}{l}\text { Noise and } \\
\text { vibration }\end{array}$ & $\begin{array}{l}\text { Propellers cavitate, } \\
\text { causing pressure } \\
\text { differences }\end{array}$ & $\begin{array}{l}\text { High, modeled noise of } 120 \\
\mathrm{~dB} \text { extended approx } 20 \mathrm{~km} \\
\text { in ocean wind turbine } \\
\text { project }\end{array}$ & $\begin{array}{l}\text { Low, vessel traffic } \\
\text { infrequent during O\&M }\end{array}$ & $\begin{array}{l}\text { Low, elevated noise } \\
\text { would occur } \\
\text { infrequently }\end{array}$ & $\begin{array}{l}\text { Austin et } \\
\text { al. } 2009\end{array}$ \\
\hline $\begin{array}{l}\text { Operation of turbines or other moving } \\
\text { parts of devices }\end{array}$ & $\begin{array}{l}\text { Noise and } \\
\text { vibration }\end{array}$ & $\begin{array}{l}\text { Adds to existing } \\
\text { natural and man-made } \\
\text { noise in project area }\end{array}$ & $\begin{array}{l}\text { Unknown levels generated; } \\
\text { site specific attenuation and } \\
\text { ambient noise also } \\
\text { unknown }\end{array}$ & $\begin{array}{l}\text { High, noise would occur } \\
\text { over life of the project }\end{array}$ & Unknown & $\begin{array}{l}\text { Study } \\
\text { warranted }\end{array}$ \\
\hline \multicolumn{7}{|c|}{ Decommissioning } \\
\hline Boat traffic & $\begin{array}{l}\text { Noise and } \\
\text { vibration }\end{array}$ & $\begin{array}{l}\text { Propellers cavitate, } \\
\text { causing pressure } \\
\text { differences }\end{array}$ & $\begin{array}{l}\text { High, modeled noise of } 120 \\
\mathrm{~dB} \text { extended approx } 20 \mathrm{~km} \\
\text { in ocean wind turbine } \\
\text { project }\end{array}$ & $\begin{array}{l}\text { Low, vessel traffic will } \\
\text { occur over } 1 \text { to } 2 \text { years } \\
\text { during the summer }\end{array}$ & $\begin{array}{l}\text { Med, noise could be } \\
\text { elevated but would be } \\
\text { short-term }\end{array}$ & $\begin{array}{l}\text { Austin et } \\
\text { al. } 2009\end{array}$ \\
\hline $\begin{array}{l}\text { Decommissioning of structures on } \\
\text { water's surface or seabed }\end{array}$ & $\begin{array}{l}\text { Noise and } \\
\text { vibration }\end{array}$ & $\begin{array}{l}\text { Adds to existing } \\
\text { natural and man-made } \\
\text { noise in project area }\end{array}$ & $\begin{array}{l}\text { High, modeled noise of } 120 \\
\mathrm{~dB} \text { extended approx } 20 \mathrm{~km} \\
\text { in ocean wind turbine } \\
\text { project }\end{array}$ & $\begin{array}{l}\text { Low, noise will occur } \\
\text { over } 1 \text { to } 2 \text { years during } \\
\text { the summer }\end{array}$ & $\begin{array}{l}\text { Med, noise could be } \\
\text { elevated but would be } \\
\text { short-term }\end{array}$ & $\begin{array}{l}\text { Austin et } \\
\text { al. } 2009\end{array}$ \\
\hline
\end{tabular}

\begin{tabular}{lrr}
\hline Environmental Assessment for Siting Wave \& Tidal & D-17 & H. T. Harvey \& Associates \\
Energy Projects & & 24 November 2009
\end{tabular}


Appendix D - Effects of Small Commercial OPT Power Buoy Project Humboldt

Potential effects on visual environment due to Small Commercial OPT Power Buoy Project at Humboldt site

\begin{tabular}{|c|c|c|c|c|c|c|}
\hline Project activity & Action & $\begin{array}{l}\text { Description of action's } \\
\text { effect on site physical } \\
\text { attribute }\end{array}$ & $\begin{array}{l}\text { Spatial exposure of } \\
\text { attribute (low, med, } \\
\text { high) }\end{array}$ & $\begin{array}{l}\text { Temporal exposure of } \\
\text { attribute (low, med, high) }\end{array}$ & $\begin{array}{l}\text { Overall risk to } \\
\text { attribute (low, med, } \\
\text { high) }\end{array}$ & Source(s) \\
\hline \multicolumn{7}{|c|}{ Construction } \\
\hline Boat traffic & $\begin{array}{l}\text { Navigation } \\
\text { lights }\end{array}$ & $\begin{array}{l}\text { Vessel lights will be visible } \\
\text { along shipping lanes from } \\
\text { Humboldt Bay docks to the } \\
\text { project site }\end{array}$ & $\begin{array}{l}\text { Low, visibility varies } \\
\text { with atmospheric } \\
\text { conditions, will be seen } \\
\text { from docks and shore }\end{array}$ & $\begin{array}{l}\text { Low, construction vessel } \\
\text { traffic will occur over } 1 \text { to } 2 \\
\text { years during the summer }\end{array}$ & $\begin{array}{l}\text { Low, lights visible } \\
\text { when boats in shipping } \\
\text { lanes in and near } \\
\text { Humboldt Bay but } \\
\text { existing traffic } \\
\text { ameliorates effect }\end{array}$ & CSLC 2008 \\
\hline $\begin{array}{l}\text { Construction of electrical } \\
\text { collector system, } \\
\text { moorings and foundations; } \\
\text { device installation }\end{array}$ & $\begin{array}{l}\text { Construction } \\
\text { and deck lights }\end{array}$ & $\begin{array}{l}\text { Construction lights much } \\
\text { brighter than vessel lights, } \\
\text { depending on sea and } \\
\text { weather conditions, could be } \\
\text { visible from shore. }\end{array}$ & $\begin{array}{l}\text { High, construction } \\
\text { lights brighter than } \\
\text { navigation lights and } \\
\text { may be visible } 2 \text { to } 5 \\
\text { nm. }\end{array}$ & $\begin{array}{l}\text { Low, construction will occur } \\
\text { over } 1 \text { to } 2 \text { years during the } \\
\text { summer }\end{array}$ & $\begin{array}{l}\text { Med, lights may be } \\
\text { visible from shore }\end{array}$ & $\begin{array}{l}\text { IALA } \\
2008 \\
\text { NOAA } \\
2007\end{array}$ \\
\hline \multicolumn{7}{|c|}{ Operation and maintenance } \\
\hline Boat traffic & $\begin{array}{l}\text { Navigation } \\
\text { lights }\end{array}$ & $\begin{array}{l}\text { Vessel lights will be visible } \\
\text { along shipping lanes from } \\
\text { Humboldt Bay docks to the } \\
\text { project site }\end{array}$ & $\begin{array}{l}\text { Med, visibility varies } \\
\text { with atmospheric } \\
\text { conditions, will be seen } \\
\text { from docks and shore }\end{array}$ & $\begin{array}{l}\text { Med, construction vessel } \\
\text { traffic will occur over life of } \\
\text { project but at reduced } \\
\text { frequency compared to } \\
\text { construction }\end{array}$ & $\begin{array}{l}\text { Low, lights visible } \\
\text { when boats in shipping } \\
\text { lanes in and near bay } \\
\text { but existing traffic } \\
\text { ameliorates effect }\end{array}$ & CSLC 2008 \\
\hline \multirow{2}{*}{$\begin{array}{l}\text { Structures on water's } \\
\text { surface }\end{array}$} & Structure & $\begin{array}{l}\text { Multiple devices } 8 \mathrm{~m} \text { above } \\
\text { water's surface and } \\
\text { clustered }\end{array}$ & $\begin{array}{l}\text { Low, devices } 3 \mathrm{~nm} \\
\text { from shore and } \\
\text { unlikely to be visible } \\
\text { from shore }\end{array}$ & $\begin{array}{l}\text { High, will be present through } \\
\text { life of project }\end{array}$ & $\begin{array}{l}\text { Med, device profiles } \\
\text { are low and “facilities } \\
\text { will probably have little } \\
\text { visual impact” (NOAA } \\
\text { 2007) }\end{array}$ & $\begin{array}{l}\text { NOAA } \\
2007\end{array}$ \\
\hline & $\begin{array}{l}\text { Navigation } \\
\text { lights }\end{array}$ & $\begin{array}{l}\text { Devices will have } \\
\text { navigational lights visible } \\
\text { from } 2 \text { to } 5 \mathrm{~nm} .\end{array}$ & $\begin{array}{l}\text { Med, device lights will } \\
\text { be visible from } 2 \text { to } 5 \\
\text { nm. }\end{array}$ & $\begin{array}{l}\text { High, device lights will be } \\
\text { required throughout the life } \\
\text { of the project }\end{array}$ & $\begin{array}{l}\text { Low, device lights will } \\
\text { have a low profile and } \\
\text { will be } 3 \mathrm{~nm} \text { from } \\
\text { shore }\end{array}$ & CSLC 2008 \\
\hline \multicolumn{7}{|c|}{ Decommissioning } \\
\hline Boat traffic & $\begin{array}{l}\text { Navigation } \\
\text { lights }\end{array}$ & $\begin{array}{l}\text { Vessel lights will be visible } \\
\text { along shipping lanes from } \\
\text { Humboldt Bay docks to the } \\
\text { project site }\end{array}$ & $\begin{array}{l}\text { Med, visibility varies } \\
\text { with atmospheric } \\
\text { conditions, will be seen } \\
\text { from docks and shore }\end{array}$ & $\begin{array}{l}\text { Low, decommissioning } \\
\text { vessel traffic will occur over } \\
1 \text { to } 2 \text { years during the } \\
\text { summer }\end{array}$ & $\begin{array}{l}\text { Low, lights visible } \\
\text { when boats in shipping } \\
\text { lanes in and near bay } \\
\text { but existing traffic } \\
\text { ameliorates effect }\end{array}$ & CSLC 2008 \\
\hline $\begin{array}{l}\text { Decommissioning of } \\
\text { structures on water's } \\
\text { surface or seabed }\end{array}$ & $\begin{array}{l}\text { Deconstruction } \\
\text { lights }\end{array}$ & $\begin{array}{l}\text { Deconstruction lights much } \\
\text { brighter than vessel lights, } \\
\text { depending on sea and } \\
\text { weather conditions, could be } \\
\text { visible from shore }\end{array}$ & $\begin{array}{l}\text { High, deconstruction } \\
\text { lights brighter than } \\
\text { navigation lights and } \\
\text { may be visible } 2 \text { to } 5 \\
\text { nm. }\end{array}$ & $\begin{array}{l}\text { Low, deconstruction will } \\
\text { occur over } 1 \text { to } 2 \text { years } \\
\text { during the summer }\end{array}$ & $\begin{array}{l}\text { Med, lights may be } \\
\text { visible from shore }\end{array}$ & $\begin{array}{l}\text { IALA } \\
2008 \\
\text { NOAA } \\
2007\end{array}$ \\
\hline
\end{tabular}

\begin{tabular}{lr}
\hline Environmental Assessment for Siting Wave \& Tidal & D-18 \\
Energy Projects &
\end{tabular}


Appendix D - Effects of Small Commercial OPT Power Buoy Project Humboldt

Potential effects on sediment and water chemistry due to Small Commercial OPT Power Buoy Project at Humboldt site

\begin{tabular}{|c|c|c|c|c|c|c|}
\hline Project activity & Project action & $\begin{array}{l}\text { Description of action's } \\
\text { effect on attribute }\end{array}$ & $\begin{array}{l}\text { Spatial exposure of } \\
\text { attribute (low, med, } \\
\text { high) }\end{array}$ & $\begin{array}{l}\text { Temporal exposure of } \\
\text { attribute (low, med, high) }\end{array}$ & $\begin{array}{l}\text { Overall risk to } \\
\text { attribute (low, } \\
\text { med, high) }\end{array}$ & Source(s) \\
\hline \multicolumn{7}{|c|}{$\begin{array}{l}\text { Construction } \\
\end{array}$} \\
\hline Boat traffic & $\begin{array}{l}\text { Oil/chemical release, } \\
\text { assumed seepage from } \\
\text { exhaust and general } \\
\text { use, not a spill from } \\
\text { collision or other } \\
\text { release }\end{array}$ & \multirow{2}{*}{$\begin{array}{l}\text { Could add compounds } \\
\text { that change the physical } \\
\text { and chemical } \\
\text { characteristics of } \\
\text { sediment and water }\end{array}$} & $\begin{array}{l}\text { Low, seepage will } \\
\text { have a small spatial } \\
\text { and areal extent } \\
\text { relative to the project }\end{array}$ & $\begin{array}{l}\text { High, traffic and boat } \\
\text { frequency will be the } \\
\text { highest during construction }\end{array}$ & $\begin{array}{l}\text { Low, given low } \\
\text { spatial extent but } \\
\text { high boat traffic }\end{array}$ & $\begin{array}{l}\text { HBHRCD } \\
2006\end{array}$ \\
\hline $\begin{array}{l}\text { Construction of electrical } \\
\text { collector system, anchors } \\
\text { and foundations; installation } \\
\text { of devices }\end{array}$ & Oil/chemical release & & $\begin{array}{l}\text { Low, seepage will } \\
\text { have a small spatial } \\
\text { and areal extent } \\
\text { relative to the project }\end{array}$ & $\begin{array}{l}\text { Low, construction will be } 1 \\
\text { to } 2 \text { years over the } 15 \text { to } 25 \\
\text { year project life }\end{array}$ & $\begin{array}{l}\text { Low, given low } \\
\text { spatial and } \\
\text { temporal extent }\end{array}$ & $\begin{array}{l}\text { HBHRCD } \\
2006\end{array}$ \\
\hline $\begin{array}{l}\text { Directional drilling, and } \\
\text { laying cable under/on seabed } \\
\text { (assume normal conditions, } \\
\text { not a drilling mud "blow } \\
\text { out” scenario) }\end{array}$ & Seabed disturbance & $\begin{array}{l}\text { Sediment would be } \\
\text { introduced into water } \\
\text { column; deeper } \\
\text { sediments with different } \\
\text { chemistry brought to } \\
\text { seabed surface }\end{array}$ & $\begin{array}{l}\text { Med, cable length } \\
\text { approx } 3 \mathrm{~nm} \text {; project } \\
\text { area approximately } \\
2.9 \mathrm{~km}^{2} \text { or } 1.1 \mathrm{mi}^{2}\end{array}$ & $\begin{array}{l}\text { Low, increased sediment in } \\
\text { water column would mix or } \\
\text { dilute quickly. }\end{array}$ & $\begin{array}{l}\text { Low, due to quick } \\
\text { dilution of } \\
\text { sediment in water } \\
\text { column }\end{array}$ & Previsic 2009 \\
\hline \multicolumn{7}{|c|}{ Operation and Maintenance } \\
\hline Boat traffic & $\begin{array}{l}\text { Oil/chemical release, } \\
\text { assumed seepage from } \\
\text { general use, not a spill } \\
\text { from collision }\end{array}$ & $\begin{array}{l}\text { Could add compounds } \\
\text { that change the physical } \\
\text { and chemical } \\
\text { characteristics of } \\
\text { sediment and water }\end{array}$ & $\begin{array}{l}\text { Low, seepage will } \\
\text { have a small spatial } \\
\text { and areal extent } \\
\text { relative to the project }\end{array}$ & $\begin{array}{l}\text { Low, O\&M vessel traffic } \\
\text { will be much less than } \\
\text { during construction }\end{array}$ & $\begin{array}{l}\text { Low, due to low } \\
\text { seepage spatial } \\
\text { extent, and low } \\
\text { volume of vessel } \\
\text { traffic during } \\
\text { O\&M } \\
\end{array}$ & $\begin{array}{l}\text { HBHRCD } \\
2006\end{array}$ \\
\hline \multirow{2}{*}{$\begin{array}{l}\text { Structures in water column } \\
\text { and on seabed, such as } \\
\text { devices and anchors }\end{array}$} & Structure & $\begin{array}{l}\text { Concrete anchors are } \\
\text { sources of alkaline } \\
\text { elements (sodium, } \\
\text { potassium) that could } \\
\text { leach into water column }\end{array}$ & $\begin{array}{l}\text { Low, effect would } \\
\text { be localized to } \\
\text { vicinity of concrete } \\
\text { anchors }\end{array}$ & $\begin{array}{l}\text { High, any leaching would } \\
\text { occur throughout life of } \\
\text { project }\end{array}$ & $\begin{array}{l}\text { Low, effects would } \\
\text { be diluted and may } \\
\text { not be measurable }\end{array}$ & $\begin{array}{l}\text { Substructure } \\
\text { [date } \\
\text { unknown] }\end{array}$ \\
\hline & $\begin{array}{l}\text { Water circulation } \\
\text { changes affecting } \\
\text { sediment transport }\end{array}$ & $\begin{array}{l}\text { Concrete anchors could } \\
\text { change sediment } \\
\text { transport dynamics }\end{array}$ & $\begin{array}{l}\text { Low, effect would } \\
\text { likely be localized }\end{array}$ & $\begin{array}{l}\text { High, any changes to water } \\
\text { circulation would be } \\
\text { throughout the life of the } \\
\text { project }\end{array}$ & $\begin{array}{l}\text { Low, effect is } \\
\text { localized although } \\
\text { occurring over life } \\
\text { of project }\end{array}$ & $\begin{array}{l}\text { Largier et al. } \\
2008\end{array}$ \\
\hline
\end{tabular}

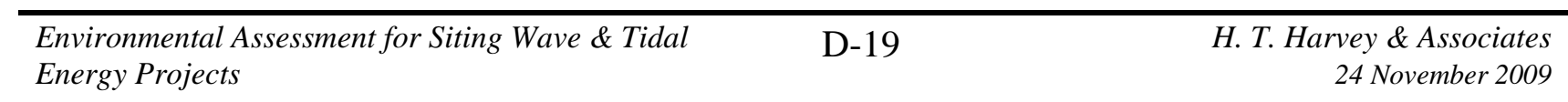


Appendix D - Effects of Small Commercial OPT Power Buoy Project Humboldt

\begin{tabular}{|c|c|c|c|c|c|c|}
\hline Project activity & Project action & $\begin{array}{l}\text { Description of action's } \\
\text { effect on attribute }\end{array}$ & $\begin{array}{l}\text { Spatial exposure of } \\
\text { attribute (low, med, } \\
\text { high) }\end{array}$ & $\begin{array}{l}\text { Temporal exposure of } \\
\text { attribute (low, med, high) }\end{array}$ & $\begin{array}{l}\text { Overall risk to } \\
\text { attribute (low, } \\
\text { med, high) }\end{array}$ & Source(s) \\
\hline \multirow{2}{*}{$\begin{array}{l}\text { Presence of structures on } \\
\text { water's surface }\end{array}$} & \multirow{2}{*}{ Structure } & $\begin{array}{l}\text { Biofouling organisms } \\
\text { slough off on to the } \\
\text { seabed surface }\end{array}$ & $\begin{array}{l}\text { Low, effect localized } \\
\text { to seabed directly } \\
\text { under devices }\end{array}$ & $\begin{array}{l}\text { Low, antifouling paint and } \\
\text { maintenance likely to } \\
\text { remove organisms before } \\
\text { sloughing }\end{array}$ & $\begin{array}{l}\text { Low, effect is } \\
\text { localized to } \\
\text { immediate vicinity } \\
\text { of seabed under } \\
\text { devices }\end{array}$ & MMS 2007 \\
\hline & & $\begin{array}{l}\text { Reduction in wave height } \\
\text { to beaches }\end{array}$ & $\begin{array}{l}\text { Low, project } \\
\text { offshore and small }\end{array}$ & $\begin{array}{l}\text { Medium, effects to beaches } \\
\text { depends on wave size, } \\
\text { larger waves affecting } \\
\text { beach dynamics probably } \\
\text { less affected. }\end{array}$ & $\begin{array}{l}\text { Low, may not be } \\
\text { measurable }\end{array}$ & $\begin{array}{l}\text { Largier et al. } \\
2008\end{array}$ \\
\hline \multicolumn{7}{|c|}{ Decommissioning } \\
\hline Boat traffic & $\begin{array}{l}\text { Oil/chemical release, } \\
\text { assumed seepage from } \\
\text { general use, not a spill } \\
\text { from collision }\end{array}$ & \multirow{2}{*}{$\begin{array}{l}\text { Could add compounds } \\
\text { that change the physical } \\
\text { and chemical } \\
\text { characteristics of } \\
\text { sediment and water }\end{array}$} & \multirow{2}{*}{$\begin{array}{l}\text { Low, seepage will } \\
\text { have a small spatial } \\
\text { and areal extent } \\
\text { relative to the project }\end{array}$} & $\begin{array}{l}\text { High, traffic and boat } \\
\text { frequency will be the high } \\
\text { during decommissioning }\end{array}$ & $\begin{array}{l}\text { Low, given low } \\
\text { spatial extent but } \\
\text { high boat traffic }\end{array}$ & $\begin{array}{l}\text { HBHRCD } \\
2006\end{array}$ \\
\hline \multirow{2}{*}{$\begin{array}{l}\text { Decommissioning of } \\
\text { structures on water's surface } \\
\text { or seabed }\end{array}$} & Oil/chemical release & & & $\begin{array}{l}\text { Low, decommissioning } \\
\text { will be } 1 \text { to } 2 \text { years over the } \\
15 \text { to } 25 \text { year project life }\end{array}$ & $\begin{array}{l}\text { Low, given low } \\
\text { spatial and } \\
\text { temporal extent }\end{array}$ & $\begin{array}{l}\text { HBHRCD } \\
2006\end{array}$ \\
\hline & Seabed disturbance & $\begin{array}{l}\text { Sediment would be } \\
\text { introduced into water } \\
\text { column when removing } \\
\text { anchors and/or subsea } \\
\text { cable }\end{array}$ & $\begin{array}{l}\text { Med, cable length } \\
\text { approx } 3 \mathrm{~nm} \text {; project } \\
\text { area approximately } \\
2.9 \mathrm{~km}^{2} \text { or } 1.1 \mathrm{mi}^{2}\end{array}$ & $\begin{array}{l}\text { Low, increased sediment in } \\
\text { water column would mix or } \\
\text { dilute quickly. }\end{array}$ & $\begin{array}{l}\text { Low, due to quick } \\
\text { dilution of } \\
\text { sediment in water } \\
\text { column }\end{array}$ & Previsic 2009 \\
\hline
\end{tabular}

\begin{tabular}{lr}
\hline Environmental Assessment for Siting Wave \& Tidal & D-20 \\
Energy Projects & H. T. Harvey \& Associates \\
24 November 2009
\end{tabular}


Appendix D - Effects of Small Commercial OPT Power Buoy Project Humboldt

Potential effects on Gray Whales due to Small Commercial OPT Power Buoy Project at Humboldt site

\begin{tabular}{|c|c|c|c|c|c|c|c|}
\hline Project activity & Project action & $\begin{array}{l}\text { Description of } \\
\text { action's effect } \\
\text { on indicator }\end{array}$ & $\begin{array}{l}\text { Spatial exposure to } \\
\text { indicator (low, med, } \\
\text { high) }\end{array}$ & $\begin{array}{l}\text { Temporal exposure } \\
\text { to (low, med, high) }\end{array}$ & $\begin{array}{l}\text { Effect's overlap with } \\
\text { critical life stage, } \\
\text { behavior, habitat, or } \\
\text { resource (low, med, } \\
\text { high) }\end{array}$ & $\begin{array}{l}\text { Overall risk to } \\
\text { indicator (low, med, } \\
\text { high, unknown) }\end{array}$ & Source(s) \\
\hline \multicolumn{8}{|c|}{ Construction } \\
\hline \multirow{3}{*}{ Boat traffic } & Direct impact & $\begin{array}{l}\text { Collision } \\
\text { injuries }\end{array}$ & $\begin{array}{l}\text { High, boat traffic } \\
\text { crosses migration } \\
\text { path along the coast }\end{array}$ & $\begin{array}{l}\text { Low, occurring } \\
\text { during the summer } \\
\text { months for } 1 \text { to } 2 \\
\text { years }\end{array}$ & $\begin{array}{l}\text { Low; most of population } \\
\text { not present during } \\
\text { construction; Med for } \\
\text { Pacific Coast Feeding } \\
\text { Aggregation (PCFA) } \\
\text { whales in region over } \\
\text { summer. }\end{array}$ & $\begin{array}{l}\text { Med-high; could affect } \\
\text { large segment of popn } \\
\text { if overlaps with north } \\
\text { migration; PCFA } \\
\text { whales present; gray } \\
\text { whale-boat collision } \\
\text { not uncommon }\end{array}$ & $\begin{array}{l}\text { Calambokidis } \\
\text { et al. 2002, } \\
\text { Sullivan et al. } \\
\text { 1983, Van } \\
\text { Waerebeek } \\
\text { and Leaper } \\
2008\end{array}$ \\
\hline & $\begin{array}{l}\text { Noise and } \\
\text { vibration }\end{array}$ & $\begin{array}{l}\text { Avoidance, } \\
\text { masking of } \\
\text { environmental } \\
\text { cues, } \\
\text { communication } \\
\text { signals }\end{array}$ & $\begin{array}{l}\text { Med to high, sound } \\
\text { travels far, animals } \\
\text { will hear outside } \\
\text { project area, but } \\
\text { affects small portion } \\
\text { of migration range as } \\
\text { they travel through }\end{array}$ & $\begin{array}{l}\text { Low, occurring } \\
\text { during the summer } \\
\text { months for } 1 \text { to } 2 \\
\text { years }\end{array}$ & $\begin{array}{l}\text { Low, PCFA gray whales } \\
\text { likely to tolerate } \\
\text { background vessel noise } \\
\text { although masking cues } \\
\text { still likely to occur. }\end{array}$ & Med-high; see above & $\begin{array}{l}\text { Richardson } \\
\text { and Wursig } \\
1997\end{array}$ \\
\hline & $\begin{array}{l}\text { Oil/chemical } \\
\text { release }\end{array}$ & $\begin{array}{l}\text { Ingestion, } \\
\text { breathing } \\
\text { exhaust fumes, } \\
\text { overall } \\
\text { accumulation of } \\
\text { toxins. } \\
\end{array}$ & $\begin{array}{l}\text { Low, significant } \\
\text { increase in existing } \\
\text { traffic but the } \\
\text { volume released low; }\end{array}$ & $\begin{array}{l}\text { Low, occurring } \\
\text { during the summer } \\
\text { months for } 1 \text { to } 2 \\
\text { years }\end{array}$ & $\begin{array}{l}\text { Low for migratory gray } \\
\text { whales; medium for } \\
\text { PCFA whales due to } \\
\text { potentially increased } \\
\text { ambient contamination } \\
\text { levels }\end{array}$ & $\begin{array}{l}\text { Low; area of exposure } \\
\text { small relative to range; } \\
\text { additional inputs may } \\
\text { compound effects of } \\
\text { elevated levels for } \\
\text { PCFA whales }\end{array}$ & \multirow{2}{*}{$\begin{array}{l}\text { Le Boeuf et } \\
\text { al. 2002, } \\
\text { Richardson } \\
\text { and Wursig } \\
1997\end{array}$} \\
\hline \multirow[t]{2}{*}{$\begin{array}{l}\text { Construction of } \\
\text { electrical } \\
\text { collector system, } \\
\text { moorings and } \\
\text { foundations; } \\
\text { device } \\
\text { installation }\end{array}$} & $\begin{array}{l}\text { Noise and } \\
\text { vibration }\end{array}$ & $\begin{array}{l}\text { Migratory and } \\
\text { PCFA whales } \\
\text { displaced from } \\
\text { project area; }\end{array}$ & $\begin{array}{l}\text { Med to high; } \\
\text { migratory whales use } \\
\text { nearshore areas, } \\
\text { PCFA whales could } \\
\text { be displaced over } \\
\text { large area of resident } \\
\text { range }\end{array}$ & $\begin{array}{l}\text { Low, occurring over } \\
1 \text { summer }\end{array}$ & $\begin{array}{l}\text { Med to high, may affect } \\
\text { large portion of the gray } \\
\text { whale population on } \\
\text { northward migration; } \\
\text { potential large area effect } \\
\text { for resident PCFA whales }\end{array}$ & $\begin{array}{l}\text { Med-high; could affect } \\
\text { large segment of popn } \\
\text { if overlaps with north } \\
\text { migration; PCFA } \\
\text { whales may be } \\
\text { displaced }\end{array}$ & \\
\hline & $\begin{array}{l}\text { Oil/chemical } \\
\text { release }\end{array}$ & $\begin{array}{l}\text { Ingestion, } \\
\text { breathing } \\
\text { exhaust fumes, } \\
\text { overall } \\
\text { accumulation of } \\
\text { toxins. }\end{array}$ & $\begin{array}{l}\text { Med, significant } \\
\text { increase in use of } \\
\text { chemicals; volume of } \\
\text { fluids released low, } \\
\text { pre-existing levels } \\
\text { elevated }\end{array}$ & $\begin{array}{l}\text { Low, occurring over } \\
1 \text { to } 2 \text { summers }\end{array}$ & $\begin{array}{l}\text { Low for migratory gray } \\
\text { whales; medium for } \\
\text { PCFA whales due to } \\
\text { potentially increased } \\
\text { ambient contamination } \\
\text { levels }\end{array}$ & $\begin{array}{l}\text { Low; area of exposure } \\
\text { small relative to range; } \\
\text { inputs may compound } \\
\text { elevated levels for } \\
\text { PCFA whales in area }\end{array}$ & $\begin{array}{l}\text { Le Boeuf et } \\
\text { al. } 2002\end{array}$ \\
\hline
\end{tabular}

\begin{tabular}{lrr}
\hline $\begin{array}{l}\text { Environmental Assessment for Siting Wave \& Tidal } \\
\text { Energy Projects }\end{array}$ & D-21 & H. T. Harvey \& Associates \\
24 November 2009
\end{tabular}


Appendix D - Effects of Small Commercial OPT Power Buoy Project Humboldt

\begin{tabular}{|c|c|c|c|c|c|c|c|}
\hline Project activity & Project action & $\begin{array}{l}\text { Description of } \\
\text { action's effect } \\
\text { on indicator } \\
\end{array}$ & $\begin{array}{l}\text { Spatial exposure to } \\
\text { indicator (low, med, } \\
\text { high) }\end{array}$ & $\begin{array}{l}\text { Temporal exposure } \\
\text { to (low, med, high) }\end{array}$ & $\begin{array}{l}\text { Effect's overlap with } \\
\text { critical life stage, } \\
\text { behavior, habitat, or } \\
\text { resource (low, med, } \\
\text { high) }\end{array}$ & $\begin{array}{l}\text { Overall risk to } \\
\text { indicator (low, med, } \\
\text { high, unknown) }\end{array}$ & Source(s) \\
\hline & $\begin{array}{l}\text { Construction } \\
\text { and deck lights }\end{array}$ & Avoidance & $\begin{array}{l}\text { Low, not likely to be } \\
\text { attracted to lighted } \\
\text { areas }\end{array}$ & $\begin{array}{l}\text { Low, occurring over } \\
1 \text { to } 2 \text { summers }\end{array}$ & $\begin{array}{l}\text { Low for migratory } \\
\text { whales. Spatial extent } \\
\text { effects low compared to } \\
\text { animal's range }\end{array}$ & $\begin{array}{l}\text { Low, effects likely to } \\
\text { be very localized } \\
\text { around project area }\end{array}$ & \\
\hline $\begin{array}{l}\text { Directional } \\
\text { drilling, and } \\
\text { laying cable } \\
\text { under/on seabed }\end{array}$ & $\begin{array}{l}\text { Noise and } \\
\text { vibration }\end{array}$ & $\begin{array}{l}\text { Migratory } \\
\text { whales } \\
\text { displaced from } \\
\text { routes around }\end{array}$ & $\begin{array}{l}\text { Med to high; } \\
\text { migratory whales use } \\
\text { nearshore areas, and } \\
\text { PCFA whales' risks } \\
\text { of collision increase } \\
\text { with operations }\end{array}$ & $\begin{array}{l}\text { Low, occurring over } \\
1 \text { summer }\end{array}$ & $\begin{array}{l}\text { Med to high, may affect } \\
\text { large portion of the gray } \\
\text { whale population during } \\
\text { northward migration. }\end{array}$ & $\begin{array}{l}\text { Med-high; High if } \\
\text { overlaps with } \\
\text { migration; med due to } \\
\text { displacement of PCFA } \\
\text { whales in summer }\end{array}$ & $\begin{array}{l}\text { Calambokidis } \\
\text { et al. 2002, } \\
\text { Sullivan et al. } \\
1983\end{array}$ \\
\hline \multicolumn{8}{|c|}{ Operation and Maintenance } \\
\hline \multirow{3}{*}{ Boat traffic } & Direct impact & $\begin{array}{l}\text { Collision } \\
\text { injuries }\end{array}$ & $\begin{array}{l}\text { High, boat traffic } \\
\text { crosses migration } \\
\text { path along the coast }\end{array}$ & $\begin{array}{l}\text { Low, occurring } \\
\text { during the summer } \\
\text { months for } 1 \text { to } 2 \\
\text { years }\end{array}$ & $\begin{array}{l}\text { Med for PCFA whales in } \\
\text { region over summer; med } \\
\text { for large portion of } \\
\text { population if overlaps } \\
\text { with northward migration } \\
\text { along coast }\end{array}$ & $\begin{array}{l}\text { Low; may affect large } \\
\text { portion of popn if } \\
\text { overlaps with north } \\
\text { migration; gray whale- } \\
\text { boat collisions not } \\
\text { uncommon }\end{array}$ & $\begin{array}{l}\text { Calambokidis } \\
\text { et al. 2002, } \\
\text { Sullivan et al. } \\
\text { 1983, Van } \\
\text { Waerebeek } \\
\text { and Leaper } \\
2008\end{array}$ \\
\hline & $\begin{array}{l}\text { Oil/chemical } \\
\text { release }\end{array}$ & $\begin{array}{l}\text { Ingestion, } \\
\text { breathing } \\
\text { exhaust fumes, } \\
\text { accumulation of } \\
\text { toxins. }\end{array}$ & $\begin{array}{l}\text { Low, increase in } \\
\text { traffic but volume of } \\
\text { fluids released low; } \\
\text { could increase } \\
\text { contamination levels } \\
\text { for PCFA whales }\end{array}$ & $\begin{array}{l}\text { Med, occurring } \\
\text { throughout project } \\
\text { duration but not } \\
\text { likely to be year } \\
\text { round action }\end{array}$ & $\begin{array}{l}\text { Low for migratory gray } \\
\text { whales; potentially } \\
\text { increased ambient } \\
\text { contamination levels for } \\
\text { PCFA whales }\end{array}$ & $\begin{array}{l}\text { Low; area of exposure } \\
\text { small relative to range; } \\
\text { inputs may compound } \\
\text { elevated levels for } \\
\text { PCFA whales }\end{array}$ & $\begin{array}{l}\text { Le Boeuf et } \\
\text { al. } 2002\end{array}$ \\
\hline & $\begin{array}{l}\text { Noise and } \\
\text { vibration }\end{array}$ & $\begin{array}{l}\text { Avoidance, } \\
\text { masks } \\
\text { environmental } \\
\text { cues, } \\
\text { communication } \\
\text { signals }\end{array}$ & $\begin{array}{l}\text { Low, limited traffic; } \\
\text { sound travels far, } \\
\text { animals will hear } \\
\text { outside project area, } \\
\text { but affects small part } \\
\text { of large range }\end{array}$ & $\begin{array}{l}\text { Low, maintenance } \\
\text { via boats occurring } \\
\text { infrequently but } \\
\text { during summer } \\
\text { months }\end{array}$ & $\begin{array}{l}\text { Low; PCFA whales likely } \\
\text { to tolerate moderate } \\
\text { increase in vessel noise. } \\
\text { Med if overlaps with } \\
\text { northward migration } \\
\text { along coast }\end{array}$ & $\begin{array}{l}\text { Low; may affect large } \\
\text { portion of popn if } \\
\text { overlaps with north } \\
\text { migration; likely to } \\
\text { cause avoidance of } \\
\text { transport corridor }\end{array}$ & $\begin{array}{l}\text { Richardson } \\
\text { and Wursig } \\
1997\end{array}$ \\
\hline $\begin{array}{l}\text { Operation of } \\
\text { turbines or other } \\
\text { moving parts of } \\
\text { devices }\end{array}$ & $\begin{array}{l}\text { Moving device } \\
\text { parts }\end{array}$ & $\begin{array}{l}\text { Movement of } \\
\text { heaving point } \\
\text { absorber and } \\
\text { mooring lines } \\
\text { could injure } \\
\text { animals }\end{array}$ & $\begin{array}{l}\text { Low, area of } \\
\text { potential contact } \\
\text { between moving } \\
\text { parts and whales } \\
\text { small, whales not } \\
\text { likely to approach }\end{array}$ & $\begin{array}{l}\text { High, movement will } \\
\text { be continuous } \\
\text { throughout the } \\
\text { duration of the } \\
\text { project }\end{array}$ & $\begin{array}{l}\text { Low, although moving } \\
\text { throughout the project } \\
\text { life, likelihood of } \\
\text { interaction between } \\
\text { animals and devices low. }\end{array}$ & $\begin{array}{l}\text { Low; unlikely to } \\
\text { approach moving parts } \\
\text { close enough to be at } \\
\text { risk }\end{array}$ & \\
\hline
\end{tabular}

\begin{tabular}{lr}
\hline Environmental Assessment for Siting Wave \& Tidal & D-22 \\
Energy Projects &
\end{tabular}


Appendix D - Effects of Small Commercial OPT Power Buoy Project Humboldt

\begin{tabular}{|c|c|c|c|c|c|c|c|}
\hline Project activity & Project action & $\begin{array}{l}\text { Description of } \\
\text { action's effect } \\
\text { on indicator } \\
\end{array}$ & $\begin{array}{l}\text { Spatial exposure to } \\
\text { indicator (low, med, } \\
\text { high) }\end{array}$ & $\begin{array}{l}\text { Temporal exposure } \\
\text { to (low, med, high) }\end{array}$ & $\begin{array}{l}\text { Effect's overlap with } \\
\text { critical life stage, } \\
\text { behavior, habitat, or } \\
\text { resource (low, med, } \\
\text { high) }\end{array}$ & $\begin{array}{l}\text { Overall risk to } \\
\text { indicator (low, med, } \\
\text { high, unknown) }\end{array}$ & Source(s) \\
\hline & $\begin{array}{l}\text { Noise and } \\
\text { vibration }\end{array}$ & $\begin{array}{l}\text { Avoidance, } \\
\text { masking of } \\
\text { environmental } \\
\text { cues, } \\
\text { communication } \\
\text { signals }\end{array}$ & $\begin{array}{l}\text { Unknown, sound } \\
\text { travels far and } \\
\text { animals will hear it } \\
\text { outside project area, } \\
\text { but affects small part } \\
\text { of large range as they } \\
\text { travel through area }\end{array}$ & $\begin{array}{l}\text { Med, occurring } \\
\text { throughout the } \\
\text { duration of the } \\
\text { project }\end{array}$ & $\begin{array}{l}\text { Low; PCFA whales likely } \\
\text { to acclimate to noise, may } \\
\text { overlap with the end of } \\
\text { the northward migration if } \\
\text { actions occur in early } \\
\text { summer/late spring }\end{array}$ & $\begin{array}{l}\text { Low; likely to affect } \\
\text { migratory and PCFA } \\
\text { whales; sound levels } \\
\text { not likely higher than } \\
\text { levels during migration } \\
\text { along coast; may cause } \\
\text { avoidance of area by } \\
\text { PCFA whales }\end{array}$ & $\begin{array}{l}\text { Richardson } \\
\text { and Wursig } \\
1997\end{array}$ \\
\hline $\begin{array}{l}\text { Structures in } \\
\text { water column and } \\
\text { on seabed, such } \\
\text { as devices and } \\
\text { moorings and } \\
\text { footings }\end{array}$ & Structure & $\begin{array}{l}\text { Collisions, } \\
\text { entanglement } \\
\text { with derelict } \\
\text { gear caught on } \\
\text { moorings; } \\
\text { artificial reef } \\
\text { could attract } \\
\text { fish } \\
\end{array}$ & $\begin{array}{l}\text { Low, home range is } \\
\text { large compared to } \\
\text { project area }\end{array}$ & $\begin{array}{l}\text { High, occurring } \\
\text { throughout project } \\
\text { duration. }\end{array}$ & $\begin{array}{l}\text { Med for PCFA resident } \\
\text { whales; low for whales } \\
\text { that migrate along coast }\end{array}$ & $\begin{array}{l}\text { Unknown; risk of } \\
\text { entanglement in } \\
\text { derelict gear, loose } \\
\text { cables or loose mooring } \\
\text { lines unknown }\end{array}$ & \\
\hline $\begin{array}{l}\text { Electricity } \\
\text { conduction } \\
\text { through cable }\end{array}$ & EMF & Unknown & $\begin{array}{l}\text { Unknown, cable } \\
\text { shielding and burial } \\
\text { provides some } \\
\text { attenuation }\end{array}$ & $\begin{array}{l}\text { High if an effect } \\
\text { because electricity } \\
\text { generated over life of } \\
\text { the project }\end{array}$ & Unknown & $\begin{array}{l}\text { Unknown; High } \\
\text { uncertainty about } \\
\text { effects on marine } \\
\text { mammals }\end{array}$ & $\begin{array}{l}\text { Boehlert et } \\
\text { al. } 2008\end{array}$ \\
\hline \multirow[t]{2}{*}{$\begin{array}{l}\text { Structures on } \\
\text { water's surface }\end{array}$} & Structure & Collision & $\begin{array}{l}\text { Low, project area is } \\
\text { small compared to } \\
\text { range }\end{array}$ & $\begin{array}{l}\text { High, occurring } \\
\text { throughout project } \\
\text { duration }\end{array}$ & $\begin{array}{l}\text { Low, for both migrants } \\
\text { and PCFA whales } \\
\text { because range is large } \\
\text { compared to project area }\end{array}$ & $\begin{array}{l}\text { Low; whales readily } \\
\text { avoid buoys; gray } \\
\text { whales encounter large } \\
\text { number of mobile and } \\
\text { stationary structures } \\
\text { during migration }\end{array}$ & \\
\hline & $\begin{array}{l}\text { Navigation } \\
\text { lights }\end{array}$ & $\begin{array}{l}\text { Low intensity; } \\
\text { likelihood of } \\
\text { impacts low to } \\
\text { none }\end{array}$ & $\begin{array}{l}\text { Low, associated with } \\
\text { navigation lights on } \\
\text { devices }\end{array}$ & $\begin{array}{l}\text { High, occurring } \\
\text { throughout project } \\
\text { duration }\end{array}$ & $\begin{array}{l}\text { Low to no effect for } \\
\text { migrants and low effect } \\
\text { for PCFA whales }\end{array}$ & $\begin{array}{l}\text { Low; navigation lights } \\
\text { relatively dim, area of } \\
\text { effect very limited }\end{array}$ & \\
\hline \multicolumn{8}{|c|}{ Decommissioning } \\
\hline
\end{tabular}

\begin{tabular}{|c|c|c|}
\hline $\begin{array}{l}\text { Environmental Assessment for Siting Wave \& Tidal } \\
\text { Energy Projects }\end{array}$ & D-23 & $\begin{array}{r}\text { H. T. Harvey \& Associates } \\
24 \text { November } 2009\end{array}$ \\
\hline
\end{tabular}


Appendix D - Effects of Small Commercial OPT Power Buoy Project Humboldt

\begin{tabular}{|c|c|c|c|c|c|c|c|}
\hline Project activity & Project action & $\begin{array}{l}\text { Description of } \\
\text { action's effect } \\
\text { on indicator } \\
\end{array}$ & $\begin{array}{l}\text { Spatial exposure to } \\
\text { indicator (low, med, } \\
\text { high) }\end{array}$ & $\begin{array}{l}\text { Temporal exposure } \\
\text { to (low, med, high) }\end{array}$ & $\begin{array}{l}\text { Effect's overlap with } \\
\text { critical life stage, } \\
\text { behavior, habitat, or } \\
\text { resource (low, med, } \\
\text { high) }\end{array}$ & $\begin{array}{l}\text { Overall risk to } \\
\text { indicator (low, med, } \\
\text { high, unknown) }\end{array}$ & Source(s) \\
\hline \multirow{3}{*}{ Boat traffic } & Direct impact & $\begin{array}{l}\text { Collision } \\
\text { injuries }\end{array}$ & $\begin{array}{l}\text { High, boat traffic } \\
\text { crosses migration } \\
\text { path along the coast }\end{array}$ & $\begin{array}{l}\text { Low, occurring } \\
\text { during the summer } \\
\text { months for } 1 \text { to } 2 \\
\text { years }\end{array}$ & $\begin{array}{l}\text { Low; for most of the } \\
\text { population is not present } \\
\text { in the region during time } \\
\text { of deconstruction; Med } \\
\text { for PCFA whales in } \\
\text { region over summer. }\end{array}$ & $\begin{array}{l}\text { Med-high; could affect } \\
\text { large segment of popn } \\
\text { if overlaps with north } \\
\text { migration; PCFA } \\
\text { whales present, gray } \\
\text { whale-boat collision } \\
\text { not uncommon }\end{array}$ & $\begin{array}{l}\text { Calambokidis } \\
\text { et al. 2002, } \\
\text { Sullivan et al. } \\
\text { 1983, Van } \\
\text { Waerebeek } \\
\text { and Leaper } \\
2008\end{array}$ \\
\hline & $\begin{array}{l}\text { Noise and } \\
\text { vibration }\end{array}$ & $\begin{array}{l}\text { Avoidance, } \\
\text { masking of } \\
\text { environmental } \\
\text { cues, } \\
\text { communication } \\
\text { signals }\end{array}$ & $\begin{array}{l}\text { Med to high, sound } \\
\text { travel far, animals } \\
\text { will hear outside } \\
\text { project area, but } \\
\text { affects small portion } \\
\text { of migration range }\end{array}$ & $\begin{array}{l}\text { Low, occurring } \\
\text { during the summer } \\
\text { months for } 1 \text { to } 2 \\
\text { years }\end{array}$ & $\begin{array}{l}\text { Low, PCFA gray whales } \\
\text { likely to tolerate } \\
\text { background vessel noise } \\
\text { although masking cues } \\
\text { still likely to occur. }\end{array}$ & $\begin{array}{l}\text { Med-high; could affect } \\
\text { large segment of } \\
\text { population if overlaps } \\
\text { with northward } \\
\text { migration; likely to } \\
\text { displace PCFA whales }\end{array}$ & $\begin{array}{l}\text { Richardson } \\
\text { and Wursig } \\
1997\end{array}$ \\
\hline & $\begin{array}{l}\text { Oil/chemical } \\
\text { release }\end{array}$ & $\begin{array}{l}\text { Ingestion, } \\
\text { breathing } \\
\text { exhaust fumes, } \\
\text { overall } \\
\text { accumulation of } \\
\text { toxins. }\end{array}$ & $\begin{array}{l}\text { Low, increase in } \\
\text { traffic but low } \\
\text { volume of fluids } \\
\text { released; medium for } \\
\text { PCFA whales due to } \\
\text { potentially increased } \\
\text { contamination levels } \\
\end{array}$ & $\begin{array}{l}\text { Low, occurring } \\
\text { during } 1 \text { to } 2 \\
\text { summers }\end{array}$ & $\begin{array}{l}\text { Low for migratory gray } \\
\text { whales; medium for } \\
\text { PCFA whales due to } \\
\text { potentially increased } \\
\text { ambient contamination } \\
\text { levels }\end{array}$ & $\begin{array}{l}\text { Low; area of exposure } \\
\text { small relative to range; } \\
\text { inputs may compound } \\
\text { elevated levels for } \\
\text { PCFA whales in area } \\
\text { for extended periods }\end{array}$ & $\begin{array}{l}\text { Le Boeuf et } \\
\text { al. } 2002\end{array}$ \\
\hline \multirow{3}{*}{$\begin{array}{l}\text { Decommissioning } \\
\text { of structures on } \\
\text { water's surface or } \\
\text { seabed }\end{array}$} & $\begin{array}{l}\text { Noise and } \\
\text { vibration }\end{array}$ & $\begin{array}{l}\text { Migratory and } \\
\text { PCFA whales } \\
\text { displaced from } \\
\text { project area; }\end{array}$ & $\begin{array}{l}\text { Med; migratory } \\
\text { whales use nearshore } \\
\text { areas, PCFA whales } \\
\text { displaced over } \\
\text { potentially large area } \\
\text { of resident range }\end{array}$ & $\begin{array}{l}\text { Low, occurring over } \\
1 \text { summer }\end{array}$ & $\begin{array}{l}\text { Med to high, may affect } \\
\text { large portion of the gray } \\
\text { whale population on } \\
\text { northward migration; } \\
\text { potential large area effect } \\
\text { for resident PCFA whales }\end{array}$ & $\begin{array}{l}\text { Med-high; could affect } \\
\text { large segment of popn } \\
\text { if overlaps with } \\
\text { migration; PCFA } \\
\text { whales may be } \\
\text { displaced }\end{array}$ & $\begin{array}{l}\text { Richardson } \\
\text { and Wursig } \\
1997\end{array}$ \\
\hline & $\begin{array}{l}\text { Oil/chemical } \\
\text { release }\end{array}$ & $\begin{array}{l}\text { Ingestion, } \\
\text { breathing } \\
\text { exhaust fumes, } \\
\text { overall } \\
\text { accumulation of } \\
\text { toxins. }\end{array}$ & $\begin{array}{l}\text { Med, significant } \\
\text { increase in use of } \\
\text { chemicals; volume of } \\
\text { fluids released low- } \\
\text { med, existing levels } \\
\text { elevated }\end{array}$ & $\begin{array}{l}\text { Low, occurring over } \\
1 \text { to } 2 \text { summers }\end{array}$ & $\begin{array}{l}\text { Low for migratory gray } \\
\text { whales; medium for } \\
\text { PCFA whales due to } \\
\text { potentially increased } \\
\text { ambient contamination } \\
\text { levels }\end{array}$ & $\begin{array}{l}\text { Low-med; area } \\
\text { exposure small relative } \\
\text { to range; additional } \\
\text { inputs may compound } \\
\text { effects of elevated } \\
\text { levels for PCFA whales }\end{array}$ & $\begin{array}{l}\text { Le Boeuf et } \\
\text { al. } 2002\end{array}$ \\
\hline & $\begin{array}{l}\text { Deconstruction } \\
\text { and deck lights }\end{array}$ & Avoidance & $\begin{array}{l}\text { Low, not likely to be } \\
\text { attracted to lighted } \\
\text { areas }\end{array}$ & $\begin{array}{l}\text { Low, occurring over } \\
1 \text { to } 2 \text { summers }\end{array}$ & $\begin{array}{l}\text { Low for migratory } \\
\text { whales. Spatial extent } \\
\text { effects low compared to } \\
\text { animal's range }\end{array}$ & $\begin{array}{l}\text { Low, effects likely to } \\
\text { be very localized } \\
\text { around project area }\end{array}$ & \\
\hline
\end{tabular}

\begin{tabular}{lr}
\hline Environmental Assessment for Siting Wave \& Tidal & D-24 \\
Energy Projects &
\end{tabular}


Appendix D - Effects of Small Commercial OPT Power Buoy Project Humboldt

Potential effects on harbor porpoise due to Small Commercial OPT Power Buoy Project at Humboldt site

\begin{tabular}{|c|c|c|c|c|c|c|c|}
\hline Project activity & Action & $\begin{array}{l}\text { Description of } \\
\text { action's effect on } \\
\text { species or group }\end{array}$ & $\begin{array}{l}\text { Spatial extent of } \\
\text { effect (low, med, } \\
\text { high) relative to } \\
\text { project }\end{array}$ & $\begin{array}{l}\text { Temporal } \\
\text { extent of effect } \\
\text { (low, med, } \\
\text { high) relative to } \\
\text { project }\end{array}$ & $\begin{array}{l}\text { Effect's overlap } \\
\text { with critical life } \\
\text { stage, behavior, } \\
\text { habitat (low, med, } \\
\text { high) relative to } \\
\text { species/group }\end{array}$ & $\begin{array}{l}\text { Overall risk to } \\
\text { indicator (low, med, } \\
\text { high, unknown) }\end{array}$ & Source(s) \\
\hline \multicolumn{8}{|c|}{ Construction } \\
\hline \multirow{3}{*}{ Boat traffic } & Direct impact & Collision injuries & $\begin{array}{l}\text { High, significant } \\
\text { increase in traffic (esp } \\
\text { in bay and jetties); } \\
\text { present inshore and at } \\
\text { harbor entrance where } \\
\text { porpoises move in and } \\
\text { out }\end{array}$ & $\begin{array}{l}\text { Low, occurs } \\
\text { over } 1 \text { to } 2 \\
\text { summers }\end{array}$ & $\begin{array}{l}\text { Low for adults; med } \\
\text { for calves foraging } \\
\text { and travel in } \\
\text { construction, } \\
\text { transport zones; area } \\
\text { of exposure small } \\
\text { relative to home } \\
\text { range; }\end{array}$ & $\begin{array}{l}\text { Low; risk at harbor } \\
\text { opening where boat } \\
\text { traffic and porpoises } \\
\text { both occur; boat- } \\
\text { porpoise collisions } \\
\text { infrequent }\end{array}$ & $\begin{array}{l}\text { Stroud and } \\
\text { Roffe } 1979\end{array}$ \\
\hline & $\begin{array}{l}\text { Noise and } \\
\text { vibration }\end{array}$ & $\begin{array}{l}\text { Avoidance, masking of } \\
\text { environmental cues. } \\
\text { Reduction of hearing } \\
\text { sensitivity (Temporary } \\
\text { Threshold Shift), could } \\
\text { increase in predation }\end{array}$ & $\begin{array}{l}\text { High, increase existing } \\
\text { traffic (esp in bay and } \\
\text { jetties); present } \\
\text { inshore and at harbor } \\
\text { entrance where } \\
\text { porpoises move in and } \\
\text { out }\end{array}$ & $\begin{array}{l}\text { Low, occurs } \\
\text { over } 1 \text { to } 2 \\
\text { summers }\end{array}$ & $\begin{array}{l}\text { Low; may affect } \\
\text { adults and calves } \\
\text { while foraging and } \\
\text { traveling parallel to } \\
\text { shore }\end{array}$ & $\begin{array}{l}\text { Med; effects greater in } \\
\text { bay near jetties and } \\
\text { project area; but short } \\
\text { duration }\end{array}$ & $\begin{array}{l}\text { Erbe and } \\
\text { Farmer } 2000\end{array}$ \\
\hline & $\begin{array}{l}\text { Oil/chemical } \\
\text { release }\end{array}$ & $\begin{array}{l}\text { Ingestion while feeding } \\
\text { or breathing, breathing } \\
\text { exhaust fumes, } \\
\text { accumulation of toxins. } \\
\text { Assuming no } \\
\text { catastrophic spills. }\end{array}$ & $\begin{array}{l}\text { Med, significant } \\
\text { increase in existing } \\
\text { traffic but the volume } \\
\text { released low and } \\
\text { dispersed }\end{array}$ & $\begin{array}{l}\text { Low, occurs } \\
\text { over } 1 \text { to } 2 \\
\text { summers }\end{array}$ & $\begin{array}{l}\text { Low-med for all age } \\
\text { classes; potential for } \\
\text { exposure while } \\
\text { foraging and while } \\
\text { resting in Bay }\end{array}$ & $\begin{array}{l}\text { Low; area of exposure } \\
\text { small relative to home } \\
\text { range; inputs } \\
\text { compound elevated } \\
\text { levels }\end{array}$ & $\begin{array}{l}\text { Brookens et al. } \\
\text { 2007, Le Boeuf } \\
\text { et al. } 2002\end{array}$ \\
\hline \multirow[t]{2}{*}{$\begin{array}{l}\text { Construction of } \\
\text { electrical } \\
\text { collector system, } \\
\text { moorings and } \\
\text { foundations, and } \\
\text { device } \\
\text { installation }\end{array}$} & $\begin{array}{l}\text { Noise and } \\
\text { vibration }\end{array}$ & $\begin{array}{l}\text { Avoidance of area, } \\
\text { affects foraging and } \\
\text { travel, masks } \\
\text { environmental cues, } \\
\text { echolocation and } \\
\text { communication signals }\end{array}$ & $\begin{array}{l}\text { Med; sound likely to } \\
\text { be detected over large } \\
\text { area (several 10s of } \\
\mathrm{km}^{2} \text { ) }\end{array}$ & $\begin{array}{l}\text { Low, only } 1 \\
\text { summer }\end{array}$ & $\begin{array}{l}\text { Med, potential } \\
\text { temporary impacts } \\
\text { on hearing if } \\
\text { porpoises are } \\
\text { attracted to fish } \\
\text { aggregates } \\
\end{array}$ & $\begin{array}{l}\text { Low; harbor porpoises } \\
\text { forage successfully } \\
\text { around similar } \\
\text { industrial activities }\end{array}$ & Todd et al. 2009 \\
\hline & $\begin{array}{l}\text { Oil/chemical } \\
\text { release }\end{array}$ & $\begin{array}{l}\text { Ingestion while feeding } \\
\text { or nursing; breathing } \\
\text { exhaust fumes; } \\
\text { accumulation of toxins. } \\
\text { Assuming no } \\
\text { catastrophic spills. }\end{array}$ & $\begin{array}{l}\text { Med, increase in use of } \\
\text { chemicals; volume of } \\
\text { fluids released low- } \\
\text { med, and pre-existing } \\
\text { ambient levels high }\end{array}$ & $\begin{array}{l}\text { Low, only } 1 \text { to } 2 \\
\text { summers }\end{array}$ & $\begin{array}{l}\text { Low for all age } \\
\text { classes; potential for } \\
\text { exposure while } \\
\text { foraging around } \\
\text { structures }\end{array}$ & $\begin{array}{l}\text { Low; area of exposure } \\
\text { small relative to home } \\
\text { range; inputs } \\
\text { compound elevated } \\
\text { contaminant levels }\end{array}$ & $\begin{array}{l}\text { Brookens et al. } \\
\text { 2007, Le Boeuf } \\
\text { et al. } 2002\end{array}$ \\
\hline
\end{tabular}

\begin{tabular}{lrr}
\hline Environmental Assessment for Siting Wave \& Tidal & D-25 & H. T. Harvey \& Associates \\
Energy Projects & & 24 November 2009
\end{tabular}


Appendix D - Effects of Small Commercial OPT Power Buoy Project Humboldt

\begin{tabular}{|c|c|c|c|c|c|c|c|}
\hline \multirow[t]{2}{*}{ Project activity } & Action & $\begin{array}{l}\text { Description of } \\
\text { action's effect on } \\
\text { species or group }\end{array}$ & $\begin{array}{l}\text { Spatial extent of } \\
\text { effect (low, med, } \\
\text { high) relative to } \\
\text { project }\end{array}$ & $\begin{array}{l}\text { Temporal } \\
\text { extent of effect } \\
\text { (low, med, } \\
\text { high) relative to } \\
\text { project }\end{array}$ & $\begin{array}{l}\text { Effect's overlap } \\
\text { with critical life } \\
\text { stage, behavior, } \\
\text { habitat (low, med, } \\
\text { high) relative to } \\
\text { species/group }\end{array}$ & $\begin{array}{l}\text { Overall risk to } \\
\text { indicator (low, med, } \\
\text { high, unknown) }\end{array}$ & Source(s) \\
\hline & $\begin{array}{l}\text { Construction } \\
\text { and deck lights }\end{array}$ & $\begin{array}{l}\text { May attract porpoise } \\
\text { prey, and indirectly } \\
\text { porpoise increasing } \\
\text { potential for impacts } \\
\text { related to other actions }\end{array}$ & $\begin{array}{l}\text { Low to med; project } \\
\text { site small relative to } \\
\text { range of porpoises; } \\
\text { lights may attract prey } \\
\text { and concentrate } \\
\text { porpoises }\end{array}$ & $\begin{array}{l}\text { Low, only } 1 \text { to } 2 \\
\text { summers }\end{array}$ & $\begin{array}{l}\text { Low; area affected } \\
\text { by lights small } \\
\text { relative to home } \\
\text { range }\end{array}$ & $\begin{array}{l}\text { Low due to high levels } \\
\text { of pre-existing boat } \\
\text { traffic }\end{array}$ & Todd et al. 2009 \\
\hline $\begin{array}{l}\text { Directional } \\
\text { drilling, and } \\
\text { laying cable } \\
\text { under/on seabed }\end{array}$ & $\begin{array}{l}\text { Noise and } \\
\text { vibration }\end{array}$ & $\begin{array}{l}\text { Avoidance of area, } \\
\text { affects foraging and } \\
\text { travel, masks } \\
\text { environmental cues, } \\
\text { echolocation and } \\
\text { communication signals }\end{array}$ & $\begin{array}{l}\text { Med; relatively small } \\
\text { footprint may affect } \\
\text { along shore movement }\end{array}$ & $\begin{array}{l}\text { Low, only } 1 \\
\text { summer }\end{array}$ & $\begin{array}{l}\text { Low, but unknown } \\
\text { effects on calves }\end{array}$ & $\begin{array}{l}\text { Med; risk with } \\
\text { concentrating impacts } \\
\text { in nearshore envir.; } \\
\text { potential for propeller } \\
\text { strike; disruption of } \\
\text { movement along shore }\end{array}$ & $\begin{array}{l}\text { Kastak et al. } \\
2005, \\
\text { Koschinski et } \\
\text { al. 2003, } \\
\text { Tougaard et al. } \\
2009 \\
\end{array}$ \\
\hline \multicolumn{8}{|c|}{ Operation and Maintenance } \\
\hline \multirow{3}{*}{ Boat traffic } & Direct impact & $\begin{array}{l}\text { Collision; behavioral } \\
\text { disruption vessels may } \\
\text { disrupt rest, foraging, } \\
\text { travel, and socialization } \\
\text { around area. }\end{array}$ & $\begin{array}{l}\text { Low-med, moderate } \\
\text { increase over existing } \\
\text { traffic (esp in bay and } \\
\text { jetties); porpoises } \\
\text { inshore and } \\
\text { concentrate at narrow } \\
\text { harbor entrance }\end{array}$ & $\begin{array}{l}\text { Low-med, } \\
\text { annual } \\
\text { maintenance } \\
\text { assumed during } \\
3 \text { months of } \\
\text { summer ( } 2-3 \\
\text { round trips/day) } \\
\end{array}$ & $\begin{array}{l}\text { Low for adults; med } \\
\text { for calves due to } \\
\text { foraging and travel } \\
\text { in and near } \\
\text { construction and } \\
\text { transport zones }\end{array}$ & $\begin{array}{l}\text { Low; area of exposure } \\
\text { small relative to home } \\
\text { range; risk at harbor } \\
\text { opening where boat } \\
\text { traffic and porpoises } \\
\text { occur; boat-porpoise } \\
\text { collisions infrequent } \\
\end{array}$ & $\begin{array}{l}\text { Stroud and } \\
\text { Roffe } 1979\end{array}$ \\
\hline & $\begin{array}{l}\text { Noise and } \\
\text { vibration }\end{array}$ & $\begin{array}{l}\text { Avoidance of area, } \\
\text { affects foraging and } \\
\text { travel, masks } \\
\text { environmental cues, } \\
\text { echolocation and } \\
\text { communication signals }\end{array}$ & $\begin{array}{l}\text { Low-med, increase in } \\
\text { traffic (esp in bay and } \\
\text { jetties); present } \\
\text { inshore and at harbor } \\
\text { entrance where } \\
\text { porpoises move in and } \\
\text { out }\end{array}$ & $\begin{array}{l}\text { Low-med, } \\
\text { annual } \\
\text { maintenance } \\
\text { assumed during } \\
3 \text { months of } \\
\text { summer ( } 2-3 \\
\text { round trips/day) } \\
\end{array}$ & $\begin{array}{l}\text { Low for adults; med } \\
\text { for calves due to } \\
\text { foraging and travel } \\
\text { in and near } \\
\text { construction and } \\
\text { transport zones }\end{array}$ & $\begin{array}{l}\text { Low; could mask cues } \\
\text { over long distance; } \\
\text { effects greater in bay } \\
\text { near jetties and around } \\
\text { project area; short } \\
\text { duration and few } \\
\text { vessels }\end{array}$ & $\begin{array}{l}\text { Erbe and } \\
\text { Farmer } 2000\end{array}$ \\
\hline & $\begin{array}{l}\text { Oil/chemical } \\
\text { release }\end{array}$ & $\begin{array}{l}\text { Ingestion while feeding } \\
\text { or breathing, breathing } \\
\text { exhaust fumes, } \\
\text { accumulation of toxins. } \\
\text { Assuming no } \\
\text { catastrophic spills. }\end{array}$ & $\begin{array}{l}\text { Low-med; increase in } \\
\text { existing traffic but the } \\
\text { volume released low } \\
\text { and dispersed }\end{array}$ & $\begin{array}{l}\text { Low, occurs } \\
\text { over } 1 \text { to } 2 \\
\text { summers }\end{array}$ & $\begin{array}{l}\text { Low-med for all age } \\
\text { classes; potential for } \\
\text { exposure while } \\
\text { foraging and while } \\
\text { resting in Bay }\end{array}$ & $\begin{array}{l}\text { Low; area of exposure } \\
\text { small relative to home } \\
\text { range; inputs } \\
\text { compound elevated } \\
\text { contaminant levels }\end{array}$ & $\begin{array}{l}\text { Brookens et al. } \\
\text { 2007, Le Boeuf } \\
\text { et al. } 2002\end{array}$ \\
\hline
\end{tabular}

\begin{tabular}{lr}
\hline Environmental Assessment for Siting Wave \& Tidal & D-26 \\
Energy Projects &
\end{tabular}


Appendix D - Effects of Small Commercial OPT Power Buoy Project Humboldt

\begin{tabular}{|c|c|c|c|c|c|c|c|}
\hline Project activity & Action & $\begin{array}{l}\text { Description of } \\
\text { action's effect on } \\
\text { species or group }\end{array}$ & $\begin{array}{l}\text { Spatial extent of } \\
\text { effect (low, med, } \\
\text { high) relative to } \\
\text { project }\end{array}$ & $\begin{array}{l}\text { Temporal } \\
\text { extent of effect } \\
\text { (low, med, } \\
\text { high) relative to } \\
\text { project }\end{array}$ & $\begin{array}{l}\text { Effect's overlap } \\
\text { with critical life } \\
\text { stage, behavior, } \\
\text { habitat (low, med, } \\
\text { high) relative to } \\
\text { species/group }\end{array}$ & $\begin{array}{l}\text { Overall risk to } \\
\text { indicator (low, med, } \\
\text { high, unknown) }\end{array}$ & Source(s) \\
\hline \multirow{2}{*}{$\begin{array}{l}\text { Operation of } \\
\text { turbines or other } \\
\text { moving parts of } \\
\text { devices }\end{array}$} & $\begin{array}{l}\text { Noise and } \\
\text { vibration }\end{array}$ & $\begin{array}{l}\text { Avoidance of area, } \\
\text { affects foraging and } \\
\text { travel, masks } \\
\text { environmental cues, } \\
\text { echolocation and } \\
\text { communication signals }\end{array}$ & $\begin{array}{l}\text { Medium, increase in } \\
\text { traffic (esp in bay and } \\
\text { jetties); present } \\
\text { inshore and at harbor } \\
\text { entrance where } \\
\text { porpoises move in and } \\
\text { out. }\end{array}$ & $\begin{array}{l}\text { Low, annual } \\
\text { maintenance } \\
\text { assumed during } \\
3 \text { months of } \\
\text { summer ( } 2-3 \\
\text { round trips/day) }\end{array}$ & $\begin{array}{l}\text { Low for adults; med } \\
\text { for calves due to } \\
\text { foraging and travel } \\
\text { in area around } \\
\text { turbines }\end{array}$ & $\begin{array}{l}\text { Low; could mask cues } \\
\text { near turbine }(<70 \mathrm{~m}) \text {; } \\
\text { porpoise less sensitive } \\
\text { to low-freq sounds and } \\
\text { sound likely inaudible } \\
\text { at }>100 \mathrm{~m}\end{array}$ & $\begin{array}{l}\text { Erbe and } \\
\text { Farmer } 2000\end{array}$ \\
\hline & $\begin{array}{l}\text { Moving device } \\
\text { parts }\end{array}$ & $\begin{array}{l}\text { Movement of the } \\
\text { heaving point absorber } \\
\text { and mooring lines } \\
\text { could injure animals }\end{array}$ & $\begin{array}{l}\text { Low, the areas of } \\
\text { movement are small } \\
\text { compared to project } \\
\text { area }\end{array}$ & $\begin{array}{l}\text { High, the } \\
\text { movement } \\
\text { occurs over the } \\
\text { life of the } \\
\text { project }\end{array}$ & $\begin{array}{l}\text { Low for all age } \\
\text { classes }\end{array}$ & $\begin{array}{l}\text { Low; porpoise highly } \\
\text { sensitive to } \\
\text { surroundings, unlikely } \\
\text { to approach regularly } \\
\text { moving parts }\end{array}$ & $\begin{array}{l}\text { Koschinski et } \\
\text { al. 2003, Stroud } \\
\text { and Roffe } 1979\end{array}$ \\
\hline $\begin{array}{l}\text { Structures in } \\
\text { water column and } \\
\text { on seabed, such } \\
\text { as devices and } \\
\text { moorings and } \\
\text { footings }\end{array}$ & Structure & $\begin{array}{l}\text { Collision, entanglement } \\
\text { with derelict gear } \\
\text { caught on moorings; } \\
\text { artificial reef could } \\
\text { attract fish and increase } \\
\text { porpoise foraging }\end{array}$ & $\begin{array}{l}\text { Low, home range large } \\
\left(210-51,000 \mathrm{~km}^{2}\right) ; \\
\text { may create new } \\
\text { habitat, depends on } \\
\text { strength of attraction }\end{array}$ & $\begin{array}{l}\text { High, any effects } \\
\text { would occur } \\
\text { over the life of } \\
\text { the project. }\end{array}$ & $\begin{array}{l}\text { Low; may } \\
\text { incrementally } \\
\text { increase foraging on } \\
\text { prey attracted to } \\
\text { structure }\end{array}$ & $\begin{array}{l}\text { Low, structure } \\
\text { unlikely to present risk } \\
\text { to porpoise }\end{array}$ & Todd et al. 2009 \\
\hline $\begin{array}{l}\text { Electricity } \\
\text { conduction } \\
\text { through cable }\end{array}$ & EMF & Unknown & $\begin{array}{l}\text { Unknown, cable } \\
\text { shielding and burial } \\
\text { provides some } \\
\text { attenuation }\end{array}$ & $\begin{array}{l}\text { High if there is } \\
\text { an effect, it } \\
\text { would occur } \\
\text { over life of the } \\
\text { project }\end{array}$ & $\begin{array}{l}\text { Unknown; any } \\
\text { effects would likely } \\
\text { impact all age } \\
\text { classes }\end{array}$ & $\begin{array}{l}\text { Unknown; High } \\
\text { uncertainty about } \\
\text { effects on marine } \\
\text { mammals }\end{array}$ & $\begin{array}{l}\text { Boehlert et al. } \\
2008\end{array}$ \\
\hline $\begin{array}{l}\text { Structures on } \\
\text { water's surface }\end{array}$ & Structure & $\begin{array}{l}\text { Artificial reef effects } \\
\text { could attract fish and } \\
\text { increase porpoise } \\
\text { foraging }\end{array}$ & $\begin{array}{l}\text { Low, reef effect area is } \\
\text { small compared to } \\
\text { home range }(210- \\
\left.51,000 \mathrm{~km}^{2}\right)\end{array}$ & $\begin{array}{l}\text { High, any effects } \\
\text { would occur } \\
\text { over life of } \\
\text { project. }\end{array}$ & $\begin{array}{l}\text { Low, may } \\
\text { incrementally } \\
\text { increase forage as de } \\
\text { facto marine reserve. }\end{array}$ & Low & Goodwin 2008 \\
\hline \multicolumn{8}{|c|}{ Decommissioning } \\
\hline Boat traffic & Direct impact & Collision injuries & $\begin{array}{l}\text { High, increase over } \\
\text { traffic (esp in bay and } \\
\text { jetties); inshore and } \\
\text { concentrated at harbor } \\
\text { entrance where } \\
\text { porpoises move in and } \\
\text { out }\end{array}$ & $\begin{array}{l}\text { Low, occurs } \\
\text { over } 1 \text { to } 2 \\
\text { summers }\end{array}$ & $\begin{array}{l}\text { Low adults; med for } \\
\text { calves due to } \\
\text { foraging and travel } \\
\text { in and near } \\
\text { construction and } \\
\text { transport zones }\end{array}$ & $\begin{array}{l}\text { Low; exposure area } \\
\text { small relative to home } \\
\text { range; risk at harbor } \\
\text { opening where boat } \\
\text { traffic and porpoises } \\
\text { occur; boat-porpoise } \\
\text { collisions infrequent }\end{array}$ & $\begin{array}{l}\text { Stroud and } \\
\text { Roffe } 1979\end{array}$ \\
\hline
\end{tabular}

\begin{tabular}{lr}
\hline Environmental Assessment for Siting Wave \& Tidal & D-27 \\
Energy Projects &
\end{tabular}


Appendix D - Effects of Small Commercial OPT Power Buoy Project Humboldt

\begin{tabular}{|c|c|c|c|c|c|c|c|}
\hline Project activity & Action & $\begin{array}{l}\text { Description of } \\
\text { action's effect on } \\
\text { species or group }\end{array}$ & $\begin{array}{l}\text { Spatial extent of } \\
\text { effect (low, med, } \\
\text { high) relative to } \\
\text { project }\end{array}$ & $\begin{array}{l}\text { Temporal } \\
\text { extent of effect } \\
\text { (low, med, } \\
\text { high) relative to } \\
\text { project }\end{array}$ & $\begin{array}{l}\text { Effect's overlap } \\
\text { with critical life } \\
\text { stage, behavior, } \\
\text { habitat (low, med, } \\
\text { high) relative to } \\
\text { species/group }\end{array}$ & $\begin{array}{l}\text { Overall risk to } \\
\text { indicator (low, med, } \\
\text { high, unknown) }\end{array}$ & Source(s) \\
\hline & $\begin{array}{l}\text { Oil/chemical } \\
\text { release }\end{array}$ & $\begin{array}{l}\text { Ingestion orally while } \\
\text { feeding or breathing, } \\
\text { breathing exhaust } \\
\text { fumes, accumulation of } \\
\text { toxins. Assumes no } \\
\text { catastrophic spills. }\end{array}$ & $\begin{array}{l}\text { Med, significant } \\
\text { increase in existing } \\
\text { traffic but the volume } \\
\text { released low and } \\
\text { dispersed }\end{array}$ & $\begin{array}{l}\text { Low, occurs } \\
\text { over } 1 \text { to } 2 \\
\text { summers }\end{array}$ & $\begin{array}{l}\text { Low for all age } \\
\text { classes; potential for } \\
\text { exposure while } \\
\text { foraging and while } \\
\text { resting in Bay }\end{array}$ & $\begin{array}{l}\text { Low; potential } \\
\text { exposure area small } \\
\text { relative to home range; } \\
\text { additional inputs } \\
\text { compound elevated } \\
\text { contaminant levels }\end{array}$ & $\begin{array}{l}\text { Brookens et al. } \\
\text { 2007, Le Boeuf } \\
\text { et al. } 2002\end{array}$ \\
\hline & $\begin{array}{l}\text { Noise and } \\
\text { vibration }\end{array}$ & $\begin{array}{l}\text { Avoidance, masking of } \\
\text { environmental cues. } \\
\text { TTS, resulting in } \\
\text { potential increase in } \\
\text { predation }\end{array}$ & $\begin{array}{l}\text { High, significant } \\
\text { increase in traffic (esp } \\
\text { in bay and jetties); } \\
\text { inshore and at harbor } \\
\text { entrance where } \\
\text { porpoises move in and } \\
\text { out }\end{array}$ & $\begin{array}{l}\text { Low, occurs } \\
\text { over } 1 \text { to } 2 \\
\text { summers }\end{array}$ & $\begin{array}{l}\text { Low, may affect } \\
\text { adults and calves } \\
\text { while foraging and } \\
\text { traveling parallel to } \\
\text { shore; }\end{array}$ & $\begin{array}{l}\text { Med; could mask } \\
\text { envir. cues over long } \\
\text { distance; effects } \\
\text { greater in bay and } \\
\text { around project area; } \\
\text { but short duration }\end{array}$ & $\begin{array}{l}\text { Erbe and } \\
\text { Farmer } 2000\end{array}$ \\
\hline \multirow{3}{*}{$\begin{array}{l}\text { Decommissioning } \\
\text { of structures on } \\
\text { water's surface or } \\
\text { seabed }\end{array}$} & $\begin{array}{l}\text { Noise and } \\
\text { vibration }\end{array}$ & $\begin{array}{l}\text { Avoidance of area, } \\
\text { affects foraging and } \\
\text { travel, masks } \\
\text { environmental cues, } \\
\text { echolocation and } \\
\text { communication signals }\end{array}$ & $\begin{array}{l}\text { Med; sound likely to } \\
\text { be detected over large } \\
\text { area (several 10s of } \\
\mathrm{km}^{2} \text { ) }\end{array}$ & $\begin{array}{l}\text { Low, only } 1 \\
\text { summer }\end{array}$ & $\begin{array}{l}\text { Med, potential } \\
\text { temporary impacts } \\
\text { on hearing if } \\
\text { porpoises attracted } \\
\text { to buoys to forage } \\
\text { on fish aggregates }\end{array}$ & $\begin{array}{l}\text { Low; harbor porpoises } \\
\text { do not appear to forage } \\
\text { around industrial } \\
\text { activities }\end{array}$ & Todd et al. 2009 \\
\hline & $\begin{array}{l}\text { Oil/chemical } \\
\text { release }\end{array}$ & $\begin{array}{l}\text { Ingestion orally while } \\
\text { feeding; breathing } \\
\text { exhaust fumes; } \\
\text { accumulation of toxins. } \\
\text { Assuming no } \\
\text { catastrophic spills. }\end{array}$ & $\begin{array}{l}\text { Med, significant } \\
\text { increase in use of } \\
\text { chemicals; volume of } \\
\text { fluids released low- } \\
\text { med, and pre-existing } \\
\text { ambient levels high }\end{array}$ & $\begin{array}{l}\text { Low, only } 1 \text { to } 2 \\
\text { summers }\end{array}$ & $\begin{array}{l}\text { Low for all age } \\
\text { classes; potential for } \\
\text { exposure while } \\
\text { foraging around } \\
\text { structures }\end{array}$ & $\begin{array}{l}\text { Low; potential } \\
\text { exposure area small } \\
\text { relative to home range; } \\
\text { additional inputs } \\
\text { compound elevated } \\
\text { contaminant levels }\end{array}$ & $\begin{array}{l}\text { Brookens et al. } \\
\text { 2007, Le Boeuf } \\
\text { et al. } 2002\end{array}$ \\
\hline & $\begin{array}{l}\text { Deconstruction } \\
\text { and deck lights }\end{array}$ & $\begin{array}{l}\text { May attract porpoise } \\
\text { prey, and indirectly } \\
\text { porpoise increasing } \\
\text { potential for impacts } \\
\text { related to other actions }\end{array}$ & $\begin{array}{l}\text { Low to med; project } \\
\text { site small relative to } \\
\text { range of porpoises; } \\
\text { lights may attract prey } \\
\text { and concentrate } \\
\text { porpoises }\end{array}$ & $\begin{array}{l}\text { Low, only } 1 \text { to } 2 \\
\text { summers }\end{array}$ & $\begin{array}{l}\text { Area affected by } \\
\text { lights small relative } \\
\text { to home range }\end{array}$ & $\begin{array}{l}\text { Low due to high levels } \\
\text { of pre-existing boat } \\
\text { traffic }\end{array}$ & Todd et al. 2009 \\
\hline
\end{tabular}

\begin{tabular}{lr}
\hline Environmental Assessment for Siting Wave \& Tidal & D-28 \\
Energy Projects &
\end{tabular}


Appendix D - Effects of Small Commercial OPT Power Buoy Project Humboldt

Potential effects on harbor seal due to Small Commercial OPT Power Buoy Project at Humboldt site

\begin{tabular}{|c|c|c|c|c|c|c|c|}
\hline Project activity & Project action & $\begin{array}{l}\text { Description of } \\
\text { action's effect on } \\
\text { indicator }\end{array}$ & $\begin{array}{l}\text { Spatial exposure } \\
\text { to indicator (low, } \\
\text { med, high) }\end{array}$ & $\begin{array}{l}\text { Temporal } \\
\text { exposure to } \\
\text { indicator (low, } \\
\text { med, high) } \\
\end{array}$ & $\begin{array}{l}\text { Effect's overlap } \\
\text { with critical life } \\
\text { stage, behavior, } \\
\text { habitat, or resource } \\
\text { (low, med, high) } \\
\end{array}$ & $\begin{array}{l}\text { Overall risk to } \\
\text { indicator (low, } \\
\text { med, high, } \\
\text { unknown) }\end{array}$ & Source(s) \\
\hline \multicolumn{8}{|c|}{ Construction } \\
\hline \multirow{3}{*}{$\begin{array}{l}\text { Boat traffic, } \\
\text { assumed to occur } \\
\text { day and night }\end{array}$} & Direct impact & Collision injuries & $\begin{array}{l}\text { High, significant } \\
\text { increase existing } \\
\text { traffic (esp in bay } \\
\text { and jetties) }\end{array}$ & $\begin{array}{l}\text { Low, occurring } \\
\text { over } 1 \text { to } 2 \\
\text { summers }\end{array}$ & $\begin{array}{l}\text { Low for adults; med } \\
\text { for pups foraging and } \\
\text { travel in construction, } \\
\text { transport zones; High } \\
\text { for pups in bay. }\end{array}$ & $\begin{array}{l}\text { Low; vessels similar } \\
\text { to existing fleet(s) } \\
\text { and do not represent } \\
\text { new types of impact }\end{array}$ & $\begin{array}{l}\text { Stroud and Roffe } \\
1979\end{array}$ \\
\hline & $\begin{array}{l}\text { Noise and } \\
\text { vibration }\end{array}$ & $\begin{array}{l}\text { Avoidance, masks } \\
\text { environmental } \\
\text { cues. Reduced } \\
\text { hearing sensitivity } \\
\text { Temporary } \\
\text { Threshold Shift } \\
\text { (TTS) could } \\
\text { increase predation }\end{array}$ & $\begin{array}{l}\text { Med, significant } \\
\text { increase in boat } \\
\text { traffic, noise } \\
\text { travels well } \\
\text { beyond project } \\
\text { area }\end{array}$ & $\begin{array}{l}\text { Low, occurring } \\
\text { over } 1 \text { to } 2 \\
\text { summers }\end{array}$ & $\begin{array}{l}\text { Low for adults; med } \\
\text { for pups due to } \\
\text { foraging and travel } \\
\text { in and near } \\
\text { construction and } \\
\text { transport zones; } \\
\text { High for pups in } \\
\text { bay. }\end{array}$ & $\begin{array}{l}\text { Low; similar species } \\
\text { (Phoca hispida) } \\
\text { show considerable } \\
\text { tolerance to similar } \\
\text { types of noise }\end{array}$ & $\begin{array}{l}\text { Blackwell et al. } \\
\text { 2004, Kastak et al. } \\
\text { 2005, Tougaard et } \\
\text { al. } 2009\end{array}$ \\
\hline & $\begin{array}{l}\text { Oil/chemical } \\
\text { release }\end{array}$ & $\begin{array}{l}\text { Ingestion, fur } \\
\text { fouling, breathing } \\
\text { exhaust fumes, } \\
\text { accumulation of } \\
\text { toxins. Assumes no } \\
\text { catastrophic spills. }\end{array}$ & $\begin{array}{l}\text { Med, significant } \\
\text { increase existing } \\
\text { traffic but the } \\
\text { volume of fluids } \\
\text { released low }\end{array}$ & $\begin{array}{l}\text { Low, occurring } \\
\text { over } 1 \text { to } 2 \\
\text { summers }\end{array}$ & $\begin{array}{l}\text { Low; potential } \\
\text { contamination in } \\
\text { Humboldt Bay and } \\
\text { near offshore site, } \\
\text { contaminants passed } \\
\text { to pups }\end{array}$ & $\begin{array}{l}\text { Low; low volume of } \\
\text { contaminants } \\
\text { expected and not } \\
\text { significantly greater } \\
\text { then background }\end{array}$ & $\begin{array}{l}\text { Brookens et al. } \\
2007\end{array}$ \\
\hline \multirow[t]{2}{*}{$\begin{array}{l}\text { Construction and } \\
\text { installation of } \\
\text { electrical collector } \\
\text { system, mooring } \\
\text { cables, anchors or } \\
\text { footings, devices }\end{array}$} & $\begin{array}{l}\text { Noise and } \\
\text { vibration }\end{array}$ & $\begin{array}{l}\text { Avoidance of area, } \\
\text { masking of } \\
\text { environmental } \\
\text { cues, TTS could } \\
\text { increase predation. }\end{array}$ & $\begin{array}{l}\text { High, sound likely } \\
\text { to be detectable up } \\
\text { to } \sim 3 \mathrm{~km} \text { from } \\
\text { project site }\end{array}$ & $\begin{array}{l}\text { Low, occurring } \\
\text { over } 1 \text { to } 2 \\
\text { summers }\end{array}$ & $\begin{array}{l}\text { Low-med for adults } \\
\text { med for pups due to } \\
\text { foraging and travel } \\
\text { in and near } \\
\text { construction zones; }\end{array}$ & $\begin{array}{l}\text { Low; similar species } \\
\text { (Phoca hispida) } \\
\text { show considerable } \\
\text { tolerance to similar } \\
\text { types of noise }\end{array}$ & $\begin{array}{l}\text { Blackwell et al. } \\
2004 \text {, Kastak et al. } \\
2005\end{array}$ \\
\hline & $\begin{array}{l}\text { Oil/chemical } \\
\text { release }\end{array}$ & $\begin{array}{l}\text { Ingestion, fur } \\
\text { fouling, breathing } \\
\text { exhaust fumes, } \\
\text { accumulation of } \\
\text { toxins. Assumes no } \\
\text { catastrophic spills. }\end{array}$ & $\begin{array}{l}\text { Med, significant } \\
\text { increase existing } \\
\text { traffic but the } \\
\text { volume of fluids } \\
\text { released low }\end{array}$ & $\begin{array}{l}\text { Low, occurring } \\
\text { over } 1 \text { to } 2 \\
\text { summers }\end{array}$ & $\begin{array}{l}\text { Low for all age } \\
\text { classes in Humboldt } \\
\text { Bay and offshore, } \\
\text { foraging and } \\
\text { pupping }\end{array}$ & $\begin{array}{l}\text { Low; low volume of } \\
\text { contaminants } \\
\text { expected and not } \\
\text { significantly greater } \\
\text { then existing } \\
\text { background levels }\end{array}$ & $\begin{array}{l}\text { Brookens et al. } \\
2007\end{array}$ \\
\hline
\end{tabular}

\begin{tabular}{lr}
\hline Environmental Assessment for Siting Wave \& Tidal & D-29 \\
Energy Projects &
\end{tabular}


Appendix D - Effects of Small Commercial OPT Power Buoy Project Humboldt

\begin{tabular}{|c|c|c|c|c|c|c|c|}
\hline Project activity & Project action & $\begin{array}{l}\text { Description of } \\
\text { action's effect on } \\
\text { indicator }\end{array}$ & $\begin{array}{l}\text { Spatial exposure } \\
\text { to indicator (low, } \\
\text { med, high) }\end{array}$ & $\begin{array}{l}\text { Temporal } \\
\text { exposure to } \\
\text { indicator (low, } \\
\text { med, high) }\end{array}$ & $\begin{array}{l}\text { Effect's overlap } \\
\text { with critical life } \\
\text { stage, behavior, } \\
\text { habitat, or resource } \\
\text { (low, med, high) }\end{array}$ & $\begin{array}{l}\text { Overall risk to } \\
\text { indicator (low, } \\
\text { med, high, } \\
\text { unknown) }\end{array}$ & Source(s) \\
\hline & $\begin{array}{l}\text { Construction } \\
\text { lights, vessel deck } \\
\text { lights and } \\
\text { spotlights. }\end{array}$ & $\begin{array}{l}\text { Visual } \\
\text { disorientation could } \\
\text { lead to collision, } \\
\text { enhance foraging } \\
\text { on species attracted } \\
\text { to light }\end{array}$ & $\begin{array}{l}\text { Low-high } \\
\text { dependant on how } \\
\text { many buoys are } \\
\text { installed } \\
\text { simultaneously, } \\
\text { and the required } \\
\text { light levels }\end{array}$ & $\begin{array}{l}\text { Low, occurring } \\
\text { over } 1 \text { to } 2 \\
\text { summers }\end{array}$ & $\begin{array}{l}\text { Low for all age } \\
\text { classes }\end{array}$ & $\begin{array}{l}\text { Low; effect highly } \\
\text { localized; may } \\
\text { enhance foraging of } \\
\text { prey attracted to } \\
\text { lights; may increase } \\
\text { exposure if attracted } \\
\text { to project activities }\end{array}$ & $\begin{array}{l}\text { Yurk and Trites } \\
2000\end{array}$ \\
\hline $\begin{array}{l}\text { Directional drilling, } \\
\text { and laying cable } \\
\text { under/on seabed }\end{array}$ & $\begin{array}{l}\text { Noise and } \\
\text { vibration }\end{array}$ & $\begin{array}{l}\text { Avoidance of area, } \\
\text { masks } \\
\text { environmental } \\
\text { cues, TTS could } \\
\text { increase predation }\end{array}$ & $\begin{array}{l}\text { Med, seals likely } \\
\text { to avoid areas } \\
\text { occupied by cable } \\
\text { and support } \\
\text { vessels }\end{array}$ & $\begin{array}{l}\text { Low, occurring } \\
\text { over } 1 \text { summer }\end{array}$ & $\begin{array}{l}\text { Low for all age } \\
\text { classes; avoidance of } \\
\text { construction area } \\
\text { occupied by vessels }\end{array}$ & $\begin{array}{l}\text { Low; activities } \\
\text { highly localized and } \\
\text { short term; seals } \\
\text { likely to acclimate } \\
\text { and tolerate noise }\end{array}$ & $\begin{array}{l}\text { Blackwell et al. } \\
2004\end{array}$ \\
\hline \multicolumn{8}{|c|}{ Operation and Maintenance } \\
\hline \multirow{3}{*}{ Boat traffic } & Direct impact & Collision injuries & $\begin{array}{l}\text { Medium, increase } \\
\text { in existing traffic } \\
\text { (esp in bay and } \\
\text { jetties) }\end{array}$ & $\begin{array}{l}\text { Med, annual } \\
\text { maintenance } \\
\text { assumed during } 3 \\
\text { months of } \\
\text { summer over } \\
\text { project duration }\end{array}$ & $\begin{array}{l}\text { Low for adults; med } \\
\text { for pups foraging and } \\
\text { travel in construction } \\
\text { and transport zones; } \\
\text { High for pups in bay. }\end{array}$ & $\begin{array}{l}\text { Low; possible sig. } \\
\text { increase in boat } \\
\text { traffic; vessels } \\
\text { similar to existing } \\
\text { and do not represent } \\
\text { new type of impact }\end{array}$ & $\begin{array}{l}\text { Stroud and Roffe } \\
1979\end{array}$ \\
\hline & $\begin{array}{l}\text { Oil/chemical } \\
\text { release }\end{array}$ & $\begin{array}{l}\text { Ingestion, fur } \\
\text { fouling, breathing } \\
\text { exhaust fumes, } \\
\text { accumulation of } \\
\text { toxins. Assumes no } \\
\text { catastrophic spills. }\end{array}$ & $\begin{array}{l}\text { Low, slight } \\
\text { increase existing } \\
\text { traffic but the } \\
\text { volume of fluids } \\
\text { released low }\end{array}$ & $\begin{array}{l}\text { Med, routine } \\
\text { maintenance } \\
\text { occurs over life } \\
\text { of the project } \\
\text { during summer }\end{array}$ & $\begin{array}{l}\text { Low to med; } \\
\text { potential } \\
\text { contamination in } \\
\text { Bay and offshore, } \\
\text { contaminants passed } \\
\text { to pups }\end{array}$ & $\begin{array}{l}\text { Low; low volume of } \\
\text { contaminants } \\
\text { expected and not } \\
\text { significantly greater } \\
\text { than background } \\
\text { levels }\end{array}$ & $\begin{array}{l}\text { Brookens et al. } \\
2007\end{array}$ \\
\hline & $\begin{array}{l}\text { Noise and } \\
\text { vibration }\end{array}$ & $\begin{array}{l}\text { Avoidance of area, } \\
\text { masking of } \\
\text { environmental } \\
\text { cues, TTS could } \\
\text { increase predation }\end{array}$ & $\begin{array}{l}\text { Med, slight } \\
\text { increase in boat } \\
\text { traffic, noise } \\
\text { travels beyond } \\
\text { project boundaries }\end{array}$ & $\begin{array}{l}\text { Med, boat traffic } \\
\text { limited to } \\
\text { summers but for } \\
\text { life of the project }\end{array}$ & $\begin{array}{l}\text { Low for adults; med } \\
\text { for pups foraging and } \\
\text { travel in construction } \\
\text { and transport zones; } \\
\text { med-high for pups in } \\
\text { bay. }\end{array}$ & $\begin{array}{l}\text { Low; similar species } \\
\text { (Phoca hispida) } \\
\text { show considerable } \\
\text { tolerance to similar } \\
\text { types of noise }\end{array}$ & $\begin{array}{l}\text { Blackwell et al. } \\
\text { 2004; Kastak et al. } \\
\text { 2005; Tougaard et } \\
\text { al. } 2009\end{array}$ \\
\hline $\begin{array}{l}\text { Operation of } \\
\text { turbines or other } \\
\text { moving parts of } \\
\text { devices }\end{array}$ & $\begin{array}{l}\text { Moving device } \\
\text { parts }\end{array}$ & $\begin{array}{l}\text { Movement of the } \\
\text { heaving point } \\
\text { absorber and } \\
\text { mooring lines could } \\
\text { injure animals }\end{array}$ & $\begin{array}{l}\text { Low, the areas of } \\
\text { movement are } \\
\text { small compared to } \\
\text { project area }\end{array}$ & $\begin{array}{l}\text { High, the } \\
\text { movement } \\
\text { occurs over the } \\
\text { life of the project }\end{array}$ & $\begin{array}{l}\text { Low for all age } \\
\text { classes, seals } \\
\text { sensitive to water } \\
\text { movement, unlikely } \\
\text { to be near moving } \\
\text { parts }\end{array}$ & $\begin{array}{l}\text { Low; seals unlikely } \\
\text { to approach moving } \\
\text { devices close } \\
\text { enough to be at } \\
\text { significant risk }\end{array}$ & $\begin{array}{l}\text { Dehnhardt et al. } \\
\text { 1998, Stroud and } \\
\text { Roffe } 1979\end{array}$ \\
\hline
\end{tabular}

\begin{tabular}{lr}
\hline Environmental Assessment for Siting Wave \& Tidal & D-30 H. T. Harvey \& Associates \\
Energy Projects & \\
24 November 2009
\end{tabular}


Appendix D - Effects of Small Commercial OPT Power Buoy Project Humboldt

\begin{tabular}{|c|c|c|c|c|c|c|c|}
\hline Project activity & Project action & $\begin{array}{l}\text { Description of } \\
\text { action's effect on } \\
\text { indicator }\end{array}$ & $\begin{array}{l}\text { Spatial exposure } \\
\text { to indicator (low, } \\
\text { med, high) }\end{array}$ & $\begin{array}{l}\text { Temporal } \\
\text { exposure to } \\
\text { indicator (low, } \\
\text { med, high) }\end{array}$ & $\begin{array}{l}\text { Effect's overlap } \\
\text { with critical life } \\
\text { stage, behavior, } \\
\text { habitat, or resource } \\
\text { (low, med, high) }\end{array}$ & $\begin{array}{l}\text { Overall risk to } \\
\text { indicator (low, } \\
\text { med, high, } \\
\text { unknown) }\end{array}$ & Source(s) \\
\hline & $\begin{array}{l}\text { Noise and } \\
\text { vibration }\end{array}$ & $\begin{array}{l}\text { Avoidance of area, } \\
\text { masking of } \\
\text { environmental } \\
\text { cues, TTS possible } \\
\text { which could } \\
\text { increase predation }\end{array}$ & $\begin{array}{l}\text { Sound propagated } \\
\text { over significant } \\
\text { distance }(\sim 3 \mathrm{~km}) \text {; } \\
\text { likely to produce } \\
\text { behavioral reaction } \\
\text { at close range } \\
(<15 \mathrm{M})\end{array}$ & $\begin{array}{l}\text { High, any effect } \\
\text { would be } \\
\text { constant over } \\
\text { project duration }\end{array}$ & $\begin{array}{l}\text { Low; structure } \\
\text { readily avoidable; } \\
\text { may exclude seals } \\
\text { from immediate area } \\
\text { of devices }\end{array}$ & $\begin{array}{l}\text { Low; similar species } \\
\text { (Phoca hispida) } \\
\text { show considerable } \\
\text { tolerance to similar } \\
\text { types of noise }\end{array}$ & $\begin{array}{l}\text { Blackwell et al. } \\
\text { 2004, Kastak et al. } \\
\text { 2005, Tougaard et } \\
\text { al. } 2009\end{array}$ \\
\hline $\begin{array}{l}\text { Structures in water } \\
\text { column and on } \\
\text { seabed, such as } \\
\text { devices and } \\
\text { moorings and } \\
\text { footings }\end{array}$ & Structure & $\begin{array}{l}\text { Artificial reef } \\
\text { effects could attract } \\
\text { fish and increase } \\
\text { foraging; collisions, } \\
\text { entanglement with } \\
\text { derelict gear caught } \\
\text { on moorings }\end{array}$ & $\begin{array}{l}\text { Low, not much } \\
\text { structure nearby } \\
\text { but home ranges } \\
\text { large. pup home } \\
\text { range } 10.4 \mathrm{~km}^{2} \\
\text { and adults travel } \\
\text { range is } 30-45 \mathrm{~km} \text {. }\end{array}$ & $\begin{array}{l}\text { High, structure } \\
\text { present over life } \\
\text { of project }\end{array}$ & $\begin{array}{l}\text { Med, structure } \\
\text { readily avoided; } \\
\text { could increase } \\
\text { foraging on prey } \\
\text { attracted to structure }\end{array}$ & $\begin{array}{l}\text { Low; effect on } \\
\text { population likely } \\
\text { insignificant; } \\
\text { possible risk to } \\
\text { individuals if they } \\
\text { approach device } \\
\text { closely }\end{array}$ & $\begin{array}{l}\text { Herder 1986, } \\
\text { Loughlin 1974, } \\
\text { Relini et al. } 2000\end{array}$ \\
\hline $\begin{array}{l}\text { Electricity } \\
\text { conduction through } \\
\text { cable }\end{array}$ & EMF & Unknown & $\begin{array}{l}\text { Unknown, cable } \\
\text { shielding and } \\
\text { burial provides } \\
\text { some attenuation }\end{array}$ & $\begin{array}{l}\text { High if an effect, } \\
\text { it would occur } \\
\text { over life of the } \\
\text { project }\end{array}$ & $\begin{array}{l}\text { Unknown; any } \\
\text { effects would likely } \\
\text { impact all age } \\
\text { classes }\end{array}$ & $\begin{array}{l}\text { Unknown; High } \\
\text { uncertainty about } \\
\text { effects on marine } \\
\text { mammals }\end{array}$ & $\begin{array}{l}\text { Boehlert et al. } \\
2008\end{array}$ \\
\hline \multirow{2}{*}{$\begin{array}{l}\text { Structures on } \\
\text { water's surface }\end{array}$} & Structure & Collision & $\begin{array}{l}\text { Low, harbor seals } \\
\text { do not haul out, } \\
\text { structure occupies } \\
\text { very small portion } \\
\text { of home range. }\end{array}$ & $\begin{array}{l}\text { High, structure } \\
\text { present over life } \\
\text { of project }\end{array}$ & $\begin{array}{l}\text { Low for all age } \\
\text { classes; harbor seals } \\
\text { do no haul out on } \\
\text { such structures }\end{array}$ & $\begin{array}{l}\text { Low, structure easily } \\
\text { avoided visually; } \\
\text { similar to existing } \\
\text { navigation buoys; }\end{array}$ & $\begin{array}{l}\text { Schusterman and } \\
\text { Balliet } 1970\end{array}$ \\
\hline & Navigation lights & $\begin{array}{l}\text { Visual } \\
\text { disorientation could } \\
\text { lead to collision, } \\
\text { could also enhance } \\
\text { foraging on species } \\
\text { attracted to light }\end{array}$ & $\begin{array}{l}\text { Low, device lights } \\
\text { of low intensity, } \\
\text { shielded, intended } \\
\text { for navigation } \\
\text { safety }\end{array}$ & $\begin{array}{l}\text { High, lights } \\
\text { shining over life } \\
\text { of the project }\end{array}$ & $\begin{array}{l}\text { Low; may have } \\
\text { effect on adults and } \\
\text { pups foraging and } \\
\text { traveling }\end{array}$ & $\begin{array}{l}\text { Low; effects not } \\
\text { expected to be } \\
\text { significant; lights } \\
\text { similar to existing } \\
\text { navigation buoy } \\
\text { lights }\end{array}$ & $\begin{array}{l}\text { Yurk and Trites } \\
2000\end{array}$ \\
\hline \multicolumn{8}{|c|}{ Decommissioning } \\
\hline Boat traffic & Direct impact & Collision injuries & $\begin{array}{l}\text { High, significant } \\
\text { increase existing } \\
\text { traffic (esp in bay } \\
\text { and jetties) }\end{array}$ & $\begin{array}{l}\text { Low, occurring } \\
\text { over } 1 \text { to } 2 \\
\text { summers }\end{array}$ & $\begin{array}{l}\text { Low for adults; med } \\
\text { for pups due to } \\
\text { foraging and travel } \\
\text { in construction and } \\
\text { transport zones; } \\
\text { High for pups in } \\
\text { bay. }\end{array}$ & $\begin{array}{l}\text { Low; possible } \\
\text { significant increase } \\
\text { in boat traffic; } \\
\text { vessels similar to } \\
\text { existing and do not } \\
\text { represent new types } \\
\text { of impact }\end{array}$ & $\begin{array}{l}\text { Stroud and Roffe } \\
1979\end{array}$ \\
\hline
\end{tabular}

\begin{tabular}{lr}
\hline Environmental Assessment for Siting Wave \& Tidal & D-31 \\
Energy Projects &
\end{tabular}


Appendix D - Effects of Small Commercial OPT Power Buoy Project Humboldt

\begin{tabular}{|c|c|c|c|c|c|c|c|}
\hline Project activity & Project action & $\begin{array}{l}\text { Description of } \\
\text { action's effect on } \\
\text { indicator }\end{array}$ & $\begin{array}{l}\text { Spatial exposure } \\
\text { to indicator (low, } \\
\text { med, high) }\end{array}$ & $\begin{array}{l}\text { Temporal } \\
\text { exposure to } \\
\text { indicator (low, } \\
\text { med, high) }\end{array}$ & $\begin{array}{l}\text { Effect's overlap } \\
\text { with critical life } \\
\text { stage, behavior, } \\
\text { habitat, or resource } \\
\text { (low, med, high) }\end{array}$ & $\begin{array}{l}\text { Overall risk to } \\
\text { indicator (low, } \\
\text { med, high, } \\
\text { unknown) }\end{array}$ & Source(s) \\
\hline & $\begin{array}{l}\text { Oil/chemical } \\
\text { release }\end{array}$ & $\begin{array}{l}\text { Ingestion, fur } \\
\text { fouling, breathing } \\
\text { exhaust fumes, } \\
\text { accumulation of } \\
\text { toxins. Assumes no } \\
\text { catastrophic spills. }\end{array}$ & $\begin{array}{l}\text { Med, significant } \\
\text { increase existing } \\
\text { traffic but the } \\
\text { volume of fluids } \\
\text { released low }\end{array}$ & $\begin{array}{l}\text { Low, occurring } \\
\text { over } 1 \text { to } 2 \\
\text { summers }\end{array}$ & $\begin{array}{l}\text { Low; for all age } \\
\text { classes potential } \\
\text { contamination in } \\
\text { Humboldt Bay and } \\
\text { near offshore site, } \\
\text { contaminants passed } \\
\text { to pups }\end{array}$ & $\begin{array}{l}\text { Low; low volume of } \\
\text { contaminants } \\
\text { expected and not } \\
\text { significantly greater } \\
\text { than background } \\
\text { levels }\end{array}$ & $\begin{array}{l}\text { Brookens et al. } \\
2007\end{array}$ \\
\hline & $\begin{array}{l}\text { Noise and } \\
\text { vibration }\end{array}$ & $\begin{array}{l}\text { Avoidance, masks } \\
\text { environmental } \\
\text { cues, TTS possible } \\
\text { which could } \\
\text { increase predation. }\end{array}$ & $\begin{array}{l}\text { Med, significant } \\
\text { increase in boat } \\
\text { traffic, noise } \\
\text { travels well } \\
\text { beyond project } \\
\text { area }\end{array}$ & $\begin{array}{l}\text { Low, occurring } \\
\text { over } 1 \text { to } 2 \\
\text { summers }\end{array}$ & $\begin{array}{l}\text { Low for adults; med } \\
\text { for pups due to } \\
\text { foraging and travel in } \\
\text { construction and } \\
\text { transport zones; High } \\
\text { for pups in bay. }\end{array}$ & $\begin{array}{l}\text { Low; similar species } \\
\text { (Phoca hispida) } \\
\text { show considerable } \\
\text { tolerance to similar } \\
\text { types of noise }\end{array}$ & $\begin{array}{l}\text { Blackwell et al. } \\
\text { 2004, Kastak et al. } \\
\text { 2005, Tougaard et } \\
\text { al. } 2009\end{array}$ \\
\hline \multirow{3}{*}{$\begin{array}{l}\text { Decommissioning } \\
\text { of structures on } \\
\text { water's surface or } \\
\text { seabed }\end{array}$} & $\begin{array}{l}\text { Noise and } \\
\text { vibration }\end{array}$ & $\begin{array}{l}\text { Avoidance, masks } \\
\text { environmental } \\
\text { cues, TTS possible } \\
\text { which could } \\
\text { increase predation. }\end{array}$ & $\begin{array}{l}\text { High, sound likely } \\
\text { to be detectable up } \\
\text { to } \sim 3 \mathrm{~km} \text { from } \\
\text { project site }\end{array}$ & $\begin{array}{l}\text { Low, occurring } \\
\text { over } 1 \text { to } 2 \\
\text { summers }\end{array}$ & $\begin{array}{l}\text { Low for adults; med } \\
\text { for pups due to } \\
\text { foraging and travel } \\
\text { in and near de- } \\
\text { construction zones }\end{array}$ & $\begin{array}{l}\text { Low; similar species } \\
\text { (Phoca hispida) } \\
\text { show considerable } \\
\text { tolerance to similar } \\
\text { types of noise }\end{array}$ & $\begin{array}{l}\text { Blackwell et al. } \\
2004 \text {, Kastak et al. } \\
2005\end{array}$ \\
\hline & $\begin{array}{l}\text { Deconstruction } \\
\text { lights, vessel deck } \\
\text { lights and } \\
\text { spotlights. }\end{array}$ & $\begin{array}{l}\text { Visual } \\
\text { disorientation could } \\
\text { lead to collision, } \\
\text { but could also } \\
\text { enhance foraging } \\
\text { on species attracted } \\
\text { to light }\end{array}$ & $\begin{array}{l}\text { Low-high } \\
\text { dependant on how } \\
\text { many buoys are } \\
\text { installed } \\
\text { simultaneously, } \\
\text { and the required } \\
\text { light levels }\end{array}$ & $\begin{array}{l}\text { Low, occurring } \\
\text { over } 1 \text { to } 2 \\
\text { summers }\end{array}$ & $\begin{array}{l}\text { Low for all age } \\
\text { classes, could be } \\
\text { adverse and } \\
\text { beneficial effects as } \\
\text { it may enhance } \\
\text { foraging on prey } \\
\text { attracted to lights }\end{array}$ & $\begin{array}{l}\text { Low; overall effect } \\
\text { localized; may } \\
\text { enhance foraging of } \\
\text { prey attracted to } \\
\text { lights; may increase } \\
\text { exposure if attracted } \\
\text { to project activities }\end{array}$ & $\begin{array}{l}\text { Yurk and Trites } \\
2000\end{array}$ \\
\hline & $\begin{array}{l}\text { Oil/chemical } \\
\text { release }\end{array}$ & $\begin{array}{l}\text { Ingestion, fur } \\
\text { fouling, breathing } \\
\text { exhaust fumes, } \\
\text { accumulation of } \\
\text { toxins. Assumes no } \\
\text { catastrophic spills. }\end{array}$ & $\begin{array}{l}\text { Med, significant } \\
\text { increase existing } \\
\text { traffic but the } \\
\text { volume of fluids } \\
\text { released low }\end{array}$ & $\begin{array}{l}\text { Low, occurring } \\
\text { over } 1 \text { to } 2 \\
\text { summers }\end{array}$ & $\begin{array}{l}\text { Low for all age } \\
\text { classes in Humboldt } \\
\text { Bay and offshore, } \\
\text { foraging and } \\
\text { pupping }\end{array}$ & $\begin{array}{l}\text { Low; low volume of } \\
\text { contaminants } \\
\text { expected and not } \\
\text { significantly greater } \\
\text { than background } \\
\text { levels }\end{array}$ & $\begin{array}{l}\text { Brookens et al. } \\
2007\end{array}$ \\
\hline
\end{tabular}

\begin{tabular}{lr}
\hline Environmental Assessment for Siting Wave \& Tidal & D-32 \\
Energy Projects & T. Harvey \& Associates \\
24 November 2009
\end{tabular}


Appendix D - Effects of Small Commercial OPT Power Buoy Project Humboldt

Potential effects on Humpback Whales due to Small Commercial OPT Power Buoy Project at Humboldt site

\begin{tabular}{|c|c|c|c|c|c|c|c|}
\hline Project activity & Project action & $\begin{array}{l}\text { Description of } \\
\text { action's effect on } \\
\text { indicator }\end{array}$ & $\begin{array}{l}\text { Spatial exposure } \\
\text { to indicator (low, } \\
\text { med, high) }\end{array}$ & $\begin{array}{l}\text { Temporal } \\
\text { exposure to } \\
\text { indicator (low, } \\
\text { med, high) } \\
\end{array}$ & $\begin{array}{l}\text { Effect's overlap } \\
\text { with critical life } \\
\text { stage, behavior, } \\
\text { habitat, or resource } \\
\text { (low, med, high) }\end{array}$ & $\begin{array}{l}\text { Overall risk to } \\
\text { indicator (low, med, } \\
\text { high, unknown) }\end{array}$ & Source(s) \\
\hline \multicolumn{8}{|c|}{ Construction } \\
\hline \multirow{3}{*}{ Boat traffic } & Direct impact & Collision injuries & $\begin{array}{l}\text { Med-high, increase } \\
\text { in existing traffic } \\
\text { but in small area of } \\
\text { their large range }\end{array}$ & $\begin{array}{l}\text { Low, occurring } \\
\text { over } 1 \text { to } 2 \\
\text { summers }\end{array}$ & $\begin{array}{l}\text { Med-high; especially } \\
\text { for summer foraging }\end{array}$ & $\begin{array}{l}\text { High; overlap in use of } \\
\text { coastal waters; } \\
\text { humpback-boat } \\
\text { collision rate one of } \\
\text { highest for cetaceans }\end{array}$ & $\begin{array}{l}\text { Van Waerebeek } \\
\text { and Leaper } 2008\end{array}$ \\
\hline & Noise and vibration & $\begin{array}{l}\text { Avoidance, masks } \\
\text { envir. cues, } \\
\text { communication } \\
\text { signals }\end{array}$ & $\begin{array}{l}\text { Med to high, } \\
\text { sound travels far, } \\
\text { animals will hear it } \\
\text { outside of the } \\
\text { project area }\end{array}$ & $\begin{array}{l}\text { Low, occurring } \\
\text { over } 1 \text { to } 2 \\
\text { summers }\end{array}$ & $\begin{array}{l}\text { Med, especially for } \\
\text { summer foraging in } \\
\text { coastal waters }\end{array}$ & $\begin{array}{l}\text { Med-high; overlap in } \\
\text { use of coastal waters } \\
\text { foraging and travel }\end{array}$ & \\
\hline & $\begin{array}{l}\text { Oil/chemical } \\
\text { release }\end{array}$ & $\begin{array}{l}\text { Ingestion, } \\
\text { breathing exhaust } \\
\text { fumes, overall } \\
\text { accumulation of } \\
\text { toxins. }\end{array}$ & $\begin{array}{l}\text { Low, significant } \\
\text { increase in existing } \\
\text { traffic but the } \\
\text { volume of fluids } \\
\text { released low }\end{array}$ & $\begin{array}{l}\text { Low, occurring } \\
\text { over } 1 \text { to } 2 \\
\text { summers }\end{array}$ & $\begin{array}{l}\text { Low for whales } \\
\text { foraging in project } \\
\text { transport corridors; } \\
\text { increased } \\
\text { contamination levels }\end{array}$ & $\begin{array}{l}\text { Low; area of potential } \\
\text { exposure small relative } \\
\text { to range; inputs may } \\
\text { compound effects of } \\
\text { elevated levels }\end{array}$ & $\begin{array}{l}\text { Le Boeuf et al. } \\
2002\end{array}$ \\
\hline \multirow{3}{*}{$\begin{array}{l}\text { Construction and } \\
\text { installation of } \\
\text { electrical collector } \\
\text { system, mooring } \\
\text { cables, anchors or } \\
\text { foundations, and } \\
\text { devices }\end{array}$} & Noise and vibration & \begin{tabular}{l|} 
Avoidance, masks \\
envir. cues, \\
communication \\
signals
\end{tabular} & $\begin{array}{l}\text { Med to high, sound } \\
\text { travels far, animals } \\
\text { will hear it outside } \\
\text { project area }\end{array}$ & $\begin{array}{l}\text { Low, occurring } \\
\text { over } 1 \text { to } 2 \\
\text { summers }\end{array}$ & $\begin{array}{l}\text { Med, especially for } \\
\text { summer foraging }\end{array}$ & $\begin{array}{l}\text { Med-high; overlap in } \\
\text { use of coastal waters; } \\
\text { may cause avoidance of } \\
\text { project area }\end{array}$ & \\
\hline & $\begin{array}{l}\text { Construction lights, } \\
\text { vessel deck lights } \\
\text { and spotlights. }\end{array}$ & $\begin{array}{l}\text { Visual disorientation } \\
\text { could lead to } \\
\text { collision, enhance } \\
\text { foraging on species } \\
\text { attracted to light } \\
\end{array}$ & Low & $\begin{array}{l}\text { Low, occurring } \\
\text { over } 1 \text { to } 2 \\
\text { summers }\end{array}$ & $\begin{array}{l}\text { Low, effects' spatial } \\
\text { extent small } \\
\text { compared to animal's } \\
\text { range }\end{array}$ & $\begin{array}{l}\text { Low; limited spatial } \\
\text { impact; relatively short } \\
\text { duration }\end{array}$ & \\
\hline & $\begin{array}{l}\text { Oil/chemical } \\
\text { release }\end{array}$ & \begin{tabular}{l|} 
Ingestion, \\
breathing exhaust \\
fumes, overall \\
accumulation of \\
toxins.
\end{tabular} & $\begin{array}{l}\text { Med, increase in } \\
\text { use of chemicals; } \\
\text { volume of fluids } \\
\text { released low }\end{array}$ & $\begin{array}{l}\text { Low, occurring } \\
\text { over } 1 \text { to } 2 \\
\text { summers }\end{array}$ & $\begin{array}{l}\text { Low; due to } \\
\text { potentially increased } \\
\text { ambient } \\
\text { contamination levels }\end{array}$ & $\begin{array}{l}\text { Low; area of exposure } \\
\text { small relative to range; } \\
\text { inputs may compound } \\
\text { elevated contaminant } \\
\text { levels }\end{array}$ & $\begin{array}{l}\text { Le Boeuf et al. } \\
2002\end{array}$ \\
\hline $\begin{array}{l}\text { Directional drilling, } \\
\text { and laying cable } \\
\text { under/on seabed }\end{array}$ & Noise and vibration & $\begin{array}{l}\text { Avoidance, masks } \\
\text { environmental } \\
\text { cues, } \\
\text { communication } \\
\text { signals }\end{array}$ & $\begin{array}{l}\text { Med to high, } \\
\text { sound travels far, } \\
\text { animals will hear it } \\
\text { outside of the } \\
\text { project area }\end{array}$ & $\begin{array}{l}\text { Low, occurring } \\
\text { over } 1 \text { summer }\end{array}$ & $\begin{array}{l}\text { Med, especially for } \\
\text { summer foraging }\end{array}$ & $\begin{array}{l}\text { Low; most activity is } \\
\text { near shore; whales will } \\
\text { readily avoid cable } \\
\text { vessel as it moves } \\
\text { offshore at low speed }\end{array}$ & \\
\hline
\end{tabular}
Operation and Maintenance

\begin{tabular}{lr}
\hline Environmental Assessment for Siting Wave \& Tidal & D-33 \\
Energy Projects & H. Harvey \& Associates \\
24 November 2009
\end{tabular}


Appendix D - Effects of Small Commercial OPT Power Buoy Project Humboldt

\begin{tabular}{|c|c|c|c|c|c|c|c|}
\hline Project activity & Project action & $\begin{array}{l}\text { Description of } \\
\text { action's effect on } \\
\text { indicator }\end{array}$ & $\begin{array}{l}\text { Spatial exposure } \\
\text { to indicator (low, } \\
\text { med, high) }\end{array}$ & $\begin{array}{l}\text { Temporal } \\
\text { exposure to } \\
\text { indicator (low, } \\
\text { med, high) }\end{array}$ & $\begin{array}{l}\text { Effect's overlap } \\
\text { with critical life } \\
\text { stage, behavior, } \\
\text { habitat, or resource } \\
\text { (low, med, high) } \\
\end{array}$ & $\begin{array}{l}\text { Overall risk to } \\
\text { indicator (low, med, } \\
\text { high, unknown) }\end{array}$ & Source(s) \\
\hline \multirow{3}{*}{ Boat traffic } & Direct impact & Collision injuries & $\begin{array}{l}\text { Low, increase in } \\
\text { existing traffic but } \\
\text { in small area of } \\
\text { large range. }\end{array}$ & $\begin{array}{l}\text { High, traffic will } \\
\text { occur during } \\
\text { summer over } \\
\text { life of project }\end{array}$ & $\begin{array}{l}\text { Low; especially for } \\
\text { summer foraging }\end{array}$ & $\begin{array}{l}\text { Low; overlap in use of } \\
\text { coastal waters; } \\
\text { humpback-boat } \\
\text { collision rate one of } \\
\text { highest for cetaceans }\end{array}$ & $\begin{array}{l}\text { Van Waerebeek } \\
\text { and Leaper } 2008\end{array}$ \\
\hline & $\begin{array}{l}\text { Oil/chemical } \\
\text { release }\end{array}$ & $\begin{array}{l}\text { Ingestion, } \\
\text { breathing exhaust } \\
\text { fumes, overall } \\
\text { accumulation of } \\
\text { toxins. }\end{array}$ & $\begin{array}{l}\text { Low, slight } \\
\text { increase existing } \\
\text { traffic but the } \\
\text { volume of fluids } \\
\text { released low } \\
\end{array}$ & $\begin{array}{l}\text { High, traffic } \\
\text { increase will } \\
\text { occur in summer } \\
\text { over life of } \\
\text { project } \\
\end{array}$ & $\begin{array}{l}\text { Low, boat frequency } \\
\text { low and limited to } \\
\text { summer, and effects } \\
\text { cover a small part of } \\
\text { large range }\end{array}$ & $\begin{array}{l}\text { Low; area of exposure } \\
\text { small relative to range; } \\
\text { inputs may compound } \\
\text { effects of elevated } \\
\text { levels }\end{array}$ & \\
\hline & Noise and vibration & $\begin{array}{l}\text { Avoidance, masks } \\
\text { of envir. cues, } \\
\text { communication } \\
\text { signals }\end{array}$ & $\begin{array}{l}\text { Med, sound travels } \\
\text { far, animals will } \\
\text { hear it outside of } \\
\text { the project area }\end{array}$ & $\begin{array}{l}\text { High, occurring } \\
\text { mostly during } \\
\text { summers over } \\
\text { life of project }\end{array}$ & $\begin{array}{l}\text { Med, especially for } \\
\text { summer foraging }\end{array}$ & $\begin{array}{l}\text { Med; project area } \\
\text { within summer foraging } \\
\text { area; increase in } \\
\text { background noise level }\end{array}$ & \\
\hline \multirow{2}{*}{$\begin{array}{l}\text { Operation of } \\
\text { turbines or other } \\
\text { moving parts of } \\
\text { devices }\end{array}$} & $\begin{array}{l}\text { Moving device } \\
\text { parts }\end{array}$ & $\begin{array}{l}\text { Movement could } \\
\text { injure animals }\end{array}$ & $\begin{array}{l}\text { Low, area of } \\
\text { devices small } \\
\text { relative to project } \\
\text { area }\end{array}$ & $\begin{array}{l}\text { High, movement } \\
\text { continuous } \\
\text { throughout life } \\
\text { of the project }\end{array}$ & $\begin{array}{l}\text { Low, interaction } \\
\text { between animals and } \\
\text { device movement } \\
\text { low. }\end{array}$ & $\begin{array}{l}\text { Low; unlikely to } \\
\text { approach close enough } \\
\text { to mechanical } \\
\text { movements to be at risk }\end{array}$ & \\
\hline & Noise and vibration & $\begin{array}{l}\text { Avoidance, masks } \\
\text { of envir. cues, } \\
\text { communication } \\
\text { signals }\end{array}$ & $\begin{array}{l}\text { Unknown, } \\
\text { attenuation over } \\
\text { large area and } \\
\text { ambient noise } \\
\text { unknown }\end{array}$ & $\begin{array}{l}\text { High, noise will } \\
\text { be continuous } \\
\text { throughout life } \\
\text { of the project }\end{array}$ & $\begin{array}{l}\text { Low; may cause } \\
\text { avoidance of project } \\
\text { area; site in small } \\
\text { area of home range }\end{array}$ & $\begin{array}{l}\text { Unknown; project area } \\
\text { within summer foraging } \\
\text { area; potential increase } \\
\text { in background noise }\end{array}$ & \\
\hline $\begin{array}{l}\text { Structures in water } \\
\text { column and on } \\
\text { seabed, such as } \\
\text { devices, moorings } \\
\text { and footings }\end{array}$ & Structure & $\begin{array}{l}\text { Collisions, } \\
\text { entanglement with } \\
\text { derelict gear caught } \\
\text { on moorings, reef } \\
\text { effects may increase } \\
\text { foraging }\end{array}$ & $\begin{array}{l}\text { Low, home range } \\
\text { large compared to } \\
\text { project area; may } \\
\text { create new habitat }\end{array}$ & $\begin{array}{l}\text { High, any } \\
\text { effects would } \\
\text { occur over life } \\
\text { of the project. }\end{array}$ & $\begin{array}{l}\text { Low, overall project } \\
\text { area covers a small } \\
\text { part of their large } \\
\text { range }\end{array}$ & $\begin{array}{l}\text { Low; humpbacks } \\
\text { readily avoid structures; } \\
\text { low risk of } \\
\text { entanglement }\end{array}$ & \\
\hline $\begin{array}{l}\text { Electricity } \\
\text { conduction through } \\
\text { cable }\end{array}$ & EMF & Unknown & $\begin{array}{l}\text { Unknown, cable } \\
\text { shielding and } \\
\text { burial provides } \\
\text { some attenuation }\end{array}$ & $\begin{array}{l}\text { High if an effect } \\
\text { electricity } \\
\text { generated over } \\
\text { life of project }\end{array}$ & Unknown & $\begin{array}{l}\text { Unknown; High } \\
\text { uncertainty about } \\
\text { effects on marine } \\
\text { mammals }\end{array}$ & $\begin{array}{l}\text { Boehlert et al. } \\
2008\end{array}$ \\
\hline
\end{tabular}

\begin{tabular}{lr}
\hline Environmental Assessment for Siting Wave \& Tidal & D-34 \\
Energy Projects &
\end{tabular}


Appendix D - Effects of Small Commercial OPT Power Buoy Project Humboldt

\begin{tabular}{|c|c|c|c|c|c|c|c|}
\hline Project activity & Project action & $\begin{array}{l}\text { Description of } \\
\text { action's effect on } \\
\text { indicator }\end{array}$ & $\begin{array}{l}\text { Spatial exposure } \\
\text { to indicator (low, } \\
\text { med, high) }\end{array}$ & $\begin{array}{l}\text { Temporal } \\
\text { exposure to } \\
\text { indicator (low, } \\
\text { med, high) }\end{array}$ & $\begin{array}{l}\text { Effect's overlap } \\
\text { with critical life } \\
\text { stage, behavior, } \\
\text { habitat, or resource } \\
\text { (low, med, high) } \\
\end{array}$ & $\begin{array}{l}\text { Overall risk to } \\
\text { indicator (low, med, } \\
\text { high, unknown) }\end{array}$ & Source(s) \\
\hline \multirow{2}{*}{$\begin{array}{l}\text { Structures on } \\
\text { water's surface }\end{array}$} & Structure & $\begin{array}{l}\text { Collision, } \\
\text { entrapment, } \\
\text { artificial reef may } \\
\text { attract krill and } \\
\text { increase foraging }\end{array}$ & $\begin{array}{l}\text { Low, home range } \\
\text { large compared to } \\
\text { project area; may } \\
\text { create new habitat }\end{array}$ & $\begin{array}{l}\text { High, positive or } \\
\text { negative effects } \\
\text { would occur } \\
\text { over life of } \\
\text { project. }\end{array}$ & $\begin{array}{l}\text { Low, effects occur in } \\
\text { tiny part of large } \\
\text { range }\end{array}$ & $\begin{array}{l}\text { Low; structure readily } \\
\text { avoided }\end{array}$ & \\
\hline & Navigation lights & $\begin{array}{l}\text { Visual } \\
\text { disorientation } \\
\text { could lead to } \\
\text { collision, enhance } \\
\text { foraging } \\
\end{array}$ & $\begin{array}{l}\text { Low, device lights } \\
\text { are low intensity, } \\
\text { and shielded. }\end{array}$ & $\begin{array}{l}\text { High, lights } \\
\text { shining over life } \\
\text { of the project }\end{array}$ & $\begin{array}{l}\text { Low, limited area of } \\
\text { effect similar to } \\
\text { existing structures } \\
\text { along coast }\end{array}$ & $\begin{array}{l}\text { Low; lights relatively } \\
\text { dim; spatial extent of } \\
\text { effect limited }\end{array}$ & \\
\hline \multicolumn{8}{|c|}{ Decommissioning } \\
\hline \multirow{3}{*}{ Boat traffic } & Direct impact & Collision injuries & $\begin{array}{l}\text { Med, significant } \\
\text { increase in existing } \\
\text { traffic in small } \\
\text { area }\end{array}$ & $\begin{array}{l}\text { Low, occurs } \\
\text { over } 1 \text { summer }\end{array}$ & $\begin{array}{l}\text { Med-high; especially } \\
\text { for summer foraging }\end{array}$ & $\begin{array}{l}\text { High; overlap in use of } \\
\text { coastal waters; } \\
\text { humpback-boat } \\
\text { collision rate one of } \\
\text { highest for cetaceans }\end{array}$ & $\begin{array}{l}\text { Van Waerebeek } \\
\text { and Leaper } 2008\end{array}$ \\
\hline & $\begin{array}{l}\text { Oil/chemical } \\
\text { release }\end{array}$ & $\begin{array}{l}\text { Ingestion, } \\
\text { breathing exhaust } \\
\text { fumes, overall } \\
\text { accumulation of } \\
\text { toxins. }\end{array}$ & $\begin{array}{l}\text { Low, significant } \\
\text { increase in existing } \\
\text { traffic but the } \\
\text { volume of fluids } \\
\text { released low }\end{array}$ & $\begin{array}{l}\text { Low, occurring } \\
\text { over } 1 \text { to } 2 \\
\text { summers }\end{array}$ & $\begin{array}{l}\text { Low for whales } \\
\text { foraging in or near } \\
\text { project transport } \\
\text { corridors; increased } \\
\text { contamination levels }\end{array}$ & $\begin{array}{l}\text { Low; area of potential } \\
\text { exposure small relative } \\
\text { to range; inputs may } \\
\text { compound elevated } \\
\text { levels }\end{array}$ & $\begin{array}{l}\text { Le Boeuf et al. } \\
2002\end{array}$ \\
\hline & Noise and vibration & $\begin{array}{l}\text { Avoidance, } \\
\text { masking of envir. } \\
\text { cues, } \\
\text { communication } \\
\text { signals }\end{array}$ & $\begin{array}{l}\text { Med to high, } \\
\text { sound travels far, } \\
\text { animals will hear it } \\
\text { outside of the } \\
\text { project area }\end{array}$ & $\begin{array}{l}\text { Low, occurring } \\
\text { over } 1 \text { to } 2 \\
\text { summers }\end{array}$ & $\begin{array}{l}\text { Med, especially for } \\
\text { summer foraging }\end{array}$ & $\begin{array}{l}\text { Med-high; overlap in } \\
\text { use of coastal waters; } \\
\text { foraging and travel; } \\
\text { increase in background } \\
\text { noise levels }\end{array}$ & \\
\hline \multirow[t]{2}{*}{$\begin{array}{l}\text { Decommissioning } \\
\text { of structures on } \\
\text { water's surface or } \\
\text { seabed }\end{array}$} & Noise and vibration & $\begin{array}{l}\text { Avoidance, } \\
\text { masking of envir. } \\
\text { cues, } \\
\text { communication } \\
\text { signals }\end{array}$ & $\begin{array}{l}\text { Med to high, } \\
\text { sound travels far, } \\
\text { animals will hear it } \\
\text { outside of the } \\
\text { project area }\end{array}$ & $\begin{array}{l}\text { Low, occurring } \\
\text { over } 1 \text { to } 2 \\
\text { summers }\end{array}$ & $\begin{array}{l}\text { Med, especially for } \\
\text { summer foraging }\end{array}$ & $\begin{array}{l}\text { Med-high; overlap in } \\
\text { use of coastal waters } \\
\text { foraging and travel; } \\
\text { may cause avoidance of } \\
\text { project area }\end{array}$ & \\
\hline & $\begin{array}{l}\text { Oil/chemical } \\
\text { release }\end{array}$ & $\begin{array}{l}\text { Ingestion, } \\
\text { breathing exhaust } \\
\text { fumes, overall } \\
\text { accumulation of } \\
\text { toxins. }\end{array}$ & $\begin{array}{l}\text { Med, increase in } \\
\text { use of chemicals; } \\
\text { volume of fluids } \\
\text { released low }\end{array}$ & $\begin{array}{l}\text { Low, occurring } \\
\text { over } 1 \text { to } 2 \\
\text { summers }\end{array}$ & $\begin{array}{l}\text { Low-med; due to } \\
\text { potentially increased } \\
\text { ambient } \\
\text { contamination levels }\end{array}$ & $\begin{array}{l}\text { Low; area of potential } \\
\text { exposure small relative } \\
\text { to range; inputs may } \\
\text { compound elevated } \\
\text { levels }\end{array}$ & $\begin{array}{l}\text { Le Boeuf et al. } \\
2002\end{array}$ \\
\hline
\end{tabular}

\begin{tabular}{|c|c|c|}
\hline $\begin{array}{l}\text { Environmental Assessment for Siting Wave \& Tidal } \\
\text { Energy Projects }\end{array}$ & D-35 & $\begin{array}{r}\text { H. T. Harvey \& Associates } \\
24 \text { November } 2009\end{array}$ \\
\hline
\end{tabular}


Appendix D - Effects of Small Commercial OPT Power Buoy Project Humboldt

\begin{tabular}{|c|c|c|c|c|c|c|c|}
\hline Project activity & Project action & $\begin{array}{l}\text { Description of } \\
\text { action's effect on } \\
\text { indicator }\end{array}$ & $\begin{array}{l}\text { Spatial exposure } \\
\text { to indicator (low, } \\
\text { med, high) }\end{array}$ & $\begin{array}{l}\text { Temporal } \\
\text { exposure to } \\
\text { indicator (low, } \\
\text { med, high) }\end{array}$ & $\begin{array}{l}\text { Effect's overlap } \\
\text { with critical life } \\
\text { stage, behavior, } \\
\text { habitat, or resource } \\
\text { (low, med, high) }\end{array}$ & $\begin{array}{l}\text { Overall risk to } \\
\text { indicator (low, med, } \\
\text { high, unknown) }\end{array}$ & Source(s) \\
\hline & $\begin{array}{l}\text { Construction lights, } \\
\text { vessel deck lights } \\
\text { and spotlights. }\end{array}$ & $\begin{array}{l}\text { Visual } \\
\text { disorientation } \\
\text { could lead to } \\
\text { collision, enhance } \\
\text { foraging }\end{array}$ & Low & $\begin{array}{l}\text { Low, occurring } \\
\text { over } 1 \text { to } 2 \\
\text { summers }\end{array}$ & $\begin{array}{l}\text { Low, effects' spatial } \\
\text { extent small } \\
\text { compared to animal's } \\
\text { range }\end{array}$ & $\begin{array}{l}\text { Low; limited spatial } \\
\text { impact; relatively short } \\
\text { duration }\end{array}$ & \\
\hline
\end{tabular}

Potential effects on Killer Whales due to Small Commercial OPT Power Buoy Project at Humboldt site

\begin{tabular}{|c|c|c|c|c|c|c|c|}
\hline Project activity & Project action & $\begin{array}{l}\text { Description of } \\
\text { action's effect on } \\
\text { indicator }\end{array}$ & $\begin{array}{l}\text { Spatial exposure } \\
\text { on indicator (low, } \\
\text { med, high) }\end{array}$ & $\begin{array}{l}\text { Temporal } \\
\text { exposure on } \\
\text { indicator (low, } \\
\text { med, high) }\end{array}$ & $\begin{array}{l}\text { Effect's overlap } \\
\text { with critical life } \\
\text { stage, behavior, } \\
\text { habitat, or } \\
\text { resource (low, } \\
\text { med, high) }\end{array}$ & $\begin{array}{l}\text { Overall risk to } \\
\text { indicator (low, } \\
\text { med, high, } \\
\text { unknown) }\end{array}$ & Source(s) \\
\hline \multicolumn{8}{|c|}{ Construction } \\
\hline \multirow{3}{*}{ Boat traffic } & Direct impact & Collision injuries & $\begin{array}{l}\text { Low, significant } \\
\text { increase in existing } \\
\text { traffic but in a } \\
\text { small area of large } \\
\text { range. }\end{array}$ & $\begin{array}{l}\text { Low, occurring } \\
\text { over } 1 \text { to } 2 \\
\text { summers }\end{array}$ & $\begin{array}{l}\text { Low, effects' } \\
\text { spatial extent small } \\
\text { compared to } \\
\text { animal's range }\end{array}$ & $\begin{array}{l}\text { Low; increase in } \\
\text { boat traffic; killer } \\
\text { whale-boat } \\
\text { collisions not } \\
\text { uncommon }\end{array}$ & $\begin{array}{l}\text { Van Waerebeek } \\
\text { and Leaper } 2008\end{array}$ \\
\hline & Noise and vibration & $\begin{array}{l}\text { Avoidance, } \\
\text { masking of } \\
\text { environmental cues, } \\
\text { communication } \\
\text { signals, } \\
\text { echolocation }\end{array}$ & $\begin{array}{l}\text { Low-Med, sound } \\
\text { travels far, animals } \\
\text { will hear outside } \\
\text { project area, but } \\
\text { affects a small part } \\
\text { of large range }\end{array}$ & $\begin{array}{l}\text { Low, occurring } \\
\text { over } 1 \text { to } 2 \\
\text { summers }\end{array}$ & $\begin{array}{l}\text { Low, small part of } \\
\text { large range. }\end{array}$ & $\begin{array}{l}\text { Low; species may } \\
\text { be displaced from } \\
\text { transport zones by } \\
\text { high amplitude } \\
\text { sound }\end{array}$ & $\begin{array}{l}\text { Morton and } \\
\text { Symonds } 2002\end{array}$ \\
\hline & $\begin{array}{l}\text { Oil/chemical } \\
\text { release }\end{array}$ & $\begin{array}{l}\text { Ingestion, breathing } \\
\text { exhaust fumes, } \\
\text { overall } \\
\text { accumulation of } \\
\text { toxins. }\end{array}$ & $\begin{array}{l}\text { Low, significant } \\
\text { increase in existing } \\
\text { traffic but the } \\
\text { volume of fluids } \\
\text { released low }\end{array}$ & $\begin{array}{l}\text { Low, occurring } \\
\text { over } 1 \text { to } 2 \\
\text { summers }\end{array}$ & $\begin{array}{l}\text { Low, effects' } \\
\text { spatial extent small } \\
\text { compared to } \\
\text { animal's range }\end{array}$ & $\begin{array}{l}\text { Low; area of } \\
\text { exposure small } \\
\text { relative to home } \\
\text { range; inputs may } \\
\text { compound elevated } \\
\text { contaminant levels }\end{array}$ & $\begin{array}{l}\text { Le Boeuf et al. } \\
2002\end{array}$ \\
\hline
\end{tabular}

\begin{tabular}{lrr}
\hline Environmental Assessment for Siting Wave \& Tidal & D-36 & H. T. Harvey \& Associates \\
Energy Projects & & 24 November 2009
\end{tabular}


Appendix D - Effects of Small Commercial OPT Power Buoy Project Humboldt

\begin{tabular}{|c|c|c|c|c|c|c|c|}
\hline Project activity & Project action & $\begin{array}{l}\text { Description of } \\
\text { action's effect on } \\
\text { indicator }\end{array}$ & $\begin{array}{l}\text { Spatial exposure } \\
\text { on indicator (low, } \\
\text { med, high) }\end{array}$ & $\begin{array}{l}\text { Temporal } \\
\text { exposure on } \\
\text { indicator (low, } \\
\text { med, high) }\end{array}$ & $\begin{array}{l}\text { Effect's overlap } \\
\text { with critical life } \\
\text { stage, behavior, } \\
\text { habitat, or } \\
\text { resource (low, } \\
\text { med, high) }\end{array}$ & $\begin{array}{l}\text { Overall risk to } \\
\text { indicator (low, } \\
\text { med, high, } \\
\text { unknown) }\end{array}$ & Source(s) \\
\hline \multirow{3}{*}{$\begin{array}{l}\text { Construction and } \\
\text { installation of } \\
\text { electrical collector } \\
\text { system, mooring } \\
\text { cables, anchors or } \\
\text { foundations, and } \\
\text { devices }\end{array}$} & Noise and vibration & $\begin{array}{l}\text { Avoidance, } \\
\text { masking of } \\
\text { environmental cues, } \\
\text { communication } \\
\text { signals, } \\
\text { echolocation } \\
\end{array}$ & $\begin{array}{l}\text { Low-Med, animals } \\
\text { will hear noise } \\
\text { outside project } \\
\text { area, but affects } \\
\text { small part of large } \\
\text { range }\end{array}$ & $\begin{array}{l}\text { Low, occurring } \\
\text { over } 1 \text { to } 2 \\
\text { summers }\end{array}$ & $\begin{array}{l}\text { Low, effects' } \\
\text { spatial extent small } \\
\text { compared to } \\
\text { animal's range }\end{array}$ & $\begin{array}{l}\text { Low; species may } \\
\text { be displaced by } \\
\text { high amplitude } \\
\text { noise; affects small } \\
\text { portion of range }\end{array}$ & $\begin{array}{l}\text { Morton and } \\
\text { Symonds } 2002\end{array}$ \\
\hline & $\begin{array}{l}\text { Oil/chemical } \\
\text { release }\end{array}$ & $\begin{array}{l}\text { Ingestion, breathing } \\
\text { exhaust fumes, } \\
\text { overall } \\
\text { accumulation of } \\
\text { toxins. }\end{array}$ & $\begin{array}{l}\text { Med, significant } \\
\text { increase in use of } \\
\text { chemicals; volume } \\
\text { released low-med, } \\
\text { and ambient levels } \\
\text { elevated }\end{array}$ & $\begin{array}{l}\text { Low, occurring } \\
\text { over } 1 \text { to } 2 \\
\text { summers }\end{array}$ & $\begin{array}{l}\text { Low, effects' } \\
\text { spatial extent small } \\
\text { compared to } \\
\text { animal's range }\end{array}$ & $\begin{array}{l}\text { Low; area of } \\
\text { potential exposure } \\
\text { small relative to } \\
\text { range; inputs may } \\
\text { compound elevated } \\
\text { contaminant levels }\end{array}$ & $\begin{array}{l}\text { Le Boeuf et al. } \\
2002\end{array}$ \\
\hline & $\begin{array}{l}\text { Construction lights, } \\
\text { vessel deck lights } \\
\text { and spotlights. }\end{array}$ & $\begin{array}{l}\text { Visual } \\
\text { disorientation could } \\
\text { lead to collision, } \\
\text { enhance foraging } \\
\text { on species attracted } \\
\text { to light }\end{array}$ & Low & $\begin{array}{l}\text { Low, occurring } \\
\text { over } 1 \text { to } 2 \\
\text { summers }\end{array}$ & $\begin{array}{l}\text { Low, effects' } \\
\text { spatial extent small } \\
\text { compared to } \\
\text { animal's range }\end{array}$ & $\begin{array}{l}\text { Low; limited } \\
\text { special and } \\
\text { temporal effect }\end{array}$ & \\
\hline $\begin{array}{l}\text { Directional drilling, } \\
\text { and laying cable } \\
\text { under/on seabed }\end{array}$ & Noise and vibration & $\begin{array}{l}\text { Avoidance, } \\
\text { masking of } \\
\text { environmental cues, } \\
\text { communication } \\
\text { signals, } \\
\text { echolocation }\end{array}$ & $\begin{array}{l}\text { Low-Med, sound } \\
\text { travels far, animals } \\
\text { will hear outside of } \\
\text { project area, but } \\
\text { affects a small part } \\
\text { of large range }\end{array}$ & $\begin{array}{l}\text { Low, occurring } \\
\text { over } 1 \text { summer }\end{array}$ & $\begin{array}{l}\text { Low, effects' } \\
\text { spatial extent small } \\
\text { compared to } \\
\text { animal's range }\end{array}$ & $\begin{array}{l}\text { Low; likely } \\
\text { displaced from } \\
\text { area but small } \\
\text { portion of range } \\
\text { and short time } \\
\text { period }\end{array}$ & $\begin{array}{l}\text { Morton and } \\
\text { Symonds } 2002\end{array}$ \\
\hline \multicolumn{8}{|c|}{ Operation and Maintenance } \\
\hline Boat traffic & Direct impact & Collision injuries & $\begin{array}{l}\text { Low, slight } \\
\text { increase in existing } \\
\text { traffic in small area } \\
\text { of their large } \\
\text { range. }\end{array}$ & $\begin{array}{l}\text { High, traffic will } \\
\text { occur over life of } \\
\text { the project in } \\
\text { summer }\end{array}$ & $\begin{array}{l}\text { Low, effects' } \\
\text { spatial extent small } \\
\text { compared to } \\
\text { animal's range }\end{array}$ & $\begin{array}{l}\text { Low; area of effect } \\
\text { small relative to } \\
\text { home range; killer } \\
\text { whale-boat } \\
\text { collisions not } \\
\text { uncommon }\end{array}$ & $\begin{array}{l}\text { Van Waerebeek } \\
\text { and Leaper } 2008\end{array}$ \\
\hline
\end{tabular}

\begin{tabular}{|c|c|c|}
\hline $\begin{array}{l}\text { Environmental Assessment for Siting Wave \& Tidal } \\
\text { Energy Projects }\end{array}$ & D-37 & $\begin{array}{r}\text { H. T. Harvey \& Associates } \\
24 \text { November } 2009\end{array}$ \\
\hline
\end{tabular}


Appendix D - Effects of Small Commercial OPT Power Buoy Project Humboldt

\begin{tabular}{|c|c|c|c|c|c|c|c|}
\hline Project activity & Project action & $\begin{array}{l}\text { Description of } \\
\text { action's effect on } \\
\text { indicator }\end{array}$ & $\begin{array}{l}\text { Spatial exposure } \\
\text { on indicator (low, } \\
\text { med, high) }\end{array}$ & $\begin{array}{l}\text { Temporal } \\
\text { exposure on } \\
\text { indicator (low, } \\
\text { med, high) }\end{array}$ & $\begin{array}{l}\text { Effect's overlap } \\
\text { with critical life } \\
\text { stage, behavior, } \\
\text { habitat, or } \\
\text { resource (low, } \\
\text { med, high) }\end{array}$ & $\begin{array}{l}\text { Overall risk to } \\
\text { indicator (low, } \\
\text { med, high, } \\
\text { unknown) }\end{array}$ & Source(s) \\
\hline & $\begin{array}{l}\text { Oil/chemical } \\
\text { release }\end{array}$ & $\begin{array}{l}\text { Ingestion, breathing } \\
\text { exhaust fumes, } \\
\text { overall } \\
\text { accumulation of } \\
\text { toxins. }\end{array}$ & $\begin{array}{l}\text { Low, increase in } \\
\text { existing traffic but } \\
\text { the volume of } \\
\text { fluids released low }\end{array}$ & $\begin{array}{l}\text { High, traffic will } \\
\text { occur over life of } \\
\text { the project in } \\
\text { summer }\end{array}$ & $\begin{array}{l}\text { Low, effects' } \\
\text { spatial extent small } \\
\text { compared to } \\
\text { animal's range }\end{array}$ & $\begin{array}{l}\text { Low; area of } \\
\text { exposure small } \\
\text { relative to home } \\
\text { range; inputs may } \\
\text { compound elevated } \\
\text { contaminant levels }\end{array}$ & $\begin{array}{l}\text { Le Boeuf et al. } \\
2002\end{array}$ \\
\hline & Noise and vibration & $\begin{array}{l}\text { Avoidance, } \\
\text { masking of } \\
\text { environmental cues, } \\
\text { communication } \\
\text { signals, } \\
\text { echolocation }\end{array}$ & $\begin{array}{l}\text { Low-Med, sound } \\
\text { travels far, animals } \\
\text { will hear it outside } \\
\text { of project area, but } \\
\text { affects a small part } \\
\text { of large range }\end{array}$ & $\begin{array}{l}\text { High, traffic will } \\
\text { occur over life of } \\
\text { the project in } \\
\text { summer }\end{array}$ & $\begin{array}{l}\text { Low, effects' } \\
\text { spatial extent small } \\
\text { compared to } \\
\text { animal's range }\end{array}$ & $\begin{array}{l}\text { Low; species } \\
\text { sensitive to noise; } \\
\text { transport corridor } \\
\text { may be avoided } \\
\text { but small area } \\
\text { compared to range }\end{array}$ & $\begin{array}{l}\text { Morton and } \\
\text { Symonds } 2002\end{array}$ \\
\hline \multirow{2}{*}{$\begin{array}{l}\text { Operation of } \\
\text { turbines or other } \\
\text { moving parts of } \\
\text { devices }\end{array}$} & $\begin{array}{l}\text { Movement of the } \\
\text { heaving point } \\
\text { absorber and } \\
\text { mooring lines }\end{array}$ & $\begin{array}{l}\text { Collision, } \\
\text { entrapment. }\end{array}$ & $\begin{array}{l}\text { Low, area of } \\
\text { devices small }\end{array}$ & $\begin{array}{l}\text { High, movement } \\
\text { will be continuous } \\
\text { throughout the life } \\
\text { of the project }\end{array}$ & $\begin{array}{l}\text { Low, low } \\
\text { interaction between } \\
\text { animals and device } \\
\text { movement }\end{array}$ & $\begin{array}{l}\text { Low; unlikely to } \\
\text { approach close } \\
\text { enough to be } \\
\text { affected by } \\
\text { movements }\end{array}$ & \\
\hline & Noise and vibration & $\begin{array}{l}\text { Avoidance, } \\
\text { masking of } \\
\text { environmental cues, } \\
\text { communication } \\
\text { signals, } \\
\text { echolocation }\end{array}$ & $\begin{array}{l}\text { Unknown, site } \\
\text { occupies small area } \\
\text { of home range; } \\
\text { attenuation over } \\
\text { large area and } \\
\text { ambient noise } \\
\text { unknown }\end{array}$ & $\begin{array}{l}\text { High, any noise } \\
\text { will be continuous } \\
\text { throughout the life } \\
\text { of the project }\end{array}$ & $\begin{array}{l}\text { Medium, } \\
\text { avoidance and } \\
\text { masking of } \\
\text { environmental cues }\end{array}$ & $\begin{array}{l}\text { Unknown; } \\
\text { significant risk if } \\
\text { noise produced } \\
\text { sufficient to } \\
\text { permanently } \\
\text { displace killer } \\
\text { whales from region }\end{array}$ & $\begin{array}{l}\text { Morton and } \\
\text { Symonds } 2002\end{array}$ \\
\hline $\begin{array}{l}\text { Structures in water } \\
\text { column and on } \\
\text { seabed, such as } \\
\text { devices and } \\
\text { moorings and } \\
\text { footings }\end{array}$ & Structure & $\begin{array}{l}\text { Collisions, } \\
\text { entanglement with } \\
\text { derelict gear caught } \\
\text { on moorings; } \\
\text { artificial reef could } \\
\text { attract prey }\end{array}$ & $\begin{array}{l}\text { Low, home range } \\
\text { large compared to } \\
\text { project area; may } \\
\text { create new habitat }\end{array}$ & $\begin{array}{l}\text { High, any positive } \\
\text { or negative effects } \\
\text { would occur over } \\
\text { the life of the } \\
\text { project. }\end{array}$ & $\begin{array}{l}\text { Low, structure } \\
\text { readily avoided; } \\
\text { loose or unattached } \\
\text { cables possible } \\
\text { entanglement risk }\end{array}$ & $\begin{array}{l}\text { Low; may attract } \\
\text { killer whales if the } \\
\text { structures } \\
\text { concentrate } \\
\text { pinnipeds }\end{array}$ & Relini et al. 2000 \\
\hline $\begin{array}{l}\text { Electricity } \\
\text { conduction through } \\
\text { cable }\end{array}$ & EMF & Unknown & $\begin{array}{l}\text { Unknown, cable } \\
\text { shielding and } \\
\text { burial provides } \\
\text { some attenuation }\end{array}$ & $\begin{array}{l}\text { High if there is an } \\
\text { effect because } \\
\text { electricity } \\
\text { generated over life } \\
\text { of the project }\end{array}$ & Unknown & $\begin{array}{l}\text { Unknown; High } \\
\text { uncertainty about } \\
\text { effects on marine } \\
\text { mammals }\end{array}$ & $\begin{array}{l}\text { Boehlert et al. } \\
2008\end{array}$ \\
\hline
\end{tabular}

\begin{tabular}{lr}
\hline Environmental Assessment for Siting Wave \& Tidal & D-38 \\
Energy Projects &
\end{tabular}


Appendix D - Effects of Small Commercial OPT Power Buoy Project Humboldt

\begin{tabular}{|c|c|c|c|c|c|c|c|}
\hline Project activity & Project action & $\begin{array}{l}\text { Description of } \\
\text { action's effect on } \\
\text { indicator }\end{array}$ & $\begin{array}{l}\text { Spatial exposure } \\
\text { on indicator (low, } \\
\text { med, high) }\end{array}$ & $\begin{array}{l}\text { Temporal } \\
\text { exposure on } \\
\text { indicator (low, } \\
\text { med, high) }\end{array}$ & $\begin{array}{l}\text { Effect's overlap } \\
\text { with critical life } \\
\text { stage, behavior, } \\
\text { habitat, or } \\
\text { resource (low, } \\
\text { med, high) }\end{array}$ & $\begin{array}{l}\text { Overall risk to } \\
\text { indicator (low, } \\
\text { med, high, } \\
\text { unknown) }\end{array}$ & Source(s) \\
\hline \multirow{2}{*}{$\begin{array}{l}\text { Structures on } \\
\text { water's surface }\end{array}$} & Structure & $\begin{array}{l}\text { Collision, artificial } \\
\text { reef effects could } \\
\text { attract fish, sea } \\
\text { lions, small } \\
\text { odontocetes }\end{array}$ & $\begin{array}{l}\text { Low, reef effect } \\
\text { area is small } \\
\text { compared to home } \\
\text { range }\end{array}$ & $\begin{array}{l}\text { High, any positive } \\
\text { or negative effects } \\
\text { would occur over } \\
\text { the life of the } \\
\text { project. }\end{array}$ & $\begin{array}{l}\text { Low, may } \\
\text { incrementally } \\
\text { increase forage as } \\
\text { marine reserve }\end{array}$ & $\begin{array}{l}\text { Low; may attract } \\
\text { killer whales if } \\
\text { pinnipeds use the } \\
\text { structure as a haul- } \\
\text { out }\end{array}$ & \\
\hline & Navigation lights & $\begin{array}{l}\text { Visual } \\
\text { disorientation could } \\
\text { lead to collision, } \\
\text { enhance foraging } \\
\text { on species attracted }\end{array}$ & $\begin{array}{l}\text { Low, device lights } \\
\text { are low intensity } \\
\text { and shielded }\end{array}$ & $\begin{array}{l}\text { High, lights } \\
\text { shining over life of } \\
\text { the project }\end{array}$ & $\begin{array}{l}\text { Low, effects' } \\
\text { spatial extent small } \\
\text { compared to } \\
\text { animal's range }\end{array}$ & Low & \\
\hline \multicolumn{8}{|c|}{ Decommissioning } \\
\hline \multirow{3}{*}{ Boat traffic } & Direct impact & Collision injuries & $\begin{array}{l}\text { Low, significant } \\
\text { increase existing } \\
\text { traffic in small area } \\
\text { of their large } \\
\text { range. }\end{array}$ & $\begin{array}{l}\text { Low, occurring } \\
\text { over } 1 \text { summer }\end{array}$ & $\begin{array}{l}\text { Low, effects' } \\
\text { spatial extent small } \\
\text { compared to } \\
\text { animal's range }\end{array}$ & $\begin{array}{l}\text { Low; area of effect } \\
\text { small relative to } \\
\text { home range; killer } \\
\text { whale-boat } \\
\text { collisions not } \\
\text { uncommon }\end{array}$ & $\begin{array}{l}\text { Van Waerebeek } \\
\text { and Leaper } 2008\end{array}$ \\
\hline & Noise and vibration & $\begin{array}{l}\text { Avoidance, } \\
\text { masking of } \\
\text { environmental cues, } \\
\text { communication } \\
\text { signals, } \\
\text { echolocation }\end{array}$ & $\begin{array}{l}\text { Low-Med, sound } \\
\text { travels far, animals } \\
\text { will hear it outside } \\
\text { of project area, but } \\
\text { affects a small part } \\
\text { of large range }\end{array}$ & $\begin{array}{l}\text { Low, occurring } \\
\text { over } 1 \text { summer }\end{array}$ & $\begin{array}{l}\text { Low, small part of } \\
\text { large range. }\end{array}$ & $\begin{array}{l}\text { Low; species may } \\
\text { be displaced from } \\
\text { transport zones by } \\
\text { high amplitude } \\
\text { noise }\end{array}$ & $\begin{array}{l}\text { Morton and } \\
\text { Symonds } 2002\end{array}$ \\
\hline & $\begin{array}{l}\text { Oil/chemical } \\
\text { release }\end{array}$ & $\begin{array}{l}\text { Ingestion, breathing } \\
\text { exhaust fumes, } \\
\text { overall } \\
\text { accumulation of } \\
\text { toxins. }\end{array}$ & $\begin{array}{l}\text { Low, significant } \\
\text { increase existing } \\
\text { traffic but the } \\
\text { volume of fluids } \\
\text { released low }\end{array}$ & $\begin{array}{l}\text { Low, occurring } \\
\text { over } 1 \text { summer }\end{array}$ & $\begin{array}{l}\text { Low, effects' } \\
\text { spatial extent small } \\
\text { compared to } \\
\text { animal's range }\end{array}$ & $\begin{array}{l}\text { Low; inputs may } \\
\text { compound elevated } \\
\text { existing levels }\end{array}$ & $\begin{array}{l}\text { Le Boeuf et al. } \\
2002\end{array}$ \\
\hline $\begin{array}{l}\text { Decommissioning of } \\
\text { structures on water's } \\
\text { surface or seabed }\end{array}$ & Noise and vibration & $\begin{array}{l}\text { Avoidance, masks } \\
\text { envir. cues, } \\
\text { communication } \\
\text { signals, } \\
\text { echolocation }\end{array}$ & $\begin{array}{l}\text { Low-Med, sound } \\
\text { travels far, will } \\
\text { hear it even if they } \\
\text { never approach }\end{array}$ & $\begin{array}{l}\text { Low, occurring } \\
\text { over } 1 \text { summer }\end{array}$ & $\begin{array}{l}\text { Low, effects' } \\
\text { spatial extent small } \\
\text { compared to } \\
\text { animal's range }\end{array}$ & $\begin{array}{l}\text { Low; species may } \\
\text { be displaced from } \\
\text { project area noise }\end{array}$ & $\begin{array}{l}\text { Morton and } \\
\text { Symonds } 2002\end{array}$ \\
\hline
\end{tabular}

\begin{tabular}{lr}
\hline Environmental Assessment for Siting Wave \& Tidal & D-39 \\
Energy Projects &
\end{tabular}


Appendix D - Effects of Small Commercial OPT Power Buoy Project Humboldt

\begin{tabular}{|c|c|c|c|c|c|c|c|}
\hline Project activity & Project action & $\begin{array}{l}\text { Description of } \\
\text { action's effect on } \\
\text { indicator }\end{array}$ & $\begin{array}{l}\text { Spatial exposure } \\
\text { on indicator (low, } \\
\text { med, high) }\end{array}$ & $\begin{array}{l}\text { Temporal } \\
\text { exposure on } \\
\text { indicator (low, } \\
\text { med, high) }\end{array}$ & $\begin{array}{l}\text { Effect's overlap } \\
\text { with critical life } \\
\text { stage, behavior, } \\
\text { habitat, or } \\
\text { resource (low, } \\
\text { med, high) }\end{array}$ & $\begin{array}{l}\text { Overall risk to } \\
\text { indicator (low, } \\
\text { med, high, } \\
\text { unknown) }\end{array}$ & Source(s) \\
\hline & $\begin{array}{l}\text { Oil/chemical } \\
\text { release }\end{array}$ & $\begin{array}{l}\text { Ingestion, breathing } \\
\text { exhaust fumes, } \\
\text { overall } \\
\text { accumulation of } \\
\text { toxins. }\end{array}$ & $\begin{array}{l}\text { Med, significant } \\
\text { increase in use of } \\
\text { chemicals, volume } \\
\text { released low-med, } \\
\text { and pre-existing } \\
\text { levels elevated }\end{array}$ & $\begin{array}{l}\text { Low, occurring } \\
\text { over } 1 \text { to } 2 \\
\text { summers }\end{array}$ & $\begin{array}{l}\text { Low, effects' } \\
\text { spatial extent small } \\
\text { compared to } \\
\text { animal's range }\end{array}$ & $\begin{array}{l}\text { Low; area of } \\
\text { exposure small } \\
\text { relative to home } \\
\text { range; inputs } \\
\text { compound elevated } \\
\text { contaminant levels }\end{array}$ & $\begin{array}{l}\text { Le Boeuf et al. } \\
2002\end{array}$ \\
\hline & $\begin{array}{l}\text { Deconstruction } \\
\text { lights (deck work } \\
\text { lights, spotlights, } \\
\text { vessel navigation } \\
\text { lights) }\end{array}$ & $\begin{array}{l}\text { Visual } \\
\text { disorientation could } \\
\text { lead to collision, } \\
\text { could also enhance } \\
\text { foraging on species } \\
\text { attracted to light }\end{array}$ & $\begin{array}{l}\text { Unknown how } \\
\text { many buoys } \\
\text { installed which } \\
\text { would increase } \\
\text { number of lights }\end{array}$ & $\begin{array}{l}\text { Low, occurring } \\
\text { over } 1 \text { summer }\end{array}$ & $\begin{array}{l}\text { Low, effects' } \\
\text { spatial extent small } \\
\text { compared to } \\
\text { animal's range }\end{array}$ & $\begin{array}{l}\text { Low; limited } \\
\text { spatial and } \\
\text { temporal effect }\end{array}$ & \\
\hline
\end{tabular}

\begin{tabular}{lrr}
\hline Environmental Assessment for Siting Wave \& Tidal & D-40 & H. T. Harvey \& Associates \\
Energy Projects & & 24 November 2009
\end{tabular}


Appendix D - Effects of Small Commercial OPT Power Buoy Project Humboldt

Potential effects on Steller sea lion due to Small Commercial OPT Power Buoy Project at Humboldt site

\begin{tabular}{|c|c|c|c|c|c|c|c|}
\hline Project activity & Project action & $\begin{array}{l}\text { Description of } \\
\text { action's effect on } \\
\text { indicator }\end{array}$ & $\begin{array}{l}\text { Spatial exposure to } \\
\text { indicator (low, med, } \\
\text { high) }\end{array}$ & $\begin{array}{l}\text { Temporal } \\
\text { exposure to } \\
\text { indicator (low, } \\
\text { med, high) } \\
\end{array}$ & $\begin{array}{l}\text { Effect's overlap } \\
\text { with critical life } \\
\text { stage, behavior, } \\
\text { habitat, or } \\
\text { resource (low, } \\
\text { med, high) }\end{array}$ & $\begin{array}{l}\text { Overall risk to } \\
\text { indicator (low, } \\
\text { med, high, } \\
\text { unknown) }\end{array}$ & Source(s) \\
\hline \multicolumn{8}{|c|}{ Construction } \\
\hline \multirow{3}{*}{$\begin{array}{l}\text { Boat traffic, } \\
\text { assumed to occur } \\
\text { day and night }\end{array}$} & Direct impact & Collision injuries & $\begin{array}{l}\text { High, significant } \\
\text { increase in existing } \\
\text { traffic (especially in bay } \\
\text { and jetties) }\end{array}$ & $\begin{array}{l}\text { Low, occurring } \\
\text { over } 1 \text { to } 2 \\
\text { summers }\end{array}$ & $\begin{array}{l}\text { Med for } \\
\text { adults/subadults, } \\
\text { high for juveniles } \\
\text { due to foraging and } \\
\text { travel }\end{array}$ & $\begin{array}{l}\text { Med-high; } \\
\text { significant } \\
\text { increase in boat } \\
\text { traffic and } \\
\text { potential for take } \\
\text { of pups and } \\
\text { juveniles }\end{array}$ & $\begin{array}{l}\text { Stroud and } \\
\text { Roffe } 1979\end{array}$ \\
\hline & $\begin{array}{l}\text { Noise and } \\
\text { vibration }\end{array}$ & $\begin{array}{l}\text { Reduction of } \\
\text { hearing sensitivity } \\
\text { TSS (Temporary } \\
\text { Threshold Shift), } \\
\text { resulting in } \\
\text { potential increase in } \\
\text { predation }\end{array}$ & $\begin{array}{l}\text { Med, significant } \\
\text { increase in boat traffic } \\
\text { between Humboldt Bay } \\
\text { and project area, and } \\
\text { within project area. } \\
\text { Noise will travel beyond } \\
\text { project area. }\end{array}$ & $\begin{array}{l}\text { Low, occurring } \\
\text { over } 1 \text { to } 2 \\
\text { summers }\end{array}$ & $\begin{array}{l}\text { Low for } \\
\text { adults/subadults, } \\
\text { and juveniles } \\
\text { related to foraging, } \\
\text { travel and predation }\end{array}$ & $\begin{array}{l}\text { Low; noise } \\
\text { detectable over } \\
\text { sign. distance } \\
\text { ( 3km); not likely } \\
\text { a threat unless } \\
\text { animals approach } \\
\text { near vessels }\end{array}$ & $\begin{array}{l}\text { Kastak et al. } \\
\text { 2005, Kastelein } \\
\text { et al. 2009, } \\
\text { Tougaard et al. } \\
2009\end{array}$ \\
\hline & $\begin{array}{l}\text { Oil/chemical } \\
\text { release }\end{array}$ & $\begin{array}{l}\text { Ingestion, fur } \\
\text { fouling, breathing } \\
\text { exhaust fumes, } \\
\text { accumulation of } \\
\text { toxins. }\end{array}$ & $\begin{array}{l}\text { Med, significant } \\
\text { increase existing traffic } \\
\text { but the volume of fluids } \\
\text { released low }\end{array}$ & $\begin{array}{l}\text { Low, occurring } \\
\text { over } 1 \text { to } 2 \\
\text { summers }\end{array}$ & $\begin{array}{l}\text { Low; could } \\
\text { contaminate } \\
\text { Humboldt Bay and } \\
\text { offshore, } \\
\text { contaminants } \\
\text { passed to pups }\end{array}$ & $\begin{array}{l}\text { Low; volume of } \\
\text { contaminants low } \\
\text { and not greater } \\
\text { than background } \\
\text { levels }\end{array}$ & $\begin{array}{l}\text { Brookens et al. } \\
\text { 2007, Le Boeuf } \\
\text { et al. } 2002\end{array}$ \\
\hline \multirow[t]{2}{*}{$\begin{array}{l}\text { Construction and } \\
\text { installation of } \\
\text { electrical collector } \\
\text { system, mooring } \\
\text { cables, anchors or } \\
\text { foundations, and } \\
\text { devices }\end{array}$} & $\begin{array}{l}\text { Noise and } \\
\text { vibration }\end{array}$ & $\begin{array}{l}\text { Avoidance of area, } \\
\text { affects foraging and } \\
\text { travel, resting } \\
\text { onshore in Bay; } \\
\text { TSS resulting in } \\
\text { potential increase in } \\
\text { predation }\end{array}$ & $\begin{array}{l}\text { High, sound likely to be } \\
\text { detectable up to } \sim 3 \mathrm{~km} \\
\text { from project site }\end{array}$ & $\begin{array}{l}\text { Low, occurring } \\
\text { over } 1 \text { to } 2 \\
\text { summers }\end{array}$ & $\begin{array}{l}\text { Low for adults; med } \\
\text { for juveniles and } \\
\text { pups due to } \\
\text { foraging and travel } \\
\text { in and near } \\
\text { construction zones; }\end{array}$ & $\begin{array}{l}\text { Low; potential for } \\
\text { noises to impact } \\
\text { hearing if sea } \\
\text { lions approach } \\
\text { and remain in } \\
\text { immediate } \\
\text { proximity }\end{array}$ & $\begin{array}{l}\text { Kastak et al. } \\
\text { 2005, Tougaard } \\
\text { et al. } 2009\end{array}$ \\
\hline & $\begin{array}{l}\text { Oil/chemical } \\
\text { release }\end{array}$ & $\begin{array}{l}\text { Ingestion, fur } \\
\text { fouling, breathing } \\
\text { exhaust fumes, } \\
\text { accumulation of } \\
\text { toxins. }\end{array}$ & $\begin{array}{l}\text { Med; significant } \\
\text { increase in use of } \\
\text { chemicals but the } \\
\text { volume of fluids } \\
\text { released low-med }\end{array}$ & $\begin{array}{l}\text { Low, occurring } \\
\text { over } 1 \text { to } 2 \\
\text { summers }\end{array}$ & $\begin{array}{l}\text { Low; for all age } \\
\text { classes, could } \\
\text { contaminate } \\
\text { Humboldt Bay and } \\
\text { offshore site, } \\
\text { contaminants } \\
\text { passed to pups }\end{array}$ & $\begin{array}{l}\text { Low; low volume } \\
\text { of contaminants } \\
\text { expected and not } \\
\text { greater than } \\
\text { background levels }\end{array}$ & $\begin{array}{l}\text { Brookens et al. } \\
\text { 2007, Le Boeuf } \\
\text { et al. } 2002\end{array}$ \\
\hline
\end{tabular}

\begin{tabular}{lr}
\hline Environmental Assessment for Siting Wave \& Tidal & D-41 \\
Energy Projects &
\end{tabular}


Appendix D - Effects of Small Commercial OPT Power Buoy Project Humboldt

\begin{tabular}{|c|c|c|c|c|c|c|c|}
\hline \multirow[t]{2}{*}{ Project activity } & Project action & $\begin{array}{l}\text { Description of } \\
\text { action's effect on } \\
\text { indicator }\end{array}$ & $\begin{array}{l}\text { Spatial exposure to } \\
\text { indicator (low, med, } \\
\text { high) }\end{array}$ & $\begin{array}{l}\text { Temporal } \\
\text { exposure to } \\
\text { indicator (low, } \\
\text { med, high) } \\
\end{array}$ & $\begin{array}{l}\text { Effect's overlap } \\
\text { with critical life } \\
\text { stage, behavior, } \\
\text { habitat, or } \\
\text { resource (low, } \\
\text { med, high) }\end{array}$ & $\begin{array}{l}\text { Overall risk to } \\
\text { indicator (low, } \\
\text { med, high, } \\
\text { unknown) }\end{array}$ & Source(s) \\
\hline & $\begin{array}{l}\text { Construction } \\
\text { lights (bright deck } \\
\text { lights and } \\
\text { spotlights) }\end{array}$ & $\begin{array}{l}\text { May be attracted to } \\
\text { construction area } \\
\text { increasing } \\
\text { likelihood of other } \\
\text { impacts, could also } \\
\text { enhance foraging } \\
\text { on prey species } \\
\text { attracted to light }\end{array}$ & $\begin{array}{l}\text { Med to high; number of } \\
\text { buoys installed } \\
\text { simultaneously would } \\
\text { affect number of lights } \\
\text { and spatial extent }\end{array}$ & $\begin{array}{l}\text { Low, occurring } \\
\text { over } 2 \text { summers }\end{array}$ & $\begin{array}{l}\text { Low; dependent on } \\
\text { scale of } \\
\text { construction } \\
\text { operations affecting } \\
\text { travel and foraging } \\
\text { at night }\end{array}$ & $\begin{array}{l}\text { Low; exposure } \\
\text { low relative to } \\
\text { home ranges and } \\
\text { short duration of } \\
\text { construction; may } \\
\text { enhance nocturnal } \\
\text { foraging on prey } \\
\text { attracted to lights }\end{array}$ & $\begin{array}{l}\text { Yurk and Trites } \\
2000\end{array}$ \\
\hline $\begin{array}{l}\text { Directional drilling, } \\
\text { and laying cable } \\
\text { under/on seabed }\end{array}$ & $\begin{array}{l}\text { Noise and } \\
\text { vibration }\end{array}$ & $\begin{array}{l}\text { Avoidance of area, } \\
\text { affects foraging and } \\
\text { travel, resting } \\
\text { onshore in Bay }\end{array}$ & $\begin{array}{l}\text { Low, activities focused } \\
\text { nearshore; localized } \\
\text { around support vessels } \\
\text { when offshore }\end{array}$ & $\begin{array}{l}\text { Low, occurring } \\
\text { over } 1 \text { summer }\end{array}$ & $\begin{array}{l}\text { Low, activities } \\
\text { focused nearshore; } \\
\text { localized around } \\
\text { support vessels } \\
\text { when offshore }\end{array}$ & $\begin{array}{l}\text { Low, because } \\
\text { exposure is low } \\
\text { relative to } \\
\text { individual home } \\
\text { range }\end{array}$ & $\begin{array}{l}\text { Kastak et al. } \\
\text { 2005, Tougaard } \\
\text { et al. } 2009\end{array}$ \\
\hline \multicolumn{8}{|c|}{ Operation and Maintenance } \\
\hline \multirow{3}{*}{ Boat traffic. } & Direct impact & Collision injuries & $\begin{array}{l}\text { Medium, increase in } \\
\text { existing traffic } \\
\text { (especially in bay and } \\
\text { jetties) }\end{array}$ & $\begin{array}{l}\text { Medium, } \\
\text { occurring during } \\
\text { summer for life of } \\
\text { the project }\end{array}$ & $\begin{array}{l}\text { Low for } \\
\text { adults/subadults, } \\
\text { med-high for } \\
\text { juveniles due to } \\
\text { foraging and travel }\end{array}$ & $\begin{array}{l}\text { Med; moderate } \\
\text { increase in boat } \\
\text { traffic during } \\
\text { summer lasting } \\
\text { project duration }\end{array}$ & $\begin{array}{l}\text { Stroud and } \\
\text { Roffe } 1979\end{array}$ \\
\hline & $\begin{array}{l}\text { Oil/chemical } \\
\text { release }\end{array}$ & $\begin{array}{l}\text { Bioaccumulation, } \\
\text { fur fouling, } \\
\text { breathing exhaust } \\
\text { fumes, Assumes no } \\
\text { catastrophic spills. }\end{array}$ & $\begin{array}{l}\text { Low, slight increase in } \\
\text { existing traffic but the } \\
\text { volume of fluids } \\
\text { released low }\end{array}$ & $\begin{array}{l}\text { Med, routine } \\
\text { maintenance } \\
\text { occurs over life of } \\
\text { the project during } \\
\text { summer }\end{array}$ & $\begin{array}{l}\text { Low; for all age } \\
\text { classes, could } \\
\text { contaminate } \\
\text { Humboldt Bay and } \\
\text { offshore site, } \\
\text { contaminants } \\
\text { passed to pups }\end{array}$ & $\begin{array}{l}\text { Low; low volume } \\
\text { of contaminants } \\
\text { expected and not } \\
\text { significantly } \\
\text { greater then } \\
\text { background levels }\end{array}$ & $\begin{array}{l}\text { Brookens et al. } \\
\text { 2007, Le Boeuf } \\
\text { et al. } 2002\end{array}$ \\
\hline & $\begin{array}{l}\text { Noise and } \\
\text { vibration }\end{array}$ & $\begin{array}{l}\text { Reduction of } \\
\text { hearing sensitivity } \\
\text { (TSS), resulting in } \\
\text { potential increases } \\
\text { in predation }\end{array}$ & $\begin{array}{l}\text { Med, noise will be } \\
\text { heard past project area } \\
\text { boundaries }(\sim 3 \mathrm{~km}) \text { but } \\
\text { increase in traffic slight. }\end{array}$ & $\begin{array}{l}\text { High, occurs } \\
\text { during summer } \\
\text { months over } \\
\text { project duration }\end{array}$ & $\begin{array}{l}\text { Low for } \\
\text { adults/subadults } \\
\text { related to effects on } \\
\text { foraging and travel; } \\
\text { med for juveniles } \\
\text { may increase } \\
\text { predation }\end{array}$ & $\begin{array}{l}\text { Low; noise } \\
\text { detectable over } \\
\text { sign. distance; not } \\
\text { likely a threat } \\
\text { unless animals } \\
\text { approach near } \\
\text { moving vessels }\end{array}$ & $\begin{array}{l}\text { Kastak et al. } \\
\text { 2005, Kastelein } \\
\text { et al. 2009, } \\
\text { Tougaard et al. } \\
2009\end{array}$ \\
\hline
\end{tabular}

\begin{tabular}{lr}
\hline Environmental Assessment for Siting Wave \& Tidal & D-42 \\
Energy Projects &
\end{tabular}


Appendix D - Effects of Small Commercial OPT Power Buoy Project Humboldt

\begin{tabular}{|c|c|c|c|c|c|c|c|}
\hline Project activity & Project action & $\begin{array}{l}\text { Description of } \\
\text { action's effect on } \\
\text { indicator }\end{array}$ & $\begin{array}{l}\text { Spatial exposure to } \\
\text { indicator (low, med, } \\
\text { high) }\end{array}$ & $\begin{array}{l}\text { Temporal } \\
\text { exposure to } \\
\text { indicator (low, } \\
\text { med, high) }\end{array}$ & $\begin{array}{l}\text { Effect's overlap } \\
\text { with critical life } \\
\text { stage, behavior, } \\
\text { habitat, or } \\
\text { resource (low, } \\
\text { med, high) }\end{array}$ & $\begin{array}{l}\text { Overall risk to } \\
\text { indicator (low, } \\
\text { med, high, } \\
\text { unknown) }\end{array}$ & Source(s) \\
\hline \multirow{2}{*}{$\begin{array}{l}\text { Operation of } \\
\text { turbines or other } \\
\text { moving parts of } \\
\text { devices }\end{array}$} & $\begin{array}{l}\text { Moving device } \\
\text { parts }\end{array}$ & $\begin{array}{l}\text { Movement of the } \\
\text { heaving point } \\
\text { absorber and } \\
\text { mooring lines could } \\
\text { injure animals }\end{array}$ & $\begin{array}{l}\text { Low, the areas of } \\
\text { movement are small } \\
\text { compared to project } \\
\text { area }\end{array}$ & $\begin{array}{l}\text { High, the } \\
\text { movement occurs } \\
\text { over the life of the } \\
\text { project }\end{array}$ & $\begin{array}{l}\text { Low for all age } \\
\text { classes; }\end{array}$ & $\begin{array}{l}\text { Low, unlikely to } \\
\text { approach close } \\
\text { enough to be at } \\
\text { risk; small area of } \\
\text { effect compared to } \\
\text { home range }\end{array}$ & Merrick 1996 \\
\hline & $\begin{array}{l}\text { Noise and } \\
\text { vibration }\end{array}$ & $\begin{array}{l}\text { Reduction of } \\
\text { hearing sensitivity } \\
\text { resulting in } \\
\text { potential increase in } \\
\text { predation, } \\
\text { avoidance, masking } \\
\text { environmental cues }\end{array}$ & $\begin{array}{l}\text { Likely to extend well be } \\
\text { project area; other } \\
\text { pinnipeds should } \\
\text { auditory response at } \\
\text { distances of } \sim 3 \mathrm{~km} \text {. }\end{array}$ & $\begin{array}{l}\text { High, if effect } \\
\text { occurs, } \\
\text { mechanical } \\
\text { movement occurs } \\
\text { over the life of the } \\
\text { project }\end{array}$ & $\begin{array}{l}\text { Low for } \\
\text { adults/subadults } \\
\text { related to effects on } \\
\text { foraging and travel; } \\
\text { med for juveniles } \\
\text { may increase } \\
\text { predation }\end{array}$ & $\begin{array}{l}\text { Low; noise } \\
\text { detectable over } \\
\text { sig. distance; not } \\
\text { likely a threat } \\
\text { unless animals } \\
\text { remain near } \\
\text { structures } \\
\end{array}$ & $\begin{array}{l}\text { Kastak et al. } \\
\text { 2005, } \\
\text { Koschinski et } \\
\text { al. 2003, } \\
\text { Tougaard et al. } \\
2009\end{array}$ \\
\hline $\begin{array}{l}\text { Structures in water } \\
\text { column and on } \\
\text { seabed, such as } \\
\text { devices and } \\
\text { moorings and } \\
\text { footings }\end{array}$ & Structure & $\begin{array}{l}\text { Collisions, } \\
\text { entanglement; } \\
\text { artificial reef effects } \\
\text { could attract fish, } \\
\text { increase forage, }\end{array}$ & $\begin{array}{l}\text { Low, currently not } \\
\text { much structure nearby } \\
\text { but home ranges are } \\
\text { large. }\end{array}$ & $\begin{array}{l}\text { High, structure } \\
\text { present over life } \\
\text { of project }\end{array}$ & $\begin{array}{l}\text { Med, possibly } \\
\text { beneficial effect by } \\
\text { increasing forage, } \\
\text { although } \\
\text { entanglement risk } \\
\text { increases }\end{array}$ & $\begin{array}{l}\text { Low; structure } \\
\text { easily avoided }\end{array}$ & $\begin{array}{l}\text { Schusterman } \\
\text { and Balliet } \\
\text { 1970, Todd et } \\
\text { al. } 2009\end{array}$ \\
\hline $\begin{array}{l}\text { Electricity } \\
\text { conduction through } \\
\text { cable }\end{array}$ & EMF & Unknown & $\begin{array}{l}\text { Unknown, cable } \\
\text { shielding provides some } \\
\text { attenuation }\end{array}$ & $\begin{array}{l}\text { High if there is an } \\
\text { effect, it would } \\
\text { occur over life of } \\
\text { the project }\end{array}$ & $\begin{array}{l}\text { Unknown; any } \\
\text { effects would likely } \\
\text { impact all age } \\
\text { classes }\end{array}$ & $\begin{array}{l}\text { Unknown; High } \\
\text { uncertainty about } \\
\text { effects on marine } \\
\text { mammals }\end{array}$ & $\begin{array}{l}\text { Boehlert et al. } \\
2008\end{array}$ \\
\hline \multirow{2}{*}{$\begin{array}{l}\text { Structures on } \\
\text { water's surface }\end{array}$} & Structure & $\begin{array}{l}\text { May provide haul } \\
\text { out structure; attract } \\
\text { sea lions and } \\
\text { increase potential } \\
\text { for other impacts }\end{array}$ & $\begin{array}{l}\text { Limited to above } \\
\text { surface structures }\end{array}$ & $\begin{array}{l}\text { High, structure } \\
\text { present over life } \\
\text { of project }\end{array}$ & $\begin{array}{l}\text { Low; may focus sea } \\
\text { lion aggregations at } \\
\text { new offshore } \\
\text { location; }\end{array}$ & $\begin{array}{l}\text { Med; may } \\
\text { increase foraging } \\
\text { on fish attracted to } \\
\text { structure }\end{array}$ & $\begin{array}{l}\text { Relini et al. } \\
2000\end{array}$ \\
\hline & Navigation lights & $\begin{array}{l}\text { Visual } \\
\text { disorientation could } \\
\text { lead to collision, } \\
\text { enhance foraging }\end{array}$ & $\begin{array}{l}\text { Low, lights associated } \\
\text { with devices and buoys } \\
\text { are low intensity, } \\
\text { affecting a small area }\end{array}$ & $\begin{array}{l}\text { High, lights would } \\
\text { be on over the life } \\
\text { of the project }\end{array}$ & $\begin{array}{l}\text { Low; may attract } \\
\text { adult/subadults } \\
\text { attracted to prey; } \\
\text { may attract curious } \\
\text { juveniles }\end{array}$ & $\begin{array}{l}\text { Low; exposure } \\
\text { low relative to } \\
\text { home range }\end{array}$ & $\begin{array}{l}\text { Yurk and Trites } \\
2000\end{array}$ \\
\hline
\end{tabular}

\begin{tabular}{lr}
\hline Environmental Assessment for Siting Wave \& Tidal & D-43 \\
Energy Projects & H. Harvey \& Associates \\
24 November 2009
\end{tabular}


Appendix D - Effects of Small Commercial OPT Power Buoy Project Humboldt

\begin{tabular}{|c|c|c|c|c|c|c|c|}
\hline Project activity & Project action & $\begin{array}{l}\text { Description of } \\
\text { action's effect on } \\
\text { indicator }\end{array}$ & $\begin{array}{l}\text { Spatial exposure to } \\
\text { indicator (low, med, } \\
\text { high) }\end{array}$ & $\begin{array}{l}\text { Temporal } \\
\text { exposure to } \\
\text { indicator (low, } \\
\text { med, high) }\end{array}$ & $\begin{array}{l}\text { Effect's overlap } \\
\text { with critical life } \\
\text { stage, behavior, } \\
\text { habitat, or } \\
\text { resource (low, } \\
\text { med, high) } \\
\end{array}$ & $\begin{array}{l}\text { Overall risk to } \\
\text { indicator (low, } \\
\text { med, high, } \\
\text { unknown) }\end{array}$ & Source(s) \\
\hline \multicolumn{8}{|c|}{ Decommissioning } \\
\hline \multirow{3}{*}{ Boat traffic } & Direct impact & Collision injuries & $\begin{array}{l}\text { High, significant } \\
\text { increase existing traffic } \\
\text { (especially in bay and } \\
\text { jetties) }\end{array}$ & $\begin{array}{l}\text { Low, occurring } \\
\text { over } 1 \text { to } 2 \\
\text { summers }\end{array}$ & $\begin{array}{l}\text { Med for } \\
\text { adults/subadults, } \\
\text { high for juveniles } \\
\text { due to foraging and } \\
\text { travel }\end{array}$ & $\begin{array}{l}\text { Med-high; } \\
\text { increase in boat } \\
\text { traffic, potential } \\
\text { for take esp. for } \\
\text { pups and juveniles }\end{array}$ & $\begin{array}{l}\text { Stroud and } \\
\text { Roffe } 1979\end{array}$ \\
\hline & $\begin{array}{l}\text { Noise and } \\
\text { vibration }\end{array}$ & $\begin{array}{l}\text { Reduction of } \\
\text { hearing sensitivity } \\
\text { (Temporary } \\
\text { Threshold Shift); } \\
\text { may increase } \\
\text { predation }\end{array}$ & $\begin{array}{l}\text { Med, sign, increase in } \\
\text { boat traffic between } \\
\text { Humboldt Bay and } \\
\text { project area, and within } \\
\text { project area. Noise } \\
\text { beyond project area. }\end{array}$ & $\begin{array}{l}\text { Low, occurring } \\
\text { over } 1 \text { to } 2 \\
\text { summers }\end{array}$ & $\begin{array}{l}\text { Med for } \\
\text { adults/subadults, } \\
\text { high for juveniles } \\
\text { related to foraging, } \\
\text { travel and predation }\end{array}$ & $\begin{array}{l}\text { Low; noise } \\
\text { detectable over } \\
\sim 3 \mathrm{~km} \text {; not likely a } \\
\text { threat unless } \\
\text { animals approach } \\
\text { moving vessels }\end{array}$ & $\begin{array}{l}\text { Kastak et al. } \\
\text { 2005, Kastelein } \\
\text { et al. } 2009\end{array}$ \\
\hline & $\begin{array}{l}\text { Oil/chemical } \\
\text { release, assuming } \\
\text { no catastrophic } \\
\text { spills. }\end{array}$ & $\begin{array}{l}\text { Ingestion, fur } \\
\text { fouling, breathing } \\
\text { exhaust fumes, } \\
\text { accumulation of } \\
\text { toxins. } \\
\end{array}$ & $\begin{array}{l}\text { Med, significant } \\
\text { increase existing traffic } \\
\text { but the volume of fluids } \\
\text { released low }\end{array}$ & $\begin{array}{l}\text { Low, occurring } \\
\text { over } 1 \text { to } 2 \\
\text { summers }\end{array}$ & $\begin{array}{l}\text { Low; could } \\
\text { contaminate bay } \\
\text { and offshore site, } \\
\text { contaminants } \\
\text { passed to pups } \\
\end{array}$ & $\begin{array}{l}\text { Low; } \\
\text { contaminants not } \\
\text { significantly } \\
\text { greater than } \\
\text { background levels }\end{array}$ & $\begin{array}{l}\text { Brookens et al. } \\
\text { 2007, Le Boeuf } \\
\text { et al. } 2002\end{array}$ \\
\hline \multirow{3}{*}{$\begin{array}{l}\text { Decommissioning } \\
\text { of structures on } \\
\text { water's surface or } \\
\text { seabed }\end{array}$} & $\begin{array}{l}\text { Noise and } \\
\text { vibration }\end{array}$ & $\begin{array}{l}\text { Avoidance of area, } \\
\text { affects foraging and } \\
\text { travel, rest onshore } \\
\text { in Bay; (TSS) could } \\
\text { increase predation }\end{array}$ & $\begin{array}{l}\text { High, sound likely to be } \\
\text { detectable up to } \sim 3 \mathrm{~km} \\
\text { from project site }\end{array}$ & $\begin{array}{l}\text { Low, occurring } \\
\text { over } 1 \text { to } 2 \\
\text { summers }\end{array}$ & $\begin{array}{l}\text { Low for adults; med } \\
\text { for juveniles/ pups } \\
\text { due to foraging and } \\
\text { travel in } \\
\text { construction zones; }\end{array}$ & $\begin{array}{l}\text { Low; noises could } \\
\text { impact hearing if } \\
\text { sea lions approach } \\
\text { and remain; may } \\
\text { cause avoidance }\end{array}$ & $\begin{array}{l}\text { Kastak et al. } \\
\text { 2005, Tougaard } \\
\text { et al. } 2009\end{array}$ \\
\hline & $\begin{array}{l}\text { Oil/chemical } \\
\text { release, assuming } \\
\text { no catastrophic } \\
\text { spills. }\end{array}$ & $\begin{array}{l}\text { Ingestion, fur } \\
\text { fouling, breathing } \\
\text { exhaust fumes, } \\
\text { accumulation of } \\
\text { toxins. }\end{array}$ & $\begin{array}{l}\text { Med; increase in use of } \\
\text { chemicals, solvents, } \\
\text { fuels, grease but the } \\
\text { volume of fluids } \\
\text { released low }\end{array}$ & $\begin{array}{l}\text { Low, occurring } \\
\text { over } 1 \text { to } 2 \\
\text { summers }\end{array}$ & $\begin{array}{l}\text { Low; could } \\
\text { contaminate Bay and } \\
\text { offshore, } \\
\text { contaminants passed } \\
\text { to pups }\end{array}$ & $\begin{array}{l}\text { Low; low volume } \\
\text { of contaminants } \\
\text { expected and not } \\
\text { more than } \\
\text { background levels }\end{array}$ & $\begin{array}{l}\text { Brookens et al. } \\
\text { 2007, Le Boeuf } \\
\text { et al. } 2002\end{array}$ \\
\hline & $\begin{array}{l}\text { Deconstruction } \\
\text { and deck lights }\end{array}$ & $\begin{array}{l}\text { Could be attracted } \\
\text { to area increasing } \\
\text { likelihood of other } \\
\text { impacts, enhance } \\
\text { foraging on prey } \\
\text { attracted to light }\end{array}$ & $\begin{array}{l}\text { Med to high; number of } \\
\text { buoys installed } \\
\text { simultaneously would } \\
\text { affect number of lights } \\
\text { and spatial extent }\end{array}$ & $\begin{array}{l}\text { Low, occurring } \\
\text { over } 2 \text { summers }\end{array}$ & $\begin{array}{l}\text { Low; operations } \\
\text { affecting travel and } \\
\text { foraging at night }\end{array}$ & $\begin{array}{l}\text { Low; exposure } \\
\text { low relative to } \\
\text { home range; short } \\
\text { duration of } \\
\text { construction }\end{array}$ & $\begin{array}{l}\text { Yurk and Trites } \\
2000\end{array}$ \\
\hline
\end{tabular}

\begin{tabular}{lr}
\hline Environmental Assessment for Siting Wave \& Tidal & D-44 \\
Energy Projects & H. Harvey \& Associates \\
24 November 2009
\end{tabular}


Appendix D - Effects of Small Commercial OPT Power Buoy Project Humboldt

Potential effects on small odontocetes (Pacific White-sided dolphin, Risso's dolphin, Dall's porpoise) due to Small Commercial OPT Power Buoy Project at Humboldt site

\begin{tabular}{|c|c|c|c|c|c|c|c|}
\hline Project activity & Project action & $\begin{array}{l}\text { Description of } \\
\text { action's effect on } \\
\text { indicator }\end{array}$ & $\begin{array}{l}\text { Spatial exposure to } \\
\text { indicator (low, med, } \\
\text { high) }\end{array}$ & $\begin{array}{l}\text { Temporal exposure } \\
\text { to indicator (low, } \\
\text { med, high) }\end{array}$ & $\begin{array}{l}\text { Effect's overlap } \\
\text { with critical life } \\
\text { stage, behavior, } \\
\text { habitat, or } \\
\text { resource (low, } \\
\text { med, high) }\end{array}$ & $\begin{array}{l}\text { Overall risk to } \\
\text { indicator (low, } \\
\text { med, high, } \\
\text { unknown) } \\
\end{array}$ & Source(s) \\
\hline \multicolumn{8}{|c|}{ Construction } \\
\hline \multirow{3}{*}{ Boat traffic } & Direct impact & $\begin{array}{l}\text { Collision injuries, } \\
\text { bow riding }\end{array}$ & $\begin{array}{l}\text { Low, significant } \\
\text { increase in existing } \\
\text { traffic in small area of } \\
\text { their large range }\end{array}$ & $\begin{array}{l}\text { Low, occurring } \\
\text { during } 1 \text { to } 2 \\
\text { summers }\end{array}$ & $\begin{array}{l}\text { Low, effects occur } \\
\text { in small part of } \\
\text { large range that is } \\
\text { mostly farther } \\
\text { offshore }\end{array}$ & $\begin{array}{l}\text { Low, because } \\
\text { exposure is low } \\
\text { relative to } \\
\text { indicator's range }\end{array}$ & $\begin{array}{l}\text { Van Waerebeek } \\
\text { and Leaper } 2008\end{array}$ \\
\hline & $\begin{array}{l}\text { Noise and } \\
\text { vibration }\end{array}$ & $\begin{array}{l}\text { Avoidance, } \\
\text { masking of } \\
\text { environmental cues, } \\
\text { communication } \\
\text { signals, } \\
\text { echolocation }\end{array}$ & $\begin{array}{l}\text { Low-Med, sound } \\
\text { travels far, animals } \\
\text { will hear it outside of } \\
\text { project area, but affects } \\
\text { a small part of large } \\
\text { range }\end{array}$ & $\begin{array}{l}\text { Low, occurring } \\
\text { during } 1 \text { to } 2 \\
\text { summers }\end{array}$ & $\begin{array}{l}\text { Low, small part of } \\
\text { large range. }\end{array}$ & $\begin{array}{l}\text { Low; may be } \\
\text { displaced from } \\
\text { transport zones } \\
\text { by high ampl. } \\
\text { sound; effect } \\
\text { area small } \\
\text { relative to range }\end{array}$ & \\
\hline & $\begin{array}{l}\text { Oil/chemical } \\
\text { release }\end{array}$ & $\begin{array}{l}\text { Ingestion, breathing } \\
\text { exhaust fumes, } \\
\text { accumulation of } \\
\text { toxins. }\end{array}$ & $\begin{array}{l}\text { Med, significant } \\
\text { increase in existing } \\
\text { traffic but the volume } \\
\text { of fluids released low }\end{array}$ & $\begin{array}{l}\text { Low, occurring } \\
\text { during } 1 \text { to } 2 \\
\text { summers }\end{array}$ & $\begin{array}{l}\text { Low, effects occur } \\
\text { in small part of } \\
\text { large range that is } \\
\text { mostly farther } \\
\text { offshore }\end{array}$ & $\begin{array}{l}\text { Low, because } \\
\text { exposure is low } \\
\text { relative to } \\
\text { indicator's range }\end{array}$ & $\begin{array}{l}\text { Le Boeuf et al. } \\
2002\end{array}$ \\
\hline \multirow[t]{2}{*}{$\begin{array}{l}\text { Construction and } \\
\text { installation of } \\
\text { electrical collector } \\
\text { system, mooring } \\
\text { cables, anchors or } \\
\text { foundations, and } \\
\text { devices }\end{array}$} & $\begin{array}{l}\text { Noise and } \\
\text { vibration }\end{array}$ & $\begin{array}{l}\text { Avoidance, } \\
\text { masking of } \\
\text { environmental cues, } \\
\text { communication } \\
\text { signals, } \\
\text { echolocation } \\
\end{array}$ & $\begin{array}{l}\text { Med to high, sound } \\
\text { travels far, animals } \\
\text { will hear noise even if } \\
\text { they never approach }\end{array}$ & $\begin{array}{l}\text { Low, occurring } \\
\text { during } 1 \text { to } 2 \\
\text { summers }\end{array}$ & $\begin{array}{l}\text { Low, effects occur } \\
\text { in small part of } \\
\text { large range that is } \\
\text { mostly farther } \\
\text { offshore }\end{array}$ & $\begin{array}{l}\text { Low, because } \\
\text { exposure is low } \\
\text { relative to } \\
\text { indicator's range }\end{array}$ & \\
\hline & $\begin{array}{l}\text { Oil/chemical } \\
\text { release }\end{array}$ & $\begin{array}{l}\text { Ingestion, breathing } \\
\text { exhaust fumes, } \\
\text { overall } \\
\text { accumulation of } \\
\text { toxins. }\end{array}$ & $\begin{array}{l}\text { Low, significant } \\
\text { increase in existing } \\
\text { traffic but the volume } \\
\text { of fluids released low }\end{array}$ & $\begin{array}{l}\text { Low, occurring } \\
\text { during } 1 \text { to } 2 \\
\text { summers }\end{array}$ & $\begin{array}{l}\text { Low, effects occur } \\
\text { in small part of } \\
\text { large range that is } \\
\text { mostly farther } \\
\text { offshore }\end{array}$ & $\begin{array}{l}\text { Low, because } \\
\text { exposure is low } \\
\text { relative to } \\
\text { indicator's range }\end{array}$ & $\begin{array}{l}\text { Le Boeuf et al. } \\
2002\end{array}$ \\
\hline
\end{tabular}

\begin{tabular}{lr}
\hline Environmental Assessment for Siting Wave \& Tidal & D-45 \\
Energy Projects & H. Harvey \& Associates \\
24 November 2009
\end{tabular}


Appendix D - Effects of Small Commercial OPT Power Buoy Project Humboldt

\begin{tabular}{|c|c|c|c|c|c|c|c|}
\hline \multirow[t]{2}{*}{ Project activity } & Project action & $\begin{array}{l}\text { Description of } \\
\text { action's effect on } \\
\text { indicator }\end{array}$ & $\begin{array}{l}\text { Spatial exposure to } \\
\text { indicator (low, med, } \\
\text { high) }\end{array}$ & $\begin{array}{l}\text { Temporal exposure } \\
\text { to indicator (low, } \\
\text { med, high) }\end{array}$ & $\begin{array}{l}\text { Effect's overlap } \\
\text { with critical life } \\
\text { stage, behavior, } \\
\text { habitat, or } \\
\text { resource (low, } \\
\text { med, high) }\end{array}$ & $\begin{array}{l}\text { Overall risk to } \\
\text { indicator (low, } \\
\text { med, high, } \\
\text { unknown) }\end{array}$ & Source(s) \\
\hline & $\begin{array}{l}\text { Construction } \\
\text { lights, vessel } \\
\text { deck lights and } \\
\text { spotlights. }\end{array}$ & $\begin{array}{l}\text { Visual } \\
\text { disorientation could } \\
\text { lead to collision, but } \\
\text { could also enhance } \\
\text { foraging on species } \\
\text { attracted to light }\end{array}$ & $\begin{array}{l}\text { Unknown area would } \\
\text { be small relative to } \\
\text { range }\end{array}$ & $\begin{array}{l}\text { Low, occurring } \\
\text { during } 1 \text { to } 2 \\
\text { summers }\end{array}$ & $\begin{array}{l}\text { Low, effects' } \\
\text { spatial extent small } \\
\text { compared to } \\
\text { animal's range }\end{array}$ & $\begin{array}{l}\text { Low, because } \\
\text { exposure is low } \\
\text { relative to } \\
\text { indicator's range }\end{array}$ & \\
\hline $\begin{array}{l}\text { Directional drilling, } \\
\text { and laying cable } \\
\text { under/on seabed }\end{array}$ & $\begin{array}{l}\text { Noise and } \\
\text { vibration }\end{array}$ & $\begin{array}{l}\text { Avoidance, } \\
\text { masking of } \\
\text { environmental cues, } \\
\text { communication } \\
\text { signals, } \\
\text { echolocation }\end{array}$ & $\begin{array}{l}\text { Med to high, sound } \\
\text { travels far, animals } \\
\text { will hear noise even if } \\
\text { they never approach, } \\
\text { but sea state can } \\
\text { attenuate }\end{array}$ & $\begin{array}{l}\text { Low, limited to } 1 \\
\text { summer }\end{array}$ & $\begin{array}{l}\text { Low, effects occur } \\
\text { in small part of } \\
\text { large range that is } \\
\text { mostly farther } \\
\text { offshore }\end{array}$ & $\begin{array}{l}\text { Low, because } \\
\text { exposure is low } \\
\text { relative to } \\
\text { indicator's range }\end{array}$ & \\
\hline \multicolumn{8}{|c|}{ Operation and Maintenance } \\
\hline \multirow{3}{*}{ Boat traffic } & Direct impact & $\begin{array}{l}\text { Collision injuries, } \\
\text { bow riding }\end{array}$ & $\begin{array}{l}\text { Low, slight increase in } \\
\text { existing traffic in a } \\
\text { small area of their } \\
\text { large range. }\end{array}$ & $\begin{array}{l}\text { High, traffic increase } \\
\text { occurs over life of the } \\
\text { project }\end{array}$ & $\begin{array}{l}\text { Low, effects occur } \\
\text { in small part of } \\
\text { large range that is } \\
\text { mostly farther } \\
\text { offshore }\end{array}$ & $\begin{array}{l}\text { Low, because } \\
\text { exposure is low } \\
\text { relative to } \\
\text { indicator's range }\end{array}$ & $\begin{array}{l}\text { Van Waerebeek } \\
\text { and Leaper } 2008\end{array}$ \\
\hline & $\begin{array}{l}\text { Oil/chemical } \\
\text { release }\end{array}$ & $\begin{array}{l}\text { Ingestion, breathing } \\
\text { exhaust fumes, } \\
\text { overall } \\
\text { accumulation of } \\
\text { toxins. }\end{array}$ & $\begin{array}{l}\text { Low, slight increase in } \\
\text { existing traffic but the } \\
\text { volume of fluids } \\
\text { released low }\end{array}$ & $\begin{array}{l}\text { High, traffic increase } \\
\text { occurs over life of the } \\
\text { project }\end{array}$ & $\begin{array}{l}\text { Low, effects occur } \\
\text { in small part of } \\
\text { large range that is } \\
\text { mostly farther } \\
\text { offshore }\end{array}$ & $\begin{array}{l}\text { Low, because } \\
\text { exposure is low } \\
\text { relative to } \\
\text { indicator's range }\end{array}$ & $\begin{array}{l}\text { Le Boeuf et al. } \\
2002\end{array}$ \\
\hline & $\begin{array}{l}\text { Noise and } \\
\text { vibration }\end{array}$ & $\begin{array}{l}\text { Avoidance, } \\
\text { masking of } \\
\text { environmental cues, } \\
\text { communication } \\
\text { signals, } \\
\text { echolocation }\end{array}$ & $\begin{array}{l}\text { Low-Med, sound } \\
\text { travels far, animals } \\
\text { will hear it outside of } \\
\text { project area, but affects } \\
\text { a small part of large } \\
\text { range }\end{array}$ & $\begin{array}{l}\text { High, traffic increase } \\
\text { occurs over life of the } \\
\text { project but vessel } \\
\text { frequency is less than } \\
\text { during construction }\end{array}$ & $\begin{array}{l}\text { Low, effects occur } \\
\text { in small part of } \\
\text { large range that is } \\
\text { mostly farther } \\
\text { offshore }\end{array}$ & $\begin{array}{l}\text { Low, because } \\
\text { exposure is low } \\
\text { relative to } \\
\text { indicator's range }\end{array}$ & \\
\hline $\begin{array}{l}\text { Operation of } \\
\text { turbines or other } \\
\text { moving parts of } \\
\text { devices }\end{array}$ & $\begin{array}{l}\text { Movement of the } \\
\text { heaving point } \\
\text { absorber and } \\
\text { mooring lines }\end{array}$ & $\begin{array}{l}\text { Collision, } \\
\text { entrapment. }\end{array}$ & $\begin{array}{l}\text { Low, the area of the } \\
\text { devices is small } \\
\text { relative to the project } \\
\text { area, and interaction } \\
\text { with animals at } \\
\text { movement area is low }\end{array}$ & $\begin{array}{l}\text { High, movement will } \\
\text { be continuous } \\
\text { throughout the life of } \\
\text { the project }\end{array}$ & $\begin{array}{l}\text { Low, although } \\
\text { moving throughout } \\
\text { the project life, } \\
\text { interaction between } \\
\text { animals and device } \\
\text { movement is low. }\end{array}$ & $\begin{array}{l}\text { Low, because } \\
\text { exposure is low } \\
\text { relative to } \\
\text { indicator's range }\end{array}$ & $\begin{array}{l}\text { Van Waerebeek } \\
\text { and Leaper } 2008\end{array}$ \\
\hline
\end{tabular}

\begin{tabular}{lr}
\hline Environmental Assessment for Siting Wave \& Tidal & D-46 \\
Energy Projects &
\end{tabular}


Appendix D - Effects of Small Commercial OPT Power Buoy Project Humboldt

\begin{tabular}{|c|c|c|c|c|c|c|c|}
\hline Project activity & Project action & $\begin{array}{l}\text { Description of } \\
\text { action's effect on } \\
\text { indicator }\end{array}$ & $\begin{array}{l}\text { Spatial exposure to } \\
\text { indicator (low, med, } \\
\text { high) }\end{array}$ & $\begin{array}{l}\text { Temporal exposure } \\
\text { to indicator (low, } \\
\text { med, high) }\end{array}$ & $\begin{array}{l}\text { Effect's overlap } \\
\text { with critical life } \\
\text { stage, behavior, } \\
\text { habitat, or } \\
\text { resource (low, } \\
\text { med, high) } \\
\end{array}$ & $\begin{array}{l}\text { Overall risk to } \\
\text { indicator (low, } \\
\text { med, high, } \\
\text { unknown) } \\
\end{array}$ & Source(s) \\
\hline & $\begin{array}{l}\text { Noise and } \\
\text { vibration }\end{array}$ & $\begin{array}{l}\text { Avoidance, } \\
\text { masking of } \\
\text { environmental cues, } \\
\text { communication } \\
\text { signals, } \\
\text { echolocation }\end{array}$ & $\begin{array}{l}\text { Unknown, but physical } \\
\text { site occupies small } \\
\text { area of total home } \\
\text { range; attenuation over } \\
\text { large area and ambient } \\
\text { noise unknown }\end{array}$ & $\begin{array}{l}\text { High, any noise will } \\
\text { be continuous } \\
\text { throughout the life of } \\
\text { the project }\end{array}$ & $\begin{array}{l}\text { Unknown because } \\
\text { animals will likely } \\
\text { acclimate over } \\
\text { time, however they } \\
\text { could still miss } \\
\text { environmental cues }\end{array}$ & $\begin{array}{l}\text { Unknown, noise } \\
\text { from devices } \\
\text { unknown, }\end{array}$ & \\
\hline $\begin{array}{l}\text { Structures in water } \\
\text { column and on } \\
\text { seabed, such as } \\
\text { devices and } \\
\text { moorings and } \\
\text { footings }\end{array}$ & Structure & $\begin{array}{l}\text { Collisions, } \\
\text { entanglement, } \\
\text { artificial reef effects } \\
\text { could increase } \\
\text { forage }\end{array}$ & $\begin{array}{l}\text { Low-Med, home range } \\
\text { is large compared to } \\
\text { project area; may } \\
\text { create new habitat, } \\
\text { depends on strength of } \\
\text { attraction }\end{array}$ & $\begin{array}{l}\text { High, any positive or } \\
\text { negative effects } \\
\text { would occur over the } \\
\text { life of the project. }\end{array}$ & $\begin{array}{l}\text { Med, entanglement } \\
\text { could increase if } \\
\text { animals attracted } \\
\text { by prey; structure } \\
\text { may incrementally } \\
\text { increase forage as } \\
\text { marine reserve }\end{array}$ & $\begin{array}{l}\text { Unknown, } \\
\text { entanglement is } \\
\text { not well } \\
\text { understood for } \\
\text { odontocetes }\end{array}$ & $\begin{array}{l}\text { Cox and Read } \\
2004\end{array}$ \\
\hline $\begin{array}{l}\text { Electricity } \\
\text { conduction through } \\
\text { cable }\end{array}$ & EMF & Unknown & $\begin{array}{l}\text { Unknown, cable } \\
\text { shielding provides } \\
\text { some attenuation }\end{array}$ & $\begin{array}{l}\text { High if an effect } \\
\text { because electricity } \\
\text { generated over life of } \\
\text { the project }\end{array}$ & Unknown & Unknown & \\
\hline \multirow{2}{*}{$\begin{array}{l}\text { Structures on } \\
\text { water's surface }\end{array}$} & Structure & $\begin{array}{l}\text { Collision, artificial } \\
\text { reef effects could } \\
\text { attract fish }\end{array}$ & $\begin{array}{l}\text { Low, fish aggregation } \\
\text { device (FAD) effect is } \\
\text { small compared to } \\
\text { indicator's home range }\end{array}$ & $\begin{array}{l}\text { High, any positive or } \\
\text { negative effects } \\
\text { would occur over the } \\
\text { life of the project. }\end{array}$ & $\begin{array}{l}\text { Low, effects occur } \\
\text { in small part of } \\
\text { large range that is } \\
\text { mostly farther } \\
\text { offshore }\end{array}$ & $\begin{array}{l}\text { Low, because } \\
\text { exposure is low } \\
\text { relative to } \\
\text { indicator's range }\end{array}$ & $\begin{array}{l}\text { Van Waerebeek } \\
\text { and Leaper } 2008\end{array}$ \\
\hline & Navigation lights & $\begin{array}{l}\text { Visual } \\
\text { disorientation could } \\
\text { lead to collision, } \\
\text { could also enhance } \\
\text { foraging on species } \\
\text { attracted to light } \\
\end{array}$ & $\begin{array}{l}\text { Low, device lights are } \\
\text { low intensity and } \\
\text { shielded. }\end{array}$ & $\begin{array}{l}\text { High, lights shining } \\
\text { over life of the } \\
\text { project }\end{array}$ & $\begin{array}{l}\text { Low, both adverse } \\
\text { and beneficial } \\
\text { effects are possible }\end{array}$ & $\begin{array}{l}\text { Low, because } \\
\text { exposure is low } \\
\text { relative to } \\
\text { indicator's range }\end{array}$ & \\
\hline \multicolumn{8}{|c|}{ Decommissioning } \\
\hline Boat traffic & Direct impact & $\begin{array}{l}\text { Collision injuries, } \\
\text { bow riding }\end{array}$ & $\begin{array}{l}\text { Low, significant } \\
\text { increase existing traffic } \\
\text { in small area of their } \\
\text { large range. }\end{array}$ & $\begin{array}{l}\text { Low, occurring } \\
\text { during } 1 \text { to } 2 \\
\text { summers }\end{array}$ & $\begin{array}{l}\text { Low, effects occur } \\
\text { in small part of } \\
\text { large range that is } \\
\text { mostly farther } \\
\text { offshore }\end{array}$ & $\begin{array}{l}\text { Low, because } \\
\text { exposure is low } \\
\text { relative to } \\
\text { indicator's range }\end{array}$ & $\begin{array}{l}\text { Van Waerebeek } \\
\text { and Leaper } 2008\end{array}$ \\
\hline
\end{tabular}

\begin{tabular}{|c|c|c|}
\hline $\begin{array}{l}\text { Environmental Assessment for Siting Wave \& Tidal } \\
\text { Energy Projects }\end{array}$ & D-47 & $\begin{array}{r}\text { H. T. Harvey \& Associates } \\
24 \text { November } 2009\end{array}$ \\
\hline
\end{tabular}


Appendix D - Effects of Small Commercial OPT Power Buoy Project Humboldt

\begin{tabular}{|c|c|c|c|c|c|c|c|}
\hline Project activity & Project action & $\begin{array}{l}\text { Description of } \\
\text { action's effect on } \\
\text { indicator }\end{array}$ & $\begin{array}{l}\text { Spatial exposure to } \\
\text { indicator (low, med, } \\
\text { high) }\end{array}$ & $\begin{array}{l}\text { Temporal exposure } \\
\text { to indicator (low, } \\
\text { med, high) }\end{array}$ & $\begin{array}{l}\text { Effect's overlap } \\
\text { with critical life } \\
\text { stage, behavior, } \\
\text { habitat, or } \\
\text { resource (low, } \\
\text { med, high) }\end{array}$ & $\begin{array}{l}\text { Overall risk to } \\
\text { indicator (low, } \\
\text { med, high, } \\
\text { unknown) }\end{array}$ & Source(s) \\
\hline & $\begin{array}{l}\text { Oil/chemical } \\
\text { release }\end{array}$ & $\begin{array}{l}\text { Ingestion, breathing } \\
\text { exhaust fumes, } \\
\text { accumulation of } \\
\text { toxins. }\end{array}$ & $\begin{array}{l}\text { Med, significant } \\
\text { increase existing traffic } \\
\text { but the volume of } \\
\text { fluids released low }\end{array}$ & $\begin{array}{l}\text { Low, occurring } \\
\text { during } 1 \text { to } 2 \\
\text { summers }\end{array}$ & $\begin{array}{l}\text { Low, effects occur } \\
\text { in small part of } \\
\text { large range that is } \\
\text { mostly farther } \\
\text { offshore }\end{array}$ & $\begin{array}{l}\text { Low, because } \\
\text { exposure is low } \\
\text { relative to } \\
\text { indicator's range }\end{array}$ & $\begin{array}{l}\text { Le Boeuf et al. } \\
2002\end{array}$ \\
\hline & $\begin{array}{l}\text { Noise and } \\
\text { vibration }\end{array}$ & $\begin{array}{l}\text { Avoidance, } \\
\text { masking of } \\
\text { environmental cues, } \\
\text { communication } \\
\text { signals, } \\
\text { echolocation } \\
\end{array}$ & $\begin{array}{l}\text { Low to med, sound } \\
\text { travels far, animals } \\
\text { will hear it outside of } \\
\text { project area, but affects } \\
\text { a small part of large } \\
\text { range }\end{array}$ & $\begin{array}{l}\text { Low, occurring } \\
\text { during } 1 \text { to } 2 \\
\text { summers }\end{array}$ & $\begin{array}{l}\text { Low, effects occur } \\
\text { in small part of } \\
\text { large range that is } \\
\text { mostly farther } \\
\text { offshore }\end{array}$ & $\begin{array}{l}\text { Low; may be } \\
\text { displaced from } \\
\text { transport zones; } \\
\text { effect area small } \\
\text { relative to range }\end{array}$ & \\
\hline \multirow{3}{*}{$\begin{array}{l}\text { Decommissioning } \\
\text { of structures on } \\
\text { water's surface and } \\
\text { seabed }\end{array}$} & $\begin{array}{l}\text { Noise and } \\
\text { vibration }\end{array}$ & $\begin{array}{l}\text { Avoidance, } \\
\text { masking of } \\
\text { environmental cues, } \\
\text { communication } \\
\text { signals, } \\
\text { echolocation }\end{array}$ & $\begin{array}{l}\text { Med to high, sound } \\
\text { travels far, animals } \\
\text { will hear noise even if } \\
\text { they never approach }\end{array}$ & $\begin{array}{l}\text { Low, occurring } \\
\text { during } 1 \text { to } 2 \\
\text { summers }\end{array}$ & $\begin{array}{l}\text { Low, effects occur } \\
\text { in small part of } \\
\text { large range that is } \\
\text { mostly farther } \\
\text { offshore }\end{array}$ & $\begin{array}{l}\text { Low; may be } \\
\text { displaced from } \\
\text { transport zones } \\
\text { by high ampl. } \\
\text { sound; effect } \\
\text { area small } \\
\text { relative to range }\end{array}$ & \\
\hline & $\begin{array}{l}\text { Oil/chemical } \\
\text { release }\end{array}$ & $\begin{array}{l}\text { Ingestion, breathing } \\
\text { exhaust fumes, } \\
\text { accumulation of } \\
\text { toxins. }\end{array}$ & $\begin{array}{l}\text { Med, significant } \\
\text { increase in existing } \\
\text { traffic but the volume } \\
\text { of fluids released low }\end{array}$ & $\begin{array}{l}\text { Low, occurring } \\
\text { during } 1 \text { to } 2 \\
\text { summers }\end{array}$ & $\begin{array}{l}\text { Low, effects occur } \\
\text { in small part of } \\
\text { large range that is } \\
\text { mostly offshore }\end{array}$ & $\begin{array}{l}\text { Low, exposure is } \\
\text { low relative to } \\
\text { indicator's range }\end{array}$ & $\begin{array}{l}\text { Le Boeuf et al. } \\
2002\end{array}$ \\
\hline & $\begin{array}{l}\text { Deconstruction } \\
\text { lights, vessel } \\
\text { deck lights and } \\
\text { spotlights. }\end{array}$ & $\begin{array}{l}\text { Visual } \\
\text { disorientation could } \\
\text { lead to collision, but } \\
\text { could also enhance } \\
\text { foraging on species } \\
\text { attracted to light }\end{array}$ & $\begin{array}{l}\text { Low, small relative to } \\
\text { range }\end{array}$ & $\begin{array}{l}\text { Low, occurring } \\
\text { during } 1 \text { to } 2 \\
\text { summers }\end{array}$ & $\begin{array}{l}\text { Low, effects' } \\
\text { spatial extent small } \\
\text { compared to } \\
\text { animal's range }\end{array}$ & $\begin{array}{l}\text { Low, exposure is } \\
\text { low relative to } \\
\text { indicator's range }\end{array}$ & \\
\hline
\end{tabular}

\begin{tabular}{lrr}
\hline Environmental Assessment for Siting Wave \& Tidal & D-48 & H. T. Harvey \& Associates \\
Energy Projects & & 24 November 2009
\end{tabular}


Appendix D - Effects of Small Commercial OPT Power Buoy Project Humboldt

Potential effects on sharks, skates, and rays due to Small Commercial OPT Power Buoy Project at Humboldt site

\begin{tabular}{|c|c|c|c|c|c|c|c|}
\hline Project activity & Action & $\begin{array}{l}\text { Description of action's } \\
\text { effect on indicator }\end{array}$ & $\begin{array}{l}\text { Spatial exposure to } \\
\text { indicator (low, med, } \\
\text { high) }\end{array}$ & $\begin{array}{l}\text { Temporal } \\
\text { exposure to } \\
\text { indicator (low, } \\
\text { med, high) } \\
\end{array}$ & $\begin{array}{l}\text { Effect's overlap with } \\
\text { critical life stage, } \\
\text { behavior, habitat or } \\
\text { resource (low, med, } \\
\text { high) }\end{array}$ & $\begin{array}{l}\text { Overall risk } \\
\text { to indicator } \\
\text { (low, med, } \\
\text { high, } \\
\text { unknown) } \\
\end{array}$ & Source(s) \\
\hline \multicolumn{8}{|c|}{ Construction } \\
\hline \multirow[t]{2}{*}{ Boat traffic } & $\begin{array}{l}\text { Oil/chemical } \\
\text { release }\end{array}$ & $\begin{array}{l}\text { Toxicity to individuals } \\
\text { and prey }\end{array}$ & $\begin{array}{l}\text { Low for typical small } \\
\text { volume releases, High } \\
\text { for large, catastrophic } \\
\text { spill }\end{array}$ & $\begin{array}{l}\text { Low, occurs over } 1 \\
\text { to } 2 \text { summers }\end{array}$ & $\begin{array}{l}\text { Low, species in water } \\
\text { column but oil floats on } \\
\text { surface }\end{array}$ & Low & $\begin{array}{l}\text { Nelson and } \\
\text { Woo } 2008\end{array}$ \\
\hline & $\begin{array}{l}\text { Noise and } \\
\text { vibration }\end{array}$ & $\begin{array}{l}\text { Possible altered } \\
\text { behavior }\end{array}$ & $\begin{array}{l}\text { Low, would occur in } \\
\text { small area relative to } \\
\text { species’ range }\end{array}$ & $\begin{array}{l}\text { Low, occurs over } 1 \\
\text { to } 2 \text { summers }\end{array}$ & $\begin{array}{l}\text { Low, species present } \\
\text { but able to avoid } \\
\text { disturbance area }\end{array}$ & Low & Cada 2008 \\
\hline \multirow{4}{*}{$\begin{array}{l}\text { Construction of } \\
\text { electrical } \\
\text { collector system, } \\
\text { moorings and } \\
\text { foundations, and } \\
\text { device } \\
\text { installation }\end{array}$} & $\begin{array}{l}\text { Noise and } \\
\text { vibration }\end{array}$ & $\begin{array}{l}\text { Possible altered } \\
\text { behavior }\end{array}$ & Low, see above & $\begin{array}{l}\text { Low, occurs over } 1 \\
\text { to } 2 \text { summers }\end{array}$ & Low, see above & Low & Cada 2008 \\
\hline & $\begin{array}{l}\text { Oil/chemical } \\
\text { release }\end{array}$ & $\begin{array}{l}\text { Toxicity to individuals } \\
\text { and prey }\end{array}$ & $\begin{array}{l}\text { Low, volume released } \\
\text { would occupy small } \\
\text { area relative to species' } \\
\text { range }\end{array}$ & $\begin{array}{l}\text { Low, occurs over } 1 \\
\text { to } 2 \text { summers }\end{array}$ & $\begin{array}{l}\text { Low, species in water } \\
\text { column but oil floats on } \\
\text { surface }\end{array}$ & Low & $\begin{array}{l}\text { Nelson and } \\
\text { Woo } 2008\end{array}$ \\
\hline & $\begin{array}{l}\text { Seabed } \\
\text { disturbance }\end{array}$ & $\begin{array}{l}\text { Temporary habitat loss, } \\
\text { changes in prey } \\
\text { resources }\end{array}$ & $\begin{array}{l}\text { Low, would occur in } \\
\text { small area relative to } \\
\text { species' range }\end{array}$ & $\begin{array}{l}\text { Low, occurs over } 1 \\
\text { to } 2 \text { summers }\end{array}$ & $\begin{array}{l}\text { Low, species present } \\
\text { but able to avoid } \\
\text { disturbance area }\end{array}$ & Low & Nelson 2008 \\
\hline & $\begin{array}{l}\text { Construction } \\
\text { lights }\end{array}$ & $\begin{array}{l}\text { Sharks may be attracted } \\
\text { due to prey aggregation }\end{array}$ & Low, see above & $\begin{array}{l}\text { Low, occurs over } 1 \\
\text { to } 2 \text { summers }\end{array}$ & $\begin{array}{l}\text { Med, if sharks attracted } \\
\text { to project area }\end{array}$ & Low & $\begin{array}{l}\text { Nightingale et } \\
\text { al. } 2006\end{array}$ \\
\hline \multirow{2}{*}{$\begin{array}{l}\text { Directional } \\
\text { drilling, and } \\
\text { laying cable } \\
\text { under/on seabed }\end{array}$} & $\begin{array}{l}\text { Seabed } \\
\text { disturbance }\end{array}$ & $\begin{array}{l}\text { Temporary habitat loss, } \\
\text { changes in prey } \\
\text { resources }\end{array}$ & Low, see above & $\begin{array}{l}\text { Low, occurs over a } \\
\text { short time period }\end{array}$ & $\begin{array}{l}\text { Low, species present } \\
\text { but able to avoid } \\
\text { disturbance area }\end{array}$ & Low & Nelson 2008 \\
\hline & $\begin{array}{l}\text { Noise and } \\
\text { vibration }\end{array}$ & $\begin{array}{l}\text { Possible altered } \\
\text { behavior }\end{array}$ & Low, see above & $\begin{array}{l}\text { Low, occurs over a } \\
\text { short time period }\end{array}$ & $\begin{array}{l}\text { Low, species present } \\
\text { but able to avoid } \\
\text { disturbance area }\end{array}$ & Low & Cada 2008 \\
\hline \multicolumn{8}{|c|}{ Operation and Maintenance } \\
\hline Boat traffic & $\begin{array}{l}\text { Oil/chemical } \\
\text { release }\end{array}$ & $\begin{array}{l}\text { Toxicity to individuals } \\
\text { and prey }\end{array}$ & $\begin{array}{l}\text { Low for typical small } \\
\text { volume releases, High } \\
\text { for large, catastrophic } \\
\text { spill }\end{array}$ & $\begin{array}{l}\text { Low, wouldn't } \\
\text { persist long-term }\end{array}$ & $\begin{array}{l}\text { Low, species in water } \\
\text { column but oil floats on } \\
\text { surface }\end{array}$ & Low & $\begin{array}{l}\text { Nelson and } \\
\text { Woo } 2008\end{array}$ \\
\hline
\end{tabular}

\begin{tabular}{lr}
\hline Environmental Assessment for Siting Wave \& Tidal & D-49 \\
Energy Projects &
\end{tabular}


Appendix D - Effects of Small Commercial OPT Power Buoy Project Humboldt

\begin{tabular}{|c|c|c|c|c|c|c|c|}
\hline Project activity & Action & $\begin{array}{l}\text { Description of action's } \\
\text { effect on indicator }\end{array}$ & $\begin{array}{l}\text { Spatial exposure to } \\
\text { indicator (low, med, } \\
\text { high) }\end{array}$ & $\begin{array}{l}\text { Temporal } \\
\text { exposure to } \\
\text { indicator (low, } \\
\text { med, high) } \\
\end{array}$ & $\begin{array}{l}\text { Effect's overlap with } \\
\text { critical life stage, } \\
\text { behavior, habitat or } \\
\text { resource (low, med, } \\
\text { high) }\end{array}$ & $\begin{array}{l}\text { Overall risk } \\
\text { to indicator } \\
\text { (low, med, } \\
\text { high, } \\
\text { unknown) } \\
\end{array}$ & Source(s) \\
\hline & $\begin{array}{l}\text { Noise and } \\
\text { vibration }\end{array}$ & $\begin{array}{l}\text { Possible altered } \\
\text { behavior }\end{array}$ & $\begin{array}{l}\text { Low, would occur in } \\
\text { small area relative to } \\
\text { species' range }\end{array}$ & $\begin{array}{l}\text { Low, wouldn't } \\
\text { persist long-term }\end{array}$ & $\begin{array}{l}\text { Low, species present } \\
\text { but able to avoid } \\
\text { disturbance area }\end{array}$ & Low & Cada 2008 \\
\hline \multirow{2}{*}{$\begin{array}{l}\text { Operation of } \\
\text { turbines or other } \\
\text { moving parts of } \\
\text { devices }\end{array}$} & $\begin{array}{l}\text { Moving device } \\
\text { parts }\end{array}$ & $\begin{array}{l}\text { None, no underwater } \\
\text { moving parts }\end{array}$ & N/A & N/A & N/A & Low & N/A \\
\hline & $\begin{array}{l}\text { Noise and } \\
\text { vibration }\end{array}$ & $\begin{array}{l}\text { Possible altered } \\
\text { behavior }\end{array}$ & $\begin{array}{l}\text { Low, would occur in } \\
\text { small area relative to } \\
\text { species' range }\end{array}$ & $\begin{array}{l}\text { High, would be } \\
\text { continuous for life } \\
\text { of project }\end{array}$ & $\begin{array}{l}\text { Low, species present } \\
\text { but able to avoid } \\
\text { disturbance area }\end{array}$ & Low & Cada 2008 \\
\hline \multirow{3}{*}{$\begin{array}{l}\text { Structures in } \\
\text { water column and } \\
\text { on seabed, such } \\
\text { as devices and } \\
\text { moorings and } \\
\text { footings }\end{array}$} & Structure & $\begin{array}{l}\text { None, prey of these } \\
\text { species not attracted to } \\
\text { subsurface structures }\end{array}$ & N/A & N/A & N/A & Low & $\begin{array}{l}\text { Boehlert et al. } \\
2008\end{array}$ \\
\hline & $\begin{array}{l}\text { Water circulation } \\
\text { changes }\end{array}$ & $\begin{array}{l}\text { None, project not large } \\
\text { enough to affect prey }\end{array}$ & N/A & N/A & N/A & Low & \\
\hline & $\begin{array}{l}\text { Oil/chemical } \\
\text { release }\end{array}$ & $\begin{array}{l}\text { Toxicity to individuals } \\
\text { and prey }\end{array}$ & $\begin{array}{l}\text { Low, would occur in } \\
\text { small area relative to } \\
\text { species' range }\end{array}$ & $\begin{array}{l}\text { Low, wouldn't } \\
\text { persist long-term }\end{array}$ & $\begin{array}{l}\text { Low, species in water } \\
\text { column while oil floats } \\
\text { on surface }\end{array}$ & Low & $\begin{array}{l}\text { Nelson and } \\
\text { Woo } 2008\end{array}$ \\
\hline $\begin{array}{l}\text { Electricity } \\
\text { conduction } \\
\text { through cable }\end{array}$ & EMF & $\begin{array}{l}\text { Possible changes in } \\
\text { orientation, behavior }\end{array}$ & Low, see above & $\begin{array}{l}\text { High, would be } \\
\text { continuous for life } \\
\text { of project }\end{array}$ & $\begin{array}{l}\text { Med, species present in } \\
\text { project area }\end{array}$ & Unknown & Nelson 2008 \\
\hline \multirow{2}{*}{$\begin{array}{l}\text { Structures on } \\
\text { water's surface }\end{array}$} & Structure & $\begin{array}{l}\text { Possible FAD effect, } \\
\text { pinniped haul-out which } \\
\text { attracts sharks }\end{array}$ & $\begin{array}{l}\text { Low, would occur in } \\
\text { small area relative to } \\
\text { species' range }\end{array}$ & $\begin{array}{l}\text { High, would be } \\
\text { continuous for life } \\
\text { of project }\end{array}$ & $\begin{array}{l}\text { Med, sharks present in } \\
\text { project area }\end{array}$ & Unknown & Nelson 2008 \\
\hline & Navigation lights & $\begin{array}{l}\text { Sharks may be attracted } \\
\text { to lights due to prey } \\
\text { aggregation }\end{array}$ & Low, see above & $\begin{array}{l}\text { High, would be } \\
\text { continuous for life } \\
\text { of project }\end{array}$ & $\begin{array}{l}\text { Med, sharks present in } \\
\text { project area }\end{array}$ & Unknown & $\begin{array}{l}\text { Nightingale et } \\
\text { al. } 2006\end{array}$ \\
\hline \multicolumn{8}{|c|}{ Decommissioning } \\
\hline Boat traffic & $\begin{array}{l}\text { Oil/chemical } \\
\text { release }\end{array}$ & $\begin{array}{l}\text { Toxicity to individuals } \\
\text { and prey }\end{array}$ & $\begin{array}{l}\text { Low for typical small } \\
\text { volume releases, High } \\
\text { for large, catastrophic } \\
\text { spill }\end{array}$ & $\begin{array}{l}\text { Low, occurs over } 1 \\
\text { to } 2 \text { summers }\end{array}$ & $\begin{array}{l}\text { Low, species in water } \\
\text { column while oil floats } \\
\text { on surface }\end{array}$ & Low & $\begin{array}{l}\text { Nelson and } \\
\text { Woo } 2008\end{array}$ \\
\hline
\end{tabular}

\begin{tabular}{lr}
\hline Environmental Assessment for Siting Wave \& Tidal & D-50 \\
Energy Projects &
\end{tabular}


Appendix D - Effects of Small Commercial OPT Power Buoy Project Humboldt

\begin{tabular}{|c|c|c|c|c|c|c|c|}
\hline Project activity & Action & $\begin{array}{l}\text { Description of action's } \\
\text { effect on indicator }\end{array}$ & $\begin{array}{l}\text { Spatial exposure to } \\
\text { indicator (low, med, } \\
\text { high) }\end{array}$ & $\begin{array}{l}\text { Temporal } \\
\text { exposure to } \\
\text { indicator (low, } \\
\text { med, high) }\end{array}$ & $\begin{array}{l}\text { Effect's overlap with } \\
\text { critical life stage, } \\
\text { behavior, habitat or } \\
\text { resource (low, med, } \\
\text { high) }\end{array}$ & $\begin{array}{l}\text { Overall risk } \\
\text { to indicator } \\
\text { (low, med, } \\
\text { high, } \\
\text { unknown) } \\
\end{array}$ & Source(s) \\
\hline & $\begin{array}{l}\text { Noise and } \\
\text { vibration }\end{array}$ & $\begin{array}{l}\text { Possible altered } \\
\text { behavior }\end{array}$ & $\begin{array}{l}\text { Low, would occur in } \\
\text { small area relative to } \\
\text { species' range }\end{array}$ & $\begin{array}{l}\text { Low, occurs over } 1 \\
\text { to } 2 \text { summers }\end{array}$ & $\begin{array}{l}\text { Low, species present } \\
\text { but able to avoid } \\
\text { disturbance area }\end{array}$ & Low & Cada 2008 \\
\hline \multirow{4}{*}{$\begin{array}{l}\text { Decommissioning } \\
\text { of structures on } \\
\text { water's surface or } \\
\text { seabed }\end{array}$} & $\begin{array}{l}\text { Noise and } \\
\text { vibration }\end{array}$ & $\begin{array}{l}\text { Possible altered } \\
\text { behavior }\end{array}$ & Low, see above & $\begin{array}{l}\text { Low, occurs over } 1 \\
\text { to } 2 \text { summers }\end{array}$ & $\begin{array}{l}\text { Low, species present } \\
\text { but able to avoid } \\
\text { disturbance area }\end{array}$ & Low & Cada 2008 \\
\hline & $\begin{array}{l}\text { Oil/chemical } \\
\text { release }\end{array}$ & $\begin{array}{l}\text { Toxicity to individuals } \\
\text { and prey }\end{array}$ & $\begin{array}{l}\text { Low, volume released } \\
\text { would occupy small } \\
\text { area }\end{array}$ & $\begin{array}{l}\text { Low, occurs over } 1 \\
\text { to } 2 \text { summers }\end{array}$ & $\begin{array}{l}\text { Low, species in water } \\
\text { column while oil floats } \\
\text { on surface }\end{array}$ & Low & $\begin{array}{l}\text { Nelson and } \\
\text { Woo } 2008\end{array}$ \\
\hline & $\begin{array}{l}\text { Seabed } \\
\text { disturbance }\end{array}$ & $\begin{array}{l}\text { Temporary habitat loss, } \\
\text { changes in prey } \\
\text { resources }\end{array}$ & $\begin{array}{l}\text { Low, would occur in } \\
\text { small area relative to } \\
\text { species' range }\end{array}$ & $\begin{array}{l}\text { Low, occurs over } 1 \\
\text { to } 2 \text { summers }\end{array}$ & $\begin{array}{l}\text { Low, species present } \\
\text { but able to avoid } \\
\text { disturbance area }\end{array}$ & Low & Nelson 2008 \\
\hline & $\begin{array}{l}\text { Deconstruction } \\
\text { lights }\end{array}$ & $\begin{array}{l}\text { Sharks may be attracted } \\
\text { due to prey aggregation }\end{array}$ & Low, see above & $\begin{array}{l}\text { Low, occurs over } 1 \\
\text { to } 2 \text { summers }\end{array}$ & $\begin{array}{l}\text { Med, sharks present in } \\
\text { project area }\end{array}$ & Low & $\begin{array}{l}\text { Nightingale et } \\
\text { al. } 2006\end{array}$ \\
\hline
\end{tabular}

\begin{tabular}{lr}
\hline Environmental Assessment for Siting Wave \& Tidal & D-51 \\
Energy Projects &
\end{tabular}


Appendix D - Effects of Small Commercial OPT Power Buoy Project Humboldt

Potential effects on flatfishes due to Small Commercial OPT Power Buoy Project at Humboldt site

\begin{tabular}{|c|c|c|c|c|c|c|c|}
\hline Project activity & Action & $\begin{array}{l}\text { Description of action's } \\
\text { effect on indicator }\end{array}$ & $\begin{array}{l}\text { Spatial exposure to } \\
\text { indicator (low, med, } \\
\text { high) }\end{array}$ & $\begin{array}{l}\text { Temporal } \\
\text { exposure to } \\
\text { indicator (low, } \\
\text { med, high) } \\
\end{array}$ & $\begin{array}{l}\text { Effect's overlap with } \\
\text { critical life stage, } \\
\text { behavior, habitat or } \\
\text { resource (low, med, } \\
\text { high) }\end{array}$ & $\begin{array}{l}\text { Overall risk } \\
\text { to indicator } \\
\text { (low, med, } \\
\text { high, } \\
\text { unknown) } \\
\end{array}$ & Source(s) \\
\hline \multicolumn{8}{|c|}{ Construction } \\
\hline \multirow[t]{2}{*}{ Boat traffic } & $\begin{array}{l}\text { Oil/chemical } \\
\text { release }\end{array}$ & $\begin{array}{l}\text { Toxicity to individuals } \\
\text { and prey }\end{array}$ & $\begin{array}{l}\text { Low for typical small } \\
\text { volume releases, High } \\
\text { for large, catastrophic } \\
\text { spill }\end{array}$ & $\begin{array}{l}\text { Low, occurs over } \\
1 \text { to } 2 \text { summers }\end{array}$ & $\begin{array}{l}\text { Low, flatfishes on } \\
\text { bottom while oil floats } \\
\text { on surface }\end{array}$ & Low & $\begin{array}{l}\text { Nelson and } \\
\text { Woo } 2008\end{array}$ \\
\hline & $\begin{array}{l}\text { Noise and } \\
\text { vibration }\end{array}$ & $\begin{array}{l}\text { None, flatfishes not very } \\
\text { sensitive to sound }\end{array}$ & N/A & N/A & N/A & Low & $\begin{array}{l}\text { Nedwell et al. } \\
2004\end{array}$ \\
\hline \multirow{4}{*}{$\begin{array}{l}\text { Construction of } \\
\text { electrical } \\
\text { collector system, } \\
\text { moorings and } \\
\text { foundations, and } \\
\text { device } \\
\text { installation }\end{array}$} & $\begin{array}{l}\text { Noise and } \\
\text { vibration }\end{array}$ & None, see above & N/A & N/A & N/A & Low & $\begin{array}{l}\text { Nedwell et al. } \\
2004\end{array}$ \\
\hline & $\begin{array}{l}\text { Oil/chemical } \\
\text { release }\end{array}$ & $\begin{array}{l}\text { Toxicity to individuals } \\
\text { and prey }\end{array}$ & $\begin{array}{l}\text { Low, volume released } \\
\text { would occupy small } \\
\text { area relative to species, } \\
\text { range }\end{array}$ & $\begin{array}{l}\text { Low, occurs over } \\
1 \text { to } 2 \text { summers }\end{array}$ & $\begin{array}{l}\text { Low, flatfishes on } \\
\text { bottom while oil floats } \\
\text { on surface }\end{array}$ & Low & $\begin{array}{l}\text { Nelson and } \\
\text { Woo } 2008\end{array}$ \\
\hline & $\begin{array}{l}\text { Seabed } \\
\text { disturbance }\end{array}$ & $\begin{array}{l}\text { Temporary habitat loss, } \\
\text { changes in prey resources }\end{array}$ & $\begin{array}{l}\text { Low, would occur in } \\
\text { small area relative to } \\
\text { species' range }\end{array}$ & $\begin{array}{l}\text { Low, occurs over } \\
1 \text { to } 2 \text { summers }\end{array}$ & $\begin{array}{l}\text { Low, flatfishes present } \\
\text { but able to avoid } \\
\text { disturbance area }\end{array}$ & Low & Nelson 2008 \\
\hline & $\begin{array}{l}\text { Construction } \\
\text { lights }\end{array}$ & $\begin{array}{l}\text { None, flatfishes not } \\
\text { known to be affected by } \\
\text { lights }\end{array}$ & N/A & N/A & N/A & Low & $\begin{array}{l}\text { Nightingale et } \\
\text { al. } 2006\end{array}$ \\
\hline \multirow{2}{*}{$\begin{array}{l}\text { Directional } \\
\text { drilling, and } \\
\text { laying cable } \\
\text { under/on seabed }\end{array}$} & $\begin{array}{l}\text { Seabed } \\
\text { disturbance }\end{array}$ & $\begin{array}{l}\text { Temporary habitat loss, } \\
\text { changes in prey resources }\end{array}$ & $\begin{array}{l}\text { Low, would occur in } \\
\text { small area relative to } \\
\text { species' range }\end{array}$ & $\begin{array}{l}\text { Low, occurs over } \\
\text { a short time } \\
\text { period }\end{array}$ & $\begin{array}{l}\text { Low, flatfishes present } \\
\text { but able to avoid } \\
\text { disturbance area }\end{array}$ & Low & Nelson 2008 \\
\hline & $\begin{array}{l}\text { Noise and } \\
\text { vibration }\end{array}$ & $\begin{array}{l}\text { None, flatfishes not very } \\
\text { sensitive to sound }\end{array}$ & N/A & N/A & N/A & Low & $\begin{array}{l}\text { Nedwell et al. } \\
2004\end{array}$ \\
\hline \multicolumn{8}{|c|}{ Operation and Maintenance } \\
\hline Boat traffic & $\begin{array}{l}\text { Oil/chemical } \\
\text { release }\end{array}$ & $\begin{array}{l}\text { Toxicity to individuals } \\
\text { and prey }\end{array}$ & $\begin{array}{l}\text { Low for typical small } \\
\text { volume releases, High } \\
\text { for large, catastrophic } \\
\text { spill }\end{array}$ & $\begin{array}{l}\text { Low, wouldn’t } \\
\text { persist long-term }\end{array}$ & $\begin{array}{l}\text { Low, flatfishes on } \\
\text { bottom while oil floats } \\
\text { on surface }\end{array}$ & Low & $\begin{array}{l}\text { Nelson and } \\
\text { Woo } 2008\end{array}$ \\
\hline
\end{tabular}

\begin{tabular}{lr}
\hline Environmental Assessment for Siting Wave \& Tidal & D-52 \\
Energy Projects &
\end{tabular}


Appendix D - Effects of Small Commercial OPT Power Buoy Project Humboldt

\begin{tabular}{|c|c|c|c|c|c|c|c|}
\hline Project activity & Action & $\begin{array}{l}\text { Description of action's } \\
\text { effect on indicator }\end{array}$ & $\begin{array}{l}\text { Spatial exposure to } \\
\text { indicator (low, med, } \\
\text { high) }\end{array}$ & $\begin{array}{l}\text { Temporal } \\
\text { exposure to } \\
\text { indicator (low, } \\
\text { med, high) } \\
\end{array}$ & $\begin{array}{l}\text { Effect's overlap with } \\
\text { critical life stage, } \\
\text { behavior, habitat or } \\
\text { resource (low, med, } \\
\text { high) }\end{array}$ & $\begin{array}{l}\text { Overall risk } \\
\text { to indicator } \\
\text { (low, med, } \\
\text { high, } \\
\text { unknown) } \\
\end{array}$ & Source(s) \\
\hline & $\begin{array}{l}\text { Noise and } \\
\text { vibration }\end{array}$ & $\begin{array}{l}\text { None, flatfishes not very } \\
\text { sensitive to sound }\end{array}$ & N/A & N/A & N/A & Low & $\begin{array}{l}\text { Nedwell et al. } \\
2004\end{array}$ \\
\hline \multirow{2}{*}{$\begin{array}{l}\text { Operation of } \\
\text { turbines or other } \\
\text { moving parts of } \\
\text { devices }\end{array}$} & $\begin{array}{l}\text { Moving device } \\
\text { parts }\end{array}$ & $\begin{array}{l}\text { None, no underwater } \\
\text { moving parts }\end{array}$ & N/A & N/A & N/A & Low & N/A \\
\hline & $\begin{array}{l}\text { Noise and } \\
\text { vibration }\end{array}$ & $\begin{array}{l}\text { None, flatfishes not very } \\
\text { sensitive to sound }\end{array}$ & N/A & N/A & N/A & Low & $\begin{array}{l}\text { Nedwell et al. } \\
2004\end{array}$ \\
\hline \multirow{3}{*}{$\begin{array}{l}\text { Structures in } \\
\text { water column and } \\
\text { on seabed, such } \\
\text { as devices and } \\
\text { moorings and } \\
\text { footings }\end{array}$} & Structure & $\begin{array}{l}\text { Possible reef effect, } \\
\text { changes in predator/prey } \\
\text { interactions }\end{array}$ & $\begin{array}{l}\text { Low, would occur in } \\
\text { small area relative to } \\
\text { species' range }\end{array}$ & $\begin{array}{l}\text { High, would be } \\
\text { continuous for } \\
\text { life of project }\end{array}$ & $\begin{array}{l}\text { Med, flatfishes in } \\
\text { sand/mud bottom } \\
\text { habitat where project } \\
\text { located }\end{array}$ & Unknown & $\begin{array}{l}\text { Boehlert et al. } \\
2008\end{array}$ \\
\hline & $\begin{array}{l}\text { Water circulation } \\
\text { changes }\end{array}$ & $\begin{array}{l}\text { None, project not large } \\
\text { enough to affect prey }\end{array}$ & N/A & N/A & N/A & Low & N/A \\
\hline & $\begin{array}{l}\text { Oil/chemical } \\
\text { release }\end{array}$ & $\begin{array}{l}\text { Toxicity to individuals } \\
\text { and prey }\end{array}$ & $\begin{array}{l}\text { Low, would occur in } \\
\text { small area relative to } \\
\text { species' range }\end{array}$ & $\begin{array}{l}\text { Low, wouldn’t } \\
\text { persist long-term }\end{array}$ & $\begin{array}{l}\text { Low, flatfishes on } \\
\text { bottom while oil floats } \\
\text { on surface }\end{array}$ & Low & $\begin{array}{l}\text { Nelson and } \\
\text { Woo } 2008\end{array}$ \\
\hline $\begin{array}{l}\text { Electricity } \\
\text { conduction } \\
\text { through cable }\end{array}$ & EMF & $\begin{array}{l}\text { Effects to flatfishes } \\
\text { unlikely, not known to } \\
\text { have sensitive } \\
\text { electroreceptive sense } \\
\text { organs }\end{array}$ & N/A & N/A & N/A & Low & Nelson 2008 \\
\hline \multirow{2}{*}{$\begin{array}{l}\text { Structures on } \\
\text { water's surface }\end{array}$} & Structure & $\begin{array}{l}\text { Possible FAD effect, } \\
\text { changes in predator/prey } \\
\text { interactions }\end{array}$ & Low, see above & $\begin{array}{l}\text { High, would be } \\
\text { continuous for } \\
\text { life of project }\end{array}$ & $\begin{array}{l}\text { Low, flatfishes on } \\
\text { bottom while effects on } \\
\text { surface }\end{array}$ & Low & Nelson 2008 \\
\hline & Navigation lights & $\begin{array}{l}\text { None, flatfishes not } \\
\text { known to be affected by } \\
\text { lights }\end{array}$ & N/A & N/A & N/A & Low & $\begin{array}{l}\text { Nightingale et } \\
\text { al. } 2006\end{array}$ \\
\hline \multicolumn{8}{|c|}{ Decommissioning } \\
\hline Boat traffic & $\begin{array}{l}\text { Oil/chemical } \\
\text { release }\end{array}$ & $\begin{array}{l}\text { Toxicity to individuals } \\
\text { and prey }\end{array}$ & $\begin{array}{l}\text { Low for typical small } \\
\text { volume releases, High } \\
\text { for large, catastrophic } \\
\text { spill }\end{array}$ & $\begin{array}{l}\text { Low, occurs over } \\
1 \text { to } 2 \text { summers }\end{array}$ & $\begin{array}{l}\text { Low, flatfishes on } \\
\text { bottom while oil floats } \\
\text { on surface }\end{array}$ & Low & $\begin{array}{l}\text { Nelson and } \\
\text { Woo } 2008\end{array}$ \\
\hline
\end{tabular}

\begin{tabular}{lr}
\hline Environmental Assessment for Siting Wave \& Tidal & D-53 \\
Energy Projects & H. Harvey \& Associates \\
24 November 2009
\end{tabular}


Appendix D - Effects of Small Commercial OPT Power Buoy Project Humboldt

\begin{tabular}{|c|c|c|c|c|c|c|c|}
\hline Project activity & Action & $\begin{array}{l}\text { Description of action's } \\
\text { effect on indicator }\end{array}$ & $\begin{array}{l}\text { Spatial exposure to } \\
\text { indicator (low, med, } \\
\text { high) }\end{array}$ & $\begin{array}{l}\text { Temporal } \\
\text { exposure to } \\
\text { indicator (low, } \\
\text { med, high) }\end{array}$ & $\begin{array}{l}\text { Effect's overlap with } \\
\text { critical life stage, } \\
\text { behavior, habitat or } \\
\text { resource (low, med, } \\
\text { high) }\end{array}$ & $\begin{array}{l}\text { Overall risk } \\
\text { to indicator } \\
\text { (low, med, } \\
\text { high, } \\
\text { unknown) } \\
\end{array}$ & Source(s) \\
\hline & $\begin{array}{l}\text { Noise and } \\
\text { vibration }\end{array}$ & $\begin{array}{l}\text { None, flatfishes not very } \\
\text { sensitive to sound }\end{array}$ & N/A & N/A & N/A & Low & $\begin{array}{l}\text { Nedwell et al. } \\
2004\end{array}$ \\
\hline \multirow{4}{*}{$\begin{array}{l}\text { Decommissioning } \\
\text { of structures on } \\
\text { water's surface or } \\
\text { seabed }\end{array}$} & $\begin{array}{l}\text { Noise and } \\
\text { vibration }\end{array}$ & None, see above & N/A & N/A & N/A & Low & $\begin{array}{l}\text { Nedwell et al. } \\
2004\end{array}$ \\
\hline & $\begin{array}{l}\text { Oil/chemical } \\
\text { release }\end{array}$ & $\begin{array}{l}\text { Toxicity to individuals } \\
\text { and prey }\end{array}$ & $\begin{array}{l}\text { Low, volume released } \\
\text { would occupy small } \\
\text { area }\end{array}$ & $\begin{array}{l}\text { Low, occurs over } \\
1 \text { to } 2 \text { summers }\end{array}$ & $\begin{array}{l}\text { Low, flatfishes on } \\
\text { bottom while oil floats } \\
\text { on surface }\end{array}$ & Low & $\begin{array}{l}\text { Nelson and } \\
\text { Woo } 2008\end{array}$ \\
\hline & $\begin{array}{l}\text { Seabed } \\
\text { disturbance }\end{array}$ & $\begin{array}{l}\text { Temporary habitat loss, } \\
\text { changes in prey resources }\end{array}$ & $\begin{array}{l}\text { Low, would occur in } \\
\text { small area relative to } \\
\text { species' range }\end{array}$ & $\begin{array}{l}\text { Low, occurs over } \\
1 \text { to } 2 \text { summers }\end{array}$ & $\begin{array}{l}\text { Low, flatfishes present } \\
\text { but able to avoid } \\
\text { disturbance area }\end{array}$ & Low & Nelson 2008 \\
\hline & $\begin{array}{l}\text { Deconstruction } \\
\text { lights }\end{array}$ & $\begin{array}{l}\text { None, flatfishes not } \\
\text { known to be affected by } \\
\text { lights }\end{array}$ & N/A & N/A & N/A & Low & $\begin{array}{l}\text { Nightingale et } \\
\text { al. } 2006\end{array}$ \\
\hline
\end{tabular}

\begin{tabular}{lr}
\hline Environmental Assessment for Siting Wave \& Tidal & D-54 \\
Energy Projects & H. T. Harvey \& Associates \\
24 November 2009
\end{tabular}


Appendix D - Effects of Small Commercial OPT Power Buoy Project Humboldt

Potential effects on rockfishes, lingcod, and cabezon due to Small Commercial OPT Power Buoy Project at Humboldt site

\begin{tabular}{|c|c|c|c|c|c|c|c|}
\hline Project activity & Action & $\begin{array}{l}\text { Description of action's } \\
\text { effect on indicator }\end{array}$ & $\begin{array}{l}\text { Spatial exposure to } \\
\text { indicator (low, med, } \\
\text { high) }\end{array}$ & $\begin{array}{l}\text { Temporal } \\
\text { exposure to } \\
\text { indicator (low, } \\
\text { med, high) } \\
\end{array}$ & $\begin{array}{l}\text { Effect's overlap with } \\
\text { critical life stage, } \\
\text { behavior, habitat or } \\
\text { resource (low, med, } \\
\text { high) }\end{array}$ & $\begin{array}{l}\text { Overall risk } \\
\text { to indicator } \\
\text { (low, med, } \\
\text { high, } \\
\text { unknown) } \\
\end{array}$ & Source(s) \\
\hline \multicolumn{8}{|c|}{ Construction } \\
\hline \multirow[t]{2}{*}{ Boat traffic } & $\begin{array}{l}\text { Oil/chemical } \\
\text { release }\end{array}$ & $\begin{array}{l}\text { Toxicity to individuals } \\
\text { and prey }\end{array}$ & $\begin{array}{l}\text { Low for typical small } \\
\text { volume releases, High } \\
\text { for large, catastrophic } \\
\text { spill }\end{array}$ & $\begin{array}{l}\text { Low, occurs over } 1 \\
\text { to } 2 \text { summers }\end{array}$ & $\begin{array}{l}\text { Low, species in water } \\
\text { column or bottom but } \\
\text { oil floats on surface }\end{array}$ & Low & $\begin{array}{l}\text { Nelson and } \\
\text { Woo } 2008\end{array}$ \\
\hline & $\begin{array}{l}\text { Noise and } \\
\text { vibration }\end{array}$ & $\begin{array}{l}\text { None, species not likely } \\
\text { to be affected }\end{array}$ & N/A & N/A & N/A & Low & N/A \\
\hline \multirow{4}{*}{$\begin{array}{l}\text { Construction of } \\
\text { electrical } \\
\text { collector system, } \\
\text { moorings and } \\
\text { foundations, and } \\
\text { device } \\
\text { installation }\end{array}$} & $\begin{array}{l}\text { Noise and } \\
\text { vibration }\end{array}$ & $\begin{array}{l}\text { None, species not } \\
\text { present in sand/mud } \\
\text { bottom habitat where } \\
\text { project located }\end{array}$ & N/A & N/A & N/A & Low & N/A \\
\hline & $\begin{array}{l}\text { Oil/chemical } \\
\text { release }\end{array}$ & None (see above) & N/A & N/A & N/A & Low & N/A \\
\hline & $\begin{array}{l}\text { Seabed } \\
\text { disturbance }\end{array}$ & None (see above) & N/A & N/A & N/A & Low & N/A \\
\hline & $\begin{array}{l}\text { Construction } \\
\text { lights }\end{array}$ & None (see above) & N/A & N/A & N/A & Low & N/A \\
\hline \multirow{2}{*}{$\begin{array}{l}\text { Directional } \\
\text { drilling, and } \\
\text { laying cable } \\
\text { under/on seabed }\end{array}$} & $\begin{array}{l}\text { Seabed } \\
\text { disturbance }\end{array}$ & None (see above) & N/A & N/A & N/A & Low & N/A \\
\hline & $\begin{array}{l}\text { Noise and } \\
\text { vibration }\end{array}$ & None (see above) & N/A & N/A & N/A & Low & N/A \\
\hline \multicolumn{8}{|c|}{ Operation and Maintenance } \\
\hline \multirow[t]{2}{*}{ Boat traffic } & $\begin{array}{l}\text { Oil/chemical } \\
\text { release }\end{array}$ & $\begin{array}{l}\text { Toxicity to individuals } \\
\text { and prey }\end{array}$ & $\begin{array}{l}\text { Low for typical small } \\
\text { volume releases, High } \\
\text { for large, catastrophic } \\
\text { spill }\end{array}$ & $\begin{array}{l}\text { Low, wouldn't } \\
\text { persist long-term }\end{array}$ & $\begin{array}{l}\text { Low, species in water } \\
\text { column or bottom but } \\
\text { oil floats on surface }\end{array}$ & Low & $\begin{array}{l}\text { Nelson and } \\
\text { Woo } 2008\end{array}$ \\
\hline & $\begin{array}{l}\text { Noise and } \\
\text { vibration }\end{array}$ & $\begin{array}{l}\text { None, species not likely } \\
\text { to be affected }\end{array}$ & N/A & N/A & N/A & Low & N/A \\
\hline $\begin{array}{l}\text { Operation of } \\
\text { turbines or other } \\
\text { moving parts of }\end{array}$ & $\begin{array}{l}\text { Moving device } \\
\text { parts }\end{array}$ & $\begin{array}{l}\text { None, no underwater } \\
\text { moving parts }\end{array}$ & N/A & N/A & N/A & Low & N/A \\
\hline
\end{tabular}

\begin{tabular}{lr}
\hline Environmental Assessment for Siting Wave \& Tidal & D-55 \\
Energy Projects & H. Tarvey \& Associates \\
24 November 2009
\end{tabular}


Appendix D - Effects of Small Commercial OPT Power Buoy Project Humboldt

\begin{tabular}{|c|c|c|c|c|c|c|c|}
\hline Project activity & Action & $\begin{array}{l}\text { Description of action's } \\
\text { effect on indicator }\end{array}$ & $\begin{array}{l}\text { Spatial exposure to } \\
\text { indicator (low, med, } \\
\text { high) }\end{array}$ & $\begin{array}{l}\text { Temporal } \\
\text { exposure to } \\
\text { indicator (low, } \\
\text { med, high) }\end{array}$ & $\begin{array}{l}\text { Effect's overlap with } \\
\text { critical life stage, } \\
\text { behavior, habitat or } \\
\text { resource (low, med, } \\
\text { high) }\end{array}$ & $\begin{array}{l}\text { Overall risk } \\
\text { to indicator } \\
\text { (low, med, } \\
\text { high, } \\
\text { unknown) } \\
\end{array}$ & Source(s) \\
\hline devices & $\begin{array}{l}\text { Noise and } \\
\text { vibration }\end{array}$ & $\begin{array}{l}\text { Possible altered } \\
\text { behavior }\end{array}$ & $\begin{array}{l}\text { Med, project area could } \\
\text { occupy individual } \\
\text { ranges }\end{array}$ & $\begin{array}{l}\text { High, would persist } \\
\text { for life of project }\end{array}$ & $\begin{array}{l}\text { Med, wave structures } \\
\text { may attract species to } \\
\text { project area }\end{array}$ & Low & Cada 2008 \\
\hline \multirow{3}{*}{$\begin{array}{l}\text { Structures in } \\
\text { water column and } \\
\text { on seabed, such } \\
\text { as devices and } \\
\text { moorings and } \\
\text { footings }\end{array}$} & Structure & $\begin{array}{l}\text { Attraction to artificial } \\
\text { reef, changes in fish } \\
\text { community }\end{array}$ & $\begin{array}{l}\text { Med, project area could } \\
\text { occupy individual } \\
\text { ranges }\end{array}$ & $\begin{array}{l}\text { High, would be } \\
\text { continuous for life } \\
\text { of project }\end{array}$ & $\begin{array}{l}\text { Med, wave structures } \\
\text { may attract species to } \\
\text { project area }\end{array}$ & Med & Nelson 2008 \\
\hline & $\begin{array}{l}\text { Water circulation } \\
\text { changes }\end{array}$ & $\begin{array}{l}\text { None, project not large } \\
\text { enough to affect prey }\end{array}$ & N/A & N/A & N/A & Low & N/A \\
\hline & $\begin{array}{l}\text { Oil/chemical } \\
\text { release }\end{array}$ & $\begin{array}{l}\text { Toxicity to individuals } \\
\text { and prey }\end{array}$ & $\begin{array}{l}\text { Med, project area could } \\
\text { occupy individual } \\
\text { ranges }\end{array}$ & $\begin{array}{l}\text { Low, wouldn't } \\
\text { persist long-term }\end{array}$ & $\begin{array}{l}\text { Low, species in water } \\
\text { column or bottom but } \\
\text { oil floats on surface }\end{array}$ & Low & $\begin{array}{l}\text { Nelson and } \\
\text { Woo } 2008\end{array}$ \\
\hline $\begin{array}{l}\text { Electricity } \\
\text { conduction } \\
\text { through cable }\end{array}$ & EMF & $\begin{array}{l}\text { Effects unlikely, species } \\
\text { not known to have } \\
\text { sensitive } \\
\text { electroreceptive sense } \\
\text { organs }\end{array}$ & N/A & N/A & N/A & Low & Nelson 2008 \\
\hline \multirow[t]{2}{*}{$\begin{array}{l}\text { Structures on } \\
\text { water's surface }\end{array}$} & Structure & $\begin{array}{l}\text { Possible FAD effect, } \\
\text { changes in } \\
\text { predator/prey } \\
\text { interactions }\end{array}$ & $\begin{array}{l}\text { Med, project area could } \\
\text { occupy individual } \\
\text { ranges }\end{array}$ & $\begin{array}{l}\text { High, would be } \\
\text { continuous for life } \\
\text { of project }\end{array}$ & $\begin{array}{l}\text { Low, species in water } \\
\text { column or bottom }\end{array}$ & Low & N/A \\
\hline & Navigation lights & $\begin{array}{l}\text { None, species don't } \\
\text { occur at surface }\end{array}$ & N/A & N/A & N/A & Low & N/A \\
\hline \multicolumn{8}{|c|}{ Decommissioning } \\
\hline \multirow[t]{2}{*}{ Boat traffic } & $\begin{array}{l}\text { Oil/chemical } \\
\text { release }\end{array}$ & $\begin{array}{l}\text { Toxicity to individuals } \\
\text { and prey }\end{array}$ & $\begin{array}{l}\text { Low for typical small } \\
\text { volume releases, High } \\
\text { for large, catastrophic } \\
\text { spill }\end{array}$ & $\begin{array}{l}\text { Low, occurs over } 1 \\
\text { to } 2 \text { summers }\end{array}$ & $\begin{array}{l}\text { Low, species in water } \\
\text { column or bottom but } \\
\text { oil floats on surface }\end{array}$ & Low & $\begin{array}{l}\text { Nelson and } \\
\text { Woo } 2008\end{array}$ \\
\hline & $\begin{array}{l}\text { Noise and } \\
\text { vibration }\end{array}$ & $\begin{array}{l}\text { None, species not likely } \\
\text { to be affected }\end{array}$ & N/A & N/A & N/A & Low & N/A \\
\hline $\begin{array}{l}\text { Decommissioning } \\
\text { of structures on } \\
\text { water's surface or }\end{array}$ & $\begin{array}{l}\text { Noise and } \\
\text { vibration }\end{array}$ & $\begin{array}{l}\text { Possible altered } \\
\text { behavior }\end{array}$ & $\begin{array}{l}\text { Med, project area could } \\
\text { occupy individual } \\
\text { ranges }\end{array}$ & $\begin{array}{l}\text { Low, occurs over } 1 \\
\text { to } 2 \text { summers }\end{array}$ & $\begin{array}{l}\text { Med, species may be } \\
\text { present due to attraction } \\
\text { to wave structures }\end{array}$ & Low & Cada 2008 \\
\hline
\end{tabular}

\begin{tabular}{lr}
\hline Environmental Assessment for Siting Wave \& Tidal & D-56 \\
Energy Projects &
\end{tabular}


Appendix D - Effects of Small Commercial OPT Power Buoy Project Humboldt

\begin{tabular}{|c|c|c|c|c|c|c|c|}
\hline Project activity & Action & $\begin{array}{l}\text { Description of action's } \\
\text { effect on indicator }\end{array}$ & $\begin{array}{l}\text { Spatial exposure to } \\
\text { indicator (low, med, } \\
\text { high) }\end{array}$ & $\begin{array}{l}\text { Temporal } \\
\text { exposure to } \\
\text { indicator (low, } \\
\text { med, high) }\end{array}$ & $\begin{array}{l}\text { Effect's overlap with } \\
\text { critical life stage, } \\
\text { behavior, habitat or } \\
\text { resource (low, med, } \\
\text { high) }\end{array}$ & $\begin{array}{l}\text { Overall risk } \\
\text { to indicator } \\
\text { (low, med, } \\
\text { high, } \\
\text { unknown) } \\
\end{array}$ & Source(s) \\
\hline \multirow[t]{3}{*}{ seabed } & $\begin{array}{l}\text { Oil/chemical } \\
\text { release }\end{array}$ & $\begin{array}{l}\text { Toxicity to individuals } \\
\text { and prey }\end{array}$ & $\begin{array}{l}\text { Med, volume released } \\
\text { could occupy individual } \\
\text { ranges }\end{array}$ & $\begin{array}{l}\text { Low, occurs over } 1 \\
\text { to } 2 \text { summers }\end{array}$ & $\begin{array}{l}\text { Low, species in water } \\
\text { column or bottom but } \\
\text { oil floats on surface }\end{array}$ & Low & $\begin{array}{l}\text { Nelson and } \\
\text { Woo } 2008\end{array}$ \\
\hline & $\begin{array}{l}\text { Seabed } \\
\text { disturbance }\end{array}$ & $\begin{array}{l}\text { Habitat loss, changes in } \\
\text { prey resources }\end{array}$ & $\begin{array}{l}\text { Med, project area could } \\
\text { occupy individual } \\
\text { ranges }\end{array}$ & $\begin{array}{l}\text { Low, occurs over } 1 \\
\text { to } 2 \text { summers }\end{array}$ & $\begin{array}{l}\text { Med, species may be } \\
\text { present due to attraction } \\
\text { to wave structures }\end{array}$ & Med & Nelson 2008 \\
\hline & $\begin{array}{l}\text { Deconstruction } \\
\text { lights }\end{array}$ & $\begin{array}{l}\text { None, species don’t } \\
\text { occur at surface }\end{array}$ & N/A & N/A & N/A & Low & N/A \\
\hline
\end{tabular}

\begin{tabular}{lrr}
\hline Environmental Assessment for Siting Wave \& Tidal & D-57 & H. T. Harvey \& Associates \\
Energy Projects & & 24 November 2009
\end{tabular}


Appendix D - Effects of Small Commercial OPT Power Buoy Project Humboldt

Potential effects on pelagic schooling fishes (mackerel, sardine, anchovy) due to Small Commercial OPT Power Buoy Project at Humboldt site

\begin{tabular}{|c|c|c|c|c|c|c|c|}
\hline Project activity & Action & $\begin{array}{l}\text { Description of action's } \\
\text { effect on indicator }\end{array}$ & $\begin{array}{l}\text { Spatial exposure to } \\
\text { indicator (low, med, } \\
\text { high) }\end{array}$ & $\begin{array}{l}\text { Temporal } \\
\text { exposure to } \\
\text { indicator (low, } \\
\text { med, high) } \\
\end{array}$ & $\begin{array}{l}\text { Effect's overlap with } \\
\text { critical life stage, } \\
\text { behavior, habitat or } \\
\text { resource (low, med, } \\
\text { high) }\end{array}$ & $\begin{array}{l}\text { Overall risk } \\
\text { to indicator } \\
\text { (low, med, } \\
\text { high, } \\
\text { unknown) } \\
\end{array}$ & Source(s) \\
\hline \multicolumn{8}{|c|}{ Construction } \\
\hline \multirow[t]{2}{*}{ Boat traffic } & $\begin{array}{l}\text { Oil/chemical } \\
\text { release }\end{array}$ & $\begin{array}{l}\text { Toxicity to individuals } \\
\text { and prey }\end{array}$ & $\begin{array}{l}\text { Low for typical small } \\
\text { volume releases, High } \\
\text { for large, catastrophic } \\
\text { spill }\end{array}$ & $\begin{array}{l}\text { Low, occurs over } 1 \\
\text { to } 2 \text { summers }\end{array}$ & $\begin{array}{l}\text { Low, species in water } \\
\text { column but oil floats on } \\
\text { surface }\end{array}$ & Low & $\begin{array}{l}\text { Nelson and } \\
\text { Woo } 2008\end{array}$ \\
\hline & $\begin{array}{l}\text { Noise and } \\
\text { vibration }\end{array}$ & $\begin{array}{l}\text { None, species too motile } \\
\text { to be affected }\end{array}$ & N/A & N/A & N/A & Low & N/A \\
\hline \multirow{4}{*}{$\begin{array}{l}\text { Construction of } \\
\text { electrical } \\
\text { collector system, } \\
\text { moorings and } \\
\text { foundations, and } \\
\text { device } \\
\text { installation }\end{array}$} & $\begin{array}{l}\text { Noise and } \\
\text { vibration }\end{array}$ & Possible altered behavior & $\begin{array}{l}\text { Low, fish have patchy } \\
\text { and wide-ranging } \\
\text { distribution }\end{array}$ & $\begin{array}{l}\text { Low, occurs over } 1 \\
\text { to } 2 \text { summers }\end{array}$ & $\begin{array}{l}\text { Low, fish are able to } \\
\text { avoid disturbance area }\end{array}$ & Low & Cada 2008 \\
\hline & $\begin{array}{l}\text { Oil/chemical } \\
\text { release }\end{array}$ & $\begin{array}{l}\text { Toxicity to individuals } \\
\text { and prey }\end{array}$ & $\begin{array}{l}\text { Low, volume released } \\
\text { would occupy small } \\
\text { area }\end{array}$ & $\begin{array}{l}\text { Low, occurs over } 1 \\
\text { to } 2 \text { summers }\end{array}$ & $\begin{array}{l}\text { Low, species in water } \\
\text { column but oil floats on } \\
\text { surface }\end{array}$ & Low & $\begin{array}{l}\text { Nelson and } \\
\text { Woo } 2008\end{array}$ \\
\hline & $\begin{array}{l}\text { Seabed } \\
\text { disturbance }\end{array}$ & $\begin{array}{l}\text { Temporary habitat loss, } \\
\text { changes in prey resources }\end{array}$ & $\begin{array}{l}\text { Low, fish have patchy } \\
\text { and wide-ranging } \\
\text { distribution }\end{array}$ & $\begin{array}{l}\text { Low, occurs over } 1 \\
\text { to } 2 \text { summers }\end{array}$ & $\begin{array}{l}\text { Low, fish are able to } \\
\text { avoid disturbance area }\end{array}$ & Low & \\
\hline & $\begin{array}{l}\text { Construction } \\
\text { lights }\end{array}$ & $\begin{array}{l}\text { Aggregation, more } \\
\text { vulnerable to predation }\end{array}$ & $\begin{array}{l}\text { Low, fish have patchy } \\
\text { and wide-ranging } \\
\text { distribution }\end{array}$ & $\begin{array}{l}\text { Low, occurs over } 1 \\
\text { to } 2 \text { summers }\end{array}$ & $\begin{array}{l}\text { Med, species could be } \\
\text { attracted to area }\end{array}$ & Low & $\begin{array}{l}\text { Nightingale et } \\
\text { al. } 2006\end{array}$ \\
\hline \multirow{2}{*}{$\begin{array}{l}\text { Directional } \\
\text { drilling, and } \\
\text { laying cable } \\
\text { under/on seabed }\end{array}$} & $\begin{array}{l}\text { Seabed } \\
\text { disturbance }\end{array}$ & $\begin{array}{l}\text { Temporary habitat loss, } \\
\text { changes in prey resources }\end{array}$ & Low, see above & $\begin{array}{l}\text { Low, occurs over } 1 \\
\text { to } 2 \text { summers }\end{array}$ & $\begin{array}{l}\text { Low, fish are able to } \\
\text { avoid disturbance area }\end{array}$ & Low & \\
\hline & $\begin{array}{l}\text { Noise and } \\
\text { vibration }\end{array}$ & Possible altered behavior & Low, see above & $\begin{array}{l}\text { Low, occurs over a } \\
\text { short time period }\end{array}$ & Low, see above & Low & Cada 2008 \\
\hline \multicolumn{8}{|c|}{ Operation and Maintenance } \\
\hline Boat traffic & $\begin{array}{l}\text { Oil/chemical } \\
\text { release }\end{array}$ & $\begin{array}{l}\text { Toxicity to individuals } \\
\text { and prey }\end{array}$ & $\begin{array}{l}\text { Low for typical small } \\
\text { volume releases, High } \\
\text { for large, catastrophic } \\
\text { spill }\end{array}$ & $\begin{array}{l}\text { Low, wouldn’t } \\
\text { persist long-term }\end{array}$ & $\begin{array}{l}\text { Low, species in water } \\
\text { column but oil floats on } \\
\text { surface }\end{array}$ & Low & $\begin{array}{l}\text { Nelson and } \\
\text { Woo } 2008\end{array}$ \\
\hline
\end{tabular}

\begin{tabular}{lr}
\hline $\begin{array}{l}\text { Environmental Assessment for Siting Wave \& Tidal } \\
\text { Energy Projects }\end{array}$ & D-58 H. T. Harvey \& Associates \\
24 November 2009
\end{tabular}


Appendix D - Effects of Small Commercial OPT Power Buoy Project Humboldt

\begin{tabular}{|c|c|c|c|c|c|c|c|}
\hline Project activity & Action & $\begin{array}{l}\text { Description of action's } \\
\text { effect on indicator }\end{array}$ & $\begin{array}{l}\text { Spatial exposure to } \\
\text { indicator (low, med, } \\
\text { high) }\end{array}$ & $\begin{array}{l}\text { Temporal } \\
\text { exposure to } \\
\text { indicator (low, } \\
\text { med, high) } \\
\end{array}$ & $\begin{array}{l}\text { Effect's overlap with } \\
\text { critical life stage, } \\
\text { behavior, habitat or } \\
\text { resource (low, med, } \\
\text { high) }\end{array}$ & $\begin{array}{l}\text { Overall risk } \\
\text { to indicator } \\
\text { (low, med, } \\
\text { high, } \\
\text { unknown) } \\
\end{array}$ & Source(s) \\
\hline & $\begin{array}{l}\text { Noise and } \\
\text { vibration }\end{array}$ & $\begin{array}{l}\text { None, species too motile } \\
\text { to be affected }\end{array}$ & N/A & N/A & N/A & Low & N/A \\
\hline \multirow{2}{*}{$\begin{array}{l}\text { Operation of } \\
\text { turbines or other } \\
\text { moving parts of } \\
\text { devices }\end{array}$} & $\begin{array}{l}\text { Moving device } \\
\text { parts }\end{array}$ & $\begin{array}{l}\text { None, no underwater } \\
\text { moving parts }\end{array}$ & N/A & N/A & N/A & Low & N/A \\
\hline & $\begin{array}{l}\text { Noise and } \\
\text { vibration }\end{array}$ & Possible altered behavior & $\begin{array}{l}\text { Low, fish have patchy } \\
\text { and wide-ranging } \\
\text { distribution }\end{array}$ & $\begin{array}{l}\text { High, would be } \\
\text { continuous for life } \\
\text { of project }\end{array}$ & $\begin{array}{l}\text { Low, fish patchy and } \\
\text { able to avoid } \\
\text { disturbance area }\end{array}$ & Low & Cada 2008 \\
\hline \multirow{3}{*}{$\begin{array}{l}\text { Structures in } \\
\text { water column and } \\
\text { on seabed, such } \\
\text { as devices and } \\
\text { moorings and } \\
\text { footings }\end{array}$} & Structure & $\begin{array}{l}\text { Possible reef effect, more } \\
\text { vulnerable to predation }\end{array}$ & Low, see above & $\begin{array}{l}\text { High, would be } \\
\text { continuous for life } \\
\text { of project }\end{array}$ & Low, see above & Low & Nelson 2008 \\
\hline & $\begin{array}{l}\text { Water circulation } \\
\text { changes }\end{array}$ & $\begin{array}{l}\text { None, project not large } \\
\text { enough to affect prey }\end{array}$ & N/A & N/A & N/A & Low & N/A \\
\hline & $\begin{array}{l}\text { Oil/chemical } \\
\text { release }\end{array}$ & $\begin{array}{l}\text { Toxicity to individuals } \\
\text { and prey }\end{array}$ & $\begin{array}{l}\text { Low, fish have patchy } \\
\text { and wide-ranging } \\
\text { distribution }\end{array}$ & $\begin{array}{l}\text { Low, wouldn't } \\
\text { persist long-term }\end{array}$ & $\begin{array}{l}\text { Low, species in water } \\
\text { column but oil floats on } \\
\text { surface }\end{array}$ & Low & $\begin{array}{l}\text { Nelson and } \\
\text { Woo } 2008\end{array}$ \\
\hline $\begin{array}{l}\text { Electricity } \\
\text { conduction } \\
\text { through cable }\end{array}$ & EMF & $\begin{array}{l}\text { Effects unlikely, species } \\
\text { not known to have } \\
\text { sensitive electroreceptive } \\
\text { sense organs }\end{array}$ & N/A & N/A & N/A & Low & Nelson 2008 \\
\hline \multirow{2}{*}{$\begin{array}{l}\text { Structures on } \\
\text { water's surface }\end{array}$} & Structure & $\begin{array}{l}\text { Possible FAD effect, } \\
\text { changes in predator/prey } \\
\text { interactions }\end{array}$ & Low, see above & $\begin{array}{l}\text { High, would be } \\
\text { continuous for life } \\
\text { of project }\end{array}$ & $\begin{array}{l}\text { Low, species } \\
\text { distribution patchy }\end{array}$ & Low & Nelson 2008 \\
\hline & Navigation lights & $\begin{array}{l}\text { Aggregation, more } \\
\text { vulnerable to predation }\end{array}$ & Low, see above & $\begin{array}{l}\text { High, would be } \\
\text { continuous for life } \\
\text { of project }\end{array}$ & $\begin{array}{l}\text { Low, species } \\
\text { distribution patchy }\end{array}$ & Low & $\begin{array}{l}\text { Nightingale et } \\
\text { al. } 2006\end{array}$ \\
\hline \multicolumn{8}{|c|}{ Decommissioning } \\
\hline \multirow[t]{2}{*}{ Boat traffic } & $\begin{array}{l}\text { Oil/chemical } \\
\text { release }\end{array}$ & $\begin{array}{l}\text { Toxicity to individuals } \\
\text { and prey }\end{array}$ & $\begin{array}{l}\text { Low for typical small } \\
\text { volume releases, High } \\
\text { for large, catastrophic } \\
\text { spill }\end{array}$ & $\begin{array}{l}\text { Low, occurs over } 1 \\
\text { to } 2 \text { summers }\end{array}$ & $\begin{array}{l}\text { Low, species in water } \\
\text { column but oil floats on } \\
\text { surface }\end{array}$ & Low & $\begin{array}{l}\text { Nelson and } \\
\text { Woo } 2008\end{array}$ \\
\hline & $\begin{array}{l}\text { Noise and } \\
\text { vibration }\end{array}$ & $\begin{array}{l}\text { None, species too motile } \\
\text { to be affected }\end{array}$ & N/A & N/A & N/A & Low & N/A \\
\hline
\end{tabular}

\begin{tabular}{lr}
\hline Environmental Assessment for Siting Wave \& Tidal & D-59 \\
Energy Projects &
\end{tabular}


Appendix D - Effects of Small Commercial OPT Power Buoy Project Humboldt

\begin{tabular}{|c|c|c|c|c|c|c|c|}
\hline Project activity & Action & $\begin{array}{l}\text { Description of action's } \\
\text { effect on indicator }\end{array}$ & $\begin{array}{l}\text { Spatial exposure to } \\
\text { indicator (low, med, } \\
\text { high) }\end{array}$ & $\begin{array}{l}\text { Temporal } \\
\text { exposure to } \\
\text { indicator (low, } \\
\text { med, high) }\end{array}$ & $\begin{array}{l}\text { Effect's overlap with } \\
\text { critical life stage, } \\
\text { behavior, habitat or } \\
\text { resource (low, med, } \\
\text { high) }\end{array}$ & $\begin{array}{l}\text { Overall risk } \\
\text { to indicator } \\
\text { (low, med, } \\
\text { high, } \\
\text { unknown) } \\
\end{array}$ & Source(s) \\
\hline \multirow{4}{*}{$\begin{array}{l}\text { Decommissioning } \\
\text { of structures on } \\
\text { water's surface or } \\
\text { seabed }\end{array}$} & $\begin{array}{l}\text { Noise and } \\
\text { vibration }\end{array}$ & Possible altered behavior & $\begin{array}{l}\text { Low, fish have patchy } \\
\text { and wide-ranging } \\
\text { distribution }\end{array}$ & $\begin{array}{l}\text { Low, occurs over } 1 \\
\text { to } 2 \text { summers }\end{array}$ & $\begin{array}{l}\text { Low, fish are able to } \\
\text { avoid disturbance area }\end{array}$ & Low & Cada 2008 \\
\hline & $\begin{array}{l}\text { Oil/chemical } \\
\text { release }\end{array}$ & $\begin{array}{l}\text { Toxicity to individuals } \\
\text { and prey }\end{array}$ & $\begin{array}{l}\text { Low, volume released } \\
\text { would occupy small } \\
\text { area }\end{array}$ & $\begin{array}{l}\text { Low, occurs over } 1 \\
\text { to } 2 \text { summers }\end{array}$ & $\begin{array}{l}\text { Low, species in water } \\
\text { column but oil floats on } \\
\text { surface }\end{array}$ & Low & $\begin{array}{l}\text { Nelson and } \\
\text { Woo } 2008\end{array}$ \\
\hline & $\begin{array}{l}\text { Seabed } \\
\text { disturbance }\end{array}$ & $\begin{array}{l}\text { None, species too motile } \\
\text { and near surface to be } \\
\text { affected }\end{array}$ & N/A & N/A & N/A & Low & N/A \\
\hline & $\begin{array}{l}\text { Deconstruction } \\
\text { lights }\end{array}$ & $\begin{array}{l}\text { Aggregation, more } \\
\text { vulnerable to predation }\end{array}$ & $\begin{array}{l}\text { Low, fish have patchy } \\
\text { and wide-ranging } \\
\text { distribution }\end{array}$ & $\begin{array}{l}\text { Low, occurs over } 1 \\
\text { to } 2 \text { summers }\end{array}$ & $\begin{array}{l}\text { Med, species could be } \\
\text { attracted to area }\end{array}$ & Low & $\begin{array}{l}\text { Nightingale et } \\
\text { al. } 2006\end{array}$ \\
\hline
\end{tabular}

\begin{tabular}{|c|c|c|}
\hline $\begin{array}{l}\text { Environmental Assessment for Siting Wave \& Tidal } \\
\text { Energy Projects }\end{array}$ & D-60 & $\begin{array}{r}\text { H. T. Harvey \& Associates } \\
24 \text { November } 2009\end{array}$ \\
\hline
\end{tabular}


Appendix D - Effects of Small Commercial OPT Power Buoy Project Humboldt

Potential effects on juvenile salmonids due to Small Commercial OPT Power Buoy Project at Humboldt site

\begin{tabular}{|c|c|c|c|c|c|c|c|}
\hline Project activity & Action & $\begin{array}{l}\text { Description of action's } \\
\text { effect on indicator }\end{array}$ & $\begin{array}{l}\text { Spatial exposure to } \\
\text { indicator (low, med, } \\
\text { high) }\end{array}$ & $\begin{array}{l}\text { Temporal } \\
\text { exposure to } \\
\text { indicator (low, } \\
\text { med, high) } \\
\end{array}$ & $\begin{array}{l}\text { Effect's overlap with } \\
\text { critical life stage, } \\
\text { behavior, habitat or } \\
\text { resource (low, med, } \\
\text { high) }\end{array}$ & $\begin{array}{l}\text { Overall risk } \\
\text { to indicator } \\
\text { (low, med, } \\
\text { high, } \\
\text { unknown) } \\
\end{array}$ & Source(s) \\
\hline \multicolumn{8}{|c|}{ Construction } \\
\hline \multirow{2}{*}{ Boat traffic } & $\begin{array}{l}\text { Oil/chemical } \\
\text { release }\end{array}$ & $\begin{array}{l}\text { Toxicity to individuals } \\
\text { and prey }\end{array}$ & $\begin{array}{l}\text { Low for typical small } \\
\text { volume releases, High } \\
\text { for large, catastrophic } \\
\text { spill }\end{array}$ & $\begin{array}{l}\text { Low, occurs over } 1 \\
\text { to } 2 \text { summers }\end{array}$ & $\begin{array}{l}\text { Low, species in water } \\
\text { column but oil floats on } \\
\text { surface }\end{array}$ & Low & $\begin{array}{l}\text { Nelson and } \\
\text { Woo } 2008\end{array}$ \\
\hline & $\begin{array}{l}\text { Noise and } \\
\text { vibration }\end{array}$ & $\begin{array}{l}\text { Possible altered } \\
\text { behavior }\end{array}$ & $\begin{array}{l}\text { Low, would occur in } \\
\text { small area relative to } \\
\text { species' range }\end{array}$ & $\begin{array}{l}\text { Low, occurs over } 1 \\
\text { to } 2 \text { summers }\end{array}$ & $\begin{array}{l}\text { Low, species able to } \\
\text { avoid disturbance area }\end{array}$ & Low & $\begin{array}{l}\text { Cada 2008, } \\
\text { Nelson } 2008\end{array}$ \\
\hline \multirow{4}{*}{$\begin{array}{l}\text { Construction of } \\
\text { electrical } \\
\text { collector system, } \\
\text { moorings and } \\
\text { foundations, and } \\
\text { device } \\
\text { installation }\end{array}$} & $\begin{array}{l}\text { Noise and } \\
\text { vibration }\end{array}$ & $\begin{array}{l}\text { Possible altered } \\
\text { behavior }\end{array}$ & Low, see above & $\begin{array}{l}\text { Low, occurs over } 1 \\
\text { to } 2 \text { summers }\end{array}$ & $\begin{array}{l}\text { Low, species able to } \\
\text { avoid disturbance area }\end{array}$ & Low & $\begin{array}{l}\text { Cada 2008, } \\
\text { Nelson } 2008\end{array}$ \\
\hline & $\begin{array}{l}\text { Oil/chemical } \\
\text { release }\end{array}$ & $\begin{array}{l}\text { Toxicity to individuals } \\
\text { and prey }\end{array}$ & $\begin{array}{l}\text { Low, volume released } \\
\text { would occupy small } \\
\text { area relative to species' } \\
\text { range }\end{array}$ & $\begin{array}{l}\text { Low, occurs over } 1 \\
\text { to } 2 \text { summers }\end{array}$ & $\begin{array}{l}\text { Low, species in water } \\
\text { column but oil floats on } \\
\text { surface }\end{array}$ & Low & $\begin{array}{l}\text { Nelson and } \\
\text { Woo } 2008\end{array}$ \\
\hline & $\begin{array}{l}\text { Seabed } \\
\text { disturbance }\end{array}$ & $\begin{array}{l}\text { None, species too } \\
\text { motile and near surface } \\
\text { to be affected }\end{array}$ & N/A & N/A & N/A & Low & N/A \\
\hline & $\begin{array}{l}\text { Construction } \\
\text { lights }\end{array}$ & $\begin{array}{l}\text { Behavioral changes, } \\
\text { more vulnerable to } \\
\text { predation }\end{array}$ & $\begin{array}{l}\text { Low, would occur in } \\
\text { small area relative to } \\
\text { species' range }\end{array}$ & $\begin{array}{l}\text { Low, occurs over } 1 \\
\text { to } 2 \text { summers }\end{array}$ & $\begin{array}{l}\text { Med, species could be } \\
\text { attracted to area }\end{array}$ & Med & $\begin{array}{l}\text { Nelson 2008, } \\
\text { Nightingale et } \\
\text { al. } 2006\end{array}$ \\
\hline \multirow{2}{*}{$\begin{array}{l}\text { Directional } \\
\text { drilling, and } \\
\text { laying cable } \\
\text { under/on seabed }\end{array}$} & $\begin{array}{l}\text { Seabed } \\
\text { disturbance }\end{array}$ & $\begin{array}{l}\text { None, these species too } \\
\text { motile and near surface } \\
\text { to be affected }\end{array}$ & N/A & N/A & N/A & Low & N/A \\
\hline & $\begin{array}{l}\text { Noise and } \\
\text { vibration }\end{array}$ & $\begin{array}{l}\text { Possible altered } \\
\text { behavior }\end{array}$ & $\begin{array}{l}\text { Low, would occur in } \\
\text { small area relative to } \\
\text { species' range }\end{array}$ & $\begin{array}{l}\text { Low, occurs over a } \\
\text { short time period }\end{array}$ & $\begin{array}{l}\text { Low, species able to } \\
\text { avoid disturbance area }\end{array}$ & Low & $\begin{array}{l}\text { Cada 2008, } \\
\text { Nelson } 2008\end{array}$ \\
\hline
\end{tabular}

\begin{tabular}{lr}
\hline Environmental Assessment for Siting Wave \& Tidal & D-61 \\
Energy Projects & H. T. Harvey \& Associates \\
24 November 2009
\end{tabular}


Appendix D - Effects of Small Commercial OPT Power Buoy Project Humboldt

\begin{tabular}{|c|c|c|c|c|c|c|c|}
\hline Project activity & Action & $\begin{array}{l}\text { Description of action's } \\
\text { effect on indicator }\end{array}$ & $\begin{array}{l}\text { Spatial exposure to } \\
\text { indicator (low, med, } \\
\text { high) }\end{array}$ & $\begin{array}{l}\text { Temporal } \\
\text { exposure to } \\
\text { indicator (low, } \\
\text { med, high) }\end{array}$ & $\begin{array}{l}\text { Effect's overlap with } \\
\text { critical life stage, } \\
\text { behavior, habitat or } \\
\text { resource (low, med, } \\
\text { high) }\end{array}$ & $\begin{array}{l}\text { Overall risk } \\
\text { to indicator } \\
\text { (low, med, } \\
\text { high, } \\
\text { unknown) } \\
\end{array}$ & Source(s) \\
\hline \multirow[t]{2}{*}{ Boat traffic } & $\begin{array}{l}\text { Oil/chemical } \\
\text { release }\end{array}$ & $\begin{array}{l}\text { Toxicity to individuals } \\
\text { and prey }\end{array}$ & $\begin{array}{l}\text { Low for typical small } \\
\text { volume releases, High } \\
\text { for large, catastrophic } \\
\text { spill }\end{array}$ & $\begin{array}{l}\text { Low, wouldn't } \\
\text { persist long-term }\end{array}$ & $\begin{array}{l}\text { Low, species in water } \\
\text { column but oil floats on } \\
\text { surface }\end{array}$ & Low & $\begin{array}{l}\text { Nelson and } \\
\text { Woo } 2008\end{array}$ \\
\hline & $\begin{array}{l}\text { Noise and } \\
\text { vibration }\end{array}$ & $\begin{array}{l}\text { Possible altered } \\
\text { behavior }\end{array}$ & $\begin{array}{l}\text { Low, would occur in } \\
\text { small area relative to } \\
\text { species' range }\end{array}$ & $\begin{array}{l}\text { Low, occurs over } 1 \\
\text { to } 2 \text { summers }\end{array}$ & $\begin{array}{l}\text { Low, species able to } \\
\text { avoid disturbance area }\end{array}$ & Low & Nelson 2008 \\
\hline \multirow{2}{*}{$\begin{array}{l}\text { Operation of } \\
\text { turbines or other } \\
\text { moving parts of } \\
\text { devices }\end{array}$} & $\begin{array}{l}\text { Moving device } \\
\text { parts }\end{array}$ & $\begin{array}{l}\text { None, no underwater } \\
\text { moving parts }\end{array}$ & N/A & N/A & N/A & Low & N/A \\
\hline & $\begin{array}{l}\text { Noise and } \\
\text { vibration }\end{array}$ & $\begin{array}{l}\text { Possible altered } \\
\text { behavior }\end{array}$ & $\begin{array}{l}\text { Low, would occur in } \\
\text { small area relative to } \\
\text { species' range }\end{array}$ & $\begin{array}{l}\text { High, would be } \\
\text { continuous for life } \\
\text { of project }\end{array}$ & $\begin{array}{l}\text { Low, species able to } \\
\text { avoid disturbance area }\end{array}$ & Low & Cada 2008 \\
\hline \multirow{3}{*}{$\begin{array}{l}\text { Structures in } \\
\text { water column and } \\
\text { on seabed, such } \\
\text { as devices and } \\
\text { moorings and } \\
\text { footings }\end{array}$} & Structure & $\begin{array}{l}\text { Possible reef effect, } \\
\text { attraction of predators } \\
\text { (e.g., rockfish) }\end{array}$ & Low, see above & $\begin{array}{l}\text { High, would be } \\
\text { continuous for life } \\
\text { of project }\end{array}$ & $\begin{array}{l}\text { Med, species present in } \\
\text { area in spring, summer } \\
\text { and fall }\end{array}$ & Unknown & Nelson 2008 \\
\hline & $\begin{array}{l}\text { Water circulation } \\
\text { changes }\end{array}$ & $\begin{array}{l}\text { None, project not large } \\
\text { enough to affect prey }\end{array}$ & N/A & N/A & N/A & Low & N/A \\
\hline & $\begin{array}{l}\text { Oil/chemical } \\
\text { release }\end{array}$ & $\begin{array}{l}\text { Toxicity to individuals } \\
\text { and prey }\end{array}$ & $\begin{array}{l}\text { Low, would occur in } \\
\text { small area relative to } \\
\text { species' range }\end{array}$ & $\begin{array}{l}\text { Low, wouldn’t } \\
\text { persist long-term }\end{array}$ & $\begin{array}{l}\text { Low, species in water } \\
\text { column but oil floats on } \\
\text { surface }\end{array}$ & Low & $\begin{array}{l}\text { Nelson and } \\
\text { Woo } 2008\end{array}$ \\
\hline $\begin{array}{l}\text { Electricity } \\
\text { conduction } \\
\text { through cable }\end{array}$ & EMF & $\begin{array}{l}\text { Possible behavior, } \\
\text { orientation changes }\end{array}$ & Low, see above & $\begin{array}{l}\text { High, would be } \\
\text { continuous for life } \\
\text { of project }\end{array}$ & $\begin{array}{l}\text { Med, species present in } \\
\text { area in spring, summer } \\
\text { and fall }\end{array}$ & Unknown & Nelson 2008 \\
\hline \multirow{2}{*}{$\begin{array}{l}\text { Structures on } \\
\text { water's surface }\end{array}$} & Structure & $\begin{array}{l}\text { Possible FAD effect, } \\
\text { attraction of predators }\end{array}$ & Low, see above & $\begin{array}{l}\text { High, would be } \\
\text { continuous for life } \\
\text { of project }\end{array}$ & $\begin{array}{l}\text { Med, species present in } \\
\text { area in spring, summer } \\
\text { and fall }\end{array}$ & Unknown & Nelson 2008 \\
\hline & Navigation lights & $\begin{array}{l}\text { Behavioral changes, } \\
\text { more vulnerable to } \\
\text { predation }\end{array}$ & Low, see above & $\begin{array}{l}\text { High, would be } \\
\text { continuous for life } \\
\text { of project }\end{array}$ & $\begin{array}{l}\text { Med, species could be } \\
\text { attracted to area }\end{array}$ & Med & $\begin{array}{l}\text { Nightingale et } \\
\text { al. } 2006\end{array}$ \\
\hline \multicolumn{8}{|c|}{ Decommissioning } \\
\hline Boat traffic & $\begin{array}{l}\text { Oil/chemical } \\
\text { release }\end{array}$ & $\begin{array}{l}\text { Toxicity to individuals } \\
\text { and prey }\end{array}$ & $\begin{array}{l}\text { Low for typical small } \\
\text { volume releases, High } \\
\text { for large, catastrophic } \\
\text { spill }\end{array}$ & $\begin{array}{l}\text { Low, occurs over } 1 \\
\text { to } 2 \text { summers }\end{array}$ & $\begin{array}{l}\text { Low, species in water } \\
\text { column but oil floats on } \\
\text { surface }\end{array}$ & Low & $\begin{array}{l}\text { Nelson and } \\
\text { Woo } 2008\end{array}$ \\
\hline
\end{tabular}

\begin{tabular}{lr}
\hline Environmental Assessment for Siting Wave \& Tidal & D-62 \\
Energy Projects &
\end{tabular}


Appendix D - Effects of Small Commercial OPT Power Buoy Project Humboldt

\begin{tabular}{|c|c|c|c|c|c|c|c|}
\hline Project activity & Action & $\begin{array}{l}\text { Description of action's } \\
\text { effect on indicator }\end{array}$ & $\begin{array}{l}\text { Spatial exposure to } \\
\text { indicator (low, med, } \\
\text { high) }\end{array}$ & $\begin{array}{l}\text { Temporal } \\
\text { exposure to } \\
\text { indicator (low, } \\
\text { med, high) }\end{array}$ & $\begin{array}{l}\text { Effect's overlap with } \\
\text { critical life stage, } \\
\text { behavior, habitat or } \\
\text { resource (low, med, } \\
\text { high) }\end{array}$ & $\begin{array}{l}\text { Overall risk } \\
\text { to indicator } \\
\text { (low, med, } \\
\text { high, } \\
\text { unknown) }\end{array}$ & Source(s) \\
\hline & $\begin{array}{l}\text { Noise and } \\
\text { vibration }\end{array}$ & $\begin{array}{l}\text { Possible altered } \\
\text { behavior }\end{array}$ & $\begin{array}{l}\text { Low, would occur in } \\
\text { small area relative to } \\
\text { species' range }\end{array}$ & $\begin{array}{l}\text { Low, occurs over a } \\
\text { short time period }\end{array}$ & $\begin{array}{l}\text { Low, species able to } \\
\text { avoid disturbance area }\end{array}$ & Low & $\begin{array}{l}\text { Cada 2008, } \\
\text { Nelson } 2008\end{array}$ \\
\hline \multirow{4}{*}{$\begin{array}{l}\text { Decommissioning } \\
\text { of structures on } \\
\text { water's surface or } \\
\text { seabed }\end{array}$} & $\begin{array}{l}\text { Noise and } \\
\text { vibration }\end{array}$ & $\begin{array}{l}\text { Possible altered } \\
\text { behavior }\end{array}$ & Low, see above & $\begin{array}{l}\text { Low, occurs over } 1 \\
\text { to } 2 \text { summers }\end{array}$ & $\begin{array}{l}\text { Low, species able to } \\
\text { avoid disturbance area }\end{array}$ & Low & $\begin{array}{l}\text { Cada 2008, } \\
\text { Nelson 2008 }\end{array}$ \\
\hline & $\begin{array}{l}\text { Oil/chemical } \\
\text { release }\end{array}$ & $\begin{array}{l}\text { Toxicity to individuals } \\
\text { and prey }\end{array}$ & $\begin{array}{l}\text { Low, volume released } \\
\text { would occupy small } \\
\text { area relative to species' } \\
\text { range }\end{array}$ & $\begin{array}{l}\text { Low, occurs over } 1 \\
\text { to } 2 \text { summers }\end{array}$ & $\begin{array}{l}\text { Low, species in water } \\
\text { column but oil floats on } \\
\text { surface }\end{array}$ & Low & $\begin{array}{l}\text { Nelson and } \\
\text { Woo } 2008\end{array}$ \\
\hline & $\begin{array}{l}\text { Seabed } \\
\text { disturbance }\end{array}$ & $\begin{array}{l}\text { None, species too } \\
\text { motile and near surface } \\
\text { to be affected }\end{array}$ & N/A & N/A & N/A & Low & N/A \\
\hline & $\begin{array}{l}\text { Deconstruction } \\
\text { lights }\end{array}$ & $\begin{array}{l}\text { Behavioral changes, } \\
\text { more vulnerable to } \\
\text { predation }\end{array}$ & $\begin{array}{l}\text { Low, would occur in } \\
\text { small area relative to } \\
\text { species' range }\end{array}$ & $\begin{array}{l}\text { Low, occurs over } 1 \\
\text { to } 2 \text { summers }\end{array}$ & $\begin{array}{l}\text { Med, species could be } \\
\text { attracted to area }\end{array}$ & Low & $\begin{array}{l}\text { Nightingale et } \\
\text { al. } 2006\end{array}$ \\
\hline
\end{tabular}

\begin{tabular}{lr}
\hline Environmental Assessment for Siting Wave \& Tidal & D-63 \\
Energy Projects & H. T. Harvey \& Associates \\
24 November 2009
\end{tabular}


Appendix D - Effects of Small Commercial OPT Power Buoy Project Humboldt

Potential effects on adult salmonids due to Small Commercial OPT Power Buoy Project at Humboldt site

\begin{tabular}{|c|c|c|c|c|c|c|c|}
\hline Project activity & Action & $\begin{array}{l}\text { Description of action's } \\
\text { effect on indicator }\end{array}$ & $\begin{array}{l}\text { Spatial exposure to } \\
\text { indicator (low, med, } \\
\text { high) }\end{array}$ & $\begin{array}{l}\text { Temporal } \\
\text { exposure to } \\
\text { indicator (low, } \\
\text { med, high) } \\
\end{array}$ & $\begin{array}{l}\text { Effect's overlap with } \\
\text { critical life stage, } \\
\text { behavior, habitat or } \\
\text { resource (low, med, } \\
\text { high) }\end{array}$ & $\begin{array}{l}\text { Overall risk } \\
\text { to indicator } \\
\text { (low, med, } \\
\text { high, } \\
\text { unknown) } \\
\end{array}$ & Source(s) \\
\hline \multicolumn{8}{|c|}{ Construction } \\
\hline \multirow[t]{2}{*}{ Boat traffic } & $\begin{array}{l}\text { Oil/chemical } \\
\text { release }\end{array}$ & $\begin{array}{l}\text { Toxicity to individuals } \\
\text { and prey }\end{array}$ & $\begin{array}{l}\text { Low for typical small } \\
\text { volume releases, High } \\
\text { for large, catastrophic } \\
\text { spill }\end{array}$ & $\begin{array}{l}\text { Low, occurs over } 1 \\
\text { to } 2 \text { summers }\end{array}$ & $\begin{array}{l}\text { Low, species in water } \\
\text { column but oil floats on } \\
\text { surface }\end{array}$ & Low & $\begin{array}{l}\text { Nelson and } \\
\text { Woo } 2008\end{array}$ \\
\hline & $\begin{array}{l}\text { Noise and } \\
\text { vibration }\end{array}$ & $\begin{array}{l}\text { Possible altered } \\
\text { behavior }\end{array}$ & $\begin{array}{l}\text { Low, would occur in } \\
\text { small area relative to } \\
\text { species' range }\end{array}$ & $\begin{array}{l}\text { Low, occurs over } 1 \\
\text { to } 2 \text { summers }\end{array}$ & $\begin{array}{l}\text { Low, species able to } \\
\text { avoid disturbance area }\end{array}$ & Low & $\begin{array}{l}\text { Cada 2008, } \\
\text { Nelson } 2008\end{array}$ \\
\hline \multirow{4}{*}{$\begin{array}{l}\text { Construction of } \\
\text { electrical } \\
\text { collector system, } \\
\text { moorings and } \\
\text { foundations, and } \\
\text { device } \\
\text { installation }\end{array}$} & $\begin{array}{l}\text { Noise and } \\
\text { vibration }\end{array}$ & $\begin{array}{l}\text { Possible altered } \\
\text { behavior }\end{array}$ & Low, see above & $\begin{array}{l}\text { Low, occurs over } 1 \\
\text { to } 2 \text { summers }\end{array}$ & $\begin{array}{l}\text { Low, species able to } \\
\text { avoid disturbance area }\end{array}$ & Low & $\begin{array}{l}\text { Cada 2008, } \\
\text { Nelson 2008 } \\
\end{array}$ \\
\hline & $\begin{array}{l}\text { Oil/chemical } \\
\text { release }\end{array}$ & $\begin{array}{l}\text { Toxicity to individuals } \\
\text { and prey }\end{array}$ & $\begin{array}{l}\text { Low, volume released } \\
\text { would occupy small } \\
\text { area relative to species' } \\
\text { range }\end{array}$ & $\begin{array}{l}\text { Low, occurs over } 1 \\
\text { to } 2 \text { summers }\end{array}$ & $\begin{array}{l}\text { Low, species in water } \\
\text { column but oil floats on } \\
\text { surface }\end{array}$ & Low & $\begin{array}{l}\text { Nelson and } \\
\text { Woo } 2008\end{array}$ \\
\hline & $\begin{array}{l}\text { Seabed } \\
\text { disturbance }\end{array}$ & $\begin{array}{l}\text { None, species too } \\
\text { motile and near surface } \\
\text { to be affected }\end{array}$ & N/A & N/A & N/A & Low & N/A \\
\hline & $\begin{array}{l}\text { Construction } \\
\text { lights }\end{array}$ & $\begin{array}{l}\text { None, not known to be } \\
\text { affected by lights }\end{array}$ & N/A & N/A & N/A & Low & $\begin{array}{l}\text { Nightingale et } \\
\text { al. } 2006\end{array}$ \\
\hline \multirow{2}{*}{$\begin{array}{l}\text { Directional } \\
\text { drilling, and } \\
\text { laying cable } \\
\text { under/on seabed }\end{array}$} & $\begin{array}{l}\text { Seabed } \\
\text { disturbance }\end{array}$ & $\begin{array}{l}\text { None, species too } \\
\text { motile and near surface } \\
\text { to be affected }\end{array}$ & N/A & N/A & N/A & Low & N/A \\
\hline & $\begin{array}{l}\text { Noise and } \\
\text { vibration }\end{array}$ & $\begin{array}{l}\text { Possible altered } \\
\text { behavior }\end{array}$ & $\begin{array}{l}\text { Low, would occur in } \\
\text { small area relative to } \\
\text { species' range }\end{array}$ & $\begin{array}{l}\text { Low, occurs over a } \\
\text { short time period }\end{array}$ & $\begin{array}{l}\text { Low, species able to } \\
\text { avoid disturbance area }\end{array}$ & Low & $\begin{array}{l}\text { Cada 2008, } \\
\text { Nelson } 2008\end{array}$ \\
\hline \multicolumn{8}{|c|}{ Operation and Maintenance } \\
\hline \multirow[t]{2}{*}{ Boat traffic } & $\begin{array}{l}\text { Oil/chemical } \\
\text { release }\end{array}$ & $\begin{array}{l}\text { Toxicity to individuals } \\
\text { and prey }\end{array}$ & $\begin{array}{l}\text { Low for typical small } \\
\text { volume releases, High } \\
\text { for large, catastrophic } \\
\text { spill }\end{array}$ & $\begin{array}{l}\text { Low, wouldn’t } \\
\text { persist long-term }\end{array}$ & $\begin{array}{l}\text { Low, species in water } \\
\text { column but oil floats on } \\
\text { surface }\end{array}$ & Low & $\begin{array}{l}\text { Nelson and } \\
\text { Woo } 2008\end{array}$ \\
\hline & $\begin{array}{l}\text { Noise and } \\
\text { vibration }\end{array}$ & $\begin{array}{l}\text { Possible altered } \\
\text { behavior }\end{array}$ & $\begin{array}{l}\text { Low, would occur in } \\
\text { small area relative to } \\
\text { species’ range }\end{array}$ & $\begin{array}{l}\text { Low, occurs over } 1 \\
\text { to } 2 \text { summers }\end{array}$ & $\begin{array}{l}\text { Low, species able to } \\
\text { avoid disturbance area }\end{array}$ & Low & Nelson 2008 \\
\hline
\end{tabular}

\begin{tabular}{lr}
\hline Environmental Assessment for Siting Wave \& Tidal & D-64 \\
Energy Projects & H. Harvey \& Associates \\
24 November 2009
\end{tabular}


Appendix D - Effects of Small Commercial OPT Power Buoy Project Humboldt

\begin{tabular}{|c|c|c|c|c|c|c|c|}
\hline Project activity & Action & $\begin{array}{l}\text { Description of action's } \\
\text { effect on indicator }\end{array}$ & $\begin{array}{l}\text { Spatial exposure to } \\
\text { indicator (low, med, } \\
\text { high) }\end{array}$ & $\begin{array}{l}\text { Temporal } \\
\text { exposure to } \\
\text { indicator (low, } \\
\text { med, high) }\end{array}$ & $\begin{array}{l}\text { Effect's overlap with } \\
\text { critical life stage, } \\
\text { behavior, habitat or } \\
\text { resource (low, med, } \\
\text { high) }\end{array}$ & $\begin{array}{l}\text { Overall risk } \\
\text { to indicator } \\
\text { (low, med, } \\
\text { high, } \\
\text { unknown) } \\
\end{array}$ & Source(s) \\
\hline \multirow{2}{*}{$\begin{array}{l}\text { Operation of } \\
\text { turbines or other } \\
\text { moving parts of } \\
\text { devices }\end{array}$} & $\begin{array}{l}\text { Moving device } \\
\text { parts }\end{array}$ & $\begin{array}{l}\text { None, no underwater } \\
\text { moving parts }\end{array}$ & N/A & N/A & N/A & Low & N/A \\
\hline & $\begin{array}{l}\text { Noise and } \\
\text { vibration }\end{array}$ & $\begin{array}{l}\text { Possible altered } \\
\text { behavior }\end{array}$ & $\begin{array}{l}\text { Low, would occur in } \\
\text { small area relative to } \\
\text { species' range }\end{array}$ & $\begin{array}{l}\text { High, would be } \\
\text { continuous for life } \\
\text { of project }\end{array}$ & $\begin{array}{l}\text { Low, species able to } \\
\text { avoid disturbance area }\end{array}$ & Low & Cada 2008 \\
\hline \multirow{3}{*}{$\begin{array}{l}\text { Structures in } \\
\text { water column and } \\
\text { on seabed, such } \\
\text { as devices and } \\
\text { moorings and } \\
\text { footings }\end{array}$} & Structure & $\begin{array}{l}\text { Possible reef effect, } \\
\text { attraction of predators }\end{array}$ & Low, see above & $\begin{array}{l}\text { High, would be } \\
\text { continuous for life } \\
\text { of project }\end{array}$ & $\begin{array}{l}\text { Med, species present in } \\
\text { area in spring, summer } \\
\text { and fall }\end{array}$ & Unknown & Nelson 2008 \\
\hline & $\begin{array}{l}\text { Water circulation } \\
\text { changes }\end{array}$ & $\begin{array}{l}\text { None, project not large } \\
\text { enough to affect prey }\end{array}$ & N/A & N/A & N/A & Low & N/A \\
\hline & $\begin{array}{l}\text { Oil/chemical } \\
\text { release }\end{array}$ & $\begin{array}{l}\text { Toxicity to individuals } \\
\text { and prey }\end{array}$ & $\begin{array}{l}\text { Low, would occur in } \\
\text { small area relative to } \\
\text { species' range }\end{array}$ & $\begin{array}{l}\text { Low, wouldn't } \\
\text { persist long-term }\end{array}$ & $\begin{array}{l}\text { Low, species in water } \\
\text { column but oil floats on } \\
\text { surface }\end{array}$ & Low & $\begin{array}{l}\text { Nelson and } \\
\text { Woo } 2008\end{array}$ \\
\hline $\begin{array}{l}\text { Electricity } \\
\text { conduction } \\
\text { through cable } \\
\end{array}$ & EMF & $\begin{array}{l}\text { Possible behavior, } \\
\text { orientation changes }\end{array}$ & Low, see above & $\begin{array}{l}\text { High, would be } \\
\text { continuous for life } \\
\text { of project }\end{array}$ & $\begin{array}{l}\text { Med, species present in } \\
\text { area in spring, summer } \\
\text { and fall }\end{array}$ & Unknown & Nelson 2008 \\
\hline \multirow{2}{*}{$\begin{array}{l}\text { Structures on } \\
\text { water's surface }\end{array}$} & Structure & $\begin{array}{l}\text { Possible FAD effect, } \\
\text { attraction of predators }\end{array}$ & Low, see above & $\begin{array}{l}\text { High, would be } \\
\text { continuous for life } \\
\text { of project }\end{array}$ & $\begin{array}{l}\text { Med, species present in } \\
\text { area in spring, summer } \\
\text { and fall }\end{array}$ & Unknown & Nelson 2008 \\
\hline & Navigation lights & $\begin{array}{l}\text { None, not known to be } \\
\text { affected by lights }\end{array}$ & N/A & N/A & N/A & Low & $\begin{array}{l}\text { Nightingale et } \\
\text { al. } 2006\end{array}$ \\
\hline \multicolumn{8}{|c|}{ Decommissioning } \\
\hline \multirow[t]{2}{*}{ Boat traffic } & $\begin{array}{l}\text { Oil/chemical } \\
\text { release }\end{array}$ & $\begin{array}{l}\text { Toxicity to individuals } \\
\text { and prey }\end{array}$ & $\begin{array}{l}\text { Low for typical small } \\
\text { volume releases, High } \\
\text { for large, catastrophic } \\
\text { spill }\end{array}$ & $\begin{array}{l}\text { Low, occurs over } 1 \\
\text { to } 2 \text { summers }\end{array}$ & $\begin{array}{l}\text { Low, species in water } \\
\text { column but oil floats on } \\
\text { surface }\end{array}$ & Low & $\begin{array}{l}\text { Nelson and } \\
\text { Woo } 2008\end{array}$ \\
\hline & $\begin{array}{l}\text { Noise and } \\
\text { vibration }\end{array}$ & $\begin{array}{l}\text { Possible altered } \\
\text { behavior }\end{array}$ & $\begin{array}{l}\text { Low, would occur in } \\
\text { small area relative to } \\
\text { species' range }\end{array}$ & $\begin{array}{l}\text { Low, occurs over } 1 \\
\text { to } 2 \text { summers }\end{array}$ & $\begin{array}{l}\text { Low, species present } \\
\text { but able to avoid } \\
\text { disturbance area }\end{array}$ & Low & $\begin{array}{l}\text { Cada 2008, } \\
\text { Nelson } 2008\end{array}$ \\
\hline \multirow{2}{*}{$\begin{array}{l}\text { Decommissioning } \\
\text { of structures on } \\
\text { water's surface or } \\
\text { seabed }\end{array}$} & $\begin{array}{l}\text { Noise and } \\
\text { vibration }\end{array}$ & $\begin{array}{l}\text { Possible altered } \\
\text { behavior }\end{array}$ & $\begin{array}{l}\text { Low, would occur in } \\
\text { small area relative to } \\
\text { species' range }\end{array}$ & $\begin{array}{l}\text { Low, occurs over } 1 \\
\text { to } 2 \text { summers }\end{array}$ & $\begin{array}{l}\text { Low, species present } \\
\text { but able to avoid } \\
\text { disturbance area } \\
\end{array}$ & Low & $\begin{array}{l}\text { Cada 2008, } \\
\text { Nelson } 2008\end{array}$ \\
\hline & $\begin{array}{l}\text { Oil/chemical } \\
\text { release }\end{array}$ & $\begin{array}{l}\text { Toxicity to individuals } \\
\text { and prey }\end{array}$ & $\begin{array}{l}\text { Low, volume released } \\
\text { would occupy small } \\
\text { area relative to species' } \\
\text { range }\end{array}$ & $\begin{array}{l}\text { Low, occurs over } 1 \\
\text { to } 2 \text { summers }\end{array}$ & $\begin{array}{l}\text { Low, species in water } \\
\text { column but oil floats on } \\
\text { surface }\end{array}$ & Low & $\begin{array}{l}\text { Nelson and } \\
\text { Woo } 2008\end{array}$ \\
\hline
\end{tabular}

\begin{tabular}{lr}
\hline Environmental Assessment for Siting Wave \& Tidal & D-65 \\
Energy Projects & H. T. Harvey \& Associates \\
24 November 2009
\end{tabular}


Appendix D - Effects of Small Commercial OPT Power Buoy Project Humboldt

\begin{tabular}{|c|c|c|c|c|c|c|c|}
\hline Project activity & Action & $\begin{array}{l}\text { Description of action's } \\
\text { effect on indicator }\end{array}$ & $\begin{array}{l}\text { Spatial exposure to } \\
\text { indicator (low, med, } \\
\text { high) }\end{array}$ & $\begin{array}{l}\text { Temporal } \\
\text { exposure to } \\
\text { indicator (low, } \\
\text { med, high) }\end{array}$ & $\begin{array}{l}\text { Effect's overlap with } \\
\text { critical life stage, } \\
\text { behavior, habitat or } \\
\text { resource (low, med, } \\
\text { high) }\end{array}$ & $\begin{array}{l}\text { Overall risk } \\
\text { to indicator } \\
\text { (low, med, } \\
\text { high, } \\
\text { unknown) }\end{array}$ & Source(s) \\
\hline & $\begin{array}{l}\text { Seabed } \\
\text { disturbance }\end{array}$ & $\begin{array}{l}\text { None, species too } \\
\text { motile and near surface } \\
\text { to be affected }\end{array}$ & N/A & N/A & N/A & Low & N/A \\
\hline & $\begin{array}{l}\text { Deconstruction } \\
\text { lights }\end{array}$ & $\begin{array}{l}\text { None, not known to be } \\
\text { affected by lights }\end{array}$ & N/A & N/A & N/A & Low & $\begin{array}{l}\text { Nightingale et } \\
\text { al. } 2006\end{array}$ \\
\hline
\end{tabular}

\begin{tabular}{lr}
\hline Environmental Assessment for Siting Wave \& Tidal & D-66 \\
Energy Projects & H. T. Harvey \& Associates \\
24 November 2009
\end{tabular}


Appendix D - Effects of Small Commercial OPT Power Buoy Project Humboldt

Potential effects on green sturgeon due to Small Commercial OPT Power Buoy Project at Humboldt site

\begin{tabular}{|c|c|c|c|c|c|c|c|}
\hline Project activity & Action & $\begin{array}{l}\text { Description of action's } \\
\text { effect on indicator }\end{array}$ & $\begin{array}{l}\text { Spatial exposure to } \\
\text { indicator (low, med, } \\
\text { high) }\end{array}$ & $\begin{array}{l}\text { Temporal } \\
\text { exposure to } \\
\text { indicator (low, } \\
\text { med, high) }\end{array}$ & $\begin{array}{l}\text { Effect's overlap with } \\
\text { critical life stage, } \\
\text { behavior, habitat or } \\
\text { resource (low, med, } \\
\text { high) }\end{array}$ & $\begin{array}{l}\text { Overall risk } \\
\text { to indicator } \\
\text { (low, med, } \\
\text { high, } \\
\text { unknown) }\end{array}$ & Source(s) \\
\hline \multicolumn{8}{|c|}{ Construction } \\
\hline \multirow[t]{2}{*}{ Boat traffic } & $\begin{array}{l}\text { Oil/chemical } \\
\text { release }\end{array}$ & $\begin{array}{l}\text { Toxicity to individuals } \\
\text { and prey }\end{array}$ & $\begin{array}{l}\text { Low for typical small } \\
\text { volume releases, High } \\
\text { for large, catastrophic } \\
\text { spill }\end{array}$ & $\begin{array}{l}\text { Low, occurs over } 1 \\
\text { to } 2 \text { summers }\end{array}$ & $\begin{array}{l}\text { Low, species on bottom } \\
\text { but oil floats on surface }\end{array}$ & Low & $\begin{array}{l}\text { Nelson and } \\
\text { Woo } 2008\end{array}$ \\
\hline & $\begin{array}{l}\text { Noise and } \\
\text { vibration }\end{array}$ & $\begin{array}{l}\text { Possible altered } \\
\text { behavior }\end{array}$ & $\begin{array}{l}\text { Low, would occur in } \\
\text { small area relative to } \\
\text { species’ range }\end{array}$ & $\begin{array}{l}\text { Low, occurs over } 1 \\
\text { to } 2 \text { summers }\end{array}$ & $\begin{array}{l}\text { Low, species motile and } \\
\text { able to avoid } \\
\text { disturbance area }\end{array}$ & Low & Cada 2008 \\
\hline \multirow{4}{*}{$\begin{array}{l}\text { Construction of } \\
\text { electrical } \\
\text { collector system, } \\
\text { moorings and } \\
\text { foundations, and } \\
\text { device } \\
\text { installation }\end{array}$} & $\begin{array}{l}\text { Noise and } \\
\text { vibration }\end{array}$ & $\begin{array}{l}\text { Possible altered } \\
\text { behavior }\end{array}$ & $\begin{array}{l}\text { Low, would occur in } \\
\text { small area relative to } \\
\text { species' range }\end{array}$ & $\begin{array}{l}\text { Low, occurs over } 1 \\
\text { to } 2 \text { summers }\end{array}$ & Low, see above & Low & Cada 2008 \\
\hline & $\begin{array}{l}\text { Oil/chemical } \\
\text { release }\end{array}$ & $\begin{array}{l}\text { Toxicity to individuals } \\
\text { and prey }\end{array}$ & $\begin{array}{l}\text { Low, volume released } \\
\text { would occupy small } \\
\text { area relative to species' } \\
\text { range }\end{array}$ & $\begin{array}{l}\text { Low, occurs over } 1 \\
\text { to } 2 \text { summers }\end{array}$ & $\begin{array}{l}\text { Low, species on bottom } \\
\text { but oil floats on surface }\end{array}$ & Low & $\begin{array}{l}\text { Nelson and } \\
\text { Woo } 2008\end{array}$ \\
\hline & $\begin{array}{l}\text { Seabed } \\
\text { disturbance }\end{array}$ & $\begin{array}{l}\text { Temporary habitat loss, } \\
\text { possible changes to } \\
\text { benthic prey resources }\end{array}$ & $\begin{array}{l}\text { Low, would occur in } \\
\text { small area relative to } \\
\text { species' range }\end{array}$ & $\begin{array}{l}\text { Low, occurs over } 1 \\
\text { to } 2 \text { summers }\end{array}$ & $\begin{array}{l}\text { Low, species motile and } \\
\text { able to avoid } \\
\text { disturbance area }\end{array}$ & Low & Nelson 2008 \\
\hline & $\begin{array}{l}\text { Construction } \\
\text { lights }\end{array}$ & $\begin{array}{l}\text { None, this species not } \\
\text { known to be attracted to } \\
\text { lights }\end{array}$ & N/A & N/A & N/A & Low & $\begin{array}{l}\text { Nightingale et } \\
\text { al. } 2006\end{array}$ \\
\hline \multirow{2}{*}{$\begin{array}{l}\text { Directional } \\
\text { drilling, and } \\
\text { laying cable } \\
\text { under/on seabed }\end{array}$} & $\begin{array}{l}\text { Seabed } \\
\text { disturbance }\end{array}$ & $\begin{array}{l}\text { Temporary habitat loss, } \\
\text { possible changes to } \\
\text { benthic prey resources }\end{array}$ & $\begin{array}{l}\text { Low, would occur in } \\
\text { small area relative to } \\
\text { species' range }\end{array}$ & $\begin{array}{l}\text { Low, occurs over a } \\
\text { short time period }\end{array}$ & $\begin{array}{l}\text { Low, species motile and } \\
\text { able to avoid } \\
\text { disturbance area }\end{array}$ & Low & Nelson 2008 \\
\hline & $\begin{array}{l}\text { Noise and } \\
\text { vibration }\end{array}$ & $\begin{array}{l}\text { Possible altered } \\
\text { behavior }\end{array}$ & $\begin{array}{l}\text { Low, would occur in } \\
\text { small area relative to } \\
\text { species' range }\end{array}$ & $\begin{array}{l}\text { Low, occurs over a } \\
\text { short time period }\end{array}$ & Low, see above & Low & Cada 2008 \\
\hline \multicolumn{8}{|c|}{ Operation and Maintenance } \\
\hline \multirow[t]{2}{*}{ Boat traffic } & $\begin{array}{l}\text { Oil/chemical } \\
\text { release }\end{array}$ & $\begin{array}{l}\text { Toxicity to individuals } \\
\text { and prey }\end{array}$ & $\begin{array}{l}\text { Low for typical small } \\
\text { volume releases, High } \\
\text { for large, catastrophic } \\
\text { spill }\end{array}$ & $\begin{array}{l}\text { Low, wouldn't } \\
\text { persist long-term }\end{array}$ & $\begin{array}{l}\text { Low, species on bottom } \\
\text { but oil floats on surface }\end{array}$ & Low & $\begin{array}{l}\text { Nelson and } \\
\text { Woo } 2008\end{array}$ \\
\hline & $\begin{array}{l}\text { Noise and } \\
\text { vibration }\end{array}$ & $\begin{array}{l}\text { Possible altered } \\
\text { behavior }\end{array}$ & $\begin{array}{l}\text { Low, would occur in } \\
\text { small area relative to } \\
\text { species’ range }\end{array}$ & $\begin{array}{l}\text { Low, wouldn't } \\
\text { persist long-term }\end{array}$ & $\begin{array}{l}\text { Low, species motile and } \\
\text { able to avoid } \\
\text { disturbance area }\end{array}$ & Low & Cada 2008 \\
\hline
\end{tabular}

\begin{tabular}{|c|c|c|}
\hline $\begin{array}{l}\text { Environmental Assessment for Siting Wave \& Tidal } \\
\text { Energy Projects }\end{array}$ & D-67 & $\begin{array}{r}\text { H. T. Harvey \& Associates } \\
24 \text { November } 2009\end{array}$ \\
\hline
\end{tabular}


Appendix D - Effects of Small Commercial OPT Power Buoy Project Humboldt

\begin{tabular}{|c|c|c|c|c|c|c|c|}
\hline Project activity & Action & $\begin{array}{l}\text { Description of action's } \\
\text { effect on indicator }\end{array}$ & $\begin{array}{l}\text { Spatial exposure to } \\
\text { indicator (low, med, } \\
\text { high) }\end{array}$ & $\begin{array}{l}\text { Temporal } \\
\text { exposure to } \\
\text { indicator (low, } \\
\text { med, high) }\end{array}$ & $\begin{array}{l}\text { Effect's overlap with } \\
\text { critical life stage, } \\
\text { behavior, habitat or } \\
\text { resource (low, med, } \\
\text { high) }\end{array}$ & $\begin{array}{l}\text { Overall risk } \\
\text { to indicator } \\
\text { (low, med, } \\
\text { high, } \\
\text { unknown) }\end{array}$ & Source(s) \\
\hline \multirow{2}{*}{$\begin{array}{l}\text { Operation of } \\
\text { turbines or other } \\
\text { moving parts of } \\
\text { devices }\end{array}$} & $\begin{array}{l}\text { Moving device } \\
\text { parts }\end{array}$ & $\begin{array}{l}\text { None, no underwater } \\
\text { moving parts }\end{array}$ & N/A & N/A & N/A & Low & N/A \\
\hline & $\begin{array}{l}\text { Noise and } \\
\text { vibration }\end{array}$ & $\begin{array}{l}\text { Possible altered } \\
\text { behavior }\end{array}$ & $\begin{array}{l}\text { Low, would occur in } \\
\text { small area relative to } \\
\text { species' range }\end{array}$ & $\begin{array}{l}\text { High, would be } \\
\text { continuous for life } \\
\text { of project }\end{array}$ & $\begin{array}{l}\text { Med, occurs in critical } \\
\text { habitat for species }\end{array}$ & Unknown & Cada 2008 \\
\hline \multirow{3}{*}{$\begin{array}{l}\text { Structures in } \\
\text { water column and } \\
\text { on seabed, such } \\
\text { as devices and } \\
\text { moorings and } \\
\text { footings }\end{array}$} & Structure & $\begin{array}{l}\text { Possible reef effect, } \\
\text { attraction of predators }\end{array}$ & $\begin{array}{l}\text { Low, would occur in } \\
\text { small area relative to } \\
\text { species' range }\end{array}$ & $\begin{array}{l}\text { High, would be } \\
\text { continuous for life } \\
\text { of project }\end{array}$ & $\begin{array}{l}\text { Med, occurs in critical } \\
\text { habitat for species }\end{array}$ & Unknown & $\begin{array}{l}\text { Boehlert et al. } \\
2008\end{array}$ \\
\hline & $\begin{array}{l}\text { Water circulation } \\
\text { changes }\end{array}$ & $\begin{array}{l}\text { None, project not large } \\
\text { enough to affect prey }\end{array}$ & N/A & N/A & N/A & Low & N/A \\
\hline & $\begin{array}{l}\text { Oil/chemical } \\
\text { release }\end{array}$ & $\begin{array}{l}\text { Toxicity to individuals } \\
\text { and prey }\end{array}$ & $\begin{array}{l}\text { Low, would occur in } \\
\text { small area relative to } \\
\text { species' range }\end{array}$ & $\begin{array}{l}\text { Low, wouldn't } \\
\text { persist long-term }\end{array}$ & $\begin{array}{l}\text { Low, species on bottom } \\
\text { but oil floats on surface }\end{array}$ & Low & $\begin{array}{l}\text { Nelson and } \\
\text { Woo } 2008\end{array}$ \\
\hline $\begin{array}{l}\text { Electricity } \\
\text { conduction } \\
\text { through cable }\end{array}$ & EMF & $\begin{array}{l}\text { Possible changes in } \\
\text { orientation, behavior }\end{array}$ & $\begin{array}{l}\text { Low, would occur in } \\
\text { small area relative to } \\
\text { species' range }\end{array}$ & $\begin{array}{l}\text { High, would be } \\
\text { continuous for life } \\
\text { of project }\end{array}$ & $\begin{array}{l}\text { Med, occurs in critical } \\
\text { habitat for species }\end{array}$ & Unknown & Nelson 2008 \\
\hline \multirow{2}{*}{$\begin{array}{l}\text { Structures on } \\
\text { water's surface }\end{array}$} & Structure & $\begin{array}{l}\text { Possible FAD effect, } \\
\text { attraction of predators }\end{array}$ & $\begin{array}{l}\text { Low, would occur in } \\
\text { small area relative to } \\
\text { species' range }\end{array}$ & $\begin{array}{l}\text { High, would be } \\
\text { continuous for life } \\
\text { of project }\end{array}$ & $\begin{array}{l}\text { Med, occurs in critical } \\
\text { habitat for species }\end{array}$ & Unknown & Nelson 2008 \\
\hline & Navigation lights & $\begin{array}{l}\text { None, this species not } \\
\text { known to be attracted to } \\
\text { lights }\end{array}$ & N/A & N/A & N/A & Low & $\begin{array}{l}\text { Nightingale et } \\
\text { al. } 2006\end{array}$ \\
\hline \multicolumn{8}{|c|}{ Decommissioning } \\
\hline \multirow[t]{2}{*}{ Boat traffic } & $\begin{array}{l}\text { Oil/chemical } \\
\text { release }\end{array}$ & $\begin{array}{l}\text { Toxicity to individuals } \\
\text { and prey }\end{array}$ & $\begin{array}{l}\text { Low for typical small } \\
\text { volume releases, High } \\
\text { for large, catastrophic } \\
\text { spill }\end{array}$ & $\begin{array}{l}\text { Low, occurs over } 1 \\
\text { to } 2 \text { summers }\end{array}$ & $\begin{array}{l}\text { Low, species on bottom } \\
\text { but oil floats on surface }\end{array}$ & Low & $\begin{array}{l}\text { Nelson and } \\
\text { Woo } 2008\end{array}$ \\
\hline & $\begin{array}{l}\text { Noise and } \\
\text { vibration }\end{array}$ & $\begin{array}{l}\text { Possible altered } \\
\text { behavior }\end{array}$ & $\begin{array}{l}\text { Low, would occur in } \\
\text { small area relative to } \\
\text { species’ range }\end{array}$ & $\begin{array}{l}\text { Low, occurs over } 1 \\
\text { to } 2 \text { summers }\end{array}$ & $\begin{array}{l}\text { Low, species motile and } \\
\text { able to avoid } \\
\text { disturbance area }\end{array}$ & Low & Cada 2008 \\
\hline \multirow{2}{*}{$\begin{array}{l}\text { Decommissioning } \\
\text { of structures on } \\
\text { water's surface or } \\
\text { seabed }\end{array}$} & $\begin{array}{l}\text { Noise and } \\
\text { vibration }\end{array}$ & $\begin{array}{l}\text { Possible altered } \\
\text { behavior }\end{array}$ & Low, see above & $\begin{array}{l}\text { Low, occurs over } 1 \\
\text { to } 2 \text { summers }\end{array}$ & Low, see above & Low & Cada 2008 \\
\hline & $\begin{array}{l}\text { Oil/chemical } \\
\text { release }\end{array}$ & $\begin{array}{l}\text { Toxicity to individuals } \\
\text { and prey }\end{array}$ & $\begin{array}{l}\text { Low, volume released } \\
\text { would occupy small } \\
\text { area relative to species' } \\
\text { range }\end{array}$ & $\begin{array}{l}\text { Low, occurs over } 1 \\
\text { to } 2 \text { summers }\end{array}$ & $\begin{array}{l}\text { Low, species on bottom } \\
\text { but oil floats on surface }\end{array}$ & Low & $\begin{array}{l}\text { Nelson and } \\
\text { Woo } 2008\end{array}$ \\
\hline
\end{tabular}

\begin{tabular}{lr}
\hline Environmental Assessment for Siting Wave \& Tidal & D-68 \\
Energy Projects & H. Harvey \& Associates \\
24 November 2009
\end{tabular}


Appendix D - Effects of Small Commercial OPT Power Buoy Project Humboldt

\begin{tabular}{|c|c|c|c|c|c|c|c|}
\hline Project activity & Action & $\begin{array}{l}\text { Description of action's } \\
\text { effect on indicator }\end{array}$ & $\begin{array}{l}\text { Spatial exposure to } \\
\text { indicator (low, med, } \\
\text { high) }\end{array}$ & $\begin{array}{l}\text { Temporal } \\
\text { exposure to } \\
\text { indicator (low, } \\
\text { med, high) }\end{array}$ & $\begin{array}{l}\text { Effect's overlap with } \\
\text { critical life stage, } \\
\text { behavior, habitat or } \\
\text { resource (low, med, } \\
\text { high) }\end{array}$ & $\begin{array}{l}\text { Overall risk } \\
\text { to indicator } \\
\text { (low, med, } \\
\text { high, } \\
\text { unknown) } \\
\end{array}$ & Source(s) \\
\hline & $\begin{array}{l}\text { Seabed } \\
\text { disturbance }\end{array}$ & $\begin{array}{l}\text { Temporary habitat loss, } \\
\text { changes in prey } \\
\text { resources }\end{array}$ & $\begin{array}{l}\text { Low, would occur in } \\
\text { small area relative to } \\
\text { species' range }\end{array}$ & $\begin{array}{l}\text { Low, occurs over } 1 \\
\text { to } 2 \text { summers }\end{array}$ & $\begin{array}{l}\text { Low, species motile and } \\
\text { able to avoid } \\
\text { disturbance area }\end{array}$ & Low & Nelson 2008 \\
\hline & $\begin{array}{l}\text { Deconstruction } \\
\text { and deck lights }\end{array}$ & $\begin{array}{l}\text { None, species not } \\
\text { known to be attracted to } \\
\text { lights }\end{array}$ & N/A & N/A & N/A & Low & $\begin{array}{l}\text { Nightingale et } \\
\text { al. } 2006\end{array}$ \\
\hline
\end{tabular}

\begin{tabular}{lr}
\hline Environmental Assessment for Siting Wave \& Tidal & D-69 \\
Energy Projects & H. T. Harvey \& Associates \\
24 November 2009
\end{tabular}


Appendix D - Effects of Small Commercial OPT Power Buoy Project Humboldt

Effects on diving ducks, and loons and grebes for a Small Commercial OPT Power Buoy Project at the Humboldt site.

\begin{tabular}{|c|c|c|c|c|c|c|c|}
\hline Project activity & Action & $\begin{array}{l}\text { Description of action's } \\
\text { effect on indicator }\end{array}$ & $\begin{array}{l}\text { Spatial exposure to } \\
\text { indicator (low, med, high) }\end{array}$ & $\begin{array}{l}\text { Temporal } \\
\text { exposure to } \\
\text { indicator (low, } \\
\text { med, high) }\end{array}$ & $\begin{array}{l}\text { Effect's overlap } \\
\text { with critical life } \\
\text { stage, behavior, } \\
\text { habitat or resource } \\
\text { (low, med, high) }\end{array}$ & $\begin{array}{l}\text { Overall risk } \\
\text { to indicator } \\
\text { (low, med, } \\
\text { high, } \\
\text { unknown) } \\
\end{array}$ & Source(s) \\
\hline \multicolumn{8}{|c|}{ Construction } \\
\hline \multirow{2}{*}{ Boat traffic } & $\begin{array}{l}\text { Noise and } \\
\text { vibration }\end{array}$ & $\begin{array}{l}\text { Possible movement away } \\
\text { from boat and disruption } \\
\text { of foraging }\end{array}$ & $\begin{array}{l}\text { Low, would occur in small } \\
\text { area relative to species' } \\
\text { range }\end{array}$ & $\begin{array}{l}\text { Low, occurs over } \\
1 \text { to } 2 \text { summers }\end{array}$ & $\begin{array}{l}\text { Low, won't occur in } \\
\text { winter when birds } \\
\text { most abundant }\end{array}$ & Low & $\begin{array}{l}\text { Harris 2006, } \\
\text { LGL Limited } \\
\text { et al. } 2009\end{array}$ \\
\hline & $\begin{array}{l}\text { Oil/chemical } \\
\text { release }\end{array}$ & $\begin{array}{l}\text { Hypothermia, toxicity to } \\
\text { individuals and prey }\end{array}$ & $\begin{array}{l}\text { Low for typical small } \\
\text { volume releases, High for } \\
\text { large, catastrophic spill }\end{array}$ & $\begin{array}{l}\text { Low, occurs over } \\
1 \text { to } 2 \text { summers }\end{array}$ & Low, see above & Low & $\begin{array}{l}\text { Harris 2006, } \\
\text { Michel et al. } \\
2007\end{array}$ \\
\hline \multirow{4}{*}{$\begin{array}{l}\text { Construction of } \\
\text { electrical collector } \\
\text { system, moorings/ } \\
\text { foundation; } \\
\text { installation of } \\
\text { devices }\end{array}$} & $\begin{array}{l}\text { Noise and } \\
\text { vibration }\end{array}$ & $\begin{array}{l}\text { Possible movement away } \\
\text { from area and disruption } \\
\text { of foraging or nesting }\end{array}$ & $\begin{array}{l}\text { Low, would occur in small } \\
\text { area relative to species' } \\
\text { range }\end{array}$ & $\begin{array}{l}\text { Low, occurs over } \\
1 \text { to } 2 \text { summers }\end{array}$ & Low, see above & Low & $\begin{array}{l}\text { Harris 2006, } \\
\text { Michel et al. } \\
2007\end{array}$ \\
\hline & $\begin{array}{l}\text { Oil/chemical } \\
\text { release }\end{array}$ & $\begin{array}{l}\text { Hypothermia, toxicity to } \\
\text { individuals and prey }\end{array}$ & $\begin{array}{l}\text { Low, volume released } \\
\text { would occupy small area } \\
\text { relative to species’ range }\end{array}$ & $\begin{array}{l}\text { Low, occurs over } \\
1 \text { to } 2 \text { summers }\end{array}$ & Low, see above & Low & $\begin{array}{l}\text { Harris 2006, } \\
\text { Michel et al. } \\
2007\end{array}$ \\
\hline & $\begin{array}{l}\text { Seabed } \\
\text { disturbance }\end{array}$ & $\begin{array}{l}\text { Possible short-term } \\
\text { changes in food resources }\end{array}$ & $\begin{array}{l}\text { Low, would occur in small } \\
\text { area relative to species' } \\
\text { range }\end{array}$ & $\begin{array}{l}\text { Low, occurs over } \\
1 \text { to } 2 \text { summers }\end{array}$ & Low, see above & Low & $\begin{array}{l}\text { Boehlert et al. } \\
2008 \text {, Harris } \\
2006\end{array}$ \\
\hline & $\begin{array}{l}\text { Construction } \\
\text { lights }\end{array}$ & $\begin{array}{l}\text { None, these species not } \\
\text { known to be attracted to } \\
\text { lights }\end{array}$ & N/A & N/A & N/A & Low & $\begin{array}{l}\text { Montevecchi } \\
2006\end{array}$ \\
\hline \multirow{2}{*}{$\begin{array}{l}\text { Directional drilling, } \\
\text { and laying cable } \\
\text { under/on seabed }\end{array}$} & $\begin{array}{l}\text { Seabed } \\
\text { disturbance }\end{array}$ & $\begin{array}{l}\text { Possible short-term } \\
\text { changes in food resources }\end{array}$ & Low, see above & $\begin{array}{l}\text { Low, occurs over } \\
\text { a short time } \\
\text { period }\end{array}$ & $\begin{array}{l}\text { Low, won't occur in } \\
\text { winter when birds } \\
\text { most abundant }\end{array}$ & Low & $\begin{array}{l}\text { Boehlert et al. } \\
2008 \text {, Harris } \\
2006\end{array}$ \\
\hline & $\begin{array}{l}\text { Noise and } \\
\text { vibration }\end{array}$ & $\begin{array}{l}\text { Possible movement away } \\
\text { from area and disruption } \\
\text { of foraging or nesting }\end{array}$ & Low, see above & $\begin{array}{l}\text { Low, occurs over } \\
\text { a short time } \\
\text { period }\end{array}$ & Low, see above & Low & $\begin{array}{l}\text { Harris 2006, } \\
\text { Michel et al. } \\
2007\end{array}$ \\
\hline \multicolumn{8}{|c|}{ Operation and Maintenance } \\
\hline Boat traffic & $\begin{array}{l}\text { Oil/chemical } \\
\text { release }\end{array}$ & $\begin{array}{l}\text { Hypothermia, toxicity to } \\
\text { individuals and prey }\end{array}$ & $\begin{array}{l}\text { Low for typical small } \\
\text { volume releases, High for } \\
\text { large, catastrophic spill }\end{array}$ & $\begin{array}{l}\text { Low, only from } \\
\text { periodic trips in } \\
\text { summer }\end{array}$ & Low, see above & Low & $\begin{array}{l}\text { Harris 2006, } \\
\text { Michel et al. } \\
2007\end{array}$ \\
\hline
\end{tabular}

\begin{tabular}{|c|c|c|}
\hline $\begin{array}{l}\text { Environmental Assessment for Siting Wave \& Tidal } \\
\text { Energy Projects }\end{array}$ & D-70 & $\begin{array}{r}\text { H. T. Harvey \& Associates } \\
24 \text { November } 2009\end{array}$ \\
\hline
\end{tabular}


Appendix D - Effects of Small Commercial OPT Power Buoy Project Humboldt

\begin{tabular}{|c|c|c|c|c|c|c|c|}
\hline Project activity & Action & $\begin{array}{l}\text { Description of action's } \\
\text { effect on indicator }\end{array}$ & $\begin{array}{l}\text { Spatial exposure to } \\
\text { indicator (low, med, high) }\end{array}$ & $\begin{array}{l}\text { Temporal } \\
\text { exposure to } \\
\text { indicator (low, } \\
\text { med, high) }\end{array}$ & $\begin{array}{l}\text { Effect's overlap } \\
\text { with critical life } \\
\text { stage, behavior, } \\
\text { habitat or resource } \\
\text { (low, med, high) } \\
\end{array}$ & $\begin{array}{l}\text { Overall risk } \\
\text { to indicator } \\
\text { (low, med, } \\
\text { high, } \\
\text { unknown) } \\
\end{array}$ & Source(s) \\
\hline & $\begin{array}{l}\text { Noise and } \\
\text { vibration }\end{array}$ & $\begin{array}{l}\text { Possible movement away } \\
\text { from boat and disruption } \\
\text { of foraging }\end{array}$ & $\begin{array}{l}\text { Low, would occur in small } \\
\text { area relative to species' } \\
\text { range }\end{array}$ & $\begin{array}{l}\text { Low, only from } \\
\text { periodic trips in } \\
\text { summer }\end{array}$ & Low, see above & Low & $\begin{array}{l}\text { Harris 2006, } \\
\text { LGL Limited } \\
\text { et al. } 2009\end{array}$ \\
\hline \multirow{2}{*}{$\begin{array}{l}\text { Operation of } \\
\text { turbines or other } \\
\text { moving parts of } \\
\text { devices }\end{array}$} & $\begin{array}{l}\text { Moving device } \\
\text { parts }\end{array}$ & $\begin{array}{l}\text { None, no underwater } \\
\text { moving parts }\end{array}$ & N/A & N/A & N/A & Low & N/A \\
\hline & $\begin{array}{l}\text { Noise and } \\
\text { vibration }\end{array}$ & $\begin{array}{l}\text { Possible avoidance of } \\
\text { project area }\end{array}$ & $\begin{array}{l}\text { Low, would occur in small } \\
\text { area relative to species' } \\
\text { range }\end{array}$ & $\begin{array}{l}\text { High, continuous } \\
\text { for life of project }\end{array}$ & $\begin{array}{l}\text { Med, would occur in } \\
\text { winter when birds } \\
\text { most abundant }\end{array}$ & Low & $\begin{array}{l}\text { Harris 2006, } \\
\text { Michel et al. } \\
2007\end{array}$ \\
\hline \multirow{3}{*}{$\begin{array}{l}\text { Structures in water } \\
\text { column and on } \\
\text { seabed, such as } \\
\text { devices and } \\
\text { moorings and } \\
\text { footings }\end{array}$} & Structure & $\begin{array}{l}\text { Possible attraction to } \\
\text { increased prey (reef } \\
\text { effect), entanglement with } \\
\text { derelict fishing gear/ } \\
\text { moorings, collisions with } \\
\text { structures while diving }\end{array}$ & Low, see above & $\begin{array}{l}\text { Med, tangled gear } \\
\text { would be } \\
\text { removed } \\
\text { periodically }\end{array}$ & $\begin{array}{l}\text { Med, birds could be } \\
\text { attracted to structure }\end{array}$ & Unknown & $\begin{array}{l}\text { Harris 2006, } \\
\text { Michel et al. } \\
2007\end{array}$ \\
\hline & $\begin{array}{l}\text { Water } \\
\text { circulation } \\
\text { changes }\end{array}$ & $\begin{array}{l}\text { None, project not large } \\
\text { enough to affect prey }\end{array}$ & N/A & N/A & N/A & Low & \\
\hline & $\begin{array}{l}\text { Oil/chemical } \\
\text { release }\end{array}$ & $\begin{array}{l}\text { Hypothermia, toxicity to } \\
\text { individuals and prey }\end{array}$ & $\begin{array}{l}\text { Low for typical small } \\
\text { volume releases, High for } \\
\text { large, catastrophic release } \\
\text { from device failure }\end{array}$ & $\begin{array}{l}\text { Low, likely } \\
\text { sporadic or } \\
\text { intermittent for } \\
\text { life of project }\end{array}$ & $\begin{array}{l}\text { Low, could occur in } \\
\text { winter when birds } \\
\text { most abundant }\end{array}$ & Low & $\begin{array}{l}\text { Harris 2006, } \\
\text { Michel et al. } \\
2007\end{array}$ \\
\hline \multirow{2}{*}{$\begin{array}{l}\text { Structures on } \\
\text { water's surface }\end{array}$} & Structure & $\begin{array}{l}\text { Possible attraction to } \\
\text { increased prey (FAD } \\
\text { effect), collision while } \\
\text { flying }\end{array}$ & $\begin{array}{l}\text { Low, would occur in small } \\
\text { area relative to species' } \\
\text { range }\end{array}$ & $\begin{array}{l}\text { High, continuous } \\
\text { for life of project }\end{array}$ & $\begin{array}{l}\text { Med, birds could be } \\
\text { attracted to structure }\end{array}$ & Unknown & $\begin{array}{l}\text { Harris 2006, } \\
\text { LGL Limited } \\
\text { et al. } 2009\end{array}$ \\
\hline & $\begin{array}{l}\text { Navigation } \\
\text { lights }\end{array}$ & $\begin{array}{l}\text { None, these species not } \\
\text { known to be attracted to } \\
\text { lights }\end{array}$ & N/A & N/A & N/A & Low & $\begin{array}{l}\text { Montevecchi } \\
2006\end{array}$ \\
\hline
\end{tabular}

\begin{tabular}{lr}
\hline Environmental Assessment for Siting Wave \& Tidal & D-71 \\
Energy Projects & H. T. Harvey \& Associates \\
24 November 2009
\end{tabular}


Appendix D - Effects of Small Commercial OPT Power Buoy Project Humboldt

\begin{tabular}{|c|c|c|c|c|c|c|c|}
\hline Project activity & Action & $\begin{array}{l}\text { Description of action's } \\
\text { effect on indicator }\end{array}$ & $\begin{array}{l}\text { Spatial exposure to } \\
\text { indicator (low, med, high) }\end{array}$ & $\begin{array}{l}\text { Temporal } \\
\text { exposure to } \\
\text { indicator (low, } \\
\text { med, high) }\end{array}$ & $\begin{array}{l}\text { Effect's overlap } \\
\text { with critical life } \\
\text { stage, behavior, } \\
\text { habitat or resource } \\
\text { (low, med, high) }\end{array}$ & $\begin{array}{l}\text { Overall risk } \\
\text { to indicator } \\
\text { (low, med, } \\
\text { high, } \\
\text { unknown) } \\
\end{array}$ & Source(s) \\
\hline \multirow{2}{*}{ Boat traffic } & $\begin{array}{l}\text { Oil/chemical } \\
\text { release }\end{array}$ & $\begin{array}{l}\text { Hypothermia, toxicity to } \\
\text { individuals and prey }\end{array}$ & $\begin{array}{l}\text { Low for typical small } \\
\text { volume releases, High for } \\
\text { large, catastrophic spill }\end{array}$ & $\begin{array}{l}\text { Low, occurs over } \\
1 \text { to } 2 \text { summers }\end{array}$ & $\begin{array}{l}\text { Low, won't occur in } \\
\text { winter when birds } \\
\text { most abundant }\end{array}$ & Low & $\begin{array}{l}\text { Harris 2006, } \\
\text { Michel et al. } \\
2007\end{array}$ \\
\hline & $\begin{array}{l}\text { Noise and } \\
\text { vibration }\end{array}$ & $\begin{array}{l}\text { Possible movement away } \\
\text { from boat and disruption } \\
\text { of foraging }\end{array}$ & $\begin{array}{l}\text { Low, would occur in small } \\
\text { area relative to species' } \\
\text { range }\end{array}$ & $\begin{array}{l}\text { Low, occurs over } \\
1 \text { to } 2 \text { summers }\end{array}$ & Low, see above & Low & $\begin{array}{l}\text { Harris } 2006 \text {, } \\
\text { LGL Limited } \\
\text { et al. } 2009\end{array}$ \\
\hline \multirow{4}{*}{$\begin{array}{l}\text { Decommissioning of } \\
\text { structures on water's } \\
\text { surface or seabed }\end{array}$} & $\begin{array}{l}\text { Noise and } \\
\text { vibration }\end{array}$ & $\begin{array}{l}\text { Possible movement away } \\
\text { from area and disruption } \\
\text { of foraging or nesting }\end{array}$ & Low, see above & $\begin{array}{l}\text { Low, occurs over } \\
1 \text { to } 2 \text { summers }\end{array}$ & Low, see above & Low & $\begin{array}{l}\text { Harris 2006, } \\
\text { Michel et al. } \\
2007\end{array}$ \\
\hline & $\begin{array}{l}\text { Oil/chemical } \\
\text { release }\end{array}$ & $\begin{array}{l}\text { Hypothermia, toxicity to } \\
\text { individuals and prey }\end{array}$ & $\begin{array}{l}\text { Low, volume released } \\
\text { would occupy small area } \\
\text { relative to species’ range }\end{array}$ & $\begin{array}{l}\text { Low, occurs over } \\
1 \text { to } 2 \text { summers }\end{array}$ & Low, see above & Low & $\begin{array}{l}\text { Harris 2006, } \\
\text { Michel et al. } \\
2007\end{array}$ \\
\hline & $\begin{array}{l}\text { Seabed } \\
\text { disturbance }\end{array}$ & $\begin{array}{l}\text { Possible short-term } \\
\text { changes in food resources }\end{array}$ & $\begin{array}{l}\text { Low, would occur in small } \\
\text { area relative to species' } \\
\text { range }\end{array}$ & $\begin{array}{l}\text { Low, occurs over } \\
1 \text { to } 2 \text { summers }\end{array}$ & Low, see above & Low & $\begin{array}{l}\text { Boehlert et al. } \\
2008 \text {, Harris } \\
2006\end{array}$ \\
\hline & $\begin{array}{l}\text { Construction } \\
\text { lights }\end{array}$ & $\begin{array}{l}\text { None, these species not } \\
\text { known to be attracted to } \\
\text { lights }\end{array}$ & N/A & N/A & N/A & Low & $\begin{array}{l}\text { Montevecchi } \\
2006\end{array}$ \\
\hline
\end{tabular}

\begin{tabular}{|c|c|c|}
\hline $\begin{array}{l}\text { Environmental Assessment for Siting Wave \& Tidal } \\
\text { Energy Projects }\end{array}$ & D-72 & $\begin{array}{r}\text { H. T. Harvey \& Associates } \\
24 \text { November } 2009\end{array}$ \\
\hline
\end{tabular}


Appendix D - Effects of Small Commercial OPT Power Buoy Project Humboldt

Effects on shearwaters and storm-petrels for a Small Commercial OPT Power Buoy Project at the Humboldt site.

\begin{tabular}{|c|c|c|c|c|c|c|c|}
\hline Project activity & Action & $\begin{array}{l}\text { Description of action's } \\
\text { effect on indicator }\end{array}$ & $\begin{array}{l}\text { Spatial exposure to } \\
\text { indicator (low, med, } \\
\text { high) }\end{array}$ & $\begin{array}{l}\text { Temporal } \\
\text { exposure to } \\
\text { indicator (low, } \\
\text { med, high) }\end{array}$ & $\begin{array}{l}\text { Effect's overlap } \\
\text { with critical life } \\
\text { stage, behavior, } \\
\text { habitat or resource } \\
\text { (low, med, high) }\end{array}$ & $\begin{array}{l}\text { Overall risk } \\
\text { to indicator } \\
\text { (low, med, } \\
\text { high, } \\
\text { unknown) } \\
\end{array}$ & Source(s) \\
\hline \multicolumn{8}{|c|}{ Construction } \\
\hline \multirow{2}{*}{ Boat traffic } & $\begin{array}{l}\text { Noise and } \\
\text { vibration }\end{array}$ & $\begin{array}{l}\text { Possible movement away } \\
\text { from boat and disruption of } \\
\text { foraging }\end{array}$ & $\begin{array}{l}\text { Low, would occur in } \\
\text { small area relative to } \\
\text { species' range }\end{array}$ & $\begin{array}{l}\text { Low, occurs over } \\
1 \text { to } 2 \text { summers }\end{array}$ & $\begin{array}{l}\text { Low, some species } \\
\text { common in summer } \\
\text { but most farther } \\
\text { offshore }\end{array}$ & Low & $\begin{array}{l}\text { Harris 2006, } \\
\text { LGL Limited } \\
\text { et al. } 2009\end{array}$ \\
\hline & $\begin{array}{l}\text { Oil/chemical } \\
\text { release }\end{array}$ & $\begin{array}{l}\text { Hypothermia, toxicity to } \\
\text { individuals and prey }\end{array}$ & $\begin{array}{l}\text { Low for typical small } \\
\text { volume releases, High for } \\
\text { large, catastrophic spill }\end{array}$ & $\begin{array}{l}\text { Low, occurs over } \\
1 \text { to } 2 \text { summers }\end{array}$ & Low, see above & Low & $\begin{array}{l}\text { Harris 2006, } \\
\text { Michel et al. } \\
2007\end{array}$ \\
\hline \multirow{4}{*}{$\begin{array}{l}\text { Construction of } \\
\text { electrical collector } \\
\text { system, moorings/ } \\
\text { foundation; } \\
\text { installation of } \\
\text { devices }\end{array}$} & $\begin{array}{l}\text { Noise and } \\
\text { vibration }\end{array}$ & $\begin{array}{l}\text { Possible movement away } \\
\text { from area and disruption of } \\
\text { foraging or nesting }\end{array}$ & $\begin{array}{l}\text { Low, would occur in } \\
\text { small area relative to } \\
\text { species' range }\end{array}$ & $\begin{array}{l}\text { Low, occurs over } \\
1 \text { to } 2 \text { summers }\end{array}$ & Low, see above & Low & $\begin{array}{l}\text { Harris 2006, } \\
\text { Michel et al. } \\
2007\end{array}$ \\
\hline & $\begin{array}{l}\text { Oil/chemical } \\
\text { release }\end{array}$ & $\begin{array}{l}\text { Hypothermia, toxicity to } \\
\text { individuals and prey }\end{array}$ & Low, see above & $\begin{array}{l}\text { Low, occurs over } \\
1 \text { to } 2 \text { summers }\end{array}$ & Low, see above & Low & $\begin{array}{l}\text { Harris 2006, } \\
\text { Michel et al. } \\
2007\end{array}$ \\
\hline & $\begin{array}{l}\text { Seabed } \\
\text { disturbance }\end{array}$ & $\begin{array}{l}\text { None, these are surface- } \\
\text { feeders }\end{array}$ & N/A & N/A & N/A & Low & USFWS 2005 \\
\hline & $\begin{array}{l}\text { Construction } \\
\text { lights }\end{array}$ & $\begin{array}{l}\text { Attraction/ disorientation, } \\
\text { collisions }\end{array}$ & $\begin{array}{l}\text { Low, would occur in } \\
\text { small area relative to } \\
\text { species' range }\end{array}$ & $\begin{array}{l}\text { Low, occurs over } \\
1 \text { to } 2 \text { summers }\end{array}$ & $\begin{array}{l}\text { Low, some species } \\
\text { common in summer } \\
\text { but most farther } \\
\text { offshore }\end{array}$ & Low & $\begin{array}{l}\text { Harris 2006, } \\
\text { Montevecchi } \\
2006\end{array}$ \\
\hline \multirow[b]{2}{*}{$\begin{array}{l}\text { Directional drilling, } \\
\text { and laying cable } \\
\text { under/on seabed }\end{array}$} & $\begin{array}{l}\text { Seabed } \\
\text { disturbance }\end{array}$ & $\begin{array}{l}\text { None, these are surface- } \\
\text { feeders }\end{array}$ & N/A & N/A & N/A & Low & USFWS 2005 \\
\hline & $\begin{array}{l}\text { Noise and } \\
\text { vibration }\end{array}$ & $\begin{array}{l}\text { Possible movement away } \\
\text { from area and disruption of } \\
\text { foraging or nesting }\end{array}$ & $\begin{array}{l}\text { Low, would occur in } \\
\text { small area relative to } \\
\text { species' range }\end{array}$ & $\begin{array}{l}\text { Low, occurs over } \\
\text { a short time } \\
\text { period }\end{array}$ & $\begin{array}{l}\text { Low, some species } \\
\text { common in summer } \\
\text { but most farther } \\
\text { offshore }\end{array}$ & Low & $\begin{array}{l}\text { Harris 2006, } \\
\text { Michel et al. } \\
2007\end{array}$ \\
\hline \multicolumn{8}{|c|}{ Operation and Maintenance } \\
\hline Boat traffic & $\begin{array}{l}\text { Oil/chemical } \\
\text { release }\end{array}$ & $\begin{array}{l}\text { Hypothermia, toxicity to } \\
\text { individuals and prey }\end{array}$ & $\begin{array}{l}\text { Low for typical small } \\
\text { volume releases, High for } \\
\text { large, catastrophic spill }\end{array}$ & $\begin{array}{l}\text { Low, only from } \\
\text { periodic trips in } \\
\text { summer }\end{array}$ & Low, see above & Low & $\begin{array}{l}\text { Harris 2006, } \\
\text { Michel et al. } \\
2007\end{array}$ \\
\hline
\end{tabular}

\begin{tabular}{lr}
\hline Environmental Assessment for Siting Wave \& Tidal & D-73 \\
Energy Projects & H. Harvey \& Associates \\
24 November 2009
\end{tabular}


Appendix D - Effects of Small Commercial OPT Power Buoy Project Humboldt

\begin{tabular}{|c|c|c|c|c|c|c|c|}
\hline Project activity & Action & $\begin{array}{l}\text { Description of action's } \\
\text { effect on indicator }\end{array}$ & $\begin{array}{l}\text { Spatial exposure to } \\
\text { indicator (low, med, } \\
\text { high) }\end{array}$ & $\begin{array}{l}\text { Temporal } \\
\text { exposure to } \\
\text { indicator (low, } \\
\text { med, high) } \\
\end{array}$ & $\begin{array}{l}\text { Effect's overlap } \\
\text { with critical life } \\
\text { stage, behavior, } \\
\text { habitat or resource } \\
\text { (low, med, high) }\end{array}$ & $\begin{array}{l}\text { Overall risk } \\
\text { to indicator } \\
\text { (low, med, } \\
\text { high, } \\
\text { unknown) } \\
\end{array}$ & Source(s) \\
\hline & $\begin{array}{l}\text { Noise and } \\
\text { vibration }\end{array}$ & $\begin{array}{l}\text { Possible movement away } \\
\text { from boat and disruption of } \\
\text { foraging }\end{array}$ & $\begin{array}{l}\text { Low, would occur in } \\
\text { small area relative to } \\
\text { species' range }\end{array}$ & $\begin{array}{l}\text { Low, only from } \\
\text { periodic trips in } \\
\text { summer }\end{array}$ & Low, see above & Low & $\begin{array}{l}\text { Harris 2006, } \\
\text { LGL Limited } \\
\text { et al. } 2009\end{array}$ \\
\hline \multirow{2}{*}{$\begin{array}{l}\text { Operation of } \\
\text { turbines or other } \\
\text { moving parts of } \\
\text { devices }\end{array}$} & $\begin{array}{l}\text { Moving device } \\
\text { parts }\end{array}$ & $\begin{array}{l}\text { None, no underwater } \\
\text { moving parts }\end{array}$ & N/A & N/A & N/A & Low & N/A \\
\hline & $\begin{array}{l}\text { Noise and } \\
\text { vibration }\end{array}$ & $\begin{array}{l}\text { Possible avoidance of } \\
\text { project area }\end{array}$ & $\begin{array}{l}\text { Low, would occur in } \\
\text { small area relative to } \\
\text { species' range }\end{array}$ & $\begin{array}{l}\text { High, continuous } \\
\text { for life of project }\end{array}$ & $\begin{array}{l}\text { Low, most species } \\
\text { farther offshore }\end{array}$ & Low & $\begin{array}{l}\text { Harris 2006, } \\
\text { Michel et al. } \\
2007\end{array}$ \\
\hline \multirow{3}{*}{$\begin{array}{l}\text { Structures in water } \\
\text { column and on } \\
\text { seabed, such as } \\
\text { devices and } \\
\text { moorings and } \\
\text { footings }\end{array}$} & Structure & $\begin{array}{l}\text { None, these species feed on } \\
\text { surface and do not dive }\end{array}$ & N/A & N/A & N/A & Low & USFWS 2005 \\
\hline & $\begin{array}{l}\text { Water } \\
\text { circulation } \\
\text { changes }\end{array}$ & $\begin{array}{l}\text { None, project not large } \\
\text { enough to affect prey }\end{array}$ & N/A & N/A & N/A & Low & N/A \\
\hline & $\begin{array}{l}\text { Oil/chemical } \\
\text { release }\end{array}$ & $\begin{array}{l}\text { Hypothermia, toxicity to } \\
\text { individuals and prey }\end{array}$ & $\begin{array}{l}\text { Low for typical small } \\
\text { volume releases, High for } \\
\text { large, catastrophic release } \\
\text { from device failure }\end{array}$ & $\begin{array}{l}\text { Med, continuous } \\
\text { and intermittent } \\
\text { for life of project }\end{array}$ & $\begin{array}{l}\text { Low, most species } \\
\text { farther offshore }\end{array}$ & Low & $\begin{array}{l}\text { Harris 2006, } \\
\text { Michel et al. } \\
2007\end{array}$ \\
\hline \multirow{2}{*}{$\begin{array}{l}\text { Structures on } \\
\text { water's surface }\end{array}$} & Structure & $\begin{array}{l}\text { Possible collision while } \\
\text { flying }\end{array}$ & $\begin{array}{l}\text { Low, would occur in } \\
\text { small area relative to } \\
\text { species’ range }\end{array}$ & $\begin{array}{l}\text { High, continuous } \\
\text { for life of project }\end{array}$ & Low, see above & Low & $\begin{array}{l}\text { Harris 2006, } \\
\text { LGL Limited } \\
\text { et al. } 2009\end{array}$ \\
\hline & $\begin{array}{l}\text { Navigation } \\
\text { lights }\end{array}$ & $\begin{array}{l}\text { Attraction/ disorientation, } \\
\text { collisions }\end{array}$ & Low, see above & $\begin{array}{l}\text { High, continuous } \\
\text { for life of project }\end{array}$ & Low, see above & Low & $\begin{array}{l}\text { Harris 2006, } \\
\text { Montevecchi } \\
2006 \\
\end{array}$ \\
\hline \multicolumn{8}{|c|}{ Decommissioning } \\
\hline \multirow{2}{*}{ Boat traffic } & $\begin{array}{l}\text { Oil/chemical } \\
\text { release }\end{array}$ & $\begin{array}{l}\text { Hypothermia, toxicity to } \\
\text { individuals and prey }\end{array}$ & $\begin{array}{l}\text { Low for typical small } \\
\text { volume releases, High for } \\
\text { large, catastrophic spill }\end{array}$ & $\begin{array}{l}\text { Low, occurs over } \\
1 \text { to } 2 \text { summers }\end{array}$ & Low, see above & Low & $\begin{array}{l}\text { Harris 2006, } \\
\text { Michel et al. } \\
2007\end{array}$ \\
\hline & $\begin{array}{l}\text { Noise and } \\
\text { vibration }\end{array}$ & $\begin{array}{l}\text { Possible movement away } \\
\text { from boat and disruption of } \\
\text { foraging }\end{array}$ & $\begin{array}{l}\text { Low, would occur in } \\
\text { small area relative to } \\
\text { species' range }\end{array}$ & $\begin{array}{l}\text { Low, occurs over } \\
1 \text { to } 2 \text { summers }\end{array}$ & Low, see above & Low & $\begin{array}{l}\text { Harris 2006, } \\
\text { LGL Limited } \\
\text { et al. } 2009\end{array}$ \\
\hline
\end{tabular}

\begin{tabular}{lr}
\hline Environmental Assessment for Siting Wave \& Tidal & D-74 \\
Energy Projects & H. T. Harvey \& Associates \\
24 November 2009
\end{tabular}


Appendix D - Effects of Small Commercial OPT Power Buoy Project Humboldt

\begin{tabular}{|c|c|c|c|c|c|c|c|}
\hline Project activity & Action & $\begin{array}{l}\text { Description of action's } \\
\text { effect on indicator }\end{array}$ & $\begin{array}{l}\text { Spatial exposure to } \\
\text { indicator (low, med, } \\
\text { high) }\end{array}$ & $\begin{array}{l}\text { Temporal } \\
\text { exposure to } \\
\text { indicator (low, } \\
\text { med, high) }\end{array}$ & $\begin{array}{l}\text { Effect's overlap } \\
\text { with critical life } \\
\text { stage, behavior, } \\
\text { habitat or resource } \\
\text { (low, med, high) }\end{array}$ & $\begin{array}{l}\text { Overall risk } \\
\text { to indicator } \\
\text { (low, med, } \\
\text { high, } \\
\text { unknown) }\end{array}$ & Source(s) \\
\hline \multirow{4}{*}{$\begin{array}{l}\text { Decommissioning of } \\
\text { structures on water's } \\
\text { surface or seabed }\end{array}$} & $\begin{array}{l}\text { Noise and } \\
\text { vibration }\end{array}$ & $\begin{array}{l}\text { Possible movement away } \\
\text { from area and disruption of } \\
\text { foraging or nesting }\end{array}$ & Low, see above & $\begin{array}{l}\text { Low, occurs over } \\
1 \text { to } 2 \text { summers }\end{array}$ & Low, see above & Low & $\begin{array}{l}\text { Harris 2006, } \\
\text { Michel et al. } \\
2007\end{array}$ \\
\hline & $\begin{array}{l}\text { Oil/chemical } \\
\text { release }\end{array}$ & $\begin{array}{l}\text { Hypothermia, toxicity to } \\
\text { individuals and prey }\end{array}$ & $\begin{array}{l}\text { Low, volume released } \\
\text { would occupy small area }\end{array}$ & $\begin{array}{l}\text { Low, occurs over } \\
1 \text { to } 2 \text { summers }\end{array}$ & Low, see above & Low & $\begin{array}{l}\text { Harris 2006, } \\
\text { Michel et al. } \\
2007\end{array}$ \\
\hline & $\begin{array}{l}\begin{array}{l}\text { Seabed } \\
\text { disturbance }\end{array} \\
\end{array}$ & $\begin{array}{l}\text { None, these are surface- } \\
\text { feeders }\end{array}$ & N/A & N/A & N/A & Low & USFWS 2005 \\
\hline & $\begin{array}{l}\text { Construction } \\
\text { lights }\end{array}$ & $\begin{array}{l}\text { Attraction/ disorientation, } \\
\text { collisions }\end{array}$ & $\begin{array}{l}\text { Low, would occur in } \\
\text { small area relative to } \\
\text { species' range }\end{array}$ & $\begin{array}{l}\text { Low, occurs over } \\
1 \text { to } 2 \text { summers }\end{array}$ & $\begin{array}{l}\text { Low, some species } \\
\text { common in summer } \\
\text { but most farther } \\
\text { offshore }\end{array}$ & Low & $\begin{array}{l}\text { Harris 2006, } \\
\text { Montevecchi } \\
2006\end{array}$ \\
\hline
\end{tabular}


Appendix D - Effects of Small Commercial OPT Power Buoy Project Humboldt

Effects on gulls and pelicans for a Small Commercial OPT Power Buoy Project at the Humboldt site.

\begin{tabular}{|c|c|c|c|c|c|c|c|}
\hline Project activity & Action & $\begin{array}{l}\text { Description of action's } \\
\text { effect on indicator }\end{array}$ & $\begin{array}{l}\text { Spatial exposure to } \\
\text { indicator (low, med, } \\
\text { high) }\end{array}$ & $\begin{array}{l}\text { Temporal } \\
\text { exposure to } \\
\text { indicator (low, } \\
\text { med, high) } \\
\end{array}$ & $\begin{array}{l}\text { Effect's overlap } \\
\text { with critical life } \\
\text { stage, behavior, } \\
\text { habitat or resource } \\
\text { (low, med, high) }\end{array}$ & $\begin{array}{l}\text { Overall risk } \\
\text { to indicator } \\
\text { (low, med, } \\
\text { high, } \\
\text { unknown) } \\
\end{array}$ & Source(s) \\
\hline \multicolumn{8}{|c|}{ Construction } \\
\hline \multirow{2}{*}{ Boat traffic } & $\begin{array}{l}\text { Noise and } \\
\text { vibration }\end{array}$ & $\begin{array}{l}\text { None, gulls and pelicans } \\
\text { follow boats and do not } \\
\text { appear to be disturbed by } \\
\text { boat noise }\end{array}$ & N/A & N/A & N/A & Low & Harris 2006 \\
\hline & $\begin{array}{l}\text { Oil/chemical } \\
\text { release }\end{array}$ & $\begin{array}{l}\text { Hypothermia, toxicity to } \\
\text { individuals and prey }\end{array}$ & $\begin{array}{l}\text { Low for typical small } \\
\text { volume releases, High for } \\
\text { large, catastrophic spill }\end{array}$ & $\begin{array}{l}\text { Low, occurs over } \\
1 \text { to } 2 \text { summers }\end{array}$ & $\begin{array}{l}\text { Med, boats could } \\
\text { pass through } \\
\text { foraging areas }\end{array}$ & Low & $\begin{array}{l}\text { Harris 2006, } \\
\text { Michel et al. } \\
2007\end{array}$ \\
\hline \multirow{4}{*}{$\begin{array}{l}\text { Construction of } \\
\text { electrical collector } \\
\text { system, moorings/ } \\
\text { foundation; } \\
\text { installation of } \\
\text { devices }\end{array}$} & $\begin{array}{l}\text { Noise and } \\
\text { vibration }\end{array}$ & $\begin{array}{l}\text { Possible movement away } \\
\text { from area and disruption of } \\
\text { foraging or nesting }\end{array}$ & $\begin{array}{l}\text { Low, would occur in } \\
\text { small area relative to } \\
\text { species' range }\end{array}$ & $\begin{array}{l}\text { Low, occurs over } \\
1 \text { to } 2 \text { summers }\end{array}$ & $\begin{array}{l}\text { Low, project not near } \\
\text { nesting colony or } \\
\text { known foraging area }\end{array}$ & Low & $\begin{array}{l}\text { Harris 2006, } \\
\text { Michel et al. } \\
2007\end{array}$ \\
\hline & $\begin{array}{l}\text { Oil/chemical } \\
\text { release }\end{array}$ & $\begin{array}{l}\text { Hypothermia, toxicity to } \\
\text { individuals and prey }\end{array}$ & $\begin{array}{l}\text { Low, volume released } \\
\text { would occupy small area } \\
\text { relative to species' range }\end{array}$ & $\begin{array}{l}\text { Low, occurs over } \\
1 \text { to } 2 \text { summers }\end{array}$ & Low, see above & Low & $\begin{array}{l}\text { Harris 2006, } \\
\text { Michel et al. } \\
2007\end{array}$ \\
\hline & $\begin{array}{l}\text { Seabed } \\
\text { disturbance }\end{array}$ & $\begin{array}{l}\text { None, these are surface- } \\
\text { feeders }\end{array}$ & N/A & N/A & N/A & Low & USFWS 2005 \\
\hline & $\begin{array}{l}\text { Construction } \\
\text { lights }\end{array}$ & $\begin{array}{l}\text { None, gulls and pelicans not } \\
\text { known to be attracted to } \\
\text { lights }\end{array}$ & N/A & N/A & N/A & Low & $\begin{array}{l}\text { Montevecchi } \\
2006\end{array}$ \\
\hline \multirow{2}{*}{$\begin{array}{l}\text { Directional drilling, } \\
\text { and laying cable } \\
\text { under/on seabed }\end{array}$} & $\begin{array}{l}\text { Seabed } \\
\text { disturbance }\end{array}$ & $\begin{array}{l}\text { None, these are surface- } \\
\text { feeders }\end{array}$ & N/A & N/A & N/A & Low & USFWS 2005 \\
\hline & $\begin{array}{l}\text { Noise and } \\
\text { vibration }\end{array}$ & $\begin{array}{l}\text { Possible movement away } \\
\text { from area and disruption of } \\
\text { foraging or nesting }\end{array}$ & $\begin{array}{l}\text { Low, would occur in } \\
\text { small area relative to } \\
\text { species' range }\end{array}$ & $\begin{array}{l}\text { Low, occurs over } \\
\text { a short time } \\
\text { period }\end{array}$ & $\begin{array}{l}\text { Low, project not near } \\
\text { nesting colony or } \\
\text { known foraging area }\end{array}$ & Low & $\begin{array}{l}\text { Harris 2006, } \\
\text { Michel et al. } \\
2007\end{array}$ \\
\hline \multicolumn{8}{|c|}{ Operation and Maintenance } \\
\hline Boat traffic & $\begin{array}{l}\text { Noise and } \\
\text { vibration }\end{array}$ & $\begin{array}{l}\text { None, gulls and pelicans } \\
\text { follow boats and do not } \\
\text { appear to be disturbed by } \\
\text { boat noise }\end{array}$ & N/A & N/A & N/A & Low & Harris 2006 \\
\hline
\end{tabular}

\begin{tabular}{|c|c|c|}
\hline $\begin{array}{l}\text { Environmental Assessment for Siting Wave \& Tidal } \\
\text { Energy Projects }\end{array}$ & D-76 & $\begin{array}{r}\text { H. T. Harvey \& Associates } \\
24 \text { November } 2009\end{array}$ \\
\hline
\end{tabular}


Appendix D - Effects of Small Commercial OPT Power Buoy Project Humboldt

\begin{tabular}{|c|c|c|c|c|c|c|c|}
\hline Project activity & Action & $\begin{array}{l}\text { Description of action's } \\
\text { effect on indicator }\end{array}$ & $\begin{array}{l}\text { Spatial exposure to } \\
\text { indicator (low, med, } \\
\text { high) }\end{array}$ & $\begin{array}{l}\text { Temporal } \\
\text { exposure to } \\
\text { indicator (low, } \\
\text { med, high) }\end{array}$ & $\begin{array}{l}\text { Effect's overlap } \\
\text { with critical life } \\
\text { stage, behavior, } \\
\text { habitat or resource } \\
\text { (low, med, high) }\end{array}$ & $\begin{array}{l}\text { Overall risk } \\
\text { to indicator } \\
\text { (low, med, } \\
\text { high, } \\
\text { unknown) } \\
\end{array}$ & Source(s) \\
\hline & $\begin{array}{l}\text { Oil/chemical } \\
\text { release }\end{array}$ & $\begin{array}{l}\text { Hypothermia, toxicity to } \\
\text { individuals and prey }\end{array}$ & $\begin{array}{l}\text { Low for typical small } \\
\text { volume releases, High for } \\
\text { large, catastrophic spill }\end{array}$ & $\begin{array}{l}\text { Low, wouldn't } \\
\text { persist long-term }\end{array}$ & $\begin{array}{l}\text { Med, boats could } \\
\text { pass through } \\
\text { foraging areas }\end{array}$ & Low & $\begin{array}{l}\text { Harris 2006, } \\
\text { Michel et al. } \\
2007\end{array}$ \\
\hline \multirow{2}{*}{$\begin{array}{l}\text { Operation of } \\
\text { turbines or other } \\
\text { moving parts of } \\
\text { devices }\end{array}$} & $\begin{array}{l}\text { Moving device } \\
\text { parts }\end{array}$ & $\begin{array}{l}\text { None, no underwater } \\
\text { moving parts }\end{array}$ & N/A & N/A & N/A & Low & N/A \\
\hline & $\begin{array}{l}\text { Noise and } \\
\text { vibration }\end{array}$ & $\begin{array}{l}\text { Possible avoidance of } \\
\text { project area }\end{array}$ & $\begin{array}{l}\text { Low, would occur in } \\
\text { small area relative to } \\
\text { species' range }\end{array}$ & $\begin{array}{l}\text { High, would be } \\
\text { continuous for life } \\
\text { of project }\end{array}$ & $\begin{array}{l}\text { Low, project not near } \\
\text { nesting colony or } \\
\text { known foraging area }\end{array}$ & Low & $\begin{array}{l}\text { Harris 2006, } \\
\text { Michel et al. } \\
2007\end{array}$ \\
\hline \multirow{3}{*}{$\begin{array}{l}\text { Structures in water } \\
\text { column and on } \\
\text { seabed, such as } \\
\text { devices and } \\
\text { moorings and } \\
\text { footings }\end{array}$} & Structure & $\begin{array}{l}\text { None, these species do not } \\
\text { dive deep enough }\end{array}$ & N/A & N/A & N/A & Low & USFWS 2005 \\
\hline & $\begin{array}{l}\text { Water } \\
\text { circulation } \\
\text { changes }\end{array}$ & $\begin{array}{l}\text { None, project not large } \\
\text { enough to affect prey }\end{array}$ & N/A & N/A & N/A & Low & N/A \\
\hline & $\begin{array}{l}\text { Oil/chemical } \\
\text { release }\end{array}$ & $\begin{array}{l}\text { Hypothermia, toxicity to } \\
\text { individuals and prey }\end{array}$ & $\begin{array}{l}\text { Low for typical small } \\
\text { volume releases, High for } \\
\text { large, catastrophic release } \\
\text { from device failure }\end{array}$ & $\begin{array}{l}\text { Med, could be } \\
\text { continuous and } \\
\text { intermittent for } \\
\text { life of project }\end{array}$ & $\begin{array}{l}\text { Med, species } \\
\text { common in area and } \\
\text { could be attracted to } \\
\text { structures }\end{array}$ & Low & $\begin{array}{l}\text { Harris 2006, } \\
\text { Michel et al. } \\
2007\end{array}$ \\
\hline \multirow[t]{2}{*}{$\begin{array}{l}\text { Structures on } \\
\text { water's surface }\end{array}$} & Structure & $\begin{array}{l}\text { Possible roosting habitat, } \\
\text { attraction to increased prey } \\
\text { (FAD effect), collision } \\
\text { while flying }\end{array}$ & $\begin{array}{l}\text { Low, would occur in } \\
\text { small area relative to } \\
\text { species' range }\end{array}$ & $\begin{array}{l}\text { High, would be } \\
\text { continuous for life } \\
\text { of project }\end{array}$ & Med, see above & Unknown & $\begin{array}{l}\text { Harris 2006, } \\
\text { LGL Limited } \\
\text { et al. 2009, } \\
\text { Michel et al. } \\
2007\end{array}$ \\
\hline & $\begin{array}{l}\text { Navigation } \\
\text { lights }\end{array}$ & $\begin{array}{l}\text { None, gulls and pelicans not } \\
\text { known to be attracted to } \\
\text { lights }\end{array}$ & N/A & N/A & N/A & Low & $\begin{array}{l}\text { Montevecchi } \\
2006\end{array}$ \\
\hline \multicolumn{8}{|c|}{ Decommissioning } \\
\hline \multirow{2}{*}{ Boat traffic } & $\begin{array}{l}\text { Oil/chemical } \\
\text { release }\end{array}$ & $\begin{array}{l}\text { Hypothermia, toxicity to } \\
\text { individuals and prey }\end{array}$ & $\begin{array}{l}\text { Low for typical small } \\
\text { volume releases, High for } \\
\text { large, catastrophic spill }\end{array}$ & $\begin{array}{l}\text { Low, occurs over } \\
1 \text { to } 2 \text { summers }\end{array}$ & $\begin{array}{l}\text { Med, boats could } \\
\text { pass through } \\
\text { foraging areas }\end{array}$ & Low & $\begin{array}{l}\text { Harris 2006, } \\
\text { Michel et al. } \\
2007\end{array}$ \\
\hline & $\begin{array}{l}\text { Noise and } \\
\text { vibration }\end{array}$ & $\begin{array}{l}\text { None, gulls and pelicans } \\
\text { follow boats and do not } \\
\text { appear to be disturbed by } \\
\text { boat noise }\end{array}$ & N/A & N/A & N/A & Low & Harris 2006 \\
\hline
\end{tabular}

\begin{tabular}{|c|c|c|}
\hline $\begin{array}{l}\text { Environmental Assessment for Siting Wave \& Tidal } \\
\text { Energy Projects }\end{array}$ & D-77 & $\begin{array}{r}\text { H. T. Harvey \& Associates } \\
24 \text { November } 2009\end{array}$ \\
\hline
\end{tabular}


Appendix D - Effects of Small Commercial OPT Power Buoy Project Humboldt

\begin{tabular}{|c|c|c|c|c|c|c|c|}
\hline Project activity & Action & $\begin{array}{l}\text { Description of action's } \\
\text { effect on indicator }\end{array}$ & $\begin{array}{l}\text { Spatial exposure to } \\
\text { indicator (low, med, } \\
\text { high) }\end{array}$ & $\begin{array}{l}\text { Temporal } \\
\text { exposure to } \\
\text { indicator (low, } \\
\text { med, high) }\end{array}$ & $\begin{array}{l}\text { Effect's overlap } \\
\text { with critical life } \\
\text { stage, behavior, } \\
\text { habitat or resource } \\
\text { (low, med, high) }\end{array}$ & $\begin{array}{l}\text { Overall risk } \\
\text { to indicator } \\
\text { (low, med, } \\
\text { high, } \\
\text { unknown) } \\
\end{array}$ & Source(s) \\
\hline \multirow{4}{*}{$\begin{array}{l}\text { Decommissioning of } \\
\text { structures on water's } \\
\text { surface or seabed }\end{array}$} & $\begin{array}{l}\text { Noise and } \\
\text { vibration }\end{array}$ & $\begin{array}{l}\text { Possible movement away } \\
\text { from area and disruption of } \\
\text { foraging or nesting }\end{array}$ & $\begin{array}{l}\text { Low, would occur in } \\
\text { small area relative to } \\
\text { species' range }\end{array}$ & $\begin{array}{l}\text { Low, occurs over } \\
1 \text { to } 2 \text { summers }\end{array}$ & $\begin{array}{l}\text { Low, project not near } \\
\text { nesting colony or } \\
\text { known foraging area }\end{array}$ & Low & $\begin{array}{l}\text { Harris 2006, } \\
\text { Michel et al. } \\
2007\end{array}$ \\
\hline & $\begin{array}{l}\text { Oil/chemical } \\
\text { release }\end{array}$ & $\begin{array}{l}\text { Hypothermia, toxicity to } \\
\text { individuals and prey }\end{array}$ & $\begin{array}{l}\text { Low, volume released } \\
\text { would occupy small area } \\
\text { relative to species' range } \\
\end{array}$ & $\begin{array}{l}\text { Low, occurs over } \\
1 \text { to } 2 \text { summers }\end{array}$ & Low, see above & Low & $\begin{array}{l}\text { Harris 2006, } \\
\text { Michel et al. } \\
2007\end{array}$ \\
\hline & $\begin{array}{l}\text { Seabed } \\
\text { disturbance }\end{array}$ & $\begin{array}{l}\text { None, these are surface- } \\
\text { feeders }\end{array}$ & N/A & N/A & N/A & Low & USFWS 2005 \\
\hline & $\begin{array}{l}\text { Construction } \\
\text { lights }\end{array}$ & $\begin{array}{l}\text { None, gulls and pelicans not } \\
\text { known to be attracted to } \\
\text { lights }\end{array}$ & N/A & N/A & N/A & Low & $\begin{array}{l}\text { Montevecchi } \\
2006\end{array}$ \\
\hline
\end{tabular}

\begin{tabular}{lr}
\hline Environmental Assessment for Siting Wave \& Tidal & D-78 \\
Energy Projects &
\end{tabular}


Appendix D - Effects of Small Commercial OPT Power Buoy Project Humboldt

Effects on cormorants for a Small Commercial OPT Power Buoy Project at the Humboldt site.

\begin{tabular}{|c|c|c|c|c|c|c|c|}
\hline Project activity & Action & $\begin{array}{l}\text { Description of action's } \\
\text { effect on indicator }\end{array}$ & $\begin{array}{l}\text { Spatial exposure to } \\
\text { indicator (low, med, } \\
\text { high) }\end{array}$ & $\begin{array}{l}\text { Temporal } \\
\text { exposure to } \\
\text { indicator (low, } \\
\text { med, high) } \\
\end{array}$ & $\begin{array}{l}\text { Effect's overlap } \\
\text { with critical life } \\
\text { stage, behavior, } \\
\text { habitat or resource } \\
\text { (low, med, high) }\end{array}$ & $\begin{array}{l}\text { Overall risk } \\
\text { to indicator } \\
\text { (low, med, } \\
\text { high, } \\
\text { unknown) } \\
\end{array}$ & Source(s) \\
\hline \multicolumn{8}{|c|}{ Construction } \\
\hline \multirow{2}{*}{ Boat traffic } & $\begin{array}{l}\text { Noise and } \\
\text { vibration }\end{array}$ & $\begin{array}{l}\text { Possible movement away } \\
\text { from boat and disruption of } \\
\text { foraging }\end{array}$ & $\begin{array}{l}\text { Low, would occur in } \\
\text { small area relative to } \\
\text { species' range }\end{array}$ & $\begin{array}{l}\text { Low, occurs over } \\
1 \text { to } 2 \text { summers }\end{array}$ & $\begin{array}{l}\text { Med, boats could } \\
\text { pass through } \\
\text { foraging areas }\end{array}$ & Low & $\begin{array}{l}\text { Harris } 2006 \text {, } \\
\text { LGL Limited } \\
\text { et al. } 2009\end{array}$ \\
\hline & $\begin{array}{l}\text { Oil/chemical } \\
\text { release }\end{array}$ & $\begin{array}{l}\text { Hypothermia, toxicity to } \\
\text { individuals and prey }\end{array}$ & $\begin{array}{l}\text { Low for typical small } \\
\text { volume releases, High for } \\
\text { large, catastrophic spill }\end{array}$ & $\begin{array}{l}\text { Low, occurs over } \\
1 \text { to } 2 \text { summers }\end{array}$ & Med, see above & Low & $\begin{array}{l}\text { Harris 2006, } \\
\text { Michel et al. } \\
2007\end{array}$ \\
\hline \multirow{4}{*}{$\begin{array}{l}\text { Construction of } \\
\text { electrical collector } \\
\text { system, moorings/ } \\
\text { foundation; } \\
\text { installation of } \\
\text { devices }\end{array}$} & $\begin{array}{l}\text { Noise and } \\
\text { vibration }\end{array}$ & $\begin{array}{l}\text { Possible movement away } \\
\text { from area and disruption of } \\
\text { foraging or nesting }\end{array}$ & $\begin{array}{l}\text { Low, would occur in } \\
\text { small area relative to } \\
\text { species' range }\end{array}$ & $\begin{array}{l}\text { Low, occurs over } \\
1 \text { to } 2 \text { summers }\end{array}$ & $\begin{array}{l}\text { Low, project not near } \\
\text { nesting colony or } \\
\text { known foraging area }\end{array}$ & Low & $\begin{array}{l}\text { Harris 2006, } \\
\text { Michel et al. } \\
2007\end{array}$ \\
\hline & $\begin{array}{l}\text { Oil/chemical } \\
\text { release }\end{array}$ & $\begin{array}{l}\text { Hypothermia, toxicity to } \\
\text { individuals and prey }\end{array}$ & $\begin{array}{l}\text { Low, volume released } \\
\text { would occupy small area } \\
\text { relative to species' range }\end{array}$ & $\begin{array}{l}\text { Low, occurs over } \\
1 \text { to } 2 \text { summers }\end{array}$ & Low, see above & Low & $\begin{array}{l}\text { Harris 2006, } \\
\text { Michel et al. } \\
2007\end{array}$ \\
\hline & $\begin{array}{l}\text { Seabed } \\
\text { disturbance }\end{array}$ & $\begin{array}{l}\text { Possible short-term changes } \\
\text { in food resources }\end{array}$ & $\begin{array}{l}\text { Low, would occur in } \\
\text { small area relative to } \\
\text { species’ range }\end{array}$ & $\begin{array}{l}\text { Low, occurs over } \\
1 \text { to } 2 \text { summers }\end{array}$ & Low, see above & Low & $\begin{array}{l}\text { Boehlert et al. } \\
\text { 2008, Harris } \\
2006 \\
\end{array}$ \\
\hline & $\begin{array}{l}\text { Construction } \\
\text { lights }\end{array}$ & $\begin{array}{l}\text { None, cormorants not } \\
\text { known to be attracted to } \\
\text { lights }\end{array}$ & N/A & N/A & N/A & Low & $\begin{array}{l}\text { Montevecchi } \\
2006\end{array}$ \\
\hline \multirow{2}{*}{$\begin{array}{l}\text { Directional drilling, } \\
\text { and laying cable } \\
\text { under/on seabed }\end{array}$} & $\begin{array}{l}\text { Seabed } \\
\text { disturbance }\end{array}$ & $\begin{array}{l}\text { Possible short-term changes } \\
\text { in food resources }\end{array}$ & $\begin{array}{l}\text { Low, would occur in } \\
\text { small area relative to } \\
\text { species' range }\end{array}$ & $\begin{array}{l}\text { Low, occurs over } \\
\text { a short time } \\
\text { period }\end{array}$ & $\begin{array}{l}\text { Low, project not near } \\
\text { nesting colony or } \\
\text { known foraging area }\end{array}$ & Low & $\begin{array}{l}\text { Boehlert et al. } \\
\text { 2008, Harris } \\
2006\end{array}$ \\
\hline & $\begin{array}{l}\text { Noise and } \\
\text { vibration }\end{array}$ & $\begin{array}{l}\text { Possible movement away } \\
\text { from area and disruption of } \\
\text { foraging or nesting }\end{array}$ & Low, see above & $\begin{array}{l}\text { Low, occurs over } \\
\text { a short time } \\
\text { period }\end{array}$ & Low, see above & Low & $\begin{array}{l}\text { Harris 2006, } \\
\text { Michel et al. } \\
2007\end{array}$ \\
\hline \multicolumn{8}{|c|}{ Operation and Maintenance } \\
\hline Boat traffic & $\begin{array}{l}\text { Noise and } \\
\text { vibration }\end{array}$ & $\begin{array}{l}\text { Possible movement away } \\
\text { from boat and disruption of } \\
\text { foraging }\end{array}$ & Low, see above & $\begin{array}{l}\text { Low, only from } \\
\text { periodic trips in } \\
\text { summer }\end{array}$ & $\begin{array}{l}\text { Med, boats could } \\
\text { pass through } \\
\text { foraging areas }\end{array}$ & Low & $\begin{array}{l}\text { Harris 2006, } \\
\text { LGL Limited } \\
\text { et al. } 2009 \\
\end{array}$ \\
\hline
\end{tabular}

\begin{tabular}{lr}
\hline Environmental Assessment for Siting Wave \& Tidal & D-79 \\
Energy Projects & H. T. Harvey \& Associates \\
24 November 2009
\end{tabular}


Appendix D - Effects of Small Commercial OPT Power Buoy Project Humboldt

\begin{tabular}{|c|c|c|c|c|c|c|c|}
\hline Project activity & Action & $\begin{array}{l}\text { Description of action's } \\
\text { effect on indicator }\end{array}$ & $\begin{array}{l}\text { Spatial exposure to } \\
\text { indicator (low, med, } \\
\text { high) }\end{array}$ & $\begin{array}{l}\text { Temporal } \\
\text { exposure to } \\
\text { indicator (low, } \\
\text { med, high) }\end{array}$ & $\begin{array}{l}\text { Effect's overlap } \\
\text { with critical life } \\
\text { stage, behavior, } \\
\text { habitat or resource } \\
\text { (low, med, high) } \\
\end{array}$ & $\begin{array}{l}\text { Overall risk } \\
\text { to indicator } \\
\text { (low, med, } \\
\text { high, } \\
\text { unknown) }\end{array}$ & Source(s) \\
\hline & $\begin{array}{l}\text { Oil/chemical } \\
\text { release }\end{array}$ & $\begin{array}{l}\text { Hypothermia, toxicity to } \\
\text { individuals and prey }\end{array}$ & $\begin{array}{l}\text { Low for typical small } \\
\text { volume releases, High for } \\
\text { large, catastrophic spill }\end{array}$ & $\begin{array}{l}\text { Low, wouldn’t } \\
\text { persist long-term }\end{array}$ & Med, see above & Low & $\begin{array}{l}\text { Harris 2006, } \\
\text { Michel et al. } \\
2007\end{array}$ \\
\hline \multirow{2}{*}{$\begin{array}{l}\text { Operation of } \\
\text { turbines or other } \\
\text { moving parts of } \\
\text { devices }\end{array}$} & $\begin{array}{l}\text { Moving device } \\
\text { parts }\end{array}$ & $\begin{array}{l}\text { None, no underwater } \\
\text { moving parts }\end{array}$ & N/A & N/A & N/A & Low & N/A \\
\hline & $\begin{array}{l}\text { Noise and } \\
\text { vibration }\end{array}$ & $\begin{array}{l}\text { Possible avoidance of } \\
\text { project area }\end{array}$ & $\begin{array}{l}\text { Low, would occur in } \\
\text { small area relative to } \\
\text { species' range }\end{array}$ & $\begin{array}{l}\text { High, would be } \\
\text { continuous for life } \\
\text { of project }\end{array}$ & $\begin{array}{l}\text { Low, project not near } \\
\text { nesting colony or } \\
\text { known foraging area }\end{array}$ & Low & $\begin{array}{l}\text { Harris 2006, } \\
\text { Michel et al. } \\
2007\end{array}$ \\
\hline \multirow{3}{*}{$\begin{array}{l}\text { Structures in water } \\
\text { column and on } \\
\text { seabed, such as } \\
\text { devices and } \\
\text { moorings and } \\
\text { footings }\end{array}$} & Structure & $\begin{array}{l}\text { Possible attraction to } \\
\text { increased prey (reef effect), } \\
\text { entanglement with derelict } \\
\text { fishing gear/ moorings, } \\
\text { collisions with structures } \\
\text { while diving }\end{array}$ & Low, see above & $\begin{array}{l}\text { Med, tangled gear } \\
\text { would be } \\
\text { removed } \\
\text { periodically }\end{array}$ & $\begin{array}{l}\text { Med, cormorants } \\
\text { common in area and } \\
\text { could be attracted to } \\
\text { structures }\end{array}$ & Unknown & $\begin{array}{l}\text { Harris 2006, } \\
\text { Michel et al. } \\
2007\end{array}$ \\
\hline & $\begin{array}{l}\text { Water } \\
\text { circulation } \\
\text { changes }\end{array}$ & $\begin{array}{l}\text { None, project not large } \\
\text { enough to affect prey }\end{array}$ & N/A & N/A & N/A & Low & N/A \\
\hline & $\begin{array}{l}\text { Oil/chemical } \\
\text { release }\end{array}$ & $\begin{array}{l}\text { Hypothermia, toxicity to } \\
\text { individuals and prey }\end{array}$ & $\begin{array}{l}\text { Low for typical small } \\
\text { volume releases, High for } \\
\text { large, catastrophic release } \\
\text { from device failure }\end{array}$ & $\begin{array}{l}\text { Med, could be } \\
\text { continuous and } \\
\text { intermittent for } \\
\text { life of project }\end{array}$ & $\begin{array}{l}\text { Med, cormorants } \\
\text { common in area and } \\
\text { could be attracted to } \\
\text { structures }\end{array}$ & Low & $\begin{array}{l}\text { Harris 2006, } \\
\text { Michel et al. } \\
2007\end{array}$ \\
\hline \multirow{2}{*}{$\begin{array}{l}\text { Structures on } \\
\text { water's surface }\end{array}$} & Structure & $\begin{array}{l}\text { Possible roosting habitat, } \\
\text { attraction to increased prey } \\
\text { (FAD effect), collision } \\
\text { while flying }\end{array}$ & $\begin{array}{l}\text { Low, would occur in } \\
\text { small area relative to } \\
\text { species' range }\end{array}$ & $\begin{array}{l}\text { High, continuous } \\
\text { for life of project }\end{array}$ & Med, see above & Unknown & $\begin{array}{l}\text { Boehlert et al. } \\
\text { 2008, Harris } \\
\text { 2006, Michel } \\
\text { et al. } 2007 \\
\end{array}$ \\
\hline & $\begin{array}{l}\text { Navigation } \\
\text { lights }\end{array}$ & $\begin{array}{l}\text { None, cormorants not } \\
\text { known to be attracted to } \\
\text { lights }\end{array}$ & N/A & N/A & N/A & Low & $\begin{array}{l}\text { Montevecchi } \\
2006\end{array}$ \\
\hline
\end{tabular}

\begin{tabular}{lr}
\hline Environmental Assessment for Siting Wave \& Tidal & D-80 \\
Energy Projects & H. T. Harvey \& Associates \\
24 November 2009
\end{tabular}


Appendix D - Effects of Small Commercial OPT Power Buoy Project Humboldt

\begin{tabular}{|c|c|c|c|c|c|c|c|}
\hline Project activity & Action & $\begin{array}{l}\text { Description of action's } \\
\text { effect on indicator }\end{array}$ & $\begin{array}{l}\text { Spatial exposure to } \\
\text { indicator (low, med, } \\
\text { high) }\end{array}$ & $\begin{array}{l}\text { Temporal } \\
\text { exposure to } \\
\text { indicator (low, } \\
\text { med, high) }\end{array}$ & $\begin{array}{l}\text { Effect's overlap } \\
\text { with critical life } \\
\text { stage, behavior, } \\
\text { habitat or resource } \\
\text { (low, med, high) }\end{array}$ & $\begin{array}{l}\text { Overall risk } \\
\text { to indicator } \\
\text { (low, med, } \\
\text { high, } \\
\text { unknown) } \\
\end{array}$ & Source(s) \\
\hline \multirow{2}{*}{ Boat traffic } & $\begin{array}{l}\text { Oil/chemical } \\
\text { release }\end{array}$ & $\begin{array}{l}\text { Hypothermia, toxicity to } \\
\text { individuals and prey }\end{array}$ & $\begin{array}{l}\text { Low for typical small } \\
\text { volume releases, High for } \\
\text { large, catastrophic spill }\end{array}$ & $\begin{array}{l}\text { Low, occurs over } \\
1 \text { to } 2 \text { summers }\end{array}$ & $\begin{array}{l}\text { Med, boats could } \\
\text { pass through } \\
\text { foraging areas }\end{array}$ & Low & $\begin{array}{l}\text { Harris 2006, } \\
\text { Michel et al. } \\
2007\end{array}$ \\
\hline & $\begin{array}{l}\text { Noise and } \\
\text { vibration }\end{array}$ & $\begin{array}{l}\text { Possible movement away } \\
\text { from boat and disruption of } \\
\text { foraging }\end{array}$ & $\begin{array}{l}\text { Low, would occur in } \\
\text { small area relative to } \\
\text { species' range }\end{array}$ & $\begin{array}{l}\text { Low, occurs over } \\
1 \text { to } 2 \text { summers }\end{array}$ & Med, see above & Low & $\begin{array}{l}\text { Harris } 2006 \text {, } \\
\text { LGL Limited } \\
\text { et al. } 2009\end{array}$ \\
\hline \multirow{4}{*}{$\begin{array}{l}\text { Decommissioning of } \\
\text { structures on water's } \\
\text { surface or seabed }\end{array}$} & $\begin{array}{l}\text { Noise and } \\
\text { vibration }\end{array}$ & $\begin{array}{l}\text { Possible movement away } \\
\text { from area and disruption of } \\
\text { foraging or nesting }\end{array}$ & $\begin{array}{l}\text { Low, would occur in } \\
\text { small area relative to } \\
\text { species' range }\end{array}$ & $\begin{array}{l}\text { Low, occurs over } \\
1 \text { to } 2 \text { summers }\end{array}$ & $\begin{array}{l}\text { Low, project not near } \\
\text { nesting colony or } \\
\text { known foraging area }\end{array}$ & Low & $\begin{array}{l}\text { Harris 2006, } \\
\text { Michel et al. } \\
2007\end{array}$ \\
\hline & $\begin{array}{l}\text { Oil/chemical } \\
\text { release }\end{array}$ & $\begin{array}{l}\text { Hypothermia, toxicity to } \\
\text { individuals and prey }\end{array}$ & $\begin{array}{l}\text { Low, volume released } \\
\text { would occupy small area } \\
\text { relative to species' range }\end{array}$ & $\begin{array}{l}\text { Low, occurs over } \\
1 \text { to } 2 \text { summers }\end{array}$ & Low, see above & Low & $\begin{array}{l}\text { Harris 2006, } \\
\text { Michel et al. } \\
2007\end{array}$ \\
\hline & $\begin{array}{l}\text { Seabed } \\
\text { disturbance }\end{array}$ & $\begin{array}{l}\text { Possible short-term changes } \\
\text { in food resources }\end{array}$ & $\begin{array}{l}\text { Low, would occur in } \\
\text { small area relative to } \\
\text { species' range }\end{array}$ & $\begin{array}{l}\text { Low, occurs over } \\
1 \text { to } 2 \text { summers }\end{array}$ & Low, see above & Low & $\begin{array}{l}\text { Boehlert et al. } \\
2008 \text {, Harris } \\
2006\end{array}$ \\
\hline & $\begin{array}{l}\text { Construction } \\
\text { lights }\end{array}$ & $\begin{array}{l}\text { None, cormorants not } \\
\text { known to be attracted to } \\
\text { lights }\end{array}$ & N/A & N/A & N/A & Low & $\begin{array}{l}\text { Montevecchi } \\
2006\end{array}$ \\
\hline
\end{tabular}

\begin{tabular}{lr}
\hline Environmental Assessment for Siting Wave \& Tidal & D-81 \\
Energy Projects & H. T. Harvey \& Associates \\
24 November 2009
\end{tabular}


Appendix D - Effects of Small Commercial OPT Power Buoy Project Humboldt

Effects on alcids for a Small Commercial OPT Power Buoy Project at the Humboldt site.

\begin{tabular}{|c|c|c|c|c|c|c|c|}
\hline Project activity & Action & $\begin{array}{l}\text { Description of action's } \\
\text { effect on indicator }\end{array}$ & $\begin{array}{l}\text { Spatial exposure to } \\
\text { indicator (low, med, } \\
\text { high) }\end{array}$ & $\begin{array}{l}\text { Temporal } \\
\text { exposure to } \\
\text { indicator (low, } \\
\text { med, high) }\end{array}$ & $\begin{array}{l}\text { Effect's overlap } \\
\text { with critical life } \\
\text { stage, behavior, } \\
\text { habitat or resource } \\
\text { (low, med, high) }\end{array}$ & $\begin{array}{l}\text { Overall risk } \\
\text { to indicator } \\
\text { (low, med, } \\
\text { high, } \\
\text { unknown) } \\
\end{array}$ & Source(s) \\
\hline \multicolumn{8}{|c|}{ Construction } \\
\hline \multirow{2}{*}{ Boat traffic } & $\begin{array}{l}\text { Noise and } \\
\text { vibration }\end{array}$ & $\begin{array}{l}\text { Possible movement away } \\
\text { from boat and disruption of } \\
\text { foraging }\end{array}$ & $\begin{array}{l}\text { Low, would occur in } \\
\text { small area relative to } \\
\text { species' range }\end{array}$ & $\begin{array}{l}\text { Low, occurs over } \\
1 \text { to } 2 \text { summers }\end{array}$ & $\begin{array}{l}\text { Med, boats could } \\
\text { pass through } \\
\text { foraging areas }\end{array}$ & Low & $\begin{array}{l}\text { Harris 2006, } \\
\text { LGL Limited } \\
\text { et al. } 2009\end{array}$ \\
\hline & $\begin{array}{l}\text { Oil/chemical } \\
\text { release }\end{array}$ & $\begin{array}{l}\text { Hypothermia, toxicity to } \\
\text { individuals and prey }\end{array}$ & $\begin{array}{l}\text { Low for typical small } \\
\text { volume releases, High for } \\
\text { large, catastrophic spill }\end{array}$ & $\begin{array}{l}\text { Low, occurs over } \\
1 \text { to } 2 \text { summers }\end{array}$ & Med, see above & Low & $\begin{array}{l}\text { Harris 2006, } \\
\text { Michel et al. } \\
2007\end{array}$ \\
\hline \multirow{4}{*}{$\begin{array}{l}\text { Construction of } \\
\text { electrical collector } \\
\text { system, moorings/ } \\
\text { foundation; } \\
\text { installation of } \\
\text { devices }\end{array}$} & $\begin{array}{l}\text { Noise and } \\
\text { vibration }\end{array}$ & $\begin{array}{l}\text { Possible movement away } \\
\text { from area and disruption of } \\
\text { foraging or nesting }\end{array}$ & $\begin{array}{l}\text { Low, would occur in } \\
\text { small area relative to } \\
\text { species' range }\end{array}$ & $\begin{array}{l}\text { Low, occurs over } \\
1 \text { to } 2 \text { summers }\end{array}$ & $\begin{array}{l}\text { Low, project not } \\
\text { near nesting colony } \\
\text { or known foraging } \\
\text { area }\end{array}$ & Low & $\begin{array}{l}\text { Harris 2006, } \\
\text { Michel et al. } \\
2007\end{array}$ \\
\hline & $\begin{array}{l}\text { Oil/chemical } \\
\text { release }\end{array}$ & $\begin{array}{l}\text { Hypothermia, toxicity to } \\
\text { individuals and prey }\end{array}$ & $\begin{array}{l}\text { Low, volume released } \\
\text { would occupy small area } \\
\text { relative to species’ range }\end{array}$ & $\begin{array}{l}\text { Low, occurs over } \\
1 \text { to } 2 \text { summers }\end{array}$ & Low, see above & Low & $\begin{array}{l}\text { Harris 2006, } \\
\text { Michel et al. } \\
2007\end{array}$ \\
\hline & $\begin{array}{l}\text { Seabed } \\
\text { disturbance }\end{array}$ & $\begin{array}{l}\text { Possible short-term changes } \\
\text { in food resources }\end{array}$ & $\begin{array}{l}\text { Low, would occur in } \\
\text { small area relative to } \\
\text { species' range }\end{array}$ & $\begin{array}{l}\text { Low, occurs over } \\
1 \text { to } 2 \text { summers }\end{array}$ & Low, see above & Low & $\begin{array}{l}\text { Boehlert et al. } \\
2008 \text {, Harris } \\
2006\end{array}$ \\
\hline & $\begin{array}{l}\text { Construction } \\
\text { lights }\end{array}$ & $\begin{array}{l}\text { Attraction/ disorientation, } \\
\text { collisions }\end{array}$ & Low, see above & $\begin{array}{l}\text { Low, occurs over } \\
1 \text { to } 2 \text { summers }\end{array}$ & Low, see above & Low & $\begin{array}{l}\text { Harris 2006, } \\
\text { Montevecchi } \\
2006\end{array}$ \\
\hline \multirow{2}{*}{$\begin{array}{l}\text { Directional drilling, } \\
\text { and laying cable } \\
\text { under/on seabed }\end{array}$} & $\begin{array}{l}\text { Seabed } \\
\text { disturbance }\end{array}$ & $\begin{array}{l}\text { Possible short-term changes } \\
\text { in food resources }\end{array}$ & Low, see above & $\begin{array}{l}\text { Low, occurs over } \\
\text { a short time } \\
\text { period }\end{array}$ & Low, see above & Low & $\begin{array}{l}\text { Boehlert et al. } \\
2008 \text {, Harris } \\
2006\end{array}$ \\
\hline & $\begin{array}{l}\text { Noise and } \\
\text { vibration }\end{array}$ & $\begin{array}{l}\text { Possible movement away } \\
\text { from area and disruption of } \\
\text { foraging or nesting }\end{array}$ & $\begin{array}{l}\text { Low, would occur in } \\
\text { small area relative to } \\
\text { species' range }\end{array}$ & $\begin{array}{l}\text { Low, occurs over } \\
\text { a short time } \\
\text { period }\end{array}$ & Low, see above & Low & $\begin{array}{l}\text { Harris 2006, } \\
\text { Michel et al. } \\
2007\end{array}$ \\
\hline \multicolumn{8}{|c|}{ Operation and Maintenance } \\
\hline Boat traffic & $\begin{array}{l}\text { Oil/chemical } \\
\text { release }\end{array}$ & $\begin{array}{l}\text { Hypothermia, toxicity to } \\
\text { individuals and prey }\end{array}$ & $\begin{array}{l}\text { Low for typical small } \\
\text { volume releases, High for } \\
\text { large, catastrophic spill }\end{array}$ & $\begin{array}{l}\text { Low, wouldn't } \\
\text { persist long-term }\end{array}$ & $\begin{array}{l}\text { Med, boats could } \\
\text { pass through } \\
\text { foraging areas }\end{array}$ & Low & $\begin{array}{l}\text { Harris 2006, } \\
\text { Michel et al. } \\
2007\end{array}$ \\
\hline
\end{tabular}

\begin{tabular}{lr}
\hline Environmental Assessment for Siting Wave \& Tidal & D-82 \\
Energy Projects & H. Harvey \& Associates \\
24 November 2009
\end{tabular}


Appendix D - Effects of Small Commercial OPT Power Buoy Project Humboldt

\begin{tabular}{|c|c|c|c|c|c|c|c|}
\hline Project activity & Action & $\begin{array}{l}\text { Description of action's } \\
\text { effect on indicator }\end{array}$ & $\begin{array}{l}\text { Spatial exposure to } \\
\text { indicator (low, med, } \\
\text { high) }\end{array}$ & $\begin{array}{l}\text { Temporal } \\
\text { exposure to } \\
\text { indicator (low, } \\
\text { med, high) }\end{array}$ & $\begin{array}{l}\text { Effect's overlap } \\
\text { with critical life } \\
\text { stage, behavior, } \\
\text { habitat or resource } \\
\text { (low, med, high) } \\
\end{array}$ & $\begin{array}{l}\text { Overall risk } \\
\text { to indicator } \\
\text { (low, med, } \\
\text { high, } \\
\text { unknown) } \\
\end{array}$ & Source(s) \\
\hline & $\begin{array}{l}\text { Noise and } \\
\text { vibration }\end{array}$ & $\begin{array}{l}\text { Possible movement away } \\
\text { from boat and disruption of } \\
\text { foraging }\end{array}$ & $\begin{array}{l}\text { Low, would occur in } \\
\text { small area relative to } \\
\text { species' range }\end{array}$ & $\begin{array}{l}\text { Low, only from } \\
\text { periodic trips in } \\
\text { summer }\end{array}$ & Med, see above & Low & $\begin{array}{l}\text { Harris 2006, } \\
\text { LGL Limited } \\
\text { et al. } 2009 \\
\end{array}$ \\
\hline \multirow{2}{*}{$\begin{array}{l}\text { Operation of } \\
\text { turbines or other } \\
\text { moving parts of } \\
\text { devices }\end{array}$} & $\begin{array}{l}\text { Moving device } \\
\text { parts }\end{array}$ & $\begin{array}{l}\text { None, no underwater } \\
\text { moving parts }\end{array}$ & N/A & N/A & N/A & Low & N/A \\
\hline & $\begin{array}{l}\text { Noise and } \\
\text { vibration }\end{array}$ & $\begin{array}{l}\text { Possible avoidance of } \\
\text { project area }\end{array}$ & $\begin{array}{l}\text { Low, would occur in } \\
\text { small area relative to } \\
\text { species' range }\end{array}$ & $\begin{array}{l}\text { High, would be } \\
\text { continuous for life } \\
\text { of project }\end{array}$ & $\begin{array}{l}\text { Low, project not } \\
\text { near nesting colony } \\
\text { or known foraging } \\
\text { area }\end{array}$ & Low & $\begin{array}{l}\text { Harris 2006, } \\
\text { Michel et al. } \\
2007\end{array}$ \\
\hline \multirow{3}{*}{$\begin{array}{l}\text { Structures in water } \\
\text { column and on } \\
\text { seabed, such as } \\
\text { devices and } \\
\text { moorings and } \\
\text { footings }\end{array}$} & Structure & $\begin{array}{l}\text { Possible attraction to } \\
\text { increased prey (reef effect), } \\
\text { entanglement with derelict } \\
\text { fishing gear/ moorings } \\
\text { while diving }\end{array}$ & Low, see above & $\begin{array}{l}\text { Med, tangled gear } \\
\text { would be } \\
\text { removed } \\
\text { periodically }\end{array}$ & Low, see above & Unknown & $\begin{array}{l}\text { Harris 2006, } \\
\text { Michel et al. } \\
2007\end{array}$ \\
\hline & $\begin{array}{l}\text { Water } \\
\text { circulation } \\
\text { changes }\end{array}$ & $\begin{array}{l}\text { None, project not large } \\
\text { enough to affect prey }\end{array}$ & N/A & N/A & N/A & Low & N/A \\
\hline & $\begin{array}{l}\text { Oil/chemical } \\
\text { release }\end{array}$ & $\begin{array}{l}\text { Hypothermia, toxicity to } \\
\text { individuals and prey }\end{array}$ & $\begin{array}{l}\text { Low for typical small } \\
\text { volume releases, High for } \\
\text { large, catastrophic release } \\
\text { from device failure }\end{array}$ & $\begin{array}{l}\text { Med, continuous } \\
\text { and intermittent } \\
\text { for life of project }\end{array}$ & $\begin{array}{l}\text { Low, project not } \\
\text { near nesting colony } \\
\text { or known foraging } \\
\text { area }\end{array}$ & Low & $\begin{array}{l}\text { Harris 2006, } \\
\text { Michel et al. } \\
2007\end{array}$ \\
\hline \multirow{2}{*}{$\begin{array}{l}\text { Structures on } \\
\text { water's surface }\end{array}$} & $\begin{array}{l}\text { Navigation } \\
\text { lights }\end{array}$ & $\begin{array}{l}\text { Attraction/ disorientation, } \\
\text { collisions }\end{array}$ & $\begin{array}{l}\text { Low, would occur in } \\
\text { small area relative to } \\
\text { species' range }\end{array}$ & $\begin{array}{l}\text { High, continuous } \\
\text { for life of project }\end{array}$ & $\begin{array}{l}\text { Med, alcids present } \\
\text { in area year-round } \\
\text { and could be } \\
\text { attracted to lights }\end{array}$ & Med & $\begin{array}{l}\text { Harris 2006, } \\
\text { Montevecchi } \\
2006\end{array}$ \\
\hline & Structure & $\begin{array}{l}\text { Possible collision while } \\
\text { flying, attraction to } \\
\text { increased prey (FAD effect) }\end{array}$ & Low, see above & $\begin{array}{l}\text { High, continuous } \\
\text { for life of project }\end{array}$ & Med, see above & Unknown & $\begin{array}{l}\text { Boehlert et al. } \\
2008 \text {, Harris } \\
2006\end{array}$ \\
\hline \multicolumn{8}{|c|}{ Decommissioning } \\
\hline Boat traffic & $\begin{array}{l}\text { Oil/chemical } \\
\text { release }\end{array}$ & $\begin{array}{l}\text { Hypothermia, toxicity to } \\
\text { individuals and prey }\end{array}$ & $\begin{array}{l}\text { Low for typical small } \\
\text { volume releases, High for } \\
\text { large, catastrophic spill }\end{array}$ & $\begin{array}{l}\text { Low, occurs over } \\
1 \text { to } 2 \text { summers }\end{array}$ & $\begin{array}{l}\text { Med, boats could } \\
\text { pass through } \\
\text { foraging areas }\end{array}$ & Low & $\begin{array}{l}\text { Harris 2006, } \\
\text { Michel et al. } \\
2007\end{array}$ \\
\hline
\end{tabular}

\begin{tabular}{lr}
\hline Environmental Assessment for Siting Wave \& Tidal & D-83 \\
Energy Projects & H. T. Harvey \& Associates \\
24 November 2009
\end{tabular}


Appendix D - Effects of Small Commercial OPT Power Buoy Project Humboldt

\begin{tabular}{|c|c|c|c|c|c|c|c|}
\hline Project activity & Action & $\begin{array}{l}\text { Description of action's } \\
\text { effect on indicator }\end{array}$ & $\begin{array}{l}\text { Spatial exposure to } \\
\text { indicator (low, med, } \\
\text { high) }\end{array}$ & $\begin{array}{l}\text { Temporal } \\
\text { exposure to } \\
\text { indicator (low, } \\
\text { med, high) }\end{array}$ & $\begin{array}{l}\text { Effect's overlap } \\
\text { with critical life } \\
\text { stage, behavior, } \\
\text { habitat or resource } \\
\text { (low, med, high) }\end{array}$ & $\begin{array}{l}\text { Overall risk } \\
\text { to indicator } \\
\text { (low, med, } \\
\text { high, } \\
\text { unknown) } \\
\end{array}$ & Source(s) \\
\hline & $\begin{array}{l}\text { Noise and } \\
\text { vibration }\end{array}$ & $\begin{array}{l}\text { Possible movement away } \\
\text { from boat and disruption of } \\
\text { foraging }\end{array}$ & $\begin{array}{l}\text { Low, would occur in } \\
\text { small area relative to } \\
\text { species' range }\end{array}$ & $\begin{array}{l}\text { Low, occurs over } \\
1 \text { to } 2 \text { summers }\end{array}$ & Med, see above & Low & $\begin{array}{l}\text { Harris } 2006 \text {, } \\
\text { LGL Limited } \\
\text { et al. } 2009\end{array}$ \\
\hline \multirow{4}{*}{$\begin{array}{l}\text { Decommissioning of } \\
\text { structures on water's } \\
\text { surface or seabed }\end{array}$} & $\begin{array}{l}\text { Noise and } \\
\text { vibration }\end{array}$ & $\begin{array}{l}\text { Possible movement away } \\
\text { from area and disruption of } \\
\text { foraging or nesting }\end{array}$ & Low, see above & $\begin{array}{l}\text { Low, occurs over } \\
1 \text { to } 2 \text { summers }\end{array}$ & $\begin{array}{l}\text { Low, project not } \\
\text { near nesting colony } \\
\text { or known foraging } \\
\text { area }\end{array}$ & Low & $\begin{array}{l}\text { Harris 2006, } \\
\text { Michel et al. } \\
2007\end{array}$ \\
\hline & $\begin{array}{l}\text { Oil/chemical } \\
\text { release }\end{array}$ & $\begin{array}{l}\text { Hypothermia, toxicity to } \\
\text { individuals and prey }\end{array}$ & $\begin{array}{l}\text { Low, volume released } \\
\text { would occupy small area } \\
\text { relative to species' range }\end{array}$ & $\begin{array}{l}\text { Low, occurs over } \\
1 \text { to } 2 \text { summers }\end{array}$ & Low, see above & Low & $\begin{array}{l}\text { Harris 2006, } \\
\text { Michel et al. } \\
2007\end{array}$ \\
\hline & $\begin{array}{l}\text { Seabed } \\
\text { disturbance }\end{array}$ & $\begin{array}{l}\text { Possible short-term changes } \\
\text { in food resources }\end{array}$ & $\begin{array}{l}\text { Low, would occur in } \\
\text { small area relative to } \\
\text { species' range }\end{array}$ & $\begin{array}{l}\text { Low, occurs over } \\
1 \text { to } 2 \text { summers }\end{array}$ & Low, see above & Low & $\begin{array}{l}\text { Boehlert et al. } \\
2008 \text {, Harris } \\
2006\end{array}$ \\
\hline & $\begin{array}{l}\text { Construction } \\
\text { lights }\end{array}$ & $\begin{array}{l}\text { Attraction/ disorientation, } \\
\text { collisions }\end{array}$ & Low, see above & $\begin{array}{l}\text { Low, occurs over } \\
1 \text { to } 2 \text { summers }\end{array}$ & Low, see above & Low & $\begin{array}{l}\text { Harris 2006, } \\
\text { Montevecchi } \\
2006\end{array}$ \\
\hline
\end{tabular}

\begin{tabular}{lr}
\hline Environmental Assessment for Siting Wave \& Tidal & D-84 \\
Energy Projects & H. T. Harvey \& Associates \\
24 November 2009
\end{tabular}


Appendix D - Effects of Small Commercial OPT Power Buoy Project Humboldt

Effects on Marbled Murrelets for a Small Commercial OPT Power Buoy Project at the Humboldt site.

\begin{tabular}{|c|c|c|c|c|c|c|c|}
\hline Project activity & Action & $\begin{array}{l}\text { Description of action's } \\
\text { effect on indicator }\end{array}$ & $\begin{array}{l}\text { Spatial exposure to } \\
\text { indicator (low, med, } \\
\text { high) }\end{array}$ & $\begin{array}{l}\text { Temporal } \\
\text { exposure to } \\
\text { indicator (low, } \\
\text { med, high) } \\
\end{array}$ & $\begin{array}{l}\text { Effect's overlap } \\
\text { with critical life } \\
\text { stage, behavior, } \\
\text { habitat or resource } \\
\text { (low, med, high) }\end{array}$ & $\begin{array}{l}\text { Overall risk } \\
\text { to indicator } \\
\text { (low, med, } \\
\text { high, } \\
\text { unknown) } \\
\end{array}$ & Source(s) \\
\hline \multicolumn{8}{|c|}{ Construction } \\
\hline \multirow[t]{2}{*}{ Boat traffic } & $\begin{array}{l}\text { Noise and } \\
\text { vibration }\end{array}$ & $\begin{array}{l}\text { Possible movement away } \\
\text { from boat and disruption of } \\
\text { foraging }\end{array}$ & $\begin{array}{l}\text { Low, would occur in } \\
\text { small area relative to } \\
\text { species' range }\end{array}$ & $\begin{array}{l}\text { Low, occurs over } \\
1 \text { to } 2 \text { summers }\end{array}$ & $\begin{array}{l}\text { Med, boats will pass } \\
\text { through murrelet } \\
\text { foraging areas }\end{array}$ & Low & $\begin{array}{l}\text { Hébert and } \\
\text { Golightly } \\
\text { 2006, LGL } \\
\text { Limited et } \\
\text { al. 2009, } \\
\text { Ralph and } \\
\text { Miller } 1995\end{array}$ \\
\hline & $\begin{array}{l}\text { Oil/chemical } \\
\text { release }\end{array}$ & $\begin{array}{l}\text { Hypothermia, toxicity to } \\
\text { individuals and prey }\end{array}$ & $\begin{array}{l}\text { Low for typical small } \\
\text { volume releases, High for } \\
\text { large, catastrophic spill }\end{array}$ & $\begin{array}{l}\text { Low, occurs over } \\
1 \text { to } 2 \text { summers }\end{array}$ & Med, see above & Low & $\begin{array}{l}\text { Michel et al. } \\
2007\end{array}$ \\
\hline \multirow{4}{*}{$\begin{array}{l}\text { Construction of } \\
\text { electrical collector } \\
\text { system, } \\
\text { moorings/foundation; } \\
\text { installation of } \\
\text { devices }\end{array}$} & $\begin{array}{l}\text { Noise and } \\
\text { vibration }\end{array}$ & $\begin{array}{l}\text { Possible movement away } \\
\text { from area and disruption of } \\
\text { foraging or nesting }\end{array}$ & $\begin{array}{l}\text { Low, would occur in } \\
\text { small area relative to } \\
\text { species' range }\end{array}$ & $\begin{array}{l}\text { Low, occurs over } \\
1 \text { to } 2 \text { summers }\end{array}$ & $\begin{array}{l}\text { Low, } 90 \% \text { of } \\
\text { murrelet locations } \\
\text { closer to shore than } \\
\text { project site }\end{array}$ & Low & $\begin{array}{l}\text { Hébert and } \\
\text { Golightly } \\
2006, \\
\text { Michel et al. } \\
\text { 2007, Ralph } \\
\text { and Miller } \\
1995 \\
\end{array}$ \\
\hline & $\begin{array}{l}\text { Oil/chemical } \\
\text { release }\end{array}$ & $\begin{array}{l}\text { Hypothermia, toxicity to } \\
\text { individuals and prey }\end{array}$ & $\begin{array}{l}\text { Low, volume released } \\
\text { would occupy small area }\end{array}$ & $\begin{array}{l}\text { Low, occurs over } \\
1 \text { to } 2 \text { summers }\end{array}$ & Low, see above & Low & $\begin{array}{l}\text { Michel et al. } \\
2007\end{array}$ \\
\hline & $\begin{array}{l}\text { Seabed } \\
\text { disturbance }\end{array}$ & $\begin{array}{l}\text { Possible short-term changes } \\
\text { in food resources }\end{array}$ & $\begin{array}{l}\text { Low, would occur in } \\
\text { small area relative to } \\
\text { species' range }\end{array}$ & $\begin{array}{l}\text { Low, occurs over } \\
1 \text { to } 2 \text { summers }\end{array}$ & Low, see above & Low & $\begin{array}{l}\text { Boehlert et } \\
\text { al. } 2008\end{array}$ \\
\hline & $\begin{array}{l}\text { Construction } \\
\text { lights }\end{array}$ & $\begin{array}{l}\text { Attraction/ disorientation, } \\
\text { collisions }\end{array}$ & Low, see above & $\begin{array}{l}\text { Low, occurs over } \\
1 \text { to } 2 \text { summers } \\
\end{array}$ & Low, see above & Low & $\begin{array}{l}\text { Montevecchi } \\
2006\end{array}$ \\
\hline $\begin{array}{l}\text { Directional drilling, } \\
\text { and laying cable } \\
\text { under/on seabed }\end{array}$ & $\begin{array}{l}\text { Seabed } \\
\text { disturbance }\end{array}$ & $\begin{array}{l}\text { Possible short-term changes } \\
\text { in food resources }\end{array}$ & Low, see above & $\begin{array}{l}\text { Low, occurs over } \\
\text { very short time } \\
\text { period }\end{array}$ & $\begin{array}{l}\text { High, would occur } \\
\text { in murrelet foraging } \\
\text { zone }(<2 \mathrm{~km} \text { from } \\
\text { shore) }\end{array}$ & Med & $\begin{array}{l}\text { Boehlert et } \\
\text { al. } 2008\end{array}$ \\
\hline
\end{tabular}

\begin{tabular}{lrr}
\hline Environmental Assessment for Siting Wave \& Tidal & D-85 & H. T. Harvey \& Associates \\
Energy Projects & & 24 November 2009
\end{tabular}


Appendix D - Effects of Small Commercial OPT Power Buoy Project Humboldt

\begin{tabular}{|c|c|c|c|c|c|c|c|}
\hline Project activity & Action & $\begin{array}{l}\text { Description of action's } \\
\text { effect on indicator }\end{array}$ & $\begin{array}{l}\text { Spatial exposure to } \\
\text { indicator (low, med, } \\
\text { high) }\end{array}$ & $\begin{array}{l}\text { Temporal } \\
\text { exposure to } \\
\text { indicator (low, } \\
\text { med, high) } \\
\end{array}$ & $\begin{array}{l}\text { Effect's overlap } \\
\text { with critical life } \\
\text { stage, behavior, } \\
\text { habitat or resource } \\
\text { (low, med, high) } \\
\end{array}$ & $\begin{array}{l}\text { Overall risk } \\
\text { to indicator } \\
\text { (low, med, } \\
\text { high, } \\
\text { unknown) } \\
\end{array}$ & Source(s) \\
\hline & $\begin{array}{l}\text { Noise and } \\
\text { vibration }\end{array}$ & $\begin{array}{l}\text { Possible movement away } \\
\text { from area and disruption of } \\
\text { foraging or nesting }\end{array}$ & Low, see above & $\begin{array}{l}\text { Low, occurs over } \\
\text { very short time } \\
\text { period }\end{array}$ & High, see above & Med & $\begin{array}{l}\text { Michel et al. } \\
2007\end{array}$ \\
\hline \multicolumn{8}{|c|}{ Operation and Maintenance } \\
\hline \multirow[t]{2}{*}{ Boat traffic } & $\begin{array}{l}\text { Oil/chemical } \\
\text { release }\end{array}$ & $\begin{array}{l}\text { Hypothermia, toxicity to } \\
\text { individuals and prey }\end{array}$ & $\begin{array}{l}\text { Low for typical small } \\
\text { volume releases, High for } \\
\text { large, catastrophic spill }\end{array}$ & $\begin{array}{l}\text { Low, wouldn't } \\
\text { persist long-term }\end{array}$ & $\begin{array}{l}\text { Med, boats will pass } \\
\text { through murrelet } \\
\text { foraging areas }\end{array}$ & Low & $\begin{array}{l}\text { Hébert and } \\
\text { Golightly } \\
\text { 2006, } \\
\text { Michel et al. } \\
\text { 2007, Ralph } \\
\text { and Miller } \\
1995\end{array}$ \\
\hline & $\begin{array}{l}\text { Noise and } \\
\text { vibration }\end{array}$ & $\begin{array}{l}\text { Possible movement away } \\
\text { from boat and disruption of } \\
\text { foraging }\end{array}$ & $\begin{array}{l}\text { Low, would occur in } \\
\text { small area relative to } \\
\text { species' range }\end{array}$ & $\begin{array}{l}\text { Low, only from } \\
\text { periodic trips in } \\
\text { summer }\end{array}$ & Med, see above & Low & $\begin{array}{l}\text { LGL } \\
\text { Limited et } \\
\text { al. } 2009\end{array}$ \\
\hline \multirow[b]{2}{*}{$\begin{array}{l}\text { Operation of turbines } \\
\text { or other moving parts } \\
\text { of devices }\end{array}$} & $\begin{array}{l}\text { Moving device } \\
\text { parts }\end{array}$ & $\begin{array}{l}\text { None, no underwater } \\
\text { moving parts }\end{array}$ & N/A & N/A & N/A & Low & N/A \\
\hline & $\begin{array}{l}\text { Noise and } \\
\text { vibration }\end{array}$ & $\begin{array}{l}\text { Possible movement away } \\
\text { from area and disruption of } \\
\text { foraging or nesting }\end{array}$ & $\begin{array}{l}\text { Low, would occur in } \\
\text { small area relative to } \\
\text { species' range }\end{array}$ & $\begin{array}{l}\text { High, continuous } \\
\text { for life of project }\end{array}$ & $\begin{array}{l}\text { Low, } 90 \% \text { of } \\
\text { murrelet locations } \\
\text { closer to shore than } \\
\text { project site }\end{array}$ & Low & $\begin{array}{l}\text { Hébert and } \\
\text { Golightly } \\
\text { 2006, } \\
\text { Michel et al. } \\
\text { 2007, Ralph } \\
\text { and Miller } \\
1995\end{array}$ \\
\hline $\begin{array}{l}\text { Structures in water } \\
\text { column and on } \\
\text { seabed, such as } \\
\text { devices and } \\
\text { moorings and } \\
\text { footings }\end{array}$ & Structure & $\begin{array}{l}\text { Possible attraction to } \\
\text { increased prey (reef effect), } \\
\text { entanglement with derelict } \\
\text { fishing gear/ moorings, } \\
\text { collisions with structures } \\
\text { while diving }\end{array}$ & $\begin{array}{l}\text { Low, would occur in } \\
\text { small area relative to } \\
\text { species' range }\end{array}$ & $\begin{array}{l}\text { Med, tangled gear } \\
\text { would be } \\
\text { removed } \\
\text { periodically }\end{array}$ & Low, see above & Low & $\begin{array}{l}\text { Michel et al. } \\
2007\end{array}$ \\
\hline
\end{tabular}

\begin{tabular}{lr}
\hline Environmental Assessment for Siting Wave \& Tidal & D-86 \\
Energy Projects &
\end{tabular}


Appendix D - Effects of Small Commercial OPT Power Buoy Project Humboldt

\begin{tabular}{|c|c|c|c|c|c|c|c|}
\hline Project activity & Action & $\begin{array}{l}\text { Description of action's } \\
\text { effect on indicator }\end{array}$ & $\begin{array}{l}\text { Spatial exposure to } \\
\text { indicator (low, med, } \\
\text { high) }\end{array}$ & $\begin{array}{l}\text { Temporal } \\
\text { exposure to } \\
\text { indicator (low, } \\
\text { med, high) }\end{array}$ & $\begin{array}{l}\text { Effect's overlap } \\
\text { with critical life } \\
\text { stage, behavior, } \\
\text { habitat or resource } \\
\text { (low, med, high) }\end{array}$ & $\begin{array}{l}\text { Overall risk } \\
\text { to indicator } \\
\text { (low, med, } \\
\text { high, } \\
\text { unknown) } \\
\end{array}$ & Source(s) \\
\hline & $\begin{array}{l}\text { Water } \\
\text { circulation } \\
\text { changes }\end{array}$ & $\begin{array}{l}\text { None, project not large } \\
\text { enough to affect prey }\end{array}$ & N/A & N/A & N/A & Low & N/A \\
\hline & $\begin{array}{l}\text { Oil/chemical } \\
\text { release }\end{array}$ & $\begin{array}{l}\text { Hypothermia, toxicity to } \\
\text { individuals and prey }\end{array}$ & $\begin{array}{l}\text { Low for typical small } \\
\text { volume releases, High for } \\
\text { large, catastrophic release } \\
\text { from device failure }\end{array}$ & $\begin{array}{l}\text { Med, continuous } \\
\text { and intermittent } \\
\text { for life of project }\end{array}$ & $\begin{array}{l}\text { Low, } 90 \% \text { of } \\
\text { murrelet locations } \\
\text { closer to shore than } \\
\text { project site }\end{array}$ & Low & $\begin{array}{l}\text { Hébert and } \\
\text { Golightly } \\
\text { 2006, } \\
\text { Michel et al. } \\
\text { 2007, Ralph } \\
\text { and Miller } \\
\text { 1995 }\end{array}$ \\
\hline \multirow{2}{*}{$\begin{array}{l}\text { Structures on water's } \\
\text { surface }\end{array}$} & $\begin{array}{l}\text { Navigation } \\
\text { lights }\end{array}$ & $\begin{array}{l}\text { Attraction/ disorientation, } \\
\text { collisions }\end{array}$ & $\begin{array}{l}\text { Low, would occur in } \\
\text { small area relative to } \\
\text { species' range }\end{array}$ & $\begin{array}{l}\text { High, continuous } \\
\text { for life of project }\end{array}$ & Low, see above & Low & $\begin{array}{l}\text { Montevecchi } \\
2006\end{array}$ \\
\hline & Structure & $\begin{array}{l}\text { Possible collision while } \\
\text { flying, attraction to } \\
\text { increased prey (FAD effect) }\end{array}$ & $\begin{array}{l}\text { Low, would occur in } \\
\text { small area relative to } \\
\text { species’ range }\end{array}$ & $\begin{array}{l}\text { High, continuous } \\
\text { for life of project }\end{array}$ & Low, see above & Low & $\begin{array}{l}\text { Boehlert et } \\
\text { al. } 2008\end{array}$ \\
\hline \multicolumn{8}{|c|}{ Decommissioning } \\
\hline \multirow[t]{2}{*}{ Boat traffic } & $\begin{array}{l}\text { Oil/chemical } \\
\text { release }\end{array}$ & $\begin{array}{l}\text { Hypothermia, toxicity to } \\
\text { individuals and prey }\end{array}$ & $\begin{array}{l}\text { Low for typical small } \\
\text { volume releases, High for } \\
\text { large, catastrophic spill }\end{array}$ & $\begin{array}{l}\text { Low, occurs over } \\
1 \text { to } 2 \text { summers }\end{array}$ & $\begin{array}{l}\text { Med, boats will pass } \\
\text { through murrelet at- } \\
\text { sea areas }\end{array}$ & Low & $\begin{array}{l}\text { Hébert and } \\
\text { Golightly } \\
\text { 2006, } \\
\text { Michel et al. } \\
\text { 2007, Ralph } \\
\text { and Miller } \\
1995\end{array}$ \\
\hline & $\begin{array}{l}\text { Noise and } \\
\text { vibration }\end{array}$ & $\begin{array}{l}\text { Possible movement away } \\
\text { from boat and disruption of } \\
\text { foraging }\end{array}$ & $\begin{array}{l}\text { Low, would occur in } \\
\text { small area relative to } \\
\text { species' range }\end{array}$ & $\begin{array}{l}\text { Low, occurs over } \\
1 \text { to } 2 \text { summers }\end{array}$ & Med, see above & Low & $\begin{array}{l}\text { LGL } \\
\text { Limited et } \\
\text { al. } 2009\end{array}$ \\
\hline $\begin{array}{l}\text { Decommissioning of } \\
\text { structures on water's } \\
\text { surface or seabed }\end{array}$ & $\begin{array}{l}\text { Noise and } \\
\text { vibration }\end{array}$ & $\begin{array}{l}\text { Possible movement away } \\
\text { from area and disruption of } \\
\text { foraging or nesting }\end{array}$ & Low, see above & $\begin{array}{l}\text { Low, occurs over } \\
1 \text { to } 2 \text { summers }\end{array}$ & $\begin{array}{l}\text { Low, } 90 \% \text { of } \\
\text { murrelet locations } \\
\text { closer to shore than } \\
\text { project site }\end{array}$ & Low & $\begin{array}{l}\text { Hébert and } \\
\text { Golightly } \\
\text { 2006, Ralph } \\
\text { and Miller } \\
1995\end{array}$ \\
\hline
\end{tabular}

\begin{tabular}{|c|c|c|}
\hline $\begin{array}{l}\text { Environmental Assessment for Siting Wave \& Tidal } \\
\text { Energy Projects }\end{array}$ & D-87 & $\begin{array}{r}\text { H. T. Harvey \& Associates } \\
24 \text { November } 2009\end{array}$ \\
\hline
\end{tabular}


Appendix D - Effects of Small Commercial OPT Power Buoy Project Humboldt

\begin{tabular}{|c|c|c|c|c|c|c|c|}
\hline Project activity & Action & $\begin{array}{l}\text { Description of action's } \\
\text { effect on indicator }\end{array}$ & $\begin{array}{l}\text { Spatial exposure to } \\
\text { indicator (low, med, } \\
\text { high) }\end{array}$ & $\begin{array}{l}\text { Temporal } \\
\text { exposure to } \\
\text { indicator (low, } \\
\text { med, high) }\end{array}$ & $\begin{array}{l}\text { Effect's overlap } \\
\text { with critical life } \\
\text { stage, behavior, } \\
\text { habitat or resource } \\
\text { (low, med, high) }\end{array}$ & $\begin{array}{l}\text { Overall risk } \\
\text { to indicator } \\
\text { (low, med, } \\
\text { high, } \\
\text { unknown) } \\
\end{array}$ & Source(s) \\
\hline & $\begin{array}{l}\text { Oil/chemical } \\
\text { release }\end{array}$ & $\begin{array}{l}\text { Hypothermia, toxicity to } \\
\text { individuals and prey }\end{array}$ & $\begin{array}{l}\text { Low, volume released } \\
\text { would occupy small area }\end{array}$ & $\begin{array}{l}\text { Low, occurs over } \\
1 \text { to } 2 \text { summers }\end{array}$ & Low, see above & Low & $\begin{array}{l}\text { Michel et al. } \\
2007\end{array}$ \\
\hline & $\begin{array}{l}\text { Construction } \\
\text { lights }\end{array}$ & $\begin{array}{l}\text { Attraction/ disorientation, } \\
\text { collisions }\end{array}$ & $\begin{array}{l}\text { Low, would occur in } \\
\text { small area relative to } \\
\text { species' range }\end{array}$ & $\begin{array}{l}\text { Low, occurs over } \\
1 \text { to } 2 \text { summers }\end{array}$ & Low, see above & Low & $\begin{array}{l}\text { Montevecchi } \\
2006\end{array}$ \\
\hline & $\begin{array}{l}\text { Seabed } \\
\text { disturbance }\end{array}$ & $\begin{array}{l}\text { Possible short-term changes } \\
\text { in food resources }\end{array}$ & Low, see above & $\begin{array}{l}\text { Low, occurs over } \\
1 \text { to } 2 \text { summers }\end{array}$ & Low, see above & Low & $\begin{array}{l}\text { Boehlert et } \\
\text { al. } 2008\end{array}$ \\
\hline
\end{tabular}


Appendix D - Effects of Small Commercial OPT Power Buoy Project Humboldt

Potential effects on Dungeness crab due to Small Commercial OPT Power Buoy Project at Humboldt site

\begin{tabular}{|c|c|c|c|c|c|c|c|}
\hline Project activity & Action & $\begin{array}{l}\text { Description of } \\
\text { action's effect on } \\
\text { indicator }\end{array}$ & $\begin{array}{l}\text { Spatial exposure to } \\
\text { indicator (low, med, } \\
\text { high) }\end{array}$ & $\begin{array}{l}\text { Temporal exposure } \\
\text { to indicator (low, } \\
\text { med, high) }\end{array}$ & $\begin{array}{l}\text { Effect's overlap } \\
\text { with critical life } \\
\text { stage, behavior, } \\
\text { habitat or resource } \\
\text { (low, med, high) } \\
\end{array}$ & $\begin{array}{l}\text { Overall risk } \\
\text { to indicator } \\
\text { (low, med, } \\
\text { high, } \\
\text { unknown) } \\
\end{array}$ & Source(s) \\
\hline \multicolumn{8}{|c|}{ Construction } \\
\hline Boat traffic & $\begin{array}{l}\text { Oil/chemical } \\
\text { release }\end{array}$ & $\begin{array}{l}\text { Toxicity to } \\
\text { individuals and prey }\end{array}$ & $\begin{array}{l}\text { Low for typical small } \\
\text { volume releases, High for } \\
\text { large, catastrophic spill }\end{array}$ & $\begin{array}{l}\text { Low, occurs over } 1 \text { to } \\
2 \text { summers }\end{array}$ & $\begin{array}{l}\text { Low for adults on } \\
\text { bottom. High for } \\
\text { megalopae on surface } \\
\text { at night, since oil } \\
\text { floats on surface }\end{array}$ & Low & $\begin{array}{l}\text { Nelson and } \\
\text { Woo } 2008\end{array}$ \\
\hline \multirow{3}{*}{$\begin{array}{l}\text { Construction of } \\
\text { electrical } \\
\text { collector system, } \\
\text { moorings and } \\
\text { foundations, and } \\
\text { device } \\
\text { installation }\end{array}$} & $\begin{array}{l}\text { Oil/chemical } \\
\text { release }\end{array}$ & $\begin{array}{l}\text { Toxicity to } \\
\text { individuals and prey }\end{array}$ & $\begin{array}{l}\text { Low, volume released } \\
\text { would occupy small area } \\
\text { relative to species' range }\end{array}$ & $\begin{array}{l}\text { Low, occurs over } 1 \text { to } \\
2 \text { summers }\end{array}$ & $\begin{array}{l}\text { Low for adults, High } \\
\text { for megalopae (see } \\
\text { above) }\end{array}$ & Low & $\begin{array}{l}\text { Emmett et al. } \\
\text { 1991, Nelson } \\
\text { and Woo } 2008\end{array}$ \\
\hline & $\begin{array}{l}\text { Seabed } \\
\text { disturbance }\end{array}$ & $\begin{array}{l}\text { Temporary habitat } \\
\text { loss, possible changes } \\
\text { to benthic prey } \\
\text { resources }\end{array}$ & $\begin{array}{l}\text { Low, would occur in } \\
\text { small area relative to } \\
\text { species' range }\end{array}$ & $\begin{array}{l}\text { Low, occurs over } 1 \text { to } \\
2 \text { summers }\end{array}$ & $\begin{array}{l}\text { Med, crabs occur on } \\
\text { bottom in project area }\end{array}$ & Low & Nelson 2008 \\
\hline & $\begin{array}{l}\text { Construction } \\
\text { lights }\end{array}$ & $\begin{array}{l}\text { Adult crabs not } \\
\text { known to be affected } \\
\text { by lights; megalopae } \\
\text { attracted to light }\end{array}$ & Low, see above & $\begin{array}{l}\text { Low, occurs over } 1 \text { to } \\
2 \text { summers }\end{array}$ & $\begin{array}{l}\text { Low for adults on } \\
\text { bottom. High for } \\
\text { megalopae on surface } \\
\text { at night. }\end{array}$ & Low & $\begin{array}{l}\text { Miller and } \\
\text { Shanks 2005, } \\
\text { Moore et al. } \\
\text { 2006, Porter et } \\
\text { al. 2008 } \\
\end{array}$ \\
\hline $\begin{array}{l}\text { Directional } \\
\text { drilling, and } \\
\text { laying cable } \\
\text { under/on seabed }\end{array}$ & $\begin{array}{l}\text { Seabed } \\
\text { disturbance }\end{array}$ & $\begin{array}{l}\text { Temporary habitat } \\
\text { loss, possible changes } \\
\text { to benthic prey } \\
\text { resources }\end{array}$ & Low, see above & $\begin{array}{l}\text { Low, occurs over a } \\
\text { short time period }\end{array}$ & $\begin{array}{l}\text { Med, crabs occur on } \\
\text { bottom in project area }\end{array}$ & Low & Nelson 2008 \\
\hline \multicolumn{8}{|c|}{ Operation and Maintenance } \\
\hline Boat traffic & $\begin{array}{l}\text { Oil/chemical } \\
\text { release }\end{array}$ & $\begin{array}{l}\text { Toxicity to } \\
\text { individuals and prey }\end{array}$ & $\begin{array}{l}\text { Low for typical small } \\
\text { volume releases, High for } \\
\text { large, catastrophic spill }\end{array}$ & $\begin{array}{l}\text { Low, wouldn't persist } \\
\text { long-term }\end{array}$ & $\begin{array}{l}\text { Low for adults on } \\
\text { bottom. High for } \\
\text { megalopae on surface } \\
\text { at night, since oil } \\
\text { floats on surface }\end{array}$ & Low & $\begin{array}{l}\text { Nelson and } \\
\text { Woo } 2008\end{array}$ \\
\hline $\begin{array}{l}\text { Operation of } \\
\text { turbines or other } \\
\text { moving parts of } \\
\text { devices }\end{array}$ & $\begin{array}{l}\text { None expected } \\
\text { that would affect } \\
\text { crabs }\end{array}$ & N/A & N/A & N/A & N/A & Low & N/A \\
\hline
\end{tabular}

\begin{tabular}{lr}
\hline Environmental Assessment for Siting Wave \& Tidal & D-89 \\
Energy Projects & H. T. Harvey \& Associates \\
24 November 2009
\end{tabular}


Appendix D - Effects of Small Commercial OPT Power Buoy Project Humboldt

\begin{tabular}{|c|c|c|c|c|c|c|c|}
\hline Project activity & Action & $\begin{array}{l}\text { Description of } \\
\text { action's effect on } \\
\text { indicator }\end{array}$ & $\begin{array}{l}\text { Spatial exposure to } \\
\text { indicator (low, med, } \\
\text { high) }\end{array}$ & $\begin{array}{l}\text { Temporal exposure } \\
\text { to indicator (low, } \\
\text { med, high) }\end{array}$ & $\begin{array}{l}\text { Effect's overlap } \\
\text { with critical life } \\
\text { stage, behavior, } \\
\text { habitat or resource } \\
\text { (low, med, high) }\end{array}$ & $\begin{array}{l}\text { Overall risk } \\
\text { to indicator } \\
\text { (low, med, } \\
\text { high, } \\
\text { unknown) }\end{array}$ & Source(s) \\
\hline \multirow{3}{*}{$\begin{array}{l}\text { Structures in } \\
\text { water column and } \\
\text { on seabed, such } \\
\text { as devices and } \\
\text { moorings and } \\
\text { footings }\end{array}$} & Structure & $\begin{array}{l}\text { Possible changes in } \\
\text { predator/prey } \\
\text { abundance, } \\
\text { interactions (reef } \\
\text { effect) }\end{array}$ & $\begin{array}{l}\text { Low, would occur in } \\
\text { small area relative to } \\
\text { species' range }\end{array}$ & $\begin{array}{l}\text { High, would be } \\
\text { continuous for life of } \\
\text { project }\end{array}$ & $\begin{array}{l}\text { Med for adult crabs } \\
\text { on bottom in project } \\
\text { area, Low for } \\
\text { megalopae on surface }\end{array}$ & Unknown & $\begin{array}{l}\text { Boehlert et al. } \\
2008\end{array}$ \\
\hline & $\begin{array}{l}\text { Water circulation } \\
\text { changes }\end{array}$ & $\begin{array}{l}\text { None, project not } \\
\text { large enough to affect } \\
\text { prey }\end{array}$ & N/A & N/A & N/A & Low & N/A \\
\hline & $\begin{array}{l}\text { Oil/chemical } \\
\text { release }\end{array}$ & $\begin{array}{l}\text { Toxicity to } \\
\text { individuals and prey }\end{array}$ & $\begin{array}{l}\text { Low, would occur in } \\
\text { small area relative to } \\
\text { species' range }\end{array}$ & $\begin{array}{l}\text { Low, wouldn't persist } \\
\text { long-term }\end{array}$ & $\begin{array}{l}\text { Low for adults on } \\
\text { bottom. High for } \\
\text { megalopae on surface } \\
\text { at night, since oil } \\
\text { floats on surface }\end{array}$ & Low & $\begin{array}{l}\text { Nelson and } \\
\text { Woo } 2008\end{array}$ \\
\hline $\begin{array}{l}\text { Electricity } \\
\text { conduction } \\
\text { through cable }\end{array}$ & EMF & $\begin{array}{l}\text { Unknown, could } \\
\text { include behavior, } \\
\text { orientation changes }\end{array}$ & Low, see above & $\begin{array}{l}\text { High, would be } \\
\text { continuous for life of } \\
\text { project }\end{array}$ & $\begin{array}{l}\text { Med, crabs occur on } \\
\text { bottom in project area }\end{array}$ & Unknown & Nelson 2008 \\
\hline \multirow{2}{*}{$\begin{array}{l}\text { Structures on } \\
\text { water's surface }\end{array}$} & Structure & $\begin{array}{l}\text { Possible changes in } \\
\text { predator/prey } \\
\text { abundance, } \\
\text { interactions (FAD } \\
\text { effect) }\end{array}$ & Low, see above & $\begin{array}{l}\text { High, would be } \\
\text { continuous for life of } \\
\text { project }\end{array}$ & Med, see above & Unknown & Nelson 2008 \\
\hline & Navigation lights & $\begin{array}{l}\text { Adult crabs not } \\
\text { known to be affected } \\
\text { by lights; megalopae } \\
\text { attracted to light }\end{array}$ & Low, see above & $\begin{array}{l}\text { High, would be } \\
\text { continuous for life of } \\
\text { project }\end{array}$ & $\begin{array}{l}\text { Low for adults on } \\
\text { bottom. High for } \\
\text { megalopae on surface } \\
\text { at night. }\end{array}$ & Med & $\begin{array}{l}\text { Miller and } \\
\text { Shanks 2005, } \\
\text { Moore et al. } \\
\text { 2006, Porter et } \\
\text { al. } 2008\end{array}$ \\
\hline \multicolumn{8}{|c|}{ Decommissioning } \\
\hline Boat traffic & $\begin{array}{l}\text { Oil/chemical } \\
\text { release }\end{array}$ & $\begin{array}{l}\text { Toxicity to } \\
\text { individuals and prey }\end{array}$ & $\begin{array}{l}\text { Low for typical small } \\
\text { volume releases, High for } \\
\text { large, catastrophic spill }\end{array}$ & $\begin{array}{l}\text { Low, occurs over } 1 \text { to } \\
2 \text { summers }\end{array}$ & $\begin{array}{l}\text { Low for adults on } \\
\text { bottom. High for } \\
\text { megalopae on surface } \\
\text { at night, since oil } \\
\text { floats on surface }\end{array}$ & Low & $\begin{array}{l}\text { Nelson and } \\
\text { Woo } 2008\end{array}$ \\
\hline $\begin{array}{l}\text { Decommissioning } \\
\text { of structures on } \\
\text { water's surface or }\end{array}$ & $\begin{array}{l}\text { Oil/chemical } \\
\text { release }\end{array}$ & $\begin{array}{l}\text { Toxicity to } \\
\text { individuals and prey }\end{array}$ & $\begin{array}{l}\text { Low, volume released } \\
\text { would occupy small area }\end{array}$ & $\begin{array}{l}\text { Low, occurs over } 1 \text { to } \\
2 \text { summers }\end{array}$ & $\begin{array}{l}\text { Low for adults, High } \\
\text { for megalopae (see } \\
\text { above) }\end{array}$ & Low & $\begin{array}{l}\text { Nelson and } \\
\text { Woo } 2008\end{array}$ \\
\hline
\end{tabular}

\begin{tabular}{lr}
\hline Environmental Assessment for Siting Wave \& Tidal & D-90 \\
Energy Projects &
\end{tabular}


Appendix D - Effects of Small Commercial OPT Power Buoy Project Humboldt

\begin{tabular}{|c|c|c|c|c|c|c|c|}
\hline Project activity & Action & $\begin{array}{l}\text { Description of } \\
\text { action's effect on } \\
\text { indicator }\end{array}$ & $\begin{array}{l}\text { Spatial exposure to } \\
\text { indicator (low, med, } \\
\text { high) }\end{array}$ & $\begin{array}{l}\text { Temporal exposure } \\
\text { to indicator (low, } \\
\text { med, high) }\end{array}$ & $\begin{array}{l}\text { Effect's overlap } \\
\text { with critical life } \\
\text { stage, behavior, } \\
\text { habitat or resource } \\
\text { (low, med, high) }\end{array}$ & $\begin{array}{l}\text { Overall risk } \\
\text { to indicator } \\
\text { (low, med, } \\
\text { high, } \\
\text { unknown) }\end{array}$ & Source(s) \\
\hline \multirow[t]{2}{*}{ seabed } & $\begin{array}{l}\text { Seabed } \\
\text { disturbance }\end{array}$ & $\begin{array}{l}\text { Temporary habitat } \\
\text { loss, changes in prey } \\
\text { resources }\end{array}$ & $\begin{array}{l}\text { Low, would occur in } \\
\text { small area relative to } \\
\text { species' range }\end{array}$ & $\begin{array}{l}\text { Low, occurs over } 1 \text { to } \\
2 \text { summers }\end{array}$ & $\begin{array}{l}\text { Med, crabs occur on } \\
\text { bottom in project area }\end{array}$ & Low & Nelson 2008 \\
\hline & $\begin{array}{l}\text { Deconstruction } \\
\text { lights }\end{array}$ & $\begin{array}{l}\text { Adult crabs not } \\
\text { known to be affected } \\
\text { by lights; megalopae } \\
\text { attracted to light }\end{array}$ & Low, see above & $\begin{array}{l}\text { Low, occurs over } 1 \text { to } \\
2 \text { summers }\end{array}$ & $\begin{array}{l}\text { Low for adults on } \\
\text { bottom. High for } \\
\text { megalopae on surface } \\
\text { at night. }\end{array}$ & Low & $\begin{array}{l}\text { Miller and } \\
\text { Shanks 2005, } \\
\text { Moore et al. } \\
\text { 2006, Porter et } \\
\text { al. } 2008\end{array}$ \\
\hline
\end{tabular}

\begin{tabular}{lr}
\hline Environmental Assessment for Siting Wave \& Tidal & D-91 \\
Energy Projects & H. T. Harvey \& Associates \\
24 November 2009
\end{tabular}


Appendix D - Effects of Small Commercial OPT Power Buoy Project Humboldt

\section{References}

Adams PB, Churchill BG, Lindley ST, Moser ML. 2002. Status review for North American green sturgeon, Acipenser medirostris. National Marine Fisheries Service.

Allen LG, Cross JN. 2006. Surface waters. In: Allen LG, Pondella II DJ, Horn MH, editors. The ecology of marine fishes, California and adjacent waters. Berkeley and Los Angeles, CA: University of California Press; p. 320-341.

Allen LG, Yoklavich MM, Cailliet GM, Horn MH. 2006. Surf zone, coastal pelagic zone, and harbors. In: Allen LG, Pondella II DJ, Horn MH, editors. The ecology of marine fishes, California and adjacent waters. Berkeley and Los Angeles, CA: University of California Press; p. 149-166.

Allen MJ. 2006. Continental shelf and upper slope. In: Allen LG, Pondella II DJ, Horn MH, editors. The ecology of marine fishes, California and adjacent waters. Berkeley and Los Angeles, CA: University of California Press; p. 167-202.

Allen MJ, Leos R. 2001. Sanddabs. In: Leet WS, Dewees CM, Klingbeil R, Larson EJ, editors. California's living marine resources: A status report. Sacramento, CA: California Department of Fish and Game; p. 201-202.

Angell T. 1982. Marine birds and mammals of Puget Sound. Seattle, WA: University of Washington Press.

Angliss RP, Allen BM. 2009. Alaska marine mammal stock assessments, 2008. Seattle, WA: U.S. Department of Commerce. NOAA Technical Memorandum NMFS-AFSC-193.

Austin M, Delarue J, Johnston HA, Laurinolli M, Leary D, MacGillivray A, O'Neill C, Sneddon H, Warner G. 2009. NaiKun Offshore Wind Energy Project environmental assessment, Volume 4 - Noise and vibration. NaiKun Wind Development Inc. and JASCO Applied Sciences.

Blackwell SB, Lawson JW, Williams MT. 2004. Tolerance by ringed seals (Phoca hispida) to impact pipe-driving and construction sounds at an oil production island. Journal of the Acoustical Society of America. 115(5):2346-2357.

Bodznick D, Montgomery J, Tricas TC. 2003. Electroreception: Extracting behaviorally important signals from noise. In: Collin SP, Marshall NJ, editors. Sensory processing in aquatic environments. New York, NY: Springer-Verlag; p. 389-403.

Boehlert GW, McMurray GR, Tortorici CE. 2008. Ecological effects of wave energy development in the Pacific Northwest. National Oceanic and Atmospheric Administration. NMFS-F/SPO-92.

Brodeur RD, Emmett RL, Fisher JP, Casillas E, Teel DJ, Miller TW. 2004. Juvenile salmonids distribution, growth, condition, origin, and environmental and species associations in the northern California Current. Fishery Bulletin. 102:25-46. 
Appendix D - Effects of Small Commercial OPT Power Buoy Project Humboldt

Brookens TJ, Harvey JT, O'Hara TM. 2007. Trace element concentrations in the Pacific harbor seal (Phoca vitulina richardii) in central and northern California. Science of the Total Environment. 372:676-692.

Burger J, Gochfeld M. 2002. Bonaparte's gull (Larus philadlphia) [Internet]. Ithaca, NY: Cornell Lab of Ornithology; [cited 21 October 2009]. Available from: http://bna.birds.cornell.edu/bna/species/634/articles/introduction.

Cada GF. 2008. The potential environmental impacts of marine and hydrokinetic renewable energy technologies. Washington, DC: Wind and Hydropower Technologies Program, U.S. Department of Energy. ER08-1040.

Calambokidis J, Darling JD, Deecke V, Gearin PJ, Gosho ME, Megill W, Tombach CM, Goley D, Toropova C, Gisborne B. 2002. Abundance, range and movements of a feeding aggregation of gray whales (Eschrichtius robustus) from California to southeastern Alaska in 1998. Journal of Cetacean Research and Management. 4(3):267-276.

Carretta JV, Forney KA, Lowry MS, Barlow J, Baker J, Johnston D, Hanson B, Muto MM, Lynch D, Carswell L. 2009. U.S. Pacific marine mammal stock assessments: 2008. U.S. Department of Commerce. NOAA-TM-NMFS-SWFSC-434.

(CDFG) California Department of Fish and Game. 2008. Review of some California fisheries for 2007: Coastal pelagic finfish, market squid, Dungeness crab, California spiny lobster, highly migratory species, ocean salmon, groundfish, California halibut, hagfish, Pacific herring, and recreational. California Cooperative Oceanic Fisheries Investigations Reports. 49:15-38.

Colwell MA, Jehl Jr. JR. 1994. Wilson's phalarope (Phalaropus tricolor) [Internet]. Ithaca, NY: Cornell Lab of Ornithology; [cited 20 October 2009]. Available from: http://bna.birds.cornell.edu/bna/species/083/articles/introduction.

Cox TM, Read AJ. 2004. Echolocation behavior of harbor porpoises Phocoena phocoena around chemically enhanced gill nets. Marine Ecology Progess Series. 279:275-282.

(CPUC) California Public Utilities Commission. 2009. Sacramento natural gas storage project EIR [Internet]. [cited 14 September 2009]. Available from:

http://www.cpuc.ca.gov/environment/info/dudek/sngs/Appendices/Section\%20D9\%20Noise\%20 and\%20Vibration.pdf.

(CSLC) California State Lands Commission. 2008. Draft Environmental Impact Report for the AT\&T Asia America gateway fiber optic cable project. State Clearinghouse No. 2007111029.

Cuthbert FJ, Wires LR. 1999. Caspian tern (Sterna caspia) [Internet]. Ithaca, NY: Cornell Lab of Ornithology; [cited 21 October 2009]. Available from: http://bna.birds.cornell.edu/bna/species/403/articles/introduction.

Dehnhardt G, Mauck B, Bleckmann H. 1998. Seal whiskers detect water movements. Nature. 394:235-236. 
Ebert D. 2001. Soupfin shark. In: Leet WS, Dewees CM, Klingbeil R, Larson EJ, editors. California's living marine resources: A status report. Sacramento, CA: California Department of Fish and Game; p. 255-256.

Emmett RL, Hinton SA, Stone SL, Monaco ME. 1991. Distribution and abundance of fishes and invertebrates in west coast estuaries, Volume II: Species life history summaries. Silver Spring, MD: NOAA/NOS Strategic Environmental Assessments Division. ELMR Report No. 8.

Erbe C, Farmer DM. 2000. Zones of impact around icebreakers affecting beluga whales in the Beaufort Sea. Journal of the Acoustical Society of America. 108(3):1332-1340.

Erickson DL, Hightower JE. 2007. Oceanic distribution and behavior of green sturgeon (Acipenser medirostris). In: Munro J, Hatin D, Hightower JE, McKown K, Sulak KJ, Kahnle AW, Caron F, editors. Anadromous sturgeons: habitats, threats, and management. Bethesda, MD: American Fisheries Society Symposium; p. 197-211.

Fritzsche R, Cavanagh WJ. 1995. A guide to the fishes of Humboldt Bay. Arcata, CA: Humboldt State University.

Goodwin L. 2008. Diurnal and tidal variations in habitat use of the harbour porpoise (Phocoena phocoena) in southwest Britain. Aquatic Mammals. 34(1):44-53.

Harris SW. 2006. Northwestern California Birds. 3rd, 2nd Printing. Klamath River, CA: Living Gold Press.

Hatch SA, Robertson GJ, Baird PH. 2009. Black-legged kittiwake (Rissa tridactyla) [Internet]. Ithaca, NY: Cornell Lab of Ornithology; [cited 21 October 2009]. Available from: http://bna.birds.cornell.edu/bna/species/092/articles/introduction.

Hayward JL, Verbeek NA. 2008. Glaucous-winged gull (Larus glaucescens) [Internet]. Ithaca, NY: Cornell Lab of Ornithology; [cited 21 October 2009]. Available from: http://bna.birds.cornell.edu/bna/species/059/articles/introduction.

(HBHRCD) Humboldt Bay Harbor Recreation and Conservation District. 2006. Humboldt Bay management plan - Draft environmental impact report. Eureka, CA: Humboldt Bay Harbor, Recreation and Conservation District. SCH \# 2005082040.

Hébert PN, Golightly RT. 2006. Movements, nesting, and response to anthropogenic disturbance of Marbled Murrelets (Brachyramphus marmoratus) in Redwood National and State Parks, California. Arcata, CA and Sacramento, CA: Department of Wildlife, Humboldt State University and California Department of Fish and Game. Report 2006-02.

Herder MJ. 1986. Seasonal movements and hauling site fidelity of harbor seals, Phoca vitulina richardsi, tagged at the Klamath River, California [master's thesis]. [Arcata, CA]: Humboldt State University.

Horn MH, Stephens Jr. JS. 2006. Climate change and overexploitation. In: Allen LG, Pondella II DJ, Horn MH, editors. The ecology of marine fishes, California and adjacent waters. Berkeley and Los Angeles, CA: University of California Press; p. 621-635. 
Appendix D - Effects of Small Commercial OPT Power Buoy Project Humboldt

(IALA) International Association of Marine Aids to Navigation and Lighthouse Authorities. 2008. IALA recommendation O-139 on the marking of man-made offshore structures. Saint Germain en Laye, France: IALA-AISM.

Kastak D, Southall BL, Schusterman RJ, Reichmuth-Kastak CJ. 2005. Underwater temporary threshold shift in pinnipeds: Effects of noise level and duration. Journal of the Acoustical Society of America. 118(5):3154-3163.

Kastelein RA, Wensveen PJ, Hoek L, Terhune JM. 2009. Underwater hearing sensitivity of harbor seals (Phoca vitulina) of narrow noise bands between 0.2 and $80 \mathrm{kHz}$. Journal of the Acoustical Society of America. 1:476-483.

Klimley AP, Le Boeuf BJ, Cantara KM, Richert JE, Davis SF, Van Sommeran S, Kelly JT. 2001. The hunting strategy of white sharks (Carcharodon carcharias) near a seal colony. Marine Biology. 138(3):617-636.

Koschinski S, Culik BM, Henriksen OD, Tregenza NC, Ellis GM, Jansen C, Kathe G. 2003. Behavioral reactions of free-ranging porpoises and seals to the noise of a simulated $2 \mathrm{MW}$ windpower generator. Marine Ecology Progess Series. 265:263-273.

Largier J, Behrens D, Robart M. 2008. The potential impact of WEC development on nearshore and shoreline environments through a reduction in nearshore wave energy. In: Nelson PA, editors. Developing wave energy in coastal California: Potential socio-economic and environmental effects. Sacramento, CA: California Energy Commission, PIER Energy-Related Environmental Research Program \& California Ocean Protection Council; p. 57-82.

Le Boeuf BJ, Giesy JP, Kannan K, Kajiwara N, Tanabe S, Debier C. 2002. Organochloride pesticides in California sea lions revisited. [Internet]. BioMed Central Ecology. 2(11):[cited 3 November 2009]. Available from: http://www.biomedcentral.com/1472-6785/3/2.

Lea RN, McAllister RD, VenTresca DA. 1999. Biological aspects of nearshore rockfishes of the genus Sebastes from central California. California Department of Fish and Game - Fish Bulletin. 177.

LGL Limited, KS Biological Services, Pottinger Gaherty Environmental Consultants. 2009. NaiKun Offshore Wind Energy Project environmental assessment, Volume 8 - Marine birds and sea turtles. NaiKun Wind Development Inc.

Lindley ST, Moser ML, Erickson DL, Belchik M, Welch DW, Rechisky E, Kelly JT, Heublein J, Klimley AP. 2008. Marine migration of North American green sturgeon. Transactions of the American Fisheries Society. 137(1):182-194.

Loughlin TR. 1974. The distribution and ecology of the harbor seal in Humboldt Bay, California [master's thesis]. [Arcata, CA]: Humboldt State University.

Love MS. 1996. Probably more than you want to know about the fishes of the Pacific coast. 2nd. Santa Barbara, CA: Really Big Press.

Love MS, Carr MH, Haldorson LJ. 1991. The ecology of substrate-associated juveniles of the genus Sebastes. Environmental Biology of Fishes. 30:225-243. 
Mcnicholl MK, Lowther PE, Hall JA. 2001. Forster's tern (Sterna forsteri) [Internet]. Ithaca, NY: Cornell Lab of Ornithology; [cited 21 October 2009]. Available from: http://bna.birds.cornell.edu/bna/species/595/articles/introduction.

Merrick RL. 1996. The relationship of the foraging ecology of Steller sea lions (Eumetopias jubatus) to their population decline in Alaska [dissertation]. [Seattle, WA]: University of Washington.

Michel J, Dunagan H, Boring C, Healy E, Evans W, Dean JM, McGillis A, Hain J. 2007. Worldwide synthesis and analysis of existing information regarding environmental effects of alternative energy uses on the Outer Continental Shelf. Herndon, VA: U.S. Department of the Interior, Minerals Management Service. MMS 2007-038.

Miller DJ, Lea RN. 1976. Guide to the coastal marine fishes of California. Sacramento, CA: California Department of Fish and Game.

Miller JA, Shanks AL. 2005. Abundance and distribution of larval and juvenile fish in Coos Bay, Oregon: Time-series analysis based on light-trap collections. Marine Ecology Progress Series. 305:177-191.

(MMS) Minerals Management Service. 2007. Programmatic environmental impact statement for alternative energy development and production and alternate use of facilities on the Outer Continental Shelf. U.S. Department of the Interior. MMS 2007-046.

Montevecchi WA. 2006. Influences of artificial light on marine birds. In: Rich C, Longcore T, editors. Ecological consequences of artificial night lighting. Washington, D.C.: Island Press; p. Chapter 5.

Moore MV, Kohler SJ, Cheers MS. 2006. Artificial light at night in freshwater habitats and its potential ecological effects. In: Rich C, Longcore T, editors. Ecological consequences of artificial night lighting. Washington, D.C.: Island Press; p. 365-384.

Morton AB, Symonds HK. 2002. Displacement of Orincus orca (L.) by high amplitude sound in British Columbia, Canada. ICES Journal of Marine Science. 59(1):71-80.

Moyle PB. 2002. Inland Fishes of California. Berkeley and Los Angeles, CA: University of California Press.

Nedwell JR, Edwards B, Turnpenny AWH, Gordon J. 2004. Fish and marine mammal audiograms: A summary of available information. Subacoustech Report ref: 534R0214

Nelson PA. 2008. Ecological effects of wave energy conversion technology on California's marine and anadromous fishes. In: Nelson PA, editors. Developing wave energy in coastal California: Potential socio-economic and environmental effects. Sacramento, CA: California Energy Commission, PIER Energy-Related Environmental Research Program \& California Ocean Protection Council; p. 111-135.

Nelson PA, Woo S. 2008. Developing wave energy in coastal California: Potential socioeconomic and environmental effects: Introduction. In: Nelson PA, editors. Developing wave 
Appendix D - Effects of Small Commercial OPT Power Buoy Project Humboldt

energy in coastal California: Potential socio-economic and environmental effects. Sacramento, CA: California Energy Commission, PIER Energy-Related Environmental Research Program \& California Ocean Protection Council; p. 7-21

Nightingale B, Longcore T, Simenstad CA. 2006. Artificial night lighting and fishes. In: Rich C, Longcore T, editors. Ecological consequences of artificial night lighting. Washington, D.C.: Island Press; p. Chapter 11.

Nisbet IC. 2002. Common tern (Sterna hirundo) [Internet]. Ithaca, NY: Cornell Lab of Ornithology; [cited 21 October 2009]. Available from: http://bna.birds.cornell.edu/bna/species/618/articles/introduction.

(NMFS) National Marine Fisheries Service. 1985. Threatened fish and wildlife; Guadalupe fur seal: Final rule. Federal Register 50:51252-51256.

(NMFS) National Marine Fisheries Service. 1988. North Pacific fur seal; Pribilof Island population; Designation as depleted: Final rule. Federal Register 5317888-17899.

(NMFS) National Marine Fisheries Service. 1990. Listing of Steller sea lions as threatened under the Endangered Species Act. Federal Register 55(227):49204-49241.

(NMFS) National Marine Fisheries Service. 1993. Designated critical habitat; Steller sea lion. Federal Register 58(165):45269-45285.

(NMFS) National Marine Fisheries Service. 1997. Endangered and threatened species; Threatened status for southern Oregon/northern California coast Evolutionary Significant Unit (ESU) of coho salmon. Federal Register 62(87):24588-24609.

(NMFS) National Marine Fisheries Service. 1999. Designated critical habitat; Central California cast and southern Oregon/northern California coasts coho salmon. Federal Register 64:2404924062.

(NMFS) National Marine Fisheries Service. 2000. Endangered and threatened species: threatened status for one steelhead Evolutionarily Significant Unit (ESU) in California. Federal Register 65:36074-36094.

(NMFS) National Marine Fisheries Service. 2005a. Endangered and threatened species: Designation of critical habitat for seven Evolutionarily Significant Units of Pacific salmon and steelhead in California; final rule. Federal Register 70:52488-52627.

(NMFS) National Marine Fisheries Service. 2005b. Endangered and threatened species: final listing determinations for 16 ESUs of west coast salmon, and final 4(d) protective regulations of threatened salmon ESUs. Federal Register 70:37160-37204.

(NMFS) National Marine Fisheries Service. 2005c. Endangered and threatened wildlife and plants: Endangered status for southern resident killer whales. Federal Register 70:69903-69912.

(NMFS) National Marine Fisheries Service. 2005d. Green sturgeon (Acipenser medirostris) status review update. Santa Cruz, CA: NOAA Fisheries, Southwest Fisheries Science Center. 
(NMFS) National Marine Fisheries Service. 2006. Endangered and threatened species: final listing determinations for 10 distinct population segments of west coast steelhead. Federal Register 71(3):834-862.

(NMFS) National Marine Fisheries Service. 2008. Endangered and threatened species; endangered status for north Pacific and north Atlantic right whales: Final rule. Federal Register 73:12024-12030.

(NOAA) National Oceanic and Atmospheric Administration. 2007. Wave power: Looking to the ocean for electricity in Oregon [Internet]. U.S. Department of Commerce; [updated March 2007; cited 15 September 2009]. Available from: http://celebrating200years.noaa.gov/magazine/wave energy/welcome.html\#answer.

(NOAA) National Oceanic and Atmospheric Administration. 2009a. Cetaceans: Whales, dolphins, and porpoises [Internet]. NOAA Fisheries, Office of Protected Resources; [cited 8 July 2009]. Available from: http://www.nmfs.noaa.gov/pr/species/mammals/cetaceans/.

(NOAA) National Oceanic and Atmospheric Administration. 2009b. Pinnipeds: Seals, sea lions, and walruses [Internet]. NOAA Fisheries, Office of Protected Resources; [cited 8 July 2009]. Available from: http://www.nmfs.noaa.gov/pr/species/mammals/pinnipeds/.

Pequegnat JE, Mondeel-Jarvis D, Borgeld JC, Bott L. 1990. Sediment characteristics, benthic infauna, demersal fish and macroinvertebrates: Analysis of communities found offshore in water between 18 and 73 meters deep west of Humboldt Bay, California, and at the nearshore disposal site (August 1989, November 1989, and March 1990). San Francisco: U.S. Army Corps of Engineers.

Pequegnat JE, Mondeel-Jarvis D, Bott L, Matos J. 1995. Sediment characteristics, benthic infauna, demersal fish and macroinvertebrates sampled September 1994 - Volume 1. Humboldt County, CA: Lousiana-Pacific Corporation.

Pierotti RJ, Annett CA. 1995. Western gull (Larus occidentalis) [Internet]. Ithaca, NY: Cornell Lab of Ornithology; [cited 21 October 2009]. Available from: http://bna.birds.cornell.edu/bna/species/174/articles/introduction.

Poole AF, Bierregaard RO, Martell MS. 2002. Osprey (Pandion haliaetus) [Internet]. Ithaca, NY: Cornell Lab of Ornithology; [cited 20 October 2009]. Available from: http://bna.birds.cornell.edu/bna/species/683/articles/introduction.

Previsic M. 2009. Working draft - Scenario based analysis of environmental and navigation impacts: Wave power scenario descriptions.

Ralph CJ, Miller SL. 1995. Offshore population estimates of marbled murrelets in California. Albany, CA: U. S. Department of Agriculture, Forest Service, Pacific Southwest Research Station. General Technical Report PSW-GTR-152.

Relini G, Relini M, Montanari M. 2000. An offshore buoy as a small artificial island and a fishaggregating device (FAD) in the Mediterranean. Hydrobiologia. 440:65-80. 
Richardson WJ, Wursig B. 1997. Influences of man-made noise and other human actions on cetacean behaviour. Marine and Freshwater Behaviour and Physiology. 29:183-209.

Rubega MA, Schamel D, Tracy DM. 2000. Red-necked phalarope (Phalaropus lobatus) [Internet]. Ithaca, NY: Cornell Lab of Ornithology; [cited 20 October 2009]. Available from: http://bna.birds.cornell.edu/bna/species/538/articles/introduction.

Schusterman RJ, Balliet RF. 1970. Visual acuity of the harbour seal and the steller sea lion under water. Nature. 226(5245):563-564.

Stroud RK, Roffe TJ. 1979. Causes of death in marine mammals stranded along the Oregon coast. Journal of Wildlife Diseases. 15:91-97.

Substructure. [date unknown]. How is concrete affected in a marine environment? [Internet]. Portsmouth, NH: [cited 15 September 2009]. Available from:

http://www.substructure.com/education/how_is_concrete_affected_in_a_marine_environment.ht $\underline{\mathrm{ml}}$.

Sullivan RM, Stack JD, Houck WJ. 1983. Observations of gray whales (Eschrichtius robustus) along northern California. Journal of Mammalogy. 64(4):689-692.

Todd VLG, Pearse WD, Tregenza NC, Lepper PA, Todd IB. 2009. Diel echolocation activity of harbour porpoises (Phocoena phocoena) around North Sea offshore gas installations. ICES Journal of Marine Science. 66:734-745.

Tougaard J, Henriksen OD, Miller LA. 2009. Underwater noise from three types of offshore wind turbines: Estimation of impact zones for harbor porpoises and harbor seals. Journal of the Acoustical Society of America. 125(6):3766-3773.

(USFWS) U.S. Fish and Wildlife Service. 1970. Conservation of endangered species and other fish or wildlife: List of endangered foreign fish and wildlife. Federal Register 35(233):1831918322.

(USFWS) U.S. Fish and Wildlife Service. 1994. Final rule to remove the Eastern North Pacific population of gray whale from the list of endangered wildlife. Federal Register 59:31094-31095.

(USFWS) U.S. Fish and Wildlife Service. 2005. Regional Seabird Conservation Plan, Pacific Region. Portland, Oregon: U.S. Fish and Wildlife Service, Migratory Birds and Habitat Programs, Pacific Region.

(USFWS) U.S. Fish and Wildlife Service. 2006. Endangered and threatened wildlife and plants; Revised critical habitat for the tidewater goby (Eucyclogobius newberryi); Proposed rule. Federal Register 71(228):68914-68995.

(USFWS) U.S. Fish and Wildlife Service. 2008. Endangered and threatened wildlife and plants: Proposed rulemaking to designate critical habitat for the threatened southern distinct population segment of North American green sturgeon; Proposed rule. Federal Register 73(174):5208452110. 


\section{Appendix D - Effects of Small Commercial OPT Power Buoy Project Humboldt}

(USFWS) U.S. Fish and Wildlife Service. 2009. Endangered and threatened wildlife and plants: Proposed rulemaking to establish take prohibitions for the threatened southern distinct population segment of North American green sturgeon. Federal Register 74(97):23822-23837.

Van Waerebeek K, Leaper R. 2008. Second report of the IWC vessel strike data standardisation working group. In: editors. IWC 60th Annual Meeting; Santiago, Chile. p. 8.

White CM, Clum NJ, Cade TJ, Hunt WG. 2002. Peregrine falcon (Falco peregrinus) [Internet]. Ithaca, NY: Cornell Lab of Ornithology; [cited 20 October 2009]. Available from: http://bna.birds.cornell.edu/bna/species/660/articles/introduction.

Wiersma P, Piersma T, Van Eerden MR. 1995. Food intake of great crested grebes Podiceps cristatus wintering on cold water as a function of various cost factors. Ardea. 83:339-350.

Yurk H, Trites AW. 2000. Experimental attempts to reduce predation by harbor seals on outmigrating juvenile salmonids. Transactions of the American Fisheries Society. 129:1360-1366. 


\section{Appendix E. Effects of Small Commercial Pelamis at Makapu'u}

Included in Appendix E are 1) a project description of the small commercial Pelamis at Makapu'u project site; 2) an effects analysis of the project on site physical and biological indicators in tabular format; and 3) a list of references used to complete the effects analysis.

\section{Project description for construction, operations and maintenance, and decommissioning phases for Small Commercial Pelamis} P-2 project at the Makapu'u site.

\begin{tabular}{|c|c|c|}
\hline Project phase & Project activity or characteristic & Description \\
\hline \multirow{14}{*}{ Construction } & Location and deployment depths & Makapu’u Point, Oahu. In 50 m water depth, 1 to 2 mi from shore \\
\hline & Footprint & $\begin{array}{l}13 \text { devices would be in organized in } 2 \text { rows, with an array length of } 2.5 \mathrm{~km} \text {. Width } \\
\text { is } 0.5 \mathrm{~m} \text {. }\end{array}$ \\
\hline & Loading ports and dock locations & $\begin{array}{l}\text { Honolulu Harbor; smaller vessels could use Makai pier near Makapu’u Point and } \\
\text { Waimanalo Bay. }\end{array}$ \\
\hline & Shipping routes for delivery and installation & Unknown, assume from Honolulu to the project site \\
\hline & Ship types and sizes & Drill rig, cable installation vessel, supply boat, derrick barge, tug boats \\
\hline & Installation and assembly procedures & $\begin{array}{l}\text { Subsea cable bolted to sea floor (rock bottom). Caternary mooring system installed } \\
\text { by barge, with devices towed to site }\end{array}$ \\
\hline & Installation equipment & Directional drill rig, cable installation vessel, supply boat, derrick barge, tug boats \\
\hline & Temporary structures & None \\
\hline & $\begin{array}{l}\text { Types, composition, locations, and numbers of } \\
\text { anchoring and mooring systems }\end{array}$ & $\begin{array}{l}\text { Caternary mooring configuration with slack to allow device to move so it points into } \\
\text { the waves. Design of embedment anchors on seabed unknown. Each device } \\
\text { requires } 4 \text { anchors and } 4 \text { cables }\end{array}$ \\
\hline & Installation schedule and phasing & $\begin{array}{l}1 \text { to } 2 \text { years, in May through early September. For the } 10 \text { devices, which is close to } \\
\text { small commercial project scale, installation is assumed to occur day and night, } 7 \\
\text { days/week }\end{array}$ \\
\hline & Chemicals and fuels used & Hydraulic fluids, gearbox oil, bearing grease, boat fuel, antifouling paint \\
\hline & Sources and levels of noise & $\begin{array}{l}\text { Boat and barge traffic noise, drilling, construction/installation of subsea cable, } \\
\text { moorings, anchors, and devices }\end{array}$ \\
\hline & Sources, levels, and characteristics of light & $\begin{array}{l}\text { Navigational lights (lower intensity) on boats and devices, construction lights } \\
\text { (bright, higher intensity) on barges }\end{array}$ \\
\hline & Number of vessel trips & Unknown \\
\hline \multirow[t]{3}{*}{$\begin{array}{l}\text { Operations and } \\
\text { Maintenance }\end{array}$} & General description of technology & $\begin{array}{l}\text { Each unit has } 4 \text { steel tubes connected in series by a heave and sway joint. Steel } \\
\text { tubes contain hydraulic motors that capture energy. }\end{array}$ \\
\hline & O\&M procedures and schedule & $\begin{array}{l}\text { Annual inspections and maintenance: detach devices from mooring, tow devices to } \\
\text { pier and inspect and repair. }\end{array}$ \\
\hline & $\begin{array}{l}\text { Operating equipment other than wave/tidal } \\
\text { device(s) }\end{array}$ & $\begin{array}{l}\text { Custom vessel for annual recovery and re-deployment, ROV for visual inspection of } \\
\text { underwater components every } 4 \text { to } 5 \text { years. }\end{array}$ \\
\hline
\end{tabular}


Appendix E- Effects of Small Commercial Pelamis Project at Makapu’u

\begin{tabular}{|c|c|c|}
\hline Project phase & Project activity or characteristic & Description \\
\hline & Listing of all moving parts & $\begin{array}{l}\text { Heave and sway joints between } 4 \text { steel tubes, for each device, approximately } 13 \\
\text { devices }\end{array}$ \\
\hline & Listing of all structures on surface and below & 13 (each 180 x 6 m) on surface, moored via multiple underwater cables and anchors \\
\hline & $\begin{array}{l}\text { Vessel routes and schedule for operation and } \\
\text { maintenance }\end{array}$ & Unknown, assumed from Honolulu \\
\hline & Ship types and sizes & Types include custom vessel, derrick barge, tugboats, supply boat. Size unknown \\
\hline & Potential emergency conditions and procedures & $\begin{array}{l}\text { Oil/chemical spills or releases from boats, equipment, or devices. Device becomes } \\
\text { lose from moorings }\end{array}$ \\
\hline & $\begin{array}{l}\text { Chemicals used by devices, e.g., hydraulic } \\
\text { fluids, antifouling paints. }\end{array}$ & $\begin{array}{l}\text { Hydraulic fluids, bearing grease, gearbox oil, anti-fouling paint, boat fuel. } 12,800 \\
\text { liters hydraulic fluid in each device. }\end{array}$ \\
\hline & Sources and levels of noise & $\begin{array}{l}\text { Noise and vibration associated with movement of devices, sound of vessels } \\
\text { servicing the devices }\end{array}$ \\
\hline & Sources and levels of light & Navigational lights on boats, navigational lights on devices \\
\hline \multirow{13}{*}{ Decommissioning } & $\begin{array}{l}\text { Description of equipment or structures } \\
\text { removed }\end{array}$ & Devices, mooring cables and anchors, transmission cable on seabed surface \\
\hline & $\begin{array}{l}\text { Description of equipment or structures to be } \\
\text { left in place }\end{array}$ & None \\
\hline & $\begin{array}{l}\text { Monitoring procedure and schedule for } \\
\text { equipment left in place }\end{array}$ & None \\
\hline & Shipping routes for equipment removed & Unknown, assumed from Honolulu \\
\hline & Ship types and size & $\begin{array}{l}\text { Supply boat, custom vessel for devices, derrick barge, tug boats, cable handling } \\
\text { vessel }\end{array}$ \\
\hline & Decommissioning and disassembly procedures & $\begin{array}{l}\text { Recover devices, mooring cables and anchors, and remove collector system and } \\
\text { subsea cable. }\end{array}$ \\
\hline & Decommissioning equipment & $\begin{array}{l}\text { Supply boat, custom vessel for devices, derrick barge, tug boats, cable handling } \\
\text { vessel }\end{array}$ \\
\hline & Temporary structures & None \\
\hline & Decommissioning schedule and phasing & $\begin{array}{l}\text { Would likely take place over } 1 \text { to } 2 \text { summer seasons, } 6 \text { to } 8 \text { weeks total, after a } \\
\text { project life of } 15 \text { to } 25 \text { years }\end{array}$ \\
\hline & Chemicals and fuels used & Hydraulic fluids, gearbox oil, bearing grease, boat fuel \\
\hline & Sources and levels of noise & $\begin{array}{l}\text { Boat and barge traffic noise, removal and recovery of subsea cable, moorings, } \\
\text { anchors, and devices }\end{array}$ \\
\hline & Vessels required, number of trips & See decommissioning equipment, numbers of trips unknown \\
\hline & Sources, levels, and characteristics of light & $\begin{array}{l}\text { Navigational lights (lower intensity) on boats and devices, deconstruction lights } \\
\text { (bright, higher intensity) on barges }\end{array}$ \\
\hline
\end{tabular}


Appendix E- Effects of Small Commercial Pelamis Project at Makapu'u

\section{Effects of Small Commercial Pelamis at Makapu'u on physical and biological indicators}

Potential effects on the visual environment due to Small Commercial Pelamis at Makapu'u site

\begin{tabular}{|c|c|c|c|c|c|c|}
\hline Project activity & Project action & $\begin{array}{l}\text { Description of action's } \\
\text { effect on site physical } \\
\text { attribute }\end{array}$ & $\begin{array}{l}\text { Spatial exposure of } \\
\text { attribute (low, med, } \\
\text { high) }\end{array}$ & $\begin{array}{l}\text { Temporal exposure } \\
\text { of attribute (low, } \\
\text { med, high) }\end{array}$ & $\begin{array}{l}\text { Overall risk to } \\
\text { attribute (low, med, } \\
\text { high or unknown) } \\
\end{array}$ & Source(s) \\
\hline \multicolumn{7}{|c|}{ Construction } \\
\hline Boat traffic & Navigation lights & $\begin{array}{l}\text { Vessel lights will be } \\
\text { visible along shipping } \\
\text { lanes from Honolulu } \\
\text { Harbor and Makai pier to } \\
\text { the project site }\end{array}$ & $\begin{array}{l}\text { Low, visibility varies } \\
\text { with atmospheric } \\
\text { conditions, will be seen } \\
\text { from docks and shore }\end{array}$ & $\begin{array}{l}\text { Low, boat traffic will } \\
\text { occur during May- } \\
\text { Sept. for 1-2 years }\end{array}$ & $\begin{array}{l}\text { Low, increase may } \\
\text { not be noticeable with } \\
\text { existing boat traffic }\end{array}$ & MMS 2007 \\
\hline $\begin{array}{l}\text { Construction of } \\
\text { electrical collector } \\
\text { system, moorings and } \\
\text { foundations; device } \\
\text { installation }\end{array}$ & Construction lights & $\begin{array}{l}\text { Construction lights much } \\
\text { brighter than vessel lights, } \\
\text { depending on sea and } \\
\text { weather conditions, could } \\
\text { be visible from shore. }\end{array}$ & $\begin{array}{l}\text { High, construction } \\
\text { lights may be visible } 2 \\
\text { to } 5 \mathrm{~nm} \text {. }\end{array}$ & $\begin{array}{l}\text { Low, construction } \\
\text { will occur during } \\
\text { May-Sept. for 1-2 } \\
\text { years }\end{array}$ & $\begin{array}{l}\text { Med, lights may be } \\
\text { visible from shore }\end{array}$ & IALA 2008 \\
\hline \multicolumn{7}{|c|}{ Operation and Maintenance } \\
\hline Boat traffic & Navigation lights & $\begin{array}{l}\text { Vessel lights will be } \\
\text { visible along shipping } \\
\text { lines from Honolulu } \\
\text { Harbor and Makai pier to } \\
\text { the project site }\end{array}$ & $\begin{array}{l}\text { Low, visibility varies } \\
\text { with atmospheric } \\
\text { conditions, will be seen } \\
\text { from docks and shore }\end{array}$ & $\begin{array}{l}\text { Med, vessel traffic } \\
\text { will occur over life of } \\
\text { project but at reduced } \\
\text { frequency compared } \\
\text { to construction }\end{array}$ & $\begin{array}{l}\text { Low, increase may } \\
\text { not be noticeable with } \\
\text { existing boat traffic }\end{array}$ & MMS 2007 \\
\hline \multirow{2}{*}{$\begin{array}{l}\text { Structures on water's } \\
\text { surface }\end{array}$} & Structure & $\begin{array}{l}\text { Devices may be visible } \\
\text { from shore }\end{array}$ & $\begin{array}{l}\text { Low, devices } 0.5 \mathrm{~km} \\
\text { from shore but profiles } \\
\text { very low }\end{array}$ & $\begin{array}{l}\text { High, devices will be } \\
\text { present } 15-25 \text { years }\end{array}$ & $\begin{array}{l}\text { Low, device profiles } \\
\text { are low and "facilities } \\
\text { will probably have } \\
\text { little visual impact" }\end{array}$ & NOAA 2007 \\
\hline & Navigation lights & $\begin{array}{l}\text { Devices will have lights } \\
\text { for navigational safety } \\
\text { visible from } 2 \text { to } 5 \mathrm{~nm} \text {. }\end{array}$ & $\begin{array}{l}\text { Med, device lights will } \\
\text { be visible from } 2 \text { to } 5 \\
\text { nm. }\end{array}$ & $\begin{array}{l}\text { High, device lights } \\
\text { will be required } \\
\text { throughout the life of } \\
\text { the project }\end{array}$ & $\begin{array}{l}\text { Med, device lights } \\
\text { will have a low } \\
\text { profile and visible } 1 \\
\text { to } 2 \text { mi from shore }\end{array}$ & IALA 2008 \\
\hline \multicolumn{7}{|c|}{ Decommissioning } \\
\hline Boat traffic & Navigation lights & $\begin{array}{l}\text { Vessel lights will be } \\
\text { visible along shipping } \\
\text { lines from Honolulu } \\
\text { Harbor and Makai pier to } \\
\text { the project site }\end{array}$ & $\begin{array}{l}\text { Low, visibility varies } \\
\text { with atmospheric } \\
\text { conditions, will be seen } \\
\text { from docks and shore }\end{array}$ & $\begin{array}{l}\text { Low, boat traffic will } \\
\text { occur during May- } \\
\text { Sept. for 1-2 years }\end{array}$ & $\begin{array}{l}\text { Low, increase may } \\
\text { not be noticeable } \\
\text { within existing boat } \\
\text { traffic }\end{array}$ & MMS 2007 \\
\hline $\begin{array}{l}\text { Decommissioning of } \\
\text { structures on water's } \\
\text { surface or seabed }\end{array}$ & Deconstruction lights & $\begin{array}{l}\text { Deconstruction lights } \\
\text { brighter than vessel lights, } \\
\text { depending on sea and } \\
\text { weather conditions, could } \\
\text { be visible from shore. }\end{array}$ & $\begin{array}{l}\text { High, deconstruction } \\
\text { may be visible } 2 \text { to } 5 \\
\text { nm. }\end{array}$ & $\begin{array}{l}\text { Low, deconstruction } \\
\text { will occur will occur } \\
\text { during May-Sept. for } \\
\text { 1-2 years }\end{array}$ & $\begin{array}{l}\text { Med, lights may be } \\
\text { visible from shore }\end{array}$ & $\begin{array}{l}\text { IALA 2008, } \\
\text { NOAA } 2007\end{array}$ \\
\hline
\end{tabular}


Appendix E- Effects of Small Commercial Pelamis Project at Makapu’u

Potential effects on acoustic environment due to Small Commercial Pelamis Project at Makapu'u Point, Hawaii

\begin{tabular}{|c|c|c|c|c|c|c|}
\hline Project activity & Project action & $\begin{array}{l}\text { Description of action's } \\
\text { effect on site physical } \\
\text { attribute }\end{array}$ & \begin{tabular}{|l|} 
Spatial exposure of \\
attribute (low, med, \\
high)
\end{tabular} & $\begin{array}{l}\text { Temporal exposure } \\
\text { of attribute (low, } \\
\text { med, high) }\end{array}$ & $\begin{array}{l}\text { Overall risk to } \\
\text { attribute (low, med, } \\
\text { high or unknown) }\end{array}$ & Source(s) \\
\hline \multicolumn{7}{|c|}{ Construction } \\
\hline Boat traffic & \multirow{3}{*}{ Noise and vibration } & $\begin{array}{l}\text { Propellers cavitate, } \\
\text { causing pressure } \\
\text { differences }\end{array}$ & $\begin{array}{l}\text { High, modeled noise of } \\
120 \mathrm{~dB} \text { extended approx } \\
20 \mathrm{~km} \text { in ocean wind } \\
\text { turbine project }\end{array}$ & $\begin{array}{l}\text { Low, boat traffic will } \\
\text { occur during May- } \\
\text { Sept. for 1-2 years }\end{array}$ & $\begin{array}{l}\text { Med, noise could be } \\
\text { elevated but short- } \\
\text { term and attenuated } \\
\text { by sea conditions and } \\
\text { ambient noise }\end{array}$ & $\begin{array}{l}\text { Austin et al. } \\
\text { 2009, USN } 2007\end{array}$ \\
\hline $\begin{array}{l}\text { Construction of } \\
\text { electrical collector } \\
\text { system, moorings and } \\
\text { foundations; device } \\
\text { installation }\end{array}$ & & $\begin{array}{l}\text { Adds to existing natural } \\
\text { and man-made noise in } \\
\text { project area }\end{array}$ & $\begin{array}{l}\text { High, modeled noise of } \\
120 \mathrm{~dB} \text { extended approx } \\
20 \mathrm{~km} \text { in ocean wind } \\
\text { turbine project }\end{array}$ & $\begin{array}{l}\text { Low, construction } \\
\text { will occur during } \\
\text { May-Sept. for 1-2 } \\
\text { years }\end{array}$ & $\begin{array}{l}\text { Med, noise could be } \\
\text { elevated but short- } \\
\text { term and attenuated } \\
\text { by sea conditions and } \\
\text { ambient noise }\end{array}$ & $\begin{array}{l}\text { Austin et al. } \\
\text { 2009, USN } 2007\end{array}$ \\
\hline $\begin{array}{l}\text { Directional drilling, and } \\
\text { laying cable under/on } \\
\text { seabed }\end{array}$ & & $\begin{array}{l}\text { Vibration of immediate } \\
\text { area being drilled }\end{array}$ & $\begin{array}{l}\text { Low, vibration could be } \\
\text { localized, assuming } \\
\text { similar to directional } \\
\text { drilling on land } \\
\end{array}$ & $\begin{array}{l}\text { Low, drilling will } \\
\text { occur for 1-2 weeks } \\
\text { in summer }\end{array}$ & $\begin{array}{l}\text { Low, noise expected } \\
\text { to be localized and } \\
\text { short-term }\end{array}$ & CPUC 2009 \\
\hline \multicolumn{7}{|c|}{ Operation and Maintenance } \\
\hline Boat traffic & \multirow{2}{*}{ Noise and vibration } & $\begin{array}{l}\text { Propellers cavitate, } \\
\text { causing pressure } \\
\text { differences }\end{array}$ & $\begin{array}{l}\text { High, modeled noise of } \\
120 \mathrm{~dB} \text { extended approx } \\
20 \mathrm{~km} \text { in ocean wind } \\
\text { turbine project } \\
\end{array}$ & $\begin{array}{l}\text { Low, vessel traffic } \\
\text { infrequent during } \\
\text { O\&M }\end{array}$ & $\begin{array}{l}\text { Low, noise would } \\
\text { occur infrequently }\end{array}$ & USN 2007 \\
\hline $\begin{array}{l}\text { Operation of turbines or } \\
\text { other moving parts of } \\
\text { devices }\end{array}$ & & $\begin{array}{l}\text { Adds to existing natural } \\
\text { and man-made noise in } \\
\text { project area }\end{array}$ & $\begin{array}{l}\text { Unknown levels } \\
\text { generated; site specific } \\
\text { attenuation and ambient } \\
\text { noise also unknown }\end{array}$ & $\begin{array}{l}\text { High, noise would } \\
\text { occur over life of the } \\
\text { project }\end{array}$ & Unknown & $\begin{array}{l}\text { Austin et al. } \\
\text { 2009, Study } \\
\text { warranted }\end{array}$ \\
\hline \multicolumn{7}{|c|}{ Decommissioning } \\
\hline Boat traffic & Noise and vibration & $\begin{array}{l}\text { Propellers cavitate, } \\
\text { causing pressure } \\
\text { differences }\end{array}$ & $\begin{array}{l}\text { High, modeled noise of } \\
120 \mathrm{~dB} \text { extended approx } \\
20 \mathrm{~km} \text { in ocean wind } \\
\text { turbine project }\end{array}$ & $\begin{array}{l}\text { Low, boat traffic will } \\
\text { occur during May- } \\
\text { Sept. for 1-2 years }\end{array}$ & $\begin{array}{l}\text { Med, noise could be } \\
\text { elevated but short- } \\
\text { term and attenuated } \\
\text { by sea conditions and } \\
\text { ambient noise }\end{array}$ & $\begin{array}{l}\text { Austin et al. } \\
\text { 2009, USN } 2007\end{array}$ \\
\hline $\begin{array}{l}\text { Decommissioning of } \\
\text { structures on water's } \\
\text { surface or seabed }\end{array}$ & Noise and vibration & $\begin{array}{l}\text { Adds to existing natural } \\
\text { and man-made noise in } \\
\text { project area }\end{array}$ & $\begin{array}{l}\text { High, modeled noise of } \\
120 \mathrm{~dB} \text { extended approx } \\
20 \mathrm{~km} \text { in ocean wind } \\
\text { turbine project }\end{array}$ & $\begin{array}{l}\text { Low, deconstruction } \\
\text { will occur during } \\
\text { May-Sept. for 1-2 } \\
\text { years }\end{array}$ & $\begin{array}{l}\text { Med, noise could be } \\
\text { elevated but short- } \\
\text { term and attenuated } \\
\text { by sea conditions and } \\
\text { ambient noise }\end{array}$ & $\begin{array}{l}\text { Austin et al. } \\
\text { 2009, USN } 2007\end{array}$ \\
\hline
\end{tabular}

\begin{tabular}{lrr}
\hline $\begin{array}{l}\text { Environmental Assessment for Siting Wave \& Tidal } \\
\text { Energy Projects }\end{array}$ & E-4 & H. T. Harvey \& Associates \\
24 November 2009
\end{tabular}


Appendix E- Effects of Small Commercial Pelamis Project at Makapu'u

Potential effects on sediment and water chemistry due to Small Commercial Pelamis at Makapu'u site

\begin{tabular}{|c|c|c|c|c|c|c|}
\hline Project activity & Project action & $\begin{array}{l}\text { Description of action's } \\
\text { effect on site physical } \\
\text { attribute } \\
\end{array}$ & $\begin{array}{l}\text { Spatial exposure of } \\
\text { attribute (low, med, } \\
\text { high) } \\
\end{array}$ & $\begin{array}{l}\text { Temporal exposure } \\
\text { of attribute (low, } \\
\text { med, high) }\end{array}$ & $\begin{array}{l}\text { Overall risk to } \\
\text { attribute (low, med, } \\
\text { high, or unknown) }\end{array}$ & Source(s) \\
\hline \multicolumn{7}{|c|}{ Construction } \\
\hline Boat traffic & $\begin{array}{l}\text { Oil/chemical release, } \\
\text { assumed seepage from } \\
\text { exhaust and general } \\
\text { use, not a spill from } \\
\text { collision or other } \\
\text { release }\end{array}$ & \multirow{2}{*}{$\begin{array}{l}\text { Could add compounds that } \\
\text { change the physical and } \\
\text { chemical characteristics of } \\
\text { sediment and water }\end{array}$} & $\begin{array}{l}\text { Low, seepage will have } \\
\text { a small spatial and areal } \\
\text { extent relative to the } \\
\text { project }\end{array}$ & $\begin{array}{l}\text { High, traffic and boat } \\
\text { frequency will be the } \\
\text { highest during } \\
\text { construction }\end{array}$ & $\begin{array}{l}\text { Low, given low } \\
\text { spatial and temporal } \\
\text { extent }\end{array}$ & USN 2007 \\
\hline $\begin{array}{l}\text { Construction of } \\
\text { electrical collector } \\
\text { system, moorings and } \\
\text { foundations; device } \\
\text { installation }\end{array}$ & Oil/chemical release & & $\begin{array}{l}\text { Low, seepage will have } \\
\text { a small spatial and areal } \\
\text { extent relative to the } \\
\text { project }\end{array}$ & $\begin{array}{l}\text { Low, construction } \\
\text { will be } 1 \text { to } 2 \text { years } \\
\text { over the } 15 \text { to } 25 \text { year } \\
\text { project life }\end{array}$ & $\begin{array}{l}\text { Low, given low } \\
\text { spatial and temporal } \\
\text { extent }\end{array}$ & USN 2007 \\
\hline $\begin{array}{l}\text { Directional drilling, and } \\
\text { laying cable under/on } \\
\text { seabed (assume normal } \\
\text { conditions, not a drilling } \\
\text { mud "blow out" } \\
\text { scenario) }\end{array}$ & Seabed disturbance & $\begin{array}{l}\text { Ocean floor is primarily } \\
\text { limestone; cables likely } \\
\text { laid on exposed limestone } \\
\text { and secured with rock } \\
\text { bolts. Sediment could be } \\
\text { introduced into water } \\
\text { column. }\end{array}$ & $\begin{array}{l}\text { Med, cable length } \\
\text { approx } 1 \text { to } 2 \text { miles; }\end{array}$ & $\begin{array}{l}\text { Low, little sediment } \\
\text { introduced in water } \\
\text { column and would } \\
\text { mix or dilute quickly. }\end{array}$ & $\begin{array}{l}\text { Low, due to surface } \\
\text { cable and quick } \\
\text { dilution of sediment } \\
\text { in water column }\end{array}$ & MMS 2007 \\
\hline \multicolumn{7}{|c|}{ Operation and Maintenance } \\
\hline Boat traffic & $\begin{array}{l}\text { Oil/chemical release, } \\
\text { assumed seepage from } \\
\text { general use, not a spill } \\
\text { from collision }\end{array}$ & $\begin{array}{l}\text { Could add compounds that } \\
\text { change the physical and } \\
\text { chemical characteristics of } \\
\text { sediment and water }\end{array}$ & $\begin{array}{l}\text { Low, seepage will have } \\
\text { a small spatial and areal } \\
\text { extent relative to the } \\
\text { project }\end{array}$ & $\begin{array}{l}\text { Low, O\&M vessel } \\
\text { traffic will be much } \\
\text { less than during } \\
\text { construction }\end{array}$ & $\begin{array}{l}\text { Low, due to low } \\
\text { seepage spatial } \\
\text { extent, and low } \\
\text { volume of vessel } \\
\text { traffic during O\&M } \\
\end{array}$ & USN 2007 \\
\hline \multirow[t]{2}{*}{$\begin{array}{l}\text { Structures in water } \\
\text { column and on seabed, } \\
\text { such as devices and } \\
\text { moorings and footings }\end{array}$} & Structure & $\begin{array}{l}\text { Concrete footings are } \\
\text { sources of alkaline } \\
\text { elements (sodium, } \\
\text { potassium) that could } \\
\text { leach into water column; } \\
\text { however, footings in rocky } \\
\text { seabed unknown and may } \\
\text { not be concrete }\end{array}$ & $\begin{array}{l}\text { Low, effect would be } \\
\text { localized to vicinity of } \\
\text { concrete footings; } \\
\text { project area } \sim 1.6 \mathrm{~km} \text { by } \\
0.5 \mathrm{~km} \text { or } 0.8 \mathrm{~km}^{2}(0.3 \\
\left.\mathrm{mi}^{2}\right)\end{array}$ & $\begin{array}{l}\text { High, any leaching } \\
\text { would occur } \\
\text { throughout life of } \\
\text { project }\end{array}$ & $\begin{array}{l}\text { Low, effects would } \\
\text { be diluted and may } \\
\text { not be measurable }\end{array}$ & $\begin{array}{l}\text { Substructure } \\
\text { [date unknown] }\end{array}$ \\
\hline & $\begin{array}{l}\text { Water circulation } \\
\text { changes }\end{array}$ & $\begin{array}{l}\text { Erosion in lee of footings } \\
\text { possible but much slower } \\
\text { in rocky seabed substrate }\end{array}$ & $\begin{array}{l}\text { Low, effect would be } \\
\text { localized to lee side of } \\
\text { footings }\end{array}$ & $\begin{array}{l}\text { Med, any erosion } \\
\text { occurring would be } \\
\text { throughout the life of } \\
\text { the project }\end{array}$ & $\begin{array}{l}\text { Low, effect is small } \\
\text { and localized, } \\
\text { although it would } \\
\text { occur over life of } \\
\text { project }\end{array}$ & USN 2007 \\
\hline
\end{tabular}

Environmental Assessment for Siting Wave \& Tidal Energy Projects
E-5

H. T. Harvey \& Associates

24 November 2009 
Appendix E- Effects of Small Commercial Pelamis Project at Makapu'u

\begin{tabular}{|c|c|c|c|c|c|c|}
\hline Project activity & Project action & $\begin{array}{l}\text { Description of action's } \\
\text { effect on site physical } \\
\text { attribute }\end{array}$ & $\begin{array}{l}\text { Spatial exposure of } \\
\text { attribute (low, med, } \\
\text { high) }\end{array}$ & $\begin{array}{l}\text { Temporal exposure } \\
\text { of attribute (low, } \\
\text { med, high) }\end{array}$ & $\begin{array}{l}\text { Overall risk to } \\
\text { attribute (low, med, } \\
\text { high, or unknown) }\end{array}$ & Source(s) \\
\hline $\begin{array}{l}\text { Structures on water's } \\
\text { surface }\end{array}$ & Structure & $\begin{array}{l}\text { Biofouling organisms } \\
\text { slough off on to the seabed } \\
\text { surface; potential for } \\
\text { hydraulic fluids release } \\
\text { from devices, } 12800 \text { liters } \\
\text { ( } 3381 \text { gal) per device }\end{array}$ & $\begin{array}{l}\text { Low, effect localized to } \\
\text { seabed directly under } \\
\text { devices }\end{array}$ & $\begin{array}{l}\text { Low, antifouling } \\
\text { paint and } \\
\text { maintenance likely to } \\
\text { remove organisms } \\
\text { before sloughing }\end{array}$ & $\begin{array}{l}\text { Low, effect is } \\
\text { localized to } \\
\text { immediate vicinity of } \\
\text { seabed under devices }\end{array}$ & MMS 2007 \\
\hline \multicolumn{7}{|c|}{ Decommissioning } \\
\hline Boat traffic & $\begin{array}{l}\text { Oil/chemical release, } \\
\text { assumed seepage from } \\
\text { general use, not a spill } \\
\text { from collision }\end{array}$ & \multirow{2}{*}{$\begin{array}{l}\text { Could add compounds that } \\
\text { change the physical and } \\
\text { chemical characteristics of } \\
\text { sediment and water }\end{array}$} & \multirow{2}{*}{$\begin{array}{l}\text { Low, seepage will have } \\
\text { a small spatial/area } \\
\text { extent relative to the } \\
\text { project }\end{array}$} & $\begin{array}{l}\text { High, traffic and boat } \\
\text { frequency will be } \\
\text { high during } \\
\text { decommissioning }\end{array}$ & $\begin{array}{l}\text { Low, given low spatial } \\
\text { and temporal extent }\end{array}$ & USN 2007 \\
\hline \multirow{2}{*}{$\begin{array}{l}\text { Decommissioning of } \\
\text { structures on water's } \\
\text { surface or seabed }\end{array}$} & Oil/chemical release & & & $\begin{array}{l}\text { Low, } \\
\text { decommissioning } \\
\text { will be } 1 \text { to } 2 \text { years } \\
\text { over the } 15 \text { to } 25 \text { year } \\
\text { project life }\end{array}$ & $\begin{array}{l}\text { Low, given low spatial } \\
\text { and temporal extent }\end{array}$ & USN 2007 \\
\hline & Seabed disturbance & $\begin{array}{l}\text { Sediment could be } \\
\text { introduced into water } \\
\text { column when removing } \\
\text { anchors or footings and/or } \\
\text { surface cable }\end{array}$ & $\begin{array}{l}\text { Med, cable length } \\
\text { approx } 1 \text { to } 2 \text { miles; } \\
\text { project area } \sim 1.6 \mathrm{~km} \text { by } \\
0.5 \mathrm{~km} \text { or } 0.8 \mathrm{~km}^{2}(0.3 \\
\left.\mathrm{mi}^{2}\right)\end{array}$ & $\begin{array}{l}\text { Low, increased } \\
\text { sediment in water } \\
\text { column would mix or } \\
\text { dilute quickly. }\end{array}$ & $\begin{array}{l}\text { Low, due to quick } \\
\text { dilution of sediment in } \\
\text { water column }\end{array}$ & MMS 2007 \\
\hline
\end{tabular}

Environmental Assessment for Siting Wave \& Tidal Energy Projects
E-6

H. T. Harvey \& Associates 
Appendix E- Effects of Small Commercial Pelamis Project at Makapu’u

Potential effects on Humpback Whales due to Small Commercial Pelamis at Makupu'u site

\begin{tabular}{|c|c|c|c|c|c|c|c|}
\hline Project activity & Project action & $\begin{array}{l}\text { Description of } \\
\text { action's effect on } \\
\text { indicator }\end{array}$ & $\begin{array}{l}\text { Spatial exposure } \\
\text { to indicator (low, } \\
\text { med, high) }\end{array}$ & $\begin{array}{l}\text { Temporal } \\
\text { exposure to } \\
\text { indicator (low, } \\
\text { med, high) }\end{array}$ & $\begin{array}{l}\text { Effect's overlap } \\
\text { with critical life } \\
\text { stage, behavior, } \\
\text { habitat or } \\
\text { resource (low, } \\
\text { med, high) }\end{array}$ & $\begin{array}{l}\text { Overall risk to indicator } \\
\text { (low, med, high or } \\
\text { unknown) }\end{array}$ & Source(s) \\
\hline \multicolumn{8}{|c|}{ Construction } \\
\hline \multirow{3}{*}{ Boat traffic } & Direct impact & Collision injuries & $\begin{array}{l}\text { High, significant } \\
\text { increase of traffic } \\
\text { traveling along } \\
\text { coast from } \\
\text { Honolulu to } \\
\text { project site } \\
\end{array}$ & $\begin{array}{l}\text { Low, occurring } \\
\text { when few if any } \\
\text { humpbacks are in } \\
\text { area. }\end{array}$ & $\begin{array}{l}\text { Low, few in area, } \\
\text { May-Sept during } \\
\text { breeding, some } \\
\text { calves in early } \\
\text { May. }\end{array}$ & $\begin{array}{l}\text { Low; few in area during } \\
\text { construction; med due to } \\
\text { humpback whale-boat } \\
\text { collisions one of the } \\
\text { highest rates among } \\
\text { marine mammals }\end{array}$ & $\begin{array}{l}\text { Laist et al. } \\
\text { 2001, Van } \\
\text { Waerebeek } \\
\text { and Leaper } \\
2008\end{array}$ \\
\hline & $\begin{array}{l}\text { Noise and } \\
\text { vibration }\end{array}$ & $\begin{array}{l}\text { Avoidance, masking } \\
\text { of environmental } \\
\text { cues, song and } \\
\text { communication } \\
\text { signals }\end{array}$ & $\begin{array}{l}\text { Med, increase of } \\
\text { traffic from } \\
\text { Honolulu, sound } \\
\text { travels beyond } \\
\text { project area }\end{array}$ & $\begin{array}{l}\text { Low, occurs when } \\
\text { few if any } \\
\text { humpbacks are in } \\
\text { area. }\end{array}$ & $\begin{array}{l}\text { Low, starts at end } \\
\text { of breeding when } \\
\text { few if any } \\
\text { humpbacks in } \\
\text { area. }\end{array}$ & $\begin{array}{l}\text { Low; few in area during } \\
\text { construction; whales may } \\
\text { flee approaching boat } \\
\text { while still distant }\end{array}$ & $\begin{array}{l}\text { Craig and } \\
\text { Herman 1997, } \\
\text { Richardson } \\
\text { and Wursig } \\
1997 \\
\end{array}$ \\
\hline & $\begin{array}{l}\text { Oil/chemical } \\
\text { release }\end{array}$ & $\begin{array}{l}\text { Ingestion, breathing } \\
\text { exhaust fumes, } \\
\text { overall accumulation } \\
\text { of toxins. }\end{array}$ & $\begin{array}{l}\text { Low, significant } \\
\text { increase in } \\
\text { existing traffic but } \\
\text { volume of fluids } \\
\text { released low }\end{array}$ & $\begin{array}{l}\text { Low, occurs when } \\
\text { few if any } \\
\text { humpbacks are in } \\
\text { area. }\end{array}$ & $\begin{array}{l}\text { Low, starts at end } \\
\text { of breeding when } \\
\text { few if any } \\
\text { humpbacks in } \\
\text { area. }\end{array}$ & $\begin{array}{l}\text { Low; few in area during } \\
\text { construction; low volume } \\
\text { of contaminants expected }\end{array}$ & $\begin{array}{l}\text { Craig and } \\
\text { Herman } 1997\end{array}$ \\
\hline \multirow{3}{*}{$\begin{array}{l}\text { Construction and } \\
\text { installation of electrical } \\
\text { collector system, } \\
\text { mooring cables, anchors } \\
\text { or foundations, and } \\
\text { devices }\end{array}$} & $\begin{array}{l}\text { Noise and } \\
\text { vibration }\end{array}$ & $\begin{array}{l}\text { Avoidance, masking } \\
\text { of environmental } \\
\text { cues, song and } \\
\text { communication } \\
\text { signals }\end{array}$ & $\begin{array}{l}\text { Med-High, sound } \\
\text { travels far, } \\
\text { animals will hear } \\
\text { it outside of the } \\
\text { project area }\end{array}$ & $\begin{array}{l}\text { Low, occurs when } \\
\text { few if any } \\
\text { humpbacks are in } \\
\text { area. }\end{array}$ & $\begin{array}{l}\text { Low, starts at end } \\
\text { of breeding when } \\
\text { few if any } \\
\text { humpbacks in } \\
\text { area. }\end{array}$ & $\begin{array}{l}\text { Low; few in area during } \\
\text { construction; may } \\
\text { interfere with male } \\
\text { vocalization in spring } \\
\text { (March-April) }\end{array}$ & $\begin{array}{l}\text { Craig and } \\
\text { Herman 1997, } \\
\text { Richardson } \\
\text { and Wursig } \\
1997\end{array}$ \\
\hline & $\begin{array}{l}\text { Construction } \\
\text { lights, vessel } \\
\text { deck lights and } \\
\text { spotlights. }\end{array}$ & $\begin{array}{l}\text { Visual disorientation } \\
\text { could lead to } \\
\text { collision, could also } \\
\text { enhance foraging on } \\
\text { fish attracted to light }\end{array}$ & Unknown & $\begin{array}{l}\text { Low, occurs when } \\
\text { few if any } \\
\text { humpbacks are in } \\
\text { area. }\end{array}$ & $\begin{array}{l}\text { Low, starts at end } \\
\text { of breeding when } \\
\text { few if any } \\
\text { humpbacks in } \\
\text { area. }\end{array}$ & $\begin{array}{l}\text { Low; few in area during } \\
\text { construction; limited area } \\
\text { of effect }\end{array}$ & $\begin{array}{l}\text { Craig and } \\
\text { Herman } 1997\end{array}$ \\
\hline & $\begin{array}{l}\text { Oil/chemical } \\
\text { release }\end{array}$ & $\begin{array}{l}\text { Ingestion, breathing } \\
\text { exhaust fumes, } \\
\text { overall accumulation } \\
\text { of toxins. }\end{array}$ & $\begin{array}{l}\text { Med; increase in } \\
\text { use of chemicals, } \\
\text { but volume of } \\
\text { fluids released } \\
\text { low-med }\end{array}$ & $\begin{array}{l}\text { Low, occurs when } \\
\text { few if any } \\
\text { humpbacks are in } \\
\text { area. }\end{array}$ & $\begin{array}{l}\text { Low, starts at end } \\
\text { of breeding when } \\
\text { few if any } \\
\text { humpbacks in } \\
\text { area. }\end{array}$ & $\begin{array}{l}\text { Low; few in area during } \\
\text { construction; low volume } \\
\text { of contaminants expected }\end{array}$ & $\begin{array}{l}\text { Craig and } \\
\text { Herman } 1997\end{array}$ \\
\hline
\end{tabular}

\begin{tabular}{lrr}
\hline $\begin{array}{l}\text { Environmental Assessment for Siting Wave \& Tidal } \\
\text { Energy Projects }\end{array}$ & E-7 & H. T. Harvey \& Associates \\
24 November 2009
\end{tabular}


Appendix E- Effects of Small Commercial Pelamis Project at Makapu’u

\begin{tabular}{|c|c|c|c|c|c|c|c|}
\hline Project activity & Project action & $\begin{array}{l}\text { Description of } \\
\text { action's effect on } \\
\text { indicator }\end{array}$ & $\begin{array}{l}\text { Spatial exposure } \\
\text { to indicator (low, } \\
\text { med, high) }\end{array}$ & $\begin{array}{l}\text { Temporal } \\
\text { exposure to } \\
\text { indicator (low, } \\
\text { med, high) }\end{array}$ & $\begin{array}{l}\text { Effect's overlap } \\
\text { with critical life } \\
\text { stage, behavior, } \\
\text { habitat or } \\
\text { resource (low, } \\
\text { med, high) }\end{array}$ & $\begin{array}{l}\text { Overall risk to indicator } \\
\text { (low, med, high or } \\
\text { unknown) }\end{array}$ & Source(s) \\
\hline $\begin{array}{l}\text { Directional drilling, and } \\
\text { laying cable under/on } \\
\text { seabed }\end{array}$ & $\begin{array}{l}\text { Noise and } \\
\text { vibration }\end{array}$ & $\begin{array}{l}\text { Avoidance, masking } \\
\text { of environmental } \\
\text { cues, song and } \\
\text { communication } \\
\text { signals }\end{array}$ & $\begin{array}{l}\text { Med-High, sound } \\
\text { travels far, } \\
\text { animals will hear } \\
\text { it outside of the } \\
\text { project area }\end{array}$ & $\begin{array}{l}\text { Low, occurs when } \\
\text { few if any } \\
\text { humpbacks are in } \\
\text { area. }\end{array}$ & $\begin{array}{l}\text { Low, starts at end } \\
\text { of breeding when } \\
\text { few if any } \\
\text { humpbacks in } \\
\text { area. }\end{array}$ & $\begin{array}{l}\text { Low; few in area during } \\
\text { construction; may } \\
\text { interfere with male } \\
\text { vocalization in spring } \\
\text { (March-April); short } \\
\text { overall duration of } \\
\text { activity }\end{array}$ & $\begin{array}{l}\text { Craig and } \\
\text { Herman } 1997\end{array}$ \\
\hline \multicolumn{8}{|c|}{ Operation and Maintenance } \\
\hline \multirow{3}{*}{$\begin{array}{l}\text { Boat traffic. (Assuming } \\
\text { maintenance done } \\
\text { throughout the year) }\end{array}$} & Direct impact & Collision injuries & $\begin{array}{l}\text { Med, increase of } \\
\text { traffic traveling } \\
\text { along coast from } \\
\text { Honolulu to } \\
\text { project site }\end{array}$ & $\begin{array}{l}\text { Med during Dec- } \\
\text { May breeding } \\
\text { season, over life of } \\
\text { project }\end{array}$ & $\begin{array}{l}\text { Med, during Dec- } \\
\text { May breeding } \\
\text { season }\end{array}$ & $\begin{array}{l}\text { Med-high; boat traffic } \\
\text { likely to occur in area } \\
\text { frequented by } \\
\text { humpbacks; boat } \\
\text { collision/ strikes for } \\
\text { species one of the } \\
\text { highest among cetaceans }\end{array}$ & $\begin{array}{l}\text { Laist et al. } \\
\text { 2001, Van } \\
\text { Waerebeek } \\
\text { and Leaper } \\
2008\end{array}$ \\
\hline & $\begin{array}{l}\text { Oil/chemical } \\
\text { release }\end{array}$ & $\begin{array}{l}\text { Ingestion, breathing } \\
\text { exhaust fumes, } \\
\text { overall accumulation } \\
\text { of toxins. }\end{array}$ & $\begin{array}{l}\text { Low, increase in } \\
\text { traffic but the } \\
\text { volume of fluids } \\
\text { released low }\end{array}$ & $\begin{array}{l}\text { Med during Dec- } \\
\text { May breeding } \\
\text { season, over life of } \\
\text { project }\end{array}$ & $\begin{array}{l}\text { Med, during Dec- } \\
\text { May breeding } \\
\text { season }\end{array}$ & $\begin{array}{l}\text { Low; humpbacks } \\
\text { abundant in winter; } \\
\text { individuals show site } \\
\text { fidelity; low volume of } \\
\text { contaminants expected }\end{array}$ & $\begin{array}{l}\text { Craig and } \\
\text { Herman } 1997\end{array}$ \\
\hline & $\begin{array}{l}\text { Noise and } \\
\text { vibration }\end{array}$ & $\begin{array}{l}\text { Avoidance, masking } \\
\text { of environmental } \\
\text { cues, song and } \\
\text { communication } \\
\text { signals }\end{array}$ & $\begin{array}{l}\text { Med, sound } \\
\text { travels far, } \\
\text { animals will hear } \\
\text { it outside of the } \\
\text { project area }\end{array}$ & $\begin{array}{l}\text { Med during Dec- } \\
\text { May breeding } \\
\text { season, over life of } \\
\text { project }\end{array}$ & $\begin{array}{l}\text { Med, during Dec- } \\
\text { May breeding } \\
\text { season }\end{array}$ & $\begin{array}{l}\text { Med-high; humpbacks } \\
\text { abundant in winter; } \\
\text { individuals show site } \\
\text { fidelity; whales may flee } \\
\text { approaching boat while } \\
\text { still distant }\end{array}$ & $\begin{array}{l}\text { Craig and } \\
\text { Herman 1997, } \\
\text { Richardson } \\
\text { and Wursig } \\
1997\end{array}$ \\
\hline \multirow{2}{*}{$\begin{array}{l}\text { Operation of turbines or } \\
\text { other moving parts of } \\
\text { devices }\end{array}$} & $\begin{array}{l}\text { Moving device } \\
\text { parts }\end{array}$ & $\begin{array}{l}\text { Movement of device } \\
\text { and mooring lines } \\
\text { could injure animals }\end{array}$ & $\begin{array}{l}\text { Low, area of the } \\
\text { devices small and } \\
\text { interaction with } \\
\text { animals low }\end{array}$ & $\begin{array}{l}\text { Med, movement } \\
\text { continuous through } \\
\text { life of project but } \\
\text { whales there only } \\
\text { Dec-May }\end{array}$ & $\begin{array}{l}\text { Med during Dec- } \\
\text { May breeding } \\
\text { season when } \\
\text { calves present. }\end{array}$ & $\begin{array}{l}\text { Low; small area of } \\
\text { impact; large whales } \\
\text { unlikely to approach } \\
\text { moving parts close } \\
\text { enough to be at risk }\end{array}$ & $\begin{array}{l}\text { Mazzuca et } \\
\text { al. } 1998\end{array}$ \\
\hline & $\begin{array}{l}\text { Noise and } \\
\text { vibration }\end{array}$ & $\begin{array}{l}\text { Avoidance, masking } \\
\text { of environmental } \\
\text { cues, song and } \\
\text { communication } \\
\text { signals }\end{array}$ & $\begin{array}{l}\text { Med, sound } \\
\text { travels far, } \\
\text { animals will hear } \\
\text { it outside of the } \\
\text { project area }\end{array}$ & $\begin{array}{l}\text { Med during Dec- } \\
\text { May breeding } \\
\text { season, over life of } \\
\text { project }\end{array}$ & $\begin{array}{l}\text { Med during Dec- } \\
\text { May breeding } \\
\text { season }\end{array}$ & $\begin{array}{l}\text { Unknown; noise in area } \\
\text { frequented by } \\
\text { humpbacks; may interfere } \\
\text { with male vocalization; } \\
\text { potential long term } \\
\text { reduction in use of area }\end{array}$ & $\begin{array}{l}\text { Craig and } \\
\text { Herman 1997, } \\
\text { Richardson } \\
\text { and Wursig } \\
1997\end{array}$ \\
\hline
\end{tabular}

\begin{tabular}{|c|c|c|}
\hline $\begin{array}{l}\text { Environmental Assessment for Siting Wave \& Tidal } \\
\text { Energy Projects }\end{array}$ & E-8 & $\begin{array}{r}\text { H. T. Harvey \& Associates } \\
24 \text { November } 2009\end{array}$ \\
\hline
\end{tabular}


Appendix E- Effects of Small Commercial Pelamis Project at Makapu’u

\begin{tabular}{|c|c|c|c|c|c|c|c|}
\hline Project activity & Project action & $\begin{array}{l}\text { Description of } \\
\text { action's effect on } \\
\text { indicator }\end{array}$ & $\begin{array}{l}\text { Spatial exposure } \\
\text { to indicator (low, } \\
\text { med, high) }\end{array}$ & $\begin{array}{l}\text { Temporal } \\
\text { exposure to } \\
\text { indicator (low, } \\
\text { med, high) }\end{array}$ & $\begin{array}{l}\text { Effect's overlap } \\
\text { with critical life } \\
\text { stage, behavior, } \\
\text { habitat or } \\
\text { resource (low, } \\
\text { med, high) } \\
\end{array}$ & $\begin{array}{l}\text { Overall risk to indicator } \\
\text { (low, med, high or } \\
\text { unknown) }\end{array}$ & Source(s) \\
\hline $\begin{array}{l}\text { Structures in water } \\
\text { column and on seabed, } \\
\text { such as devices and } \\
\text { moorings and footings }\end{array}$ & Structure & $\begin{array}{l}\text { Collision, } \\
\text { entanglement with } \\
\text { devices or derelict } \\
\text { fishing gear }\end{array}$ & $\begin{array}{l}\text { Med-High, project } \\
\text { in coastal } \\
\text { breeding area } \\
\text { where whales } \\
\text { concentrate. }\end{array}$ & $\begin{array}{l}\text { Med during Dec- } \\
\text { May breeding } \\
\text { season, over life of } \\
\text { project }\end{array}$ & $\begin{array}{l}\text { Med-High, } \\
\text { humpbacks very } \\
\text { coastal during } \\
\text { breeding season, } \\
\text { esp. calves }\end{array}$ & $\begin{array}{l}\text { Low for adults; high risk } \\
\text { of entanglement for } \\
\text { young of the year }\end{array}$ & $\begin{array}{l}\text { Lien et al. } \\
\text { 1992, } \\
\text { Mazzuca et } \\
\text { al. 1998, } \\
\text { SIMON } 2008 \\
\end{array}$ \\
\hline $\begin{array}{l}\text { Electricity conduction } \\
\text { through cable }\end{array}$ & EMF & Unknown & $\begin{array}{l}\text { Unknown, cable } \\
\text { shielding and } \\
\text { burial provides } \\
\text { some attenuation }\end{array}$ & $\begin{array}{l}\text { High if an effect } \\
\text { electricity/EMF } \\
\text { generated over } \\
\text { project duration }\end{array}$ & Unknown & $\begin{array}{l}\text { Unknown; High } \\
\text { uncertainty about effects } \\
\text { on marine mammals }\end{array}$ & $\begin{array}{l}\text { Boehlert et al. } \\
2008\end{array}$ \\
\hline \multirow{2}{*}{$\begin{array}{l}\text { Structures on water's } \\
\text { surface }\end{array}$} & Structure & Collision & $\begin{array}{l}\text { Med-High, project } \\
\text { in coastal } \\
\text { breeding area } \\
\text { where whales } \\
\text { concentrate. }\end{array}$ & $\begin{array}{l}\text { Med during Dec- } \\
\text { May breeding } \\
\text { season, over life of } \\
\text { project }\end{array}$ & $\begin{array}{l}\text { Med-High, } \\
\text { humpbacks very } \\
\text { coastal during } \\
\text { breeding season, } \\
\text { esp. calves } \\
\end{array}$ & $\begin{array}{l}\text { Low; low potential for } \\
\text { collision with structure } \\
\text { because on water's } \\
\text { surface }\end{array}$ & $\begin{array}{l}\text { Lien et al. } \\
\text { 1992, } \\
\text { Mazzuca et } \\
\text { al. 1998, } \\
\text { SIMON } 2008 \\
\end{array}$ \\
\hline & $\begin{array}{l}\text { Navigation } \\
\text { lights }\end{array}$ & $\begin{array}{l}\text { Visual disorientation } \\
\text { could lead to } \\
\text { collision }\end{array}$ & $\begin{array}{l}\text { Low, device lights } \\
\text { of low intensity, } \\
\text { shielded, intended } \\
\text { for navigation } \\
\text { safety }\end{array}$ & $\begin{array}{l}\text { Med during Dec- } \\
\text { May breeding } \\
\text { season, over life of } \\
\text { project }\end{array}$ & $\begin{array}{l}\text { Med, humpbacks } \\
\text { very coastal during } \\
\text { breeding season, } \\
\text { esp. calves }\end{array}$ & $\begin{array}{l}\text { Low; low intensity of } \\
\text { lights; similar to existing } \\
\text { navigation lights on boats } \\
\text { and buoys encountered } \\
\text { around Hawaiian islands }\end{array}$ & $\begin{array}{l}\text { Baker and } \\
\text { Herman } 1981\end{array}$ \\
\hline \multicolumn{8}{|c|}{ Decommissioning } \\
\hline \multirow{3}{*}{ Boat traffic } & Direct impact & Collision injuries & $\begin{array}{l}\text { High, significant } \\
\text { increase of traffic } \\
\text { along coast from } \\
\text { Honolulu to } \\
\text { project site }\end{array}$ & $\begin{array}{l}\text { Low, occurring } \\
\text { when few if any } \\
\text { humpbacks are in } \\
\text { area. }\end{array}$ & $\begin{array}{l}\text { Low, few in area } \\
\text { May-Sept during } \\
\text { breeding, some } \\
\text { calves in early } \\
\text { May. }\end{array}$ & $\begin{array}{l}\text { Low; few in area during } \\
\text { deconstruction; } \\
\text { humpback whale-boat } \\
\text { collisions one of the } \\
\text { highest among marine } \\
\text { mammals }\end{array}$ & $\begin{array}{l}\text { Laist et al. } \\
\text { 2001, Van } \\
\text { Waerebeek } \\
\text { and Leaper } \\
2008\end{array}$ \\
\hline & $\begin{array}{l}\text { Noise and } \\
\text { vibration }\end{array}$ & $\begin{array}{l}\text { Avoidance, masking } \\
\text { of environmental } \\
\text { cues, song and } \\
\text { communication } \\
\text { signals } \\
\end{array}$ & $\begin{array}{l}\text { Med, sound } \\
\text { travels far, } \\
\text { animals will hear } \\
\text { it outside of the } \\
\text { project area }\end{array}$ & $\begin{array}{l}\text { Low, occurring } \\
\text { when few if any } \\
\text { humpbacks are in } \\
\text { area. }\end{array}$ & $\begin{array}{l}\text { Low, starts at end } \\
\text { of breeding when } \\
\text { few if any } \\
\text { humpbacks in } \\
\text { area. }\end{array}$ & $\begin{array}{l}\text { Low; few in area during } \\
\text { deconstruction; whales } \\
\text { may flee approaching } \\
\text { boat while still distant }\end{array}$ & $\begin{array}{l}\text { Craig and } \\
\text { Herman 1997, } \\
\text { Richardson and } \\
\text { Wursig } 1997\end{array}$ \\
\hline & $\begin{array}{l}\text { Oil/chemical } \\
\text { release }\end{array}$ & $\begin{array}{l}\text { Ingestion, breathing } \\
\text { exhaust fumes, } \\
\text { overall accumulation } \\
\text { of toxins. }\end{array}$ & $\begin{array}{l}\text { Low, significant } \\
\text { increase in } \\
\text { existing traffic but } \\
\text { volume of fluids } \\
\text { released low }\end{array}$ & $\begin{array}{l}\text { Low, occurring } \\
\text { when few if any } \\
\text { humpbacks are in } \\
\text { area. }\end{array}$ & $\begin{array}{l}\text { Low, starts at end } \\
\text { of breeding when } \\
\text { few if any } \\
\text { humpbacks in } \\
\text { area. }\end{array}$ & $\begin{array}{l}\text { Low; few in area during } \\
\text { deconstruction; low } \\
\text { volume of contaminants } \\
\text { expected }\end{array}$ & $\begin{array}{l}\text { Craig and } \\
\text { Herman } 1997\end{array}$ \\
\hline
\end{tabular}

Environmental Assessment for Siting Wave \& Tidal Energy Projects
E-9
H. T. Harvey \& Associates 24 November 2009 
Appendix E- Effects of Small Commercial Pelamis Project at Makapu'u

\begin{tabular}{|c|c|c|c|c|c|c|c|}
\hline Project activity & Project action & $\begin{array}{l}\text { Description of } \\
\text { action's effect on } \\
\text { indicator }\end{array}$ & $\begin{array}{l}\text { Spatial exposure } \\
\text { to indicator (low, } \\
\text { med, high) }\end{array}$ & $\begin{array}{l}\text { Temporal } \\
\text { exposure to } \\
\text { indicator (low, } \\
\text { med, high) }\end{array}$ & $\begin{array}{l}\text { Effect's overlap } \\
\text { with critical life } \\
\text { stage, behavior, } \\
\text { habitat or } \\
\text { resource (low, } \\
\text { med, high) }\end{array}$ & $\begin{array}{l}\text { Overall risk to indicator } \\
\text { (low, med, high or } \\
\text { unknown) }\end{array}$ & Source(s) \\
\hline \multirow{3}{*}{$\begin{array}{l}\text { Decommissioning of } \\
\text { structures on water's } \\
\text { surface or seabed }\end{array}$} & $\begin{array}{l}\text { Noise and } \\
\text { vibration }\end{array}$ & $\begin{array}{l}\text { Avoidance, masking } \\
\text { of environmental } \\
\text { cues, song and } \\
\text { communication } \\
\text { signals }\end{array}$ & $\begin{array}{l}\text { Med-High, sound } \\
\text { travels far, } \\
\text { animals will hear } \\
\text { it outside of the } \\
\text { project area }\end{array}$ & $\begin{array}{l}\text { Low, occurring } \\
\text { when few if any } \\
\text { humpbacks are in } \\
\text { area. }\end{array}$ & $\begin{array}{l}\text { Low, starts at end } \\
\text { of breeding when } \\
\text { few if any } \\
\text { humpbacks in } \\
\text { area. }\end{array}$ & $\begin{array}{l}\text { Low; few in area during } \\
\text { deconstruction; may } \\
\text { interfere with male } \\
\text { vocalization in spring } \\
\text { (March-April); potential } \\
\text { long term reduction in use } \\
\text { of areas }\end{array}$ & $\begin{array}{l}\text { Craig and } \\
\text { Herman } 1997, \\
\text { Richardson } \\
\text { and Wursig } \\
1997\end{array}$ \\
\hline & $\begin{array}{l}\text { Oil/chemical } \\
\text { release }\end{array}$ & $\begin{array}{l}\text { Visual disorientation } \\
\text { could lead to } \\
\text { collision, enhance } \\
\text { foraging on species } \\
\text { attracted to light }\end{array}$ & Unknown & $\begin{array}{l}\text { Low, occurring } \\
\text { when few if any } \\
\text { humpbacks are in } \\
\text { area. }\end{array}$ & $\begin{array}{l}\text { Low, starts at end } \\
\text { of breeding when } \\
\text { few if any } \\
\text { humpbacks in } \\
\text { area. }\end{array}$ & $\begin{array}{l}\text { Low; few in area during } \\
\text { deconstruction; volume } \\
\text { of contaminants expected } \\
\text { to be low }\end{array}$ & $\begin{array}{l}\text { Craig and } \\
\text { Herman } 1997\end{array}$ \\
\hline & $\begin{array}{l}\text { Construction } \\
\text { lights, vessel } \\
\text { deck lights and } \\
\text { spotlights. }\end{array}$ & $\begin{array}{l}\text { Ingestion, breathing } \\
\text { exhaust fumes, } \\
\text { overall accumulation } \\
\text { of toxins. }\end{array}$ & $\begin{array}{l}\text { Low, significant } \\
\text { increase in traffic } \\
\text { but low volume } \\
\text { of fluids released }\end{array}$ & $\begin{array}{l}\text { Low, occurring } \\
\text { when few if any } \\
\text { humpbacks are in } \\
\text { area. }\end{array}$ & $\begin{array}{l}\text { Low, starts at end } \\
\text { of breeding when } \\
\text { few if any } \\
\text { humpbacks }\end{array}$ & $\begin{array}{l}\text { Low; few in area during } \\
\text { deconstruction; limited } \\
\text { area of effect }\end{array}$ & $\begin{array}{l}\text { Craig and } \\
\text { Herman } 1997\end{array}$ \\
\hline
\end{tabular}

Environmental Assessment for Siting Wave \& Tidal Energy Projects
E-10

H. T. Harvey \& Associates 
Appendix E- Effects of Small Commercial Pelamis Project at Makapu'u

Potential effects on False Killer whales due to Small Commercial Pelamis system at Makupu'u site

\begin{tabular}{|c|c|c|c|c|c|c|c|}
\hline Project activity & Project action & $\begin{array}{l}\text { Description of } \\
\text { action's effect on } \\
\text { indicator }\end{array}$ & $\begin{array}{l}\text { Spatial exposure to } \\
\text { indicator (low, med, } \\
\text { high) }\end{array}$ & $\begin{array}{l}\text { Temporal } \\
\text { exposure to } \\
\text { indicator (low, } \\
\text { med, high) }\end{array}$ & $\begin{array}{l}\text { Effect's overlap } \\
\text { with critical life } \\
\text { stage, behavior, } \\
\text { habitat or resource } \\
\text { (low, med, high) } \\
\end{array}$ & $\begin{array}{l}\text { Overall risk to the } \\
\text { indicator (low, med, } \\
\text { high or unknown) }\end{array}$ & Source(s) \\
\hline \multicolumn{8}{|c|}{ Construction } \\
\hline \multirow{3}{*}{$\begin{array}{l}\text { Boat traffic, assumed } \\
\text { to occur day and night }\end{array}$} & Direct impact & Collision injuries & $\begin{array}{l}\text { High, significant } \\
\text { increase of boat } \\
\text { traffic traveling along } \\
\text { coast from Honolulu } \\
\text { to project site }\end{array}$ & $\begin{array}{l}\text { Low, occurring } \\
\text { over } 1 \text { to } 2 \\
\text { summers }\end{array}$ & $\begin{array}{l}\text { Low; species tends to } \\
\text { use deeper water than } \\
\text { project area; potential } \\
\text { overlap as boats travel } \\
\text { through near shore } \\
\text { deep water along SE } \\
\text { edge of Oahu }\end{array}$ & $\begin{array}{l}\text { Low; limited } \\
\text { potential for } \\
\text { interaction; false- } \\
\text { killer whale-boat } \\
\text { collisions apparently } \\
\text { rare }\end{array}$ & $\begin{array}{l}\text { Mobley Jr. et } \\
\text { al. 2000, Van } \\
\text { Waerebeek and } \\
\text { Leaper } 2008\end{array}$ \\
\hline & $\begin{array}{l}\text { Noise and } \\
\text { vibration }\end{array}$ & Avoidance of area & $\begin{array}{l}\text { High, significant } \\
\text { increase of traffic } \\
\text { traveling along coast } \\
\text { from Honolulu to } \\
\text { project site }\end{array}$ & $\begin{array}{l}\text { Low, occurring } \\
\text { over } 1 \text { to } 2 \\
\text { summers }\end{array}$ & $\begin{array}{l}\text { Low; false killer } \\
\text { whales tend to utilize } \\
\text { water deeper than } \\
\text { project area; may be } \\
\text { attracted to vessels in } \\
\text { search of hooked fish }\end{array}$ & $\begin{array}{l}\text { Low; not likely in } \\
\text { project area for } \\
\text { extended periods; } \\
\text { may travel through } \\
\text { area; likely in deep } \\
\text { water offshore }\end{array}$ & $\begin{array}{l}\text { HMRG [date } \\
\text { unknown], } \\
\text { Mobley Jr. et } \\
\text { al. } 2000\end{array}$ \\
\hline & $\begin{array}{l}\text { Oil/chemical } \\
\text { release }\end{array}$ & $\begin{array}{l}\text { Bioaccumulation, } \\
\text { direct ingestion of } \\
\text { toxins, breathing } \\
\text { exhaust fumes, } \\
\text { Assuming no } \\
\text { catastrophic }\end{array}$ & $\begin{array}{l}\text { Med, significant } \\
\text { increase in boat } \\
\text { traffic but the volume } \\
\text { of fluids released low }\end{array}$ & $\begin{array}{l}\text { Low, occurring } \\
\text { over } 1 \text { to } 2 \\
\text { summers }\end{array}$ & $\begin{array}{l}\text { Low; limited } \\
\text { potential for direct } \\
\text { exposure; }\end{array}$ & $\begin{array}{l}\text { Low; limited direct } \\
\text { exposure; false killer } \\
\text { whales show some of } \\
\text { highest levels of } \\
\text { accumulated toxins } \\
\text { among marine } \\
\text { mammals }\end{array}$ & $\begin{array}{l}\text { Stacey et al. } \\
1994\end{array}$ \\
\hline \multirow[t]{2}{*}{$\begin{array}{l}\text { Construction and } \\
\text { installation of } \\
\text { electrical collector } \\
\text { system, mooring } \\
\text { cables, anchors or } \\
\text { foundations, and } \\
\text { devices }\end{array}$} & $\begin{array}{l}\text { Noise and } \\
\text { vibration }\end{array}$ & Avoidance of area & $\begin{array}{l}\text { Low-med; false killer } \\
\text { whales likely to be } \\
\text { less sensitive to } \\
\text { lower frequency } \\
\text { noises (below 2kHz) }\end{array}$ & $\begin{array}{l}\text { Low, occurring } \\
\text { over } 1 \text { to } 2 \\
\text { summers }\end{array}$ & $\begin{array}{l}\text { Low; likely to be less } \\
\text { sensitive to lower } \\
\text { frequency noise; } \\
\text { higher frequency } \\
\text { sounds may cause } \\
\text { avoidance of project } \\
\text { area }\end{array}$ & $\begin{array}{l}\text { Low; area sub- } \\
\text { optimal habitat for } \\
\text { species; likely } \\
\text { audible to animals in } \\
\text { deep water } \\
\text { immediately offshore } \\
\text { of project site }\end{array}$ & $\begin{array}{l}\text { HMRG [date } \\
\text { unknown], } \\
\text { Mobley Jr. et } \\
\text { al. 2000, Nitta } \\
\text { and Henderson } \\
\text { 1993, Van } \\
\text { Waerebeek and } \\
\text { Leaper 2008 }\end{array}$ \\
\hline & $\begin{array}{l}\text { Oil/chemical } \\
\text { release }\end{array}$ & $\begin{array}{l}\text { Bioaccumulation, } \\
\text { direct ingestion of } \\
\text { toxins, breathing } \\
\text { exhaust fumes, } \\
\text { Assuming no } \\
\text { catastrophic spills. }\end{array}$ & $\begin{array}{l}\text { Med; significant } \\
\text { increase in use of } \\
\text { chemicals, solvents, } \\
\text { fuels, grease but the } \\
\text { volume of fluids } \\
\text { released low-med }\end{array}$ & $\begin{array}{l}\text { Low, occurring } \\
\text { over } 1 \text { to } 2 \\
\text { summers }\end{array}$ & $\begin{array}{l}\text { Low-med; limited } \\
\text { potential for direct } \\
\text { exposure }\end{array}$ & $\begin{array}{l}\text { Low; limited direct } \\
\text { exposure; false killer } \\
\text { whales show some of } \\
\text { the highest levels of } \\
\text { accumulated toxins } \\
\text { among marine } \\
\text { mammals }\end{array}$ & $\begin{array}{l}\text { Stacey et al. } \\
1994\end{array}$ \\
\hline
\end{tabular}

\begin{tabular}{|c|c|c|}
\hline $\begin{array}{l}\text { Environmental Assessment for Siting Wave \& Tidal } \\
\text { Energy Projects }\end{array}$ & E-11 & $\begin{array}{r}\text { H. T. Harvey \& Associates } \\
24 \text { November } 2009\end{array}$ \\
\hline
\end{tabular}


Appendix E- Effects of Small Commercial Pelamis Project at Makapu’u

\begin{tabular}{|c|c|c|c|c|c|c|c|}
\hline \multirow[t]{2}{*}{ Project activity } & Project action & $\begin{array}{l}\text { Description of } \\
\text { action's effect on } \\
\text { indicator }\end{array}$ & $\begin{array}{l}\text { Spatial exposure to } \\
\text { indicator (low, med, } \\
\text { high) }\end{array}$ & $\begin{array}{l}\text { Temporal } \\
\text { exposure to } \\
\text { indicator (low, } \\
\text { med, high) }\end{array}$ & $\begin{array}{l}\text { Effect's overlap } \\
\text { with critical life } \\
\text { stage, behavior, } \\
\text { habitat or resource } \\
\text { (low, med, high) } \\
\end{array}$ & $\begin{array}{l}\text { Overall risk to the } \\
\text { indicator (low, med, } \\
\text { high or unknown) }\end{array}$ & Source(s) \\
\hline & $\begin{array}{l}\text { Construction } \\
\text { lights, vessel } \\
\text { deck lights and } \\
\text { spotlights. }\end{array}$ & $\begin{array}{l}\text { Visual disorientation } \\
\text { could lead to } \\
\text { collision, could also } \\
\text { enhance foraging on } \\
\text { species attracted to } \\
\text { light }\end{array}$ & $\begin{array}{l}\text { Med to low extent } \\
\text { beyond project site. }\end{array}$ & $\begin{array}{l}\text { Low, occurring } \\
\text { over } 1 \text { to } 2 \\
\text { summers }\end{array}$ & $\begin{array}{l}\text { Low; species } \\
\text { presumably nocturnal } \\
\text { foragers; provide } \\
\text { benefit by } \\
\text { concentrating prey }\end{array}$ & $\begin{array}{l}\text { Low; species } \\
\text { infrequently in } \\
\text { project area; if lights } \\
\text { attract prey could } \\
\text { increase risk of } \\
\text { interactions }\end{array}$ & $\begin{array}{l}\text { Mobley Jr. et } \\
\text { al. } 2000 \text {, Stacey } \\
\text { et al. } 1994\end{array}$ \\
\hline $\begin{array}{l}\text { Directional drilling, } \\
\text { and laying cable } \\
\text { under/on seabed }\end{array}$ & $\begin{array}{l}\text { Noise and } \\
\text { vibration from } \\
\text { drilling and } \\
\text { support vessels }\end{array}$ & $\begin{array}{l}\text { Avoidance of } \\
\text { nearshore area and } \\
\text { support vessels }\end{array}$ & $\begin{array}{l}\text { Low; false killer } \\
\text { whales less sensitive } \\
\text { to lower frequency } \\
\text { noise }\end{array}$ & $\begin{array}{l}\text { Low, occurring } \\
\text { over } 1 \text { summer }\end{array}$ & $\begin{array}{l}\text { Low; effect area } \\
\text { relatively small and } \\
\text { close to shore }\end{array}$ & $\begin{array}{l}\text { Low; species rarely } \\
\text { observed in nearshore } \\
\text { waters around Hawaii }\end{array}$ & $\begin{array}{l}\text { Mobley Jr. et } \\
\text { al. 2000, } \\
\text { Thomas et al. } \\
1988\end{array}$ \\
\hline \multicolumn{8}{|c|}{ Operations and maintenance } \\
\hline \multirow{3}{*}{$\begin{array}{l}\text { Boat traffic. } \\
\text { (Assuming } \\
\text { maintenance done } \\
\text { throughout the year) }\end{array}$} & Direct impact & Collision injuries & $\begin{array}{l}\text { Low-Med; increase } \\
\text { in boat traffic } \\
\text { traveling along coast } \\
\text { from Honolulu to } \\
\text { project site }\end{array}$ & $\begin{array}{l}\text { med, occurring } \\
\text { at low frequency } \\
\text { over project } \\
\text { duration }\end{array}$ & $\begin{array}{l}\text { Low; species tend to } \\
\text { use deeper waters than } \\
\text { project area; potential } \\
\text { overlap as vessels } \\
\text { travel through } \\
\text { nearshore deep water } \\
\text { along SE edge of Oahu }\end{array}$ & $\begin{array}{l}\text { Low; limited } \\
\text { potential for } \\
\text { interaction; false } \\
\text { killer whale-boat } \\
\text { collisions apparently } \\
\text { rare }\end{array}$ & $\begin{array}{l}\text { Mobley Jr. et } \\
\text { al. 2000, Van } \\
\text { Waerebeek and } \\
\text { Leaper } 2008\end{array}$ \\
\hline & $\begin{array}{l}\text { Oil/chemical } \\
\text { release }\end{array}$ & $\begin{array}{l}\text { Bioaccumulation, } \\
\text { direct ingestion of } \\
\text { toxins, breathing } \\
\text { exhaust fumes, } \\
\text { Assuming no } \\
\text { catastrophic spills. }\end{array}$ & $\begin{array}{l}\text { Med; significant } \\
\text { increase in use of } \\
\text { chemicals, solvents, } \\
\text { fuels, grease but the } \\
\text { volume of fluids } \\
\text { released low-med }\end{array}$ & $\begin{array}{l}\text { High; occurring } \\
\text { over project } \\
\text { duration }\end{array}$ & $\begin{array}{l}\text { Low; limited } \\
\text { potential for direct } \\
\text { exposure }\end{array}$ & $\begin{array}{l}\text { Low; limited direct } \\
\text { exposure; species has } \\
\text { one of highest levels } \\
\text { of accumulated } \\
\text { toxins among marine } \\
\text { mammals }\end{array}$ & $\begin{array}{l}\text { Stacey et al. } \\
1994\end{array}$ \\
\hline & $\begin{array}{l}\text { Noise and } \\
\text { vibration }\end{array}$ & Avoidance of area & $\begin{array}{l}\text { Low-Med, increased } \\
\text { boat traffic traveling } \\
\text { along coast from } \\
\text { Honolulu to project } \\
\text { site }\end{array}$ & $\begin{array}{l}\text { Low, occurring } \\
\text { at low frequency } \\
\text { over project } \\
\text { duration }\end{array}$ & $\begin{array}{l}\text { Low; species tends to } \\
\text { use water deeper then } \\
\text { project area; may be } \\
\text { attracted to vessels }\end{array}$ & $\begin{array}{l}\text { Low; species not likely } \\
\text { in project area but may } \\
\text { travel through; likely } \\
\text { audible in deep water } \\
\text { offshore of project site }\end{array}$ & $\begin{array}{l}\text { HMRG [date } \\
\text { unknown], } \\
\text { Mobley Jr. et } \\
\text { al. } 2000\end{array}$ \\
\hline $\begin{array}{l}\text { Operation of turbines } \\
\text { or other moving parts } \\
\text { of devices }\end{array}$ & $\begin{array}{l}\text { Moving device } \\
\text { parts }\end{array}$ & $\begin{array}{l}\text { Movement of } \\
\text { devices and mooring } \\
\text { lines could injure } \\
\text { animals }\end{array}$ & $\begin{array}{l}\text { Low-Med; project } \\
\text { location likely to be } \\
\text { traveled through }\end{array}$ & $\begin{array}{l}\text { High, the } \\
\text { movement } \\
\text { occurs over } \\
\text { project duration }\end{array}$ & $\begin{array}{l}\text { Low; false killer } \\
\text { whales tend to use } \\
\text { water deeper then } \\
\text { project area }\end{array}$ & $\begin{array}{l}\text { Low; moving parts } \\
\text { readily avoided; } \\
\text { expected infrequently } \\
\text { in project area }\end{array}$ & $\begin{array}{l}\text { Mobley Jr. et } \\
\text { al. } 2000\end{array}$ \\
\hline
\end{tabular}

\begin{tabular}{|c|c|c|}
\hline $\begin{array}{l}\text { Environmental Assessment for Siting Wave \& Tidal } \\
\text { Energy Projects }\end{array}$ & E-12 & $\begin{array}{r}\text { H. T. Harvey \& Associates } \\
24 \text { November } 2009\end{array}$ \\
\hline
\end{tabular}


Appendix E- Effects of Small Commercial Pelamis Project at Makapu’u

\begin{tabular}{|c|c|c|c|c|c|c|c|}
\hline Project activity & Project action & $\begin{array}{l}\text { Description of } \\
\text { action's effect on } \\
\text { indicator }\end{array}$ & $\begin{array}{l}\text { Spatial exposure to } \\
\text { indicator (low, med, } \\
\text { high) }\end{array}$ & $\begin{array}{l}\text { Temporal } \\
\text { exposure to } \\
\text { indicator (low, } \\
\text { med, high) }\end{array}$ & $\begin{array}{l}\text { Effect's overlap } \\
\text { with critical life } \\
\text { stage, behavior, } \\
\text { habitat or resource } \\
\text { (low, med, high) } \\
\end{array}$ & $\begin{array}{l}\text { Overall risk to the } \\
\text { indicator (low, med, } \\
\text { high or unknown) }\end{array}$ & Source(s) \\
\hline & $\begin{array}{l}\text { Noise and } \\
\text { vibration }\end{array}$ & Avoidance of area & $\begin{array}{l}\text { Noise likely to be } \\
\text { low frequency and } \\
\text { not likely to invoke } \\
\text { behavioral response } \\
\text { by false killer whales } \\
\text { unless very close to } \\
\text { equipment }\end{array}$ & $\begin{array}{l}\text { High, noise } \\
\text { level would be } \\
\text { constant over } \\
\text { project duration }\end{array}$ & $\begin{array}{l}\text { Low; likely less } \\
\text { sensitive to lower } \\
\text { frequency noise } \\
\text { (below } 2 \mathrm{kHz} \text { ); higher } \\
\text { frequency sounds } \\
\text { may cause avoidance } \\
\text { of project area }\end{array}$ & $\begin{array}{l}\text { Low; area sub- } \\
\text { optimal habitat for } \\
\text { species; noise likely } \\
\text { audible in deep water } \\
\text { immediately offshore } \\
\text { of project site }\end{array}$ & $\begin{array}{l}\text { HMRG [date } \\
\text { unknown], } \\
\text { Mobley Jr. et } \\
\text { al. 2000, Nitta } \\
\text { and Henderson } \\
\text { 1993, Thomas } \\
\text { et al. 1988, Van } \\
\text { Waerebeek and } \\
\text { Leaper } 2008\end{array}$ \\
\hline $\begin{array}{l}\text { Structures in water } \\
\text { column and on seabed, } \\
\text { such as devices and } \\
\text { moorings and footings }\end{array}$ & Structure & $\begin{array}{l}\text { Biofouling inverts } \\
\text { could create artificial } \\
\text { reef and attract fish; } \\
\text { entanglement with } \\
\text { devices or derelict } \\
\text { fishing gear }\end{array}$ & $\begin{array}{l}\text { Low, structures likely } \\
\text { to be avoided as false } \\
\text { killer whales travel } \\
\text { through project area }\end{array}$ & $\begin{array}{l}\text { High, structure } \\
\text { present over } \\
\text { project duration }\end{array}$ & $\begin{array}{l}\text { Low; structures } \\
\text { should be readily } \\
\text { avoided }\end{array}$ & $\begin{array}{l}\text { Low; may be } \\
\text { attracted to fish } \\
\text { aggregations; risk of } \\
\text { entanglement if } \\
\text { derelict gear, cables, } \\
\text { lines present }\end{array}$ & $\begin{array}{l}\text { Mobley Jr. et } \\
\text { al. } 2000 \text {, Relini } \\
\text { et al. } 2000\end{array}$ \\
\hline $\begin{array}{l}\text { Electricity conduction } \\
\text { through cable }\end{array}$ & EMF & Unknown & $\begin{array}{l}\text { Unknown, cable } \\
\text { shielding and burial } \\
\text { provides some } \\
\text { attenuation }\end{array}$ & $\begin{array}{l}\text { High if effect; } \\
\text { electricity/ EMF } \\
\text { generated over } \\
\text { project duration }\end{array}$ & $\begin{array}{l}\text { Unknown; false killer } \\
\text { whales tend to utilize } \\
\text { water deeper then } \\
\text { cable area }\end{array}$ & $\begin{array}{l}\text { Unknown; High } \\
\text { uncertainty about } \\
\text { effects on marine } \\
\text { mammals }\end{array}$ & $\begin{array}{l}\text { Boehlert et al. } \\
2008\end{array}$ \\
\hline \multirow{2}{*}{$\begin{array}{l}\text { Structures on water's } \\
\text { surface }\end{array}$} & Structure & Collision & $\begin{array}{l}\text { Low, structures likely } \\
\text { to be avoided as false } \\
\text { killer whales travel } \\
\text { through project area }\end{array}$ & $\begin{array}{l}\text { High, structure } \\
\text { present over } \\
\text { project duration }\end{array}$ & $\begin{array}{l}\text { Low; structures } \\
\text { should be readily } \\
\text { avoided; }\end{array}$ & $\begin{array}{l}\text { Low; may enhance } \\
\text { foraging for large fish } \\
\text { attracted to artificial } \\
\text { reef; species expected } \\
\text { to occur infrequently } \\
\text { in project area }\end{array}$ & $\begin{array}{l}\text { Mobley Jr. et } \\
\text { al. } 2000 \text {, Relini } \\
\text { et al. } 2000\end{array}$ \\
\hline & $\begin{array}{l}\text { Navigation } \\
\text { lights }\end{array}$ & $\begin{array}{l}\text { Visual disorientation } \\
\text { could lead to } \\
\text { collision, but could } \\
\text { also enhance } \\
\text { foraging on species } \\
\text { attracted to light } \\
\end{array}$ & $\begin{array}{l}\text { Low, device lights of } \\
\text { low intensity, } \\
\text { shielded, intended } \\
\text { for navigation safety }\end{array}$ & $\begin{array}{l}\text { High, lights } \\
\text { shining over } \\
\text { project duration }\end{array}$ & $\begin{array}{l}\text { Low; false killer } \\
\text { whales presumably } \\
\text { are nocturnal foragers } \\
\text { but navigational } \\
\text { lights low intensity }\end{array}$ & $\begin{array}{l}\text { Low; unlikely lights } \\
\text { will attract fish } \\
\text { aggregations due to } \\
\text { low intensity; species } \\
\text { expected to be rare at } \\
\text { project site }\end{array}$ & $\begin{array}{l}\text { Stacey et al. } \\
1994\end{array}$ \\
\hline \multicolumn{8}{|c|}{ Decommissioning } \\
\hline Boat traffic & Direct impact & Collision injuries & $\begin{array}{l}\text { High, significant } \\
\text { increase of boat } \\
\text { traffic traveling along } \\
\text { coast from Honolulu } \\
\text { to project site }\end{array}$ & $\begin{array}{l}\text { Low, occurring } \\
\text { over } 1 \text { to } 2 \\
\text { summers }\end{array}$ & $\begin{array}{l}\text { Low; species uses } \\
\text { water deeper than } \\
\text { project area; potential } \\
\text { overlap as boats travel } \\
\text { through near shore } \\
\text { deep water SE of Oahu }\end{array}$ & $\begin{array}{l}\text { Low; limited } \\
\text { potential for } \\
\text { interaction; false- } \\
\text { killer whale-boat } \\
\text { collisions apparently } \\
\text { rare }\end{array}$ & $\begin{array}{l}\text { Mobley Jr. et } \\
\text { al. 2000, Van } \\
\text { Waerebeek and } \\
\text { Leaper } 2008\end{array}$ \\
\hline
\end{tabular}

\begin{tabular}{|c|c|c|}
\hline $\begin{array}{l}\text { Environmental Assessment for Siting Wave \& Tidal } \\
\text { Energy Projects }\end{array}$ & E-13 & $\begin{array}{r}\text { H. T. Harvey \& Associates } \\
24 \text { November } 2009\end{array}$ \\
\hline
\end{tabular}


Appendix E- Effects of Small Commercial Pelamis Project at Makapu’u

\begin{tabular}{|c|c|c|c|c|c|c|c|}
\hline Project activity & Project action & $\begin{array}{l}\text { Description of } \\
\text { action's effect on } \\
\text { indicator }\end{array}$ & $\begin{array}{l}\text { Spatial exposure to } \\
\text { indicator (low, med, } \\
\text { high) }\end{array}$ & $\begin{array}{l}\text { Temporal } \\
\text { exposure to } \\
\text { indicator (low, } \\
\text { med, high) }\end{array}$ & $\begin{array}{l}\text { Effect's overlap } \\
\text { with critical life } \\
\text { stage, behavior, } \\
\text { habitat or resource } \\
\text { (low, med, high) } \\
\end{array}$ & $\begin{array}{l}\text { Overall risk to the } \\
\text { indicator (low, med, } \\
\text { high or unknown) }\end{array}$ & Source(s) \\
\hline & $\begin{array}{l}\text { Oil/chemical } \\
\text { release }\end{array}$ & $\begin{array}{l}\text { Bioaccumulation } \\
\text { (forage on large } \\
\text { fish), direct } \\
\text { ingestion of toxins, } \\
\text { breathing exhaust } \\
\text { fumes, Assuming no } \\
\text { catastrophic }\end{array}$ & $\begin{array}{l}\text { Med, significant } \\
\text { increase in boat } \\
\text { traffic but the volume } \\
\text { of fluids released low }\end{array}$ & $\begin{array}{l}\text { Low, occurring } \\
\text { over } 1 \text { to } 2 \\
\text { summers }\end{array}$ & $\begin{array}{l}\text { Low; limited } \\
\text { potential for direct } \\
\text { exposure }\end{array}$ & $\begin{array}{l}\text { Low; limited direct } \\
\text { exposure; false killer } \\
\text { whales show some of } \\
\text { highest levels of } \\
\text { accumulated toxins } \\
\text { among marine } \\
\text { mammals }\end{array}$ & $\begin{array}{l}\text { Stacey et al. } \\
1994\end{array}$ \\
\hline & $\begin{array}{l}\text { Noise and } \\
\text { vibration }\end{array}$ & Avoidance of area & $\begin{array}{l}\text { High, significant } \\
\text { increase of traffic } \\
\text { traveling along coast } \\
\text { from Honolulu to } \\
\text { project site }\end{array}$ & $\begin{array}{l}\text { Low, occurring } \\
\text { over } 1 \text { to } 2 \\
\text { summers }\end{array}$ & $\begin{array}{l}\text { Low; false killer } \\
\text { whales tend to utilize } \\
\text { water deeper then } \\
\text { project area; may be } \\
\text { attracted to vessels in } \\
\text { search of hooked fish }\end{array}$ & $\begin{array}{l}\text { Low; area sub- } \\
\text { optimal habitat for } \\
\text { species; noise likely } \\
\text { audible in deep water } \\
\text { immediately offshore } \\
\text { of project site }\end{array}$ & $\begin{array}{l}\text { HMRG [date } \\
\text { unknown], } \\
\text { Mobley Jr. et } \\
\text { al. 2000, Nitta } \\
\text { and Henderson } \\
\text { 1993, Van } \\
\text { Waerebeek and } \\
\text { Leaper } 2008\end{array}$ \\
\hline \multirow{3}{*}{$\begin{array}{l}\text { Decommissioning of } \\
\text { structures on water's } \\
\text { surface or seabed }\end{array}$} & $\begin{array}{l}\text { Noise and } \\
\text { vibration }\end{array}$ & Avoidance of area & $\begin{array}{l}\text { Low-med; false killer } \\
\text { whales likely to be } \\
\text { less sensitive to } \\
\text { lower frequency } \\
\text { noises (below } 2 \mathrm{kHz} \text { ) }\end{array}$ & $\begin{array}{l}\text { Low, occurring } \\
\text { over } 1 \text { to } 2 \\
\text { summers }\end{array}$ & $\begin{array}{l}\text { Low; likely to be less } \\
\text { sensitive to lower } \\
\text { frequency noise; } \\
\text { higher frequency } \\
\text { sounds may cause } \\
\text { avoidance of project } \\
\text { area }\end{array}$ & $\begin{array}{l}\text { Low; area sub- } \\
\text { optimal habitat for } \\
\text { species; noise likely } \\
\text { audible in deep water } \\
\text { immediately offshore } \\
\text { of project site }\end{array}$ & $\begin{array}{l}\text { HMRG [date } \\
\text { unknown], } \\
\text { Mobley Jr. et } \\
\text { al. 2000, Nitta } \\
\text { and Henderson } \\
\text { 1993, Thomas } \\
\text { et al. 1988, Van } \\
\text { Waerebeek and } \\
\text { Leaper } 2008 \\
\end{array}$ \\
\hline & $\begin{array}{l}\text { Deconstruction } \\
\text { lights, vessel } \\
\text { deck lights and } \\
\text { spotlights. }\end{array}$ & $\begin{array}{l}\text { Visual disorientation } \\
\text { could lead to } \\
\text { collision, could also } \\
\text { enhance foraging on } \\
\text { species attracted to } \\
\text { light }\end{array}$ & $\begin{array}{l}\text { Med to low extent } \\
\text { beyond project site. }\end{array}$ & $\begin{array}{l}\text { Low, occurring } \\
\text { over } 1 \text { to } 2 \\
\text { summers }\end{array}$ & $\begin{array}{l}\text { Low; species } \\
\text { presumably nocturnal } \\
\text { foragers; provide } \\
\text { benefit by } \\
\text { concentrating prey }\end{array}$ & $\begin{array}{l}\text { Low; lights could } \\
\text { attract prey and false } \\
\text { killer whales; if lights } \\
\text { attract prey could } \\
\text { may increase risk of } \\
\text { interactions } \\
\end{array}$ & $\begin{array}{l}\text { Mobley Jr. et } \\
\text { al. } 2000 \text {, Stacey } \\
\text { et al. } 1994\end{array}$ \\
\hline & $\begin{array}{l}\text { Oil/chemical } \\
\text { release }\end{array}$ & $\begin{array}{l}\text { Bioaccumulation, } \\
\text { direct ingestion of } \\
\text { toxins, breathing } \\
\text { exhaust fumes, } \\
\text { Assuming no } \\
\text { catastrophic spills. }\end{array}$ & $\begin{array}{l}\text { Med; significant } \\
\text { increase in use of } \\
\text { chemicals, solvents, } \\
\text { fuels, grease but the } \\
\text { volume of fluids } \\
\text { released low-med }\end{array}$ & $\begin{array}{l}\text { Low, occurring } \\
\text { over } 1 \text { to } 2 \\
\text { summers }\end{array}$ & $\begin{array}{l}\text { Low; limited direct } \\
\text { exposure; med } \\
\text { bioaccumulation } \\
\text { effect }\end{array}$ & $\begin{array}{l}\text { Low; limited direct } \\
\text { exposure; false killer } \\
\text { whales show some of } \\
\text { the highest levels of } \\
\text { accumulated toxins } \\
\text { among marine } \\
\text { mammals }\end{array}$ & $\begin{array}{l}\text { Stacey et al. } \\
1994\end{array}$ \\
\hline
\end{tabular}

\begin{tabular}{|c|c|c|}
\hline $\begin{array}{l}\text { Environmental Assessment for Siting Wave \& Tidal } \\
\text { Energy Projects }\end{array}$ & E-14 & $\begin{array}{r}\text { H. T. Harvey \& Associates } \\
24 \text { November } 2009\end{array}$ \\
\hline
\end{tabular}


Appendix E- Effects of Small Commercial Pelamis Project at Makapu’u

Potential effects on Bottlenose dolphins due to Small Commercial Pelamis system at Makupu'u site

\begin{tabular}{|c|c|c|c|c|c|c|c|}
\hline Project activity & Project action & $\begin{array}{l}\text { Description of } \\
\text { action's effect on } \\
\text { indicator }\end{array}$ & $\begin{array}{l}\text { Spatial exposure tp } \\
\text { indicator(low, med, } \\
\text { high) }\end{array}$ & $\begin{array}{l}\text { Temporal } \\
\text { exposure to } \\
\text { indicator (low, } \\
\text { med, high) }\end{array}$ & $\begin{array}{l}\text { Effect's overlap } \\
\text { with critical life } \\
\text { stage, behavior, } \\
\text { habitat or resource } \\
\text { (low, med, high) }\end{array}$ & $\begin{array}{l}\text { Overall risk to } \\
\text { indicator (low, med, } \\
\text { high, unknown) }\end{array}$ & Source(s) \\
\hline \multicolumn{8}{|c|}{ Construction } \\
\hline \multirow{3}{*}{$\begin{array}{l}\text { Boat traffic, assumed } \\
\text { to occur day and night }\end{array}$} & Direct impact & Collision injuries & $\begin{array}{l}\text { High, significant } \\
\text { increase of boat } \\
\text { traffic traveling along } \\
\text { coast from Honolulu } \\
\text { to project site }\end{array}$ & $\begin{array}{l}\text { Low, occurring } \\
\text { over } 1 \text { to } 2 \\
\text { summers }\end{array}$ & $\begin{array}{l}\text { Low; effects } \\
\text { amplified if vessels } \\
\text { are close to shore or } \\
\text { as dolphins travel to } \\
\text { and from deeper } \\
\text { waters }\end{array}$ & $\begin{array}{l}\text { Med-high; bottlenose } \\
\text { dolphin-boat collision } \\
\text { rate one of the highest } \\
\text { among dolphin } \\
\text { species; risk high if } \\
\text { boat travel along coast } \\
\text { from Honolulu to site }\end{array}$ & $\begin{array}{l}\text { Mobley Jr. et } \\
\text { al. 2000, Van } \\
\text { Waerebeek } \\
\text { and Leaper } \\
2008\end{array}$ \\
\hline & $\begin{array}{l}\text { Noise and } \\
\text { vibration }\end{array}$ & Avoidance of area & $\begin{array}{l}\text { High, significant } \\
\text { increase of traffic } \\
\text { traveling along coast } \\
\text { from Honolulu to } \\
\text { project site }\end{array}$ & $\begin{array}{l}\text { Low, occurring } \\
\text { over } 1 \text { to } 2 \\
\text { summers }\end{array}$ & $\begin{array}{l}\text { Low; effects } \\
\text { amplified if vessels } \\
\text { close to shore or as } \\
\text { dolphins travel to and } \\
\text { from deeper waters; } \\
\text { may not react to } \\
\text { boats unless } \\
\text { harassed. }\end{array}$ & $\begin{array}{l}\text { Low; significant } \\
\text { increase in boat } \\
\text { traffic; increase risk if } \\
\text { boats travel along } \\
\text { shore from Honolulu } \\
\text { to project site }\end{array}$ & $\begin{array}{l}\text { Irvine et al. } \\
\text { 1981, Mobley } \\
\text { Jr. et al. 2000, } \\
\text { Tyack et al. } \\
\text { 1993, Van } \\
\text { Waerebeek } \\
\text { and Leaper } \\
2008 \\
\end{array}$ \\
\hline & $\begin{array}{l}\text { Oil/chemical } \\
\text { release }\end{array}$ & $\begin{array}{l}\text { Ingestion, overall } \\
\text { accumulation of } \\
\text { toxins, breathing } \\
\text { exhaust fumes, } \\
\text { Assuming no } \\
\text { catastrophic spills. }\end{array}$ & $\begin{array}{l}\text { Med, significant } \\
\text { increase in boat } \\
\text { traffic but the volume } \\
\text { of fluids released low }\end{array}$ & $\begin{array}{l}\text { Low, occurring } \\
\text { over } 1 \text { to } 2 \\
\text { summers }\end{array}$ & $\begin{array}{l}\text { Low; possible contact } \\
\text { as dolphins pass } \\
\text { through project area } \\
\text { traveling to and from } \\
\text { deeper waters }\end{array}$ & $\begin{array}{l}\text { Low; low contaminant } \\
\text { volume expected; } \\
\text { exposure along coast } \\
\text { or traveling through } \\
\text { project sites on way } \\
\text { to offshore waters }\end{array}$ & $\begin{array}{l}\text { Mobley Jr. et } \\
\text { al. } 2000\end{array}$ \\
\hline \multirow[t]{2}{*}{$\begin{array}{l}\text { Construction and } \\
\text { installation of } \\
\text { electrical collector } \\
\text { system, mooring } \\
\text { cables, anchors or } \\
\text { foundations, and } \\
\text { devices }\end{array}$} & $\begin{array}{l}\text { Noise and } \\
\text { vibration }\end{array}$ & $\begin{array}{l}\text { Avoidance, masking } \\
\text { environmental cues. } \\
\text { Reduction of hearing } \\
\text { sensitivity (TTS), } \\
\text { resulting in } \\
\text { potential increase in } \\
\text { predation }\end{array}$ & $\begin{array}{l}\text { Low-med; dolphins } \\
\text { likely to be less } \\
\text { sensitive to lower } \\
\text { frequency noises } \\
\text { unless they are within } \\
\text { the immediate } \\
\text { project area }\end{array}$ & $\begin{array}{l}\text { Low, occurring } \\
\text { over } 1 \text { to } 2 \\
\text { summers }\end{array}$ & $\begin{array}{l}\text { Low; likely to be less } \\
\text { sensitive to lower } \\
\text { frequency noise; } \\
\text { higher frequency } \\
\text { sounds may cause } \\
\text { avoidance of project } \\
\text { area }\end{array}$ & $\begin{array}{l}\text { Low; activities far } \\
\text { enough from shore to } \\
\text { reduce noise impacts } \\
\text { on species while } \\
\text { nearshore; potential } \\
\text { temporary impact to } \\
\text { hearing if dolphins in } \\
\text { area }\end{array}$ & $\begin{array}{l}\text { Irvine et al. } \\
\text { 1981, Tyack et } \\
\text { al. 1993, } \\
\text { Schlundt et al. } \\
2000\end{array}$ \\
\hline & $\begin{array}{l}\text { Oil/chemical } \\
\text { release }\end{array}$ & $\begin{array}{l}\text { Ingestion, overall } \\
\text { accumulation of } \\
\text { toxins, breathing } \\
\text { exhaust fumes, } \\
\text { Assuming no } \\
\text { catastrophic spills. }\end{array}$ & $\begin{array}{l}\text { Med; significant } \\
\text { increase in use of } \\
\text { chemicals, solvents, } \\
\text { fuels, grease but the } \\
\text { volume of fluids } \\
\text { released low-med }\end{array}$ & $\begin{array}{l}\text { Low, occurring } \\
\text { over } 1 \text { to } 2 \\
\text { summers }\end{array}$ & $\begin{array}{l}\text { Low; possible contact } \\
\text { as dolphins pass } \\
\text { through project area } \\
\text { traveling to and from } \\
\text { deeper waters }\end{array}$ & $\begin{array}{l}\text { Low; exposure risk as } \\
\text { dolphins travel } \\
\text { through and around } \\
\text { the project area; low } \\
\text { level of contaminants } \\
\text { expected }\end{array}$ & $\begin{array}{l}\text { Mobley Jr. et } \\
\text { al. } 2000\end{array}$ \\
\hline
\end{tabular}

\begin{tabular}{lrr}
\hline $\begin{array}{l}\text { Environmental Assessment for Siting Wave \& Tidal } \\
\text { Energy Projects }\end{array}$ & E-15 & H. T. Harvey \& Associates \\
24 November 2009
\end{tabular}


Appendix E- Effects of Small Commercial Pelamis Project at Makapu’u

\begin{tabular}{|c|c|c|c|c|c|c|c|}
\hline \multirow[t]{2}{*}{ Project activity } & Project action & $\begin{array}{l}\text { Description of } \\
\text { action's effect on } \\
\text { indicator } \\
\end{array}$ & $\begin{array}{l}\text { Spatial exposure tp } \\
\text { indicator(low, med, } \\
\text { high) }\end{array}$ & $\begin{array}{l}\text { Temporal } \\
\text { exposure to } \\
\text { indicator (low, } \\
\text { med, high) }\end{array}$ & $\begin{array}{l}\text { Effect's overlap } \\
\text { with critical life } \\
\text { stage, behavior, } \\
\text { habitat or resource } \\
\text { (low, med, high) }\end{array}$ & $\begin{array}{l}\text { Overall risk to } \\
\text { indicator (low, med, } \\
\text { high, unknown) }\end{array}$ & Source(s) \\
\hline & $\begin{array}{l}\text { Construction } \\
\text { lights, vessel } \\
\text { deck lights and } \\
\text { spotlights. }\end{array}$ & $\begin{array}{l}\text { Visual disorientation } \\
\text { could lead to } \\
\text { collision, could also } \\
\text { enhance foraging on } \\
\text { species attracted to } \\
\text { light }\end{array}$ & $\begin{array}{l}\text { Med to low extent } \\
\text { beyond project site. }\end{array}$ & $\begin{array}{l}\text { Low, occurring } \\
\text { over } 1 \text { to } 2 \\
\text { summers }\end{array}$ & $\begin{array}{l}\text { Low, Bottlenose } \\
\text { dolphins do forage at } \\
\text { night but home range } \\
\left(\sim 125 \mathrm{~km}^{2}\right) \text { is much } \\
\text { larger than project } \\
\text { area }\end{array}$ & $\begin{array}{l}\text { Low; area of effect } \\
\text { low relative to range; } \\
\text { may attract dolphins } \\
\text { pursuing prey } \\
\text { attracted to lights }\end{array}$ & $\begin{array}{l}\text { Day and } \\
\text { Defran 1995, } \\
\text { Klatsky et al. } \\
2007 \text {, } \\
\text { Reynolds III et } \\
\text { al. } 2000\end{array}$ \\
\hline $\begin{array}{l}\text { Directional drilling, } \\
\text { and laying cable } \\
\text { under/on seabed }\end{array}$ & $\begin{array}{l}\text { Noise and } \\
\text { vibration from } \\
\text { drilling and } \\
\text { support vessels }\end{array}$ & $\begin{array}{l}\text { Avoidance of } \\
\text { nearshore area and } \\
\text { support vessels; and } \\
\text { area around support } \\
\text { vessels moving } \\
\text { offshore }\end{array}$ & $\begin{array}{l}\text { Low; bottlenose } \\
\text { dolphins less } \\
\text { sensitive to lower } \\
\text { frequency noise }\end{array}$ & $\begin{array}{l}\text { Low, occurring } \\
\text { over } 1 \text { summer }\end{array}$ & $\begin{array}{l}\text { Low; effect area } \\
\text { small relative to large } \\
\text { home range }(\sim 125 \\
\left.\mathrm{km}^{2}\right)\end{array}$ & $\begin{array}{l}\text { Low; may displace } \\
\text { dolphins form } \\
\text { nearshore area; } \\
\text { increased potential of } \\
\text { propeller strike from } \\
\text { boats in small area } \\
\text { nearshore }\end{array}$ & $\begin{array}{l}\text { Reynolds III et } \\
\text { al. 2000, } \\
\text { Tyack et al. } \\
\text { 1993, Van } \\
\text { Waerebeek } \\
\text { and Leaper } \\
2008\end{array}$ \\
\hline \multicolumn{8}{|c|}{ Operations and maintenance } \\
\hline \multirow{3}{*}{$\begin{array}{l}\text { Boat traffic. } \\
\text { (Assuming } \\
\text { maintenance done } \\
\text { throughout the year) }\end{array}$} & Direct impact & Collision injuries & $\begin{array}{l}\text { Low-Med; increase } \\
\text { in boat traffic } \\
\text { traveling along coast } \\
\text { from Honolulu to } \\
\text { project site }\end{array}$ & $\begin{array}{l}\text { med, occurring } \\
\text { at low frequency } \\
\text { over project } \\
\text { duration }\end{array}$ & $\begin{array}{l}\text { Low; effects } \\
\text { amplified if vessels } \\
\text { are close to shore or } \\
\text { as dolphins travel to } \\
\text { and from deeper } \\
\text { waters }\end{array}$ & $\begin{array}{l}\text { Low; moderate } \\
\text { increase in boat traffic; } \\
\text { bottlenose dolphin- } \\
\text { boat collision rate one } \\
\text { of highest among } \\
\text { dolphin spp.; risk } \\
\text { elevated if traffic along } \\
\text { coast from Honolulu to } \\
\text { project site }\end{array}$ & $\begin{array}{l}\text { Mobley Jr. et } \\
\text { al. 2000, Van } \\
\text { Waerebeek } \\
\text { and Leaper } \\
2008\end{array}$ \\
\hline & $\begin{array}{l}\text { Oil/chemical } \\
\text { release }\end{array}$ & $\begin{array}{l}\text { Ingestion, } \\
\text { accumulation of } \\
\text { toxins, breathing } \\
\text { exhaust fumes } \\
\text { Assuming no } \\
\text { catastrophic spills. }\end{array}$ & $\begin{array}{l}\text { Low; increased boat } \\
\text { traffic but the volume } \\
\text { of fluids released low }\end{array}$ & $\begin{array}{l}\text { Low, occurring } \\
\text { at low frequency } \\
\text { over project } \\
\text { duration }\end{array}$ & $\begin{array}{l}\text { Dolphins may pass } \\
\text { through project area } \\
\text { traveling to and from } \\
\text { foraging areas } \\
\text { increasing potential } \\
\text { for exposure }\end{array}$ & $\begin{array}{l}\text { Low; contaminant } \\
\text { volume expected to be } \\
\text { low; possible exposure } \\
\text { along coast or while } \\
\text { traveling to offshore } \\
\text { waters }\end{array}$ & $\begin{array}{l}\text { Mobley Jr. et } \\
\text { al. } 2000\end{array}$ \\
\hline & $\begin{array}{l}\text { Noise and } \\
\text { vibration }\end{array}$ & Avoidance of area & $\begin{array}{l}\text { Low-Med, increased } \\
\text { boat traffic traveling } \\
\text { along coast from } \\
\text { Honolulu to project } \\
\text { site }\end{array}$ & $\begin{array}{l}\text { Low, occurring } \\
\text { at low frequency } \\
\text { over project } \\
\text { duration }\end{array}$ & $\begin{array}{l}\text { Low; effects amplified } \\
\text { if boats near shore or } \\
\text { as dolphins travel to } \\
\text { and from deeper } \\
\text { waters; may not react } \\
\text { to sound unless } \\
\text { harassed }\end{array}$ & $\begin{array}{l}\text { Low; moderate } \\
\text { increase in boat } \\
\text { traffic; potential } \\
\text { impact greater if } \\
\text { boats travel along } \\
\text { shore from Honolulu } \\
\text { to project site }\end{array}$ & $\begin{array}{l}\text { Irvine et al. } \\
1981 \text {, Mobley } \\
\text { Jr. et al. 2000, } \\
\text { Tyack et al. } \\
1993\end{array}$ \\
\hline
\end{tabular}

\begin{tabular}{|c|c|c|}
\hline $\begin{array}{l}\text { Environmental Assessment for Siting Wave \& Tidal } \\
\text { Energy Projects }\end{array}$ & E-16 & $\begin{array}{r}\text { H. T. Harvey \& Associates } \\
24 \text { November } 2009\end{array}$ \\
\hline
\end{tabular}


Appendix E- Effects of Small Commercial Pelamis Project at Makapu’u

\begin{tabular}{|c|c|c|c|c|c|c|c|}
\hline Project activity & Project action & $\begin{array}{l}\text { Description of } \\
\text { action's effect on } \\
\text { indicator }\end{array}$ & $\begin{array}{l}\text { Spatial exposure tp } \\
\text { indicator(low, med, } \\
\text { high) }\end{array}$ & $\begin{array}{l}\text { Temporal } \\
\text { exposure to } \\
\text { indicator (low, } \\
\text { med, high) }\end{array}$ & $\begin{array}{l}\text { Effect's overlap } \\
\text { with critical life } \\
\text { stage, behavior, } \\
\text { habitat or resource } \\
\text { (low, med, high) }\end{array}$ & $\begin{array}{l}\text { Overall risk to } \\
\text { indicator (low, med, } \\
\text { high, unknown) }\end{array}$ & Source(s) \\
\hline \multirow[b]{2}{*}{$\begin{array}{l}\text { Operation of turbines } \\
\text { or other moving parts } \\
\text { of devices }\end{array}$} & $\begin{array}{l}\text { Moving device } \\
\text { parts }\end{array}$ & $\begin{array}{l}\text { Movement of } \\
\text { devices and mooring } \\
\text { lines could injure } \\
\text { animals }\end{array}$ & $\begin{array}{l}\text { Low-Med; project } \\
\text { location likely to be } \\
\text { traveled through }\end{array}$ & $\begin{array}{l}\text { High, the } \\
\text { movement } \\
\text { occurs over } \\
\text { project duration }\end{array}$ & $\begin{array}{l}\text { Low; area occupied } \\
\text { by moving parts } \\
\text { small relative to the } \\
\text { animal's home range } \\
\left(\sim 125 \mathrm{~km}^{2}\right)\end{array}$ & $\begin{array}{l}\text { Low; dolphins } \\
\text { sensitive to } \\
\text { surrounding envir., } \\
\text { unlikely to approach } \\
\text { close enough }\end{array}$ & $\begin{array}{l}\text { Reynolds III et } \\
\text { al. } 2000\end{array}$ \\
\hline & $\begin{array}{l}\text { Noise and } \\
\text { vibration }\end{array}$ & Avoidance of area & $\begin{array}{l}\text { Noise likely to be } \\
\text { low frequency and } \\
\text { not likely to invoke } \\
\text { behavioral response } \\
\text { by bottlenose } \\
\text { dolphins unless very } \\
\text { close to equipment }\end{array}$ & $\begin{array}{l}\text { High, noise } \\
\text { level would be } \\
\text { constant over } \\
\text { project duration }\end{array}$ & $\begin{array}{l}\text { Low; frequency and } \\
\text { sound pressure levels } \\
\text { expected to be below } \\
\text { hearing sensitivity of } \\
\text { dolphins unless they } \\
\text { are very close to } \\
\text { equipment }\end{array}$ & $\begin{array}{l}\text { Low; not expected to } \\
\text { be audible to dolphins } \\
\text { in nearshore waters; } \\
\text { likely detectable to } \\
\text { dolphins within area } \\
\text { immediately around } \\
\text { machinery }\end{array}$ & $\begin{array}{l}\text { Tyack et al. } \\
1993\end{array}$ \\
\hline $\begin{array}{l}\text { Structures in water } \\
\text { column and on seabed, } \\
\text { such as devices and } \\
\text { moorings and footings }\end{array}$ & Structure & $\begin{array}{l}\text { Biofouling } \\
\text { invertebrates could } \\
\text { create artificial reef } \\
\text { and attract fish; } \\
\text { entanglement with } \\
\text { devices or derelict } \\
\text { fishing gear }\end{array}$ & $\begin{array}{l}\text { Low, structures likely } \\
\text { to be avoided as } \\
\text { dolphins travel to and } \\
\text { from deeper waters }\end{array}$ & $\begin{array}{l}\text { High, structure } \\
\text { present over } \\
\text { project duration }\end{array}$ & $\begin{array}{l}\text { Low; structures } \\
\text { should be readily } \\
\text { avoided; may } \\
\text { enhance foraging } \\
\text { opportunities }\end{array}$ & $\begin{array}{l}\text { Low; dolphins highly } \\
\text { sensitive to } \\
\text { surrounding } \\
\text { environment; structure } \\
\text { readily avoided }\end{array}$ & $\begin{array}{l}\text { Mobley Jr. et } \\
\text { al. 2000, } \\
\text { Relini et al. } \\
2000\end{array}$ \\
\hline $\begin{array}{l}\text { Electricity conduction } \\
\text { through cable }\end{array}$ & EMF & Unknown & $\begin{array}{l}\text { Unknown, cable } \\
\text { shielding and burial } \\
\text { provides some } \\
\text { attenuation }\end{array}$ & $\begin{array}{l}\text { High if an } \\
\text { effect, } \\
\text { electricity/ EMF } \\
\text { generated over } \\
\text { project duration } \\
\end{array}$ & $\begin{array}{l}\text { Unknown; large } \\
\text { home range may } \\
\text { reduce extent of } \\
\text { effects is there are } \\
\text { any }\end{array}$ & $\begin{array}{l}\text { Unknown; High } \\
\text { uncertainty about } \\
\text { effects on marine } \\
\text { mammals }\end{array}$ & $\begin{array}{l}\text { Boehlert et al. } \\
2008\end{array}$ \\
\hline \multirow[b]{2}{*}{$\begin{array}{l}\text { Structures on water's } \\
\text { surface }\end{array}$} & Structure & Collision & $\begin{array}{l}\text { Low, structures likely } \\
\text { to be avoided as } \\
\text { dolphins travel to and } \\
\text { from deeper waters }\end{array}$ & $\begin{array}{l}\text { High, structure } \\
\text { present over } \\
\text { project duration }\end{array}$ & $\begin{array}{l}\text { Low; structures } \\
\text { readily avoided }\end{array}$ & $\begin{array}{l}\text { Low; structure readily } \\
\text { avoided }\end{array}$ & $\begin{array}{l}\text { Mobley Jr. et } \\
\text { al. 2000, } \\
\text { Relini et al. } \\
2000\end{array}$ \\
\hline & $\begin{array}{l}\text { Navigation } \\
\text { lights }\end{array}$ & $\begin{array}{l}\text { Visual disorientation } \\
\text { could lead to } \\
\text { collision, could } \\
\text { enhance foraging on } \\
\text { species attracted to } \\
\text { light }\end{array}$ & $\begin{array}{l}\text { Low, device lights of } \\
\text { low intensity, } \\
\text { shielded, intended } \\
\text { for navigation safety }\end{array}$ & $\begin{array}{l}\text { High, lights } \\
\text { shining over } \\
\text { project duration }\end{array}$ & $\begin{array}{l}\text { Low, species forages } \\
\text { at night but home } \\
\text { range }\left(\sim 125 \mathrm{~km}^{2}\right) \\
\text { much larger than } \\
\text { project area }\end{array}$ & $\begin{array}{l}\text { Low; area of effect } \\
\text { low relative to range; } \\
\text { lights not unlike other } \\
\text { common navigational } \\
\text { light sources }\end{array}$ & $\begin{array}{l}\text { Day and Defran } \\
\text { 1995, Klatsky et } \\
\text { al. 2007, } \\
\text { Reynolds III et } \\
\text { al. } 2000\end{array}$ \\
\hline
\end{tabular}

Environmental Assessment for Siting Wave \& Tidal Energy Projects
E-17

H. T. Harvey \& Associates

24 November 2009 
Appendix E- Effects of Small Commercial Pelamis Project at Makapu’u

\begin{tabular}{|c|c|c|c|c|c|c|c|}
\hline Project activity & Project action & $\begin{array}{l}\text { Description of } \\
\text { action's effect on } \\
\text { indicator }\end{array}$ & $\begin{array}{l}\text { Spatial exposure tp } \\
\text { indicator(low, med, } \\
\text { high) }\end{array}$ & $\begin{array}{l}\text { Temporal } \\
\text { exposure to } \\
\text { indicator (low, } \\
\text { med, high) }\end{array}$ & $\begin{array}{l}\text { Effect's overlap } \\
\text { with critical life } \\
\text { stage, behavior, } \\
\text { habitat or resource } \\
\text { (low, med, high) }\end{array}$ & $\begin{array}{l}\text { Overall risk to } \\
\text { indicator (low, med, } \\
\text { high, unknown) }\end{array}$ & Source(s) \\
\hline \multirow{3}{*}{ Boat traffic } & Direct impact & Collision injuries & $\begin{array}{l}\text { High, significant } \\
\text { increase of boat } \\
\text { traffic traveling along } \\
\text { coast from Honolulu } \\
\text { to project site }\end{array}$ & $\begin{array}{l}\text { Low, occurring } \\
\text { over } 1 \text { to } 2 \\
\text { summers }\end{array}$ & $\begin{array}{l}\text { Low; effects } \\
\text { amplified if vessels } \\
\text { are close to shore or } \\
\text { as dolphins travel to } \\
\text { and from deeper } \\
\text { waters }\end{array}$ & $\begin{array}{l}\text { Med-high; bottlenose } \\
\text { dolphin boat collision } \\
\text { rate one of highest } \\
\text { among dolphin spp; } \\
\text { risk high if travel along } \\
\text { coast to project site }\end{array}$ & $\begin{array}{l}\text { Mobley Jr. et } \\
\text { al. 2000, Van } \\
\text { Waerebeek } \\
\text { and Leaper } \\
2008\end{array}$ \\
\hline & $\begin{array}{l}\text { Oil/chemical } \\
\text { release }\end{array}$ & $\begin{array}{l}\text { Ingestion, } \\
\text { accumulation of } \\
\text { toxins, breathing } \\
\text { exhaust fumes, } \\
\text { Assuming no } \\
\text { catastrophic spills. }\end{array}$ & $\begin{array}{l}\text { Med, significant } \\
\text { increase in boat } \\
\text { traffic but the volume } \\
\text { of fluids released low }\end{array}$ & $\begin{array}{l}\text { Low, occurring } \\
\text { over } 1 \text { to } 2 \\
\text { summers }\end{array}$ & $\begin{array}{l}\text { Low; possible contact } \\
\text { as dolphins pass } \\
\text { through project area } \\
\text { traveling to and from } \\
\text { deeper waters }\end{array}$ & $\begin{array}{l}\text { Low; low contaminant } \\
\text { volume expected; } \\
\text { exposure along coast } \\
\text { or traveling through } \\
\text { project sites }\end{array}$ & $\begin{array}{l}\text { Mobley Jr. et } \\
\text { al. } 2000\end{array}$ \\
\hline & $\begin{array}{l}\text { Noise and } \\
\text { vibration }\end{array}$ & Avoidance of area & $\begin{array}{l}\text { High, significant } \\
\text { increase of traffic } \\
\text { traveling along coast } \\
\text { from Honolulu to } \\
\text { project site }\end{array}$ & $\begin{array}{l}\text { Low, occurring } \\
\text { over } 1 \text { to } 2 \\
\text { summers }\end{array}$ & $\begin{array}{l}\text { Low; effects } \\
\text { amplified if boats } \\
\text { near shore or as } \\
\text { dolphins travel to and } \\
\text { from deeper waters }\end{array}$ & $\begin{array}{l}\text { Low; increase risk if } \\
\text { boats travel along } \\
\text { shore from Honolulu } \\
\text { to project site; may } \\
\text { not react to boats } \\
\text { unless harassed. }\end{array}$ & $\begin{array}{l}\text { Irvine et al. } \\
\text { 1981, Mobley Jr } \\
\text { et al. 2000, } \\
\text { Tyack et al. } \\
\text { 1993, Van } \\
\text { Waerebeek and } \\
\text { Leaper } 2008\end{array}$ \\
\hline \multirow{3}{*}{$\begin{array}{l}\text { Decommissioning of } \\
\text { structures on water's } \\
\text { surface or seabed }\end{array}$} & $\begin{array}{l}\text { Noise and } \\
\text { vibration }\end{array}$ & $\begin{array}{l}\text { Avoidance, masking } \\
\text { environmental cues. } \\
\text { Reduction of hearing } \\
\text { sensitivity (TTS), } \\
\text { could increase } \\
\text { predation }\end{array}$ & $\begin{array}{l}\text { Low-med; dolphins } \\
\text { likely to be less } \\
\text { sensitive to lower } \\
\text { frequency noises } \\
\text { unless within project } \\
\text { area }\end{array}$ & $\begin{array}{l}\text { Low, occurring } \\
\text { over } 1 \text { to } 2 \\
\text { summers }\end{array}$ & $\begin{array}{l}\text { Low; likely less } \\
\text { sensitive to lower } \\
\text { freq noise; higher } \\
\text { freq sounds may } \\
\text { cause avoidance of } \\
\text { project area }\end{array}$ & $\begin{array}{l}\text { Low; far enough from } \\
\text { shore to reduce noise } \\
\text { impacts; potential } \\
\text { impact if dolphins } \\
\text { remain in project area }\end{array}$ & $\begin{array}{l}\text { Irvine et al. } \\
\text { 1981, } \\
\text { Schlundt et al. } \\
\text { 2000, Tyack et } \\
\text { al. } 1993\end{array}$ \\
\hline & $\begin{array}{l}\text { Deconstruction } \\
\text { lights, vessel } \\
\text { deck lights and } \\
\text { spotlights. }\end{array}$ & $\begin{array}{l}\text { Visual disorientation } \\
\text { could lead to } \\
\text { collision, could also } \\
\text { enhance foraging on } \\
\text { species attracted to } \\
\text { light }\end{array}$ & $\begin{array}{l}\text { Med to low extent } \\
\text { beyond project site. }\end{array}$ & $\begin{array}{l}\text { Low, occurring } \\
\text { over } 1 \text { to } 2 \\
\text { summers }\end{array}$ & $\begin{array}{l}\text { Low, species forages } \\
\text { at night but home } \\
\text { range }\left(\sim 125 \mathrm{~km}^{2}\right) \\
\text { much larger than } \\
\text { project area }\end{array}$ & $\begin{array}{l}\text { Low; area of effect } \\
\text { low relative to range; } \\
\text { may attract dolphins } \\
\text { pursuing prey } \\
\text { attracted to lights }\end{array}$ & $\begin{array}{l}\text { Day and } \\
\text { Defran 1995, } \\
\text { Klatsky et al. } \\
2007, \\
\text { Reynolds III et } \\
\text { al. } 2000 \\
\end{array}$ \\
\hline & $\begin{array}{l}\text { Oil/chemical } \\
\text { release }\end{array}$ & $\begin{array}{l}\text { Ingestion, } \\
\text { accumulation of } \\
\text { toxins, breathing } \\
\text { exhaust fumes; } \\
\text { Assuming no } \\
\text { catastrophic spills. }\end{array}$ & $\begin{array}{l}\text { Med; significant } \\
\text { increase in use of } \\
\text { chemicals, but } \\
\text { volume of fluids } \\
\text { released low-med }\end{array}$ & $\begin{array}{l}\text { Low, occurring } \\
\text { over } 1 \text { to } 2 \\
\text { summers }\end{array}$ & $\begin{array}{l}\text { Low; possible contact } \\
\text { as dolphins pass } \\
\text { through project area } \\
\text { traveling to and from } \\
\text { deeper waters }\end{array}$ & $\begin{array}{l}\text { Low; exposure risk as } \\
\text { dolphins travel } \\
\text { through project area; } \\
\text { low level of } \\
\text { contaminants expected }\end{array}$ & $\begin{array}{l}\text { Mobley Jr. et } \\
\text { al. } 2000\end{array}$ \\
\hline
\end{tabular}

\begin{tabular}{|c|c|c|}
\hline $\begin{array}{l}\text { Environmental Assessment for Siting Wave \& Tidal } \\
\text { Energy Projects }\end{array}$ & E-18 & $\begin{array}{r}\text { H. T. Harvey \& Associates } \\
24 \text { November } 2009\end{array}$ \\
\hline
\end{tabular}


Appendix E- Effects of Small Commercial Pelamis Project at Makapu’u

Potential effects on Hawaiian spinner dolphin due to Small Commercial Pelamis at Makupu'u site

\begin{tabular}{|c|c|c|c|c|c|c|c|}
\hline Project activity & Project action & $\begin{array}{l}\text { Description of } \\
\text { action's effect on } \\
\text { indicator }\end{array}$ & $\begin{array}{l}\text { Spatial exposure to } \\
\text { indicator (low, med, } \\
\text { high) }\end{array}$ & $\begin{array}{l}\text { Temporal } \\
\text { exposure to } \\
\text { indicator (low, } \\
\text { med, high) }\end{array}$ & $\begin{array}{l}\text { Effect's overlap } \\
\text { with critical life } \\
\text { stage, behavior, } \\
\text { habitat or resource } \\
\text { (low, med, high) } \\
\end{array}$ & $\begin{array}{l}\text { Overall risk to } \\
\text { indicator (low, med, } \\
\text { high, unknown) }\end{array}$ & Source(s) \\
\hline \multicolumn{8}{|c|}{ Construction } \\
\hline \multirow{3}{*}{$\begin{array}{l}\text { Boat traffic, assumed } \\
\text { to occur day and night }\end{array}$} & Direct impact & Collision injuries & $\begin{array}{l}\text { High, significant } \\
\text { increase of traffic } \\
\text { traveling along coast } \\
\text { from Honolulu to } \\
\text { project site }\end{array}$ & $\begin{array}{l}\text { Low, occurring } \\
\text { over } 1 \text { to } 2 \\
\text { summers }\end{array}$ & $\begin{array}{l}\text { Low; effects } \\
\text { amplified if vessels } \\
\text { close to shore or as } \\
\text { dolphins travel to and } \\
\text { from offshore } \\
\text { foraging areas }\end{array}$ & $\begin{array}{l}\text { Low; collisions } \\
\text { between spinner } \\
\text { dolphins and boats } \\
\text { relatively infrequent }\end{array}$ & $\begin{array}{l}\text { Camargo and } \\
\text { Bellini 2007, } \\
\text { Van } \\
\text { Waerebeek } \\
\text { and Leaper } \\
2008\end{array}$ \\
\hline & $\begin{array}{l}\text { Noise and } \\
\text { vibration }\end{array}$ & $\begin{array}{l}\text { Repeated disturbance } \\
\text { may disrupt daytime } \\
\text { resting period and } \\
\text { cause avoidance of } \\
\text { area. }\end{array}$ & $\begin{array}{l}\text { Med, significant } \\
\text { increase in boat } \\
\text { traffic }\end{array}$ & $\begin{array}{l}\text { Low, occurring } \\
\text { over } 1 \text { to } 2 \\
\text { summers }\end{array}$ & $\begin{array}{l}\text { Low; effects amplified } \\
\text { if vessels close to } \\
\text { shore or as dolphins } \\
\text { travel between resting } \\
\text { and foraging areas }\end{array}$ & $\begin{array}{l}\text { Low; risk increased if } \\
\text { boats travel close to } \\
\text { nearshore resting areas }\end{array}$ & $\begin{array}{l}\text { Danil et al. } \\
2005\end{array}$ \\
\hline & $\begin{array}{l}\text { Oil/chemical } \\
\text { release }\end{array}$ & $\begin{array}{l}\text { Ingestion, } \\
\text { accumulation of } \\
\text { toxins, breathing } \\
\text { exhaust fumes, } \\
\text { Assuming no } \\
\text { catastrophic spills. }\end{array}$ & $\begin{array}{l}\text { Med, significant } \\
\text { increase in boat } \\
\text { traffic but the volume } \\
\text { of fluids released low }\end{array}$ & $\begin{array}{l}\text { Low, occurring } \\
\text { over } 1 \text { to } 2 \\
\text { summers }\end{array}$ & $\begin{array}{l}\text { Low; possible contact } \\
\text { as dolphins pass } \\
\text { through project area } \\
\text { traveling to and from } \\
\text { foraging areas }\end{array}$ & $\begin{array}{l}\text { Low; volume of } \\
\text { contaminants expected } \\
\text { to be low; area of } \\
\text { potential impact } \\
\text { relatively small } \\
\text { compared to range }\end{array}$ & $\begin{array}{l}\text { Norris et al. } \\
1994\end{array}$ \\
\hline \multirow[t]{2}{*}{$\begin{array}{l}\text { Construction and } \\
\text { installation of } \\
\text { electrical collector } \\
\text { system, mooring } \\
\text { cables, anchors or } \\
\text { foundations, and } \\
\text { devices }\end{array}$} & $\begin{array}{l}\text { Noise and } \\
\text { vibration }\end{array}$ & $\begin{array}{l}\text { Avoidance, masks } \\
\text { environmental cues. } \\
\text { Reduction of hearing } \\
\text { sensitivity (TTS), } \\
\text { resulting in } \\
\text { potential increase in } \\
\text { predation }\end{array}$ & $\begin{array}{l}\text { Low-med; dolphins } \\
\text { likely to be less } \\
\text { sensitive to low } \\
\text { frequency unless they } \\
\text { are within the } \\
\text { immediate project } \\
\text { area; }\end{array}$ & $\begin{array}{l}\text { Low, occurring } \\
\text { over } 1 \text { to } 2 \\
\text { summers }\end{array}$ & $\begin{array}{l}\text { Low; likely to be less } \\
\text { sensitive to noise in } \\
\text { low frequencies; } \\
\text { higher frequency may } \\
\text { cause avoidance of } \\
\text { project area }\end{array}$ & $\begin{array}{l}\text { Low; construction } \\
\text { activities far enough } \\
\text { from shore to reduce } \\
\text { impacts on resting } \\
\text { dolphins; transit } \\
\text { through/past project } \\
\text { area regular but brief }\end{array}$ & $\begin{array}{l}\text { Norris et al. } \\
1994\end{array}$ \\
\hline & $\begin{array}{l}\text { Oil/chemical } \\
\text { release }\end{array}$ & $\begin{array}{l}\text { Ingestion, } \\
\text { accumulation of } \\
\text { toxins, breathing } \\
\text { exhaust fumes, } \\
\text { Assuming no } \\
\text { catastrophic spills. }\end{array}$ & $\begin{array}{l}\text { Med; significant } \\
\text { increase in use of } \\
\text { chemicals, solvents, } \\
\text { fuels, grease but the } \\
\text { volume of fluids } \\
\text { released low-med }\end{array}$ & $\begin{array}{l}\text { Low, occurring } \\
\text { over } 1 \text { to } 2 \\
\text { summers }\end{array}$ & $\begin{array}{l}\text { Dolphins may pass } \\
\text { through project area } \\
\text { traveling to and from } \\
\text { foraging areas } \\
\text { increasing potential } \\
\text { for exposure }\end{array}$ & $\begin{array}{l}\text { Low; volume of } \\
\text { contaminants expected } \\
\text { to be low; area of } \\
\text { potential impact } \\
\text { relatively small } \\
\text { compared to range }\end{array}$ & $\begin{array}{l}\text { Norris et al. } \\
1994\end{array}$ \\
\hline
\end{tabular}

\begin{tabular}{lrr}
\hline $\begin{array}{l}\text { Environmental Assessment for Siting Wave \& Tidal } \\
\text { Energy Projects }\end{array}$ & E-19 & H. T. Harvey \& Associates \\
24 November 2009
\end{tabular}


Appendix E- Effects of Small Commercial Pelamis Project at Makapu’u

\begin{tabular}{|c|c|c|c|c|c|c|c|}
\hline Project activity & Project action & $\begin{array}{l}\text { Description of } \\
\text { action's effect on } \\
\text { indicator }\end{array}$ & $\begin{array}{l}\text { Spatial exposure to } \\
\text { indicator (low, med, } \\
\text { high) }\end{array}$ & $\begin{array}{l}\text { Temporal } \\
\text { exposure to } \\
\text { indicator (low, } \\
\text { med, high) }\end{array}$ & $\begin{array}{l}\text { Effect's overlap } \\
\text { with critical life } \\
\text { stage, behavior, } \\
\text { habitat or resource } \\
\text { (low, med, high) }\end{array}$ & $\begin{array}{l}\text { Overall risk to } \\
\text { indicator (low, med, } \\
\text { high, unknown) }\end{array}$ & Source(s) \\
\hline & $\begin{array}{l}\text { Construction } \\
\text { lights, vessel } \\
\text { deck lights and } \\
\text { spotlights. }\end{array}$ & $\begin{array}{l}\text { Visual disorientation } \\
\text { could lead to } \\
\text { collision, but could } \\
\text { also enhance } \\
\text { foraging on species } \\
\text { attracted to light }\end{array}$ & $\begin{array}{l}\text { Med to low extent } \\
\text { beyond project site. }\end{array}$ & $\begin{array}{l}\text { Low, occurring } \\
\text { over } 1 \text { to } 2 \\
\text { summers }\end{array}$ & $\begin{array}{l}\text { Spinner dolphins are } \\
\text { nocturnal foragers } \\
\text { but typically forage } \\
\text { further offshore than } \\
\text { project location }\end{array}$ & $\begin{array}{l}\text { Low; may disrupt } \\
\text { dolphins traveling from } \\
\text { nocturnal foraging } \\
\text { areas to nearshore } \\
\text { resting areas; not likely } \\
\text { to impact foraging }\end{array}$ & $\begin{array}{l}\text { Norris et al. } \\
1994\end{array}$ \\
\hline $\begin{array}{l}\text { Directional drilling, } \\
\text { and laying cable } \\
\text { under/on seabed }\end{array}$ & $\begin{array}{l}\text { Noise and } \\
\text { vibration from } \\
\text { drilling and } \\
\text { support vessels }\end{array}$ & $\begin{array}{l}\text { Avoidance of } \\
\text { nearshore area, } \\
\text { disruption of resting } \\
\text { (spinner dolphins } \\
\text { highly sensitive to } \\
\text { boats and people } \\
\text { while resting) }\end{array}$ & $\begin{array}{l}\text { High, dolphins will } \\
\text { avoid areas they may } \\
\text { regularly use for } \\
\text { resting due to noise } \\
\text { levels and presence } \\
\text { of support vessels }\end{array}$ & $\begin{array}{l}\text { Low, occurring } \\
\text { over } 1 \text { summer }\end{array}$ & $\begin{array}{l}\text { High; Spinner dolphins } \\
\text { rest in shallow waters } \\
\text { during the day; vessels } \\
\text { likely significant } \\
\text { disturbance to resting } \\
\text { animals }\end{array}$ & $\begin{array}{l}\text { Low; low if drilling at } \\
\text { location not used by } \\
\text { resting dolphins; high } \\
\text { if occurs in area used } \\
\text { by resting dolphins }\end{array}$ & $\begin{array}{l}\text { Danil et al. } \\
\text { 2005, Norris } \\
\text { et al. 1994, } \\
\text { Richardson } \\
\text { and Wursig } \\
1997\end{array}$ \\
\hline \multicolumn{8}{|c|}{ Operation and Maintenance } \\
\hline \multirow{3}{*}{$\begin{array}{l}\text { Boat traffic. } \\
\text { (Assuming } \\
\text { maintenance done } \\
\text { throughout the year) }\end{array}$} & Direct impact & Collision injuries & $\begin{array}{l}\text { Low-med; moderate } \\
\text { increase in boat } \\
\text { traffic traveling along } \\
\text { coast from Honolulu } \\
\text { to project site }\end{array}$ & $\begin{array}{l}\text { Med, occurring } \\
\text { at low frequency } \\
\text { over project } \\
\text { duration }\end{array}$ & $\begin{array}{l}\text { Low; effects amplified } \\
\text { if boats close to shore } \\
\text { or cross paths of } \\
\text { dolphins traveling to } \\
\text { and from offshore } \\
\text { foraging areas }\end{array}$ & $\begin{array}{l}\text { Low; collisions } \\
\text { between spinner } \\
\text { dolphins and boats } \\
\text { relatively infrequent; } \\
\text { level of traffic expected } \\
\text { to be relatively low }\end{array}$ & $\begin{array}{l}\text { Camargo and } \\
\text { Bellini 2007, } \\
\text { Van } \\
\text { Waerebeek } \\
\text { and Leaper } \\
2008\end{array}$ \\
\hline & $\begin{array}{l}\text { Oil/chemical } \\
\text { release }\end{array}$ & $\begin{array}{l}\text { Ingestion, } \\
\text { accumulation of } \\
\text { toxins, breathing } \\
\text { exhaust fumes, } \\
\text { overall Assuming no } \\
\text { catastrophic spills. }\end{array}$ & $\begin{array}{l}\text { Low-Med, increased } \\
\text { boat traffic but the } \\
\text { volume of fluids } \\
\text { released low }\end{array}$ & $\begin{array}{l}\text { Low, occurring } \\
\text { at low frequency } \\
\text { over project } \\
\text { duration }\end{array}$ & $\begin{array}{l}\text { Dolphins may pass } \\
\text { through project area } \\
\text { traveling to and from } \\
\text { foraging areas } \\
\text { increasing potential } \\
\text { for exposure }\end{array}$ & $\begin{array}{l}\text { Low; volume of } \\
\text { contaminants expected } \\
\text { to be low; area of } \\
\text { potential impact } \\
\text { relatively small } \\
\text { compared to range }\end{array}$ & $\begin{array}{l}\text { Norris et al. } \\
1994\end{array}$ \\
\hline & $\begin{array}{l}\text { Noise and } \\
\text { vibration }\end{array}$ & $\begin{array}{l}\text { Repeated } \\
\text { disturbance may } \\
\text { disrupt daytime } \\
\text { resting and cause } \\
\text { avoidance of area. } \\
\end{array}$ & $\begin{array}{l}\text { Med, significant } \\
\text { increase in boat } \\
\text { traffic }\end{array}$ & $\begin{array}{l}\text { Low, occurring } \\
\text { over } 1 \text { to } 2 \\
\text { summers }\end{array}$ & $\begin{array}{l}\text { Low; effects amplified } \\
\text { if boats close to shore } \\
\text { or as dolphins travel } \\
\text { between resting and } \\
\text { offshore foraging }\end{array}$ & $\begin{array}{l}\text { Low; risk increased if } \\
\text { boats travel close to } \\
\text { shore; may result in } \\
\text { disruption of rest } \\
\text { period }\end{array}$ & $\begin{array}{l}\text { Danil et al. } \\
2005\end{array}$ \\
\hline $\begin{array}{l}\text { Operation of turbines } \\
\text { or other moving parts } \\
\text { of devices }\end{array}$ & $\begin{array}{l}\text { Moving device } \\
\text { parts }\end{array}$ & $\begin{array}{l}\text { Movement of devices } \\
\text { and mooring lines } \\
\text { could injure animals }\end{array}$ & $\begin{array}{l}\text { Low-Med; project } \\
\text { location likely to be } \\
\text { traveled through, but } \\
\text { not used for foraging } \\
\text { or resting }\end{array}$ & $\begin{array}{l}\text { High, the } \\
\text { movement } \\
\text { occurs over } \\
\text { project duration }\end{array}$ & $\begin{array}{l}\text { Low; area occupied } \\
\text { by moving parts } \\
\text { small relative to the } \\
\text { animal's home range; }\end{array}$ & $\begin{array}{l}\text { Low; species sensitive to } \\
\text { surrounding environment } \\
\text { but not likely to approach } \\
\text { close enough to be at risk }\end{array}$ & $\begin{array}{l}\text { Norris et al. } \\
1994\end{array}$ \\
\hline
\end{tabular}

\begin{tabular}{|c|c|c|}
\hline $\begin{array}{l}\text { Environmental Assessment for Siting Wave \& Tidal } \\
\text { Energy Projects }\end{array}$ & E-20 & $\begin{array}{r}\text { H. T. Harvey \& Associates } \\
24 \text { November } 2009\end{array}$ \\
\hline
\end{tabular}


Appendix E- Effects of Small Commercial Pelamis Project at Makapu’u

\begin{tabular}{|c|c|c|c|c|c|c|c|}
\hline Project activity & Project action & $\begin{array}{l}\text { Description of } \\
\text { action's effect on } \\
\text { indicator }\end{array}$ & $\begin{array}{l}\text { Spatial exposure to } \\
\text { indicator (low, med, } \\
\text { high) }\end{array}$ & $\begin{array}{l}\text { Temporal } \\
\text { exposure to } \\
\text { indicator (low, } \\
\text { med, high) }\end{array}$ & $\begin{array}{l}\text { Effect's overlap } \\
\text { with critical life } \\
\text { stage, behavior, } \\
\text { habitat or resource } \\
\text { (low, med, high) }\end{array}$ & $\begin{array}{l}\text { Overall risk to } \\
\text { indicator (low, med, } \\
\text { high, unknown) }\end{array}$ & Source(s) \\
\hline & $\begin{array}{l}\text { Noise and } \\
\text { vibration }\end{array}$ & $\begin{array}{l}\text { Repeated } \\
\text { disturbance may } \\
\text { disrupt daytime } \\
\text { resting period and } \\
\text { cause avoidance of } \\
\text { area. }\end{array}$ & $\begin{array}{l}\text { Noise likely to be } \\
\text { low frequency and } \\
\text { not audible by } \\
\text { spinner dolphins, } \\
\text { beyond project area }\end{array}$ & $\begin{array}{l}\text { High, noise } \\
\text { level would be } \\
\text { constant over } \\
\text { project duration }\end{array}$ & $\begin{array}{l}\text { Low; frequency and } \\
\text { sound pressure levels } \\
\text { expected to be below } \\
\text { hearing sensitivity of } \\
\text { dolphins unless very } \\
\text { close to equipment }\end{array}$ & $\begin{array}{l}\text { Low; generators } \\
\text { sufficiently far from } \\
\text { shore to reduce impacts } \\
\text { of noise; transit } \\
\text { through/past project } \\
\text { area regular but brief }\end{array}$ & $\begin{array}{l}\text { Norris et al. } \\
1994\end{array}$ \\
\hline $\begin{array}{l}\text { Structures in water } \\
\text { column and on seabed, } \\
\text { such as devices and } \\
\text { moorings and footings }\end{array}$ & Structure & $\begin{array}{l}\text { Biofouling inverts } \\
\text { could create artificial } \\
\text { reef and attract fish; } \\
\text { entanglement with } \\
\text { devices or derelict } \\
\text { fishing gear }\end{array}$ & $\begin{array}{l}\text { Low, structures likely } \\
\text { to be avoided as } \\
\text { animals pass between } \\
\text { during travel to and } \\
\text { from foraging areas }\end{array}$ & $\begin{array}{l}\text { High, structure } \\
\text { present over } \\
\text { project duration }\end{array}$ & $\begin{array}{l}\text { Low; structures } \\
\text { should be readily } \\
\text { avoided while } \\
\text { traveling to and from } \\
\text { foraging areas }\end{array}$ & $\begin{array}{l}\text { Low; may enhance } \\
\text { foraging on prey } \\
\text { species attracted to } \\
\text { floating structures }\end{array}$ & $\begin{array}{l}\text { Relini et al. } \\
2000\end{array}$ \\
\hline $\begin{array}{l}\text { Electricity conduction } \\
\text { through cable }\end{array}$ & EMF & Unknown & $\begin{array}{l}\text { Unknown, cable } \\
\text { shielding and burial } \\
\text { provides some } \\
\text { attenuation } \\
\end{array}$ & $\begin{array}{l}\text { High if an effect, } \\
\text { electricity } \\
\text { generated over } \\
\text { project duration }\end{array}$ & $\begin{array}{l}\text { Unknown; effects } \\
\text { amplified if power } \\
\text { cable brought onshore } \\
\text { across resting area }\end{array}$ & $\begin{array}{l}\text { Unknown; High } \\
\text { uncertainty about } \\
\text { effects on marine } \\
\text { mammals }\end{array}$ & $\begin{array}{l}\text { Boehlert et al. } \\
2008\end{array}$ \\
\hline \multirow[b]{2}{*}{$\begin{array}{l}\text { Structures on water's } \\
\text { surface }\end{array}$} & Structure & Collision & $\begin{array}{l}\text { Low, structures } \\
\text { readily avoided as } \\
\text { animals pass between } \\
\text { during travel to and } \\
\text { from foraging areas }\end{array}$ & $\begin{array}{l}\text { High, structure } \\
\text { present over } \\
\text { project duration }\end{array}$ & $\begin{array}{l}\text { Low; structures } \\
\text { readily avoided while } \\
\text { traveling to and from } \\
\text { foraging areas }\end{array}$ & $\begin{array}{l}\text { Low; structures } \\
\text { detected and avoided }\end{array}$ & $\begin{array}{l}\text { Norris et al. } \\
\text { 1994, Relini } \\
\text { et al. } 2000\end{array}$ \\
\hline & $\begin{array}{l}\text { Navigation } \\
\text { lights }\end{array}$ & $\begin{array}{l}\text { Visual disorientation } \\
\text { could lead to } \\
\text { collision, could also } \\
\text { enhance foraging on } \\
\text { species attracted to } \\
\text { light }\end{array}$ & $\begin{array}{l}\text { Low, device lights of } \\
\text { low intensity, } \\
\text { shielded, intended } \\
\text { for navigation safety }\end{array}$ & $\begin{array}{l}\text { High, lights } \\
\text { shining over } \\
\text { project duration }\end{array}$ & $\begin{array}{l}\text { Spinner dolphins are } \\
\text { nocturnal foragers } \\
\text { but typically forage } \\
\text { further offshore than } \\
\text { project location }\end{array}$ & $\begin{array}{l}\text { Low; light intensity } \\
\text { low and like other } \\
\text { vessel/buoy navigation } \\
\text { lights; not expected to } \\
\text { impact foraging or } \\
\text { travel }\end{array}$ & $\begin{array}{l}\text { Norris et al. } \\
1994\end{array}$ \\
\hline \multicolumn{8}{|c|}{ Decommissioning } \\
\hline Boat traffic & Direct impact & Collision injuries & $\begin{array}{l}\text { High, significant } \\
\text { increase of traffic } \\
\text { traveling along coast } \\
\text { from Honolulu to } \\
\text { project site }\end{array}$ & $\begin{array}{l}\text { Low, occurring } \\
\text { over } 1 \text { to } 2 \\
\text { summers }\end{array}$ & $\begin{array}{l}\text { Low; effects amplified } \\
\text { if vessels close to } \\
\text { shore or as dolphins } \\
\text { travel to and from } \\
\text { offshore foraging areas }\end{array}$ & $\begin{array}{l}\text { Low; collisions } \\
\text { between spinner } \\
\text { dolphins and boats } \\
\text { relatively infrequent }\end{array}$ & $\begin{array}{l}\text { Camargo and } \\
\text { Bellini 2007, } \\
\text { Van } \\
\text { Waerebeek } \\
\text { and Leaper } \\
2008\end{array}$ \\
\hline
\end{tabular}

\begin{tabular}{lrr}
\hline Environmental Assessment for Siting Wave \& Tidal & E-21 & H. T. Harvey \& Associates \\
Energy Projects & & 24 November 2009
\end{tabular}


Appendix E- Effects of Small Commercial Pelamis Project at Makapu’u

\begin{tabular}{|c|c|c|c|c|c|c|c|}
\hline Project activity & Project action & $\begin{array}{l}\text { Description of } \\
\text { action's effect on } \\
\text { indicator }\end{array}$ & $\begin{array}{l}\text { Spatial exposure to } \\
\text { indicator (low, med, } \\
\text { high) }\end{array}$ & $\begin{array}{l}\text { Temporal } \\
\text { exposure to } \\
\text { indicator (low, } \\
\text { med, high) }\end{array}$ & $\begin{array}{l}\text { Effect's overlap } \\
\text { with critical life } \\
\text { stage, behavior, } \\
\text { habitat or resource } \\
\text { (low, med, high) } \\
\end{array}$ & $\begin{array}{l}\text { Overall risk to } \\
\text { indicator (low, med, } \\
\text { high, unknown) }\end{array}$ & Source(s) \\
\hline & $\begin{array}{l}\text { Oil/chemical } \\
\text { release }\end{array}$ & $\begin{array}{l}\text { Ingestion, } \\
\text { accumulation of } \\
\text { toxins, breathing } \\
\text { exhaust fumes, } \\
\text { Assuming no } \\
\text { catastrophic spills. }\end{array}$ & $\begin{array}{l}\text { Med, significant } \\
\text { increase in boat } \\
\text { traffic but the volume } \\
\text { of fluids released low }\end{array}$ & $\begin{array}{l}\text { Low, occurring } \\
\text { over } 1 \text { to } 2 \\
\text { summers }\end{array}$ & $\begin{array}{l}\text { Low; possible contact } \\
\text { as dolphins pass } \\
\text { through project area } \\
\text { traveling to and from } \\
\text { foraging areas }\end{array}$ & $\begin{array}{l}\text { Low; volume of } \\
\text { contaminants expected } \\
\text { to be low; area of } \\
\text { potential impact } \\
\text { relatively small } \\
\text { compared to range }\end{array}$ & $\begin{array}{l}\text { Norris et al. } \\
1994\end{array}$ \\
\hline & $\begin{array}{l}\text { Noise and } \\
\text { vibration }\end{array}$ & $\begin{array}{l}\text { Repeated } \\
\text { disturbance may } \\
\text { disrupt daytime } \\
\text { resting and cause } \\
\text { avoidance of area. }\end{array}$ & $\begin{array}{l}\text { Med, significant } \\
\text { increase in boat } \\
\text { traffic }\end{array}$ & $\begin{array}{l}\text { Low, occurring } \\
\text { over } 1 \text { to } 2 \\
\text { summers }\end{array}$ & $\begin{array}{l}\text { Low; effects amplified } \\
\text { if boats close to shore } \\
\text { or as dolphins travel } \\
\text { between resting and } \\
\text { offshore foraging areas }\end{array}$ & $\begin{array}{l}\text { Low; risk increased if } \\
\text { boats travel close to } \\
\text { nearshore resting areas }\end{array}$ & $\begin{array}{l}\text { Danil et al. } \\
2005\end{array}$ \\
\hline \multirow{3}{*}{$\begin{array}{l}\text { Decommissioning of } \\
\text { structures on water's } \\
\text { surface or seabed }\end{array}$} & $\begin{array}{l}\text { Noise and } \\
\text { vibration }\end{array}$ & $\begin{array}{l}\text { Repeated } \\
\text { disturbance may } \\
\text { disrupt daytime } \\
\text { resting period and } \\
\text { cause avoidance of } \\
\text { area. }\end{array}$ & $\begin{array}{l}\text { Low-med; dolphins } \\
\text { likely to be less } \\
\text { sensitive to low } \\
\text { frequency unless they } \\
\text { are within immediate } \\
\text { project area }\end{array}$ & $\begin{array}{l}\text { Low, occurring } \\
\text { over } 1 \text { to } 2 \\
\text { summers }\end{array}$ & $\begin{array}{l}\text { Low; likely to be less } \\
\text { sensitive to noise in } \\
\text { low frequencies; } \\
\text { higher frequency } \\
\text { sounds may disrupt } \\
\text { resting period }\end{array}$ & $\begin{array}{l}\text { Low; deconstruction } \\
\text { far enough from shore } \\
\text { to reduce noise } \\
\text { impacts; transit through } \\
\text { project area regular but } \\
\text { brief }\end{array}$ & $\begin{array}{l}\text { Norris et al. } \\
1994\end{array}$ \\
\hline & $\begin{array}{l}\text { Deconstruction } \\
\text { lights, vessel } \\
\text { deck lights and } \\
\text { spotlights. }\end{array}$ & $\begin{array}{l}\text { Visual disorientation } \\
\text { could lead to } \\
\text { collision, could also } \\
\text { enhance foraging on } \\
\text { species attracted to } \\
\text { light }\end{array}$ & $\begin{array}{l}\text { Med to low extent } \\
\text { beyond project site. }\end{array}$ & $\begin{array}{l}\text { Low, occurring } \\
\text { over } 1 \text { to } 2 \\
\text { summers }\end{array}$ & $\begin{array}{l}\text { Spinner dolphins are } \\
\text { nocturnal foragers } \\
\text { but typically forage } \\
\text { further offshore than } \\
\text { project location }\end{array}$ & $\begin{array}{l}\text { Low; may disrupt } \\
\text { dolphins traveling from } \\
\text { nocturnal foraging } \\
\text { areas offshore to } \\
\text { nearshore resting areas; } \\
\text { not likely to impact } \\
\text { foraging }\end{array}$ & $\begin{array}{l}\text { Norris et al. } \\
1994\end{array}$ \\
\hline & $\begin{array}{l}\text { Oil/chemical } \\
\text { release }\end{array}$ & $\begin{array}{l}\text { Ingestion, } \\
\text { accumulation of } \\
\text { toxins, breathing } \\
\text { exhaust fumes; } \\
\text { Assuming no } \\
\text { catastrophic spills. }\end{array}$ & $\begin{array}{l}\text { Med; significant } \\
\text { increase in use of } \\
\text { chemicals, solvents, } \\
\text { fuels, grease but the } \\
\text { volume of fluids } \\
\text { released low-med }\end{array}$ & $\begin{array}{l}\text { Low, occurring } \\
\text { over } 1 \text { to } 2 \\
\text { summers }\end{array}$ & $\begin{array}{l}\text { Dolphins may pass } \\
\text { through project area } \\
\text { traveling to and from } \\
\text { foraging areas } \\
\text { increasing potential } \\
\text { for exposure }\end{array}$ & $\begin{array}{l}\text { Low; volume of } \\
\text { contaminants expected } \\
\text { to be low; area of } \\
\text { potential impact } \\
\text { relatively small } \\
\text { compared to range }\end{array}$ & $\begin{array}{l}\text { Norris et al. } \\
1994\end{array}$ \\
\hline
\end{tabular}

Environmental Assessment for Siting Wave \& Tidal

E-22

H. T. Harvey \& Associates

Energy Projects

24 November 2009 
Appendix E- Effects of Small Commercial Pelamis Project at Makapu’u

Potential effects on Hawaiian monk seal due to Small Commercial Pelamis at Makupu'u site

\begin{tabular}{|c|c|c|c|c|c|c|c|}
\hline Project activity & Project action & $\begin{array}{l}\text { Description of } \\
\text { action's effect on } \\
\text { indicator }\end{array}$ & $\begin{array}{l}\text { Spatial exposure to } \\
\text { indicator (low, med, } \\
\text { high) }\end{array}$ & $\begin{array}{l}\text { Temporal } \\
\text { exposure to } \\
\text { indicator (low, } \\
\text { med, high) }\end{array}$ & $\begin{array}{l}\text { Effect's overlap } \\
\text { with critical life } \\
\text { stage, behavior, } \\
\text { habitat, or resource } \\
\text { (low, med, high) }\end{array}$ & $\begin{array}{l}\text { Overall risk to } \\
\text { indicator (low, med, } \\
\text { high or unknown) }\end{array}$ & Source(s) \\
\hline \multicolumn{8}{|c|}{ Construction } \\
\hline \multirow{3}{*}{$\begin{array}{l}\text { Boat traffic, assumed } \\
\text { to occur day and night }\end{array}$} & Direct impact & Collision injuries & $\begin{array}{l}\text { High, significant } \\
\text { increase of traffic } \\
\text { traveling along coast } \\
\text { from Honolulu to } \\
\text { project site }\end{array}$ & $\begin{array}{l}\text { Low, occurring } \\
\text { over } 1 \text { to } 2 \\
\text { summers }\end{array}$ & $\begin{array}{l}\text { Low due to relative } \\
\text { infrequent } \\
\text { occurrence of monk } \\
\text { seals near Oahu }\end{array}$ & $\begin{array}{l}\text { Low-high; likelihood } \\
\text { of collision low as } \\
\text { there are few seals in } \\
\text { the area and collisions } \\
\text { infrequent; high risk } \\
\text { due to potential for } \\
\text { loss of a single } \\
\text { individual of a species } \\
\text { at risk of extinction }\end{array}$ & $\begin{array}{l}\text { Antonelis et } \\
\text { al. 2006, } \\
\text { Baker and } \\
\text { Johanos } \\
2004\end{array}$ \\
\hline & $\begin{array}{l}\text { Noise and } \\
\text { vibration }\end{array}$ & $\begin{array}{l}\text { Repeated disturbance } \\
\text { will cause permanent } \\
\text { avoidance of area. } \\
\text { Reduced hearing } \\
\text { sensitivity could } \\
\text { mask envir. cues }\end{array}$ & $\begin{array}{l}\text { Med, significant } \\
\text { increase in boat } \\
\text { traffic, low } \\
\text { frequency sound } \\
\text { detectable for several } \\
\text { km }\end{array}$ & $\begin{array}{l}\text { Low, occurring } \\
\text { over } 1 \text { to } 2 \\
\text { summers }\end{array}$ & $\begin{array}{l}\text { Potential for } \\
\text { reducing from low } \\
\text { occurrence } \\
\text { frequency of monk } \\
\text { seals to complete } \\
\text { avoidance of area }\end{array}$ & $\begin{array}{l}\text { Low; risk of avoidance } \\
\text { of area during } \\
\text { construction; numbers } \\
\text { of seals observed in } \\
\text { the region is low }\end{array}$ & $\begin{array}{l}\text { Antonelis et } \\
\text { al. 2006, } \\
\text { Baker and } \\
\text { Johanos } \\
\text { 2004, Kastak } \\
\text { et al. 2005, } \\
\text { Tougaard et } \\
\text { al. } 2009\end{array}$ \\
\hline & $\begin{array}{l}\text { Oil/chemical } \\
\text { release }\end{array}$ & $\begin{array}{l}\text { Ingestion, fur } \\
\text { fouling, breathing } \\
\text { exhaust fumes, } \\
\text { accumulation of } \\
\text { toxins. Assuming no } \\
\text { catastrophic spills. }\end{array}$ & $\begin{array}{l}\text { Med, significant } \\
\text { increase existing } \\
\text { traffic but the } \\
\text { volume of fluids } \\
\text { released low }\end{array}$ & $\begin{array}{l}\text { Low, occurring } \\
\text { over } 1 \text { to } 2 \\
\text { summers }\end{array}$ & $\begin{array}{l}\text { Low due to relative } \\
\text { infrequent } \\
\text { occurrence of monk } \\
\text { seals near Oahu }\end{array}$ & $\begin{array}{l}\text { Low; monk seals } \\
\text { infrequent in project } \\
\text { area; additional inputs } \\
\text { may compound } \\
\text { elevated contaminant } \\
\text { levels in few monk } \\
\text { seals present }\end{array}$ & $\begin{array}{l}\text { Baker and } \\
\text { Johanos } \\
\text { 2004, Ylitalo } \\
\text { et al. } 2008\end{array}$ \\
\hline $\begin{array}{l}\text { Construction and } \\
\text { installation of } \\
\text { electrical collector } \\
\text { system, mooring } \\
\text { cables, anchors or } \\
\text { foundations, and } \\
\text { devices }\end{array}$ & $\begin{array}{l}\text { Noise and } \\
\text { vibration }\end{array}$ & $\begin{array}{l}\text { Repeated disturbance } \\
\text { will cause permanent } \\
\text { avoidance of area. } \\
\text { Reduction of hearing } \\
\text { sensitivity could } \\
\text { mask envir. cues }\end{array}$ & $\begin{array}{l}\text { High, seals will } \\
\text { avoid project area } \\
\text { depending on sound } \\
\text { propagation; low } \\
\text { freq. sound } \\
\text { detectable for several } \\
\mathrm{km}\end{array}$ & $\begin{array}{l}\text { Low, occurring } \\
\text { over } 1 \text { to } 2 \\
\text { summers }\end{array}$ & $\begin{array}{l}\text { Med-high; potential } \\
\text { for reducing monk } \\
\text { seal use of water } \\
\text { around Oahu }\end{array}$ & $\begin{array}{l}\text { Unknown; potential } \\
\text { for reducing } \\
\text { occurrence of monk } \\
\text { seals from low } \\
\text { frequency to complete } \\
\text { avoidance of area }\end{array}$ & $\begin{array}{l}\text { Baker and } \\
\text { Johanos } \\
2004\end{array}$ \\
\hline
\end{tabular}

\begin{tabular}{lrr}
\hline $\begin{array}{l}\text { Environmental Assessment for Siting Wave \& Tidal } \\
\text { Energy Projects }\end{array}$ & E-23 & H. T. Harvey \& Associates \\
24 November 2009
\end{tabular}


Appendix E- Effects of Small Commercial Pelamis Project at Makapu’u

\begin{tabular}{|c|c|c|c|c|c|c|c|}
\hline \multirow[t]{3}{*}{ Project activity } & Project action & $\begin{array}{l}\text { Description of } \\
\text { action's effect on } \\
\text { indicator }\end{array}$ & $\begin{array}{l}\text { Spatial exposure to } \\
\text { indicator (low, med, } \\
\text { high) }\end{array}$ & $\begin{array}{l}\text { Temporal } \\
\text { exposure to } \\
\text { indicator (low, } \\
\text { med, high) }\end{array}$ & $\begin{array}{l}\text { Effect's overlap } \\
\text { with critical life } \\
\text { stage, behavior, } \\
\text { habitat, or resource } \\
\text { (low, med, high) }\end{array}$ & $\begin{array}{l}\text { Overall risk to } \\
\text { indicator (low, med, } \\
\text { high or unknown) }\end{array}$ & Source(s) \\
\hline & $\begin{array}{l}\text { Oil/chemical } \\
\text { release }\end{array}$ & $\begin{array}{l}\text { Ingestion, fur } \\
\text { fouling, breathing } \\
\text { exhaust fumes, } \\
\text { overall accumulation } \\
\text { of toxins. Assuming } \\
\text { no catastrophic } \\
\text { spills. }\end{array}$ & $\begin{array}{l}\text { Med; significant } \\
\text { increase in use of } \\
\text { chemicals, solvents, } \\
\text { fuels, grease but the } \\
\text { volume of fluids } \\
\text { released low-med }\end{array}$ & $\begin{array}{l}\text { Low, occurring } \\
\text { over } 1 \text { to } 2 \\
\text { summers }\end{array}$ & $\begin{array}{l}\text { Low due to relative } \\
\text { infrequent } \\
\text { occurrence of monk } \\
\text { seals near Oahu }\end{array}$ & $\begin{array}{l}\text { Low; monk seals } \\
\text { infrequent in project } \\
\text { area; additional inputs } \\
\text { may compound } \\
\text { elevated contaminant } \\
\text { levels in few monk } \\
\text { seals present }\end{array}$ & $\begin{array}{l}\text { Baker and } \\
\text { Johanos } \\
\text { 2004, Ylitalo } \\
\text { et al. } 2008\end{array}$ \\
\hline & $\begin{array}{l}\text { Construction } \\
\text { lights, vessel } \\
\text { deck lights and } \\
\text { spotlights. }\end{array}$ & $\begin{array}{l}\text { Visual disorientation } \\
\text { could lead to } \\
\text { collision, but could } \\
\text { also enhance } \\
\text { foraging on species } \\
\text { attracted to light }\end{array}$ & $\begin{array}{l}\text { Med to low extent } \\
\text { beyond project site. }\end{array}$ & $\begin{array}{l}\text { Low, occurring } \\
\text { over } 1 \text { to } 2 \\
\text { summers }\end{array}$ & $\begin{array}{l}\text { Low due to relative } \\
\text { infrequent } \\
\text { occurrence of monk } \\
\text { seals near Oahu }\end{array}$ & $\begin{array}{l}\text { Unknown; monk seals } \\
\text { forage at night; lights } \\
\text { may hinder or result in } \\
\text { avoidance; or could } \\
\text { enhance foraging on } \\
\text { prey attracted to lights }\end{array}$ & $\begin{array}{l}\text { Baker and } \\
\text { Johanos } \\
2004, \\
\text { Goodman- } \\
\text { Lowe } 1998\end{array}$ \\
\hline $\begin{array}{l}\text { Directional drilling, } \\
\text { and laying cable } \\
\text { under/on seabed }\end{array}$ & $\begin{array}{l}\text { Noise and } \\
\text { vibration from } \\
\text { drilling and } \\
\text { support vessels }\end{array}$ & $\begin{array}{l}\text { Avoidance of area, } \\
\text { masks environmental } \\
\text { cues, affecting } \\
\text { foraging, predation, } \\
\text { and threat avoidance }\end{array}$ & $\begin{array}{l}\text { High, seals will } \\
\text { avoid the project } \\
\text { area and beyond } \\
\text { depending on } \\
\text { propagation; sound } \\
\text { detectable for several } \\
\text { km. } \\
\end{array}$ & $\begin{array}{l}\text { Low, occurring } \\
\text { over } 1 \text { summer }\end{array}$ & $\begin{array}{l}\text { Potential for } \\
\text { reducing from low } \\
\text { occurrence } \\
\text { frequency of monk } \\
\text { seals to complete } \\
\text { avoidance of area }\end{array}$ & $\begin{array}{l}\text { Low; monk seals occur } \\
\text { infrequently in area } \\
\text { may haul out on beach } \\
\text { where drilling is to } \\
\text { occur; any disturbance } \\
\text { constitutes "take" of } \\
\text { this species }\end{array}$ & $\begin{array}{l}\text { Antonelis et } \\
\text { al. } 2006\end{array}$ \\
\hline \multicolumn{8}{|c|}{ Operations and maintenance } \\
\hline \multirow[t]{2}{*}{$\begin{array}{l}\text { Boat traffic. } \\
\text { (Assuming } \\
\text { maintenance done } \\
\text { throughout the year) }\end{array}$} & Direct impact & Collision injuries & $\begin{array}{l}\text { High, significant } \\
\text { increase existing } \\
\text { traffic traveling } \\
\text { along coast from } \\
\text { Honolulu to project } \\
\text { site }\end{array}$ & $\begin{array}{l}\text { Med, occurring } \\
\text { at low } \\
\text { frequency over } \\
\text { project duration }\end{array}$ & $\begin{array}{l}\text { Low due to relative } \\
\text { infrequent } \\
\text { occurrence of monk } \\
\text { seals near Oahu }\end{array}$ & $\begin{array}{l}\text { Low-high; likelihood } \\
\text { of collision low } \\
\text { because few seals in } \\
\text { area, low boat traffic } \\
\text { expected and collisions } \\
\text { infrequent; high risk } \\
\text { due to potential for } \\
\text { loss of a single } \\
\text { individual }\end{array}$ & $\begin{array}{l}\text { Antonelis et } \\
\text { al. 2006, } \\
\text { Baker and } \\
\text { Johanos } \\
2004\end{array}$ \\
\hline & $\begin{array}{l}\text { Oil/chemical } \\
\text { release }\end{array}$ & $\begin{array}{l}\text { Ingestion, fur } \\
\text { fouling, breathing } \\
\text { exhaust fumes, } \\
\text { accumulation of } \\
\text { toxins. Assumes no } \\
\text { catastrophic spills. }\end{array}$ & $\begin{array}{l}\text { Med, significant } \\
\text { increase existing } \\
\text { traffic but the } \\
\text { volume of fluids } \\
\text { released low }\end{array}$ & $\begin{array}{l}\text { Med, occurring } \\
\text { at low } \\
\text { frequency over } \\
\text { project duration }\end{array}$ & $\begin{array}{l}\text { Low due to relative } \\
\text { infrequent } \\
\text { occurrence of monk } \\
\text { seals near Oahu }\end{array}$ & $\begin{array}{l}\text { Low; monk seals } \\
\text { infrequent in project } \\
\text { area; additional inputs } \\
\text { may compound } \\
\text { elevated levels in few } \\
\text { monk seals present }\end{array}$ & $\begin{array}{l}\text { Baker and } \\
\text { Johanos } \\
\text { 2004, Ylitalo } \\
\text { et al. } 2008\end{array}$ \\
\hline
\end{tabular}

\begin{tabular}{|c|c|c|}
\hline $\begin{array}{l}\text { Environmental Assessment for Siting Wave \& Tidal } \\
\text { Energy Projects }\end{array}$ & E-24 & $\begin{array}{r}\text { H. T. Harvey \& Associates } \\
24 \text { November } 2009\end{array}$ \\
\hline
\end{tabular}


Appendix E- Effects of Small Commercial Pelamis Project at Makapu’u

\begin{tabular}{|c|c|c|c|c|c|c|c|}
\hline Project activity & Project action & $\begin{array}{l}\text { Description of } \\
\text { action's effect on } \\
\text { indicator }\end{array}$ & $\begin{array}{l}\text { Spatial exposure to } \\
\text { indicator (low, med, } \\
\text { high) }\end{array}$ & $\begin{array}{l}\text { Temporal } \\
\text { exposure to } \\
\text { indicator (low, } \\
\text { med, high) }\end{array}$ & $\begin{array}{l}\text { Effect's overlap } \\
\text { with critical life } \\
\text { stage, behavior, } \\
\text { habitat, or resource } \\
\text { (low, med, high) } \\
\end{array}$ & $\begin{array}{l}\text { Overall risk to } \\
\text { indicator (low, med, } \\
\text { high or unknown) }\end{array}$ & Source(s) \\
\hline & $\begin{array}{l}\text { Noise and } \\
\text { vibration }\end{array}$ & $\begin{array}{l}\text { Repeated disturbance } \\
\text { will cause permanent } \\
\text { avoidance of area. } \\
\text { Reduction of hearing } \\
\text { sensitivity could } \\
\text { mask environmental } \\
\text { cues }\end{array}$ & $\begin{array}{l}\text { Med, significant } \\
\text { increase in boat } \\
\text { traffic, sound } \\
\text { detectable for several } \\
\text { km. }\end{array}$ & $\begin{array}{l}\text { Med, occurring } \\
\text { at low } \\
\text { frequency over } \\
\text { project duration }\end{array}$ & $\begin{array}{l}\text { Potential for } \\
\text { reducing from low } \\
\text { occurrence } \\
\text { frequency of monk } \\
\text { seals to complete } \\
\text { avoidance of area. }\end{array}$ & $\begin{array}{l}\text { Low; may cause } \\
\text { avoidance of area; } \\
\text { occurrence currently } \\
\text { rare but present; level } \\
\text { of boat traffic expected } \\
\text { to be relatively low }\end{array}$ & $\begin{array}{l}\text { Antonelis et } \\
\text { al. 2006, } \\
\text { Baker and } \\
\text { Johanos } \\
\text { 2004, Kastak } \\
\text { et al. 2005, } \\
\text { Tougaard et } \\
\text { al. } 2009\end{array}$ \\
\hline \multirow{2}{*}{$\begin{array}{l}\text { Operation of turbines } \\
\text { or other moving parts } \\
\text { of devices }\end{array}$} & $\begin{array}{l}\text { Moving device } \\
\text { parts }\end{array}$ & $\begin{array}{l}\text { Movement of } \\
\text { devices and mooring } \\
\text { lines could injure } \\
\text { animals }\end{array}$ & $\begin{array}{l}\text { Med, areas of } \\
\text { movement small } \\
\text { compared to project } \\
\text { area but monk seals } \\
\text { show highest rates of } \\
\text { entanglement among } \\
\text { pinnipeds }\end{array}$ & $\begin{array}{l}\text { High, the } \\
\text { movement } \\
\text { occurs over } \\
\text { project duration }\end{array}$ & $\begin{array}{l}\text { Low due to relative } \\
\text { infrequent } \\
\text { occurrence of monk } \\
\text { seals near Oahu }\end{array}$ & $\begin{array}{l}\text { Low; rare occurrence } \\
\text { in the area; moving } \\
\text { parts small relative to } \\
\text { range and readily } \\
\text { avoidable }\end{array}$ & $\begin{array}{l}\text { Baker and } \\
\text { Johanos } \\
2004\end{array}$ \\
\hline & $\begin{array}{l}\text { Noise and } \\
\text { vibration }\end{array}$ & $\begin{array}{l}\text { Repeated disturbance } \\
\text { will cause permanent } \\
\text { avoidance of area. } \\
\text { Reduction of hearing } \\
\text { sensitivity could } \\
\text { mask environmental } \\
\text { cues }\end{array}$ & $\begin{array}{l}\text { Unknown noise } \\
\text { levels, but } \\
\text { attenuation } \\
\text { dependent on sea } \\
\text { state. sound } \\
\text { detectable for several } \\
\text { km. }\end{array}$ & $\begin{array}{l}\text { High, noise } \\
\text { level would be } \\
\text { constant over } \\
\text { project duration }\end{array}$ & $\begin{array}{l}\text { Med-high; potential } \\
\text { for reducing monk } \\
\text { seal use of water } \\
\text { around Oahu }\end{array}$ & $\begin{array}{l}\text { Med-high; may cause } \\
\text { permanent avoidance } \\
\text { of area; may affect rate } \\
\text { of reestablishment } \\
\text { on Oahu; occurrence } \\
\text { currently rare but they } \\
\text { are present }\end{array}$ & $\begin{array}{l}\text { Antonelis et } \\
\text { al. 2006, } \\
\text { Baker and } \\
\text { Johanos } \\
\text { 2004, Kastak } \\
\text { et al. 2005, } \\
\text { Tougaard et } \\
\text { al. 2009 }\end{array}$ \\
\hline $\begin{array}{l}\text { Structures in water } \\
\text { column and on } \\
\text { seabed, such as } \\
\text { devices and moorings } \\
\text { and footings }\end{array}$ & Structure & $\begin{array}{l}\text { Artificial reef and } \\
\text { attract fish; } \\
\text { entanglement with } \\
\text { devices or derelict } \\
\text { fishing gear }\end{array}$ & $\begin{array}{l}\text { Med, the project area } \\
\text { is small but monk } \\
\text { seals show highest } \\
\text { rates of } \\
\text { entanglement among } \\
\text { pinnipeds }\end{array}$ & $\begin{array}{l}\text { High, structure } \\
\text { present over } \\
\text { project duration }\end{array}$ & $\begin{array}{l}\text { Low; possibly } \\
\text { beneficial effect by } \\
\text { increasing forage on } \\
\text { fishes, relative } \\
\text { infrequent } \\
\text { occurrence of monk } \\
\text { seals near Oahu } \\
\end{array}$ & $\begin{array}{l}\text { High; monk seals show } \\
\text { highest rates of } \\
\text { entanglement among } \\
\text { pinnipeds }\end{array}$ & $\begin{array}{l}\text { Baker and } \\
\text { Johanos } \\
\text { 2004, Relini } \\
\text { et al. } 2000\end{array}$ \\
\hline $\begin{array}{l}\text { Electricity conduction } \\
\text { through cable }\end{array}$ & EMF & Unknown & $\begin{array}{l}\text { Unknown, cable } \\
\text { shielding and burial } \\
\text { provides some } \\
\text { attenuation }\end{array}$ & $\begin{array}{l}\text { High if an effect } \\
\text { because } \\
\text { electricity } \\
\text { generated over } \\
\text { project duration } \\
\end{array}$ & $\begin{array}{l}\text { Unknown; effects } \\
\text { amplified if power } \\
\text { cable brought } \\
\text { onshore across a } \\
\text { resting area }\end{array}$ & $\begin{array}{l}\text { Unknown; High } \\
\text { uncertainty about } \\
\text { effects on marine } \\
\text { mammals }\end{array}$ & $\begin{array}{l}\text { Boehlert et } \\
\text { al. } 2008\end{array}$ \\
\hline
\end{tabular}

\begin{tabular}{lrr}
\hline $\begin{array}{l}\text { Environmental Assessment for Siting Wave \& Tidal } \\
\text { Energy Projects }\end{array}$ & E-25 & $\begin{array}{r}\text { H. T. Harvey \& Associates } \\
24 \text { November } 2009\end{array}$
\end{tabular}


Appendix E- Effects of Small Commercial Pelamis Project at Makapu’u

\begin{tabular}{|c|c|c|c|c|c|c|c|}
\hline Project activity & Project action & $\begin{array}{l}\text { Description of } \\
\text { action's effect on } \\
\text { indicator }\end{array}$ & $\begin{array}{l}\text { Spatial exposure to } \\
\text { indicator (low, med, } \\
\text { high) }\end{array}$ & $\begin{array}{l}\text { Temporal } \\
\text { exposure to } \\
\text { indicator (low, } \\
\text { med, high) }\end{array}$ & $\begin{array}{l}\text { Effect's overlap } \\
\text { with critical life } \\
\text { stage, behavior, } \\
\text { habitat, or resource } \\
\text { (low, med, high) }\end{array}$ & $\begin{array}{l}\text { Overall risk to } \\
\text { indicator (low, med, } \\
\text { high or unknown) }\end{array}$ & Source(s) \\
\hline \multirow[b]{2}{*}{$\begin{array}{l}\text { Structures on water's } \\
\text { surface }\end{array}$} & Structure & Collision & $\begin{array}{l}\text { Low, monk seals } \\
\text { prefer sandy beach } \\
\text { haulouts, not likely } \\
\text { to use buoys for } \\
\text { resting }\end{array}$ & $\begin{array}{l}\text { High, structure } \\
\text { present over } \\
\text { project duration }\end{array}$ & Low & $\begin{array}{l}\text { Low; rare occurrence } \\
\text { in the area; structures } \\
\text { readily avoidable }\end{array}$ & $\begin{array}{l}\text { Baker and } \\
\text { Johanos } \\
\text { 2004, Relini } \\
\text { et al. } 2000\end{array}$ \\
\hline & Navigation lights & $\begin{array}{l}\text { Visual disorientation } \\
\text { could lead to } \\
\text { collision, could also } \\
\text { enhance foraging on } \\
\text { species attracted to } \\
\text { light }\end{array}$ & $\begin{array}{l}\text { Low, device lights of } \\
\text { low intensity, } \\
\text { shielded, intended } \\
\text { for navigation safety }\end{array}$ & $\begin{array}{l}\text { High, lights } \\
\text { shining over } \\
\text { project duration }\end{array}$ & $\begin{array}{l}\text { Low due to relative } \\
\text { infrequent } \\
\text { occurrence of monk } \\
\text { seals near Oahu }\end{array}$ & $\begin{array}{l}\text { Low; low light } \\
\text { intensity; similar to } \\
\text { lights on existing } \\
\text { buoys and boats }\end{array}$ & $\begin{array}{l}\text { Baker and } \\
\text { Johanos } \\
2004\end{array}$ \\
\hline \multicolumn{8}{|c|}{ Decommissioning } \\
\hline \multirow{3}{*}{ Boat traffic } & Direct impact & Collision injuries & $\begin{array}{l}\text { High, significant } \\
\text { increase of traffic } \\
\text { traveling along coast } \\
\text { from Honolulu to } \\
\text { project site }\end{array}$ & $\begin{array}{l}\text { Low, occurring } \\
\text { over } 1 \text { to } 2 \\
\text { summers }\end{array}$ & $\begin{array}{l}\text { Low due to relative } \\
\text { infrequent } \\
\text { occurrence of monk } \\
\text { seals near Oahu }\end{array}$ & $\begin{array}{l}\text { Low-high; likelihood } \\
\text { of collision low as } \\
\text { there are few seals in } \\
\text { area and collisions } \\
\text { infrequent; high risk } \\
\text { due to potential for } \\
\text { loss of a single } \\
\text { individual of species at } \\
\text { risk of extinction }\end{array}$ & $\begin{array}{l}\text { Antonelis et } \\
\text { al. 2006, } \\
\text { Baker and } \\
\text { Johanos } \\
2004\end{array}$ \\
\hline & $\begin{array}{l}\text { Oil/chemical } \\
\text { release }\end{array}$ & $\begin{array}{l}\text { Ingestion, fur } \\
\text { fouling, breathing } \\
\text { exhaust fumes, } \\
\text { accumulation of } \\
\text { toxins. Assuming no } \\
\text { catastrophic spills. }\end{array}$ & $\begin{array}{l}\text { Med, significant } \\
\text { increase existing } \\
\text { traffic but the } \\
\text { volume of fluids } \\
\text { released low }\end{array}$ & $\begin{array}{l}\text { Low, occurring } \\
\text { over } 1 \text { to } 2 \\
\text { summers }\end{array}$ & $\begin{array}{l}\text { Low due to relative } \\
\text { infrequent } \\
\text { occurrence of monk } \\
\text { seals near Oahu }\end{array}$ & $\begin{array}{l}\text { Low; monk seals occur } \\
\text { infrequently in project } \\
\text { area; additional inputs } \\
\text { may compound } \\
\text { elevated levels in few } \\
\text { monk seals present }\end{array}$ & $\begin{array}{l}\text { Baker and } \\
\text { Johanos } \\
\text { 2004, Ylitalo } \\
\text { et al. } 2008\end{array}$ \\
\hline & $\begin{array}{l}\text { Noise and } \\
\text { vibration }\end{array}$ & $\begin{array}{l}\text { Repeated disturbance } \\
\text { will cause permanent } \\
\text { avoidance of area. } \\
\text { Reduction of hearing } \\
\text { sensitivity could } \\
\text { mask environmental } \\
\text { cues }\end{array}$ & $\begin{array}{l}\text { Med, significant } \\
\text { increase in boat } \\
\text { traffic, sound } \\
\text { detectable for several } \\
\text { km. }\end{array}$ & $\begin{array}{l}\text { Low, occurring } \\
\text { over } 1 \text { to } 2 \\
\text { summers }\end{array}$ & $\begin{array}{l}\text { Potential for } \\
\text { reducing from low } \\
\text { occurrence } \\
\text { frequency of monk } \\
\text { seals to complete } \\
\text { avoidance of area }\end{array}$ & $\begin{array}{l}\text { Low; risk of avoidance } \\
\text { of area; numbers of } \\
\text { seals in the region is } \\
\text { low }\end{array}$ & $\begin{array}{l}\text { Antonelis et } \\
\text { al. 2006, } \\
\text { Baker and } \\
\text { Johanos } \\
\text { 2004, Kastak } \\
\text { et al. 2005, } \\
\text { Tougaard et } \\
\text { al. } 2009\end{array}$ \\
\hline
\end{tabular}

\begin{tabular}{|c|c|c|}
\hline $\begin{array}{l}\text { Environmental Assessment for Siting Wave \& Tidal } \\
\text { Energy Projects }\end{array}$ & E-26 & $\begin{array}{r}\text { H. T. Harvey \& Associates } \\
24 \text { November } 2009\end{array}$ \\
\hline
\end{tabular}


Appendix E- Effects of Small Commercial Pelamis Project at Makapu’u

\begin{tabular}{|c|c|c|c|c|c|c|c|}
\hline Project activity & Project action & $\begin{array}{l}\text { Description of } \\
\text { action's effect on } \\
\text { indicator }\end{array}$ & $\begin{array}{l}\text { Spatial exposure to } \\
\text { indicator (low, med, } \\
\text { high) }\end{array}$ & $\begin{array}{l}\text { Temporal } \\
\text { exposure to } \\
\text { indicator (low, } \\
\text { med, high) }\end{array}$ & $\begin{array}{l}\text { Effect's overlap } \\
\text { with critical life } \\
\text { stage, behavior, } \\
\text { habitat, or resource } \\
\text { (low, med, high) } \\
\end{array}$ & $\begin{array}{l}\text { Overall risk to } \\
\text { indicator (low, med, } \\
\text { high or unknown) }\end{array}$ & Source(s) \\
\hline \multirow{4}{*}{$\begin{array}{l}\text { Decommissioning of } \\
\text { structures on water's } \\
\text { surface or seabed }\end{array}$} & $\begin{array}{l}\text { Noise and } \\
\text { vibration }\end{array}$ & $\begin{array}{l}\text { Repeated disturbance } \\
\text { will cause permanent } \\
\text { avoidance of area. } \\
\text { Reduction of hearing } \\
\text { sensitivity could } \\
\text { mask environmental } \\
\text { cues }\end{array}$ & $\begin{array}{l}\text { High, seals will } \\
\text { avoid the project } \\
\text { area and beyond } \\
\text { depending on } \\
\text { propagation; sound } \\
\text { detectable for several } \\
\text { km. }\end{array}$ & $\begin{array}{l}\text { Low, occurring } \\
\text { over } 1 \text { to } 2 \\
\text { summers }\end{array}$ & $\begin{array}{l}\text { Med-high; potential } \\
\text { for reducing monk } \\
\text { seal use of water } \\
\text { around Oahu }\end{array}$ & $\begin{array}{l}\text { Med-high; potential } \\
\text { for reducing } \\
\text { occurrence of monk } \\
\text { seals from low } \\
\text { frequency to complete } \\
\text { avoidance of area }\end{array}$ & $\begin{array}{l}\text { Antonelis et } \\
\text { al. 2006, } \\
\text { Bodson et al. } \\
\text { 2007, Kastak } \\
\text { et al. 2005, } \\
\text { Kastelein et } \\
\text { al. 2009, } \\
\text { Tougaard et } \\
\text { al. 2009 }\end{array}$ \\
\hline & $\begin{array}{l}\text { Deconstruction } \\
\text { lights, vessel } \\
\text { deck lights and } \\
\text { spotlights. }\end{array}$ & $\begin{array}{l}\text { Visual disorientation } \\
\text { could lead to } \\
\text { collision, but could } \\
\text { also enhance } \\
\text { foraging on species } \\
\text { attracted to light }\end{array}$ & $\begin{array}{l}\text { Med to low extent } \\
\text { beyond project site. }\end{array}$ & $\begin{array}{l}\text { Low, occurring } \\
\text { over } 1 \text { to } 2 \\
\text { summers }\end{array}$ & $\begin{array}{l}\text { Low due to relative } \\
\text { infrequent } \\
\text { occurrence of monk } \\
\text { seals near Oahu }\end{array}$ & $\begin{array}{l}\text { Unknown; monk seals } \\
\text { forage at night; lights } \\
\text { may hinder or result in } \\
\text { avoidance; lights may } \\
\text { enhance foraging on } \\
\text { prey attracted to lights }\end{array}$ & $\begin{array}{l}\text { Baker and } \\
\text { Johanos } \\
\text { 2004, } \\
\text { Goodman- } \\
\text { Lowe } 1998\end{array}$ \\
\hline & $\begin{array}{l}\text { Oil/chemical } \\
\text { release }\end{array}$ & $\begin{array}{l}\text { Ingestion, fur } \\
\text { fouling, breathing } \\
\text { exhaust fumes, } \\
\text { overall accumulation } \\
\text { of toxins. Assuming } \\
\text { no catastrophic } \\
\text { spills. }\end{array}$ & $\begin{array}{l}\text { Med, significant } \\
\text { increase in use of } \\
\text { chemicals, solvents, } \\
\text { fuels, grease but the } \\
\text { volume of fluids } \\
\text { released low-med }\end{array}$ & $\begin{array}{l}\text { Low, occurring } \\
\text { over } 1 \text { to } 2 \\
\text { summers }\end{array}$ & $\begin{array}{l}\text { Low due to relative } \\
\text { infrequent } \\
\text { occurrence of monk } \\
\text { seals near Oahu }\end{array}$ & $\begin{array}{l}\text { Low; monk seals occur } \\
\text { infrequently in project } \\
\text { area; additional inputs } \\
\text { may compound } \\
\text { elevated levels in few } \\
\text { monk seals in the area }\end{array}$ & $\begin{array}{l}\text { Baker and } \\
\text { Johanos } \\
\text { 2004, Ylitalo } \\
\text { et al. } 2008\end{array}$ \\
\hline & $\begin{array}{l}\text { Decommissioning } \\
\text { of cables and } \\
\text { mooring }\end{array}$ & $\begin{array}{l}\text { Entanglement in } \\
\text { debris if not removed } \\
\text { from project area }\end{array}$ & $\begin{array}{l}\text { Med, depending on } \\
\text { thoroughness of } \\
\text { removal; }\end{array}$ & $\begin{array}{l}\text { Low to Med; } \\
\text { depending on } \\
\text { extent of debris }\end{array}$ & $\begin{array}{l}\text { Low to med due to } \\
\text { relative infrequent } \\
\text { occurrence of monk } \\
\text { seals near Oahu }\end{array}$ & $\begin{array}{l}\text { Low-high depending } \\
\text { on extent of removal; } \\
\text { monk seals show } \\
\text { highest rates of } \\
\text { entanglement among } \\
\text { pinnipeds; any debris } \\
\text { presents potentially } \\
\text { significant risk }\end{array}$ & $\begin{array}{l}\text { Antonelis et } \\
\text { al. 2006, } \\
\text { Baker and } \\
\text { Johanos } \\
2004 \text {, } \\
\text { Henderson } \\
2001\end{array}$ \\
\hline
\end{tabular}

Environmental Assessment for Siting Wave \& Tidal Energy Projects
E-27

H. T. Harvey \& Associates

24 November 2009 
Appendix E- Effects of Small Commercial Pelamis Project at Makapu'u

Potential effects on bottomfishes due to small commercial Pelamis project at Makapu'u site

\begin{tabular}{|c|c|c|c|c|c|c|c|}
\hline Project activity & Action & $\begin{array}{l}\text { Description of action's } \\
\text { effect on indicator }\end{array}$ & $\begin{array}{l}\text { Spatial exposure to } \\
\text { indicator (low, med, } \\
\text { high) }\end{array}$ & $\begin{array}{l}\text { Temporal } \\
\text { exposure to } \\
\text { indicator (low, } \\
\text { med, high) }\end{array}$ & $\begin{array}{l}\text { Effect's overlap with } \\
\text { critical life stage, } \\
\text { behavior, habitat or } \\
\text { resource (low, med, } \\
\text { high) }\end{array}$ & $\begin{array}{l}\text { Overall risk } \\
\text { to indicator } \\
\text { (low, med, } \\
\text { high, } \\
\text { unknown) }\end{array}$ & Source(s) \\
\hline \multicolumn{8}{|c|}{ Construction } \\
\hline \multirow{2}{*}{ Boat traffic } & $\begin{array}{l}\text { Oil/chemical } \\
\text { release }\end{array}$ & $\begin{array}{l}\text { Toxicity to individuals } \\
\text { and prey }\end{array}$ & $\begin{array}{l}\text { Low for typical small } \\
\text { volume releases, High } \\
\text { for large, catastrophic } \\
\text { spill }\end{array}$ & $\begin{array}{l}\text { Low, May-Sept. for } \\
1-2 \text { years }\end{array}$ & $\begin{array}{l}\text { Low, species in water } \\
\text { column but oil floats on } \\
\text { surface }\end{array}$ & Low & $\begin{array}{l}\text { Nelson and } \\
\text { Woo } 2008\end{array}$ \\
\hline & $\begin{array}{l}\text { Noise and } \\
\text { vibration }\end{array}$ & $\begin{array}{l}\text { Possible altered } \\
\text { behavior, may interact } \\
\text { with some species } \\
\text { communication }\end{array}$ & $\begin{array}{l}\text { Low, would occur in } \\
\text { very small area although } \\
\text { noise travels far in water }\end{array}$ & $\begin{array}{l}\text { Low, May-Sept. for } \\
1-2 \text { years }\end{array}$ & $\begin{array}{l}\text { Low, species occur on } \\
\text { or near bottom }\end{array}$ & Low & Cada 2008 \\
\hline \multirow{4}{*}{$\begin{array}{l}\text { Construction of } \\
\text { electrical } \\
\text { collector system, } \\
\text { moorings and } \\
\text { foundations, and } \\
\text { device } \\
\text { installation }\end{array}$} & $\begin{array}{l}\text { Noise and } \\
\text { vibration }\end{array}$ & $\begin{array}{l}\text { Possible altered } \\
\text { behavior, may interact } \\
\text { with some species } \\
\text { communication }\end{array}$ & $\begin{array}{l}\text { Low, would occur in } \\
\text { very small area, } \\
\text { although noise travels } \\
\text { far in water }\end{array}$ & $\begin{array}{l}\text { Low, May-Sept. for } \\
1-2 \text { years }\end{array}$ & $\begin{array}{l}\text { Med, species present in } \\
\text { area during construction }\end{array}$ & Low & Cada 2008 \\
\hline & $\begin{array}{l}\text { Oil/chemical } \\
\text { release }\end{array}$ & $\begin{array}{l}\text { Toxicity to individuals } \\
\text { and prey }\end{array}$ & $\begin{array}{l}\text { Low, volume released } \\
\text { would occupy small } \\
\text { area }\end{array}$ & $\begin{array}{l}\text { Low, May-Sept. for } \\
1-2 \text { years }\end{array}$ & $\begin{array}{l}\text { Low, species in water } \\
\text { column but oil floats on } \\
\text { surface }\end{array}$ & Low & $\begin{array}{l}\text { Nelson and } \\
\text { Woo } 2008\end{array}$ \\
\hline & $\begin{array}{l}\text { Seabed } \\
\text { disturbance }\end{array}$ & $\begin{array}{l}\text { Temporary habitat loss, } \\
\text { changes in prey } \\
\text { resources }\end{array}$ & $\begin{array}{l}\text { Low, would occur in } \\
\text { very small area }\end{array}$ & $\begin{array}{l}\text { Low, May-Sept. for } \\
1-2 \text { years }\end{array}$ & $\begin{array}{l}\text { Med, species present in } \\
\text { these habitats during } \\
\text { construction }\end{array}$ & Low & Nelson 2008 \\
\hline & $\begin{array}{l}\text { Construction } \\
\text { lights }\end{array}$ & $\begin{array}{l}\text { Attraction to prey } \\
\text { aggregation, beneficial } \\
\text { effect }\end{array}$ & $\begin{array}{l}\text { Low, would occur in } \\
\text { very small area }\end{array}$ & $\begin{array}{l}\text { Low, May-Sept. for } \\
1-2 \text { years }\end{array}$ & $\begin{array}{l}\text { Low, likely prey } \\
\text { attracted to surface not } \\
\text { bottom where species } \\
\text { occur }\end{array}$ & Low & $\begin{array}{l}\text { Nightingale } \\
\text { et al. } 2006\end{array}$ \\
\hline \multirow{2}{*}{$\begin{array}{l}\text { Directional } \\
\text { drilling, and } \\
\text { laying cable } \\
\text { under/on seabed }\end{array}$} & $\begin{array}{l}\text { Seabed } \\
\text { disturbance }\end{array}$ & $\begin{array}{l}\text { Temporary habitat loss, } \\
\text { changes in prey } \\
\text { resources }\end{array}$ & $\begin{array}{l}\text { Low, would occur in } \\
\text { very small area }\end{array}$ & $\begin{array}{l}\text { Low, occurs over a } \\
\text { short time period }\end{array}$ & $\begin{array}{l}\text { Med, species present in } \\
\text { area when drilling } \\
\text { and/or cable laying }\end{array}$ & Low & Nelson 2008 \\
\hline & $\begin{array}{l}\text { Noise and } \\
\text { vibration }\end{array}$ & $\begin{array}{l}\text { Possible altered } \\
\text { behavior, may interact } \\
\text { with some species } \\
\text { communication }\end{array}$ & $\begin{array}{l}\text { Low, would occur in } \\
\text { very small area although } \\
\text { noise travels far in water }\end{array}$ & $\begin{array}{l}\text { Low, occurs over a } \\
\text { short time period }\end{array}$ & $\begin{array}{l}\text { Med, species present in } \\
\text { area when drilling } \\
\text { and/or cable laying }\end{array}$ & Low & Cada 2008 \\
\hline \multicolumn{8}{|c|}{ Operation and Maintenance } \\
\hline Boat traffic & $\begin{array}{l}\text { Oil/chemical } \\
\text { release }\end{array}$ & $\begin{array}{l}\text { Toxicity to individuals } \\
\text { and prey }\end{array}$ & $\begin{array}{l}\text { Low for typical small } \\
\text { volume releases, High } \\
\text { for large, catastrophic } \\
\text { spill }\end{array}$ & $\begin{array}{l}\text { Low, wouldn't } \\
\text { persist long-term }\end{array}$ & $\begin{array}{l}\text { Low, species in water } \\
\text { column but oil floats on } \\
\text { surface }\end{array}$ & Low & $\begin{array}{l}\text { Nelson and } \\
\text { Woo } 2008\end{array}$ \\
\hline
\end{tabular}

\begin{tabular}{|c|c|c|}
\hline $\begin{array}{l}\text { Environmental Assessment for Siting Wave \& Tidal } \\
\text { Energy Projects }\end{array}$ & E-28 & $\begin{array}{r}\text { H. T. Harvey \& Associates } \\
24 \text { November } 2009\end{array}$ \\
\hline
\end{tabular}


Appendix E- Effects of Small Commercial Pelamis Project at Makapu’u

\begin{tabular}{|c|c|c|c|c|c|c|c|}
\hline Project activity & Action & $\begin{array}{l}\text { Description of action's } \\
\text { effect on indicator }\end{array}$ & $\begin{array}{l}\text { Spatial exposure to } \\
\text { indicator (low, med, } \\
\text { high) }\end{array}$ & $\begin{array}{l}\text { Temporal } \\
\text { exposure to } \\
\text { indicator (low, } \\
\text { med, high) }\end{array}$ & $\begin{array}{l}\text { Effect's overlap with } \\
\text { critical life stage, } \\
\text { behavior, habitat or } \\
\text { resource (low, med, } \\
\text { high) }\end{array}$ & $\begin{array}{l}\text { Overall risk } \\
\text { to indicator } \\
\text { (low, med, } \\
\text { high, } \\
\text { unknown) }\end{array}$ & Source(s) \\
\hline & $\begin{array}{l}\text { Noise and } \\
\text { vibration }\end{array}$ & $\begin{array}{l}\text { Possible altered } \\
\text { behavior, may interact } \\
\text { with some species } \\
\text { communication }\end{array}$ & $\begin{array}{l}\text { Low, would occur in } \\
\text { very small area although } \\
\text { noise travels far in water }\end{array}$ & $\begin{array}{l}\text { Low, wouldn't } \\
\text { persist long-term }\end{array}$ & $\begin{array}{l}\text { Low, species occur on } \\
\text { or near bottom }\end{array}$ & Low & Cada 2008 \\
\hline \multirow{2}{*}{$\begin{array}{l}\text { Operation of } \\
\text { turbines or other } \\
\text { moving parts of } \\
\text { devices }\end{array}$} & $\begin{array}{l}\text { Moving device } \\
\text { parts }\end{array}$ & $\begin{array}{l}\text { None, moving parts on } \\
\text { water's surface, fish on } \\
\text { bottom }\end{array}$ & N/A & N/A & N/A & Low & \\
\hline & $\begin{array}{l}\text { Noise and } \\
\text { vibration }\end{array}$ & $\begin{array}{l}\text { Possible altered } \\
\text { behavior, may interact } \\
\text { with some species } \\
\text { communication }\end{array}$ & $\begin{array}{l}\text { Low, would occur in } \\
\text { very small area although } \\
\text { noise travels far in water }\end{array}$ & $\begin{array}{l}\text { High, would be } \\
\text { continuous for life } \\
\text { of project }\end{array}$ & $\begin{array}{l}\text { Low, species occur on } \\
\text { or near bottom }\end{array}$ & Low & Cada 2008 \\
\hline \multirow{3}{*}{$\begin{array}{l}\text { Structures in } \\
\text { water column and } \\
\text { on seabed, such } \\
\text { as devices and } \\
\text { moorings and } \\
\text { footings }\end{array}$} & Structure & $\begin{array}{l}\text { Structure in water } \\
\text { column acts similarly to } \\
\text { FAD, no effect of } \\
\text { seafloor structure }\end{array}$ & $\begin{array}{l}\text { Low, would occur in } \\
\text { very small area }\end{array}$ & $\begin{array}{l}\text { High, would be } \\
\text { continuous for life } \\
\text { of project }\end{array}$ & $\begin{array}{l}\text { Med, wave structures } \\
\text { may attract species to } \\
\text { project area }\end{array}$ & Unknown & Nelson 2008 \\
\hline & $\begin{array}{l}\text { Water circulation } \\
\text { changes }\end{array}$ & $\begin{array}{l}\text { None, project not large } \\
\text { enough to affect prey }\end{array}$ & N/A & N/A & N/A & Low & N/A \\
\hline & $\begin{array}{l}\text { Oil/chemical } \\
\text { release }\end{array}$ & $\begin{array}{l}\text { Toxicity to individuals } \\
\text { and prey }\end{array}$ & $\begin{array}{l}\text { Low, would occur in } \\
\text { very small area }\end{array}$ & $\begin{array}{l}\text { Low, wouldn’t } \\
\text { persist long-term }\end{array}$ & $\begin{array}{l}\text { Low, species in water } \\
\text { column while oil floats } \\
\text { on surface }\end{array}$ & Low & $\begin{array}{l}\text { Nelson and } \\
\text { Woo } 2008\end{array}$ \\
\hline $\begin{array}{l}\text { Electricity } \\
\text { conduction } \\
\text { through cable }\end{array}$ & EMF & $\begin{array}{l}\text { Effects on species } \\
\text { unknown, could include } \\
\text { behavior, orientation } \\
\text { changes }\end{array}$ & $\begin{array}{l}\text { Low, would occur in } \\
\text { very small area }\end{array}$ & $\begin{array}{l}\text { High, would be } \\
\text { continuous for life } \\
\text { of project }\end{array}$ & $\begin{array}{l}\text { Med, wave structures } \\
\text { may attract species to } \\
\text { project area }\end{array}$ & Unknown & Nelson 2008 \\
\hline \multirow{2}{*}{$\begin{array}{l}\text { Structures on } \\
\text { water's surface }\end{array}$} & Structure & $\begin{array}{l}\text { FAD, changes in } \\
\text { predator/prey } \\
\text { abundance, interactions }\end{array}$ & $\begin{array}{l}\text { Med, would occur in } \\
\text { very small area }\end{array}$ & $\begin{array}{l}\text { High, would be } \\
\text { continuous for life } \\
\text { of project }\end{array}$ & $\begin{array}{l}\text { Low, species in water } \\
\text { column or bottom }\end{array}$ & Unknown & Nelson 2008 \\
\hline & Navigation lights & $\begin{array}{l}\text { None, species don't } \\
\text { occur at surface }\end{array}$ & N/A & N/A & N/A & Low & N/A \\
\hline \multicolumn{8}{|c|}{ Decommissioning } \\
\hline \multirow{2}{*}{ Boat traffic } & $\begin{array}{l}\text { Oil/chemical } \\
\text { release }\end{array}$ & $\begin{array}{l}\text { Toxicity to individuals } \\
\text { and prey }\end{array}$ & $\begin{array}{l}\text { Low for typical small } \\
\text { volume releases, High } \\
\text { for large, catastrophic } \\
\text { spill }\end{array}$ & $\begin{array}{l}\text { Low, May-Sept. for } \\
1-2 \text { years }\end{array}$ & $\begin{array}{l}\text { Low, species in water } \\
\text { column while oil floats } \\
\text { on surface }\end{array}$ & Low & $\begin{array}{l}\text { Nelson and } \\
\text { Woo } 2008\end{array}$ \\
\hline & $\begin{array}{l}\text { Noise and } \\
\text { vibration }\end{array}$ & $\begin{array}{l}\text { Possible altered } \\
\text { behavior, may interact } \\
\text { with some species } \\
\text { communication }\end{array}$ & $\begin{array}{l}\text { Low, would occur in } \\
\text { very small area although } \\
\text { noise travels far in water }\end{array}$ & $\begin{array}{l}\text { Low, May-Sept. for } \\
1-2 \text { years }\end{array}$ & $\begin{array}{l}\text { Low, species occur on } \\
\text { or near bottom }\end{array}$ & Low & Cada 2008 \\
\hline
\end{tabular}

\begin{tabular}{|c|c|c|}
\hline $\begin{array}{l}\text { Environmental Assessment for Siting Wave \& Tidal } \\
\text { Energy Projects }\end{array}$ & E-29 & $\begin{array}{r}\text { H. T. Harvey \& Associates } \\
24 \text { November } 2009\end{array}$ \\
\hline
\end{tabular}


Appendix E- Effects of Small Commercial Pelamis Project at Makapu’u

\begin{tabular}{|c|c|c|c|c|c|c|c|}
\hline Project activity & Action & $\begin{array}{l}\text { Description of action's } \\
\text { effect on indicator }\end{array}$ & $\begin{array}{l}\text { Spatial exposure to } \\
\text { indicator (low, med, } \\
\text { high) }\end{array}$ & $\begin{array}{l}\text { Temporal } \\
\text { exposure to } \\
\text { indicator (low, } \\
\text { med, high) }\end{array}$ & $\begin{array}{l}\text { Effect's overlap with } \\
\text { critical life stage, } \\
\text { behavior, habitat or } \\
\text { resource (low, med, } \\
\text { high) }\end{array}$ & $\begin{array}{l}\text { Overall risk } \\
\text { to indicator } \\
\text { (low, med, } \\
\text { high, } \\
\text { unknown) } \\
\end{array}$ & Source(s) \\
\hline \multirow{4}{*}{$\begin{array}{l}\text { Decommissioning } \\
\text { of structures on } \\
\text { water's surface or } \\
\text { seabed }\end{array}$} & $\begin{array}{l}\text { Noise and } \\
\text { vibration }\end{array}$ & $\begin{array}{l}\text { Possible altered } \\
\text { behavior, may interact } \\
\text { with some species } \\
\text { communication }\end{array}$ & $\begin{array}{l}\text { Low, would occur in } \\
\text { very small area although } \\
\text { noise travels far in water }\end{array}$ & $\begin{array}{l}\text { Low, May-Sept. for } \\
1-2 \text { years }\end{array}$ & $\begin{array}{l}\text { Med, species present in } \\
\text { area during } \\
\text { decommissioning }\end{array}$ & Low & Cada 2008 \\
\hline & $\begin{array}{l}\text { Oil/chemical } \\
\text { release }\end{array}$ & $\begin{array}{l}\text { Toxicity to individuals } \\
\text { and prey }\end{array}$ & $\begin{array}{l}\text { Low, volume released } \\
\text { would occupy small } \\
\text { area }\end{array}$ & $\begin{array}{l}\text { Low, May-Sept. for } \\
1-2 \text { years }\end{array}$ & $\begin{array}{l}\text { Low, species in water } \\
\text { column while oil floats } \\
\text { on surface }\end{array}$ & Low & $\begin{array}{l}\text { Nelson and } \\
\text { Woo } 2008\end{array}$ \\
\hline & $\begin{array}{l}\text { Seabed } \\
\text { disturbance }\end{array}$ & $\begin{array}{l}\text { Temporary habitat loss, } \\
\text { changes in prey } \\
\text { resources }\end{array}$ & $\begin{array}{l}\text { Low, would occur in } \\
\text { very small area }\end{array}$ & $\begin{array}{l}\text { Low, May-Sept. for } \\
1-2 \text { years }\end{array}$ & $\begin{array}{l}\text { Med, species present in } \\
\text { area during } \\
\text { decommissioning }\end{array}$ & Low & Nelson 2008 \\
\hline & $\begin{array}{l}\text { Deconstruction } \\
\text { lights }\end{array}$ & $\begin{array}{l}\text { Attraction to prey } \\
\text { aggregation, beneficial } \\
\text { effect }\end{array}$ & $\begin{array}{l}\text { Low, would occur in } \\
\text { very small area }\end{array}$ & $\begin{array}{l}\text { Low, May-Sept. for } \\
1-2 \text { years }\end{array}$ & $\begin{array}{l}\text { Low, likely prey } \\
\text { attracted to surface not } \\
\text { bottom where species } \\
\text { occur }\end{array}$ & Low & $\begin{array}{l}\text { Nightingale } \\
\text { et al. } 2006\end{array}$ \\
\hline
\end{tabular}

\begin{tabular}{lrr}
\hline Environmental Assessment for Siting Wave \& Tidal & E-30 & H. T. Harvey \& Associates \\
Energy Projects & & 24 November 2009
\end{tabular}


Appendix E- Effects of Small Commercial Pelamis Project at Makapu’u

Potential effects on mahimahi and scombrids (tunas, wahoo) due to Small Commercial Pelamis project at Makapu'u site

\begin{tabular}{|c|c|c|c|c|c|c|c|}
\hline Project activity & Action & $\begin{array}{l}\text { Description of action's } \\
\text { effect on indicator }\end{array}$ & $\begin{array}{l}\text { Spatial exposure to } \\
\text { indicator (low, med, } \\
\text { high) }\end{array}$ & $\begin{array}{l}\text { Temporal } \\
\text { exposure to } \\
\text { indicator (low, } \\
\text { med, high) }\end{array}$ & $\begin{array}{l}\text { Effect's overlap with } \\
\text { critical life stage, } \\
\text { behavior, habitat or } \\
\text { resource (low, med, } \\
\text { high) }\end{array}$ & $\begin{array}{l}\text { Overall risk } \\
\text { to indicator } \\
\text { (low, med, } \\
\text { high, } \\
\text { unknown) }\end{array}$ & Source(s) \\
\hline \multicolumn{8}{|c|}{ Construction } \\
\hline \multirow[t]{2}{*}{ Boat traffic } & $\begin{array}{l}\text { Oil/chemical } \\
\text { release }\end{array}$ & $\begin{array}{l}\text { Toxicity to individuals } \\
\text { and prey }\end{array}$ & $\begin{array}{l}\text { Low for typical small } \\
\text { volume releases, High } \\
\text { for large, catastrophic } \\
\text { spill }\end{array}$ & $\begin{array}{l}\text { Low, May-Sept. for } \\
1-2 \text { years }\end{array}$ & $\begin{array}{l}\text { Low, species in water } \\
\text { column but oil floats on } \\
\text { surface }\end{array}$ & Low & $\begin{array}{l}\text { Nelson and } \\
\text { Woo } 2008\end{array}$ \\
\hline & $\begin{array}{l}\text { Noise and } \\
\text { vibration }\end{array}$ & $\begin{array}{l}\text { Possible altered } \\
\text { behavior }\end{array}$ & $\begin{array}{l}\text { Low, would occur in } \\
\text { very small area }\end{array}$ & $\begin{array}{l}\text { Low, May-Sept. for } \\
1-2 \text { years }\end{array}$ & $\begin{array}{l}\text { Med, species present in } \\
\text { area during construction } \\
\text { although highly mobile }\end{array}$ & Low & $\begin{array}{l}\text { Cada 2008, } \\
\text { Sara et al. } \\
2007\end{array}$ \\
\hline \multirow{4}{*}{$\begin{array}{l}\text { Construction of } \\
\text { electrical } \\
\text { collector system, } \\
\text { moorings and } \\
\text { foundations, and } \\
\text { device } \\
\text { installation }\end{array}$} & $\begin{array}{l}\text { Noise and } \\
\text { vibration }\end{array}$ & $\begin{array}{l}\text { Possible altered } \\
\text { behavior }\end{array}$ & $\begin{array}{l}\text { Low, would occur in } \\
\text { very small area }\end{array}$ & $\begin{array}{l}\text { Low, May-Sept. for } \\
1-2 \text { years }\end{array}$ & $\begin{array}{l}\text { Med, species present in } \\
\text { area during construction } \\
\text { although highly mobile }\end{array}$ & Low & $\begin{array}{l}\text { Cada 2008, } \\
\text { Sara et al. } \\
2007\end{array}$ \\
\hline & $\begin{array}{l}\text { Oil/chemical } \\
\text { release }\end{array}$ & $\begin{array}{l}\text { Toxicity to individuals } \\
\text { and prey }\end{array}$ & $\begin{array}{l}\text { Low, volume released } \\
\text { would occupy small } \\
\text { area }\end{array}$ & $\begin{array}{l}\text { Low, May-Sept. for } \\
1-2 \text { years }\end{array}$ & $\begin{array}{l}\text { Low, species in water } \\
\text { column but oil floats on } \\
\text { surface }\end{array}$ & Low & $\begin{array}{l}\text { Nelson and } \\
\text { Woo } 2008\end{array}$ \\
\hline & $\begin{array}{l}\text { Seabed } \\
\text { disturbance }\end{array}$ & $\begin{array}{l}\text { None, species too motile } \\
\text { and near surface to be } \\
\text { affected }\end{array}$ & N/A & N/A & N/A & Low & N/A \\
\hline & $\begin{array}{l}\text { Construction } \\
\text { lights }\end{array}$ & $\begin{array}{l}\text { Attraction to prey } \\
\text { aggregation, beneficial } \\
\text { effect }\end{array}$ & $\begin{array}{l}\text { Low, would occur in } \\
\text { very small area }\end{array}$ & $\begin{array}{l}\text { Low, May-Sept. for } \\
1-2 \text { years }\end{array}$ & $\begin{array}{l}\text { Med, species present in } \\
\text { area during construction }\end{array}$ & Low & $\begin{array}{l}\text { Nightingale } \\
\text { et al. } 2006\end{array}$ \\
\hline \multirow{2}{*}{$\begin{array}{l}\text { Directional } \\
\text { drilling, and } \\
\text { laying cable } \\
\text { under/on seabed }\end{array}$} & $\begin{array}{l}\text { Seabed } \\
\text { disturbance }\end{array}$ & $\begin{array}{l}\text { Temporary habitat loss, } \\
\text { changes in prey } \\
\text { resources }\end{array}$ & $\begin{array}{l}\text { Low, would occur in } \\
\text { very small area }\end{array}$ & $\begin{array}{l}\text { Low, occurs over a } \\
\text { short time period }\end{array}$ & $\begin{array}{l}\text { Low, species are highly } \\
\text { mobile }\end{array}$ & Low & Nelson 2008 \\
\hline & $\begin{array}{l}\text { Noise and } \\
\text { vibration }\end{array}$ & $\begin{array}{l}\text { Possible altered } \\
\text { behavior }\end{array}$ & $\begin{array}{l}\text { Low, would occur in } \\
\text { very small area }\end{array}$ & $\begin{array}{l}\text { Low, occurs over a } \\
\text { short time period }\end{array}$ & $\begin{array}{l}\text { Med, species present in } \\
\text { area during construction } \\
\text { although highly mobile }\end{array}$ & Low & $\begin{array}{l}\text { Cada 2008, } \\
\text { Sara et al. } \\
2007 \\
\end{array}$ \\
\hline \multicolumn{8}{|c|}{ Operation and Maintenance } \\
\hline \multirow[t]{2}{*}{ Boat traffic } & $\begin{array}{l}\text { Oil/chemical } \\
\text { release }\end{array}$ & $\begin{array}{l}\text { Toxicity to individuals } \\
\text { and prey }\end{array}$ & $\begin{array}{l}\text { Low for typical small } \\
\text { volume releases, High } \\
\text { for large, catastrophic } \\
\text { spill }\end{array}$ & $\begin{array}{l}\text { Low, wouldn't } \\
\text { persist long-term }\end{array}$ & $\begin{array}{l}\text { Low, species in water } \\
\text { column but oil floats on } \\
\text { surface }\end{array}$ & Low & $\begin{array}{l}\text { Nelson and } \\
\text { Woo } 2008\end{array}$ \\
\hline & $\begin{array}{l}\text { Noise and } \\
\text { vibration }\end{array}$ & $\begin{array}{l}\text { Possible altered } \\
\text { behavior }\end{array}$ & $\begin{array}{l}\text { Low, would occur in } \\
\text { very small area }\end{array}$ & $\begin{array}{l}\text { Low, wouldn't } \\
\text { persist long-term }\end{array}$ & $\begin{array}{l}\text { Med, species present in } \\
\text { area although highly } \\
\text { mobile }\end{array}$ & Low & $\begin{array}{l}\text { Cada 2008, } \\
\text { Sara et al. } \\
2007\end{array}$ \\
\hline $\begin{array}{l}\text { Operation of } \\
\text { turbines or other }\end{array}$ & $\begin{array}{l}\text { Moving device } \\
\text { parts }\end{array}$ & $\begin{array}{l}\text { None, species very } \\
\text { mobile }\end{array}$ & N/A & N/A & N/A & Low & N/A \\
\hline
\end{tabular}

\begin{tabular}{|c|c|c|}
\hline $\begin{array}{l}\text { Environmental Assessment for Siting Wave \& Tidal } \\
\text { Energy Projects }\end{array}$ & E-31 & $\begin{array}{r}\text { H. T. Harvey \& Associates } \\
24 \text { November } 2009\end{array}$ \\
\hline
\end{tabular}


Appendix E- Effects of Small Commercial Pelamis Project at Makapu’u

\begin{tabular}{|c|c|c|c|c|c|c|c|}
\hline Project activity & Action & $\begin{array}{l}\text { Description of action's } \\
\text { effect on indicator }\end{array}$ & $\begin{array}{l}\text { Spatial exposure to } \\
\text { indicator (low, med, } \\
\text { high) }\end{array}$ & $\begin{array}{l}\text { Temporal } \\
\text { exposure to } \\
\text { indicator (low, } \\
\text { med, high) }\end{array}$ & $\begin{array}{l}\text { Effect's overlap with } \\
\text { critical life stage, } \\
\text { behavior, habitat or } \\
\text { resource (low, med, } \\
\text { high) }\end{array}$ & $\begin{array}{l}\text { Overall risk } \\
\text { to indicator } \\
\text { (low, med, } \\
\text { high, } \\
\text { unknown) }\end{array}$ & Source(s) \\
\hline $\begin{array}{l}\text { moving parts of } \\
\text { devices }\end{array}$ & $\begin{array}{l}\text { Noise and } \\
\text { vibration }\end{array}$ & $\begin{array}{l}\text { Possible altered } \\
\text { behavior }\end{array}$ & $\begin{array}{l}\text { Low, would occur in } \\
\text { very small area }\end{array}$ & $\begin{array}{l}\text { High, would be } \\
\text { continuous for life } \\
\text { of project }\end{array}$ & $\begin{array}{l}\text { Med, species present in } \\
\text { area although highly } \\
\text { mobile }\end{array}$ & Low & $\begin{array}{l}\text { Cada 2008, } \\
\text { Sara et al. } \\
2007 \\
\end{array}$ \\
\hline \multirow{3}{*}{$\begin{array}{l}\text { Structures in } \\
\text { water column and } \\
\text { on seabed, such } \\
\text { as devices and } \\
\text { moorings and } \\
\text { footings }\end{array}$} & Structure & $\begin{array}{l}\text { Structure in water } \\
\text { column acts similarly to } \\
\text { FAD, no effect of } \\
\text { seafloor structure }\end{array}$ & $\begin{array}{l}\text { Med, would occur in } \\
\text { very small area }\end{array}$ & $\begin{array}{l}\text { High, would be } \\
\text { continuous for life } \\
\text { of project }\end{array}$ & $\begin{array}{l}\text { High, species exhibit } \\
\text { attraction to FADs on } \\
\text { Oahu }\end{array}$ & Unknown & $\begin{array}{l}\text { Dagorn et al. } \\
2007 \text {, Nelson } \\
2008\end{array}$ \\
\hline & $\begin{array}{l}\text { Water circulation } \\
\text { changes }\end{array}$ & $\begin{array}{l}\text { None, project not large } \\
\text { enough to affect prey }\end{array}$ & N/A & N/A & N/A & Low & N/A \\
\hline & $\begin{array}{l}\text { Oil/chemical } \\
\text { release }\end{array}$ & $\begin{array}{l}\text { Toxicity to individuals } \\
\text { and prey }\end{array}$ & $\begin{array}{l}\text { Low, would occur in } \\
\text { very small area }\end{array}$ & $\begin{array}{l}\text { Low, wouldn't } \\
\text { persist long-term }\end{array}$ & $\begin{array}{l}\text { Low, species in water } \\
\text { column while oil floats } \\
\text { on surface }\end{array}$ & & $\begin{array}{l}\text { Nelson and } \\
\text { Woo } 2008\end{array}$ \\
\hline $\begin{array}{l}\text { Electricity } \\
\text { conduction } \\
\text { through cable }\end{array}$ & EMF & $\begin{array}{l}\text { Changes in orientation, } \\
\text { behavior }\end{array}$ & $\begin{array}{l}\text { Low, would occur in } \\
\text { very small area }\end{array}$ & $\begin{array}{l}\text { High, would be } \\
\text { continuous for life } \\
\text { of project }\end{array}$ & $\begin{array}{l}\text { Unknown, laboratory } \\
\text { studies show tunas can } \\
\text { detect magnetic fields }\end{array}$ & Unknown & $\begin{array}{l}\text { Nelson 2008, } \\
\text { Walker } 1984\end{array}$ \\
\hline \multirow{2}{*}{$\begin{array}{l}\text { Structures on } \\
\text { water's surface }\end{array}$} & Structure & $\begin{array}{l}\text { FAD, changes in } \\
\text { predator/prey } \\
\text { abundance, interactions }\end{array}$ & $\begin{array}{l}\text { Med, would occur in } \\
\text { very small area }\end{array}$ & $\begin{array}{l}\text { High, would be } \\
\text { continuous for life } \\
\text { of project }\end{array}$ & $\begin{array}{l}\text { High, species exhibit } \\
\text { attraction to FADs on } \\
\text { Oahu }\end{array}$ & Unknown & $\begin{array}{l}\text { Dagorn et al. } \\
2007 \text {, Nelson } \\
2008\end{array}$ \\
\hline & Navigation lights & $\begin{array}{l}\text { Attraction to prey } \\
\text { aggregation, beneficial } \\
\text { effect }\end{array}$ & $\begin{array}{l}\text { Low, would occur in } \\
\text { very small area }\end{array}$ & $\begin{array}{l}\text { High, would be } \\
\text { continuous for life } \\
\text { of project }\end{array}$ & $\begin{array}{l}\text { Med, species present in } \\
\text { area }\end{array}$ & Med & $\begin{array}{l}\text { Nightingale } \\
\text { et al. } 2006\end{array}$ \\
\hline \multicolumn{8}{|c|}{ Decommissioning } \\
\hline \multirow[t]{2}{*}{ Boat traffic } & $\begin{array}{l}\text { Oil/chemical } \\
\text { release }\end{array}$ & $\begin{array}{l}\text { Toxicity to individuals } \\
\text { and prey }\end{array}$ & $\begin{array}{l}\text { Low for typical small } \\
\text { volume releases, High } \\
\text { for large, catastrophic } \\
\text { spill }\end{array}$ & $\begin{array}{l}\text { Low, May-Sept. for } \\
1-2 \text { years }\end{array}$ & $\begin{array}{l}\text { Low, species in water } \\
\text { column while oil floats } \\
\text { on surface }\end{array}$ & Low & $\begin{array}{l}\text { Nelson and } \\
\text { Woo } 2008\end{array}$ \\
\hline & $\begin{array}{l}\text { Noise and } \\
\text { vibration }\end{array}$ & $\begin{array}{l}\text { Possible altered } \\
\text { behavior }\end{array}$ & $\begin{array}{l}\text { Low, would occur in } \\
\text { very small area }\end{array}$ & $\begin{array}{l}\text { Low, May-Sept. for } \\
1-2 \text { years }\end{array}$ & $\begin{array}{l}\text { Med, species present in } \\
\text { area although highly } \\
\text { mobile }\end{array}$ & Low & $\begin{array}{l}\text { Cada 2008, } \\
\text { Sara et al. } \\
2007\end{array}$ \\
\hline \multirow{3}{*}{$\begin{array}{l}\text { Decommissioning } \\
\text { of structures on } \\
\text { water's surface or } \\
\text { seabed }\end{array}$} & $\begin{array}{l}\text { Noise and } \\
\text { vibration }\end{array}$ & $\begin{array}{l}\text { Possible altered } \\
\text { behavior }\end{array}$ & $\begin{array}{l}\text { Low, would occur in } \\
\text { very small area }\end{array}$ & $\begin{array}{l}\text { Low, May-Sept. for } \\
1-2 \text { years }\end{array}$ & $\begin{array}{l}\text { Med, species present in } \\
\text { area although highly } \\
\text { mobile }\end{array}$ & Low & $\begin{array}{l}\text { Cada 2008, } \\
\text { Sara et al. } \\
2007 \\
\end{array}$ \\
\hline & $\begin{array}{l}\text { Oil/chemical } \\
\text { release }\end{array}$ & $\begin{array}{l}\text { Toxicity to individuals } \\
\text { and prey }\end{array}$ & $\begin{array}{l}\text { Low, volume released } \\
\text { would occupy small } \\
\text { area }\end{array}$ & $\begin{array}{l}\text { Low, May-Sept. for } \\
1-2 \text { years }\end{array}$ & $\begin{array}{l}\text { Low, species in water } \\
\text { column while oil floats } \\
\text { on surface }\end{array}$ & Low & $\begin{array}{l}\text { Nelson and } \\
\text { Woo } 2008\end{array}$ \\
\hline & $\begin{array}{l}\text { Seabed } \\
\text { disturbance }\end{array}$ & $\begin{array}{l}\text { Temporary habitat loss, } \\
\text { changes in prey } \\
\text { resources }\end{array}$ & $\begin{array}{l}\text { Low, would occur in } \\
\text { very small area }\end{array}$ & $\begin{array}{l}\text { Low, May-Sept. for } \\
1-2 \text { years }\end{array}$ & $\begin{array}{l}\text { Low, species are highly } \\
\text { mobile }\end{array}$ & Low & Nelson 2008 \\
\hline
\end{tabular}

\begin{tabular}{|c|c|c|}
\hline $\begin{array}{l}\text { Environmental Assessment for Siting Wave \& Tidal } \\
\text { Energy Projects }\end{array}$ & E-32 & $\begin{array}{r}\text { H. T. Harvey \& Associates } \\
24 \text { November } 2009\end{array}$ \\
\hline
\end{tabular}


Appendix E- Effects of Small Commercial Pelamis Project at Makapu'u

\begin{tabular}{|c|c|c|c|c|c|c|c|}
\hline Project activity & Action & $\begin{array}{l}\text { Description of action's } \\
\text { effect on indicator }\end{array}$ & $\begin{array}{l}\text { Spatial exposure to } \\
\text { indicator (low, med, } \\
\text { high) }\end{array}$ & $\begin{array}{l}\text { Temporal } \\
\text { exposure to } \\
\text { indicator (low, } \\
\text { med, high) }\end{array}$ & $\begin{array}{l}\text { Effect's overlap with } \\
\text { critical life stage, } \\
\text { behavior, habitat or } \\
\text { resource (low, med, } \\
\text { high) }\end{array}$ & $\begin{array}{l}\text { Overall risk } \\
\text { to indicator } \\
\text { (low, med, } \\
\text { high, } \\
\text { unknown) } \\
\end{array}$ & Source(s) \\
\hline & $\begin{array}{l}\text { Deconstruction } \\
\text { lights }\end{array}$ & $\begin{array}{l}\text { Attraction to prey } \\
\text { aggregation beneficial } \\
\text { effect }\end{array}$ & $\begin{array}{l}\text { Low, would occur in } \\
\text { very small area }\end{array}$ & $\begin{array}{l}\text { Low, May-Sept. for } \\
1-2 \text { years }\end{array}$ & $\begin{array}{l}\text { Med, species present in } \\
\text { area }\end{array}$ & Low & $\begin{array}{l}\text { Nightingale } \\
\text { et al. } 2006\end{array}$ \\
\hline
\end{tabular}


Appendix E- Effects of Small Commercial Pelamis Project at Makapu’u

Potential effects on Swordfish and Billfishes due to Small Commercial Pelamis project at Makapu'u site

\begin{tabular}{|c|c|c|c|c|c|c|c|}
\hline Project activity & Action & $\begin{array}{l}\text { Description of action's } \\
\text { effect on indicator }\end{array}$ & $\begin{array}{l}\text { Spatial exposure to } \\
\text { indicator (low, med, } \\
\text { high) }\end{array}$ & $\begin{array}{l}\text { Temporal } \\
\text { exposure to } \\
\text { indicator (low, } \\
\text { med, high) }\end{array}$ & $\begin{array}{l}\text { Effect's overlap with } \\
\text { critical life stage, } \\
\text { behavior, habitat or } \\
\text { resource (low, med, } \\
\text { high) }\end{array}$ & $\begin{array}{l}\text { Overall risk } \\
\text { to indicator } \\
\text { (low, med, } \\
\text { high, } \\
\text { unknown) }\end{array}$ & Source(s) \\
\hline \multicolumn{8}{|c|}{ Construction } \\
\hline \multirow[t]{2}{*}{ Boat traffic } & $\begin{array}{l}\text { Oil/chemical } \\
\text { release }\end{array}$ & $\begin{array}{l}\text { Toxicity to individuals } \\
\text { and prey }\end{array}$ & $\begin{array}{l}\text { Low for typical small } \\
\text { volume releases, } \\
\text { Medium for large, } \\
\text { catastrophic spill } \\
\text { because they are } \\
\text { migratory/mobile }\end{array}$ & $\begin{array}{l}\text { Low, May-Sept. for } \\
1-2 \text { years }\end{array}$ & $\begin{array}{l}\text { Low, species in water } \\
\text { column but oil floats on } \\
\text { surface }\end{array}$ & Low & $\begin{array}{l}\text { Nelson and } \\
\text { Woo } 2008\end{array}$ \\
\hline & $\begin{array}{l}\text { Noise and } \\
\text { vibration }\end{array}$ & $\begin{array}{l}\text { Possible altered } \\
\text { behavior }\end{array}$ & $\begin{array}{l}\text { Low, would occur in } \\
\text { very small area }\end{array}$ & $\begin{array}{l}\text { Low, May-Sept. for } \\
1-2 \text { years }\end{array}$ & $\begin{array}{l}\text { Low, species distributed } \\
\text { predominantly offshore } \\
\text { of the project area }\end{array}$ & Low & Cada 2008 \\
\hline \multirow{4}{*}{$\begin{array}{l}\text { Construction of } \\
\text { electrical } \\
\text { collector system, } \\
\text { moorings and } \\
\text { foundations, and } \\
\text { device } \\
\text { installation }\end{array}$} & $\begin{array}{l}\text { Noise and } \\
\text { vibration }\end{array}$ & $\begin{array}{l}\text { Possible altered } \\
\text { behavior }\end{array}$ & $\begin{array}{l}\text { Low, would occur in } \\
\text { very small area }\end{array}$ & $\begin{array}{l}\text { Low, May-Sept. for } \\
1-2 \text { years }\end{array}$ & $\begin{array}{l}\text { Low, species distributed } \\
\text { predominantly offshore } \\
\text { of the project area }\end{array}$ & Low & Cada 2008 \\
\hline & $\begin{array}{l}\text { Oil/chemical } \\
\text { release }\end{array}$ & $\begin{array}{l}\text { Toxicity to individuals } \\
\text { and prey }\end{array}$ & $\begin{array}{l}\text { Low, volume released } \\
\text { would occupy small } \\
\text { area }\end{array}$ & $\begin{array}{l}\text { Low, May-Sept. for } \\
1-2 \text { years }\end{array}$ & $\begin{array}{l}\text { Low, species in water } \\
\text { column but oil floats on } \\
\text { surface }\end{array}$ & Low & $\begin{array}{l}\text { Nelson and } \\
\text { Woo } 2008\end{array}$ \\
\hline & $\begin{array}{l}\text { Seabed } \\
\text { disturbance }\end{array}$ & $\begin{array}{l}\text { Temporary habitat loss, } \\
\text { changes in prey } \\
\text { resources }\end{array}$ & $\begin{array}{l}\text { Low, would occur in } \\
\text { very small area }\end{array}$ & $\begin{array}{l}\text { Low, May-Sept. for } \\
1-2 \text { years }\end{array}$ & $\begin{array}{l}\text { Low, species distributed } \\
\text { predominantly offshore } \\
\text { of the project area }\end{array}$ & Low & Nelson 2008 \\
\hline & $\begin{array}{l}\text { Construction } \\
\text { lights }\end{array}$ & $\begin{array}{l}\text { Attraction to prey } \\
\text { aggregation }\end{array}$ & $\begin{array}{l}\text { Low, would occur in } \\
\text { very small area }\end{array}$ & $\begin{array}{l}\text { Low, May-Sept. for } \\
1-2 \text { years }\end{array}$ & $\begin{array}{l}\text { Low, species distributed } \\
\text { predominantly offshore } \\
\text { of the project area }\end{array}$ & Low & $\begin{array}{l}\text { Nightingale } \\
\text { et al. } 2006\end{array}$ \\
\hline \multirow{2}{*}{$\begin{array}{l}\text { Directional } \\
\text { drilling, and } \\
\text { laying cable } \\
\text { under/on seabed }\end{array}$} & $\begin{array}{l}\text { Seabed } \\
\text { disturbance }\end{array}$ & $\begin{array}{l}\text { Temporary habitat loss, } \\
\text { changes in prey } \\
\text { resources }\end{array}$ & $\begin{array}{l}\text { Low, would occur in } \\
\text { very small area }\end{array}$ & $\begin{array}{l}\text { Low, occurs over a } \\
\text { short time period }\end{array}$ & $\begin{array}{l}\text { Low, species distributed } \\
\text { predominantly offshore } \\
\text { of the project area }\end{array}$ & Low & Nelson 2008 \\
\hline & $\begin{array}{l}\text { Noise and } \\
\text { vibration }\end{array}$ & $\begin{array}{l}\text { Possible altered } \\
\text { behavior }\end{array}$ & $\begin{array}{l}\text { Low, would occur in } \\
\text { very small area }\end{array}$ & $\begin{array}{l}\text { Low, occurs over a } \\
\text { short time period }\end{array}$ & $\begin{array}{l}\text { Low, species distributed } \\
\text { predominantly offshore } \\
\text { of the project area }\end{array}$ & Low & Cada 2008 \\
\hline \multicolumn{8}{|c|}{ Operation and Maintenance } \\
\hline \multirow[t]{2}{*}{ Boat traffic } & $\begin{array}{l}\text { Oil/chemical } \\
\text { release }\end{array}$ & $\begin{array}{l}\text { Toxicity to individuals } \\
\text { and prey }\end{array}$ & $\begin{array}{l}\text { Low for typical small } \\
\text { volume releases, High } \\
\text { for large, catastrophic } \\
\text { spill }\end{array}$ & $\begin{array}{l}\text { Low, wouldn't } \\
\text { persist long-term }\end{array}$ & $\begin{array}{l}\text { Low, species in water } \\
\text { column but oil floats on } \\
\text { surface }\end{array}$ & Low & $\begin{array}{l}\text { Nelson and } \\
\text { Woo } 2008\end{array}$ \\
\hline & $\begin{array}{l}\text { Noise and } \\
\text { vibration }\end{array}$ & $\begin{array}{l}\text { Possible altered } \\
\text { behavior }\end{array}$ & $\begin{array}{l}\text { Low, would occur in } \\
\text { very small area }\end{array}$ & $\begin{array}{l}\text { Low, wouldn’t } \\
\text { persist long-term }\end{array}$ & $\begin{array}{l}\text { Low, species distributed } \\
\text { predominantly offshore } \\
\text { of the project area }\end{array}$ & Low & Cada 2008 \\
\hline
\end{tabular}

\begin{tabular}{|c|c|c|}
\hline $\begin{array}{l}\text { Environmental Assessment for Siting Wave \& Tidal } \\
\text { Energy Projects }\end{array}$ & E-34 & $\begin{array}{r}\text { H. T. Harvey \& Associates } \\
24 \text { November } 2009\end{array}$ \\
\hline
\end{tabular}


Appendix E- Effects of Small Commercial Pelamis Project at Makapu’u

\begin{tabular}{|c|c|c|c|c|c|c|c|}
\hline Project activity & Action & $\begin{array}{l}\text { Description of action's } \\
\text { effect on indicator }\end{array}$ & $\begin{array}{l}\text { Spatial exposure to } \\
\text { indicator (low, med, } \\
\text { high) }\end{array}$ & $\begin{array}{l}\text { Temporal } \\
\text { exposure to } \\
\text { indicator (low, } \\
\text { med, high) }\end{array}$ & $\begin{array}{l}\text { Effect's overlap with } \\
\text { critical life stage, } \\
\text { behavior, habitat or } \\
\text { resource (low, med, } \\
\text { high) }\end{array}$ & $\begin{array}{l}\text { Overall risk } \\
\text { to indicator } \\
\text { (low, med, } \\
\text { high, } \\
\text { unknown) }\end{array}$ & Source(s) \\
\hline \multirow{2}{*}{$\begin{array}{l}\text { Operation of } \\
\text { turbines or other } \\
\text { moving parts of } \\
\text { devices }\end{array}$} & $\begin{array}{l}\text { Moving device } \\
\text { parts }\end{array}$ & $\begin{array}{l}\text { None, species very } \\
\text { mobile }\end{array}$ & N/A & N/A & N/A & Low & N/A \\
\hline & $\begin{array}{l}\text { Noise and } \\
\text { vibration }\end{array}$ & Disturbance & $\begin{array}{l}\text { Low, would occur in } \\
\text { very small area }\end{array}$ & $\begin{array}{l}\text { High, would be } \\
\text { continuous for life } \\
\text { of project }\end{array}$ & $\begin{array}{l}\text { Low, species distributed } \\
\text { predominantly offshore } \\
\text { of the project area }\end{array}$ & Low & Cada 2008 \\
\hline \multirow{3}{*}{$\begin{array}{l}\text { Structures in } \\
\text { water column and } \\
\text { on seabed, such } \\
\text { as devices and } \\
\text { moorings and } \\
\text { footings }\end{array}$} & Structure & $\begin{array}{l}\text { Structure in water } \\
\text { column acts similarly to } \\
\text { FAD, no effect of } \\
\text { seafloor structure }\end{array}$ & $\begin{array}{l}\text { Med, would occur in } \\
\text { very small area }\end{array}$ & $\begin{array}{l}\text { High, would be } \\
\text { continuous for life } \\
\text { of project }\end{array}$ & $\begin{array}{l}\text { High, species known to } \\
\text { be attracted to FADs }\end{array}$ & Unknown & $\begin{array}{l}\text { Dempster } \\
\text { and Taquet } \\
2004\end{array}$ \\
\hline & $\begin{array}{l}\text { Water circulation } \\
\text { changes }\end{array}$ & $\begin{array}{l}\text { None, project not large } \\
\text { enough to affect prey }\end{array}$ & N/A & N/A & N/A & Low & N/A \\
\hline & $\begin{array}{l}\text { Oil/chemical } \\
\text { release }\end{array}$ & $\begin{array}{l}\text { Toxicity to individuals } \\
\text { and prey }\end{array}$ & $\begin{array}{l}\text { Low, would occur in } \\
\text { very small area }\end{array}$ & $\begin{array}{l}\text { Low, wouldn’t } \\
\text { persist long-term }\end{array}$ & $\begin{array}{l}\text { Low, species in water } \\
\text { column while oil floats } \\
\text { on surface }\end{array}$ & Low & $\begin{array}{l}\text { Nelson and } \\
\text { Woo } 2008\end{array}$ \\
\hline $\begin{array}{l}\text { Electricity } \\
\text { conduction } \\
\text { through cable } \\
\end{array}$ & EMF & $\begin{array}{l}\text { Changes in orientation, } \\
\text { behavior }\end{array}$ & $\begin{array}{l}\text { Low, would occur in } \\
\text { very small area }\end{array}$ & $\begin{array}{l}\text { High, would be } \\
\text { continuous for life } \\
\text { of project }\end{array}$ & $\begin{array}{l}\text { Low, species distributed } \\
\text { predominantly offshore } \\
\text { of the project area }\end{array}$ & Low & Nelson 2008 \\
\hline \multirow{2}{*}{$\begin{array}{l}\text { Structures on } \\
\text { water's surface }\end{array}$} & Structure & FAD & $\begin{array}{l}\text { Med, would occur in } \\
\text { very small area }\end{array}$ & $\begin{array}{l}\text { High, would be } \\
\text { continuous for life } \\
\text { of project }\end{array}$ & $\begin{array}{l}\text { High, species known to } \\
\text { be attracted to FADs }\end{array}$ & Med & Nelson 2008 \\
\hline & Navigation lights & $\begin{array}{l}\text { Attraction to prey } \\
\text { aggregation }\end{array}$ & $\begin{array}{l}\text { Low, would occur in } \\
\text { very small area }\end{array}$ & $\begin{array}{l}\text { Low, occurs over a } \\
\text { short time period }\end{array}$ & $\begin{array}{l}\text { Low, species distributed } \\
\text { predominantly offshore } \\
\text { of the project area }\end{array}$ & Low & $\begin{array}{l}\text { Nightingale } \\
\text { et al. } 2006\end{array}$ \\
\hline \multicolumn{8}{|c|}{ Decommissioning } \\
\hline \multirow[t]{2}{*}{ Boat traffic } & $\begin{array}{l}\text { Oil/chemical } \\
\text { release }\end{array}$ & $\begin{array}{l}\text { Toxicity to individuals } \\
\text { and prey }\end{array}$ & $\begin{array}{l}\text { Low for typical small } \\
\text { volume releases, High } \\
\text { for large, catastrophic } \\
\text { spill }\end{array}$ & $\begin{array}{l}\text { Low, May-Sept. for } \\
1 \text {-2 years }\end{array}$ & $\begin{array}{l}\text { Low, species in water } \\
\text { column while oil floats } \\
\text { on surface }\end{array}$ & Low & $\begin{array}{l}\text { Nelson and } \\
\text { Woo } 2008\end{array}$ \\
\hline & $\begin{array}{l}\text { Noise and } \\
\text { vibration }\end{array}$ & $\begin{array}{l}\text { Possible altered } \\
\text { behavior }\end{array}$ & $\begin{array}{l}\text { Low, would occur in } \\
\text { very small area }\end{array}$ & $\begin{array}{l}\text { Low, May-Sept. for } \\
1-2 \text { years }\end{array}$ & $\begin{array}{l}\text { Low, species distributed } \\
\text { predominantly offshore } \\
\text { of the project area }\end{array}$ & Low & Cada 2008 \\
\hline \multirow{2}{*}{$\begin{array}{l}\text { Decommissioning } \\
\text { of structures on } \\
\text { water's surface or } \\
\text { seabed }\end{array}$} & $\begin{array}{l}\text { Noise and } \\
\text { vibration }\end{array}$ & $\begin{array}{l}\text { Possible altered } \\
\text { behavior }\end{array}$ & $\begin{array}{l}\text { Low, would occur in } \\
\text { very small area }\end{array}$ & $\begin{array}{l}\text { Low, May-Sept. for } \\
1-2 \text { years }\end{array}$ & $\begin{array}{l}\text { Low, species distributed } \\
\text { predominantly offshore } \\
\text { of the project area }\end{array}$ & Low & Cada 2008 \\
\hline & $\begin{array}{l}\text { Oil/chemical } \\
\text { release }\end{array}$ & $\begin{array}{l}\text { Toxicity to individuals } \\
\text { and prey }\end{array}$ & $\begin{array}{l}\text { Low, volume released } \\
\text { would occupy small } \\
\text { area }\end{array}$ & $\begin{array}{l}\text { Low, May-Sept. for } \\
1-2 \text { years }\end{array}$ & $\begin{array}{l}\text { Low, species in water } \\
\text { column while oil floats } \\
\text { on surface }\end{array}$ & Low & $\begin{array}{l}\text { Nelson and } \\
\text { Woo } 2008\end{array}$ \\
\hline
\end{tabular}

\begin{tabular}{|c|c|c|}
\hline $\begin{array}{l}\text { Environmental Assessment for Siting Wave \& Tidal } \\
\text { Energy Projects }\end{array}$ & E-35 & $\begin{array}{r}\text { H. T. Harvey \& Associates } \\
24 \text { November } 2009\end{array}$ \\
\hline
\end{tabular}


Appendix E- Effects of Small Commercial Pelamis Project at Makapu'u

\begin{tabular}{|c|c|c|c|c|c|c|c|}
\hline Project activity & Action & $\begin{array}{l}\text { Description of action's } \\
\text { effect on indicator }\end{array}$ & $\begin{array}{l}\text { Spatial exposure to } \\
\text { indicator (low, med, } \\
\text { high) }\end{array}$ & $\begin{array}{l}\text { Temporal } \\
\text { exposure to } \\
\text { indicator (low, } \\
\text { med, high) }\end{array}$ & $\begin{array}{l}\text { Effect's overlap with } \\
\text { critical life stage, } \\
\text { behavior, habitat or } \\
\text { resource (low, med, } \\
\text { high) }\end{array}$ & $\begin{array}{l}\text { Overall risk } \\
\text { to indicator } \\
\text { (low, med, } \\
\text { high, } \\
\text { unknown) } \\
\end{array}$ & Source(s) \\
\hline & $\begin{array}{l}\text { Seabed } \\
\text { disturbance }\end{array}$ & $\begin{array}{l}\text { Temporary habitat loss, } \\
\text { changes in prey } \\
\text { resources }\end{array}$ & $\begin{array}{l}\text { Low, would occur in } \\
\text { very small area }\end{array}$ & $\begin{array}{l}\text { Low, May-Sept. for } \\
1-2 \text { years }\end{array}$ & $\begin{array}{l}\text { Low, species distributed } \\
\text { predominantly offshore } \\
\text { of the project area }\end{array}$ & Low & \\
\hline & $\begin{array}{l}\text { Deconstruction } \\
\text { lights }\end{array}$ & $\begin{array}{l}\text { Attraction to prey } \\
\text { aggregation }\end{array}$ & $\begin{array}{l}\text { Low, would occur in } \\
\text { very small area }\end{array}$ & $\begin{array}{l}\text { Low, May-Sept. for } \\
1-2 \text { years }\end{array}$ & $\begin{array}{l}\text { Low, species distributed } \\
\text { predominantly offshore } \\
\text { of the project area }\end{array}$ & Low & $\begin{array}{l}\text { Nightingale } \\
\text { et al. } 2006\end{array}$ \\
\hline
\end{tabular}


Appendix E- Effects of Small Commercial Pelamis Project at Makapu'u

Potential effects on sharks (e.g., Oceanic whitetip, Tiger, Shortfin mako, and Longfin mako) due to Small Commercial Pelamis project at Makapu'u site

\begin{tabular}{|c|c|c|c|c|c|c|c|}
\hline Project activity & Action & $\begin{array}{l}\text { Description of action's } \\
\text { effect on indicator }\end{array}$ & $\begin{array}{l}\text { Spatial exposure to } \\
\text { indicator (low, med, } \\
\text { high) }\end{array}$ & $\begin{array}{l}\text { Temporal } \\
\text { exposure to } \\
\text { indicator (low, } \\
\text { med, high) }\end{array}$ & $\begin{array}{l}\text { Effect's overlap with } \\
\text { critical life stage, } \\
\text { behavior, habitat or } \\
\text { resource (low, med, } \\
\text { high) }\end{array}$ & $\begin{array}{l}\text { Overall risk } \\
\text { to indicator } \\
\text { (low, med, } \\
\text { high, } \\
\text { unknown) }\end{array}$ & Source(s) \\
\hline \multicolumn{8}{|c|}{ Construction } \\
\hline \multirow{2}{*}{ Boat traffic } & $\begin{array}{l}\text { Oil/chemical } \\
\text { release }\end{array}$ & $\begin{array}{l}\text { Toxicity to individuals } \\
\text { and prey }\end{array}$ & $\begin{array}{l}\text { Low for typical small } \\
\text { volume releases, High } \\
\text { for large spill }\end{array}$ & $\begin{array}{l}\text { Low, May-Sept. for } \\
\text { 1-2 years }\end{array}$ & $\begin{array}{l}\text { Low, species in water } \\
\text { column but oil floats on } \\
\text { surface }\end{array}$ & Low & $\begin{array}{l}\text { Nelson and } \\
\text { Woo } 2008\end{array}$ \\
\hline & $\begin{array}{l}\text { Noise and } \\
\text { vibration }\end{array}$ & $\begin{array}{l}\text { Possible altered } \\
\text { behavior }\end{array}$ & $\begin{array}{l}\text { Low, would occur in } \\
\text { very small area }\end{array}$ & $\begin{array}{l}\text { Low, May-Sept. for } \\
1-2 \text { years }\end{array}$ & $\begin{array}{l}\text { Med, species present in } \\
\text { area during construction } \\
\text { although highly mobile }\end{array}$ & Low & $\begin{array}{l}\text { Cada 2008, } \\
\text { Parrish and } \\
\text { Goto } 1997 \\
\end{array}$ \\
\hline \multirow{4}{*}{$\begin{array}{l}\text { Construction of } \\
\text { electrical } \\
\text { collector system, } \\
\text { moorings and } \\
\text { foundations, and } \\
\text { device } \\
\text { installation }\end{array}$} & $\begin{array}{l}\text { Noise and } \\
\text { vibration }\end{array}$ & $\begin{array}{l}\text { Possible altered } \\
\text { behavior }\end{array}$ & $\begin{array}{l}\text { Low, would occur in } \\
\text { very small area }\end{array}$ & $\begin{array}{l}\text { Low, May-Sept. for } \\
1-2 \text { years }\end{array}$ & $\begin{array}{l}\text { Med, species present in } \\
\text { area during construction } \\
\text { although highly mobile }\end{array}$ & Low & $\begin{array}{l}\text { Cada 2008, } \\
\text { Parrish and } \\
\text { Goto } 1997 \\
\end{array}$ \\
\hline & $\begin{array}{l}\text { Oil/chemical } \\
\text { release }\end{array}$ & $\begin{array}{l}\text { Toxicity to individuals } \\
\text { and prey }\end{array}$ & $\begin{array}{l}\text { Low, volume released } \\
\text { would occupy small } \\
\text { area }\end{array}$ & $\begin{array}{l}\text { Low, May-Sept. for } \\
1-2 \text { years }\end{array}$ & $\begin{array}{l}\text { Low, species in water } \\
\text { column but oil floats on } \\
\text { surface }\end{array}$ & Low & $\begin{array}{l}\text { Nelson and } \\
\text { Woo } 2008\end{array}$ \\
\hline & $\begin{array}{l}\text { Seabed } \\
\text { disturbance }\end{array}$ & $\begin{array}{l}\text { Temporary habitat loss, } \\
\text { changes in prey } \\
\text { resources }\end{array}$ & $\begin{array}{l}\text { Low, would occur in } \\
\text { very small area }\end{array}$ & $\begin{array}{l}\text { Low, May-Sept. for } \\
1-2 \text { years }\end{array}$ & $\begin{array}{l}\text { Med, species present in } \\
\text { area during construction } \\
\text { although highly mobile }\end{array}$ & Low & Nelson 2008 \\
\hline & $\begin{array}{l}\text { Construction } \\
\text { lights }\end{array}$ & $\begin{array}{l}\text { Attraction to prey } \\
\text { aggregation }\end{array}$ & $\begin{array}{l}\text { Low, would occur in } \\
\text { very small area }\end{array}$ & $\begin{array}{l}\text { Low, May-Sept. for } \\
1-2 \text { years }\end{array}$ & $\begin{array}{l}\text { Med, species present in } \\
\text { area during construction } \\
\text { although highly mobile }\end{array}$ & Low & $\begin{array}{l}\text { Nightingale } \\
\text { et al. } 2006\end{array}$ \\
\hline \multirow{2}{*}{$\begin{array}{l}\text { Directional } \\
\text { drilling, and } \\
\text { laying cable } \\
\text { under/on seabed }\end{array}$} & $\begin{array}{l}\text { Seabed } \\
\text { disturbance }\end{array}$ & $\begin{array}{l}\text { Temporary habitat loss, } \\
\text { changes in prey } \\
\text { resources }\end{array}$ & $\begin{array}{l}\text { Low, would occur in } \\
\text { very small area }\end{array}$ & $\begin{array}{l}\text { Low, occurs over a } \\
\text { short time period }\end{array}$ & $\begin{array}{l}\text { Med, species present in } \\
\text { area during construction } \\
\text { although highly mobile }\end{array}$ & Low & Nelson 2008 \\
\hline & $\begin{array}{l}\text { Noise and } \\
\text { vibration }\end{array}$ & $\begin{array}{l}\text { Possible altered } \\
\text { behavior }\end{array}$ & $\begin{array}{l}\text { Low, would occur in } \\
\text { very small area }\end{array}$ & $\begin{array}{l}\text { Low, occurs over a } \\
\text { short time period }\end{array}$ & $\begin{array}{l}\text { Med, species present in } \\
\text { area during construction } \\
\text { although highly mobile }\end{array}$ & Low & Cada 2008 \\
\hline \multicolumn{8}{|c|}{ Operation and Maintenance } \\
\hline \multirow{2}{*}{ Boat traffic } & $\begin{array}{l}\text { Oil/chemical } \\
\text { release }\end{array}$ & $\begin{array}{l}\text { Toxicity to individuals } \\
\text { and prey }\end{array}$ & $\begin{array}{l}\text { Low for typical small } \\
\text { volume releases, High } \\
\text { for large spill }\end{array}$ & $\begin{array}{l}\text { Low, wouldn't } \\
\text { persist long-term }\end{array}$ & $\begin{array}{l}\text { Low, species in water } \\
\text { column but oil floats on } \\
\text { surface }\end{array}$ & Low & $\begin{array}{l}\text { Nelson and } \\
\text { Woo } 2008\end{array}$ \\
\hline & $\begin{array}{l}\text { Noise and } \\
\text { vibration }\end{array}$ & $\begin{array}{l}\text { Possible altered } \\
\text { behavior }\end{array}$ & $\begin{array}{l}\text { Low, would occur in } \\
\text { very small area }\end{array}$ & $\begin{array}{l}\text { Low, wouldn't } \\
\text { persist long-term }\end{array}$ & $\begin{array}{l}\text { Med, species present in } \\
\text { area although highly } \\
\text { mobile }\end{array}$ & Low & Cada 2008 \\
\hline $\begin{array}{l}\text { Operation of } \\
\text { turbines or other }\end{array}$ & $\begin{array}{l}\text { Moving device } \\
\text { parts }\end{array}$ & $\begin{array}{l}\text { None, species very } \\
\text { mobile }\end{array}$ & N/A & N/A & N/A & Low & N/A \\
\hline
\end{tabular}

\begin{tabular}{|c|c|c|}
\hline $\begin{array}{l}\text { Environmental Assessment for Siting Wave \& Tidal } \\
\text { Energy Projects }\end{array}$ & E-37 & $\begin{array}{r}\text { H. T. Harvey \& Associates } \\
24 \text { November } 2009\end{array}$ \\
\hline
\end{tabular}


Appendix E- Effects of Small Commercial Pelamis Project at Makapu’u

\begin{tabular}{|c|c|c|c|c|c|c|c|}
\hline Project activity & Action & $\begin{array}{l}\text { Description of action's } \\
\text { effect on indicator }\end{array}$ & $\begin{array}{l}\text { Spatial exposure to } \\
\text { indicator (low, med, } \\
\text { high) }\end{array}$ & $\begin{array}{l}\text { Temporal } \\
\text { exposure to } \\
\text { indicator (low, } \\
\text { med, high) }\end{array}$ & $\begin{array}{l}\text { Effect's overlap with } \\
\text { critical life stage, } \\
\text { behavior, habitat or } \\
\text { resource (low, med, } \\
\text { high) }\end{array}$ & $\begin{array}{l}\text { Overall risk } \\
\text { to indicator } \\
\text { (low, med, } \\
\text { high, } \\
\text { unknown) }\end{array}$ & Source(s) \\
\hline $\begin{array}{l}\text { moving parts of } \\
\text { devices }\end{array}$ & $\begin{array}{l}\text { Noise and } \\
\text { vibration }\end{array}$ & $\begin{array}{l}\text { Possible altered } \\
\text { behavior }\end{array}$ & $\begin{array}{l}\text { Low, would occur in } \\
\text { very small area }\end{array}$ & $\begin{array}{l}\text { High, would be } \\
\text { continuous for life } \\
\text { of project }\end{array}$ & $\begin{array}{l}\text { Med, species present in } \\
\text { area although highly } \\
\text { mobile }\end{array}$ & Low & Cada 2008 \\
\hline \multirow{3}{*}{$\begin{array}{l}\text { Structures in } \\
\text { water column and } \\
\text { on seabed, such } \\
\text { as devices and } \\
\text { moorings and } \\
\text { footings }\end{array}$} & Structure & $\begin{array}{l}\text { Structure in water } \\
\text { column acts similar to } \\
\text { FAD, no effect of } \\
\text { seafloor structure }\end{array}$ & $\begin{array}{l}\text { Med, would occur in } \\
\text { very small area }\end{array}$ & $\begin{array}{l}\text { High, would be } \\
\text { continuous for life } \\
\text { of project }\end{array}$ & $\begin{array}{l}\text { High, species known to } \\
\text { be attracted to FADs }\end{array}$ & Unknown & $\begin{array}{l}\text { Dempster } \\
\text { and Taquet } \\
2004\end{array}$ \\
\hline & $\begin{array}{l}\text { Water circulation } \\
\text { changes }\end{array}$ & $\begin{array}{l}\text { None, project not large } \\
\text { enough to affect prey }\end{array}$ & N/A & N/A & N/A & Low & N/A \\
\hline & $\begin{array}{l}\text { Oil/chemical } \\
\text { release }\end{array}$ & $\begin{array}{l}\text { Toxicity to individuals } \\
\text { and prey }\end{array}$ & $\begin{array}{l}\text { Low, would occur in } \\
\text { very small area }\end{array}$ & $\begin{array}{l}\text { Low, wouldn’t } \\
\text { persist long-term }\end{array}$ & $\begin{array}{l}\text { Low, species in water } \\
\text { column while oil floats } \\
\text { on surface }\end{array}$ & Low & $\begin{array}{l}\text { Nelson and } \\
\text { Woo } 2008\end{array}$ \\
\hline $\begin{array}{l}\text { Electricity } \\
\text { conduction } \\
\text { through cable }\end{array}$ & EMF & $\begin{array}{l}\text { Changes in orientation, } \\
\text { behavior }\end{array}$ & $\begin{array}{l}\text { Low, would occur in } \\
\text { very small area }\end{array}$ & $\begin{array}{l}\text { High, would be } \\
\text { continuous for life } \\
\text { of project }\end{array}$ & $\begin{array}{l}\text { High, species known to } \\
\text { be able to detect EMFs, } \\
\text { although actual effect on } \\
\text { behavior unknown }\end{array}$ & Unknown & Nelson 2008 \\
\hline \multirow{2}{*}{$\begin{array}{l}\text { Structures on } \\
\text { water's surface }\end{array}$} & Structure & FAD & $\begin{array}{l}\text { Med, would occur in } \\
\text { very small area }\end{array}$ & $\begin{array}{l}\text { High, would be } \\
\text { continuous for life } \\
\text { of project }\end{array}$ & $\begin{array}{l}\text { High, species known to } \\
\text { be attracted to FADs }\end{array}$ & Med & Nelson 2008 \\
\hline & Navigation lights & $\begin{array}{l}\text { Attraction to prey } \\
\text { aggregation }\end{array}$ & $\begin{array}{l}\text { Low, would occur in } \\
\text { very small area }\end{array}$ & $\begin{array}{l}\text { High, would be } \\
\text { continuous for life } \\
\text { of project }\end{array}$ & $\begin{array}{l}\text { Med, species present in } \\
\text { area although highly } \\
\text { mobile }\end{array}$ & Med & $\begin{array}{l}\text { Nightingale } \\
\text { et al. } 2006\end{array}$ \\
\hline \multicolumn{8}{|c|}{ Decommissioning } \\
\hline \multirow{2}{*}{ Boat traffic } & $\begin{array}{l}\text { Oil/chemical } \\
\text { release }\end{array}$ & $\begin{array}{l}\text { Toxicity to individuals } \\
\text { and prey }\end{array}$ & $\begin{array}{l}\text { Low for typical small } \\
\text { volume releases, High } \\
\text { for large spill }\end{array}$ & $\begin{array}{l}\text { Low, May-Sept. for } \\
1-2 \text { years }\end{array}$ & $\begin{array}{l}\text { Low, species in water } \\
\text { column while oil floats } \\
\text { on surface }\end{array}$ & Low & Nelson 2008 \\
\hline & $\begin{array}{l}\text { Noise and } \\
\text { vibration }\end{array}$ & $\begin{array}{l}\text { Possible altered } \\
\text { behavior }\end{array}$ & $\begin{array}{l}\text { Low, would occur in } \\
\text { very small area }\end{array}$ & $\begin{array}{l}\text { Low, May-Sept. for } \\
1-2 \text { years }\end{array}$ & $\begin{array}{l}\text { Med, species present in } \\
\text { area although highly } \\
\text { mobile }\end{array}$ & Low & Cada 2008 \\
\hline \multirow{3}{*}{$\begin{array}{l}\text { Decommissioning } \\
\text { of structures on } \\
\text { water's surface or } \\
\text { seabed }\end{array}$} & $\begin{array}{l}\text { Noise and } \\
\text { vibration }\end{array}$ & $\begin{array}{l}\text { Possible altered } \\
\text { behavior }\end{array}$ & $\begin{array}{l}\text { Low, would occur in } \\
\text { very small area }\end{array}$ & $\begin{array}{l}\text { Low, May-Sept. for } \\
1-2 \text { years }\end{array}$ & $\begin{array}{l}\text { Med, species present in } \\
\text { area although highly } \\
\text { mobile }\end{array}$ & Low & Cada 2008 \\
\hline & $\begin{array}{l}\text { Oil/chemical } \\
\text { release }\end{array}$ & $\begin{array}{l}\text { Toxicity to individuals } \\
\text { and prey }\end{array}$ & $\begin{array}{l}\text { Low, volume released } \\
\text { would occupy small } \\
\text { area }\end{array}$ & $\begin{array}{l}\text { Low, May-Sept. for } \\
1-2 \text { years }\end{array}$ & $\begin{array}{l}\text { Low, species in water } \\
\text { column while oil floats } \\
\text { on surface }\end{array}$ & Low & $\begin{array}{l}\text { Nelson and } \\
\text { Woo } 2008\end{array}$ \\
\hline & $\begin{array}{l}\text { Seabed } \\
\text { disturbance }\end{array}$ & $\begin{array}{l}\text { Temporary habitat loss, } \\
\text { changes in prey } \\
\text { resources }\end{array}$ & $\begin{array}{l}\text { Low, would occur in } \\
\text { very small area }\end{array}$ & $\begin{array}{l}\text { Low, May-Sept. for } \\
1-2 \text { years }\end{array}$ & $\begin{array}{l}\text { Medium, species present } \\
\text { in area although highly } \\
\text { mobile }\end{array}$ & Low & Nelson 2008 \\
\hline
\end{tabular}

\begin{tabular}{|c|c|c|}
\hline $\begin{array}{l}\text { Environmental Assessment for Siting Wave \& Tidal } \\
\text { Energy Projects }\end{array}$ & E-38 & $\begin{array}{r}\text { H. T. Harvey \& Associates } \\
24 \text { November } 2009\end{array}$ \\
\hline
\end{tabular}


Appendix E- Effects of Small Commercial Pelamis Project at Makapu'u

\begin{tabular}{|c|c|c|c|c|c|c|c|}
\hline Project activity & Action & $\begin{array}{l}\text { Description of action's } \\
\text { effect on indicator }\end{array}$ & $\begin{array}{l}\text { Spatial exposure to } \\
\text { indicator (low, med, } \\
\text { high) }\end{array}$ & $\begin{array}{l}\text { Temporal } \\
\text { exposure to } \\
\text { indicator (low, } \\
\text { med, high) }\end{array}$ & $\begin{array}{l}\text { Effect's overlap with } \\
\text { critical life stage, } \\
\text { behavior, habitat or } \\
\text { resource (low, med, } \\
\text { high) }\end{array}$ & $\begin{array}{l}\text { Overall risk } \\
\text { to indicator } \\
\text { (low, med, } \\
\text { high, } \\
\text { unknown) } \\
\end{array}$ & Source(s) \\
\hline & $\begin{array}{l}\text { Deconstruction } \\
\text { lights }\end{array}$ & $\begin{array}{l}\text { Attraction to prey } \\
\text { aggregation }\end{array}$ & $\begin{array}{l}\text { Low, would occur in } \\
\text { very small area }\end{array}$ & $\begin{array}{l}\text { Low, May-Sept. for } \\
1-2 \text { years }\end{array}$ & $\begin{array}{l}\text { Medium, species present } \\
\text { in area although highly } \\
\text { mobile }\end{array}$ & Low & $\begin{array}{l}\text { Nightingale } \\
\text { et al. } 2006\end{array}$ \\
\hline
\end{tabular}


Appendix E- Effects of Small Commercial Pelamis Project at Makapu’u

Effects on green sea turtles and hawksbill sea turtles from Small Commercial Pelamis project at Makapu'u site

\begin{tabular}{|c|c|c|c|c|c|c|c|}
\hline Project activity & Action & $\begin{array}{l}\text { Description of } \\
\text { action's effect on } \\
\text { indicator }\end{array}$ & $\begin{array}{l}\text { Spatial exposure to } \\
\text { indicator (low, med, } \\
\text { high) }\end{array}$ & $\begin{array}{l}\text { Temporal } \\
\text { exposure to } \\
\text { indicator (low, } \\
\text { med, high) }\end{array}$ & $\begin{array}{l}\text { Effect's overlap with } \\
\text { critical life stage, } \\
\text { behavior, habitat or } \\
\text { resource (low, med, } \\
\text { high) }\end{array}$ & $\begin{array}{l}\text { Overall risk to } \\
\text { indicator (low, } \\
\text { med, high, } \\
\text { unknown) }\end{array}$ & Source(s) \\
\hline \multicolumn{8}{|c|}{ Construction } \\
\hline \multirow{3}{*}{ Boat traffic } & Direct impact & $\begin{array}{l}\text { Collision and injury } \\
\text { or mortality }\end{array}$ & $\begin{array}{l}\text { Med, species nearshore } \\
\text { where boats would } \\
\text { pass }\end{array}$ & $\begin{array}{l}\text { Low, May-Sept. } \\
\text { for } 1-2 \text { years }\end{array}$ & $\begin{array}{l}\text { Med, these species } \\
\text { nest on Oahu and } \\
\text { forage nearshore }\end{array}$ & Med & $\begin{array}{l}\text { Michel et al. } \\
\text { 2007, USN } 2005\end{array}$ \\
\hline & $\begin{array}{l}\text { Noise and } \\
\text { vibration }\end{array}$ & $\begin{array}{l}\text { Disturbance, } \\
\text { avoidance of area }\end{array}$ & $\begin{array}{l}\text { Med, species nearshore } \\
\text { where boats would } \\
\text { pass }\end{array}$ & $\begin{array}{l}\text { Low, May-Sept. } \\
\text { for 1-2 years }\end{array}$ & $\begin{array}{l}\text { Med, these species } \\
\text { nest on Oahu and } \\
\text { forage nearshore }\end{array}$ & Med & $\begin{array}{l}\text { Michel et al. } \\
\text { 2007, USN } 2005\end{array}$ \\
\hline & $\begin{array}{l}\text { Oil/chemical } \\
\text { release }\end{array}$ & $\begin{array}{l}\text { Toxicity to } \\
\text { individuals and } \\
\text { prey }\end{array}$ & $\begin{array}{l}\text { Low for typical small } \\
\text { volume releases, High } \\
\text { for large, catastrophic } \\
\text { spill }\end{array}$ & $\begin{array}{l}\text { Low, May-Sept. } \\
\text { for 1-2 years }\end{array}$ & $\begin{array}{l}\text { Low, species in water } \\
\text { column, oil floats on } \\
\text { surface }\end{array}$ & Low & $\begin{array}{l}\text { Michel et al. } \\
\text { 2007, USN } 2005\end{array}$ \\
\hline \multirow{4}{*}{$\begin{array}{l}\text { Construction and } \\
\text { installation of } \\
\text { electrical collector } \\
\text { system, mooring } \\
\text { cables, anchors or } \\
\text { foundations, and } \\
\text { devices }\end{array}$} & $\begin{array}{l}\text { Noise and } \\
\text { vibration }\end{array}$ & $\begin{array}{l}\text { Disturbance, } \\
\text { avoidance of area }\end{array}$ & $\begin{array}{l}\text { Med, species nearshore } \\
\text { where construction } \\
\text { would occur }\end{array}$ & $\begin{array}{l}\text { Low, May-Sept. } \\
\text { for 1-2 years }\end{array}$ & $\begin{array}{l}\text { Med, these species } \\
\text { nest on Oahu and } \\
\text { forage nearshore }\end{array}$ & Med & $\begin{array}{l}\text { Michel et al. } \\
\text { 2007, USN } 2005\end{array}$ \\
\hline & $\begin{array}{l}\text { Oil/chemical } \\
\text { release }\end{array}$ & $\begin{array}{l}\text { Toxicity to } \\
\text { individuals and } \\
\text { prey }\end{array}$ & $\begin{array}{l}\text { Low, volume released } \\
\text { would occupy small } \\
\text { area }\end{array}$ & $\begin{array}{l}\text { Low, May-Sept. } \\
\text { for } 1-2 \text { years }\end{array}$ & $\begin{array}{l}\text { Low, species in water } \\
\text { column, oil floats on } \\
\text { surface }\end{array}$ & Low & $\begin{array}{l}\text { Michel et al. } \\
\text { 2007, USN } 2005\end{array}$ \\
\hline & $\begin{array}{l}\text { Seabed } \\
\text { disturbance }\end{array}$ & $\begin{array}{l}\text { Temporary loss of } \\
\text { foraging habitat }\end{array}$ & $\begin{array}{l}\text { Med, species nearshore } \\
\text { where construction } \\
\text { would occur }\end{array}$ & $\begin{array}{l}\text { Low, May-Sept. } \\
\text { for 1-2 years }\end{array}$ & $\begin{array}{l}\text { Med, these species } \\
\text { nest on Oahu and } \\
\text { forage nearshore }\end{array}$ & Med & $\begin{array}{l}\text { Michel et al. } \\
\text { 2007, USN } 2005\end{array}$ \\
\hline & $\begin{array}{l}\text { Construction } \\
\text { lights }\end{array}$ & $\begin{array}{l}\text { Attraction, } \\
\text { disorientation of } \\
\text { hatchlings }\end{array}$ & Med, see above & $\begin{array}{l}\text { Low, May-Sept. } \\
\text { for } 1-2 \text { years }\end{array}$ & $\begin{array}{l}\text { High, hatching occurs } \\
\text { in summer and fall }\end{array}$ & Med & $\begin{array}{l}\text { Michel et al. } \\
\text { 2007, USN } 2005\end{array}$ \\
\hline \multirow{2}{*}{$\begin{array}{l}\text { Directional drilling, } \\
\text { and laying cable } \\
\text { under/on seabed }\end{array}$} & $\begin{array}{l}\text { Seabed } \\
\text { disturbance }\end{array}$ & $\begin{array}{l}\text { Temporary loss of } \\
\text { foraging habitat }\end{array}$ & Med, see above & $\begin{array}{l}\text { Low, short-term } \\
\text { disturbance }\end{array}$ & $\begin{array}{l}\text { Med, these species } \\
\text { nest on Oahu and } \\
\text { forage nearshore }\end{array}$ & Med & $\begin{array}{l}\text { Michel et al. } \\
\text { 2007, USN } 2005\end{array}$ \\
\hline & $\begin{array}{l}\text { Noise and } \\
\text { vibration }\end{array}$ & $\begin{array}{l}\text { Disturbance, } \\
\text { avoidance of area }\end{array}$ & Med, see above & $\begin{array}{l}\text { Low, short-term } \\
\text { disturbance }\end{array}$ & $\begin{array}{l}\text { Med, these species } \\
\text { nest on Oahu and } \\
\text { forage nearshore }\end{array}$ & Med & $\begin{array}{l}\text { Michel et al. } \\
\text { 2007, USN } 2005\end{array}$ \\
\hline \multicolumn{8}{|c|}{ Operation and Maintenance } \\
\hline Boat traffic & Direct impact & $\begin{array}{l}\text { Collision and injury } \\
\text { or mortality }\end{array}$ & $\begin{array}{l}\text { Low, would occur in } \\
\text { small area }\end{array}$ & $\begin{array}{l}\text { Low, only from } \\
\text { periodic trips }\end{array}$ & $\begin{array}{l}\text { Med, these species } \\
\text { nest on Oahu and } \\
\text { forage nearshore }\end{array}$ & Med & $\begin{array}{l}\text { Michel et al. } \\
\text { 2007, USN } 2005\end{array}$ \\
\hline
\end{tabular}

\begin{tabular}{lrr}
\hline $\begin{array}{l}\text { Environmental Assessment for Siting Wave \& Tidal } \\
\text { Energy Projects }\end{array}$ & E-40 & H. T. Harvey \& Associates \\
24 November 2009
\end{tabular}


Appendix E- Effects of Small Commercial Pelamis Project at Makapu’u

\begin{tabular}{|c|c|c|c|c|c|c|c|}
\hline Project activity & Action & $\begin{array}{l}\text { Description of } \\
\text { action's effect on } \\
\text { indicator }\end{array}$ & $\begin{array}{l}\text { Spatial exposure to } \\
\text { indicator (low, med, } \\
\text { high) }\end{array}$ & $\begin{array}{l}\text { Temporal } \\
\text { exposure to } \\
\text { indicator (low, } \\
\text { med, high) }\end{array}$ & $\begin{array}{l}\text { Effect's overlap with } \\
\text { critical life stage, } \\
\text { behavior, habitat or } \\
\text { resource (low, med, } \\
\text { high) }\end{array}$ & $\begin{array}{l}\text { Overall risk to } \\
\text { indicator (low, } \\
\text { med, high, } \\
\text { unknown) }\end{array}$ & Source(s) \\
\hline & $\begin{array}{l}\text { Oil/chemical } \\
\text { release }\end{array}$ & $\begin{array}{l}\text { Toxicity to } \\
\text { individuals and } \\
\text { prey }\end{array}$ & $\begin{array}{l}\text { Low for typical small } \\
\text { volume releases, High } \\
\text { for large, catastrophic } \\
\text { spill }\end{array}$ & $\begin{array}{l}\text { Low, wouldn’t } \\
\text { persist long-term }\end{array}$ & $\begin{array}{l}\text { Low, species in water } \\
\text { column, oil floats on } \\
\text { surface }\end{array}$ & Low & $\begin{array}{l}\text { Michel et al. } \\
\text { 2007, USN } 2005\end{array}$ \\
\hline & $\begin{array}{l}\text { Noise and } \\
\text { vibration }\end{array}$ & $\begin{array}{l}\text { Disturbance, } \\
\text { avoidance of area }\end{array}$ & $\begin{array}{l}\text { Low, would occur in } \\
\text { small area }\end{array}$ & $\begin{array}{l}\text { Low, only from } \\
\text { periodic trips }\end{array}$ & $\begin{array}{l}\text { Med, these species } \\
\text { nest on Oahu and } \\
\text { forage nearshore }\end{array}$ & Med & $\begin{array}{l}\text { Michel et al. } \\
\text { 2007, USN } 2005\end{array}$ \\
\hline \multirow{2}{*}{$\begin{array}{l}\text { Operation of turbines } \\
\text { or other moving parts } \\
\text { of devices }\end{array}$} & $\begin{array}{l}\text { Moving device } \\
\text { parts }\end{array}$ & $\begin{array}{l}\text { Effects unlikely, } \\
\text { moving parts on } \\
\text { surface, turtles } \\
\text { underwater } \\
\end{array}$ & N/A & N/A & N/A & Low & $\begin{array}{l}\text { Cada 2008, USN } \\
2005\end{array}$ \\
\hline & $\begin{array}{l}\text { Noise and } \\
\text { vibration }\end{array}$ & $\begin{array}{l}\text { Disturbance, } \\
\text { avoidance of area }\end{array}$ & $\begin{array}{l}\text { Low, would occur in } \\
\text { small area }\end{array}$ & $\begin{array}{l}\text { High, continuous } \\
\text { for life of project }\end{array}$ & $\begin{array}{l}\text { Med, these species } \\
\text { nest on Oahu and } \\
\text { forage nearshore }\end{array}$ & Unknown & $\begin{array}{l}\text { Michel et al. } \\
\text { 2007, USN } 2005\end{array}$ \\
\hline \multirow{2}{*}{$\begin{array}{l}\text { Structures in water } \\
\text { column and on } \\
\text { seabed, such as } \\
\text { devices and moorings } \\
\text { and footings }\end{array}$} & Structure & $\begin{array}{l}\text { Entanglement with } \\
\text { devices or derelict } \\
\text { fishing gear }\end{array}$ & $\begin{array}{l}\text { Low, would occupy } \\
\text { small area }\end{array}$ & $\begin{array}{l}\text { High, continuous } \\
\text { for life of project }\end{array}$ & $\begin{array}{l}\text { Med, these species } \\
\text { nest on Oahu and } \\
\text { forage nearshore }\end{array}$ & High & $\begin{array}{l}\text { Cada 2008, USN } \\
2005\end{array}$ \\
\hline & $\begin{array}{l}\text { Oil/chemical } \\
\text { release }\end{array}$ & $\begin{array}{l}\text { Hypothermia, } \\
\text { toxicity to } \\
\text { individuals and } \\
\text { prey }\end{array}$ & $\begin{array}{l}\text { Low for typical small } \\
\text { volume releases, High } \\
\text { for large release from } \\
\text { device failure }\end{array}$ & $\begin{array}{l}\text { Low, wouldn’t } \\
\text { persist long-term }\end{array}$ & $\begin{array}{l}\text { Low, species in water } \\
\text { column, oil floats on } \\
\text { surface }\end{array}$ & Low & $\begin{array}{l}\text { Michel et al. } \\
\text { 2007, USFWS } \\
2005\end{array}$ \\
\hline $\begin{array}{l}\text { Electricity } \\
\text { conduction through } \\
\text { cable }\end{array}$ & EMF & $\begin{array}{l}\text { Disorientation, } \\
\text { alteration of } \\
\text { behavior } \\
\end{array}$ & $\begin{array}{l}\text { Low, would occur in } \\
\text { small area }\end{array}$ & $\begin{array}{l}\text { High, continuous } \\
\text { for life of project }\end{array}$ & $\begin{array}{l}\text { Med, these species } \\
\text { nest on Oahu and } \\
\text { forage nearshore }\end{array}$ & Med & $\begin{array}{l}\text { Michel et al. } \\
\text { 2007, USN } 2005\end{array}$ \\
\hline \multirow{2}{*}{$\begin{array}{l}\text { Structures on water's } \\
\text { surface }\end{array}$} & $\begin{array}{l}\text { Navigation } \\
\text { lights }\end{array}$ & $\begin{array}{l}\text { Attraction, } \\
\text { disorientation of } \\
\text { hatchlings } \\
\end{array}$ & $\begin{array}{l}\text { Low, would occur in } \\
\text { small area }\end{array}$ & $\begin{array}{l}\text { High, continuous } \\
\text { for life of project }\end{array}$ & $\begin{array}{l}\text { High, hatching occurs } \\
\text { in summer and fall }\end{array}$ & High & $\begin{array}{l}\text { Michel et al. } \\
\text { 2007, USN } 2005\end{array}$ \\
\hline & Structure & $\begin{array}{l}\text { Effects unlikely, } \\
\text { turtles do not feed } \\
\text { at surface }\end{array}$ & N/A & N/A & N/A & Low & $\begin{array}{l}\text { Michel et al. } \\
\text { 2007, USN } 2005\end{array}$ \\
\hline \multicolumn{8}{|c|}{ Decommissioning } \\
\hline Boat traffic & Direct impact & $\begin{array}{l}\text { Collision and injury } \\
\text { or mortality }\end{array}$ & $\begin{array}{l}\text { Low, would occur in } \\
\text { very small area }\end{array}$ & $\begin{array}{l}\text { Low, May-Sept. } \\
\text { for } 1-2 \text { years }\end{array}$ & $\begin{array}{l}\text { Med, these species } \\
\text { nest on Oahu and } \\
\text { forage nearshore }\end{array}$ & Med & $\begin{array}{l}\text { Michel et al. } \\
\text { 2007, USN } 2005\end{array}$ \\
\hline
\end{tabular}

\begin{tabular}{|c|c|c|}
\hline $\begin{array}{l}\text { Environmental Assessment for Siting Wave \& Tidal } \\
\text { Energy Projects }\end{array}$ & E-41 & $\begin{array}{r}\text { H. T. Harvey \& Associates } \\
24 \text { November } 2009\end{array}$ \\
\hline
\end{tabular}


Appendix E- Effects of Small Commercial Pelamis Project at Makapu’u

\begin{tabular}{|c|c|c|c|c|c|c|c|}
\hline Project activity & Action & $\begin{array}{l}\text { Description of } \\
\text { action's effect on } \\
\text { indicator }\end{array}$ & $\begin{array}{l}\text { Spatial exposure to } \\
\text { indicator (low, med, } \\
\text { high) }\end{array}$ & $\begin{array}{l}\text { Temporal } \\
\text { exposure to } \\
\text { indicator (low, } \\
\text { med, high) }\end{array}$ & $\begin{array}{l}\text { Effect's overlap with } \\
\text { critical life stage, } \\
\text { behavior, habitat or } \\
\text { resource (low, med, } \\
\text { high) }\end{array}$ & $\begin{array}{l}\text { Overall risk to } \\
\text { indicator (low, } \\
\text { med, high, } \\
\text { unknown) }\end{array}$ & Source(s) \\
\hline & $\begin{array}{l}\text { Oil/chemical } \\
\text { release }\end{array}$ & $\begin{array}{l}\text { Toxicity to } \\
\text { individuals and } \\
\text { prey }\end{array}$ & $\begin{array}{l}\text { Low for typical small } \\
\text { volume releases, High } \\
\text { for large, catastrophic } \\
\text { spill }\end{array}$ & $\begin{array}{l}\text { Low, May-Sept. } \\
\text { for 1-2 years }\end{array}$ & $\begin{array}{l}\text { Low, species in water } \\
\text { column, oil floats on } \\
\text { surface }\end{array}$ & Low & $\begin{array}{l}\text { Michel et al. } \\
\text { 2007, USN } 2005\end{array}$ \\
\hline & $\begin{array}{l}\text { Noise and } \\
\text { vibration }\end{array}$ & $\begin{array}{l}\text { Disturbance, } \\
\text { avoidance of area }\end{array}$ & $\begin{array}{l}\text { Low, would occur in } \\
\text { very small area }\end{array}$ & $\begin{array}{l}\text { Low, May-Sept. } \\
\text { for } 1-2 \text { years }\end{array}$ & $\begin{array}{l}\text { Med, these species } \\
\text { nest on Oahu and } \\
\text { forage nearshore }\end{array}$ & Med & $\begin{array}{l}\text { Michel et al. } \\
\text { 2007, USN } 2005\end{array}$ \\
\hline \multirow{4}{*}{$\begin{array}{l}\text { Decommissioning of } \\
\text { structures on water's } \\
\text { surface or seabed }\end{array}$} & $\begin{array}{l}\text { Noise and } \\
\text { vibration }\end{array}$ & $\begin{array}{l}\text { Disturbance, } \\
\text { avoidance of area }\end{array}$ & $\begin{array}{l}\text { Low, would occur in } \\
\text { very small area }\end{array}$ & $\begin{array}{l}\text { Low, May-Sept. } \\
\text { for } 1-2 \text { years }\end{array}$ & $\begin{array}{l}\text { Med, these species } \\
\text { nest on Oahu and } \\
\text { forage nearshore }\end{array}$ & Med & $\begin{array}{l}\text { Michel et al. } \\
\text { 2007, USN } 2005\end{array}$ \\
\hline & $\begin{array}{l}\text { Oil/chemical } \\
\text { release }\end{array}$ & $\begin{array}{l}\text { Toxicity to } \\
\text { individuals and } \\
\text { prey }\end{array}$ & $\begin{array}{l}\text { Low, volume released } \\
\text { would occupy small } \\
\text { area }\end{array}$ & $\begin{array}{l}\text { Low, May-Sept. } \\
\text { for } 1-2 \text { years }\end{array}$ & $\begin{array}{l}\text { Low, species in water } \\
\text { column, oil floats on } \\
\text { surface }\end{array}$ & Low & $\begin{array}{l}\text { Michel et al. } \\
\text { 2007, USN } 2005\end{array}$ \\
\hline & $\begin{array}{l}\text { Seabed } \\
\text { disturbance }\end{array}$ & $\begin{array}{l}\text { Temporary loss of } \\
\text { foraging habitat }\end{array}$ & $\begin{array}{l}\text { Low, would occur in } \\
\text { very small area }\end{array}$ & $\begin{array}{l}\text { Low, May-Sept. } \\
\text { for } 1-2 \text { years }\end{array}$ & $\begin{array}{l}\text { Med, these species } \\
\text { nest on Oahu and } \\
\text { forage nearshore }\end{array}$ & Med & $\begin{array}{l}\text { Michel et al. } \\
\text { 2007, USN } 2005\end{array}$ \\
\hline & $\begin{array}{l}\text { Deconstruction } \\
\text { lights }\end{array}$ & $\begin{array}{l}\text { Attraction, } \\
\text { disorientation of } \\
\text { hatchlings }\end{array}$ & $\begin{array}{l}\text { Low, would occur in } \\
\text { very small area }\end{array}$ & $\begin{array}{l}\text { Low, May-Sept. } \\
\text { for 1-2 years }\end{array}$ & $\begin{array}{l}\text { High, hatching occurs } \\
\text { in summer and fall }\end{array}$ & Med & $\begin{array}{l}\text { Michel et al. } \\
\text { 2007, USN } 2005\end{array}$ \\
\hline
\end{tabular}

\begin{tabular}{|c|c|c|}
\hline $\begin{array}{l}\text { Environmental Assessment for Siting Wave \& Tidal } \\
\text { Energy Projects }\end{array}$ & E-42 & $\begin{array}{r}\text { H. T. Harvey \& Associates } \\
24 \text { November } 2009\end{array}$ \\
\hline
\end{tabular}


Appendix E- Effects of Small Commercial Pelamis Project at Makapu’u

Effects on albatrosses, shearwaters, and storm-petrels from Small Commercial Pelamis project at Makapu'u site

\begin{tabular}{|c|c|c|c|c|c|c|c|}
\hline Project activity & Action & $\begin{array}{l}\text { Description of action's } \\
\text { effect on indicator }\end{array}$ & $\begin{array}{l}\text { Spatial exposure to } \\
\text { indicator (low, med, } \\
\text { high) }\end{array}$ & $\begin{array}{l}\text { Temporal } \\
\text { exposure to } \\
\text { indicator (low, } \\
\text { med, high) }\end{array}$ & $\begin{array}{l}\text { Effect's overlap with } \\
\text { critical life stage, } \\
\text { behavior, habitat or } \\
\text { resource (low, med, high) }\end{array}$ & $\begin{array}{l}\text { Overall risk } \\
\text { to indicator } \\
\text { (low, med, } \\
\text { high, } \\
\text { unknown) } \\
\end{array}$ & Source(s) \\
\hline \multicolumn{8}{|c|}{ Construction } \\
\hline \multirow[b]{2}{*}{ Boat traffic } & $\begin{array}{l}\text { Noise and } \\
\text { vibration }\end{array}$ & $\begin{array}{l}\text { None, species do not } \\
\text { forage nearshore where } \\
\text { boats would pass }\end{array}$ & N/A & N/A & N/A & Low & $\begin{array}{l}\text { USFWS } \\
2005\end{array}$ \\
\hline & $\begin{array}{l}\text { Oil/chemical } \\
\text { release }\end{array}$ & $\begin{array}{l}\text { Hypothermia, toxicity to } \\
\text { individuals and prey }\end{array}$ & $\begin{array}{l}\text { Low for typical small } \\
\text { volume releases, High } \\
\text { for large, catastrophic } \\
\text { spill }\end{array}$ & $\begin{array}{l}\text { Low, May-Sept. } \\
\text { for } 1-2 \text { years }\end{array}$ & $\begin{array}{l}\text { None for small release, } \\
\text { Low for large release } \\
\text { because these species } \\
\text { forage far offshore and } \\
\text { unlikely to contact oil }\end{array}$ & Low & $\begin{array}{l}\text { USFWS } \\
2005\end{array}$ \\
\hline \multirow{4}{*}{$\begin{array}{l}\text { Construction of } \\
\text { electrical collector } \\
\text { system, moorings/ } \\
\text { foundation; } \\
\text { installation of devices }\end{array}$} & $\begin{array}{l}\text { Noise and } \\
\text { vibration }\end{array}$ & $\begin{array}{l}\text { None, species do not } \\
\text { forage in project area }\end{array}$ & N/A & N/A & N/A & Low & $\begin{array}{l}\text { USFWS } \\
2005\end{array}$ \\
\hline & $\begin{array}{l}\text { Oil/chemical } \\
\text { release }\end{array}$ & $\begin{array}{l}\text { None, species do not } \\
\text { forage in project area }\end{array}$ & N/A & N/A & N/A & Low & $\begin{array}{l}\text { USFWS } \\
2005\end{array}$ \\
\hline & $\begin{array}{l}\text { Seabed } \\
\text { disturbance }\end{array}$ & $\begin{array}{l}\text { None, species are } \\
\text { surface-feeders and do } \\
\text { not forage in project } \\
\text { area }\end{array}$ & N/A & N/A & N/A & Low & $\begin{array}{l}\text { USFWS } \\
2005\end{array}$ \\
\hline & $\begin{array}{l}\text { Construction } \\
\text { lights }\end{array}$ & $\begin{array}{l}\text { Attraction/ } \\
\text { disorientation }\end{array}$ & $\begin{array}{l}\text { Med, species may } \\
\text { congregate nearshore } \\
\text { at night }\end{array}$ & $\begin{array}{l}\text { Low, May-Sept. } \\
\text { for } 1-2 \text { years }\end{array}$ & $\begin{array}{l}\text { Med, these species nest on } \\
\text { Oahu Island and could be } \\
\text { attracted to lights while } \\
\text { attending nests }\end{array}$ & Med & $\begin{array}{l}\text { Montevecchi } \\
\text { 2006, } \\
\text { USFWS } \\
2005\end{array}$ \\
\hline \multirow{2}{*}{$\begin{array}{l}\text { Directional drilling, } \\
\text { and laying cable } \\
\text { under/on seabed }\end{array}$} & $\begin{array}{l}\text { Seabed } \\
\text { disturbance }\end{array}$ & $\begin{array}{l}\text { None, species are } \\
\text { surface-feeders and do } \\
\text { not forage in project } \\
\text { area }\end{array}$ & N/A & N/A & N/A & Low & $\begin{array}{l}\text { USFWS } \\
2005\end{array}$ \\
\hline & $\begin{array}{l}\text { Noise and } \\
\text { vibration }\end{array}$ & $\begin{array}{l}\text { None, species do not } \\
\text { forage in project area }\end{array}$ & N/A & N/A & N/A & Low & $\begin{array}{l}\text { USFWS } \\
2005\end{array}$ \\
\hline \multicolumn{8}{|c|}{ Operation and Maintenance } \\
\hline Boat traffic & $\begin{array}{l}\text { Oil/chemical } \\
\text { release }\end{array}$ & $\begin{array}{l}\text { Hypothermia, toxicity to } \\
\text { individuals and prey }\end{array}$ & $\begin{array}{l}\text { Low for typical small } \\
\text { volume releases, High } \\
\text { for large, catastrophic } \\
\text { spill }\end{array}$ & $\begin{array}{l}\text { Low, from } \\
\text { periodic trips } \\
\text { only }\end{array}$ & $\begin{array}{l}\text { None for small release, } \\
\text { Low for large release } \\
\text { because these species } \\
\text { forage far offshore and } \\
\text { unlikely to contact oil }\end{array}$ & Low & $\begin{array}{l}\text { Michel et al. } \\
\text { 2007, } \\
\text { USFWS } \\
2005\end{array}$ \\
\hline
\end{tabular}

\begin{tabular}{lr}
\hline Environmental Assessment for Siting Wave \& Tidal & E-43 \\
Energy Projects &
\end{tabular}


Appendix E- Effects of Small Commercial Pelamis Project at Makapu’u

\begin{tabular}{|c|c|c|c|c|c|c|c|}
\hline Project activity & Action & $\begin{array}{l}\text { Description of action's } \\
\text { effect on indicator }\end{array}$ & $\begin{array}{l}\text { Spatial exposure to } \\
\text { indicator (low, med, } \\
\text { high) }\end{array}$ & $\begin{array}{l}\text { Temporal } \\
\text { exposure to } \\
\text { indicator (low, } \\
\text { med, high) }\end{array}$ & $\begin{array}{l}\text { Effect's overlap with } \\
\text { critical life stage, } \\
\text { behavior, habitat or } \\
\text { resource (low, med, high) }\end{array}$ & $\begin{array}{l}\text { Overall risk } \\
\text { to indicator } \\
\text { (low, med, } \\
\text { high, } \\
\text { unknown) } \\
\end{array}$ & Source(s) \\
\hline & $\begin{array}{l}\text { Noise and } \\
\text { vibration }\end{array}$ & $\begin{array}{l}\text { None, species do not } \\
\text { forage nearshore where } \\
\text { boats would pass }\end{array}$ & N/A & N/A & N/A & Low & $\begin{array}{l}\text { USFWS } \\
2005\end{array}$ \\
\hline \multirow{2}{*}{$\begin{array}{l}\text { Operation of turbines } \\
\text { or other moving parts } \\
\text { of devices }\end{array}$} & $\begin{array}{l}\text { Moving device } \\
\text { parts }\end{array}$ & $\begin{array}{l}\text { None, species do not } \\
\text { forage in project area }\end{array}$ & N/A & N/A & N/A & Low & $\begin{array}{l}\text { USFWS } \\
2005\end{array}$ \\
\hline & $\begin{array}{l}\text { Noise and } \\
\text { vibration }\end{array}$ & $\begin{array}{l}\text { None, species do not } \\
\text { forage in project area }\end{array}$ & N/A & N/A & N/A & Low & $\begin{array}{l}\text { USFWS } \\
2005\end{array}$ \\
\hline \multirow{3}{*}{$\begin{array}{l}\text { Structures in water } \\
\text { column and on } \\
\text { seabed, such as } \\
\text { devices and moorings } \\
\text { and footings }\end{array}$} & Structure & $\begin{array}{l}\text { None, species do not } \\
\text { forage in project area }\end{array}$ & N/A & N/A & N/A & Low & $\begin{array}{l}\text { USFWS } \\
2005\end{array}$ \\
\hline & $\begin{array}{l}\text { Water } \\
\text { circulation } \\
\text { changes }\end{array}$ & $\begin{array}{l}\text { None, species do not } \\
\text { forage in project area }\end{array}$ & N/A & N/A & N/A & Low & $\begin{array}{l}\text { USFWS } \\
2005\end{array}$ \\
\hline & $\begin{array}{l}\text { Oil/chemical } \\
\text { release }\end{array}$ & $\begin{array}{l}\text { Hypothermia, toxicity to } \\
\text { individuals and prey }\end{array}$ & $\begin{array}{l}\text { Low for typical small } \\
\text { volume releases, High } \\
\text { for large, catastrophic } \\
\text { release from device } \\
\text { failure }\end{array}$ & $\begin{array}{l}\text { Low, wouldn't } \\
\text { persist long-term }\end{array}$ & $\begin{array}{l}\text { None for small release, } \\
\text { Low for large release } \\
\text { because these species } \\
\text { forage far offshore and } \\
\text { unlikely to contact oil }\end{array}$ & Low & $\begin{array}{l}\text { Michel et al. } \\
\text { 2007, } \\
\text { USFWS } \\
2005\end{array}$ \\
\hline \multirow{2}{*}{$\begin{array}{l}\text { Structures on water's } \\
\text { surface }\end{array}$} & $\begin{array}{l}\text { Navigation } \\
\text { lights }\end{array}$ & $\begin{array}{l}\text { Attraction/ } \\
\text { disorientation }\end{array}$ & $\begin{array}{l}\text { Low, would occur in } \\
\text { very small area }\end{array}$ & $\begin{array}{l}\text { High, would be } \\
\text { continuous for } \\
\text { life of project }\end{array}$ & $\begin{array}{l}\text { High, these species nest on } \\
\text { Oahu Island and could be } \\
\text { attracted to lights while } \\
\text { attending nests }\end{array}$ & High & $\begin{array}{l}\text { Montevecchi } \\
2006, \\
\text { USFWS } \\
2005\end{array}$ \\
\hline & Structure & $\begin{array}{l}\text { Possible collision while } \\
\text { flying }\end{array}$ & $\begin{array}{l}\text { Low, would occur in } \\
\text { very small area }\end{array}$ & $\begin{array}{l}\text { High, would be } \\
\text { continuous for } \\
\text { life of project }\end{array}$ & $\begin{array}{l}\text { Med, these species nest on } \\
\text { Oahu Island and attraction } \\
\text { to lights could increase } \\
\text { collision risk }\end{array}$ & Unknown & $\begin{array}{l}\text { LGL Limited } \\
\text { et al. 2009, } \\
\text { USFWS } \\
2005\end{array}$ \\
\hline \multicolumn{8}{|c|}{ Decommissioning } \\
\hline \multirow[t]{2}{*}{ Boat traffic } & $\begin{array}{l}\text { Oil/chemical } \\
\text { release }\end{array}$ & $\begin{array}{l}\text { Hypothermia, toxicity to } \\
\text { individuals and prey }\end{array}$ & $\begin{array}{l}\text { Low for typical small } \\
\text { volume releases, High } \\
\text { for large, catastrophic } \\
\text { spill }\end{array}$ & $\begin{array}{l}\text { Low, May-Sept. } \\
\text { for } 1-2 \text { years }\end{array}$ & $\begin{array}{l}\text { None for small release, } \\
\text { Low for large release } \\
\text { because these species } \\
\text { forage far offshore and } \\
\text { unlikely to contact oil }\end{array}$ & Low & $\begin{array}{l}\text { USFWS } \\
2005\end{array}$ \\
\hline & $\begin{array}{l}\text { Noise and } \\
\text { vibration }\end{array}$ & $\begin{array}{l}\text { None, these species do } \\
\text { not forage nearshore } \\
\text { where boats would pass }\end{array}$ & N/A & N/A & N/A & Low & $\begin{array}{l}\text { USFWS } \\
2005\end{array}$ \\
\hline
\end{tabular}

\begin{tabular}{|c|c|c|}
\hline $\begin{array}{l}\text { Environmental Assessment for Siting Wave \& Tidal } \\
\text { Energy Projects }\end{array}$ & E-44 & $\begin{array}{r}\text { H. T. Harvey \& Associates } \\
24 \text { November } 2009\end{array}$ \\
\hline
\end{tabular}


Appendix E- Effects of Small Commercial Pelamis Project at Makapu’u

\begin{tabular}{|c|c|c|c|c|c|c|c|}
\hline Project activity & Action & $\begin{array}{l}\text { Description of action's } \\
\text { effect on indicator }\end{array}$ & $\begin{array}{l}\text { Spatial exposure to } \\
\text { indicator (low, med, } \\
\text { high) }\end{array}$ & $\begin{array}{l}\text { Temporal } \\
\text { exposure to } \\
\text { indicator (low, } \\
\text { med, high) }\end{array}$ & $\begin{array}{l}\text { Effect's overlap with } \\
\text { critical life stage, } \\
\text { behavior, habitat or } \\
\text { resource (low, med, high) }\end{array}$ & $\begin{array}{l}\text { Overall risk } \\
\text { to indicator } \\
\text { (low, med, } \\
\text { high, } \\
\text { unknown) }\end{array}$ & Source(s) \\
\hline \multirow{4}{*}{$\begin{array}{l}\text { Decommissioning of } \\
\text { structures on water's } \\
\text { surface or seabed }\end{array}$} & $\begin{array}{l}\text { Noise and } \\
\text { vibration }\end{array}$ & $\begin{array}{l}\text { None, species do not } \\
\text { forage in project area }\end{array}$ & N/A & N/A & N/A & Low & $\begin{array}{l}\text { USFWS } \\
2005\end{array}$ \\
\hline & $\begin{array}{l}\text { Oil/chemical } \\
\text { release }\end{array}$ & $\begin{array}{l}\text { None, species do not } \\
\text { forage in project area }\end{array}$ & N/A & N/A & N/A & Low & $\begin{array}{l}\text { USFWS } \\
2005\end{array}$ \\
\hline & $\begin{array}{l}\text { Deconstruction } \\
\text { and deck lights }\end{array}$ & $\begin{array}{l}\text { Attraction/ } \\
\text { disorientation }\end{array}$ & $\begin{array}{l}\text { Med, species may } \\
\text { congregate nearshore } \\
\text { at night }\end{array}$ & $\begin{array}{l}\text { Low, May-Sept. } \\
\text { for } 1-2 \text { years }\end{array}$ & $\begin{array}{l}\text { Med, these species nest on } \\
\text { Oahu Island and could be } \\
\text { attracted to lights while } \\
\text { attending nests }\end{array}$ & Med & $\begin{array}{l}\text { Montevecchi } \\
\text { 2006, } \\
\text { USFWS } \\
2005\end{array}$ \\
\hline & $\begin{array}{l}\text { Seabed } \\
\text { disturbance }\end{array}$ & $\begin{array}{l}\text { None, species are } \\
\text { surface-feeders and do } \\
\text { not forage in project } \\
\text { area }\end{array}$ & N/A & N/A & N/A & Low & $\begin{array}{l}\text { USFWS } \\
2005\end{array}$ \\
\hline
\end{tabular}

Environmental Assessment for Siting Wave \& Tidal Energy Projects
E-45

H. T. Harvey \& Associates 
Appendix E- Effects of Small Commercial Pelamis Project at Makapu’u

Effects on noddies and terns from Small Commercial Pelamis project at Makapu'u site

\begin{tabular}{|c|c|c|c|c|c|c|c|}
\hline Project activity & Action & $\begin{array}{l}\text { Description of action's } \\
\text { effect on indicator }\end{array}$ & $\begin{array}{l}\text { Spatial exposure to } \\
\text { indicator (low, med, } \\
\text { high) }\end{array}$ & $\begin{array}{l}\text { Temporal } \\
\text { exposure to } \\
\text { indicator (low, } \\
\text { med, high) }\end{array}$ & $\begin{array}{l}\text { Effect's overlap } \\
\text { with critical life } \\
\text { stage, behavior, } \\
\text { habitat or resource } \\
\text { (low, med, high) }\end{array}$ & $\begin{array}{l}\text { Overall risk } \\
\text { to indicator } \\
\text { (low, med, } \\
\text { high, } \\
\text { unknown) } \\
\end{array}$ & Source(s) \\
\hline \multicolumn{8}{|c|}{ Construction } \\
\hline \multirow{2}{*}{ Boat traffic } & $\begin{array}{l}\text { Noise and } \\
\text { vibration }\end{array}$ & $\begin{array}{l}\text { Possible movement away } \\
\text { from area and disruption } \\
\text { of foraging }\end{array}$ & $\begin{array}{l}\text { Low, would only } \\
\text { pass by foraging } \\
\text { areas }\end{array}$ & $\begin{array}{l}\text { Low, May-Sept. } \\
\text { for 1-2 years }\end{array}$ & $\begin{array}{l}\text { High, these species } \\
\text { nest on Oahu Island } \\
\text { and forage inshore }\end{array}$ & Med & $\begin{array}{l}\text { LGL Limited et } \\
\text { al. 2009, } \\
\text { USFWS } 2005\end{array}$ \\
\hline & $\begin{array}{l}\text { Oil/chemical } \\
\text { release }\end{array}$ & $\begin{array}{l}\text { Hypothermia, toxicity to } \\
\text { individuals and prey }\end{array}$ & $\begin{array}{l}\text { Low for typical small } \\
\text { volume releases, } \\
\text { High for large, } \\
\text { catastrophic spill }\end{array}$ & $\begin{array}{l}\text { Low, wouldn't } \\
\text { persist long-term }\end{array}$ & High, see above & Low & $\begin{array}{l}\text { Michel et al. } \\
\text { 2007, USFWS } \\
2005\end{array}$ \\
\hline \multirow{4}{*}{$\begin{array}{l}\text { Construction of } \\
\text { electrical collector } \\
\text { system, } \\
\text { moorings/foundation; } \\
\text { installation of devices }\end{array}$} & $\begin{array}{l}\text { Noise and } \\
\text { vibration }\end{array}$ & $\begin{array}{l}\text { Possible movement away } \\
\text { from area and disruption } \\
\text { of foraging or nesting }\end{array}$ & $\begin{array}{l}\text { Med, may occur in } \\
\text { foraging areas }\end{array}$ & $\begin{array}{l}\text { Low, May-Sept. } \\
\text { for } 1-2 \text { years }\end{array}$ & High, see above & Med & $\begin{array}{l}\text { Michel et al. } \\
\text { 2007, USFWS } \\
2005\end{array}$ \\
\hline & $\begin{array}{l}\text { Oil/chemical } \\
\text { release }\end{array}$ & $\begin{array}{l}\text { Hypothermia, toxicity to } \\
\text { individuals and prey }\end{array}$ & $\begin{array}{l}\text { Low, volume } \\
\text { released would } \\
\text { occupy small area }\end{array}$ & $\begin{array}{l}\text { Low, May-Sept. } \\
\text { for 1-2 years }\end{array}$ & High, see above & Low & $\begin{array}{l}\text { Michel et al. } \\
\text { 2007, USFWS } \\
2005\end{array}$ \\
\hline & $\begin{array}{l}\text { Seabed } \\
\text { disturbance }\end{array}$ & $\begin{array}{l}\text { None, species are } \\
\text { surface-feeders }\end{array}$ & N/A & N/A & N/A & Low & USFWS 2005 \\
\hline & $\begin{array}{l}\text { Construction } \\
\text { lights }\end{array}$ & $\begin{array}{l}\text { None, species are not } \\
\text { known to be attracted to } \\
\text { lights }\end{array}$ & N/A & N/A & N/A & Low & $\begin{array}{l}\text { Montevecchi } \\
2006\end{array}$ \\
\hline \multirow{2}{*}{$\begin{array}{l}\text { Directional drilling, } \\
\text { and laying cable } \\
\text { under/on seabed }\end{array}$} & $\begin{array}{l}\text { Seabed } \\
\text { disturbance }\end{array}$ & $\begin{array}{l}\text { None, species are } \\
\text { surface-feeders }\end{array}$ & N/A & N/A & N/A & Low & USFWS 2005 \\
\hline & $\begin{array}{l}\text { Noise and } \\
\text { vibration }\end{array}$ & $\begin{array}{l}\text { Possible movement away } \\
\text { from area and disruption } \\
\text { of foraging or nesting }\end{array}$ & $\begin{array}{l}\text { Med, may occur in } \\
\text { foraging areas }\end{array}$ & $\begin{array}{l}\text { Low, short-term } \\
\text { disturbance }\end{array}$ & $\begin{array}{l}\text { High, these species } \\
\text { nest on Oahu Island } \\
\text { and forage inshore }\end{array}$ & Med & $\begin{array}{l}\text { Michel et al. } \\
2007 \text {, USFWS } \\
2005\end{array}$ \\
\hline \multicolumn{8}{|c|}{ Operation and Maintenance } \\
\hline Boat traffic & $\begin{array}{l}\text { Oil/chemical } \\
\text { release }\end{array}$ & $\begin{array}{l}\text { Hypothermia, toxicity to } \\
\text { individuals and prey }\end{array}$ & $\begin{array}{l}\text { Low for typical small } \\
\text { volume releases, } \\
\text { High for large, } \\
\text { catastrophic spill }\end{array}$ & $\begin{array}{l}\text { Low, wouldn’t } \\
\text { persist long-term }\end{array}$ & High, see above & Low & $\begin{array}{l}\text { Michel et al. } \\
\text { 2007, USFWS } \\
2005\end{array}$ \\
\hline
\end{tabular}

\begin{tabular}{lrr}
\hline $\begin{array}{l}\text { Environmental Assessment for Siting Wave \& Tidal } \\
\text { Energy Projects }\end{array}$ & E-46 & H. T. Harvey \& Associates \\
24 November 2009
\end{tabular}


Appendix E- Effects of Small Commercial Pelamis Project at Makapu’u

\begin{tabular}{|c|c|c|c|c|c|c|c|}
\hline Project activity & Action & $\begin{array}{l}\text { Description of action's } \\
\text { effect on indicator }\end{array}$ & $\begin{array}{l}\text { Spatial exposure to } \\
\text { indicator (low, med, } \\
\text { high) }\end{array}$ & $\begin{array}{l}\text { Temporal } \\
\text { exposure to } \\
\text { indicator (low, } \\
\text { med, high) }\end{array}$ & $\begin{array}{l}\text { Effect's overlap } \\
\text { with critical life } \\
\text { stage, behavior, } \\
\text { habitat or resource } \\
\text { (low, med, high) }\end{array}$ & $\begin{array}{l}\text { Overall risk } \\
\text { to indicator } \\
\text { (low, med, } \\
\text { high, } \\
\text { unknown) } \\
\end{array}$ & Source(s) \\
\hline & $\begin{array}{l}\text { Noise and } \\
\text { vibration }\end{array}$ & $\begin{array}{l}\text { Possible movement away } \\
\text { from area and disruption } \\
\text { of foraging or nesting }\end{array}$ & $\begin{array}{l}\text { Low, would only } \\
\text { pass by foraging } \\
\text { areas }\end{array}$ & $\begin{array}{l}\text { Low, only from } \\
\text { periodic trips in } \\
\text { summer }\end{array}$ & High, see above & Low & $\begin{array}{l}\text { LGL Limited et } \\
\text { al. 2009, } \\
\text { USFWS } 2005\end{array}$ \\
\hline \multirow{2}{*}{$\begin{array}{l}\text { Operation of turbines } \\
\text { or other moving parts } \\
\text { of devices }\end{array}$} & $\begin{array}{l}\text { Moving device } \\
\text { parts }\end{array}$ & $\begin{array}{l}\text { None, moving parts at the } \\
\text { surface and visible to } \\
\text { birds }\end{array}$ & N/A & N/A & N/A & Low & N/A \\
\hline & $\begin{array}{l}\text { Noise and } \\
\text { vibration }\end{array}$ & $\begin{array}{l}\text { Possible movement away } \\
\text { from area and disruption } \\
\text { of foraging }\end{array}$ & $\begin{array}{l}\text { Med, may occur in } \\
\text { foraging areas }\end{array}$ & $\begin{array}{l}\text { High, would be } \\
\text { continuous for life } \\
\text { of project }\end{array}$ & $\begin{array}{l}\text { High, these species } \\
\text { nest on Oahu Island } \\
\text { and forage inshore }\end{array}$ & Med & $\begin{array}{l}\text { Michel et al. } \\
\text { 2007, USFWS } \\
2005\end{array}$ \\
\hline \multirow{3}{*}{$\begin{array}{l}\text { Structures in water } \\
\text { column and on } \\
\text { seabed, such as } \\
\text { devices and moorings } \\
\text { and footings }\end{array}$} & Structure & $\begin{array}{l}\text { None, these species are } \\
\text { surface-feeders }\end{array}$ & N/A & N/A & N/A & Low & USFWS 2005 \\
\hline & $\begin{array}{l}\text { Water } \\
\text { circulation } \\
\text { changes }\end{array}$ & $\begin{array}{l}\text { None, these species are } \\
\text { surface-feeders }\end{array}$ & N/A & N/A & N/A & Low & USFWS 2005 \\
\hline & $\begin{array}{l}\text { Oil/chemical } \\
\text { release }\end{array}$ & $\begin{array}{l}\text { Hypothermia, toxicity to } \\
\text { individuals and prey }\end{array}$ & $\begin{array}{l}\text { Low for typical small } \\
\text { volume releases, } \\
\text { High for large release } \\
\text { from device failure }\end{array}$ & $\begin{array}{l}\text { Low, wouldn't } \\
\text { persist long-term }\end{array}$ & $\begin{array}{l}\text { High, these species } \\
\text { nest on Oahu Island } \\
\text { and forage inshore }\end{array}$ & Low & $\begin{array}{l}\text { Michel et al. } \\
\text { 2007, USFWS } \\
2005\end{array}$ \\
\hline \multirow[b]{2}{*}{$\begin{array}{l}\text { Structures on water's } \\
\text { surface }\end{array}$} & $\begin{array}{l}\text { Navigation } \\
\text { lights }\end{array}$ & $\begin{array}{l}\text { None, these species not } \\
\text { known to be attracted to } \\
\text { lights }\end{array}$ & N/A & N/A & N/A & Low & $\begin{array}{l}\text { Montevecchi } \\
2006\end{array}$ \\
\hline & Structure & $\begin{array}{l}\text { Possible attractant to } \\
\text { birds due to increased } \\
\text { prey abundance, collision } \\
\text { while flying }\end{array}$ & $\begin{array}{l}\text { Med, may occur in } \\
\text { foraging areas }\end{array}$ & $\begin{array}{l}\text { High, would be } \\
\text { continuous for life } \\
\text { of project }\end{array}$ & $\begin{array}{l}\text { High, these species } \\
\text { nest on Oahu Island } \\
\text { and forage inshore }\end{array}$ & Unknown & $\begin{array}{l}\text { Boehlert et al. } \\
\text { 2008, USFWS } \\
\text { 2005, LGL } \\
\text { Limited et al. } \\
2009 \\
\end{array}$ \\
\hline \multicolumn{8}{|c|}{ Decommissioning } \\
\hline Boat traffic & $\begin{array}{l}\text { Oil/chemical } \\
\text { release }\end{array}$ & $\begin{array}{l}\text { Hypothermia, toxicity to } \\
\text { individuals and prey }\end{array}$ & $\begin{array}{l}\text { Low for typical small } \\
\text { volume releases, } \\
\text { High for large, } \\
\text { catastrophic spill }\end{array}$ & $\begin{array}{l}\text { Low, wouldn't } \\
\text { persist long-term }\end{array}$ & High, see above & Low & $\begin{array}{l}\text { Michel et al. } \\
\text { 2007, USFWS } \\
2005\end{array}$ \\
\hline
\end{tabular}

\begin{tabular}{|c|c|c|}
\hline $\begin{array}{l}\text { Environmental Assessment for Siting Wave \& Tidal } \\
\text { Energy Projects }\end{array}$ & E-47 & $\begin{array}{r}\text { H. T. Harvey \& Associates } \\
24 \text { November } 2009\end{array}$ \\
\hline
\end{tabular}


Appendix E- Effects of Small Commercial Pelamis Project at Makapu’u

\begin{tabular}{|c|c|c|c|c|c|c|c|}
\hline Project activity & Action & $\begin{array}{l}\text { Description of action's } \\
\text { effect on indicator }\end{array}$ & $\begin{array}{l}\text { Spatial exposure to } \\
\text { indicator (low, med, } \\
\text { high) }\end{array}$ & $\begin{array}{l}\text { Temporal } \\
\text { exposure to } \\
\text { indicator (low, } \\
\text { med, high) }\end{array}$ & $\begin{array}{l}\text { Effect's overlap } \\
\text { with critical life } \\
\text { stage, behavior, } \\
\text { habitat or resource } \\
\text { (low, med, high) }\end{array}$ & $\begin{array}{l}\text { Overall risk } \\
\text { to indicator } \\
\text { (low, med, } \\
\text { high, } \\
\text { unknown) } \\
\end{array}$ & Source(s) \\
\hline & $\begin{array}{l}\text { Noise and } \\
\text { vibration }\end{array}$ & $\begin{array}{l}\text { Possible movement away } \\
\text { from area and disruption } \\
\text { of foraging or nesting }\end{array}$ & $\begin{array}{l}\text { Low, would only } \\
\text { pass by foraging } \\
\text { areas }\end{array}$ & $\begin{array}{l}\text { Low, May-Sept. } \\
\text { for } 1-2 \text { years }\end{array}$ & High, see above & Med & $\begin{array}{l}\text { LGL Limited et } \\
\text { al. 2009, } \\
\text { USFWS } 2005\end{array}$ \\
\hline \multirow{4}{*}{$\begin{array}{l}\text { Decommissioning of } \\
\text { structures on water's } \\
\text { surface or seabed }\end{array}$} & $\begin{array}{l}\text { Noise and } \\
\text { vibration }\end{array}$ & $\begin{array}{l}\text { Possible movement away } \\
\text { from area and disruption } \\
\text { of foraging or nesting }\end{array}$ & $\begin{array}{l}\text { Med, may occur in } \\
\text { foraging areas }\end{array}$ & $\begin{array}{l}\text { Low, May-Sept. } \\
\text { for 1-2 years }\end{array}$ & High, see above & Med & $\begin{array}{l}\text { Michel et al. } \\
2007, \text { USFWS } \\
2005\end{array}$ \\
\hline & $\begin{array}{l}\text { Oil/chemical } \\
\text { release }\end{array}$ & $\begin{array}{l}\text { Hypothermia, toxicity to } \\
\text { individuals and prey }\end{array}$ & $\begin{array}{l}\text { Low, volume } \\
\text { released would } \\
\text { occupy small area }\end{array}$ & $\begin{array}{l}\text { Low, wouldn't } \\
\text { persist long-term }\end{array}$ & High, see above & Low & $\begin{array}{l}\text { Michel et al. } \\
\text { 2007, USFWS } \\
2005\end{array}$ \\
\hline & $\begin{array}{l}\text { Construction } \\
\text { lights }\end{array}$ & $\begin{array}{l}\text { None, these species not } \\
\text { known to be attracted to } \\
\text { lights }\end{array}$ & N/A & N/A & N/A & Low & $\begin{array}{l}\text { Montevecchi } \\
2006\end{array}$ \\
\hline & $\begin{array}{l}\text { Seabed } \\
\text { disturbance }\end{array}$ & $\begin{array}{l}\text { None, these species are } \\
\text { surface-feeders }\end{array}$ & N/A & N/A & N/A & Low & USFWS 2005 \\
\hline
\end{tabular}

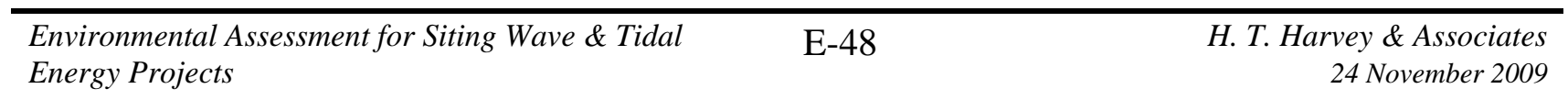


Appendix E- Effects of Small Commercial Pelamis Project at Makapu’u

Effects on white-tailed tropicbird, brown booby, and great frigatebirds from Small Commercial Pelamis project at Makapu'u site

\begin{tabular}{|c|c|c|c|c|c|c|c|}
\hline Project activity & Action & $\begin{array}{l}\text { Description of action's } \\
\text { effect on indicator }\end{array}$ & $\begin{array}{l}\text { Spatial exposure to } \\
\text { indicator (low, med, } \\
\text { high) }\end{array}$ & $\begin{array}{l}\text { Temporal } \\
\text { exposure to } \\
\text { indicator (low, } \\
\text { med, high) }\end{array}$ & $\begin{array}{l}\text { Effect's overlap } \\
\text { with critical life } \\
\text { stage, behavior, } \\
\text { habitat or resource } \\
\text { (low, med, high) }\end{array}$ & $\begin{array}{l}\text { Overall risk to } \\
\text { indicator (low, } \\
\text { med, high, } \\
\text { unknown) }\end{array}$ & Source(s) \\
\hline \multicolumn{8}{|c|}{ Construction } \\
\hline \multirow{2}{*}{ Boat traffic } & $\begin{array}{l}\text { Noise and } \\
\text { vibration }\end{array}$ & $\begin{array}{l}\text { Possible movement } \\
\text { away from area and } \\
\text { disruption of foraging }\end{array}$ & $\begin{array}{l}\text { Low, would occur in } \\
\text { small area relative to } \\
\text { species' range }\end{array}$ & $\begin{array}{l}\text { Low, May-Sept. } \\
\text { for 1-2 years }\end{array}$ & $\begin{array}{l}\text { Med, these species } \\
\text { nest on Oahu Island } \\
\text { and could forage in } \\
\text { project area }\end{array}$ & Low & $\begin{array}{l}\text { LGL Limited et } \\
\text { al. 2009, } \\
\text { USFWS } 2005\end{array}$ \\
\hline & $\begin{array}{l}\text { Oil/chemical } \\
\text { release }\end{array}$ & $\begin{array}{l}\text { Hypothermia, toxicity } \\
\text { to individuals and prey }\end{array}$ & $\begin{array}{l}\text { Low for typical small } \\
\text { volume releases, } \\
\text { High for large, } \\
\text { catastrophic spill }\end{array}$ & $\begin{array}{l}\text { Low, wouldn't } \\
\text { persist long-term }\end{array}$ & Med, see above & Low & $\begin{array}{l}\text { Michel et al. } \\
2007 \text {, USFWS } \\
2005\end{array}$ \\
\hline \multirow{4}{*}{$\begin{array}{l}\text { Construction of } \\
\text { electrical collector } \\
\text { system, moorings/ } \\
\text { foundation; } \\
\text { installation of } \\
\text { devices }\end{array}$} & $\begin{array}{l}\text { Noise and } \\
\text { vibration }\end{array}$ & $\begin{array}{l}\text { Possible movement } \\
\text { away from area and } \\
\text { disruption of foraging }\end{array}$ & $\begin{array}{l}\text { Low, would occur in } \\
\text { small area relative to } \\
\text { species' range }\end{array}$ & $\begin{array}{l}\text { Low, May-Sept. } \\
\text { for 1-2 years }\end{array}$ & Med, see above & Low & $\begin{array}{l}\text { Michel et al. } \\
2007 \text {, USFWS } \\
2005\end{array}$ \\
\hline & $\begin{array}{l}\text { Oil/chemical } \\
\text { release }\end{array}$ & $\begin{array}{l}\text { Hypothermia, toxicity } \\
\text { to individuals and prey }\end{array}$ & $\begin{array}{l}\text { Low, volume } \\
\text { released would } \\
\text { occupy small area } \\
\text { relative to species' } \\
\text { range }\end{array}$ & $\begin{array}{l}\text { Low, May-Sept. } \\
\text { for 1-2 years }\end{array}$ & Med, see above & Low & $\begin{array}{l}\text { Michel et al. } \\
2007 \text {, USFWS } \\
2005\end{array}$ \\
\hline & $\begin{array}{l}\text { Seabed } \\
\text { disturbance }\end{array}$ & $\begin{array}{l}\text { None, species are } \\
\text { surface-feeders }\end{array}$ & N/A & N/A & N/A & Low & USFWS 2005 \\
\hline & $\begin{array}{l}\text { Construction } \\
\text { lights }\end{array}$ & $\begin{array}{l}\text { None, species not } \\
\text { known to be attracted to } \\
\text { lights }\end{array}$ & N/A & N/A & N/A & Low & $\begin{array}{l}\text { Montevecchi } \\
2006\end{array}$ \\
\hline \multirow{2}{*}{$\begin{array}{l}\text { Directional drilling, } \\
\text { and laying cable } \\
\text { under/on seabed }\end{array}$} & $\begin{array}{l}\text { Seabed } \\
\text { disturbance }\end{array}$ & $\begin{array}{l}\text { None, species are } \\
\text { surface-feeders }\end{array}$ & N/A & N/A & N/A & Low & USFWS 2005 \\
\hline & $\begin{array}{l}\text { Noise and } \\
\text { vibration }\end{array}$ & $\begin{array}{l}\text { Possible movement } \\
\text { away from area and } \\
\text { disruption of foraging } \\
\text { or nesting }\end{array}$ & $\begin{array}{l}\text { Low, would occur in } \\
\text { small area relative to } \\
\text { species' range }\end{array}$ & $\begin{array}{l}\text { Low, occurs over } \\
\text { short time-period }\end{array}$ & $\begin{array}{l}\text { Med, these species } \\
\text { nest on Oahu Island } \\
\text { and could forage in } \\
\text { project area }\end{array}$ & Low & $\begin{array}{l}\text { Michel et al. } \\
\text { 2007, USFWS } \\
2005\end{array}$ \\
\hline \multicolumn{8}{|c|}{ Operation and Maintenance } \\
\hline Boat traffic & $\begin{array}{l}\text { Oil/chemical } \\
\text { release }\end{array}$ & $\begin{array}{l}\text { Hypothermia, toxicity } \\
\text { to individuals and prey }\end{array}$ & $\begin{array}{l}\text { Low for typical small } \\
\text { volume releases, } \\
\text { High for large, } \\
\text { catastrophic spill }\end{array}$ & $\begin{array}{l}\text { Low, wouldn't } \\
\text { persist long-term }\end{array}$ & Med, see above & Low & $\begin{array}{l}\text { Michel et al. } \\
2007 \text {, USFWS } \\
2005\end{array}$ \\
\hline
\end{tabular}

\begin{tabular}{|c|c|c|}
\hline $\begin{array}{l}\text { Environmental Assessment for Siting Wave \& Tidal } \\
\text { Energy Projects }\end{array}$ & E-49 & $\begin{array}{r}\text { H. T. Harvey \& Associates } \\
24 \text { November } 2009\end{array}$ \\
\hline
\end{tabular}


Appendix E- Effects of Small Commercial Pelamis Project at Makapu’u

\begin{tabular}{|c|c|c|c|c|c|c|c|}
\hline Project activity & Action & $\begin{array}{l}\text { Description of action's } \\
\text { effect on indicator }\end{array}$ & $\begin{array}{l}\text { Spatial exposure to } \\
\text { indicator (low, med, } \\
\text { high) }\end{array}$ & $\begin{array}{l}\text { Temporal } \\
\text { exposure to } \\
\text { indicator (low, } \\
\text { med, high) }\end{array}$ & $\begin{array}{l}\text { Effect's overlap } \\
\text { with critical life } \\
\text { stage, behavior, } \\
\text { habitat or resource } \\
\text { (low, med, high) }\end{array}$ & $\begin{array}{l}\text { Overall risk to } \\
\text { indicator (low, } \\
\text { med, high, } \\
\text { unknown) }\end{array}$ & Source(s) \\
\hline & $\begin{array}{l}\text { Noise and } \\
\text { vibration }\end{array}$ & $\begin{array}{l}\text { Possible movement } \\
\text { away from area and } \\
\text { disruption of foraging } \\
\text { or nesting }\end{array}$ & $\begin{array}{l}\text { Low, would occur in } \\
\text { small area relative to } \\
\text { species' range }\end{array}$ & $\begin{array}{l}\text { Low, only from } \\
\text { periodic trips in } \\
\text { summer }\end{array}$ & Med, see above & Low & $\begin{array}{l}\text { LGL Limited et } \\
\text { al. 2009, } \\
\text { USFWS } 2005\end{array}$ \\
\hline \multirow{2}{*}{$\begin{array}{l}\text { Operation of } \\
\text { turbines or other } \\
\text { moving parts of } \\
\text { devices }\end{array}$} & $\begin{array}{l}\text { Moving device } \\
\text { parts }\end{array}$ & $\begin{array}{l}\text { None, moving parts at } \\
\text { the surface and visible } \\
\text { to birds }\end{array}$ & N/A & N/A & N/A & Low & N/A \\
\hline & $\begin{array}{l}\text { Noise and } \\
\text { vibration }\end{array}$ & $\begin{array}{l}\text { Possible movement } \\
\text { away from area and } \\
\text { disruption of foraging }\end{array}$ & $\begin{array}{l}\text { Low, would occur in } \\
\text { small area relative to } \\
\text { species' range }\end{array}$ & $\begin{array}{l}\text { High, would be } \\
\text { continuous for life } \\
\text { of project }\end{array}$ & $\begin{array}{l}\text { Med, these species } \\
\text { could forage in } \\
\text { project area }\end{array}$ & Low & $\begin{array}{l}\text { Michel et al. } \\
2007 \text {, USFWS } \\
2005\end{array}$ \\
\hline \multirow{3}{*}{$\begin{array}{l}\text { Structures in water } \\
\text { column and on } \\
\text { seabed, such as } \\
\text { devices and } \\
\text { moorings and } \\
\text { footings }\end{array}$} & Structure & $\begin{array}{l}\text { None, species are } \\
\text { surface-feeders }\end{array}$ & N/A & N/A & N/A & Low & USFWS 2005 \\
\hline & $\begin{array}{l}\text { Water circulation } \\
\text { changes }\end{array}$ & $\begin{array}{l}\text { None, species are } \\
\text { surface-feeders }\end{array}$ & N/A & N/A & N/A & Low & USFWS 2005 \\
\hline & $\begin{array}{l}\text { Oil/chemical } \\
\text { release }\end{array}$ & $\begin{array}{l}\text { Hypothermia, toxicity } \\
\text { to individuals and prey }\end{array}$ & $\begin{array}{l}\text { Low for typical small } \\
\text { volume releases, } \\
\text { High for large release } \\
\text { from device failure }\end{array}$ & $\begin{array}{l}\text { Low, wouldn’t } \\
\text { persist long-term }\end{array}$ & $\begin{array}{l}\text { Med, these species } \\
\text { could forage in } \\
\text { project area }\end{array}$ & Low & $\begin{array}{l}\text { Michel et al. } \\
\text { 2007, USFWS } \\
2005\end{array}$ \\
\hline \multirow[b]{2}{*}{$\begin{array}{l}\text { Structures on } \\
\text { water's surface }\end{array}$} & Navigation lights & $\begin{array}{l}\text { None, these species not } \\
\text { known to be attracted to } \\
\text { lights }\end{array}$ & N/A & N/A & N/A & Low & $\begin{array}{l}\text { Montevecchi } \\
2006\end{array}$ \\
\hline & Structure & $\begin{array}{l}\text { Possible attractant to } \\
\text { birds due to increased } \\
\text { prey abundance, } \\
\text { collision while flying }\end{array}$ & $\begin{array}{l}\text { Low, would occur in } \\
\text { small area relative to } \\
\text { species' range }\end{array}$ & $\begin{array}{l}\text { High, would be } \\
\text { continuous for life } \\
\text { of project }\end{array}$ & $\begin{array}{l}\text { Med, frigatebirds } \\
\text { could attracted to and } \\
\text { steal prey from other } \\
\text { seabirds, other } \\
\text { species could be } \\
\text { attracted to FAD }\end{array}$ & Unknown & $\begin{array}{l}\text { Boehlert et al. } \\
\text { 2008, USFWS } \\
\text { 2005, LGL } \\
\text { Limited et al. } \\
2009\end{array}$ \\
\hline
\end{tabular}

Environmental Assessment for Siting Wave \& Tidal Energy Projects
E-50

H. T. Harvey \& Associates

24 November 2009 
Appendix E- Effects of Small Commercial Pelamis Project at Makapu’u

\begin{tabular}{|c|c|c|c|c|c|c|c|}
\hline Project activity & Action & $\begin{array}{l}\text { Description of action's } \\
\text { effect on indicator }\end{array}$ & $\begin{array}{l}\text { Spatial exposure to } \\
\text { indicator (low, med, } \\
\text { high) }\end{array}$ & $\begin{array}{l}\text { Temporal } \\
\text { exposure to } \\
\text { indicator (low, } \\
\text { med, high) }\end{array}$ & $\begin{array}{l}\text { Effect's overlap } \\
\text { with critical life } \\
\text { stage, behavior, } \\
\text { habitat or resource } \\
\text { (low, med, high) }\end{array}$ & $\begin{array}{l}\text { Overall risk to } \\
\text { indicator (low, } \\
\text { med, high, } \\
\text { unknown) }\end{array}$ & Source(s) \\
\hline \multirow{2}{*}{ Boat traffic } & $\begin{array}{l}\text { Oil/chemical } \\
\text { release }\end{array}$ & $\begin{array}{l}\text { Hypothermia, toxicity } \\
\text { to individuals and prey }\end{array}$ & $\begin{array}{l}\text { Low for typical small } \\
\text { volume releases, } \\
\text { High for large, } \\
\text { catastrophic spill } \\
\end{array}$ & $\begin{array}{l}\text { Low, wouldn't } \\
\text { persist long-term }\end{array}$ & $\begin{array}{l}\text { Med, these species } \\
\text { nest on Oahu Island } \\
\text { and could forage in } \\
\text { project area }\end{array}$ & Low & $\begin{array}{l}\text { Michel et al. } \\
\text { 2007, USFWS } \\
2005\end{array}$ \\
\hline & $\begin{array}{l}\text { Noise and } \\
\text { vibration }\end{array}$ & $\begin{array}{l}\text { Possible movement } \\
\text { away from area and } \\
\text { disruption of foraging } \\
\text { or nesting }\end{array}$ & $\begin{array}{l}\text { Low, would occur in } \\
\text { small area relative to } \\
\text { species' range }\end{array}$ & $\begin{array}{l}\text { Low, May-Sept. } \\
\text { for 1-2 years }\end{array}$ & Med, see above & Low & $\begin{array}{l}\text { LGL Limited et } \\
\text { al. 2009, } \\
\text { USFWS } 2005\end{array}$ \\
\hline \multirow{4}{*}{$\begin{array}{l}\text { Decommissioning of } \\
\text { structures on water's } \\
\text { surface or seabed }\end{array}$} & $\begin{array}{l}\text { Noise and } \\
\text { vibration }\end{array}$ & $\begin{array}{l}\text { Possible movement } \\
\text { away from area and } \\
\text { disruption of foraging } \\
\text { or nesting }\end{array}$ & $\begin{array}{l}\text { Low, would occur in } \\
\text { small area relative to } \\
\text { species' range }\end{array}$ & $\begin{array}{l}\text { Low, May-Sept. } \\
\text { for 1-2 years }\end{array}$ & Med, see above & Low & $\begin{array}{l}\text { Michel et al. } \\
\text { 2007, USFWS } \\
2005\end{array}$ \\
\hline & $\begin{array}{l}\text { Oil/chemical } \\
\text { release }\end{array}$ & $\begin{array}{l}\text { Hypothermia, toxicity } \\
\text { to individuals and prey }\end{array}$ & $\begin{array}{l}\text { Low, volume } \\
\text { released would } \\
\text { occupy small area } \\
\text { relative to species' } \\
\text { range }\end{array}$ & $\begin{array}{l}\text { Low, wouldn't } \\
\text { persist long-term }\end{array}$ & Med, see above & Low & $\begin{array}{l}\text { Michel et al. } \\
\text { 2007, USFWS } \\
2005\end{array}$ \\
\hline & $\begin{array}{l}\text { Construction } \\
\text { lights }\end{array}$ & $\begin{array}{l}\text { None, species not } \\
\text { known to be attracted to } \\
\text { lights }\end{array}$ & N/A & N/A & N/A & Low & $\begin{array}{l}\text { Montevecchi } \\
2006\end{array}$ \\
\hline & $\begin{array}{l}\text { Seabed } \\
\text { disturbance }\end{array}$ & $\begin{array}{l}\text { None, species are } \\
\text { surface-feeders }\end{array}$ & N/A & N/A & N/A & Low & USFWS 2005 \\
\hline
\end{tabular}

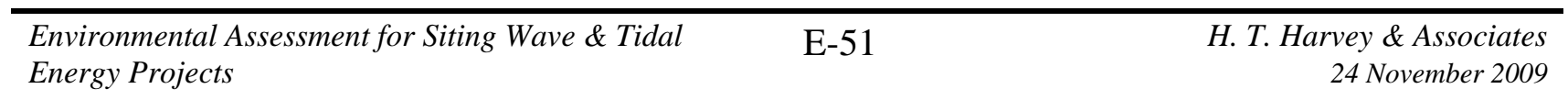




\section{References}

Antonelis GA, Baker JD, Johanos TC, Braun RC, Harting AL. 2006. Hawaiian monk seal (Monachus schauinslandi): Status and conservation issues. Atoll Research Bulletin. 543:75-101.

Austin M, Delarue J, Johnston HA, Laurinolli M, Leary D, MacGillivray A, O'Neill C, Sneddon H, Warner G. 2009. NaiKun Offshore Wind Energy Project environmental assessment, Volume 4 - Noise and vibration. NaiKun Wind Development Inc. and JASCO Applied Sciences.

Baker JD, Johanos TC. 2004. Abundance of the Hawaiian monk seal in the main Hawaiian Islands. Biological Conservation. 116(1):103-110.

Baker SC, Herman LM. 1981. Migration and local movement of humpback whales (Megaptera novaeangliae) through Hawaiian waters. Canadian Journal of Zoology. 59:460-469.

Bodson A, Miersch L, Dehnhardt G. 2007. Underwater localization of pure tones by harbor seals (Phoca vitulina). Journal of the Acoustical Society of America. 122(4):2263-2269.

Boehlert GW, McMurray GR, Tortorici CE. 2008. Ecological effects of wave energy development in the Pacific Northwest. National Oceanic and Atmospheric Administration. NMFS-F/SPO-92.

Cada GF. 2008. The potential environmental impacts of marine and hydrokinetic renewable energy technologies. Washington, DC: Wind and Hydropower Technologies Program, U.S. Department of Energy. ER08-1040.

Camargo FS, Bellini C. 2007. Report on the collision between a spinner dolphin and a boat in the Fernando de Noronha Archipelago, Western Equatorial Atlantic, Brazil. Biota Neotropica. 7(1):209-211.

(CPUC) California Public Utilities Commission. 2009. Sacramento natural gas storage project EIR [Internet]. [cited 14 September 2009]. Available from: http://www.cpuc.ca.gov/environment/info/dudek/sngs/Appendices/Section\%20D9\%20Noise\%20 and\%20Vibration.pdf.

Craig AS, Herman LM. 1997. Sex differences in site fidelity and migration of humpback whales (Megaptera novaeangliae) to the Hawaiian islands. Canadian Journal of Zoology. 75:1923-1933.

Dagorn L, Holland KN, Itano DG. 2007. Behavior of yellowfin (Thunnus albacares) and bigeye (T. obesus) tuna in a network of fish aggregating devices (FADs). Marine Biology. 151:595-606.

Danil K, Maldini D, Marten K. 2005. Patterns of use of Makua Beach, Oahu, Hawaii, by spinner dolphins (Stenella longirostris) and potential effects of swimmers on their behavior. Aquatic Mammals. 31(4):403-412.

Day JR, Defran RH. 1995. Nocturnal activity of Pacific coast bottlenose dolphins (Tursiops truncatus) in California. Orlando, FL. 
Dempster T, Taquet M. 2004. Fish aggregation device (FAD) research: Gaps in current knowledge and future directions for ecological studies. Reviews in Fish Biology and Fisheries. 14:21-42.

Goodman-Lowe GD. 1998. Diet of the Hawaiian monk seal (Monachus schauinslandi) from the Northwestern Hawaiian islands during 1991 to 1994. Marine Biology. 132:535-546.

Henderson JR. 2001. A pre- and post-MARPOL Annex V summary of Hawaiian monk seal entanglements and marine debris accumulation in the Northwestern Hawaiian Islands, 19821998. Marine Pollution Bulletin. 42(7):584-589.

(HMRG) Hawaii Mapping Research Group. [date unknown]. Main Hawaiian Islands multibeam synthesis - HI15821 [Internet]. School of Ocean and Earth Science and Technology, HMRG; [cited 11 November 2009]. Available from: http://www.soest.hawaii.edu/HMRG/Multibeam/grids/HI15821.php.

(IALA) International Association of Marine Aids to Navigation and Lighthouse Authorities. 2008. IALA recommendation O-139 on the marking of man-made offshore structures. Saint Germain en Laye, France: IALA-AISM.

Irvine AB, Scott MD, Wells RS, Kaufman JH. 1981. Movements and activities of the Atlantic bottlenose dolphin, Tursiops truncatus, near Sarasota, Florida. Fishery Bulletin. 79:671-688.

Kastak D, Southall BL, Schusterman RJ, Reichmuth-Kastak CJ. 2005. Underwater temporary threshold shift in pinnipeds: Effects of noise level and duration. Journal of the Acoustical Society of America. 118(5):3154-3163.

Kastelein RA, Wensveen PJ, Hoek L, Terhune JM. 2009. Underwater hearing sensitivity of harbor seals (Phoca vitulina) of narrow noise bands between 0.2 and $80 \mathrm{kHz}$. Journal of the Acoustical Society of America. 1:476-483.

Klatsky LJ, Wells RS, Sweeney JC. 2007. Offshore bottlenose dolphins (Tursiops truncatus): Movement and dive behavior near the Bermuda Pedestal. Journal of Mammalogy. 88(1):59-66.

Laist DW, Knowlton AR, Mead JG, Collet AS, Podesta M. 2001. Collisions between ships and whales. Marine Mammal Science. 17(1):35-75.

LGL Limited, KS Biological Services, Pottinger Gaherty Environmental Consultants. 2009. NaiKun Offshore Wind Energy Project environmental assessment, Volume 8 - Marine birds and sea turtles. NaiKun Wind Development Inc.

Lien J, Barney W, Todd S, Seton R, Guzzwell J. 1992. Effects of adding sounds to cod traps on the probability of collisions by humpback whales. In: Thomas JA, Kastelein RA, Supin A, editors. Marine mammal sensory systems. New York, NY: Plenum Press; p. 701-708.

Mazzuca L, Atkinson S, Nitta E. 1998. Deaths and entanglements of humpback whales, Megaptera novaeangliae, in the main Hawaiian Islands, 1972-1996. Pacific Science. 52(1):1-13.

Michel J, Dunagan H, Boring C, Healy E, Evans W, Dean JM, McGillis A, Hain J. 2007. Worldwide synthesis and analysis of existing information regarding environmental effects of 
alternative energy uses on the Outer Continental Shelf. Herndon, VA: U.S. Department of the Interior, Minerals Management Service. MMS 2007-038.

(MMS) Minerals Management Service. 2007. Programmatic environmental impact statement for alternative energy development and production and alternate use of facilities on the Outer Continental Shelf. U.S. Department of the Interior. MMS 2007-046.

Mobley Jr. JR, Spitz SS, Forney KA, Grotefendt R, Forestell PH. 2000. Distribution and abundance of odontocete species in Hawaiian waters: Preliminary results of 1993-98 aerial surveys. La Jolla, CA: National Marine Fisheries Service, Southwest Fisheries Science Center. Administrative Report LJ-00-14C.

Montevecchi WA. 2006. Influences of artificial light on marine birds. In: Rich C, Longcore T, editors. Ecological consequences of artificial night lighting. Washington, D.C.: Island Press; p. Chapter 5.

Nelson PA. 2008. Ecological effects of wave energy conversion technology on California's marine and anadromous fishes. In: Nelson PA, editors. Developing wave energy in coastal California: Potential socio-economic and environmental effects. Sacramento, CA: California Energy Commission, PIER Energy-Related Environmental Research Program \& California Ocean Protection Council; p. 111-135

Nelson PA, Woo S. 2008. Developing wave energy in coastal California: Potential socioeconomic and environmental effects: Introduction. In: Nelson PA, editors. Developing wave energy in coastal California: Potential socio-economic and environmental effects. Sacramento, CA: California Energy Commission, PIER Energy-Related Environmental Research Program \& California Ocean Protection Council; p. 7-21

Nightingale B, Longcore T, Simenstad CA. 2006. Artificial night lighting and fishes. In: Rich C, Longcore T, editors. Ecological consequences of artificial night lighting. Washington, D.C.: Island Press; p. Chapter 11.

Nitta ET, Henderson JR. 1993. A review of interactions between Hawaii's fisheries and protected species. Marine Fisheries Review. 55(2):83-92.

(NOAA) National Oceanic and Atmospheric Administration. 2007. Wave power: Looking to the ocean for electricity in Oregon [Internet]. U.S. Department of Commerce; [updated March 2007; cited 15 September 2009]. Available from:

http://celebrating200years.noaa.gov/magazine/wave energy/welcome.html\#answer.

Norris KS, Wursig B, Wells RS, Wursig M. 1994. The Hawaiian spinner dolphin. Berkeley and Los Angeles, CA: University of California Press.

Parrish FA, Goto RS. 1997. Patterns of insular shark dynamics based on fishery bycatch and lifeguard surveillance at Oahu, Hawaii 1983-1992. Bulletin of Marine Science. 61(3):763-777.

Relini G, Relini M, Montanari M. 2000. An offshore buoy as a small artificial island and a fishaggregating device (FAD) in the Mediterranean. Hydrobiologia. 440:65-80. 
Reynolds III JE, Wells RS, Eide SD. 2000. The bottlenose dolphin: Biology and conservation. 1st. Gainsville, FL: University Press of Florida.

Richardson WJ, Wursig B. 1997. Influences of man-made noise and other human actions on cetacean behaviour. Marine and Freshwater Behaviour and Physiology. 29:183-209.

Sara G, Dean JM, D'Amato D, Buscaino G, Oliveri A, Genovese S, Ferro S, Buffa G, Lo Martire M, Mazzola S. 2007. Effect of boat noise on the behaviour of bluefin tuna Thunnus thynnus in the Mediterranean Sea. Marine Ecology Progress Series. 331:243-253.

Schlundt CE, Finneran JJ, Carder DA, Ridgway SH. 2000. Temporary shift in masked hearing thresholds of bottlenose dolphins, Tursiops truncatus, and white whales, Delphinapterus leucas, after exposure to intense tones. Journal of the Acoustical Society of America. 107(6):3496-3508.

(SIMON) Sanctuary Integrated Monitoring Network. 2008. Whale entanglement [Internet]. National Oceanic and Atmospheric Administration, Monterey Bay National Marine Sanctuary; [updated June 2008; cited 27 October 2009]. Available from:

http://sanctuarysimon.com/monterey/sections/other/sporadic_we.php.

Stacey PJ, Leatherwood S, Baird RW. 1994. Pseudorca crassidens. Mammalian Species. 456:1-6.

Substructure. [date unknown]. How is concrete affected in a marine environment? [Internet]. Portsmouth, NH: [cited 15 September 2009]. Available from: http://www.substructure.com/education/how_is_concrete_affected_in_a_marine_environment.ht $\underline{\mathrm{ml}}$.

Thomas J, Chun N, Au WWL, Pugh K. 1988. Underwater audiogram of a false killer whale (Pseudorca crassidens). Journal of the Acoustical Society of America. 84(3):936-940.

Tougaard J, Henriksen OD, Miller LA. 2009. Underwater noise from three types of offshore wind turbines: Estimation of impact zones for harbor porpoises and harbor seals. Journal of the Acoustical Society of America. 125(6):3766-3773.

Tyack PL, Wells R, Read A, Howald T, Spradlin T. 1993. Experimental playback of low frequency noise to bottlenose dolphins, Tursiops truncatus. In: editors. Tenth Biennial Conference on the Biology of Marine Mammals; Galveston, TX. p. p. 3 Abstract.

(USFWS) U.S. Fish and Wildlife Service. 2005. Regional Seabird Conservation Plan, Pacific Region. Portland, Oregon: U.S. Fish and Wildlife Service, Migratory Birds and Habitat Programs, Pacific Region.

(USN) U.S. Department of the Navy. 2005. Marine Resources Assessment for the Hawaiian Islands Operating Area. Pacific Division, Naval Facilities Engineering Command, Pearl Harbor, HI: Department of the Navy, Commander, U.S. Pacific Fleet. Contract \# N62470-02-D-9997, CTO 0026.

(USN) U.S. Department of the Navy. 2007. Hawaii range complex - Draft environmental impact statement/Overseas environmental impact statement - Volume 1 of 3. Kekaha, HI: U.S. Department of Defense, Department of the Navy. 
Van Waerebeek K, Leaper R. 2008. Second report of the IWC vessel strike data standardisation working group. In: editors. IWC 60th Annual Meeting; Santiago, Chile. p. 8.

Walker MM. 1984. Learned magnetic field discrimination in yellowfin tuna, Thunnus albacares. Journal of Comparative Physiology A. 155:673-679.

Ylitalo GM, Myers M, Stewart BS, Yochem PK, Braun RC, Kashinsky L, Boyd D, Antonelis GA, Atkinson S, Aguirre AA, et al. 2008. Organochlorine contaminants in endangered Hawaiian monk seals from four subpopulations in the Northwestern Hawaiian Islands. Marine Pollution Bulletin. 56(2):231-244. 


\section{Appendix F. Effects of Pilot Scale MCT SeaGen project at Tacoma Narrows}

Included in Appendix F are 1) a project description of the pilot scale MCT SeaGen project at the Tacoma Narrows site; 2) an effects analysis of the project on site physical and biological indicators in tabular format; and 3) a list of references used to complete the effects analysis.

\section{Project description for construction, operations and maintenance, and decommissioning phases for Pilot Scale MCT SeaGen} project at the Tacoma Narrows site.

\begin{tabular}{|c|c|c|}
\hline Project phase & Project activity or characteristic & Information specific to a pilot scale MCT Seagen at Tacoma Narrows \\
\hline \multirow{14}{*}{ Construction } & Location and deployment depths & Tacoma Narrows. Deployment depth approximately $34 \mathrm{~m}$. \\
\hline & Footprint & 1 device, monopole footprint $7 \mathrm{~m}^{2}$ \\
\hline & Loading ports and dock locations & Port of Tacoma \\
\hline & $\begin{array}{l}\text { Shipping routes for delivery and } \\
\text { installation }\end{array}$ & from Port of Tacoma to project site \\
\hline & Ship types and sizes & 1 drill rig, 2 tug boats, 1 derrick barge, 1 supply boat \\
\hline & Installation and assembly procedures & $\begin{array}{l}\text { Cable directionally drilled from land to water, subsea cable installed to turbine site } \\
\text { Tug brings barge, barge is moored into place, piling hole is drilled, the piling is installed and } \\
\text { grouted. Turbine cross arm installed onto pile, top of device installed, turbine unit lowered } \\
\text { into water }\end{array}$ \\
\hline & Installation equipment & Barge with crane \\
\hline & Temporary structures & Barge with crane \\
\hline & $\begin{array}{l}\text { Types, composition, locations, and } \\
\text { numbers of anchoring and mooring } \\
\text { systems }\end{array}$ & $\begin{array}{l}\text { Barge is moored with } 2 \text { or more moors controlled by hydraulic winches. Monopile does not } \\
\text { require mooring cables. }\end{array}$ \\
\hline & Installation schedule and phasing & $\begin{array}{l}\text { Directional drilling from sea to land power, subsea cable installation, pile fabrication and } \\
\text { drilling, pile and device installation, installation of power equipment, connect subsea cable to } \\
\text { turbine, commissioning, approximately } 4 \text { months }\end{array}$ \\
\hline & Chemicals and fuels used & Hydraulic fluids, gearbox oil, bearing grease, boat fuel \\
\hline & Sources and levels of noise & Pile driving, vessel traffic, directional drilling \\
\hline & Sources, levels, and characteristics of light & Navigational lights on boats, construction lights on decks \\
\hline & Number of vessel trips & Unknown \\
\hline \multirow{2}{*}{$\begin{array}{l}\text { Operations and } \\
\text { Maintenance }\end{array}$} & General description of technology & 1 dual-rotor turbine supported by a mono-pile foundation \\
\hline & O\&M procedures and schedule & $\begin{array}{l}\text { Annual inspections and maintenance, access via small craft (i.e., rigid inflatable boat). } \\
\text { Includes replacement of gearbox oil, application of bearing grease, changing oil filters, } \\
\text { inspection \& repairs if needed of electrical equipment. For larger and infrequent, components } \\
\text { hoisted out with crane or winch, placed on barge (brought by tug-boat) and repaired. }\end{array}$ \\
\hline
\end{tabular}


Appendix F - Effects of Pilot Scale MCT SeaGen Project at the Tacoma Narrows Site

\begin{tabular}{|c|c|c|}
\hline Project phase & Project activity or characteristic & Information specific to a pilot scale MCT Seagen at Tacoma Narrows \\
\hline & $\begin{array}{l}\text { Operating equipment other than tidal } \\
\text { device(s) }\end{array}$ & e \\
\hline & Listing of all moving parts & 2 underwater turbine blades \\
\hline & $\begin{array}{l}\text { Listing of all structures on surface and } \\
\text { below }\end{array}$ & $\begin{array}{l}\text { Mono-pile foundation with dual rotors attached, minimum of } 15 \mathrm{~m} \text { below water's surface in } \\
50 \mathrm{~m} \text { water depth. The pile pierces the surface. }\end{array}$ \\
\hline & $\begin{array}{l}\text { Vessel routes and schedule for operation } \\
\text { and maintenance }\end{array}$ & $\begin{array}{l}\text { Routes unknown but assumed from Port of Tacoma to project site. See above for O\&M } \\
\text { schedule. }\end{array}$ \\
\hline & Ship types and sizes & $\begin{array}{l}\text { Rigid inflatable boat for annual inspections and maintenance. Tug and barge for large repairs } \\
\text { (infrequent, as needed) }\end{array}$ \\
\hline & $\begin{array}{l}\text { Potential emergency conditions and } \\
\text { procedures }\end{array}$ & Oil or hydraulic fluid releases from boats or devices \\
\hline & $\begin{array}{l}\text { Chemicals used by devices, e.g., hydraulic } \\
\text { fluids, antifouling paints. }\end{array}$ & Hydraulic fluids, bearing grease, gearbox oil, anti-fouling paint, boat fuel \\
\hline & Sources and levels of noise & Sources are the turbine blades, level of noise unknown \\
\hline & Sources and levels of light & Navigational lights on boats and on device monopile that pierces surface \\
\hline \multirow{13}{*}{ Decommissioning } & $\begin{array}{l}\text { Description of equipment or structures } \\
\text { removed }\end{array}$ & Electrical and mechanical equipment, pile above seabed, subsea cable \\
\hline & $\begin{array}{l}\text { Description of equipment or structures to } \\
\text { be left in place }\end{array}$ & Pile foundation (in seabed) \\
\hline & $\begin{array}{l}\text { Monitoring procedure and schedule for } \\
\text { equipment left in place }\end{array}$ & None \\
\hline & Shipping routes for equipment removed & Assumed from Port of Tacoma \\
\hline & Ship types and size & Derrick barge, 2 tug boats, 1 supply boat. Sizes unknown \\
\hline & $\begin{array}{l}\text { Decommissioning and disassembly } \\
\text { procedures }\end{array}$ & $\begin{array}{l}\text { Electrical and mechanical equipment removed with barge and crane, pile above grade cut off } \\
\text { and recovered, subsea cable removed, shore landing from directional drilling capped }\end{array}$ \\
\hline & Decommissioning equipment & Derrick barge, crane \\
\hline & Temporary structures & None \\
\hline & Decommissioning schedule and phasing & $\begin{array}{l}\text { Recover electrical and mechanical components, cut off pile and recover upper portion, remove } \\
\text { subsea cable, cap shore landing of directional drilled hole. Approximately } 1 \text { to } 2 \text { months. }\end{array}$ \\
\hline & Chemicals and fuels used & Hydraulic fluids, gearbox oil, bearing grease, boat fuel \\
\hline & Sources and levels of noise & $\begin{array}{l}\text { Removal of pile’s upper portion, boat traffic, deconstruction noise while dismantling device } \\
\text { from monopile }\end{array}$ \\
\hline & Vessels required, number of trips & Derrick barge, 2 tug boats, 1 supply boat. Number of trips unknown \\
\hline & Best management practices planned & Oil and fuel handling, vessel traffic laws, \\
\hline
\end{tabular}




\section{Effects of Pilot Scale MCT SeaGen project at Tacoma Narrows on physical and biological indicators}

\begin{tabular}{|c|c|c|c|c|c|c|}
\hline Project activity & Action & $\begin{array}{l}\text { Description of action's } \\
\text { effect on site physical } \\
\text { attribute }\end{array}$ & $\begin{array}{l}\text { Spatial exposure of } \\
\text { attribute (low, med, } \\
\text { high) }\end{array}$ & $\begin{array}{l}\text { Temporal exposure of } \\
\text { attribute (low, med, } \\
\text { high) }\end{array}$ & $\begin{array}{l}\text { Overall risk to attribute } \\
\text { (low, med, high) }\end{array}$ & Source(s) \\
\hline \multicolumn{7}{|c|}{ Construction } \\
\hline Boat traffic & $\begin{array}{l}\text { Navigation } \\
\text { lights }\end{array}$ & $\begin{array}{l}\text { Vessel lights will be } \\
\text { visible along shipping } \\
\text { lanes from Port of } \\
\text { Tacoma to project site }\end{array}$ & $\begin{array}{l}\text { Low, visibility varies with } \\
\text { atmospheric conditions, } \\
\text { will be seen from shore }\end{array}$ & $\begin{array}{l}\text { Low, construction } \\
\text { vessel traffic will occur } \\
\text { over } 1 \text { to } 2 \text { years during } \\
\text { summer }\end{array}$ & $\begin{array}{l}\text { Low, boat lights visible in } \\
\text { shipping lanes but existing } \\
\text { traffic ameliorates effect }\end{array}$ & $\begin{array}{l}\text { Snohomish PUD } \\
\text { 2008, USN } 2008\end{array}$ \\
\hline $\begin{array}{l}\text { Construction of } \\
\text { electrical collector } \\
\text { system, moorings } \\
\text { and foundations; } \\
\text { device installation }\end{array}$ & $\begin{array}{l}\text { Construction } \\
\text { and deck lights }\end{array}$ & $\begin{array}{l}\text { Construction lights } \\
\text { much brighter than } \\
\text { vessel lights, will be } \\
\text { visible from shore }\end{array}$ & $\begin{array}{l}\text { High, construction lights } \\
\text { brighter than navigation } \\
\text { lights and may be visible } 2 \\
\text { to } 5 \mathrm{~nm} \text {. }\end{array}$ & $\begin{array}{l}\text { Low, construction will } \\
\text { occur over } 1 \text { to } 2 \text { years } \\
\text { during summer }\end{array}$ & $\begin{array}{l}\text { Med, lights may be visible } \\
\text { from shore but location in } \\
\text { industrial/ urban area } \\
\text { ameliorates effect }\end{array}$ & $\begin{array}{l}\text { IALA 2008, } \\
\text { Snohomish PUD } \\
2008\end{array}$ \\
\hline \multicolumn{7}{|c|}{ Operation and maintenance } \\
\hline Boat traffic & $\begin{array}{l}\text { Navigation } \\
\text { lights }\end{array}$ & $\begin{array}{l}\text { Vessel lights will be } \\
\text { visible along shipping } \\
\text { lanes from Port of } \\
\text { Tacoma to project site }\end{array}$ & $\begin{array}{l}\text { Med, visibility varies with } \\
\text { atmospheric conditions, } \\
\text { will be seen from shore }\end{array}$ & $\begin{array}{l}\text { Med, traffic over life of } \\
\text { project but at reduced } \\
\text { frequency compared to } \\
\text { construction }\end{array}$ & $\begin{array}{l}\text { Low, boat lights visible in } \\
\text { shipping lanes in Tacoma } \\
\text { Narrows, but existing traffic } \\
\text { ameliorates effect }\end{array}$ & $\begin{array}{l}\text { Snohomish PUD } \\
\text { 2008, USN } 2008\end{array}$ \\
\hline \multirow{2}{*}{$\begin{array}{l}\text { Structures on } \\
\text { water's surface }\end{array}$} & Structure & $\begin{array}{l}\text { Single device } 15 \mathrm{~m} \\
\text { above water's surface } \\
\text { and }<1 \mathrm{~km} \text { from shore }\end{array}$ & $\begin{array}{l}\text { Low, device will be } \\
\text { visible from shore but } \\
\text { only one device }\end{array}$ & $\begin{array}{l}\text { High, will be present } \\
\text { through life of project }\end{array}$ & $\begin{array}{l}\text { Low, only a single device } \\
\text { located in an industrial/urban } \\
\text { area }\end{array}$ & $\begin{array}{l}\text { Snohomish PUD } \\
2008\end{array}$ \\
\hline & $\begin{array}{l}\text { Navigation } \\
\text { lights }\end{array}$ & $\begin{array}{l}\text { Device will have } \\
\text { navigational lights } \\
\text { visible from } 2 \text { to } 5 \mathrm{~nm} \text {. }\end{array}$ & $\begin{array}{l}\text { Low, device lights from a } \\
\text { single device, will be } \\
\text { visible from shore }\end{array}$ & $\begin{array}{l}\text { High, device lights will } \\
\text { be required throughout } \\
\text { the life of the project }\end{array}$ & $\begin{array}{l}\text { Low, lights only from a } \\
\text { single device, location in } \\
\text { industrial/ urban area } \\
\text { ameliorates effect }\end{array}$ & $\begin{array}{l}\text { Snohomish PUD } \\
2008\end{array}$ \\
\hline \multicolumn{7}{|c|}{ Decommissioning } \\
\hline Boat traffic & $\begin{array}{l}\text { Navigation } \\
\text { lights }\end{array}$ & $\begin{array}{l}\text { Vessel lights will be } \\
\text { visible along shipping } \\
\text { lanes from Port of } \\
\text { Tacoma to project site }\end{array}$ & $\begin{array}{l}\text { Med, lights will be seen } \\
\text { from shore }\end{array}$ & $\begin{array}{l}\text { Low, decommissioning } \\
\text { vessel traffic will occur } \\
\text { over } 1 \text { to } 2 \text { years during } \\
\text { summer }\end{array}$ & $\begin{array}{l}\text { Low, boat lights visiblein } \\
\text { shipping lanes in Tacoma } \\
\text { Narrows but existing traffic } \\
\text { ameliorates effect }\end{array}$ & $\begin{array}{l}\text { Snohomish PUD } \\
\text { 2008, USN } 2008\end{array}$ \\
\hline $\begin{array}{l}\text { Decommissioning of } \\
\text { structures on water's } \\
\text { surface or seabed }\end{array}$ & $\begin{array}{l}\text { Deconstruction } \\
\text { and deck lights }\end{array}$ & $\begin{array}{l}\text { Deconstruction lights } \\
\text { much brighter than } \\
\text { vessel lights, will be } \\
\text { visible from shore. }\end{array}$ & $\begin{array}{l}\text { High, deconstruction } \\
\text { lights brighter than } \\
\text { navigation lights and may } \\
\text { be visible } 2 \text { to } 5 \mathrm{~nm} \text {. }\end{array}$ & $\begin{array}{l}\text { Low, deconstruction } \\
\text { will occur over } 1 \text { to } 2 \\
\text { years during summer }\end{array}$ & $\begin{array}{l}\text { Med, lights may be visible } \\
\text { from shore but location in } \\
\text { industrial/ urban area } \\
\text { ameliorates effect }\end{array}$ & $\begin{array}{l}\text { IALA 2008, } \\
\text { Snohomish PUD } \\
2008\end{array}$ \\
\hline
\end{tabular}

\begin{tabular}{lrr}
\hline $\begin{array}{l}\text { Environmental Assessment for Siting Wave \& Tidal } \\
\text { Energy Projects }\end{array}$ & F-3 & H. T. Harvey \& Associates \\
24 November 2009
\end{tabular}


Appendix F - Effects of Pilot Scale MCT SeaGen Project at the Tacoma Narrows Site

Potential effects on acoustic environment due to Pilot Scale MCT SeaGen project at Tacoma Narrows site

\begin{tabular}{|c|c|c|c|c|c|c|}
\hline Project activity & Project action & $\begin{array}{l}\text { Description of } \\
\text { action's effect on } \\
\text { site physical } \\
\text { attribute } \\
\end{array}$ & $\begin{array}{l}\text { Spatial exposure of } \\
\text { attribute (low, med, } \\
\text { high) }\end{array}$ & $\begin{array}{l}\text { Temporal exposure } \\
\text { of attribute (low, } \\
\text { med, high) }\end{array}$ & $\begin{array}{l}\text { Overall risk to attribute } \\
\text { (low, med, high or } \\
\text { unknown) }\end{array}$ & Source(s) \\
\hline \multicolumn{7}{|c|}{ Construction } \\
\hline Boat traffic & $\begin{array}{l}\text { Noise and } \\
\text { vibration }\end{array}$ & $\begin{array}{l}\text { Propellers cavitate, } \\
\text { causing pressure } \\
\text { differences }\end{array}$ & $\begin{array}{l}\text { High, modeled noise of } \\
120 \mathrm{~dB} \text { extended } \\
\text { approx } 20 \mathrm{~km} \text { in ocean } \\
\text { wind turbine project }\end{array}$ & $\begin{array}{l}\text { Low, vessel traffic } \\
\text { will occur over } 1 \text { to } 2 \\
\text { years during the } \\
\text { summer }\end{array}$ & $\begin{array}{l}\text { Low, noise could be } \\
\text { elevated but short-term, pre- } \\
\text { existing boat traffic in Puget } \\
\text { Sound ameliorates effect }\end{array}$ & $\begin{array}{l}\text { Austin et al. 2009, } \\
\text { Snohomish PUD } 2008\end{array}$ \\
\hline $\begin{array}{l}\text { Construction of } \\
\text { electrical collector } \\
\text { system, moorings and } \\
\text { foundations; device } \\
\text { installation }\end{array}$ & $\begin{array}{l}\text { Noise and } \\
\text { vibration }\end{array}$ & $\begin{array}{l}\text { Adds to existing } \\
\text { natural and man- } \\
\text { made noise in } \\
\text { project area }\end{array}$ & $\begin{array}{l}\text { High, modeled noise of } \\
120 \mathrm{~dB} \text { extended } \\
\text { approx } 20 \mathrm{~km} \text { in ocean } \\
\text { wind turbine project }\end{array}$ & $\begin{array}{l}\text { Low, construction } \\
\text { noise will occur over } \\
1 \text { to } 2 \text { years during the } \\
\text { summer }\end{array}$ & $\begin{array}{l}\text { Med, noise could be } \\
\text { elevated but short-term, pre- } \\
\text { existing noise in Puget } \\
\text { Sound ameliorates effect }\end{array}$ & Austin et al. 2009 \\
\hline $\begin{array}{l}\text { Directional drilling, } \\
\text { and laying cable } \\
\text { under/on seabed }\end{array}$ & $\begin{array}{l}\text { Noise and } \\
\text { vibration }\end{array}$ & $\begin{array}{l}\text { Vibration of } \\
\text { immediate area } \\
\text { being drilled }\end{array}$ & $\begin{array}{l}\text { Low, vibration could be } \\
\text { localized, assuming } \\
\text { similar to directional } \\
\text { drilling on land }\end{array}$ & $\begin{array}{l}\text { Low, drilling will } \\
\text { occur for } 1-2 \text { weeks }\end{array}$ & $\begin{array}{l}\text { Low, effect expected to be } \\
\text { localized and short-term }\end{array}$ & CPUC 2009 \\
\hline \multicolumn{7}{|c|}{ Operation and Maintenance } \\
\hline Boat traffic & $\begin{array}{l}\text { Noise and } \\
\text { vibration }\end{array}$ & $\begin{array}{l}\text { Propellers cavitate, } \\
\text { causing pressure } \\
\text { differences }\end{array}$ & $\begin{array}{l}\text { High, modeled noise of } \\
120 \mathrm{~dB} \text { extended } \\
\text { approx } 20 \mathrm{~km} \text { in ocean } \\
\text { wind turbine project }\end{array}$ & $\begin{array}{l}\text { Low, vessel traffic } \\
\text { infrequent during } \\
\text { O\&M }\end{array}$ & $\begin{array}{l}\text { Low, elevated noise would } \\
\text { occur infrequently, pre- } \\
\text { existing boat traffic in Puget } \\
\text { Sound ameliorates effect }\end{array}$ & Austin et al. 2009 \\
\hline $\begin{array}{l}\text { Operation of turbines } \\
\text { or other moving parts } \\
\text { of devices }\end{array}$ & $\begin{array}{l}\text { Noise and } \\
\text { vibration }\end{array}$ & $\begin{array}{l}\text { Adds to existing } \\
\text { natural and man- } \\
\text { made noise in } \\
\text { project area }\end{array}$ & $\begin{array}{l}\text { Unknown levels } \\
\text { generated; site specific } \\
\text { attenuation and ambient } \\
\text { noise also unknown }\end{array}$ & $\begin{array}{l}\text { High, noise would } \\
\text { occur over life of the } \\
\text { project }\end{array}$ & Unknown & Study warranted \\
\hline \multicolumn{7}{|c|}{ Decommissioning } \\
\hline Boat traffic & $\begin{array}{l}\text { Noise and } \\
\text { vibration }\end{array}$ & $\begin{array}{l}\text { Propellers cavitate, } \\
\text { causing pressure } \\
\text { differences }\end{array}$ & $\begin{array}{l}\text { High, modeled noise of } \\
120 \mathrm{~dB} \text { extended } \\
\text { approx } 20 \mathrm{~km} \text { in ocean } \\
\text { wind turbine project }\end{array}$ & $\begin{array}{l}\text { Low, vessel traffic } \\
\text { will occur over } 1 \text { to } 2 \\
\text { years during the } \\
\text { summer }\end{array}$ & $\begin{array}{l}\text { Low, noise could be } \\
\text { elevated but short-term, pre- } \\
\text { existing boat traffic in Puget } \\
\text { Sound ameliorates effect }\end{array}$ & Austin et al. 2009 \\
\hline $\begin{array}{l}\text { Decommissioning of } \\
\text { structures on water’s } \\
\text { surface or seabed }\end{array}$ & $\begin{array}{l}\text { Noise and } \\
\text { vibration }\end{array}$ & $\begin{array}{l}\text { Adds to existing } \\
\text { natural and man- } \\
\text { made noise in } \\
\text { project area }\end{array}$ & $\begin{array}{l}\text { High, modeled noise of } \\
120 \mathrm{~dB} \text { extended } \\
\text { approx } 20 \mathrm{~km} \text { in ocean } \\
\text { wind turbine project }\end{array}$ & $\begin{array}{l}\text { Low, noise will occur } \\
\text { over } 1 \text { to } 2 \text { years } \\
\text { during the summer }\end{array}$ & $\begin{array}{l}\text { Med, noise could be } \\
\text { elevated but short-term, pre- } \\
\text { existing noise in Puget } \\
\text { Sound ameliorates effect }\end{array}$ & Austin et al. 2009 \\
\hline
\end{tabular}

\begin{tabular}{lrr}
\hline $\begin{array}{l}\text { Environmental Assessment for Siting Wave \& Tidal } \\
\text { Energy Projects }\end{array}$ & F-4 & H. T. Harvey \& Associates \\
24 November 2009
\end{tabular}


Appendix F - Effects of Pilot Scale MCT SeaGen Project at the Tacoma Narrows Site

Potential effects on sediment and water chemistry due to Pilot Scale MCT SeaGen project at Tacoma Narrows site

\begin{tabular}{|c|c|c|c|c|c|c|}
\hline Project activity & Project action & $\begin{array}{l}\text { Description of action's } \\
\text { effect on attribute }\end{array}$ & $\begin{array}{l}\text { Spatial exposure of } \\
\text { attribute (low, med, } \\
\text { high) }\end{array}$ & $\begin{array}{l}\text { Temporal exposure of } \\
\text { attribute (low, med, } \\
\text { high) }\end{array}$ & $\begin{array}{l}\text { Overall risk to } \\
\text { attribute (low, med, } \\
\text { high) }\end{array}$ & Source(s) \\
\hline \multicolumn{7}{|c|}{ Construction } \\
\hline Boat traffic & $\begin{array}{l}\text { Oil/chemical release, } \\
\text { assumed seepage from } \\
\text { exhaust and general use, } \\
\text { not a spill from collision } \\
\text { or other release }\end{array}$ & \multirow{2}{*}{$\begin{array}{l}\text { Could add compounds } \\
\text { that change the physical } \\
\text { and chemical } \\
\text { characteristics of } \\
\text { sediment and water }\end{array}$} & $\begin{array}{l}\text { Low, seepage will } \\
\text { have a small spatial } \\
\text { and areal extent } \\
\text { relative to the project }\end{array}$ & $\begin{array}{l}\text { High, traffic and boat } \\
\text { frequency will be the } \\
\text { highest during } \\
\text { construction }\end{array}$ & $\begin{array}{l}\text { Low, given low spatial } \\
\text { exposure and high pre- } \\
\text { existing contaminants } \\
\text { and boat traffic in Puget } \\
\text { Sound }\end{array}$ & EVS 2003 \\
\hline $\begin{array}{l}\text { Construction of } \\
\text { electrical collector } \\
\text { system, moorings } \\
\text { and foundations; } \\
\text { device installation }\end{array}$ & Oil/chemical release & & $\begin{array}{l}\text { Low, seepage will } \\
\text { have a small spatial } \\
\text { and areal extent } \\
\text { relative to the project }\end{array}$ & $\begin{array}{l}\text { Low, construction will } \\
\text { occur over } 2 \text { months }\end{array}$ & $\begin{array}{l}\text { Low, given low spatial } \\
\text { and temporal extent and } \\
\text { high pre-existing } \\
\text { contaminants in Puget } \\
\text { Sound }\end{array}$ & EVS 2003 \\
\hline $\begin{array}{l}\text { Directional drilling, } \\
\text { and laying cable } \\
\text { under/on seabed } \\
\text { (assume normal } \\
\text { conditions, not a } \\
\text { drilling mud “blow } \\
\text { out” scenario) }\end{array}$ & Seabed disturbance & $\begin{array}{l}\text { Sediment would be } \\
\text { introduced into water } \\
\text { column; deeper } \\
\text { sediments with different } \\
\text { chemistry brought to } \\
\text { seabed surface }\end{array}$ & $\begin{array}{l}\text { Low, cable length }<1 \\
\mathrm{~nm}\end{array}$ & $\begin{array}{l}\text { Low, increased sediment } \\
\text { in water column would } \\
\text { mix or dilute quickly. }\end{array}$ & $\begin{array}{l}\text { Low, due to quick } \\
\text { dilution of sediment in } \\
\text { water column }\end{array}$ & Previsic 2009 \\
\hline \multicolumn{7}{|c|}{ Operation and maintenance } \\
\hline Boat traffic & $\begin{array}{l}\text { Oil/chemical release, } \\
\text { assumed seepage from } \\
\text { general use, not a spill } \\
\text { from collision }\end{array}$ & $\begin{array}{l}\text { Could add compounds } \\
\text { that change the physical } \\
\text { and chemical } \\
\text { characteristics of } \\
\text { sediment and water }\end{array}$ & $\begin{array}{l}\text { Low, seepage will } \\
\text { have a small spatial } \\
\text { and areal extent } \\
\text { relative to project }\end{array}$ & $\begin{array}{l}\text { Low, O\&M vessel traffic } \\
\text { will be much less than } \\
\text { during construction }\end{array}$ & $\begin{array}{l}\text { Low, due to low spatial } \\
\text { extent, low volume of } \\
\text { vessel traffic during } \\
\text { O\&M, and high pre- } \\
\text { existing contaminants in } \\
\text { Puget Sound }\end{array}$ & EVS 2003 \\
\hline \multirow{2}{*}{$\begin{array}{l}\text { Structures in water } \\
\text { column and on } \\
\text { seabed, such as } \\
\text { devices and } \\
\text { moorings and } \\
\text { footings }\end{array}$} & Structure & $\begin{array}{l}\text { Concrete footings are } \\
\text { sources of alkaline } \\
\text { elements (sodium, } \\
\text { potassium) that could } \\
\text { leach into water column }\end{array}$ & $\begin{array}{l}\text { Low, effect would be } \\
\text { localized to vicinity } \\
\text { of concrete footings }\end{array}$ & $\begin{array}{l}\text { High, any leaching would } \\
\text { occur throughout life of } \\
\text { project }\end{array}$ & $\begin{array}{l}\text { Low, effects would be } \\
\text { diluted and may not be } \\
\text { measurable }\end{array}$ & $\begin{array}{l}\text { Substructure } \\
\text { [date unknown] }\end{array}$ \\
\hline & Water circulation changes & $\begin{array}{l}\text { Concrete footing could } \\
\text { cause seabed erosion in } \\
\text { lee of the footings }\end{array}$ & $\begin{array}{l}\text { Low, effect would be } \\
\text { localized to lee side } \\
\text { of footings }\end{array}$ & $\begin{array}{l}\text { High, any erosion } \\
\text { occurring would be } \\
\text { throughout the life of the } \\
\text { project }\end{array}$ & $\begin{array}{l}\text { Low, effect is localized } \\
\text { although occurring over } \\
\text { life of project }\end{array}$ & $\begin{array}{l}\text { Largier et al. } \\
2008\end{array}$ \\
\hline $\begin{array}{l}\text { Structures on } \\
\text { water's surface }\end{array}$ & Structure & $\begin{array}{l}\text { Biofouling organisms } \\
\text { slough off on to the } \\
\text { seabed surface }\end{array}$ & $\begin{array}{l}\text { Low, effect localized } \\
\text { to seabed directly } \\
\text { under devices }\end{array}$ & $\begin{array}{l}\text { Low, antifouling paint } \\
\text { and maintenance likely to } \\
\text { remove organisms before } \\
\text { sloughing }\end{array}$ & $\begin{array}{l}\text { Low, effect is localized } \\
\text { to immediate vicinity of } \\
\text { seabed under devices }\end{array}$ & MMS 2007 \\
\hline
\end{tabular}

\begin{tabular}{|c|c|c|}
\hline $\begin{array}{l}\text { Environmental Assessment for Siting Wave \& Tidal } \\
\text { Energy Projects }\end{array}$ & F-5 & $\begin{array}{r}\text { H. T. Harvey \& Associates } \\
24 \text { November } 2009\end{array}$ \\
\hline
\end{tabular}


Appendix F - Effects of Pilot Scale MCT SeaGen Project at the Tacoma Narrows Site

\begin{tabular}{|c|c|c|c|c|c|c|}
\hline Project activity & Project action & $\begin{array}{l}\text { Description of action's } \\
\text { effect on attribute }\end{array}$ & $\begin{array}{l}\text { Spatial exposure of } \\
\text { attribute (low, med, } \\
\text { high) }\end{array}$ & $\begin{array}{l}\text { Temporal exposure of } \\
\text { attribute (low, med, } \\
\text { high) }\end{array}$ & $\begin{array}{l}\text { Overall risk to } \\
\text { attribute (low, med, } \\
\text { high) }\end{array}$ & Source(s) \\
\hline \multicolumn{7}{|c|}{ Decommissioning } \\
\hline Boat traffic & $\begin{array}{l}\text { Oil/chemical release, } \\
\text { assumed seepage from } \\
\text { general use, not a spill } \\
\text { from collision }\end{array}$ & \multirow{2}{*}{$\begin{array}{l}\text { Could add compounds } \\
\text { that change the physical } \\
\text { and chemical } \\
\text { characteristics of } \\
\text { sediment and water }\end{array}$} & \multirow{2}{*}{$\begin{array}{l}\text { Low, seepage will } \\
\text { have a small spatial } \\
\text { and areal extent } \\
\text { relative to the project }\end{array}$} & $\begin{array}{l}\text { High, traffic and boat } \\
\text { frequency will be the high } \\
\text { during decommissioning }\end{array}$ & $\begin{array}{l}\text { Low, given low spatial } \\
\text { extent and high pre- } \\
\text { existing boat traffic and } \\
\text { contaminants in Puget } \\
\text { Sound }\end{array}$ & EVS 2003 \\
\hline \multirow{2}{*}{$\begin{array}{l}\text { Decommissioning of } \\
\text { structures on water's } \\
\text { surface or seabed }\end{array}$} & Oil/chemical release & & & $\begin{array}{l}\text { Low, decommissioning } \\
\text { will occur over } 2 \text { months }\end{array}$ & $\begin{array}{l}\text { Low, given low spatial } \\
\text { and temporal extent and } \\
\text { high pre-existing } \\
\text { contaminants in Puget } \\
\text { Sound }\end{array}$ & EVS 2003 \\
\hline & Seabed disturbance & $\begin{array}{l}\text { Sediment would be } \\
\text { introduced into water } \\
\text { column when removing } \\
\text { footings and/or subsea } \\
\text { cable }\end{array}$ & $\begin{array}{l}\text { Low, cable length }<1 \\
n m\end{array}$ & $\begin{array}{l}\text { Low, increased sediment } \\
\text { in water column would } \\
\text { mix or dilute quickly. }\end{array}$ & $\begin{array}{l}\text { Low, due to quick } \\
\text { dilution of sediment in } \\
\text { water column }\end{array}$ & Previsic 2009 \\
\hline
\end{tabular}

Environmental Assessment for Siting Wave \& Tidal Energy Projects
F-6

H. T. Harvey \& Associates

24 November 2009 
Appendix F - Effects of Pilot Scale MCT SeaGen Project at the Tacoma Narrows Site

Potential effects on Gray Whales due to Pilot Scale MCT SeaGen project at Tacoma Narrows site

\begin{tabular}{|c|c|c|c|c|c|c|c|}
\hline Project activity & Project action & $\begin{array}{l}\text { Description of } \\
\text { action's effect on } \\
\text { indicator }\end{array}$ & $\begin{array}{l}\text { Spatial exposure to } \\
\text { indicator (low, med, } \\
\text { high) }\end{array}$ & $\begin{array}{l}\text { Temporal } \\
\text { exposure to } \\
\text { (low, med, high) }\end{array}$ & $\begin{array}{l}\text { Effect's overlap } \\
\text { with critical life } \\
\text { stage, behavior, } \\
\text { habitat, or } \\
\text { resource (low, } \\
\text { med, high) }\end{array}$ & $\begin{array}{l}\text { Overall risk to } \\
\text { indicator (low, med, } \\
\text { high, unknown) }\end{array}$ & Source(s) \\
\hline \multicolumn{8}{|c|}{ Construction } \\
\hline \multirow{3}{*}{$\begin{array}{l}\text { Boat traffic } \\
\text { including subsea } \\
\text { cable laying vessel }\end{array}$} & Direct impact & Collision injuries & $\begin{array}{l}\text { High, boat traffic } \\
\text { crosses migration } \\
\text { path along the coast }\end{array}$ & $\begin{array}{l}\text { Low, occurring } \\
\text { during summer } \\
\text { for } 1 \text { to } 2 \text { years }\end{array}$ & $\begin{array}{l}\text { Low, effects' } \\
\text { spatial extent small } \\
\text { compared to } \\
\text { animal's range }\end{array}$ & $\begin{array}{l}\text { Low; acclimation } \\
\text { likely due to high } \\
\text { boat traffic; gray } \\
\text { whale-boat collisions } \\
\text { not uncommon }\end{array}$ & $\begin{array}{l}\text { Calambokidis et } \\
\text { al. 2002, Sullivan } \\
\text { et al. 1983, Van } \\
\text { Waerebeek and } \\
\text { Leaper } 2008\end{array}$ \\
\hline & $\begin{array}{l}\text { Noise and } \\
\text { vibration }\end{array}$ & $\begin{array}{l}\text { Avoidance, masking } \\
\text { of envir. cues, } \\
\text { communication } \\
\text { signals }\end{array}$ & $\begin{array}{l}\text { Med to high, sound } \\
\text { travels far, animals } \\
\text { will hear outside } \\
\text { project area }\end{array}$ & $\begin{array}{l}\text { Low, occurring } \\
\text { during summer } \\
\text { for } 1 \text { to } 2 \text { years }\end{array}$ & $\begin{array}{l}\text { Low, effect } \\
\text { influences small } \\
\text { part of large range }\end{array}$ & $\begin{array}{l}\text { Low; likely tolerate } \\
\text { vessel noise due to } \\
\text { high levels in Puget } \\
\text { Sounds }\end{array}$ & $\begin{array}{l}\text { Richardson and } \\
\text { Wursig } 1997\end{array}$ \\
\hline & $\begin{array}{l}\text { Oil/chemical } \\
\text { release }\end{array}$ & $\begin{array}{l}\text { Ingestion, breathing } \\
\text { exhaust fumes, } \\
\text { accumulation of } \\
\text { toxins. }\end{array}$ & $\begin{array}{l}\text { Low, low volume of } \\
\text { fluids released; } \\
\text { medium for PCFA } \\
\text { whales due to } \\
\text { potentially increased } \\
\text { contamination levels }\end{array}$ & $\begin{array}{l}\text { Low, occurring } \\
\text { during } 1 \text { to } 2 \\
\text { summers }\end{array}$ & $\begin{array}{l}\text { Low, effects' } \\
\text { spatial extent small } \\
\text { compared to } \\
\text { animal's range }\end{array}$ & $\begin{array}{l}\text { Low; inputs } \\
\text { insignificant relative } \\
\text { to background levels } \\
\text { in Puget Sound }\end{array}$ & Ebbert et al. 2000 \\
\hline \multirow{3}{*}{$\begin{array}{l}\text { Construction and } \\
\text { installation of } \\
\text { electrical collector } \\
\text { system, mooring } \\
\text { cables, anchors or } \\
\text { foundations, and } \\
\text { devices }\end{array}$} & $\begin{array}{l}\text { Oil/chemical } \\
\text { release }\end{array}$ & $\begin{array}{l}\text { Ingestion, breathing } \\
\text { exhaust fumes, } \\
\text { accumulation of } \\
\text { toxins. }\end{array}$ & $\begin{array}{l}\text { Low, significant } \\
\text { increase in existing } \\
\text { traffic but volume of } \\
\text { fluids released low. }\end{array}$ & $\begin{array}{l}\text { Low, occurring } \\
\text { over } 1 \text { to } 2 \\
\text { summers }\end{array}$ & $\begin{array}{l}\text { Low, effects' } \\
\text { spatial extent small } \\
\text { compared to } \\
\text { animal's range }\end{array}$ & $\begin{array}{l}\text { Low; inputs } \\
\text { insignificant relative } \\
\text { to background levels } \\
\text { in Puget Sound }\end{array}$ & Ebbert et al. 2000 \\
\hline & $\begin{array}{l}\text { Construction } \\
\text { lights }\end{array}$ & Avoidance & $\begin{array}{l}\text { Low, not likely to be } \\
\text { attracted to lighted } \\
\text { areas }\end{array}$ & $\begin{array}{l}\text { Low, occurring } \\
\text { over } 1 \text { to } 2 \\
\text { summers }\end{array}$ & $\begin{array}{l}\text { Low, effects' } \\
\text { spatial extent small } \\
\text { compared to } \\
\text { animal's range }\end{array}$ & $\begin{array}{l}\text { Low; high levels of } \\
\text { shoreline } \\
\text { development, boat } \\
\text { traffic, industry in } \\
\text { Puget Sound }\end{array}$ & Todd et al. 2009 \\
\hline & $\begin{array}{l}\text { Noise and } \\
\text { vibration }\end{array}$ & $\begin{array}{l}\text { Avoidance, masking } \\
\text { of environmental } \\
\text { cues, communication } \\
\text { signals }\end{array}$ & $\begin{array}{l}\text { Low to med, sound } \\
\text { travels far and } \\
\text { animals will hear it } \\
\text { outside project area, } \\
\text { but affected area } \\
\text { small part of range }\end{array}$ & $\begin{array}{l}\text { Low, occurring } \\
\text { over } 1 \text { summer }\end{array}$ & $\begin{array}{l}\text { Low, effects' } \\
\text { spatial extent small } \\
\text { compared to } \\
\text { animal's range }\end{array}$ & $\begin{array}{l}\text { Low; spp. likely } \\
\text { displaced if noise } \\
\text { high; displacement } \\
\text { area small portion of } \\
\text { range and short } \\
\text { duration }\end{array}$ & $\begin{array}{l}\text { Richardson and } \\
\text { Wursig } 1997\end{array}$ \\
\hline
\end{tabular}


Appendix F - Effects of Pilot Scale MCT SeaGen Project at the Tacoma Narrows Site

\begin{tabular}{|c|c|c|c|c|c|c|c|}
\hline Project activity & Project action & $\begin{array}{l}\text { Description of } \\
\text { action's effect on } \\
\text { indicator }\end{array}$ & $\begin{array}{l}\text { Spatial exposure to } \\
\text { indicator (low, med, } \\
\text { high) }\end{array}$ & $\begin{array}{l}\text { Temporal } \\
\text { exposure to } \\
\text { (low, med, high) }\end{array}$ & $\begin{array}{l}\text { Effect's overlap } \\
\text { with critical life } \\
\text { stage, behavior, } \\
\text { habitat, or } \\
\text { resource (low, } \\
\text { med, high) }\end{array}$ & $\begin{array}{l}\text { Overall risk to } \\
\text { indicator (low, med, } \\
\text { high, unknown) }\end{array}$ & Source(s) \\
\hline $\begin{array}{l}\text { Directional drilling, } \\
\text { and laying cable } \\
\text { under/on seabed }\end{array}$ & $\begin{array}{l}\text { Noise and } \\
\text { vibration }\end{array}$ & $\begin{array}{l}\text { Avoidance, masking } \\
\text { of environmental } \\
\text { cues, communication } \\
\text { signals }\end{array}$ & $\begin{array}{l}\text { Low to med, sound } \\
\text { travels far and } \\
\text { animals will hear it } \\
\text { outside project area, } \\
\text { affected area small } \\
\text { part of range }\end{array}$ & $\begin{array}{l}\text { Low, occurring } \\
\text { over } 1 \text { summer }\end{array}$ & $\begin{array}{l}\text { Low, effects' } \\
\text { spatial extent small } \\
\text { compared to } \\
\text { animal's range }\end{array}$ & Low; see above & $\begin{array}{l}\text { Richardson and } \\
\text { Wursig } 1997\end{array}$ \\
\hline \multicolumn{8}{|c|}{ Operation and Maintenance } \\
\hline \multirow{3}{*}{ Boat traffic } & Direct impact & Collision injuries & $\begin{array}{l}\text { Low, boat traffic } \\
\text { crosses migration } \\
\text { path along the coast } \\
\text { but frequency is low }\end{array}$ & $\begin{array}{l}\text { Med, occurs in } \\
\text { summer during } \\
\text { northward } \\
\text { migration }\end{array}$ & $\begin{array}{l}\text { Low, effects' } \\
\text { spatial extent small } \\
\text { compared to } \\
\text { animal's range }\end{array}$ & $\begin{array}{l}\text { Low; insignificant } \\
\text { increase in traffic } \\
\text { over existing } \\
\text { (235,000 vessels /yr) } \\
\text { in Puget sound; gray } \\
\text { whale-boat collisions } \\
\text { not uncommon }\end{array}$ & $\begin{array}{l}\text { USCG [date } \\
\text { unknown], Van } \\
\text { Waerebeek and } \\
\text { Leaper } 2008\end{array}$ \\
\hline & $\begin{array}{l}\text { Oil/chemical } \\
\text { release }\end{array}$ & $\begin{array}{l}\text { Ingestion, breathing } \\
\text { exhaust fumes, } \\
\text { overall accumulation } \\
\text { of toxins. }\end{array}$ & $\begin{array}{l}\text { Low, low volume of } \\
\text { fluids released; could } \\
\text { increase contaminant } \\
\text { levels for PCFA } \\
\text { whales }\end{array}$ & $\begin{array}{l}\text { Med, throughout } \\
\text { project duration } \\
\text { but not likely to } \\
\text { be year round } \\
\text { action }\end{array}$ & $\begin{array}{l}\text { Low, effects' } \\
\text { spatial extent small } \\
\text { compared to } \\
\text { animal's range }\end{array}$ & $\begin{array}{l}\text { Low; exposure are } \\
\text { small relative to } \\
\text { range; inputs } \\
\text { compound elevated } \\
\text { background levels } \\
\end{array}$ & Ebbert et al. 2000 \\
\hline & $\begin{array}{l}\text { Noise and } \\
\text { vibration }\end{array}$ & $\begin{array}{l}\text { Avoidance, masking } \\
\text { of environmental } \\
\text { cues, communication } \\
\text { signals }\end{array}$ & $\begin{array}{l}\text { Low, sound travels } \\
\text { far, animals will hear } \\
\text { outside project area, } \\
\text { but affects small part } \\
\text { of large range }\end{array}$ & $\begin{array}{l}\text { Low, } \\
\text { maintenance via } \\
\text { boats occurring } \\
\text { infrequently but } \\
\text { during summer } \\
\end{array}$ & $\begin{array}{l}\text { Low, effects' } \\
\text { spatial extent small } \\
\text { compared to } \\
\text { animal's range }\end{array}$ & $\begin{array}{l}\text { Low; insignificant } \\
\text { increase in boat } \\
\text { traffic over existing } \\
\text { (235,000 vessels/yr } \\
\text { in Puget sound) }\end{array}$ & $\begin{array}{l}\text { Morton and } \\
\text { Symonds } 2002\end{array}$ \\
\hline \multirow{2}{*}{$\begin{array}{l}\text { Operation of } \\
\text { turbines, rotation of } \\
\text { rotors, or other } \\
\text { moving parts of } \\
\text { devices }\end{array}$} & Direct impact & $\begin{array}{l}\text { Direct injury or } \\
\text { mortality; secondary } \\
\text { mortality due to } \\
\text { infection, loss of } \\
\text { mobility, secondary } \\
\text { complications, } \\
\text { predation }\end{array}$ & $\begin{array}{l}\text { Low, areas of } \\
\text { movement small } \\
\text { ( 1\%) compared to } \\
\text { channel area } \\
\text { (approximated } \\
\text { channel width } x \\
\text { average depth) } \\
\end{array}$ & $\begin{array}{l}\text { High, the } \\
\text { movement } \\
\text { occurs over the } \\
\text { life of the project }\end{array}$ & $\begin{array}{l}\text { High while } \\
\text { foraging, traveling }\end{array}$ & $\begin{array}{l}\text { High; significant risk } \\
\text { of rotor strike - may } \\
\text { be less detectable } \\
\text { than ship rotor due to } \\
\text { slower speed; strike at } \\
12 \mathrm{~m} / \mathrm{s} \text { will cause } \\
\text { serious trauma }\end{array}$ & $\begin{array}{l}\text { Dadswell and } \\
\text { Rulifson 1994, } \\
\text { Todd et al. 2009, } \\
\text { Wilson et al. 2007 }\end{array}$ \\
\hline & $\begin{array}{l}\text { Noise and } \\
\text { vibration }\end{array}$ & $\begin{array}{l}\text { Avoidance, masks } \\
\text { environmental cues, } \\
\text { communication } \\
\text { signals }\end{array}$ & $\begin{array}{l}\text { Unknown, sound } \\
\text { travels far and animals } \\
\text { will hear outside } \\
\text { project area, affects } \\
\text { small part of large } \\
\text { range }\end{array}$ & $\begin{array}{l}\text { Med, occurring } \\
\text { throughout the } \\
\text { duration of the } \\
\text { project }\end{array}$ & $\begin{array}{l}\text { Low; effect not } \\
\text { expected to extend } \\
\text { beyond immediate } \\
\text { area around turbine }\end{array}$ & $\begin{array}{l}\text { Unknown; could } \\
\text { mask environmental } \\
\text { cues near turbine } \\
\text { ( }<70 \mathrm{~m}) \text {; acclimation } \\
\text { likely due to ambient } \\
\text { industrial noise }\end{array}$ & $\begin{array}{l}\text { Richardson and } \\
\text { Wursig } 1997\end{array}$ \\
\hline
\end{tabular}

Environmental Assessment for Siting Wave \& Tidal
Energy Projects
F-8

H. T. Harvey \& Associates

24 November 2009 
Appendix F - Effects of Pilot Scale MCT SeaGen Project at the Tacoma Narrows Site

\begin{tabular}{|c|c|c|c|c|c|c|c|}
\hline Project activity & Project action & $\begin{array}{l}\text { Description of } \\
\text { action's effect on } \\
\text { indicator }\end{array}$ & $\begin{array}{l}\text { Spatial exposure to } \\
\text { indicator (low, med, } \\
\text { high) }\end{array}$ & $\begin{array}{l}\text { Temporal } \\
\text { exposure to } \\
\text { (low, med, high) }\end{array}$ & $\begin{array}{l}\text { Effect's overlap } \\
\text { with critical life } \\
\text { stage, behavior, } \\
\text { habitat, or } \\
\text { resource (low, } \\
\text { med, high) }\end{array}$ & $\begin{array}{l}\text { Overall risk to } \\
\text { indicator (low, med, } \\
\text { high, unknown) }\end{array}$ & Source(s) \\
\hline $\begin{array}{l}\text { Structures in water } \\
\text { column and on } \\
\text { seabed, such as } \\
\text { devices and } \\
\text { moorings and } \\
\text { footings }\end{array}$ & Structure & $\begin{array}{l}\text { Entanglement with } \\
\text { derelict gear caught on } \\
\text { devices; artificial reef } \\
\text { effects could attract } \\
\text { fish, increase forage, } \\
\text { and attract species }\end{array}$ & $\begin{array}{l}\text { Low, home range is } \\
\text { large compared to } \\
\text { project area; }\end{array}$ & $\begin{array}{l}\text { High, occurring } \\
\text { throughout } \\
\text { project duration. }\end{array}$ & $\begin{array}{l}\text { Low, effects' } \\
\text { spatial extent small } \\
\text { compared to } \\
\text { animal's range }\end{array}$ & $\begin{array}{l}\text { Unknown; risk of } \\
\text { entanglement in } \\
\text { derelict gear } \\
\text { unknown }\end{array}$ & \\
\hline $\begin{array}{l}\text { Electricity } \\
\text { conduction through } \\
\text { cable }\end{array}$ & EMF & Unknown & $\begin{array}{l}\text { Unknown, cable } \\
\text { shielding provides } \\
\text { some attenuation }\end{array}$ & $\begin{array}{l}\text { High if there is } \\
\text { an effect, would } \\
\text { continue over } \\
\text { life of project }\end{array}$ & Unknown & $\begin{array}{l}\text { Unknown; High } \\
\text { uncertainty about } \\
\text { effects on marine } \\
\text { mammals }\end{array}$ & $\begin{array}{l}\text { Boehlert et al. } \\
2008\end{array}$ \\
\hline \multirow[b]{2}{*}{$\begin{array}{l}\text { Structures on } \\
\text { water's surface }\end{array}$} & Structure & Collision & $\begin{array}{l}\text { Low, area is small } \\
\text { compared to home } \\
\text { range }\end{array}$ & $\begin{array}{l}\text { High, occurring } \\
\text { throughout } \\
\text { project duration }\end{array}$ & $\begin{array}{l}\text { Low; no effect } \\
\text { expected }\end{array}$ & $\begin{array}{l}\text { Low; no risk } \\
\text { expected }\end{array}$ & \\
\hline & $\begin{array}{l}\text { Navigation } \\
\text { lights }\end{array}$ & $\begin{array}{l}\text { Low intensity; } \\
\text { likelihood of impacts } \\
\text { low to none }\end{array}$ & $\begin{array}{l}\text { Low, associated with } \\
\text { nav lights on devices }\end{array}$ & $\begin{array}{l}\text { High, occurring } \\
\text { throughout } \\
\text { project duration }\end{array}$ & $\begin{array}{l}\text { Low, light } \\
\text { intensity low }\end{array}$ & $\begin{array}{l}\text { Low; high levels of } \\
\text { shoreline } \\
\text { development, boat } \\
\text { traffic, industry in } \\
\text { Puget Sound }\end{array}$ & Todd et al. 2009 \\
\hline \multicolumn{8}{|c|}{ Decommissioning } \\
\hline \multirow{3}{*}{ Boat traffic } & Direct impact & Collision injuries & $\begin{array}{l}\text { High; boat traffic } \\
\text { crosses migration } \\
\text { path along the coast }\end{array}$ & $\begin{array}{l}\text { Low - Med } \\
\text { depending on } \\
\text { timing of } \\
\text { construction * }\end{array}$ & $\begin{array}{l}\text { Low, effects' } \\
\text { spatial extent small } \\
\text { compared to } \\
\text { animal's range }\end{array}$ & $\begin{array}{l}\text { Low; exposure area } \\
\text { small relative to } \\
\text { range; acclimation } \\
\text { likely due to high } \\
\text { boat traffic; gray } \\
\text { whale-boat collisions } \\
\text { not uncommon }\end{array}$ & $\begin{array}{l}\text { Calambokidis et } \\
\text { al. 2002, Sullivan } \\
\text { et al. 1983, Van } \\
\text { Waerebeek and } \\
\text { Leaper } 2008\end{array}$ \\
\hline & $\begin{array}{l}\text { Noise and } \\
\text { vibration }\end{array}$ & $\begin{array}{l}\text { Avoidance, masks } \\
\text { environmental cues, } \\
\text { communication } \\
\text { signals }\end{array}$ & $\begin{array}{l}\text { Low, sound travels } \\
\text { far, animals will hear } \\
\text { it outside project } \\
\text { area, but affects } \\
\text { small part of range }\end{array}$ & $\begin{array}{l}\text { Low, occurring } \\
\text { only during early } \\
\text { months of } 1 \text { to } 2 \\
\text { summers, }\end{array}$ & $\begin{array}{l}\text { Low, effect } \\
\text { influences small } \\
\text { part of large range }\end{array}$ & $\begin{array}{l}\text { Low; likely to } \\
\text { tolerate vessel noise } \\
\text { due to high levels of } \\
\text { background noise in } \\
\text { Puget Sound }\end{array}$ & $\begin{array}{l}\text { Richardson and } \\
\text { Wursig } 1997\end{array}$ \\
\hline & $\begin{array}{l}\text { Oil/chemical } \\
\text { release }\end{array}$ & $\begin{array}{l}\text { Ingestion, breathing } \\
\text { exhaust fumes, } \\
\text { accumulation of } \\
\text { toxins. }\end{array}$ & $\begin{array}{l}\text { Low, low volume of } \\
\text { fluids; could increase } \\
\text { contaminant levels for } \\
\text { PCFA whales }\end{array}$ & $\begin{array}{l}\text { Low, occurring } \\
\text { only during } 1 \text { to } \\
2 \text { summers }\end{array}$ & $\begin{array}{l}\text { Low, effects' } \\
\text { spatial extent small } \\
\text { compared to } \\
\text { animal's range }\end{array}$ & $\begin{array}{l}\text { Low; inputs } \\
\text { insignificant relative } \\
\text { to background levels } \\
\text { in Puget Sound }\end{array}$ & Ebbert et al. 2000 \\
\hline
\end{tabular}

\begin{tabular}{|c|c|c|}
\hline $\begin{array}{l}\text { Environmental Assessment for Siting Wave \& Tidal } \\
\text { Energy Projects }\end{array}$ & F-9 & $\begin{array}{r}\text { H. T. Harvey \& Associates } \\
24 \text { November } 2009\end{array}$ \\
\hline
\end{tabular}


Appendix F - Effects of Pilot Scale MCT SeaGen Project at the Tacoma Narrows Site

\begin{tabular}{|c|c|c|c|c|c|c|c|}
\hline Project activity & Project action & $\begin{array}{l}\text { Description of } \\
\text { action's effect on } \\
\text { indicator }\end{array}$ & $\begin{array}{l}\text { Spatial exposure to } \\
\text { indicator (low, med, } \\
\text { high) }\end{array}$ & $\begin{array}{l}\text { Temporal } \\
\text { exposure to } \\
\text { (low, med, high) }\end{array}$ & $\begin{array}{l}\text { Effect's overlap } \\
\text { with critical life } \\
\text { stage, behavior, } \\
\text { habitat, or } \\
\text { resource (low, } \\
\text { med, high) }\end{array}$ & $\begin{array}{l}\text { Overall risk to } \\
\text { indicator (low, med, } \\
\text { high, unknown) }\end{array}$ & Source(s) \\
\hline \multirow{3}{*}{$\begin{array}{l}\text { Decommissioning } \\
\text { and removal of } \\
\text { electrical collector } \\
\text { system, subsea } \\
\text { cable, mooring } \\
\text { cables, foundations } \\
\text { or anchors, and } \\
\text { devices }\end{array}$} & $\begin{array}{l}\text { Noise and } \\
\text { vibration }\end{array}$ & $\begin{array}{l}\text { Avoidance, masks } \\
\text { environmental cues, } \\
\text { communication } \\
\text { signals }\end{array}$ & $\begin{array}{l}\text { Low-med, sound } \\
\text { travels far, animals } \\
\text { will hear it outside of } \\
\text { project area, but } \\
\text { affects small part of } \\
\text { range }\end{array}$ & $\begin{array}{l}\text { Low, occurring } \\
\text { over } 1 \text { to } 2 \\
\text { summers }\end{array}$ & $\begin{array}{l}\text { Low, effects' } \\
\text { spatial extent small } \\
\text { compared to } \\
\text { animal's range }\end{array}$ & $\begin{array}{l}\text { Low; likely displaced } \\
\text { from by high sound } \\
\text { levels; displacement } \\
\text { area small portion of } \\
\text { range }\end{array}$ & $\begin{array}{l}\text { Richardson and } \\
\text { Wursig } 1997\end{array}$ \\
\hline & $\begin{array}{l}\text { Oil/chemical } \\
\text { release }\end{array}$ & $\begin{array}{l}\text { Ingestion, breathing } \\
\text { exhaust fumes, } \\
\text { accumulation of } \\
\text { toxins. }\end{array}$ & $\begin{array}{l}\text { Med, sig. potential } \\
\text { increase for exposure } \\
\text { from leakage of } \\
\text { chemicals }\end{array}$ & $\begin{array}{l}\text { Low, occurring } \\
\text { over } 1 \text { to } 2 \\
\text { summers }\end{array}$ & $\begin{array}{l}\text { Low, effects' } \\
\text { spatial extent small } \\
\text { compared to } \\
\text { animal's range }\end{array}$ & $\begin{array}{l}\text { Low; inputs } \\
\text { insignificant relative } \\
\text { to background levels } \\
\text { in Puget Sound }\end{array}$ & Ebbert et al. 2000 \\
\hline & $\begin{array}{l}\text { Construction } \\
\text { lights }\end{array}$ & Avoidance & $\begin{array}{l}\text { Low - not likely to } \\
\text { be attracted to } \\
\text { lighted areas }\end{array}$ & $\begin{array}{l}\text { Low, occurring } \\
\text { over } 1 \text { to } 2 \\
\text { summers }\end{array}$ & $\begin{array}{l}\text { Low, effects' } \\
\text { spatial extent small } \\
\text { compared to } \\
\text { animal's range }\end{array}$ & $\begin{array}{l}\text { Low; high levels of } \\
\text { shoreline } \\
\text { development, boat } \\
\text { traffic, industry in } \\
\text { Puget Sound }\end{array}$ & Todd et al. 2009 \\
\hline
\end{tabular}

\begin{tabular}{lrr}
\hline $\begin{array}{l}\text { Environmental Assessment for Siting Wave \& Tidal } \\
\text { Energy Projects }\end{array}$ & F-10 & H. T. Harvey \& Associates \\
24 November 2009
\end{tabular}


Appendix F - Effects of Pilot Scale MCT SeaGen Project at the Tacoma Narrows Site

Potential effects on Northern sea lion due to Pilot Scale MCT Seagen project at Tacoma Narrows site

\begin{tabular}{|c|c|c|c|c|c|c|c|}
\hline Project activity & Project action & $\begin{array}{l}\text { Description of } \\
\text { action's effect on } \\
\text { indicator }\end{array}$ & $\begin{array}{l}\text { Spatial exposure to } \\
\text { indicator (low, med, } \\
\text { high) }\end{array}$ & $\begin{array}{l}\text { Temporal } \\
\text { exposure to } \\
\text { indicator (low, } \\
\text { med, high) }\end{array}$ & $\begin{array}{l}\text { Effect's overlap } \\
\text { with critical life } \\
\text { stage, behavior, } \\
\text { habitat, or } \\
\text { resource (low, } \\
\text { med, high) }\end{array}$ & $\begin{array}{l}\text { Overall risk to } \\
\text { indicator (low, } \\
\text { med, high, } \\
\text { unknown) }\end{array}$ & Source(s) \\
\hline \multicolumn{8}{|c|}{ Construction } \\
\hline \multirow{3}{*}{$\begin{array}{l}\text { Boat traffic, } \\
\text { assumed to occur } \\
\text { day and night }\end{array}$} & Direct impact & Collision injuries & $\begin{array}{l}\text { Low-med; increase in } \\
\text { boat traffic between } \\
\text { project site and dock }\end{array}$ & $\begin{array}{l}\text { Low, occurring } \\
\text { over } \sim 2 \text { months }\end{array}$ & $\begin{array}{l}\text { Med; males of the } \\
\text { species only } \\
\text { normally present } \\
\text { during non-summer } \\
\text { months }\end{array}$ & $\begin{array}{l}\text { Med; increase in } \\
\text { boat traffic } \\
\text { compared to } \\
\text { existing ( 235,000 } \\
\text { vessels/yr) }\end{array}$ & $\begin{array}{l}\text { Calambokidis } \\
\text { and Baird 1994; } \\
\text { Stroud and Roffe } \\
\text { 1979, USCG } \\
\text { [date unknown] }\end{array}$ \\
\hline & $\begin{array}{l}\text { Noise and } \\
\text { vibration }\end{array}$ & $\begin{array}{l}\text { Reduction of hearing } \\
\text { sensitivity (Temporary } \\
\text { Threshold Shift), could } \\
\text { increase predation }\end{array}$ & $\begin{array}{l}\text { Low-med, increase in } \\
\text { boat traffic between } \\
\text { project site and dock; } \\
\text { sound potentially } \\
\text { detectable }(\sim 3 \mathrm{~km})\end{array}$ & $\begin{array}{l}\text { Low, occurring } \\
\text { over } 2 \text { months }\end{array}$ & $\begin{array}{l}\text { Low-med; related } \\
\text { to foraging, travel } \\
\text { and predation }\end{array}$ & $\begin{array}{l}\text { Low; noise not } \\
\text { sig. more than } \\
\text { background; } \\
\text { not likely a threat } \\
\text { unless animals } \\
\text { approach vessels }\end{array}$ & $\begin{array}{l}\text { Kastak et al. } \\
\text { 2005, Tougaard } \\
\text { et al. } 2009\end{array}$ \\
\hline & $\begin{array}{l}\text { Oil/chemical } \\
\text { release }\end{array}$ & $\begin{array}{l}\text { Ingestion, fur fouling, } \\
\text { breathing exhaust } \\
\text { fumes, accumulation of } \\
\text { toxins. }\end{array}$ & $\begin{array}{l}\text { Med, significant } \\
\text { increase in existing } \\
\text { traffic but volume of } \\
\text { fluids released low }\end{array}$ & $\begin{array}{l}\text { Low, occurring } \\
\text { over } 2 \text { months }\end{array}$ & $\begin{array}{l}\text { Low; inputs } \\
\text { insignificant relative } \\
\text { to background levels } \\
\text { in Puget Sound }\end{array}$ & $\begin{array}{l}\text { Low; low volume } \\
\text { of contaminants } \\
\text { expected }\end{array}$ & $\begin{array}{l}\text { Ebbert et al. } \\
\text { 2000, Hall } 2003 \\
\text { \& refs therein }\end{array}$ \\
\hline \multirow{3}{*}{$\begin{array}{l}\text { Construction and } \\
\text { installation of } \\
\text { electrical } \\
\text { collector system, } \\
\text { mooring cables, } \\
\text { anchors or } \\
\text { foundations, and } \\
\text { devices }\end{array}$} & $\begin{array}{l}\text { Noise and } \\
\text { vibration }\end{array}$ & $\begin{array}{l}\text { Avoidance of area, } \\
\text { affects foraging and } \\
\text { travel, haul out; TTS } \\
\text { could increase predation }\end{array}$ & $\begin{array}{l}\text { Med; increase in } \\
\text { industrial activity, pre- } \\
\text { existing noise levels } \\
\text { expected to be high }\end{array}$ & $\begin{array}{l}\text { Low, occurring } \\
\text { over } \sim 2 \text { months }\end{array}$ & $\begin{array}{l}\text { Low due to } \\
\text { foraging and } \\
\text { traveling near } \\
\text { construction site }\end{array}$ & $\begin{array}{l}\text { Low; could impact } \\
\text { hearing if sea lions } \\
\text { near; high ambient } \\
\text { noise levels }\end{array}$ & $\begin{array}{l}\text { Kastak et al. } \\
\text { 2005, Tougaard } \\
\text { et al. } 2009\end{array}$ \\
\hline & $\begin{array}{l}\text { Oil/chemical } \\
\text { release }\end{array}$ & $\begin{array}{l}\text { Ingestion, fur fouling, } \\
\text { breathing exhaust } \\
\text { fumes, accumulation of } \\
\text { toxins. }\end{array}$ & $\begin{array}{l}\text { Med; significant } \\
\text { increase in use of } \\
\text { chemicals, volume of } \\
\text { fluids released }\end{array}$ & $\begin{array}{l}\text { Low, occurring } \\
\text { over } \sim 2 \text { months }\end{array}$ & $\begin{array}{l}\text { Low; inputs } \\
\text { insignificant relative } \\
\text { to background levels } \\
\text { in Puget Sound }\end{array}$ & $\begin{array}{l}\text { Low; low volume } \\
\text { of contaminants } \\
\text { expected }\end{array}$ & $\begin{array}{l}\text { Ebbert et al. } \\
\text { 2000, Hall } 2003 \\
\text { \& refs therein }\end{array}$ \\
\hline & $\begin{array}{l}\text { Construction } \\
\text { lights (bright } \\
\text { deck lights and } \\
\text { spotlights) }\end{array}$ & $\begin{array}{l}\text { May be attracted } \\
\text { increasing other impacts, } \\
\text { could enhance foraging } \\
\text { on prey attracted to light }\end{array}$ & Med to high & $\begin{array}{l}\text { Low, occurring } \\
\text { over } \sim 2 \text { months }\end{array}$ & $\begin{array}{l}\text { Low; possibly } \\
\text { affecting travel and } \\
\text { foraging at night }\end{array}$ & $\begin{array}{l}\text { Low; exposure low } \\
\text { relative to range; } \\
\text { short duration of } \\
\text { construction }\end{array}$ & $\begin{array}{l}\text { Yurk and Trites } \\
2000\end{array}$ \\
\hline $\begin{array}{l}\text { Directional } \\
\text { drilling, and } \\
\text { laying cable } \\
\text { under/on seabed }\end{array}$ & $\begin{array}{l}\text { Noise and } \\
\text { vibration }\end{array}$ & $\begin{array}{l}\text { Avoidance of area, } \\
\text { affects foraging and } \\
\text { travel, resting onshore } \\
\text { in Bay }\end{array}$ & $\begin{array}{l}\text { Low, activities focused } \\
\text { nearshore; localized } \\
\text { around support vessels } \\
\text { when offshore }\end{array}$ & $\begin{array}{l}\text { Low, occurring } \\
\text { over } \sim 2 \text { months }\end{array}$ & $\begin{array}{l}\text { Low, activities } \\
\text { focused nearshore }\end{array}$ & $\begin{array}{l}\text { Low; increase in } \\
\text { noise but high } \\
\text { ambient noise } \\
\text { levels }\end{array}$ & $\begin{array}{l}\text { Kastak et al. } \\
\text { 2005, Tougaard } \\
\text { et al. } 2009\end{array}$ \\
\hline
\end{tabular}


Appendix F - Effects of Pilot Scale MCT SeaGen Project at the Tacoma Narrows Site

\begin{tabular}{|c|c|c|c|c|c|c|c|}
\hline Project activity & Project action & $\begin{array}{l}\text { Description of } \\
\text { action's effect on } \\
\text { indicator }\end{array}$ & $\begin{array}{l}\text { Spatial exposure to } \\
\text { indicator (low, med, } \\
\text { high) }\end{array}$ & $\begin{array}{l}\text { Temporal } \\
\text { exposure to } \\
\text { indicator (low, } \\
\text { med, high) }\end{array}$ & $\begin{array}{l}\text { Effect's overlap } \\
\text { with critical life } \\
\text { stage, behavior, } \\
\text { habitat, or } \\
\text { resource (low, } \\
\text { med, high) }\end{array}$ & $\begin{array}{l}\text { Overall risk to } \\
\text { indicator (low, } \\
\text { med, high, } \\
\text { unknown) }\end{array}$ & Source(s) \\
\hline \multirow{3}{*}{ Boat traffic. } & Direct impact & Collision injuries & $\begin{array}{l}\text { Medium, increase in } \\
\text { existing traffic } \\
\text { (especially in bay and } \\
\text { jetties) }\end{array}$ & $\begin{array}{l}\text { Medium, } \\
\text { occurring during } \\
\text { summer for life of } \\
\text { the project }\end{array}$ & $\begin{array}{l}\text { Low; low if during } \\
\text { summer when } \\
\text { animals are rare; } \\
\text { med if during non- } \\
\text { summer }\end{array}$ & $\begin{array}{l}\text { Low; boat traffic } \\
\text { not significantly } \\
\text { more than existing } \\
(\sim 235,000 \\
\text { vessels/yr) }\end{array}$ & $\begin{array}{l}\text { Calambokidis } \\
\text { and Baird 1994, } \\
\text { Stroud and Roffe } \\
\text { 1979, USCG } \\
\text { [date unknown] }\end{array}$ \\
\hline & $\begin{array}{l}\text { Oil/chemical } \\
\text { release }\end{array}$ & $\begin{array}{l}\text { Ingestion, fur fouling, } \\
\text { breathing exhaust } \\
\text { fumes, accumulation of } \\
\text { toxins. }\end{array}$ & $\begin{array}{l}\text { Low, slight increase in } \\
\text { existing traffic but } \\
\text { volume of fluids } \\
\text { released low }\end{array}$ & $\begin{array}{l}\text { Med, maintenance } \\
\text { occurs over life of } \\
\text { project during } \\
\text { summer }\end{array}$ & $\begin{array}{l}\text { Low; inputs } \\
\text { insignificant } \\
\text { relative to } \\
\text { background levels }\end{array}$ & $\begin{array}{l}\text { Low; low volume } \\
\text { of contaminants } \\
\text { expected }\end{array}$ & $\begin{array}{l}\text { Ebbert et al. } \\
\text { 2000, Hall } 2003 \\
\text { \& refs therein }\end{array}$ \\
\hline & $\begin{array}{l}\text { Noise and } \\
\text { vibration }\end{array}$ & $\begin{array}{l}\text { TTS, resulting in } \\
\text { potential increased in } \\
\text { predation }\end{array}$ & $\begin{array}{l}\text { Med, noise will be } \\
\text { heard past project area } \\
(\sim 3 \mathrm{~km}) \text { but increase in } \\
\text { traffic slight. }\end{array}$ & $\begin{array}{l}\text { High, occurs } \\
\text { during summer } \\
\text { months over } \\
\text { project duration }\end{array}$ & $\begin{array}{l}\text { Low for adults/ } \\
\text { subadults effects on } \\
\text { foraging and travel; } \\
\text { med for juv., may } \\
\text { increase predation }\end{array}$ & $\begin{array}{l}\text { Low; not likely a } \\
\text { threat unless } \\
\text { animals approach } \\
\text { vessels }\end{array}$ & $\begin{array}{l}\text { Kastak et al. } \\
\text { 2005, Tougaard } \\
\text { et al. 2009, } \\
\text { USCG [date } \\
\text { unknown] }\end{array}$ \\
\hline \multirow[t]{2}{*}{$\begin{array}{l}\text { Operation of } \\
\text { turbines or other } \\
\text { moving parts of } \\
\text { devices }\end{array}$} & Direct impact & $\begin{array}{l}\text { Direct injury or } \\
\text { mortality; secondary } \\
\text { mortality due to } \\
\text { infection, loss of } \\
\text { mobility, predation }\end{array}$ & $\begin{array}{l}\text { Low, the areas of } \\
\text { movement are small } \\
(\sim 1 \%) \text { compared to } \\
\text { channel area } \\
\text { (approximated channel } \\
\text { width x average depth) }\end{array}$ & $\begin{array}{l}\text { High, the } \\
\text { movement occurs } \\
\text { over the life of the } \\
\text { project }\end{array}$ & $\begin{array}{l}\text { High for any } \\
\text { animals foraging or } \\
\text { traveling in Tacoma } \\
\text { narrows }\end{array}$ & $\begin{array}{l}\text { High; sig. risk of } \\
\text { rotor strike- may } \\
\text { be less detectable } \\
\text { than ship rotor } \\
\text { due to slower } \\
\text { speed; strike at } 12 \\
\text { m/s will cause } \\
\text { serious trauma }\end{array}$ & $\begin{array}{l}\text { Dadswell and } \\
\text { Rulifson 1994, } \\
\text { Wilson et al. } \\
2007\end{array}$ \\
\hline & $\begin{array}{l}\text { Noise and } \\
\text { vibration }\end{array}$ & $\begin{array}{l}\text { Reduction of hearing } \\
\text { sensitivity could } \\
\text { increase predation, } \\
\text { avoidance, mask of } \\
\text { envir. cues }\end{array}$ & $\begin{array}{l}\text { Likely to extend beyond } \\
\text { project area; other } \\
\text { pinnipeds show auditory } \\
\text { response at } \sim 3 \mathrm{~km} \text {. }\end{array}$ & $\begin{array}{l}\text { High, if effect } \\
\text { occurs, movement } \\
\text { occurs over the } \\
\text { life of the project }\end{array}$ & $\begin{array}{l}\text { Low; foraging and } \\
\text { traveling in project } \\
\text { area; sounds not } \\
\text { expected to result } \\
\text { in sig. increase } \\
\end{array}$ & $\begin{array}{l}\text { Unknown; not } \\
\text { likely a threat } \\
\text { unless animals } \\
\text { close to structures }\end{array}$ & $\begin{array}{l}\text { Kastak et al. } \\
\text { 2005, Koschinski } \\
\text { et al. 2003, } \\
\text { Tougaard et al. } \\
\text { 2009 } \\
\end{array}$ \\
\hline $\begin{array}{l}\text { Structures in } \\
\text { water column and } \\
\text { on seabed, such as } \\
\text { devices and } \\
\text { moorings and } \\
\text { footings }\end{array}$ & Structure & $\begin{array}{l}\text { Entanglement with } \\
\text { derelict gear caught on } \\
\text { devices; artificial reef } \\
\text { effects could attract fish } \\
\text { and species }\end{array}$ & $\begin{array}{l}\text { Low, currently not } \\
\text { much structure nearby } \\
\text { but home ranges are } \\
\text { large. }\end{array}$ & $\begin{array}{l}\text { High, structure } \\
\text { present over life } \\
\text { of project }\end{array}$ & $\begin{array}{l}\text { Med, possibly } \\
\text { beneficial effect by } \\
\text { increasing forage }\end{array}$ & $\begin{array}{l}\text { Unknown; } \\
\text { structure easily } \\
\text { avoided but could } \\
\text { lure sea lions into } \\
\text { rotor path in } \\
\text { pursuit of prey }\end{array}$ & $\begin{array}{l}\text { Schusterman and } \\
\text { Balliet 1970, } \\
\text { Todd et al. } 2009\end{array}$ \\
\hline $\begin{array}{l}\text { Electricity } \\
\text { conduction } \\
\text { through cable }\end{array}$ & EMF & Unknown & $\begin{array}{l}\text { Unknown, cable } \\
\text { shielding provides some } \\
\text { attenuation }\end{array}$ & $\begin{array}{l}\text { High if an effect, } \\
\text { it would occur } \\
\text { over life of the } \\
\text { project }\end{array}$ & $\begin{array}{l}\text { Unknown; any } \\
\text { effects would likely } \\
\text { impact all age } \\
\text { classes }\end{array}$ & $\begin{array}{l}\text { Unknown; High } \\
\text { uncertainty about } \\
\text { effects on marine } \\
\text { mammals }\end{array}$ & $\begin{array}{l}\text { Boehlert et al. } \\
2008\end{array}$ \\
\hline
\end{tabular}

\begin{tabular}{|c|c|c|}
\hline $\begin{array}{l}\text { Environmental Assessment for Siting Wave \& Tidal } \\
\text { Energy Projects }\end{array}$ & F-12 & $\begin{array}{r}\text { H. T. Harvey \& Associates } \\
24 \text { November } 2009\end{array}$ \\
\hline
\end{tabular}


Appendix F - Effects of Pilot Scale MCT SeaGen Project at the Tacoma Narrows Site

\begin{tabular}{|c|c|c|c|c|c|c|c|}
\hline Project activity & Project action & $\begin{array}{l}\text { Description of } \\
\text { action's effect on } \\
\text { indicator }\end{array}$ & $\begin{array}{l}\text { Spatial exposure to } \\
\text { indicator (low, med, } \\
\text { high) }\end{array}$ & $\begin{array}{l}\text { Temporal } \\
\text { exposure to } \\
\text { indicator (low, } \\
\text { med, high) }\end{array}$ & $\begin{array}{l}\text { Effect's overlap } \\
\text { with critical life } \\
\text { stage, behavior, } \\
\text { habitat, or } \\
\text { resource (low, } \\
\text { med, high) } \\
\end{array}$ & $\begin{array}{l}\text { Overall risk to } \\
\text { indicator (low, } \\
\text { med, high, } \\
\text { unknown) }\end{array}$ & Source(s) \\
\hline \multirow[b]{2}{*}{$\begin{array}{l}\text { Structures on } \\
\text { water's surface }\end{array}$} & Structure & $\begin{array}{l}\text { Could provide haul out } \\
\text { structure; attract sea } \\
\text { lions and increase } \\
\text { potential impacts }\end{array}$ & $\begin{array}{l}\text { Limited to above } \\
\text { surface structures }\end{array}$ & $\begin{array}{l}\text { High, structure } \\
\text { present over life } \\
\text { of project }\end{array}$ & $\begin{array}{l}\text { Low; structure not } \\
\text { suitable for haul out }\end{array}$ & $\begin{array}{l}\text { Low; risk if } \\
\text { animals attracted } \\
\text { to structure in } \\
\text { search of haul out }\end{array}$ & $\begin{array}{l}\text { Jeffries et al. } \\
2000\end{array}$ \\
\hline & Navigation lights & $\begin{array}{l}\text { Visual disorientation } \\
\text { could lead to collision, } \\
\text { could also enhance } \\
\text { foraging on species } \\
\text { attracted to light }\end{array}$ & $\begin{array}{l}\text { Low, lights associated } \\
\text { with devices and buoys } \\
\text { are low intensity, } \\
\text { affecting a small area }\end{array}$ & $\begin{array}{l}\text { High, lights would } \\
\text { be on over the life } \\
\text { of the project }\end{array}$ & $\begin{array}{l}\text { Low; not expected } \\
\text { to effect nocturnal } \\
\text { foraging due to } \\
\text { high pre-existing } \\
\text { light levels }\end{array}$ & $\begin{array}{l}\text { Low; ambient } \\
\text { light levels high; } \\
\text { nav lights dim and } \\
\text { similar to those on } \\
\text { other structures } \\
\text { and vessels } \\
\end{array}$ & $\begin{array}{l}\text { USCG [date } \\
\text { unknown] }\end{array}$ \\
\hline \multicolumn{8}{|c|}{ Decommissioning } \\
\hline \multirow{3}{*}{ Boat traffic } & Direct impact & Collision injuries & $\begin{array}{l}\text { Low-med; moderate } \\
\text { relative increase in boat } \\
\text { traffic between project } \\
\text { site and dock }\end{array}$ & $\begin{array}{l}\text { Low, occurring } \\
\text { over } \sim 2 \text { months }\end{array}$ & $\begin{array}{l}\text { Low-med; males } \\
\text { only normally } \\
\text { present during non- } \\
\text { summer }\end{array}$ & $\begin{array}{l}\text { Low; increase in } \\
\text { boat traffic than } \\
\text { existing ( 235,000 } \\
\text { vessels/yr) }\end{array}$ & $\begin{array}{l}\text { Calambokidis } \\
\text { and Baird 1994, } \\
\text { Stroud and Roffe } \\
\text { 1979, USCG } \\
\text { [date unknown] }\end{array}$ \\
\hline & $\begin{array}{l}\text { Noise and } \\
\text { vibration }\end{array}$ & $\begin{array}{l}\text { Reduction of hearing } \\
\text { sensitivity (TTS); may } \\
\text { increase predation }\end{array}$ & $\begin{array}{l}\text { Low-med, moderate } \\
\text { increase in boat traffic; } \\
\text { sound may be } \\
\text { detectable }(\sim 3 \mathrm{~km})\end{array}$ & $\begin{array}{l}\text { Low, occurring } \\
\text { over } \sim 2 \text { months }\end{array}$ & $\begin{array}{l}\text { Low; related to } \\
\text { foraging, travel and } \\
\text { predation }\end{array}$ & $\begin{array}{l}\text { Low; noise not } \\
\text { more than } \\
\text { background }\end{array}$ & $\begin{array}{l}\text { Kastak et al. 2005, } \\
\text { Tougaard et al. } \\
\text { 2009, USCG [date } \\
\text { unknown] }\end{array}$ \\
\hline & $\begin{array}{l}\text { Oil/chemical } \\
\text { release, assumes } \\
\text { no catastrophic } \\
\text { spills. }\end{array}$ & $\begin{array}{l}\text { Ingestion, fur fouling, } \\
\text { breathing exhaust } \\
\text { fumes, accumulation of } \\
\text { toxins. }\end{array}$ & $\begin{array}{l}\text { Med, significant } \\
\text { increase existing traffic } \\
\text { but the volume of fluids } \\
\text { released low }\end{array}$ & $\begin{array}{l}\text { Low, occurring } \\
\text { over } \sim 2 \text { months }\end{array}$ & $\begin{array}{l}\text { Low; inputs } \\
\text { insignificant } \\
\text { relative to } \\
\text { background levels }\end{array}$ & $\begin{array}{l}\text { Low; low volume } \\
\text { of contaminants } \\
\text { expected }\end{array}$ & $\begin{array}{l}\text { Ebbert et al. } \\
\text { 2000, Hall } 2003 \\
\text { \& refs therein }\end{array}$ \\
\hline \multirow{3}{*}{$\begin{array}{l}\text { Decommissioning } \\
\text { and removal of } \\
\text { electrical } \\
\text { collector system, } \\
\text { subsea cable, } \\
\text { mooring cables, } \\
\text { foundations or } \\
\text { anchors, and } \\
\text { devices }\end{array}$} & $\begin{array}{l}\text { Noise and } \\
\text { vibration }\end{array}$ & $\begin{array}{l}\text { Avoidance of area, } \\
\text { affects foraging, travel, } \\
\text { resting onshore; could } \\
\text { increase predation }\end{array}$ & $\begin{array}{l}\text { Med; increase in } \\
\text { industrial activity, pre- } \\
\text { existing noise levels } \\
\text { expected to be high }\end{array}$ & $\begin{array}{l}\text { Low, occurring } \\
\text { over } \sim 2 \text { months }\end{array}$ & $\begin{array}{l}\text { Low due to } \\
\text { foraging and } \\
\text { traveling near } \\
\text { construction site } \\
\end{array}$ & $\begin{array}{l}\text { Low; could } \\
\text { impact hearing; } \\
\text { high ambient } \\
\text { noise levels }\end{array}$ & $\begin{array}{l}\text { Kastak et al. } \\
\text { 2005, Tougaard } \\
\text { et al. } 2009\end{array}$ \\
\hline & $\begin{array}{l}\text { Oil/chemical } \\
\text { release, assumes } \\
\text { no catastrophic } \\
\text { spills. }\end{array}$ & $\begin{array}{l}\text { Ingestion, fur fouling, } \\
\text { breathing exhaust } \\
\text { fumes, accumulation of } \\
\text { toxins. }\end{array}$ & $\begin{array}{l}\text { Med; significant } \\
\text { increase in use of } \\
\text { chemicals, volume of } \\
\text { fluids released low }\end{array}$ & $\begin{array}{l}\text { Low, occurring } \\
\text { over } \sim 2 \text { months }\end{array}$ & $\begin{array}{l}\text { Low; inputs } \\
\text { insignificant } \\
\text { relative to } \\
\text { background levels }\end{array}$ & $\begin{array}{l}\text { Low; low volume } \\
\text { of contaminants } \\
\text { expected }\end{array}$ & $\begin{array}{l}\text { Ebbert et al. } \\
\text { 2000, Hall } 2003 \\
\text { \& refs therein }\end{array}$ \\
\hline & $\begin{array}{l}\text { Deconstruction } \\
\text { lights }\end{array}$ & $\begin{array}{l}\text { If attracted could } \\
\text { increase impacts, } \\
\text { enhance foraging on } \\
\text { prey attracted to light }\end{array}$ & Med to high & $\begin{array}{l}\text { Low, occurring } \\
\text { over } \sim 2 \text { months }\end{array}$ & $\begin{array}{l}\text { Low; possibly } \\
\text { affecting travel and } \\
\text { foraging at night }\end{array}$ & $\begin{array}{l}\text { Low; exposure } \\
\text { low relative to } \\
\text { range; short } \\
\text { duration }\end{array}$ & $\begin{array}{l}\text { Yurk and Trites } \\
2000\end{array}$ \\
\hline
\end{tabular}

\begin{tabular}{|c|c|c|}
\hline $\begin{array}{l}\text { Environmental Assessment for Siting Wave \& Tidal } \\
\text { Energy Projects }\end{array}$ & F-13 & $\begin{array}{r}\text { H. T. Harvey \& Associates } \\
24 \text { November } 2009\end{array}$ \\
\hline
\end{tabular}


Appendix F - Effects of Pilot Scale MCT SeaGen Project at the Tacoma Narrows Site

Potential effects on harbor seal due to Pilot Scale MCT SeaGen project at Tacoma Narrows

\begin{tabular}{|c|c|c|c|c|c|c|c|}
\hline Project activity & Project action & $\begin{array}{l}\text { Description of } \\
\text { action's effect on } \\
\text { indicator }\end{array}$ & $\begin{array}{l}\text { Spatial exposure to } \\
\text { indicator (low, med, } \\
\text { high) }\end{array}$ & $\begin{array}{l}\text { Temporal } \\
\text { exposure to } \\
\text { indicator } \\
\text { (low, med, } \\
\text { high) }\end{array}$ & $\begin{array}{l}\text { Effect's overlap with } \\
\text { critical life stage, } \\
\text { behavior, habitat or } \\
\text { resource (low, med, } \\
\text { high) }\end{array}$ & $\begin{array}{l}\text { Overall risk to } \\
\text { indicator (low, med, } \\
\text { high unknown) }\end{array}$ & Source(s) \\
\hline \multicolumn{8}{|c|}{ Construction } \\
\hline \multirow{3}{*}{$\begin{array}{l}\text { Boat traffic, assumed to } \\
\text { occur day and night }\end{array}$} & Direct impact & Collision injuries & $\begin{array}{l}\text { Med; low to } \\
\text { moderate increase in } \\
\text { high levels of pre- } \\
\text { existing boat traffic }\end{array}$ & $\begin{array}{l}\text { Low, } \\
\text { occurring over } \\
1 \text { to } 2 \\
\text { summers }\end{array}$ & $\begin{array}{l}\text { Low for adults med } \\
\text { for pups due to } \\
\text { foraging and travel in } \\
\text { construction zones; } \\
\text { acclimation likely due } \\
\text { to high boat traffic in } \\
\text { Puget Sound }\end{array}$ & $\begin{array}{l}\text { Low; increase in boat } \\
\text { traffic; vessels } \\
\text { similar to existing } \\
\text { and do not represent } \\
\text { new types of impacts }\end{array}$ & $\begin{array}{l}\text { Stroud and } \\
\text { Roffe } 1979\end{array}$ \\
\hline & $\begin{array}{l}\text { Noise and } \\
\text { vibration }\end{array}$ & $\begin{array}{l}\text { Avoidance, masks } \\
\text { environmental cues. } \\
\text { Reduction of } \\
\text { hearing sensitivity } \\
\text { (TTS) could } \\
\text { increase predation }\end{array}$ & $\begin{array}{l}\text { Med; moderate } \\
\text { increase in boat } \\
\text { traffic, pre-existing } \\
\text { ambient noise levels } \\
\text { expected to be high }\end{array}$ & $\begin{array}{l}\text { Low, } \\
\text { occurring over } \\
1 \text { to } 2 \\
\text { summers }\end{array}$ & $\begin{array}{l}\text { Low-med for adults } \\
\text { med for pups; see } \\
\text { above }\end{array}$ & $\begin{array}{l}\text { Low; similar species } \\
\text { (Phoca hispida) } \\
\text { show considerable } \\
\text { tolerance to similar } \\
\text { types of noise }\end{array}$ & $\begin{array}{l}\text { Blackwell et } \\
\text { al. 2004, } \\
\text { Kastak et al. } \\
\text { 2005, } \\
\text { Tougaard et al. } \\
2009\end{array}$ \\
\hline & $\begin{array}{l}\text { Oil/chemical } \\
\text { release }\end{array}$ & $\begin{array}{l}\text { Ingestion, fur } \\
\text { fouling, breathing } \\
\text { exhaust fumes, } \\
\text { accumulation of } \\
\text { toxins. Assumes no } \\
\text { catastrophic spills. }\end{array}$ & $\begin{array}{l}\text { Low; increase in } \\
\text { existing traffic but } \\
\text { the volume of fluids } \\
\text { released low and pre- } \\
\text { existing ambient } \\
\text { levels high }\end{array}$ & $\begin{array}{l}\text { Low, } \\
\text { occurring over } \\
1 \text { to } 2 \\
\text { summers }\end{array}$ & $\begin{array}{l}\text { Low-med all age } \\
\text { classes; inputs } \\
\text { insignificant relative } \\
\text { to background levels } \\
\text { in Puget Sound }\end{array}$ & $\begin{array}{l}\text { Low; volume of } \\
\text { contaminants } \\
\text { expected to be low } \\
\text { and not significantly } \\
\text { greater than } \\
\text { background levels }\end{array}$ & $\begin{array}{l}\text { Ebbert et al. } \\
2000\end{array}$ \\
\hline \multirow[t]{2}{*}{$\begin{array}{l}\text { Construction and } \\
\text { installation of electrical } \\
\text { collector system, } \\
\text { mooring cables, } \\
\text { anchors or foundations, } \\
\text { and devices }\end{array}$} & $\begin{array}{l}\text { Noise and } \\
\text { vibration }\end{array}$ & $\begin{array}{l}\text { Avoidance of area, } \\
\text { masking of } \\
\text { environmental cues, } \\
\text { TTS possible which } \\
\text { could increase } \\
\text { predation. }\end{array}$ & $\begin{array}{l}\text { Med; moderate } \\
\text { overall increase in } \\
\text { industrial activity, } \\
\text { pre-existing ambient } \\
\text { noise levels expected } \\
\text { to be high }\end{array}$ & $\begin{array}{l}\text { Low, } \\
\text { occurring over } \\
1 \text { to } 2 \\
\text { summers }\end{array}$ & $\begin{array}{l}\text { Low for adults; med } \\
\text { for pups due to } \\
\text { foraging and travel in } \\
\text { construction zone; } \\
\text { acclimation likely due } \\
\text { to high industrial } \\
\text { noise in Puget Sound }\end{array}$ & $\begin{array}{l}\text { Low; similar species } \\
\text { (Phoca hispida) } \\
\text { show considerable } \\
\text { tolerance to similar } \\
\text { types of noise }\end{array}$ & $\begin{array}{l}\text { Blackwell et } \\
\text { al. 2004, } \\
\text { Kastak et al. } \\
\text { 2005, } \\
\text { Tougaard et al. } \\
2009\end{array}$ \\
\hline & $\begin{array}{l}\text { Oil/chemical } \\
\text { release }\end{array}$ & $\begin{array}{l}\text { Ingestion, fur } \\
\text { fouling, breathing } \\
\text { exhaust fumes, } \\
\text { accumulation of } \\
\text { toxins. Assumes no } \\
\text { catastrophic spills. }\end{array}$ & $\begin{array}{l}\text { Med, significant } \\
\text { increase in use of } \\
\text { chemicals; low } \\
\text { volume of fluids } \\
\text { released }\end{array}$ & $\begin{array}{l}\text { Low, } \\
\text { occurring over } \\
1 \text { to } 2 \\
\text { summers }\end{array}$ & Low & $\begin{array}{l}\text { Low; volume of } \\
\text { contaminants } \\
\text { expected to be low } \\
\text { and not significantly } \\
\text { greater then existing } \\
\text { background levels }\end{array}$ & $\begin{array}{l}\text { Ebbert et al. } \\
2000\end{array}$ \\
\hline
\end{tabular}

\begin{tabular}{lrr}
\hline $\begin{array}{l}\text { Environmental Assessment for Siting Wave \& Tidal } \\
\text { Energy Projects }\end{array}$ & F-14 & H. T. Harvey \& Associates \\
24 November 2009
\end{tabular}


Appendix F - Effects of Pilot Scale MCT SeaGen Project at the Tacoma Narrows Site

\begin{tabular}{|c|c|c|c|c|c|c|c|}
\hline Project activity & Project action & $\begin{array}{l}\text { Description of } \\
\text { action's effect on } \\
\text { indicator }\end{array}$ & $\begin{array}{l}\text { Spatial exposure to } \\
\text { indicator (low, med, } \\
\text { high) }\end{array}$ & $\begin{array}{l}\text { Temporal } \\
\text { exposure to } \\
\text { indicator } \\
\text { (low, med, } \\
\text { high) } \\
\end{array}$ & $\begin{array}{l}\text { Effect's overlap with } \\
\text { critical life stage, } \\
\text { behavior, habitat or } \\
\text { resource (low, med, } \\
\text { high) }\end{array}$ & $\begin{array}{l}\text { Overall risk to } \\
\text { indicator (low, med, } \\
\text { high unknown) }\end{array}$ & Source(s) \\
\hline & $\begin{array}{l}\text { Construction } \\
\text { lights, vessel } \\
\text { deck lights and } \\
\text { spotlights. }\end{array}$ & $\begin{array}{l}\text { Visual } \\
\text { disorientation could } \\
\text { lead to collision, but } \\
\text { could also enhance } \\
\text { foraging on species } \\
\text { attracted to light }\end{array}$ & $\begin{array}{l}\text { Low; increase in high } \\
\text { levels of boat traffic, } \\
\text { deck lights illuminate } \\
\text { only a small area }\end{array}$ & $\begin{array}{l}\text { Low, } \\
\text { occurring over } \\
1 \text { to } 2 \\
\text { summers }\end{array}$ & $\begin{array}{l}\text { Low all age classes } \\
\text { due to shoreline } \\
\text { development, boat } \\
\text { traffic, and industrial } \\
\text { activity in Puget } \\
\text { Sound }\end{array}$ & $\begin{array}{l}\text { Low; vessels and } \\
\text { construction } \\
\text { activities similar to } \\
\text { existing and do not } \\
\text { represent new types } \\
\text { of impact }\end{array}$ & $\begin{array}{l}\text { Yurk and } \\
\text { Trites } 2000\end{array}$ \\
\hline $\begin{array}{l}\text { Directional drilling, } \\
\text { and laying cable } \\
\text { under/on seabed }\end{array}$ & $\begin{array}{l}\text { Noise and } \\
\text { vibration }\end{array}$ & $\begin{array}{l}\text { Avoidance of area, } \\
\text { masks environmental } \\
\text { cues, TTS possible } \\
\text { which could increase } \\
\text { predation }\end{array}$ & $\begin{array}{l}\text { Med, seals likely to } \\
\text { avoid areas occupied } \\
\text { by cable and support } \\
\text { vessels }\end{array}$ & $\begin{array}{l}\text { Low, } \\
\text { occurring over } \\
1 \text { summer }\end{array}$ & $\begin{array}{l}\text { Low all age classes; } \\
\text { avoidance of } \\
\text { construction area } \\
\text { occupied by cable } \\
\text { ship and support } \\
\text { vessels }\end{array}$ & $\begin{array}{l}\text { Low; activities } \\
\text { highly localized and } \\
\text { short term; seals } \\
\text { likely to acclimate } \\
\text { and tolerate noise }\end{array}$ & $\begin{array}{l}\text { Kastak et al. } \\
2005 \text {, } \\
\text { Tougaard et al. } \\
2009\end{array}$ \\
\hline \multicolumn{8}{|c|}{ Operation and Maintenance } \\
\hline \multirow{3}{*}{ Boat traffic } & Direct impact & Collision injuries & $\begin{array}{l}\text { Med; low to } \\
\text { moderate increase in } \\
\text { already high levels of } \\
\text { pre-existing boat } \\
\text { traffic }\end{array}$ & $\begin{array}{l}\text { Med, routine } \\
\text { maintenance } \\
\text { occurs over } \\
\text { life of the } \\
\text { project } \\
\end{array}$ & $\begin{array}{l}\text { Low; boat traffic } \\
\text { infrequent relative to } \\
\text { pre-existing traffic in } \\
\text { Puget Sound }\end{array}$ & $\begin{array}{l}\text { Low; vessels similar } \\
\text { to existing fleet(s) } \\
\text { and do not represent } \\
\text { new types of impacts }\end{array}$ & $\begin{array}{l}\text { Stroud and } \\
\text { Roffe } 1979\end{array}$ \\
\hline & $\begin{array}{l}\text { Oil/chemical } \\
\text { release }\end{array}$ & $\begin{array}{l}\text { Ingestion, fur } \\
\text { fouling, breathing } \\
\text { exhaust fumes, } \\
\text { accumulation of } \\
\text { toxins. Assumes no } \\
\text { catastrophic spills. }\end{array}$ & $\begin{array}{l}\text { Low; increase in } \\
\text { existing traffic but } \\
\text { the volume of fluids } \\
\text { released low and pre- } \\
\text { existing ambient } \\
\text { levels high }\end{array}$ & $\begin{array}{l}\text { Med, routine } \\
\text { maintenance } \\
\text { occurs over } \\
\text { life of the } \\
\text { project }\end{array}$ & $\begin{array}{l}\text { Low all age classes; } \\
\text { inputs insignificant } \\
\text { relative to } \\
\text { background levels in } \\
\text { Puget Sound }\end{array}$ & $\begin{array}{l}\text { Low; low volume of } \\
\text { contaminants } \\
\text { expected and not } \\
\text { significantly greater } \\
\text { than background }\end{array}$ & $\begin{array}{l}\text { Ebbert et al. } \\
2000\end{array}$ \\
\hline & $\begin{array}{l}\text { Noise and } \\
\text { vibration }\end{array}$ & $\begin{array}{l}\text { Avoidance of area, } \\
\text { masks } \\
\text { environmental cues, } \\
\text { TTS possible which } \\
\text { could increase } \\
\text { predation }\end{array}$ & $\begin{array}{l}\text { Med; moderate } \\
\text { increase in boat } \\
\text { traffic, pre-existing } \\
\text { ambient noise levels } \\
\text { high }\end{array}$ & $\begin{array}{l}\text { Med, routine } \\
\text { maintenance } \\
\text { occurs over } \\
\text { life of the } \\
\text { project }\end{array}$ & $\begin{array}{l}\text { Low for adults; med } \\
\text { for pups due to } \\
\text { foraging and travel in } \\
\text { turbine and transport } \\
\text { zones; acclimation } \\
\text { likely due to high } \\
\text { levels of boat traffic } \\
\text { in Puget Sound }\end{array}$ & $\begin{array}{l}\text { Low; similar species } \\
\text { (Phoca hispida) } \\
\text { show considerable } \\
\text { tolerance to similar } \\
\text { types of noise }\end{array}$ & $\begin{array}{l}\text { Blackwell et } \\
\text { al. 2004, } \\
\text { Kastak et al. } \\
\text { 2005, } \\
\text { Tougaard et al. } \\
2009\end{array}$ \\
\hline
\end{tabular}

\begin{tabular}{lrr}
\hline $\begin{array}{l}\text { Environmental Assessment for Siting Wave \& Tidal } \\
\text { Energy Projects }\end{array}$ & F-15 & $\begin{array}{r}\text { H. T. Harvey \& Associates } \\
24 \text { November } 2009\end{array}$
\end{tabular}


Appendix F - Effects of Pilot Scale MCT SeaGen Project at the Tacoma Narrows Site

\begin{tabular}{|c|c|c|c|c|c|c|c|}
\hline Project activity & Project action & $\begin{array}{l}\text { Description of } \\
\text { action's effect on } \\
\text { indicator }\end{array}$ & $\begin{array}{l}\text { Spatial exposure to } \\
\text { indicator (low, med, } \\
\text { high) }\end{array}$ & $\begin{array}{l}\text { Temporal } \\
\text { exposure to } \\
\text { indicator } \\
\text { (low, med, } \\
\text { high) }\end{array}$ & $\begin{array}{l}\text { Effect's overlap with } \\
\text { critical life stage, } \\
\text { behavior, habitat or } \\
\text { resource (low, med, } \\
\text { high) }\end{array}$ & $\begin{array}{l}\text { Overall risk to } \\
\text { indicator (low, med, } \\
\text { high unknown) }\end{array}$ & Source(s) \\
\hline \multirow{2}{*}{$\begin{array}{l}\text { Operation of turbines, } \\
\text { rotation of rotors, or } \\
\text { other moving parts of } \\
\text { devices }\end{array}$} & Direct impact & $\begin{array}{l}\text { Direct injury or } \\
\text { mortality; } \\
\text { secondary mortality } \\
\text { due to infection, } \\
\text { loss of mobility, } \\
\text { secondary } \\
\text { complications, } \\
\text { predation }\end{array}$ & $\begin{array}{l}\text { Low, the areas of } \\
\text { movement are small } \\
\text { ( 1\%) compared to } \\
\text { channel area } \\
\text { (approximated } \\
\text { channel width } \mathrm{x} \\
\text { average depth) }\end{array}$ & $\begin{array}{l}\text { High, the } \\
\text { movement } \\
\text { occurs over the } \\
\text { life of the } \\
\text { project }\end{array}$ & $\begin{array}{l}\text { High for all age } \\
\text { classes }\end{array}$ & $\begin{array}{l}\text { High; significant risk } \\
\text { of rotor strike due to } \\
\text { rotational speed - may } \\
\text { be less detectable then } \\
\text { ship rotor due to } \\
\text { slower speed; strike at } \\
12 \mathrm{~m} / \mathrm{s} \text { ( } 27 \mathrm{mph}) \text { will } \\
\text { cause serious trauma }\end{array}$ & $\begin{array}{l}\text { Dadswell and } \\
\text { Rulifson 1994, } \\
\text { Wilson et al. } \\
2007\end{array}$ \\
\hline & $\begin{array}{l}\text { Noise (cavitation } \\
\text { off rotor) and } \\
\text { vibration }\end{array}$ & $\begin{array}{l}\text { Avoidance of area, } \\
\text { masks } \\
\text { environmental cues, } \\
\text { TTS possible which } \\
\text { could increase } \\
\text { predation }\end{array}$ & $\begin{array}{l}\text { Turbine sound } \\
\text { propagated over sig. } \\
\text { distance }(\sim 3 \mathrm{~km}) \text {; } \\
\text { behavioral reaction } \\
\text { likely at close range } \\
(<15 \mathrm{M})\end{array}$ & $\begin{array}{l}\text { High, any } \\
\text { effect would } \\
\text { be constant } \\
\text { over project }\end{array}$ & $\begin{array}{l}\text { Low for all age } \\
\text { classes; harbor seals } \\
\text { can detect low-freq. } \\
\text { sounds at great } \\
\text { distances; acclimation } \\
\text { likely due to noise in } \\
\text { Puget Sound }\end{array}$ & $\begin{array}{l}\text { Low; similar species } \\
\text { (Phoca hispida) } \\
\text { show considerable } \\
\text { tolerance to similar } \\
\text { types of noise }\end{array}$ & $\begin{array}{l}\text { Blackwell et } \\
\text { al. 2004, } \\
\text { Kastak et al. } \\
\text { 2005, } \\
\text { Tougaard et al. } \\
2009\end{array}$ \\
\hline $\begin{array}{l}\text { Structures in water } \\
\text { column and on seabed, } \\
\text { such as devices and } \\
\text { moorings and footings }\end{array}$ & Structure & $\begin{array}{l}\text { Entanglement with } \\
\text { derelict gear caught } \\
\text { on devices; artificial } \\
\text { reef effects could } \\
\text { attract fish, increase } \\
\text { forage, and attract } \\
\text { species }\end{array}$ & $\begin{array}{l}\text { Low, abundant man- } \\
\text { made structures } \\
\text { nearby; home ranges } \\
\text { large - harbor seal } \\
\text { pup home range } 10.4 \\
\mathrm{~km}^{2} \text { and adults travel } \\
\text { range is } 30-45 \mathrm{~km} .\end{array}$ & $\begin{array}{l}\text { High, structure } \\
\text { present over } \\
\text { life of project } \\
\text { duration }\end{array}$ & $\begin{array}{l}\text { Med, effect overlaps } \\
\text { with all age classes } \\
\text { foraging and traveling } \\
\text { in Puget Sound }\end{array}$ & $\begin{array}{l}\text { Unknown; structure } \\
\text { readily avoided } \\
\text { unless turbidity high } \\
\text { enough to decrease } \\
\text { visual acuity; high if } \\
\text { structures lure seals } \\
\text { into path of rotors in } \\
\text { pursuit of prey }\end{array}$ & $\begin{array}{l}\text { Dadswell and } \\
\text { Rulifson 1994, } \\
\text { Herder 1986, } \\
\text { Wilson et al. } \\
2007\end{array}$ \\
\hline $\begin{array}{l}\text { Electricity conduction } \\
\text { through cable }\end{array}$ & EMF & Unknown & $\begin{array}{l}\text { Unknown, cable } \\
\text { shielding and burial } \\
\text { provides some } \\
\text { attenuation }\end{array}$ & $\begin{array}{l}\text { High if an } \\
\text { effect, it would } \\
\text { occur over life } \\
\text { of the project }\end{array}$ & $\begin{array}{l}\text { Unknown; any effects } \\
\text { would likely impact } \\
\text { all age classes }\end{array}$ & Unknown & $\begin{array}{l}\text { Boehlert et al. } \\
2008\end{array}$ \\
\hline \multirow[t]{2}{*}{$\begin{array}{l}\text { Structures on water's } \\
\text { surface }\end{array}$} & Structure & Collision & $\begin{array}{l}\text { Low, no haulout } \\
\text { potential }\end{array}$ & $\begin{array}{l}\text { High, structure } \\
\text { present over } \\
\text { life of project }\end{array}$ & $\begin{array}{l}\text { Low for all age } \\
\text { classes; harbor seals } \\
\text { do no haul out on } \\
\text { such structures }\end{array}$ & $\begin{array}{l}\text { Low, structure easily } \\
\text { avoided; similar to } \\
\text { existing navigation } \\
\text { buoys; not suitable } \\
\text { for hauling out }\end{array}$ & $\begin{array}{l}\text { Schusterman } \\
\text { and Balliet } \\
1970\end{array}$ \\
\hline & Navigation lights & $\begin{array}{l}\text { Visual } \\
\text { disorientation; } \\
\text { could lure seals into } \\
\text { path of rotors if } \\
\text { prey species } \\
\text { attracted to lights }\end{array}$ & $\begin{array}{l}\text { Low, device lights of } \\
\text { low intensity, } \\
\text { shielded }\end{array}$ & $\begin{array}{l}\text { High, lights } \\
\text { shining over } \\
\text { life of the } \\
\text { project }\end{array}$ & $\begin{array}{l}\text { Low for all age } \\
\text { classes due to high } \\
\text { levels of pre-existing } \\
\text { light levels in Puget } \\
\text { Sound. }\end{array}$ & $\begin{array}{l}\text { Low; lights similar to } \\
\text { existing navigation } \\
\text { buoy lights; } \\
\text { significant ambient } \\
\text { light }\end{array}$ & $\begin{array}{l}\text { Yurk and } \\
\text { Trites } 2000\end{array}$ \\
\hline
\end{tabular}

Environmental Assessment for Siting Wave \& Tidal
Energy Projects

F-16

H. T. Harvey \& Associates

24 November 2009 
Appendix F - Effects of Pilot Scale MCT SeaGen Project at the Tacoma Narrows Site

\begin{tabular}{|c|c|c|c|c|c|c|c|}
\hline Project activity & Project action & $\begin{array}{l}\text { Description of } \\
\text { action's effect on } \\
\text { indicator }\end{array}$ & $\begin{array}{l}\text { Spatial exposure to } \\
\text { indicator (low, med, } \\
\text { high) }\end{array}$ & $\begin{array}{l}\text { Temporal } \\
\text { exposure to } \\
\text { indicator } \\
\text { (low, med, } \\
\text { high) } \\
\end{array}$ & $\begin{array}{l}\text { Effect's overlap with } \\
\text { critical life stage, } \\
\text { behavior, habitat or } \\
\text { resource (low, med, } \\
\text { high) }\end{array}$ & $\begin{array}{l}\text { Overall risk to } \\
\text { indicator (low, med, } \\
\text { high unknown) }\end{array}$ & Source(s) \\
\hline \multicolumn{8}{|c|}{ Decommissioning } \\
\hline \multirow{3}{*}{ Boat traffic } & Direct impact & Collision injuries & $\begin{array}{l}\text { Med; low to } \\
\text { moderate increase in } \\
\text { already high levels of } \\
\text { existing boat traffic }\end{array}$ & $\begin{array}{l}\text { Low, } \\
\text { occurring over } \\
1 \text { to } 2 \\
\text { summers }\end{array}$ & $\begin{array}{l}\text { Low for adults; med } \\
\text { for pups due to } \\
\text { foraging and travel in } \\
\text { construction zones; } \\
\text { acclimation likely due } \\
\text { to high boat traffic in } \\
\text { Puget Sound }\end{array}$ & $\begin{array}{l}\text { Low; vessels similar } \\
\text { to existing fleet(s) } \\
\text { and do not represent } \\
\text { new types of impacts }\end{array}$ & $\begin{array}{l}\text { Stroud and } \\
\text { Roffe } 1979\end{array}$ \\
\hline & $\begin{array}{l}\text { Oil/chemical } \\
\text { release }\end{array}$ & $\begin{array}{l}\text { Ingestion, fur } \\
\text { fouling, breathing } \\
\text { exhaust fumes, } \\
\text { accumulation of } \\
\text { toxins. }\end{array}$ & $\begin{array}{l}\text { Low; increase in } \\
\text { existing traffic but } \\
\text { low volume of fluids } \\
\text { released and pre- } \\
\text { existing levels high }\end{array}$ & $\begin{array}{l}\text { Low, } \\
\text { occurring over } \\
1 \text { to } 2 \\
\text { summers }\end{array}$ & $\begin{array}{l}\text { Low all age classes; } \\
\text { additional inputs } \\
\text { insignificant relative } \\
\text { to background levels } \\
\text { in Puget Sound }\end{array}$ & $\begin{array}{l}\text { Low; low volume of } \\
\text { contaminants } \\
\text { expected and not } \\
\text { significantly greater } \\
\text { than background } \\
\end{array}$ & $\begin{array}{l}\text { Ebbert et al. } \\
2000\end{array}$ \\
\hline & $\begin{array}{l}\text { Noise and } \\
\text { vibration }\end{array}$ & $\begin{array}{l}\text { Avoidance of area, } \\
\text { masks } \\
\text { environmental cues, } \\
\text { TTS possible which } \\
\text { could increase } \\
\text { predation }\end{array}$ & $\begin{array}{l}\text { Med; moderate } \\
\text { increase in boat } \\
\text { traffic, pre-existing } \\
\text { ambient noise levels } \\
\text { expected to be high }\end{array}$ & $\begin{array}{l}\text { Low, } \\
\text { occurring over } \\
1 \text { to } 2 \\
\text { summers }\end{array}$ & $\begin{array}{l}\text { Low for adults med } \\
\text { for pups due to } \\
\text { foraging and travel in } \\
\text { and near construction } \\
\text { zones; acclimation \& } \\
\text { likely due to boat } \\
\text { traffic in Puget Sound }\end{array}$ & $\begin{array}{l}\text { Low; similar species } \\
\text { (Phoca hispida) } \\
\text { show considerable } \\
\text { tolerance to similar } \\
\text { types of noise }\end{array}$ & $\begin{array}{l}\text { Blackwell et } \\
\text { al. 2004, } \\
\text { Kastak et al. } \\
\text { 2005, } \\
\text { Tougaard et al. } \\
2009\end{array}$ \\
\hline \multirow{3}{*}{$\begin{array}{l}\text { Decommissioning and } \\
\text { removal of electrical } \\
\text { collector system, } \\
\text { subsea cable, mooring } \\
\text { cables, foundations or } \\
\text { anchors, and devices }\end{array}$} & $\begin{array}{l}\text { Noise and } \\
\text { vibration }\end{array}$ & $\begin{array}{l}\text { Avoidance of area, } \\
\text { masks } \\
\text { environmental cues, } \\
\text { TTS could increase } \\
\text { predation }\end{array}$ & $\begin{array}{l}\text { Med; moderate } \\
\text { overall increase in } \\
\text { industrial activity, } \\
\text { pre-existing ambient } \\
\text { noise levels expected } \\
\text { to be high } \\
\end{array}$ & $\begin{array}{l}\text { Low, } \\
\text { occurring over } \\
1 \text { to } 2 \\
\text { summers }\end{array}$ & $\begin{array}{l}\text { Low-med for adults } \\
\text { med for pups; see } \\
\text { above }\end{array}$ & $\begin{array}{l}\text { Low; similar species } \\
\text { (Phoca hispida) } \\
\text { show considerable } \\
\text { tolerance to similar } \\
\text { types of noise }\end{array}$ & $\begin{array}{l}\text { Blackwell et } \\
\text { al. 2004, } \\
\text { Kastak et al. } \\
\text { 2005, } \\
\text { Tougaard et al. } \\
2009 \\
\end{array}$ \\
\hline & $\begin{array}{l}\text { Deconstruction } \\
\text { lights, vessel } \\
\text { deck lights and } \\
\text { spotlights. }\end{array}$ & $\begin{array}{l}\text { Visual } \\
\text { disorientation could } \\
\text { lead to collision, but } \\
\text { could also enhance } \\
\text { foraging on species } \\
\text { attracted to light } \\
\end{array}$ & $\begin{array}{l}\text { Low; moderate } \\
\text { increase in already } \\
\text { high levels of boat } \\
\text { traffic vessel, deck } \\
\text { lights illuminate only } \\
\text { a small area }\end{array}$ & $\begin{array}{l}\text { Low, } \\
\text { occurring over } \\
1 \text { to } 2 \\
\text { summers }\end{array}$ & $\begin{array}{l}\text { Low all age classes } \\
\text { due to high shoreline } \\
\text { development, boat } \\
\text { traffic, and industrial } \\
\text { activity in Puget } \\
\text { Sound }\end{array}$ & $\begin{array}{l}\text { Low; vessels and } \\
\text { construction similar } \\
\text { to existing activities } \\
\text { and do not represent } \\
\text { new types of impact }\end{array}$ & $\begin{array}{l}\text { Yurk and } \\
\text { Trites } 2000\end{array}$ \\
\hline & $\begin{array}{l}\text { Oil/chemical } \\
\text { release }\end{array}$ & $\begin{array}{l}\text { Ingestion, fur } \\
\text { fouling, breathing } \\
\text { exhaust fumes, } \\
\text { accumulation of } \\
\text { toxins. Assumes no } \\
\text { catastrophic spills. }\end{array}$ & $\begin{array}{l}\text { Med, significant } \\
\text { increase in use of } \\
\text { chemicals released } \\
\text { low, high pre-existing } \\
\text { levels }\end{array}$ & $\begin{array}{l}\text { Low, } \\
\text { occurring over } \\
1 \text { to } 2 \\
\text { summers }\end{array}$ & $\begin{array}{l}\text { Low all age classes; } \\
\text { inputs insignificant } \\
\text { relative to pre- } \\
\text { existing levels in } \\
\text { Puget Sound }\end{array}$ & $\begin{array}{l}\text { Low; volume of } \\
\text { contaminants } \\
\text { expected to be low } \\
\text { and not sig. greater } \\
\text { than background }\end{array}$ & $\begin{array}{l}\text { Ebbert et al. } \\
2000\end{array}$ \\
\hline
\end{tabular}

Environmental Assessment for Siting Wave \& Tidal

Energy Projects
F-17
H. T. Harvey \& Associates

24 November 2009 
Potential effects on Killer Whales due to Pilot Scale MCT SeaGen project at Tacoma Narrows site

\begin{tabular}{|c|c|c|c|c|c|c|c|}
\hline Project activity & Project action & $\begin{array}{l}\text { Description of } \\
\text { action's effect on } \\
\text { indicator }\end{array}$ & $\begin{array}{l}\text { Spatial exposure } \\
\text { on indicator (low, } \\
\text { med, high) }\end{array}$ & $\begin{array}{l}\text { Temporal } \\
\text { exposure on } \\
\text { indicator (low, } \\
\text { med, high) }\end{array}$ & $\begin{array}{l}\text { Effect's overlap } \\
\text { with critical life } \\
\text { stage, behavior, } \\
\text { habitat, or } \\
\text { resource (low, } \\
\text { med, high) }\end{array}$ & $\begin{array}{l}\text { Overall risk to } \\
\text { indicator (low, } \\
\text { med, high, } \\
\text { unknown) }\end{array}$ & Source(s) \\
\hline \multicolumn{8}{|c|}{ Construction } \\
\hline \multirow{3}{*}{ Boat traffic } & Direct impact & Collision injuries & $\begin{array}{l}\text { Low, significant } \\
\text { increase in existing } \\
\text { traffic but in a } \\
\text { small area of their } \\
\text { large range. }\end{array}$ & $\begin{array}{l}\text { Low, occurring } \\
\text { over } 1 \text { to } 2 \\
\text { summers }\end{array}$ & $\begin{array}{l}\text { Low, effects' } \\
\text { spatial extent small } \\
\text { compared to } \\
\text { animal's range }\end{array}$ & $\begin{array}{l}\text { Low; small area of } \\
\text { exposure relative to } \\
\text { home range; } \\
\text { acclimation and } \\
\text { avoidance likely due } \\
\text { to high boat traffic in } \\
\text { Puget Sound; killer } \\
\text { whale-boat collisions } \\
\text { not uncommon }\end{array}$ & $\begin{array}{l}\text { Van Waerebeek } \\
\text { and Leaper } 2008\end{array}$ \\
\hline & Noise and vibration & $\begin{array}{l}\text { Avoidance, } \\
\text { masking of } \\
\text { environmental cues, } \\
\text { communication } \\
\text { signals, } \\
\text { echolocation } \\
\end{array}$ & $\begin{array}{l}\text { Low, sound travels } \\
\text { far, animals will } \\
\text { hear noises outside } \\
\text { of project area, but } \\
\text { affects a small part } \\
\text { of large range }\end{array}$ & $\begin{array}{l}\text { Low, occurring } \\
\text { over } 1 \text { to } 2 \\
\text { summers }\end{array}$ & $\begin{array}{l}\text { Low, small part of } \\
\text { large range. }\end{array}$ & $\begin{array}{l}\text { Low; may be } \\
\text { displaced by high } \\
\text { amplitude sound; } \\
\text { background noise } \\
\text { levels high; short } \\
\text { duration }\end{array}$ & $\begin{array}{l}\text { Morton and } \\
\text { Symonds } 2002\end{array}$ \\
\hline & $\begin{array}{l}\text { Oil/chemical } \\
\text { release }\end{array}$ & $\begin{array}{l}\text { Ingestion, fur } \\
\text { fouling, breathing } \\
\text { exhaust fumes, } \\
\text { accumulation of } \\
\text { toxins. Assumes no } \\
\text { catastrophic spills. }\end{array}$ & $\begin{array}{l}\text { Low, significant } \\
\text { increase in existing } \\
\text { traffic but volume } \\
\text { of fluids released } \\
\text { low }\end{array}$ & $\begin{array}{l}\text { Low, occurring } \\
\text { over } 1 \text { to } 2 \\
\text { summers }\end{array}$ & $\begin{array}{l}\text { Low, effects' } \\
\text { spatial extent small } \\
\text { compared to } \\
\text { animal's range }\end{array}$ & $\begin{array}{l}\text { Low; additional } \\
\text { inputs insignificant } \\
\text { relative to } \\
\text { background levels }\end{array}$ & Ebbert et al. 2000 \\
\hline \multirow[t]{2}{*}{$\begin{array}{l}\text { Construction and } \\
\text { installation of } \\
\text { electrical collector } \\
\text { system, mooring } \\
\text { cables, anchors or } \\
\text { foundations, and } \\
\text { devices }\end{array}$} & Noise and vibration & $\begin{array}{l}\text { Avoidance, } \\
\text { masking of } \\
\text { environmental cues, } \\
\text { communication } \\
\text { signals, } \\
\text { echolocation } \\
\end{array}$ & $\begin{array}{l}\text { Low-Med, sound } \\
\text { travels far, animals } \\
\text { will hear outside } \\
\text { project area, but } \\
\text { affects a small part } \\
\text { of large range }\end{array}$ & $\begin{array}{l}\text { Low, occurring } \\
\text { over } 1 \text { to } 2 \\
\text { summers }\end{array}$ & $\begin{array}{l}\text { Low, effects' } \\
\text { spatial extent small } \\
\text { compared to } \\
\text { animal's range }\end{array}$ & $\begin{array}{l}\text { Low; may be } \\
\text { displaced by high } \\
\text { amplitude noise; } \\
\text { displacement area } \\
\text { small portion of } \\
\text { range }\end{array}$ & $\begin{array}{l}\text { Morton and } \\
\text { Symonds } 2002\end{array}$ \\
\hline & $\begin{array}{l}\text { Oil/chemical } \\
\text { release }\end{array}$ & $\begin{array}{l}\text { Ingestion, fur } \\
\text { fouling, breathing } \\
\text { exhaust fumes, } \\
\text { accumulation of } \\
\text { toxins. Assumes no } \\
\text { catastrophic spills. } \\
\end{array}$ & $\begin{array}{l}\text { Med, t increase in } \\
\text { use of chemicals, } \\
\text { fuels, solvents, } \\
\text { grease; ambient } \\
\text { levels elevated }\end{array}$ & $\begin{array}{l}\text { Low, occurring } \\
\text { over } 1 \text { to } 2 \\
\text { summers }\end{array}$ & $\begin{array}{l}\text { Low, effects' } \\
\text { spatial extent small } \\
\text { compared to } \\
\text { animal's range }\end{array}$ & $\begin{array}{l}\text { Low; additional } \\
\text { inputs insignificant } \\
\text { relative to } \\
\text { background levels } \\
\text { in Puget Sound }\end{array}$ & Ebbert et al. 2000 \\
\hline
\end{tabular}

\begin{tabular}{|c|c|c|}
\hline $\begin{array}{l}\text { Environmental Assessment for Siting Wave \& Tidal } \\
\text { Energy Projects }\end{array}$ & F-18 & $\begin{array}{r}\text { H. T. Harvey \& Associates } \\
24 \text { November } 2009\end{array}$ \\
\hline
\end{tabular}


Appendix F - Effects of Pilot Scale MCT SeaGen Project at the Tacoma Narrows Site

\begin{tabular}{|c|c|c|c|c|c|c|c|}
\hline \multirow[t]{2}{*}{ Project activity } & Project action & $\begin{array}{l}\text { Description of } \\
\text { action's effect on } \\
\text { indicator }\end{array}$ & $\begin{array}{l}\text { Spatial exposure } \\
\text { on indicator (low, } \\
\text { med, high) }\end{array}$ & $\begin{array}{l}\text { Temporal } \\
\text { exposure on } \\
\text { indicator (low, } \\
\text { med, high) }\end{array}$ & $\begin{array}{l}\text { Effect's overlap } \\
\text { with critical life } \\
\text { stage, behavior, } \\
\text { habitat, or } \\
\text { resource (low, } \\
\text { med, high) }\end{array}$ & $\begin{array}{l}\text { Overall risk to } \\
\text { indicator (low, } \\
\text { med, high, } \\
\text { unknown) }\end{array}$ & Source(s) \\
\hline & $\begin{array}{l}\text { Construction lights, } \\
\text { vessel deck lights } \\
\text { and spotlights. }\end{array}$ & $\begin{array}{l}\text { Visual } \\
\text { disorientation could } \\
\text { lead to collision, } \\
\text { could also enhance } \\
\text { foraging on species } \\
\text { attracted to light }\end{array}$ & Low & $\begin{array}{l}\text { Low, occurring } \\
\text { over } 1 \text { to } 2 \\
\text { summers }\end{array}$ & $\begin{array}{l}\text { Low, effects' } \\
\text { spatial extent small } \\
\text { compared to } \\
\text { animal's range }\end{array}$ & $\begin{array}{l}\text { Low; high levels of } \\
\text { shoreline } \\
\text { development, boat } \\
\text { traffic, industrial } \\
\text { activity in Sound }\end{array}$ & $\begin{array}{l}\text { USCG [date } \\
\text { unknown] }\end{array}$ \\
\hline $\begin{array}{l}\text { Directional drilling, } \\
\text { and laying cable } \\
\text { under/on seabed }\end{array}$ & Noise and vibration & $\begin{array}{l}\text { Avoidance, } \\
\text { masking of } \\
\text { environmental cues, } \\
\text { communication } \\
\text { signals, } \\
\text { echolocation }\end{array}$ & $\begin{array}{l}\text { Low-Med, sound } \\
\text { travels far, animals } \\
\text { will hear it outside } \\
\text { of project area, but } \\
\text { affects a small part } \\
\text { of large range }\end{array}$ & $\begin{array}{l}\text { Low, occurring } \\
\text { over } 1 \text { summer }\end{array}$ & $\begin{array}{l}\text { Low, effects' } \\
\text { spatial extent small } \\
\text { compared to } \\
\text { animal's range }\end{array}$ & $\begin{array}{l}\text { Low; likely } \\
\text { displaced from } \\
\text { area if noise levels } \\
\text { high; displacement } \\
\text { area small portion } \\
\text { of range and short } \\
\text { duration }\end{array}$ & $\begin{array}{l}\text { Morton and } \\
\text { Symonds } 2002\end{array}$ \\
\hline \multicolumn{8}{|c|}{ Operation and Maintenance } \\
\hline \multirow{3}{*}{ Boat traffic } & Direct impact & Collision injuries & $\begin{array}{l}\text { Low, slight } \\
\text { increase in existing } \\
\text { traffic in small area } \\
\text { of their large } \\
\text { range. }\end{array}$ & $\begin{array}{l}\text { High, traffic will } \\
\text { occur over life of } \\
\text { the project in } \\
\text { summer }\end{array}$ & $\begin{array}{l}\text { Low, effects' } \\
\text { spatial extent small } \\
\text { compared to } \\
\text { animal's range }\end{array}$ & $\begin{array}{l}\text { Low; insignificant } \\
\text { increase in boat } \\
\text { traffic ( } 235,000 \\
\text { vessels/yr); killer } \\
\text { whale-boat } \\
\text { collisions not } \\
\text { uncommon }\end{array}$ & $\begin{array}{l}\text { USCG [date } \\
\text { unknown], Van } \\
\text { Waerebeek and } \\
\text { Leaper } 2008\end{array}$ \\
\hline & $\begin{array}{l}\text { Oil/chemical } \\
\text { release }\end{array}$ & $\begin{array}{l}\text { Ingestion, fur } \\
\text { fouling, breathing } \\
\text { exhaust fumes, } \\
\text { accumulation of } \\
\text { toxins. Assumes no } \\
\text { catastrophic spills. }\end{array}$ & $\begin{array}{l}\text { Low, increase in } \\
\text { existing traffic but } \\
\text { the volume of } \\
\text { fluids released low }\end{array}$ & $\begin{array}{l}\text { High, traffic will } \\
\text { occur over life of } \\
\text { the project in } \\
\text { summer }\end{array}$ & $\begin{array}{l}\text { Low, effects' } \\
\text { spatial extent small } \\
\text { compared to } \\
\text { animal's range }\end{array}$ & $\begin{array}{l}\text { Low; area of } \\
\text { exposure small } \\
\text { relative to range; } \\
\text { inputs compound } \\
\text { elevated levels in } \\
\text { Puget Sound }\end{array}$ & Ebbert et al. 2000 \\
\hline & Noise and vibration & $\begin{array}{l}\text { Avoidance, } \\
\text { masking of } \\
\text { environmental cues, } \\
\text { communication } \\
\text { signals, } \\
\text { echolocation } \\
\end{array}$ & $\begin{array}{l}\text { Low-Med, sound } \\
\text { travels far, animals } \\
\text { will hear it outside } \\
\text { of project area, but } \\
\text { affects a small part } \\
\text { of large range }\end{array}$ & $\begin{array}{l}\text { High, traffic will } \\
\text { occur over life of } \\
\text { the project in } \\
\text { summer }\end{array}$ & $\begin{array}{l}\text { Low, effects' } \\
\text { spatial extent small } \\
\text { compared to } \\
\text { animal's range }\end{array}$ & $\begin{array}{l}\text { Low; insignificant } \\
\text { increase in boat } \\
\text { traffic over } \\
\text { existing levels } \\
\text { (235,000 vessels/yr } \\
\text { in Puget sound) }\end{array}$ & $\begin{array}{l}\text { Morton and } \\
\text { Symonds } 2002\end{array}$ \\
\hline
\end{tabular}

\begin{tabular}{|c|c|c|}
\hline $\begin{array}{l}\text { Environmental Assessment for Siting Wave \& Tidal } \\
\text { Energy Projects }\end{array}$ & F-19 & $\begin{array}{r}\text { H. T. Harvey \& Associates } \\
24 \text { November } 2009\end{array}$ \\
\hline
\end{tabular}


Appendix F - Effects of Pilot Scale MCT SeaGen Project at the Tacoma Narrows Site

\begin{tabular}{|c|c|c|c|c|c|c|c|}
\hline Project activity & Project action & $\begin{array}{l}\text { Description of } \\
\text { action's effect on } \\
\text { indicator }\end{array}$ & $\begin{array}{l}\text { Spatial exposure } \\
\text { on indicator (low, } \\
\text { med, high) }\end{array}$ & $\begin{array}{l}\text { Temporal } \\
\text { exposure on } \\
\text { indicator (low, } \\
\text { med, high) }\end{array}$ & $\begin{array}{l}\text { Effect's overlap } \\
\text { with critical life } \\
\text { stage, behavior, } \\
\text { habitat, or } \\
\text { resource (low, } \\
\text { med, high) }\end{array}$ & $\begin{array}{l}\text { Overall risk to } \\
\text { indicator (low, } \\
\text { med, high, } \\
\text { unknown) }\end{array}$ & Source(s) \\
\hline \multirow[t]{2}{*}{$\begin{array}{l}\text { Operation of } \\
\text { turbines, rotation of } \\
\text { rotors, or other } \\
\text { moving parts of } \\
\text { devices }\end{array}$} & Direct impact & $\begin{array}{l}\text { Direct injury or } \\
\text { mortality; } \\
\text { secondary mortality } \\
\text { due to infection, } \\
\text { loss of mobility, } \\
\text { secondary } \\
\text { complications, } \\
\text { predation }\end{array}$ & $\begin{array}{l}\text { Low, the areas of } \\
\text { movement are } \\
\text { small ( 1\%) } \\
\text { compared to } \\
\text { channel area } \\
\text { (approximated } \\
\text { channel width } x \\
\text { average depth) }\end{array}$ & $\begin{array}{l}\text { High, the } \\
\text { movement occurs } \\
\text { over the life of the } \\
\text { project }\end{array}$ & $\begin{array}{l}\text { High for all age } \\
\text { classes while } \\
\text { foraging, traveling; } \\
\text { increasingly } \\
\text { significant impact } \\
\text { if killer whales } \\
\text { attracted to prey } \\
\text { species attracted to } \\
\text { structure }\end{array}$ & $\begin{array}{l}\text { High; significant } \\
\text { risk of rotor strike } \\
\text { - may be less } \\
\text { detectable then a } \\
\text { ship rotor due to } \\
\text { slower rotational } \\
\text { speed; direct strike } \\
\text { at } 12 \mathrm{~m} / \mathrm{s} \text { ( } 27 \\
\text { mph) will cause } \\
\text { serious trauma }\end{array}$ & $\begin{array}{l}\text { Dadswell and } \\
\text { Rulifson 1994, } \\
\text { Wilson et al. } 2007\end{array}$ \\
\hline & Noise and vibration & $\begin{array}{l}\text { Avoidance, } \\
\text { masking of } \\
\text { environmental cues, } \\
\text { communication } \\
\text { signals, } \\
\text { echolocation }\end{array}$ & $\begin{array}{l}\text { Turbine sound will } \\
\text { likely produce } \\
\text { behavioral reaction } \\
\text { at close range (< } \\
15 \mathrm{~m} \text {; detectable at } \\
70 \mathrm{~m})\end{array}$ & $\begin{array}{l}\text { High, any effect } \\
\text { would be constant } \\
\text { over life of project }\end{array}$ & $\begin{array}{l}\text { Low for all age } \\
\text { classes; effect not } \\
\text { expected to extend } \\
\text { beyond immediate } \\
\text { area around turbine }\end{array}$ & $\begin{array}{l}\text { Unknown; could } \\
\text { mask cues near } \\
\text { turbine }(<70 \mathrm{~m}) \text {; } \\
\text { acclimation likely } \\
\text { due to ambient noise } \\
\text { in Puget Sound } \\
\end{array}$ & $\begin{array}{l}\text { Morton and } \\
\text { Symonds } 2002\end{array}$ \\
\hline $\begin{array}{l}\text { Structures in water } \\
\text { column and on } \\
\text { seabed, such as } \\
\text { devices and } \\
\text { moorings and } \\
\text { footings }\end{array}$ & Structure & $\begin{array}{l}\text { Entanglement with } \\
\text { derelict gear caught } \\
\text { on devices; } \\
\text { artificial reef effects } \\
\text { could attract fish } \\
\text { and species }\end{array}$ & $\begin{array}{l}\text { Low, home range } \\
\text { large compared to } \\
\text { project area; may } \\
\text { create new habitat } \\
\text { relative to habitat } \\
\text { already there }\end{array}$ & $\begin{array}{l}\text { High, any positive } \\
\text { or negative effects } \\
\text { would occur over } \\
\text { the life of the } \\
\text { project. }\end{array}$ & $\begin{array}{l}\text { Low-med, } \\
\text { structure readily } \\
\text { avoided; loose or } \\
\text { unattached cables }\end{array}$ & $\begin{array}{l}\text { Unknown; may } \\
\text { attract killer } \\
\text { whales if structures } \\
\text { concentrate } \\
\text { pinnipeds pursuing } \\
\text { fish prey } \\
\end{array}$ & Relini et al. 2000 \\
\hline $\begin{array}{l}\text { Electricity } \\
\text { conduction through } \\
\text { cable }\end{array}$ & EMF & Unknown & $\begin{array}{l}\text { Unknown, cable } \\
\text { shielding and } \\
\text { burial provides } \\
\text { some attenuation }\end{array}$ & $\begin{array}{l}\text { High if an effect } \\
\text { because electricity } \\
\text { generated over life } \\
\text { of the project }\end{array}$ & Unknown & $\begin{array}{l}\text { Unknown; High } \\
\text { uncertainty about } \\
\text { effects on marine } \\
\text { mammals }\end{array}$ & $\begin{array}{l}\text { Boehlert et al. } \\
2008\end{array}$ \\
\hline \multirow[b]{2}{*}{$\begin{array}{l}\text { Structures on } \\
\text { water's surface }\end{array}$} & Structure & $\begin{array}{l}\text { Collision, artificial } \\
\text { reef effects could } \\
\text { attract fish, sea } \\
\text { lions, small } \\
\text { odontocetes } \\
\end{array}$ & $\begin{array}{l}\text { Low, reef effect } \\
\text { area is small } \\
\text { compared to home } \\
\text { range }\end{array}$ & $\begin{array}{l}\text { High, any effects } \\
\text { would occur over } \\
\text { the life of the } \\
\text { project. }\end{array}$ & $\begin{array}{l}\text { Low, may } \\
\text { incrementally } \\
\text { increase forage as } \\
\text { marine reserve }\end{array}$ & $\begin{array}{l}\text { Low; structure } \\
\text { readily avoided }\end{array}$ & \\
\hline & Navigation lights & $\begin{array}{l}\text { Visual } \\
\text { disorientation could } \\
\text { lead to collision, } \\
\text { could also enhance } \\
\text { foraging on species } \\
\text { attracted to light } \\
\end{array}$ & $\begin{array}{l}\text { Low, device lights } \\
\text { are low intensity } \\
\text { and shielded }\end{array}$ & $\begin{array}{l}\text { High, lights } \\
\text { shining over life of } \\
\text { the project }\end{array}$ & $\begin{array}{l}\text { Low, light intensity } \\
\text { low }\end{array}$ & $\begin{array}{l}\text { Low due to high } \\
\text { shoreline } \\
\text { development, boat } \\
\text { traffic and industry } \\
\text { in Puget Sound }\end{array}$ & $\begin{array}{l}\text { USCG [date } \\
\text { unknown] }\end{array}$ \\
\hline
\end{tabular}

\begin{tabular}{|c|c|c|}
\hline $\begin{array}{l}\text { Environmental Assessment for Siting Wave \& Tidal } \\
\text { Energy Projects }\end{array}$ & F-20 & $\begin{array}{r}\text { H. T. Harvey \& Associates } \\
24 \text { November } 2009\end{array}$ \\
\hline
\end{tabular}


Appendix F - Effects of Pilot Scale MCT SeaGen Project at the Tacoma Narrows Site

\begin{tabular}{|c|c|c|c|c|c|c|c|}
\hline Project activity & Project action & $\begin{array}{l}\text { Description of } \\
\text { action's effect on } \\
\text { indicator }\end{array}$ & $\begin{array}{l}\text { Spatial exposure } \\
\text { on indicator (low, } \\
\text { med, high) }\end{array}$ & $\begin{array}{l}\text { Temporal } \\
\text { exposure on } \\
\text { indicator (low, } \\
\text { med, high) }\end{array}$ & $\begin{array}{l}\text { Effect's overlap } \\
\text { with critical life } \\
\text { stage, behavior, } \\
\text { habitat, or } \\
\text { resource (low, } \\
\text { med, high) }\end{array}$ & $\begin{array}{l}\text { Overall risk to } \\
\text { indicator (low, } \\
\text { med, high, } \\
\text { unknown) }\end{array}$ & Source(s) \\
\hline \multicolumn{8}{|c|}{ Decommissioning } \\
\hline \multirow{3}{*}{ Boat traffic } & Direct impact & Collision injuries & $\begin{array}{l}\text { Low, significant } \\
\text { increase existing } \\
\text { traffic in small area } \\
\text { of their large } \\
\text { range. }\end{array}$ & $\begin{array}{l}\text { Low, occurring } \\
\text { over } 1 \text { summer }\end{array}$ & $\begin{array}{l}\text { Low, effects' } \\
\text { spatial extent small } \\
\text { compared to } \\
\text { animal's range }\end{array}$ & $\begin{array}{l}\text { Low; acclimation } \\
\text { and avoidance } \\
\text { likely due to } \\
\text { existing traffic; } \\
\text { killer whale-boat } \\
\text { collisions not } \\
\text { uncommon }\end{array}$ & $\begin{array}{l}\text { Van Waerebeek } \\
\text { and Leaper } 2008\end{array}$ \\
\hline & Noise and vibration & $\begin{array}{l}\text { Avoidance, masks } \\
\text { environmental cues, } \\
\text { communication } \\
\text { signals, } \\
\text { echolocation }\end{array}$ & $\begin{array}{l}\text { Low-Med, sound } \\
\text { travels far, animals } \\
\text { will hear it outside } \\
\text { project area, but } \\
\text { affects small part } \\
\text { of large range }\end{array}$ & $\begin{array}{l}\text { Low, occurring } \\
\text { over } 1 \text { summer }\end{array}$ & $\begin{array}{l}\text { Low, small part of } \\
\text { large range. }\end{array}$ & $\begin{array}{l}\text { Low; may be } \\
\text { displaced by high } \\
\text { amplitude sound; } \\
\text { background noise } \\
\text { levels high; short } \\
\text { overall duration }\end{array}$ & $\begin{array}{l}\text { Morton and } \\
\text { Symonds } 2002\end{array}$ \\
\hline & $\begin{array}{l}\text { Oil/chemical } \\
\text { release }\end{array}$ & $\begin{array}{l}\text { Ingestion, fur } \\
\text { fouling, breathing } \\
\text { exhaust fumes, } \\
\text { accumulation of } \\
\text { toxins. Assumes no } \\
\text { catastrophic spills. }\end{array}$ & $\begin{array}{l}\text { Low, significant } \\
\text { increase existing } \\
\text { traffic but the } \\
\text { volume of fluids } \\
\text { released low }\end{array}$ & $\begin{array}{l}\text { Low, occurring } \\
\text { over } 1 \text { summer }\end{array}$ & $\begin{array}{l}\text { Low, effects' } \\
\text { spatial extent small } \\
\text { compared to } \\
\text { animal's range }\end{array}$ & $\begin{array}{l}\text { Low; additional } \\
\text { inputs insignificant } \\
\text { relative to } \\
\text { background levels } \\
\text { in Puget Sound }\end{array}$ & Ebbert et al. 2000 \\
\hline \multirow{3}{*}{$\begin{array}{l}\text { Decommissioning } \\
\text { and removal of } \\
\text { electrical collector } \\
\text { system, subsea } \\
\text { cable, mooring } \\
\text { cables, foundations } \\
\text { or anchors, and } \\
\text { devices }\end{array}$} & Noise and vibration & $\begin{array}{l}\text { Avoidance, } \\
\text { masking of } \\
\text { environmental cues, } \\
\text { communication } \\
\text { signals, } \\
\text { echolocation } \\
\end{array}$ & $\begin{array}{l}\text { Low-Med, sound } \\
\text { travels far, will } \\
\text { hear it even if they } \\
\text { never approach, } \\
\text { but small part of } \\
\text { large range }\end{array}$ & $\begin{array}{l}\text { Low, occurring } \\
\text { over } 1 \text { summer }\end{array}$ & $\begin{array}{l}\text { Low, effects' } \\
\text { spatial extent small } \\
\text { compared to } \\
\text { animal's range }\end{array}$ & $\begin{array}{l}\text { Low; may be } \\
\text { displaced by high } \\
\text { amplitude noise; } \\
\text { displacement area } \\
\text { represents small } \\
\text { portion of range }\end{array}$ & $\begin{array}{l}\text { Morton and } \\
\text { Symonds } 2002\end{array}$ \\
\hline & $\begin{array}{l}\text { Oil/chemical } \\
\text { release }\end{array}$ & $\begin{array}{l}\text { Ingestion, breathing } \\
\text { exhaust fumes, } \\
\text { accumulation of } \\
\text { toxins. }\end{array}$ & $\begin{array}{l}\text { Med, increase in } \\
\text { use of chemicals, } \\
\text { solvents, fuels, } \\
\text { grease; ambient } \\
\text { levels elevated }\end{array}$ & $\begin{array}{l}\text { Low, occurring } \\
\text { over } 1 \text { to } 2 \\
\text { summers }\end{array}$ & $\begin{array}{l}\text { Low, effects' } \\
\text { spatial extent small } \\
\text { compared to } \\
\text { animal's range }\end{array}$ & $\begin{array}{l}\text { Low; additional } \\
\text { inputs insignificant } \\
\text { relative to } \\
\text { background }\end{array}$ & Ebbert et al. 2000 \\
\hline & $\begin{array}{l}\text { Deconstruction } \\
\text { lights (deck work } \\
\text { lights and } \\
\text { spotlights, vessel } \\
\text { navigation lights) }\end{array}$ & $\begin{array}{l}\text { Visual } \\
\text { disorientation could } \\
\text { lead to collision, } \\
\text { could also enhance } \\
\text { foraging on species } \\
\text { attracted to light }\end{array}$ & Unknown & $\begin{array}{l}\text { Low, occurring } \\
\text { over } 1 \text { summer }\end{array}$ & $\begin{array}{l}\text { Low, effects' } \\
\text { spatial extent small } \\
\text { compared to } \\
\text { animal's range }\end{array}$ & $\begin{array}{l}\text { Low; high levels of } \\
\text { shoreline } \\
\text { development, boat } \\
\text { traffic and industry } \\
\text { in Puget Sound }\end{array}$ & $\begin{array}{l}\text { USCG [date } \\
\text { unknown] }\end{array}$ \\
\hline
\end{tabular}

\begin{tabular}{|c|c|c|}
\hline $\begin{array}{l}\text { Environmental Assessment for Siting Wave \& Tidal } \\
\text { Energy Projects }\end{array}$ & F-21 & $\begin{array}{r}\text { H. T. Harvey \& Associates } \\
24 \text { November } 2009\end{array}$ \\
\hline
\end{tabular}


Appendix F - Effects of Pilot Scale MCT SeaGen Project at the Tacoma Narrows Site

Potential effects on harbor porpoise and Dall's porpoise due to Pilot Scale MCT SeaGen project at Tacoma Narrows

\begin{tabular}{|c|c|c|c|c|c|c|c|}
\hline Project activity & Project action & $\begin{array}{l}\text { Description of } \\
\text { action's effect on } \\
\text { indicator }\end{array}$ & $\begin{array}{l}\text { Spatial exposure to } \\
\text { indicator (low, med, } \\
\text { high) }\end{array}$ & $\begin{array}{l}\text { Temporal } \\
\text { exposure to } \\
\text { indicator (low, } \\
\text { med, high) } \\
\end{array}$ & $\begin{array}{l}\text { Effect's overlap } \\
\text { with critical life } \\
\text { stage, behavior, } \\
\text { habitat, or } \\
\text { resource (low, } \\
\text { med, high) }\end{array}$ & $\begin{array}{l}\text { Overall risk to } \\
\text { indicator (low, med, } \\
\text { high unknown }\end{array}$ & Source(s) \\
\hline \multicolumn{8}{|c|}{$\begin{array}{l}\text { Construction } \\
\end{array}$} \\
\hline \multirow{3}{*}{$\begin{array}{l}\text { Boat traffic, } \\
\text { assumed to occur } \\
\text { day and night }\end{array}$} & Direct impact & Collision injuries & $\begin{array}{l}\text { Med; low to } \\
\text { moderate increase in } \\
\text { already high levels of } \\
\text { pre-existing boat } \\
\text { traffic. }\end{array}$ & $\begin{array}{l}\text { Low, occurring } \\
\text { over } 1 \text { to } 2 \\
\text { summers }\end{array}$ & $\begin{array}{l}\text { Low for adults; med } \\
\text { for calves due to } \\
\text { foraging and travel } \\
\text { in construction and } \\
\text { transport zones }\end{array}$ & $\begin{array}{l}\text { Low; area of exposure } \\
\text { small relative to home } \\
\text { range; acclimation and } \\
\text { avoidance likely due to } \\
\text { high boat traffic; boat } \\
\text { collisions with harbor } \\
\text { porpoise infrequent }\end{array}$ & $\begin{array}{l}\text { Stroud and } \\
\text { Roffe } 1979\end{array}$ \\
\hline & $\begin{array}{l}\text { Noise and } \\
\text { vibration }\end{array}$ & $\begin{array}{l}\text { Avoidance, masks } \\
\text { environmental cues. } \\
\text { Reduction of hearing } \\
\text { sensitivity (TTS), } \\
\text { could increase } \\
\text { predation }\end{array}$ & $\begin{array}{l}\text { Med; moderate } \\
\text { increase in boat } \\
\text { traffic, pre-existing } \\
\text { ambient noise levels } \\
\text { expected to be high }\end{array}$ & $\begin{array}{l}\text { Low, occurring } \\
\text { over } 1 \text { to } 2 \\
\text { summers }\end{array}$ & $\begin{array}{l}\text { Low for adults; med } \\
\text { for calves due to } \\
\text { foraging and travel } \\
\text { in construction and } \\
\text { transport zones }\end{array}$ & $\begin{array}{l}\text { Low; acclimation and } \\
\text { tolerance likely due } \\
\text { to pre-existing boat } \\
\text { traffic noise in Puget } \\
\text { Sound; short duration }\end{array}$ & $\begin{array}{l}\text { Tougaard et al. } \\
2009\end{array}$ \\
\hline & $\begin{array}{l}\text { Oil/chemical } \\
\text { release }\end{array}$ & $\begin{array}{l}\text { Ingestion, fur fouling, } \\
\text { breathing exhaust } \\
\text { fumes, accumulation } \\
\text { of toxins. Assumes no } \\
\text { catastrophic spills. }\end{array}$ & $\begin{array}{l}\text { Low; increase in } \\
\text { traffic but the volume } \\
\text { of fluids released low } \\
\text { and ambient levels } \\
\text { high }\end{array}$ & $\begin{array}{l}\text { Low, occurring } \\
\text { over } 1 \text { to } 2 \\
\text { summers }\end{array}$ & $\begin{array}{l}\text { Low; area of } \\
\text { potential exposure } \\
\text { small relative to } \\
\text { home range }\end{array}$ & $\begin{array}{l}\text { Low; inputs } \\
\text { insignificant relative } \\
\text { to background levels } \\
\text { in Puget Sound }\end{array}$ & $\begin{array}{l}\text { Ebbert et al. } \\
2000\end{array}$ \\
\hline \multirow{3}{*}{$\begin{array}{l}\text { Construction and } \\
\text { installation of } \\
\text { electrical } \\
\text { collector system, } \\
\text { mooring cables, } \\
\text { anchors or } \\
\text { foundations, and } \\
\text { devices }\end{array}$} & $\begin{array}{l}\text { Noise and } \\
\text { vibration }\end{array}$ & $\begin{array}{l}\text { Avoidance of area, } \\
\text { masking of } \\
\text { environmental cues, } \\
\text { TTS could increase } \\
\text { predation. }\end{array}$ & $\begin{array}{l}\text { Med; moderate } \\
\text { increase in industrial } \\
\text { activity, high pre- } \\
\text { existing noise levels }\end{array}$ & $\begin{array}{l}\text { Low, occurring } \\
\text { over } 1 \text { to } 2 \\
\text { summers }\end{array}$ & $\begin{array}{l}\text { Low-med for adults } \\
\text { med for calves due } \\
\text { to foraging and } \\
\text { travel in } \\
\text { construction zone }\end{array}$ & $\begin{array}{l}\text { Low; acclimation } \\
\text { likely due to high } \\
\text { levels of boat traffic } \\
\text { in Puget Sound; }\end{array}$ & $\begin{array}{l}\text { Blackwell et al. } \\
\text { 2004, Kastak et } \\
\text { al. 2005, Todd et } \\
\text { al. 2009, } \\
\text { Tougaard et al. } \\
2009\end{array}$ \\
\hline & $\begin{array}{l}\text { Oil/chemical } \\
\text { release }\end{array}$ & $\begin{array}{l}\text { Ingestion, skin fouling, } \\
\text { breathing exhaust } \\
\text { fumes, accumulation } \\
\text { of toxins. Assumes no } \\
\text { catastrophic spills. }\end{array}$ & $\begin{array}{l}\text { Med, increase in use } \\
\text { of chemicals; volume } \\
\text { of fluids released } \\
\text { low, pre-existing } \\
\text { levels high }\end{array}$ & $\begin{array}{l}\text { Low, occurring } \\
\text { over } 1 \text { to } 2 \\
\text { summers }\end{array}$ & $\begin{array}{l}\text { Low to med; area of } \\
\text { potential exposure } \\
\text { is small relative to } \\
\text { home range }\end{array}$ & $\begin{array}{l}\text { Low; inputs } \\
\text { insignificant relative } \\
\text { to background levels } \\
\text { in Puget Sound }\end{array}$ & $\begin{array}{l}\text { Ebbert et al. } \\
2000\end{array}$ \\
\hline & $\begin{array}{l}\text { Construction } \\
\text { lights, vessel } \\
\text { deck lights and } \\
\text { spotlights. }\end{array}$ & $\begin{array}{l}\text { Visual disorientation } \\
\text { could lead to } \\
\text { collision, enhance } \\
\text { foraging on species } \\
\text { attracted to light }\end{array}$ & $\begin{array}{l}\text { Low; area affected by } \\
\text { lights small relative } \\
\text { to home range }\end{array}$ & $\begin{array}{l}\text { Low, occurring } \\
\text { over } 1 \text { to } 2 \\
\text { summers }\end{array}$ & $\begin{array}{l}\text { Area affected by } \\
\text { lights small relative } \\
\text { to home range }\end{array}$ & $\begin{array}{l}\text { Low due to high } \\
\text { levels of shoreline } \\
\text { development, boat } \\
\text { traffic and industrial } \\
\text { activity in Sound } \\
\end{array}$ & Todd et al. 2009 \\
\hline
\end{tabular}

\begin{tabular}{|c|c|c|}
\hline $\begin{array}{l}\text { Environmental Assessment for Siting Wave \& Tidal } \\
\text { Energy Projects }\end{array}$ & F-22 & $\begin{array}{r}\text { H. T. Harvey \& Associates } \\
24 \text { November } 2009\end{array}$ \\
\hline
\end{tabular}


Appendix F - Effects of Pilot Scale MCT SeaGen Project at the Tacoma Narrows Site

\begin{tabular}{|c|c|c|c|c|c|c|c|}
\hline Project activity & Project action & $\begin{array}{l}\text { Description of } \\
\text { action's effect on } \\
\text { indicator }\end{array}$ & $\begin{array}{l}\text { Spatial exposure to } \\
\text { indicator (low, med, } \\
\text { high) }\end{array}$ & $\begin{array}{l}\text { Temporal } \\
\text { exposure to } \\
\text { indicator (low, } \\
\text { med, high) }\end{array}$ & $\begin{array}{l}\text { Effect's overlap } \\
\text { with critical life } \\
\text { stage, behavior, } \\
\text { habitat, or } \\
\text { resource (low, } \\
\text { med, high) }\end{array}$ & $\begin{array}{l}\text { Overall risk to } \\
\text { indicator (low, med, } \\
\text { high unknown }\end{array}$ & Source(s) \\
\hline $\begin{array}{l}\text { Directional } \\
\text { drilling, and } \\
\text { laying cable } \\
\text { under/on seabed }\end{array}$ & $\begin{array}{l}\text { Noise and } \\
\text { vibration }\end{array}$ & $\begin{array}{l}\text { Avoidance of area, } \\
\text { masking of envir. } \\
\text { cues, TTS could } \\
\text { increase predation }\end{array}$ & $\begin{array}{l}\text { Med, porpoises likely } \\
\text { to avoid areas } \\
\text { occupied by cable } \\
\text { and support vessels }\end{array}$ & $\begin{array}{l}\text { Low, occurring } \\
\text { over } 1 \text { summer }\end{array}$ & $\begin{array}{l}\text { Low; avoidance of } \\
\text { construction area } \\
\text { occupied by cable } \\
\text { ship and support } \\
\text { vessels }\end{array}$ & $\begin{array}{l}\text { Low; could } \\
\text { concentrate impacts } \\
\text { nearshore; disruption } \\
\text { of movement along } \\
\text { shore }\end{array}$ & $\begin{array}{l}\text { Kastak et al. } \\
\text { 2005, } \\
\text { Koschinski et al. } \\
\text { 2003, Tougaard } \\
\text { et al. } 2009\end{array}$ \\
\hline \multicolumn{8}{|c|}{ Operation and Maintenance } \\
\hline \multirow{3}{*}{ Boat traffic } & Direct impact & Collision injuries & $\begin{array}{l}\text { Med; low to } \\
\text { moderate increase in } \\
\text { already high levels of } \\
\text { pre-existing boat } \\
\text { traffic }\end{array}$ & $\begin{array}{l}\text { Med, routine } \\
\text { maintenance } \\
\text { occurs over life of } \\
\text { the project }\end{array}$ & $\begin{array}{l}\text { Low; O\&M boat } \\
\text { traffic infrequent } \\
\text { relative to pre- } \\
\text { existing traffic in } \\
\text { Puget Sound }\end{array}$ & $\begin{array}{l}\text { Low; area of exposure } \\
\text { small relative to home } \\
\text { range; acclimation } \\
\text { likely due to high level } \\
\text { of existing boat traffic; } \\
\text { boat collisions with } \\
\text { porpoise infrequent }\end{array}$ & $\begin{array}{l}\text { Stroud and } \\
\text { Roffe } 1979\end{array}$ \\
\hline & $\begin{array}{l}\text { Noise and } \\
\text { vibration }\end{array}$ & $\begin{array}{l}\text { Avoidance of area, } \\
\text { masking of } \\
\text { environmental cues, } \\
\text { TTS possible which } \\
\text { could increase } \\
\text { predation }\end{array}$ & $\begin{array}{l}\text { Med; moderate } \\
\text { increase in boat } \\
\text { traffic, pre-existing } \\
\text { ambient noise levels } \\
\text { high }\end{array}$ & $\begin{array}{l}\text { Med, routine } \\
\text { maintenance } \\
\text { occurs over life of } \\
\text { the project }\end{array}$ & $\begin{array}{l}\text { Low adults, med for } \\
\text { calves due to } \\
\text { foraging and travel } \\
\text { in and near turbine } \\
\text { and transport zones }\end{array}$ & $\begin{array}{l}\text { Low; acclimation } \\
\text { likely due to high } \\
\text { levels of pre-existing } \\
\text { boat traffic noise in } \\
\text { Puget Sound; short } \\
\text { overall duration }\end{array}$ & $\begin{array}{l}\text { Tougaard et al. } \\
2009\end{array}$ \\
\hline & $\begin{array}{l}\text { Oil/chemical } \\
\text { release }\end{array}$ & $\begin{array}{l}\text { Ingestion, skin } \\
\text { fouling, breathing } \\
\text { exhaust fumes, } \\
\text { accumulation of } \\
\text { toxins. Assumes no } \\
\text { catastrophic spills. }\end{array}$ & $\begin{array}{l}\text { Low; increase in } \\
\text { traffic but volume of } \\
\text { fluids released low } \\
\text { and pre-existing } \\
\text { levels high }\end{array}$ & $\begin{array}{l}\text { Med, routine } \\
\text { maintenance } \\
\text { occurs over life of } \\
\text { the project }\end{array}$ & Low & $\begin{array}{l}\text { Low; additional } \\
\text { inputs insignificant } \\
\text { relative to } \\
\text { background levels in } \\
\text { Puget Sound }\end{array}$ & $\begin{array}{l}\text { Ebbert et al. } \\
2000\end{array}$ \\
\hline $\begin{array}{l}\text { Operation of } \\
\text { turbines, rotation } \\
\text { of rotors, or other } \\
\text { moving parts of } \\
\text { devices }\end{array}$ & $\begin{array}{l}\text { Noise and } \\
\text { vibration }\end{array}$ & $\begin{array}{l}\text { Avoidance of area, } \\
\text { masking of } \\
\text { environmental cues, } \\
\text { TTS possible which } \\
\text { could increase } \\
\text { predation }\end{array}$ & $\begin{array}{l}\text { Turbine sound could } \\
\text { produce behavioral } \\
\text { reaction at close } \\
\text { range }(<15 \mathrm{~m} ; \\
\text { detectable at } 70 \mathrm{~m}) ; \\
\text { high pre-existing } \\
\text { noise levels expected }\end{array}$ & $\begin{array}{l}\text { High, any effect } \\
\text { would be constant } \\
\text { over life of project }\end{array}$ & $\begin{array}{l}\text { Low-med for all age } \\
\text { classes; effect not } \\
\text { expected to extend } \\
\text { beyond immediate } \\
\text { area around turbine }\end{array}$ & $\begin{array}{l}\text { Unknown; porpoises } \\
\text { less sensitive to low- } \\
\text { freq. sounds and } \\
\text { generated noise likely } \\
\text { inaudible at }>100 \mathrm{~m} \text {; } \\
\text { acclimation likely due } \\
\text { to noise in Sound }\end{array}$ & $\begin{array}{l}\text { Blackwell et al. } \\
\text { 2004, Kastak et } \\
\text { al. 2005, } \\
\text { Koschinski et al. } \\
\text { 2003, Tougaard } \\
\text { et al. } 2009\end{array}$ \\
\hline
\end{tabular}

\begin{tabular}{lrr}
\hline $\begin{array}{l}\text { Environmental Assessment for Siting Wave \& Tidal } \\
\text { Energy Projects }\end{array}$ & F-23 & H. T. Harvey \& Associates \\
24 November 2009
\end{tabular}


Appendix F - Effects of Pilot Scale MCT SeaGen Project at the Tacoma Narrows Site

\begin{tabular}{|c|c|c|c|c|c|c|c|}
\hline Project activity & Project action & $\begin{array}{l}\text { Description of } \\
\text { action's effect on } \\
\text { indicator }\end{array}$ & $\begin{array}{l}\text { Spatial exposure to } \\
\text { indicator (low, med, } \\
\text { high) }\end{array}$ & $\begin{array}{l}\text { Temporal } \\
\text { exposure to } \\
\text { indicator (low, } \\
\text { med, high) }\end{array}$ & $\begin{array}{l}\text { Effect's overlap } \\
\text { with critical life } \\
\text { stage, behavior, } \\
\text { habitat, or } \\
\text { resource (low, } \\
\text { med, high) }\end{array}$ & $\begin{array}{l}\text { Overall risk to } \\
\text { indicator (low, med, } \\
\text { high unknown }\end{array}$ & Source(s) \\
\hline & Direct impact & $\begin{array}{l}\text { Injury or mortality; } \\
\text { secondary mortality } \\
\text { due to infection, loss } \\
\text { of mobility, predation }\end{array}$ & $\begin{array}{l}\text { Low, areas of } \\
\text { movement small } \\
(\sim 1 \%) \text { compared to } \\
\text { channel area } \\
\text { (approximated } \\
\text { channel width } x \\
\text { average depth) }\end{array}$ & $\begin{array}{l}\text { High, the } \\
\text { movement occurs } \\
\text { over the life of the } \\
\text { project }\end{array}$ & $\begin{array}{l}\text { High for all age } \\
\text { classes while } \\
\text { foraging, traveling; } \\
\text { greater impact if } \\
\text { porpoises attracted } \\
\text { to prey species } \\
\text { attracted to structure }\end{array}$ & $\begin{array}{l}\text { High; significant risk } \\
\text { of rotor strike - may } \\
\text { be less detectable } \\
\text { then a ship rotor due } \\
\text { to slower rotational } \\
\text { speed; direct strike at } \\
12 \mathrm{~m} / \mathrm{s} \text { will likely } \\
\text { cause serious trauma }\end{array}$ & $\begin{array}{l}\text { Dadswell and } \\
\text { Rulifson 1994, } \\
\text { Todd et al. 2009, } \\
\text { Wilson et al. } \\
2007\end{array}$ \\
\hline $\begin{array}{l}\text { Structures in } \\
\text { water column and } \\
\text { on seabed, such as } \\
\text { devices and } \\
\text { moorings and } \\
\text { footings }\end{array}$ & Structure & $\begin{array}{l}\text { Entanglement with } \\
\text { derelict gear caught on } \\
\text { devices; artificial reef } \\
\text { could attract fish and } \\
\text { attract species }\end{array}$ & $\begin{array}{l}\text { Low, abundant man- } \\
\text { made structures } \\
\text { nearby; home range } \\
\text { large }\left(>200 \mathrm{~km}^{2}\right)\end{array}$ & $\begin{array}{l}\text { High, structure } \\
\text { present over life of } \\
\text { project }\end{array}$ & $\begin{array}{l}\text { Med; potential } \\
\text { impacts while } \\
\text { foraging, if attracted } \\
\text { to prey concentrated } \\
\text { around structure }\end{array}$ & $\begin{array}{l}\text { Unknown; structure is } \\
\text { readily avoided with } \\
\text { echolocation; but } \\
\text { could lure porpoises } \\
\text { in pursuit of prey }\end{array}$ & Todd et al. 2009 \\
\hline $\begin{array}{l}\text { Electricity } \\
\text { conduction } \\
\text { through cable }\end{array}$ & EMF & Unknown & $\begin{array}{l}\text { Unknown, cable } \\
\text { shielding and burial } \\
\text { provides some } \\
\text { attenuation }\end{array}$ & $\begin{array}{l}\text { High if there is an } \\
\text { effect, it would } \\
\text { occur over life of } \\
\text { the project }\end{array}$ & Unknown & $\begin{array}{l}\text { Unknown; High } \\
\text { uncertainty about } \\
\text { effects on marine } \\
\text { mammals }\end{array}$ & $\begin{array}{l}\text { Boehlert et al. } \\
2008\end{array}$ \\
\hline \multirow{2}{*}{$\begin{array}{l}\text { Structures on } \\
\text { water's surface }\end{array}$} & Structure & Collision & $\begin{array}{l}\text { Low, no haulout } \\
\text { potential }\end{array}$ & $\begin{array}{l}\text { High, structure } \\
\text { present over life of } \\
\text { project }\end{array}$ & $\begin{array}{l}\text { Low, structure } \\
\text { easily avoided with } \\
\text { echolocation; } \\
\text { similar to existing } \\
\text { navigation buoys } \\
\end{array}$ & $\begin{array}{l}\text { Low; structure similar } \\
\text { to other structures in } \\
\text { Puget Sound; above } \\
\text { water structure } \\
\text { immobile }\end{array}$ & $\begin{array}{l}\text { Hatakeyama and } \\
\text { Soeda } 1990\end{array}$ \\
\hline & $\begin{array}{l}\text { Navigation } \\
\text { lights }\end{array}$ & $\begin{array}{l}\text { Visual disorientation; } \\
\text { could lure porpoises } \\
\text { into path of rotors if } \\
\text { prey attracted to } \\
\text { lights }\end{array}$ & $\begin{array}{l}\text { Low, device lights of } \\
\text { low intensity, } \\
\text { shielded, intended } \\
\text { for navigation safety }\end{array}$ & $\begin{array}{l}\text { High, lights } \\
\text { shining over life of } \\
\text { the project }\end{array}$ & $\begin{array}{l}\text { Low; area affected } \\
\text { small relative to } \\
\text { home range; may } \\
\text { enhance foraging on } \\
\text { prey attracted to } \\
\text { lights }\end{array}$ & $\begin{array}{l}\text { Low due to high } \\
\text { levels of shoreline } \\
\text { development, boat } \\
\text { traffic and industrial } \\
\text { activity in Sound }\end{array}$ & Todd et al. 2009 \\
\hline \multicolumn{8}{|c|}{ Decommissioning } \\
\hline & Direct impact & Collision injuries & $\begin{array}{l}\text { Med; low to } \\
\text { moderate increase in } \\
\text { already high levels of } \\
\text { existing boat traffic }\end{array}$ & $\begin{array}{l}\text { Low, occurring } \\
\text { over } 1 \text { to } 2 \\
\text { summers }\end{array}$ & $\begin{array}{l}\text { Low for adults; med } \\
\text { for calves due to } \\
\text { foraging and travel } \\
\text { in and near } \\
\text { construction and } \\
\text { transport zones }\end{array}$ & $\begin{array}{l}\text { Low; exposure area } \\
\text { small relative to home } \\
\text { range; avoidance } \\
\text { likely due to existing } \\
\text { boat traffic; boat } \\
\text { collisions with harbor } \\
\text { porpoise infrequent }\end{array}$ & $\begin{array}{l}\text { Stroud and } \\
\text { Roffe } 1979\end{array}$ \\
\hline
\end{tabular}

\begin{tabular}{|c|c|c|}
\hline $\begin{array}{l}\text { Environmental Assessment for Siting Wave \& Tidal } \\
\text { Energy Projects }\end{array}$ & F-24 & $\begin{array}{r}\text { H. T. Harvey \& Associates } \\
24 \text { November } 2009\end{array}$ \\
\hline
\end{tabular}


Appendix F - Effects of Pilot Scale MCT SeaGen Project at the Tacoma Narrows Site

\begin{tabular}{|c|c|c|c|c|c|c|c|}
\hline Project activity & Project action & $\begin{array}{l}\text { Description of } \\
\text { action's effect on } \\
\text { indicator }\end{array}$ & $\begin{array}{l}\text { Spatial exposure to } \\
\text { indicator (low, med, } \\
\text { high) }\end{array}$ & $\begin{array}{l}\text { Temporal } \\
\text { exposure to } \\
\text { indicator (low, } \\
\text { med, high) }\end{array}$ & $\begin{array}{l}\text { Effect's overlap } \\
\text { with critical life } \\
\text { stage, behavior, } \\
\text { habitat, or } \\
\text { resource (low, } \\
\text { med, high) }\end{array}$ & $\begin{array}{l}\text { Overall risk to } \\
\text { indicator (low, med, } \\
\text { high unknown }\end{array}$ & Source(s) \\
\hline \multirow[t]{2}{*}{ Boat traffic } & $\begin{array}{l}\text { Oil/chemical } \\
\text { release }\end{array}$ & $\begin{array}{l}\text { Ingestion, skin } \\
\text { fouling, breathing } \\
\text { exhaust fumes, } \\
\text { accumulation of } \\
\text { toxins. }\end{array}$ & $\begin{array}{l}\text { Low; increase in } \\
\text { traffic but low } \\
\text { volume of fluids } \\
\text { released and pre- } \\
\text { existing levels high }\end{array}$ & $\begin{array}{l}\text { Low, occurring } \\
\text { over } 1 \text { to } 2 \\
\text { summers }\end{array}$ & $\begin{array}{l}\text { Low; area of } \\
\text { potential exposure } \\
\text { small relative to } \\
\text { home range }\end{array}$ & $\begin{array}{l}\text { Low; inputs } \\
\text { insignificant relative } \\
\text { to background levels } \\
\text { in Puget Sound }\end{array}$ & $\begin{array}{l}\text { Ebbert et al. } \\
2000\end{array}$ \\
\hline & $\begin{array}{l}\text { Noise and } \\
\text { vibration }\end{array}$ & $\begin{array}{l}\text { Avoidance, masks } \\
\text { environmental cues. } \\
\text { TTS could increase } \\
\text { predation }\end{array}$ & $\begin{array}{l}\text { Med; moderate } \\
\text { increase in boat } \\
\text { traffic, pre-existing } \\
\text { noise levels expected } \\
\text { to be high }\end{array}$ & $\begin{array}{l}\text { Low, occurring } \\
\text { over } 1 \text { to } 2 \\
\text { summers }\end{array}$ & $\begin{array}{l}\text { Low for adults; med } \\
\text { for calves due to } \\
\text { foraging and travel } \\
\text { in construction and } \\
\text { transport zones }\end{array}$ & $\begin{array}{l}\text { Low; acclimation and } \\
\text { likely due to high } \\
\text { levels of boat traffic } \\
\text { noise in Puget Sound; } \\
\text { short overall duration }\end{array}$ & $\begin{array}{l}\text { Tougaard et al. } \\
2009\end{array}$ \\
\hline \multirow{3}{*}{$\begin{array}{l}\text { Decommissioning } \\
\text { and removal of } \\
\text { electrical } \\
\text { collector system, } \\
\text { subsea cable, } \\
\text { mooring cables, } \\
\text { foundations or } \\
\text { anchors, and } \\
\text { devices }\end{array}$} & $\begin{array}{l}\text { Noise and } \\
\text { vibration }\end{array}$ & $\begin{array}{l}\text { Avoidance, masks } \\
\text { environmental cues. } \\
\text { TTS, resulting in } \\
\text { potential increase in } \\
\text { predation }\end{array}$ & $\begin{array}{l}\text { Med; moderate } \\
\text { overall increase in } \\
\text { industrial activity, } \\
\text { pre-existing noise } \\
\text { levels expected to be } \\
\text { high }\end{array}$ & $\begin{array}{l}\text { Low, occurring } \\
\text { over } 1 \text { to } 2 \\
\text { summers }\end{array}$ & $\begin{array}{l}\text { Low for adults; med } \\
\text { for calves due to } \\
\text { foraging and travel } \\
\text { in and near } \\
\text { construction zone; }\end{array}$ & $\begin{array}{l}\text { Low; acclimation and } \\
\text { tolerance likely due } \\
\text { to high levels of pre- } \\
\text { existing boat traffic in } \\
\text { Puget Sound }\end{array}$ & $\begin{array}{l}\text { Blackwell et al. } \\
\text { 2004, Kastak et } \\
\text { al. 2005, Todd et } \\
\text { al. 2009, } \\
\text { Tougaard et al. } \\
2009\end{array}$ \\
\hline & $\begin{array}{l}\text { Deconstruction } \\
\text { lights, vessel } \\
\text { deck lights and } \\
\text { spotlights. }\end{array}$ & $\begin{array}{l}\text { Visual disorientation } \\
\text { could lead to collision, } \\
\text { could enhance foraging } \\
\text { on species attracted to } \\
\text { light }\end{array}$ & $\begin{array}{l}\text { Low; moderate } \\
\text { increase in existing } \\
\text { boat traffic, deck } \\
\text { lights illuminate only } \\
\text { a small area }\end{array}$ & $\begin{array}{l}\text { Low, occurring } \\
\text { over } 1 \text { to } 2 \\
\text { summers }\end{array}$ & $\begin{array}{l}\text { Low; Area affected } \\
\text { by lights small } \\
\text { relative to home } \\
\text { range }\end{array}$ & $\begin{array}{l}\text { Low; high levels of } \\
\text { shoreline } \\
\text { development, boat } \\
\text { traffic, and industry } \\
\text { in Puget Sound }\end{array}$ & Todd et al. 2009 \\
\hline & $\begin{array}{l}\text { Oil/chemical } \\
\text { release }\end{array}$ & $\begin{array}{l}\text { Ingestion, skin fouling, } \\
\text { breathing exhaust } \\
\text { fumes, accumulation } \\
\text { of toxins. Assumes no } \\
\text { catastrophic spills. }\end{array}$ & $\begin{array}{l}\text { Med, increase in use } \\
\text { of chemicals; volume } \\
\text { of fluids released } \\
\text { low, pre-existing } \\
\text { levels high }\end{array}$ & $\begin{array}{l}\text { Low, occurring } \\
\text { over } 1 \text { to } 2 \\
\text { summers }\end{array}$ & $\begin{array}{l}\text { Low to med; area of } \\
\text { potential exposure } \\
\text { is small relative to } \\
\text { home range }\end{array}$ & $\begin{array}{l}\text { Low; inputs } \\
\text { insignificant relative } \\
\text { to background levels } \\
\text { in Puget Sound }\end{array}$ & $\begin{array}{l}\text { Ebbert et al. } \\
2000\end{array}$ \\
\hline
\end{tabular}

\begin{tabular}{lrr}
\hline $\begin{array}{l}\text { Environmental Assessment for Siting Wave \& Tidal } \\
\text { Energy Projects }\end{array}$ & F-25 & H. T. Harvey \& Associates \\
24 November 2009
\end{tabular}


Appendix F - Effects of Pilot Scale MCT SeaGen Project at the Tacoma Narrows Site

Potential effects on sharks, skates, and rays due to Pilot Scale MCT SeaGen project at Tacoma Narrows

\begin{tabular}{|c|c|c|c|c|c|c|c|}
\hline Project activity & Action & $\begin{array}{l}\text { Description of action's } \\
\text { effect on indicator }\end{array}$ & $\begin{array}{l}\text { Spatial exposure to } \\
\text { indicator (low, med, } \\
\text { high) }\end{array}$ & $\begin{array}{l}\text { Temporal } \\
\text { exposure to } \\
\text { indicator (low, } \\
\text { med, high) }\end{array}$ & $\begin{array}{l}\text { Effect's overlap with } \\
\text { critical life stage, } \\
\text { behavior, habitat or } \\
\text { resource (low, med, } \\
\text { high) }\end{array}$ & $\begin{array}{l}\text { Overall risk } \\
\text { to indicator } \\
\text { (low, med, } \\
\text { high, } \\
\text { unknown) } \\
\end{array}$ & Source(s) \\
\hline \multicolumn{8}{|c|}{ Construction } \\
\hline \multirow{2}{*}{ Boat traffic } & $\begin{array}{l}\text { Oil/chemical } \\
\text { release }\end{array}$ & $\begin{array}{l}\text { Toxicity to individuals } \\
\text { and prey }\end{array}$ & $\begin{array}{l}\text { Low for typical small } \\
\text { volume releases, High } \\
\text { for large, catastrophic } \\
\text { spill }\end{array}$ & $\begin{array}{l}\text { Low, occurs over } 2 \\
\text { months }\end{array}$ & $\begin{array}{l}\text { Low, species in water } \\
\text { column but oil floats on } \\
\text { surface }\end{array}$ & Low & $\begin{array}{l}\text { Nelson and } \\
\text { Woo } 2008\end{array}$ \\
\hline & $\begin{array}{l}\text { Noise and } \\
\text { vibration }\end{array}$ & $\begin{array}{l}\text { Possible altered } \\
\text { behavior }\end{array}$ & $\begin{array}{l}\text { Low, would occur in } \\
\text { small area relative to } \\
\text { species' range }\end{array}$ & $\begin{array}{l}\text { Low, occurs over } 2 \\
\text { months }\end{array}$ & $\begin{array}{l}\text { Low, species present but } \\
\text { able to avoid } \\
\text { disturbance area }\end{array}$ & Low & Cada 2008 \\
\hline \multirow{4}{*}{$\begin{array}{l}\text { Construction of } \\
\text { electrical } \\
\text { collector system, } \\
\text { moorings and } \\
\text { foundations, and } \\
\text { device } \\
\text { installation }\end{array}$} & $\begin{array}{l}\text { Noise and } \\
\text { vibration }\end{array}$ & $\begin{array}{l}\text { Possible altered } \\
\text { behavior }\end{array}$ & Low, see above & $\begin{array}{l}\text { Low, occurs over } 2 \\
\text { months }\end{array}$ & $\begin{array}{l}\text { Low, species present but } \\
\text { able to avoid } \\
\text { disturbance area }\end{array}$ & Low & Cada 2008 \\
\hline & $\begin{array}{l}\text { Oil/chemical } \\
\text { release }\end{array}$ & $\begin{array}{l}\text { Toxicity to individuals } \\
\text { and prey }\end{array}$ & $\begin{array}{l}\text { Low, volume released } \\
\text { would occupy small } \\
\text { area }\end{array}$ & $\begin{array}{l}\text { Low, occurs over } 2 \\
\text { months }\end{array}$ & $\begin{array}{l}\text { Low, species in water } \\
\text { column but oil floats on } \\
\text { surface }\end{array}$ & Low & $\begin{array}{l}\text { Nelson and } \\
\text { Woo } 2008\end{array}$ \\
\hline & $\begin{array}{l}\text { Seabed } \\
\text { disturbance }\end{array}$ & $\begin{array}{l}\text { Temporary habitat loss, } \\
\text { changes in prey } \\
\text { resources }\end{array}$ & $\begin{array}{l}\text { Low, would occur in } \\
\text { small area relative to } \\
\text { species' range }\end{array}$ & $\begin{array}{l}\text { Low, occurs over } 2 \\
\text { months }\end{array}$ & $\begin{array}{l}\text { Low, species present but } \\
\text { able to avoid } \\
\text { disturbance area }\end{array}$ & Low & Nelson 2008 \\
\hline & $\begin{array}{l}\text { Construction } \\
\text { lights }\end{array}$ & $\begin{array}{l}\text { Sharks may be attracted } \\
\text { due to prey aggregation }\end{array}$ & Low, see above & $\begin{array}{l}\text { Low, occurs over } 2 \\
\text { months }\end{array}$ & $\begin{array}{l}\text { Med, if sharks attracted } \\
\text { to project area }\end{array}$ & Low & $\begin{array}{l}\text { Nightingale } \\
\text { et al. } 2006\end{array}$ \\
\hline \multirow{2}{*}{$\begin{array}{l}\text { Directional } \\
\text { drilling, and } \\
\text { laying cable } \\
\text { under/on seabed }\end{array}$} & $\begin{array}{l}\text { Seabed } \\
\text { disturbance }\end{array}$ & $\begin{array}{l}\text { Temporary habitat loss, } \\
\text { changes in prey } \\
\text { resources }\end{array}$ & Low, see above & $\begin{array}{l}\text { Low, occurs over } 2 \\
\text { months }\end{array}$ & $\begin{array}{l}\text { Low, species present but } \\
\text { able to avoid } \\
\text { disturbance area }\end{array}$ & Low & Nelson 2008 \\
\hline & $\begin{array}{l}\text { Noise and } \\
\text { vibration }\end{array}$ & $\begin{array}{l}\text { Possible altered } \\
\text { behavior }\end{array}$ & Low, see above & $\begin{array}{l}\text { Low, occurs over } 2 \\
\text { months }\end{array}$ & $\begin{array}{l}\text { Low, species present but } \\
\text { able to avoid } \\
\text { disturbance area }\end{array}$ & Low & Cada 2008 \\
\hline \multicolumn{8}{|c|}{ Operation and Maintenance } \\
\hline \multirow[t]{2}{*}{ Boat traffic } & $\begin{array}{l}\text { Oil/chemical } \\
\text { release }\end{array}$ & $\begin{array}{l}\text { Toxicity to individuals } \\
\text { and prey }\end{array}$ & $\begin{array}{l}\text { Low for typical small } \\
\text { volume releases, High } \\
\text { for large, catastrophic } \\
\text { spill }\end{array}$ & $\begin{array}{l}\text { Low, wouldn’t } \\
\text { persist long-term }\end{array}$ & $\begin{array}{l}\text { Low, species in water } \\
\text { column but oil floats on } \\
\text { surface }\end{array}$ & Low & $\begin{array}{l}\text { Nelson and } \\
\text { Woo } 2008\end{array}$ \\
\hline & $\begin{array}{l}\text { Noise and } \\
\text { vibration }\end{array}$ & $\begin{array}{l}\text { Possible altered } \\
\text { behavior }\end{array}$ & $\begin{array}{l}\text { Low, would occur in } \\
\text { small area relative to } \\
\text { species' range }\end{array}$ & $\begin{array}{l}\text { Low, wouldn't } \\
\text { persist long-term }\end{array}$ & $\begin{array}{l}\text { Low, species present but } \\
\text { able to avoid } \\
\text { disturbance area }\end{array}$ & Low & Cada 2008 \\
\hline $\begin{array}{l}\text { Operation of } \\
\text { turbines or other } \\
\text { moving parts of }\end{array}$ & $\begin{array}{l}\text { Moving device } \\
\text { parts }\end{array}$ & $\begin{array}{l}\text { Possible collision with } \\
\text { turbines }\end{array}$ & Low, see above & $\begin{array}{l}\text { High, would be } \\
\text { continuous for life } \\
\text { of project }\end{array}$ & $\begin{array}{l}\text { Med, species present in } \\
\text { project area }\end{array}$ & Unknown & Nelson 2008 \\
\hline
\end{tabular}

\begin{tabular}{|c|c|c|}
\hline $\begin{array}{l}\text { Environmental Assessment for Siting Wave \& Tidal } \\
\text { Energy Projects }\end{array}$ & F-26 & $\begin{array}{r}\text { H. T. Harvey \& Associates } \\
24 \text { November } 2009\end{array}$ \\
\hline
\end{tabular}


Appendix F - Effects of Pilot Scale MCT SeaGen Project at the Tacoma Narrows Site

\begin{tabular}{|c|c|c|c|c|c|c|c|}
\hline Project activity & Action & $\begin{array}{l}\text { Description of action's } \\
\text { effect on indicator }\end{array}$ & $\begin{array}{l}\text { Spatial exposure to } \\
\text { indicator (low, med, } \\
\text { high) }\end{array}$ & $\begin{array}{l}\text { Temporal } \\
\text { exposure to } \\
\text { indicator (low, } \\
\text { med, high) }\end{array}$ & $\begin{array}{l}\text { Effect's overlap with } \\
\text { critical life stage, } \\
\text { behavior, habitat or } \\
\text { resource (low, med, } \\
\text { high) }\end{array}$ & $\begin{array}{l}\text { Overall risk } \\
\text { to indicator } \\
\text { (low, med, } \\
\text { high, } \\
\text { unknown) } \\
\end{array}$ & Source(s) \\
\hline devices & $\begin{array}{l}\text { Noise and } \\
\text { vibration }\end{array}$ & $\begin{array}{l}\text { Possible altered } \\
\text { behavior }\end{array}$ & Low, see above & $\begin{array}{l}\text { High, would be } \\
\text { continuous for life } \\
\text { of project }\end{array}$ & $\begin{array}{l}\text { Low, species present but } \\
\text { able to avoid } \\
\text { disturbance area }\end{array}$ & Low & Cada 2008 \\
\hline \multirow{3}{*}{$\begin{array}{l}\text { Structures in } \\
\text { water column and } \\
\text { on seabed, such } \\
\text { as devices and } \\
\text { moorings and } \\
\text { footings }\end{array}$} & Structure & $\begin{array}{l}\text { None, prey of these } \\
\text { species not attracted to } \\
\text { subsurface structures }\end{array}$ & N/A & N/A & N/A & Low & \\
\hline & $\begin{array}{l}\text { Water circulation } \\
\text { changes }\end{array}$ & $\begin{array}{l}\text { None, project not large } \\
\text { enough to affect prey }\end{array}$ & N/A & N/A & N/A & Low & \\
\hline & $\begin{array}{l}\text { Oil/chemical } \\
\text { release }\end{array}$ & $\begin{array}{l}\text { Toxicity to individuals } \\
\text { and prey }\end{array}$ & $\begin{array}{l}\text { Low, would occur in } \\
\text { small area relative to } \\
\text { species' range }\end{array}$ & $\begin{array}{l}\text { Low, wouldn't } \\
\text { persist long-term }\end{array}$ & $\begin{array}{l}\text { Low, species in water } \\
\text { column while oil floats } \\
\text { on surface }\end{array}$ & Low & $\begin{array}{l}\text { Nelson and } \\
\text { Woo } 2008\end{array}$ \\
\hline $\begin{array}{l}\text { Electricity } \\
\text { conduction } \\
\text { through cable }\end{array}$ & EMF & $\begin{array}{l}\text { Possible changes in } \\
\text { orientation, behavior }\end{array}$ & Low, see above & $\begin{array}{l}\text { High, would be } \\
\text { continuous for life } \\
\text { of project }\end{array}$ & $\begin{array}{l}\text { Med, species present in } \\
\text { project area }\end{array}$ & Unknown & Nelson 2008 \\
\hline \multirow{2}{*}{$\begin{array}{l}\text { Structures on } \\
\text { water's surface }\end{array}$} & Structure & $\begin{array}{l}\text { Could attract prey (FAD } \\
\text { effect) and/or pinniped } \\
\text { haul-out and attract } \\
\text { sharks }\end{array}$ & Low, see above & $\begin{array}{l}\text { High, would be } \\
\text { continuous for life } \\
\text { of project }\end{array}$ & $\begin{array}{l}\text { Med, sharks present in } \\
\text { project area }\end{array}$ & Unknown & Nelson 2008 \\
\hline & Navigation lights & $\begin{array}{l}\text { Sharks may be attracted } \\
\text { due to prey aggregation }\end{array}$ & Low, see above & $\begin{array}{l}\text { High, would be } \\
\text { continuous for life } \\
\text { of project }\end{array}$ & $\begin{array}{l}\text { Med, sharks present in } \\
\text { project area }\end{array}$ & Unknown & $\begin{array}{l}\text { Nightingale } \\
\text { et al. } 2006\end{array}$ \\
\hline \multicolumn{8}{|c|}{ Decommissioning } \\
\hline \multirow[t]{2}{*}{ Boat traffic } & $\begin{array}{l}\text { Oil/chemical } \\
\text { release }\end{array}$ & $\begin{array}{l}\text { Toxicity to individuals } \\
\text { and prey }\end{array}$ & $\begin{array}{l}\text { Low for typical small } \\
\text { volume releases, High } \\
\text { for large, catastrophic } \\
\text { spill }\end{array}$ & $\begin{array}{l}\text { Low, occurs over } 2 \\
\text { months }\end{array}$ & $\begin{array}{l}\text { Low, species in water } \\
\text { column while oil floats } \\
\text { on surface }\end{array}$ & Low & $\begin{array}{l}\text { Nelson and } \\
\text { Woo } 2008\end{array}$ \\
\hline & $\begin{array}{l}\text { Noise and } \\
\text { vibration }\end{array}$ & $\begin{array}{l}\text { Possible altered } \\
\text { behavior }\end{array}$ & $\begin{array}{l}\text { Low, would occur in } \\
\text { small area relative to } \\
\text { species’ range }\end{array}$ & $\begin{array}{l}\text { Low, occurs over } 2 \\
\text { months }\end{array}$ & $\begin{array}{l}\text { Low, species present but } \\
\text { able to avoid } \\
\text { disturbance area }\end{array}$ & Low & Cada 2008 \\
\hline \multirow{2}{*}{$\begin{array}{l}\text { Decommissioning } \\
\text { of structures on } \\
\text { water's surface or } \\
\text { seabed }\end{array}$} & $\begin{array}{l}\text { Noise and } \\
\text { vibration }\end{array}$ & $\begin{array}{l}\text { Possible altered } \\
\text { behavior }\end{array}$ & Low, see above & $\begin{array}{l}\text { Low, occurs over } 2 \\
\text { months }\end{array}$ & $\begin{array}{l}\text { Low, species present but } \\
\text { able to avoid } \\
\text { disturbance area }\end{array}$ & Low & Cada 2008 \\
\hline & $\begin{array}{l}\text { Oil/chemical } \\
\text { release }\end{array}$ & $\begin{array}{l}\text { Toxicity to individuals } \\
\text { and prey }\end{array}$ & $\begin{array}{l}\text { Low, volume released } \\
\text { would occupy small } \\
\text { area }\end{array}$ & $\begin{array}{l}\text { Low, occurs over } 2 \\
\text { months }\end{array}$ & $\begin{array}{l}\text { Low, species in water } \\
\text { column while oil floats } \\
\text { on surface }\end{array}$ & Low & $\begin{array}{l}\text { Nelson and } \\
\text { Woo } 2008\end{array}$ \\
\hline
\end{tabular}

\begin{tabular}{|c|c|c|}
\hline $\begin{array}{l}\text { Environmental Assessment for Siting Wave \& Tidal } \\
\text { Energy Projects }\end{array}$ & F-27 & $\begin{array}{r}\text { H. T. Harvey \& Associates } \\
24 \text { November } 2009\end{array}$ \\
\hline
\end{tabular}


Appendix F - Effects of Pilot Scale MCT SeaGen Project at the Tacoma Narrows Site

\begin{tabular}{|c|c|c|c|c|c|c|c|}
\hline Project activity & Action & $\begin{array}{l}\text { Description of action's } \\
\text { effect on indicator }\end{array}$ & $\begin{array}{l}\text { Spatial exposure to } \\
\text { indicator (low, med, } \\
\text { high) }\end{array}$ & $\begin{array}{l}\text { Temporal } \\
\text { exposure to } \\
\text { indicator (low, } \\
\text { med, high) }\end{array}$ & $\begin{array}{l}\text { Effect's overlap with } \\
\text { critical life stage, } \\
\text { behavior, habitat or } \\
\text { resource (low, med, } \\
\text { high) }\end{array}$ & $\begin{array}{l}\text { Overall risk } \\
\text { to indicator } \\
\text { (low, med, } \\
\text { high, } \\
\text { unknown) } \\
\end{array}$ & Source(s) \\
\hline & $\begin{array}{l}\text { Seabed } \\
\text { disturbance }\end{array}$ & $\begin{array}{l}\text { Temporary habitat loss, } \\
\text { changes in prey } \\
\text { resources }\end{array}$ & $\begin{array}{l}\text { Low, would occur in } \\
\text { small area relative to } \\
\text { species' range }\end{array}$ & $\begin{array}{l}\text { Low, occurs over } 2 \\
\text { months }\end{array}$ & $\begin{array}{l}\text { Low, species present but } \\
\text { able to avoid } \\
\text { disturbance area }\end{array}$ & Low & \\
\hline & $\begin{array}{l}\text { Deconstruction } \\
\text { lights }\end{array}$ & $\begin{array}{l}\text { Sharks may be attracted } \\
\text { due to prey aggregation }\end{array}$ & Low, see above & $\begin{array}{l}\text { Low, occurs over } 2 \\
\text { months }\end{array}$ & $\begin{array}{l}\text { Med, sharks present in } \\
\text { project area }\end{array}$ & Low & $\begin{array}{l}\text { Nightingale } \\
\text { et al. } 2006\end{array}$ \\
\hline
\end{tabular}


Appendix F - Effects of Pilot Scale MCT SeaGen Project at the Tacoma Narrows Site

Potential effects on flatfishes due to Pilot Scale MCT SeaGen project at Tacoma Narrows

\begin{tabular}{|c|c|c|c|c|c|c|c|}
\hline Project activity & Action & $\begin{array}{l}\text { Description of action's } \\
\text { effect on indicator }\end{array}$ & $\begin{array}{l}\text { Spatial exposure to } \\
\text { indicator (low, med, } \\
\text { high) }\end{array}$ & $\begin{array}{l}\text { Temporal } \\
\text { exposure to } \\
\text { indicator (low, } \\
\text { med, high) }\end{array}$ & $\begin{array}{l}\text { Effect's overlap with } \\
\text { critical life stage, } \\
\text { behavior, habitat or } \\
\text { resource (low, med, } \\
\text { high) }\end{array}$ & $\begin{array}{l}\text { Overall risk } \\
\text { to indicator } \\
\text { (low, med, } \\
\text { high, } \\
\text { unknown) }\end{array}$ & Source(s) \\
\hline \multicolumn{8}{|c|}{ Construction } \\
\hline \multirow[t]{2}{*}{ Boat traffic } & $\begin{array}{l}\text { Oil/chemical } \\
\text { release }\end{array}$ & $\begin{array}{l}\text { Toxicity to individuals } \\
\text { and prey }\end{array}$ & $\begin{array}{l}\text { Low for typical small } \\
\text { volume releases, High } \\
\text { for large, catastrophic } \\
\text { spill }\end{array}$ & $\begin{array}{l}\text { Low, occurs over } 2 \\
\text { months }\end{array}$ & $\begin{array}{l}\text { Low, flatfishes on } \\
\text { bottom while oil floats } \\
\text { on surface }\end{array}$ & Low & $\begin{array}{l}\text { Nelson and } \\
\text { Woo } 2008\end{array}$ \\
\hline & $\begin{array}{l}\text { Noise and } \\
\text { vibration }\end{array}$ & $\begin{array}{l}\text { None, flatfishes not very } \\
\text { sensitive to sound }\end{array}$ & N/A & N/A & N/A & Low & $\begin{array}{l}\text { Nedwell et } \\
\text { al. } 2004\end{array}$ \\
\hline \multirow{4}{*}{$\begin{array}{l}\text { Construction of } \\
\text { electrical } \\
\text { collector system, } \\
\text { moorings and } \\
\text { foundations, and } \\
\text { device } \\
\text { installation }\end{array}$} & $\begin{array}{l}\text { Noise and } \\
\text { vibration }\end{array}$ & $\begin{array}{l}\text { None, flatfishes not very } \\
\text { sensitive to sound }\end{array}$ & N/A & N/A & N/A & Low & $\begin{array}{l}\text { Nedwell et } \\
\text { al. } 2004\end{array}$ \\
\hline & $\begin{array}{l}\text { Oil/chemical } \\
\text { release }\end{array}$ & $\begin{array}{l}\text { Toxicity to individuals } \\
\text { and prey }\end{array}$ & $\begin{array}{l}\text { Low, volume released } \\
\text { would occupy small } \\
\text { area }\end{array}$ & $\begin{array}{l}\text { Low, occurs over } 2 \\
\text { months }\end{array}$ & $\begin{array}{l}\text { Low, flatfishes on } \\
\text { bottom while oil floats } \\
\text { on surface }\end{array}$ & Low & $\begin{array}{l}\text { Nelson and } \\
\text { Woo } 2008\end{array}$ \\
\hline & $\begin{array}{l}\text { Seabed } \\
\text { disturbance }\end{array}$ & $\begin{array}{l}\text { Temporary habitat loss, } \\
\text { changes in prey } \\
\text { resources }\end{array}$ & $\begin{array}{l}\text { Low, would occur in } \\
\text { small area relative to } \\
\text { species’ range }\end{array}$ & $\begin{array}{l}\text { Low, occurs over } 2 \\
\text { months }\end{array}$ & $\begin{array}{l}\text { Low, flatfishes able to } \\
\text { avoid disturbance area }\end{array}$ & Low & Nelson 2008 \\
\hline & $\begin{array}{l}\text { Construction } \\
\text { lights }\end{array}$ & $\begin{array}{l}\text { None, flatfishes not } \\
\text { known to be affected by } \\
\text { lights }\end{array}$ & N/A & N/A & N/A & Low & $\begin{array}{l}\text { Nightingale } \\
\text { et al. } 2006\end{array}$ \\
\hline \multirow{2}{*}{$\begin{array}{l}\text { Directional } \\
\text { drilling, and } \\
\text { laying cable } \\
\text { under/on seabed }\end{array}$} & $\begin{array}{l}\text { Seabed } \\
\text { disturbance }\end{array}$ & $\begin{array}{l}\text { Temporary habitat loss, } \\
\text { changes in prey } \\
\text { resources }\end{array}$ & $\begin{array}{l}\text { Low, would occur in } \\
\text { small area relative to } \\
\text { species' range }\end{array}$ & $\begin{array}{l}\text { Low, occurs over } 2 \\
\text { months }\end{array}$ & $\begin{array}{l}\text { Low, flatfishes able to } \\
\text { avoid disturbance area }\end{array}$ & Low & Nelson 2008 \\
\hline & $\begin{array}{l}\text { Noise and } \\
\text { vibration }\end{array}$ & $\begin{array}{l}\text { None, flatfishes not very } \\
\text { sensitive to sound }\end{array}$ & N/A & N/A & N/A & Low & $\begin{array}{l}\text { Nedwell et } \\
\text { al. } 2004\end{array}$ \\
\hline \multicolumn{8}{|c|}{ Operation and Maintenance } \\
\hline \multirow[t]{2}{*}{ Boat traffic } & $\begin{array}{l}\text { Oil/chemical } \\
\text { release }\end{array}$ & $\begin{array}{l}\text { Toxicity to individuals } \\
\text { and prey }\end{array}$ & $\begin{array}{l}\text { Low for typical small } \\
\text { volume releases, High } \\
\text { for large, catastrophic } \\
\text { spill }\end{array}$ & $\begin{array}{l}\text { Low, wouldn't } \\
\text { persist long-term }\end{array}$ & $\begin{array}{l}\text { Low, flatfishes on } \\
\text { bottom while oil floats } \\
\text { on surface }\end{array}$ & Low & $\begin{array}{l}\text { Nelson and } \\
\text { Woo } 2008\end{array}$ \\
\hline & $\begin{array}{l}\text { Noise and } \\
\text { vibration }\end{array}$ & $\begin{array}{l}\text { None, flatfishes not very } \\
\text { sensitive to sound }\end{array}$ & N/A & N/A & N/A & Low & $\begin{array}{l}\text { Nedwell et } \\
\text { al. } 2004\end{array}$ \\
\hline \multirow{2}{*}{$\begin{array}{l}\text { Operation of } \\
\text { turbines or other } \\
\text { moving parts of } \\
\text { devices }\end{array}$} & $\begin{array}{l}\text { Moving device } \\
\text { parts }\end{array}$ & $\begin{array}{l}\text { None, flatfishes occur } \\
\text { on bottom, would not } \\
\text { collide with turbines }\end{array}$ & N/A & N/A & N/A & Low & N/A \\
\hline & $\begin{array}{l}\text { Noise and } \\
\text { vibration }\end{array}$ & $\begin{array}{l}\text { None, flatfishes not very } \\
\text { sensitive to sound }\end{array}$ & N/A & N/A & N/A & Low & $\begin{array}{l}\text { Nedwell et } \\
\text { al. } 2004\end{array}$ \\
\hline
\end{tabular}

\begin{tabular}{|c|c|c|}
\hline $\begin{array}{l}\text { Environmental Assessment for Siting Wave \& Tidal } \\
\text { Energy Projects }\end{array}$ & F-29 & $\begin{array}{r}\text { H. T. Harvey \& Associates } \\
24 \text { November } 2009\end{array}$ \\
\hline
\end{tabular}


Appendix F - Effects of Pilot Scale MCT SeaGen Project at the Tacoma Narrows Site

\begin{tabular}{|c|c|c|c|c|c|c|c|}
\hline Project activity & Action & $\begin{array}{l}\text { Description of action's } \\
\text { effect on indicator }\end{array}$ & $\begin{array}{l}\text { Spatial exposure to } \\
\text { indicator (low, med, } \\
\text { high) }\end{array}$ & $\begin{array}{l}\text { Temporal } \\
\text { exposure to } \\
\text { indicator (low, } \\
\text { med, high) }\end{array}$ & $\begin{array}{l}\text { Effect's overlap with } \\
\text { critical life stage, } \\
\text { behavior, habitat or } \\
\text { resource (low, med, } \\
\text { high) }\end{array}$ & $\begin{array}{l}\text { Overall risk } \\
\text { to indicator } \\
\text { (low, med, } \\
\text { high, } \\
\text { unknown) } \\
\end{array}$ & Source(s) \\
\hline \multirow{3}{*}{$\begin{array}{l}\text { Structures in } \\
\text { water column and } \\
\text { on seabed, such } \\
\text { as devices and } \\
\text { moorings and } \\
\text { footings }\end{array}$} & Structure & $\begin{array}{l}\text { Possible reef effect, } \\
\text { changes in predator/ } \\
\text { prey interactions }\end{array}$ & $\begin{array}{l}\text { Low, would occur in } \\
\text { small area relative to } \\
\text { species' range }\end{array}$ & $\begin{array}{l}\text { High, would be } \\
\text { continuous for life } \\
\text { of project }\end{array}$ & $\begin{array}{l}\text { Med, flatfishes in } \\
\text { sand/gravel habitat } \\
\text { where project located }\end{array}$ & Unknown & $\begin{array}{l}\text { Boehlert et } \\
\text { al. } 2008\end{array}$ \\
\hline & $\begin{array}{l}\text { Water circulation } \\
\text { changes }\end{array}$ & $\begin{array}{l}\text { None, project not large } \\
\text { enough to affect prey }\end{array}$ & N/A & N/A & N/A & Low & N/A \\
\hline & $\begin{array}{l}\text { Oil/chemical } \\
\text { release }\end{array}$ & $\begin{array}{l}\text { Toxicity to individuals } \\
\text { and prey }\end{array}$ & $\begin{array}{l}\text { Low, would occur in } \\
\text { small area relative to } \\
\text { species' range }\end{array}$ & $\begin{array}{l}\text { Low, wouldn’t } \\
\text { persist long-term }\end{array}$ & $\begin{array}{l}\text { Low, flatfishes on } \\
\text { bottom while oil floats } \\
\text { on surface }\end{array}$ & Low & $\begin{array}{l}\text { Nelson and } \\
\text { Woo } 2008\end{array}$ \\
\hline $\begin{array}{l}\text { Electricity } \\
\text { conduction } \\
\text { through cable }\end{array}$ & EMF & $\begin{array}{l}\text { Effects to flatfishes } \\
\text { unlikely, not known to } \\
\text { have sensitive } \\
\text { electroreceptive sense } \\
\text { organs }\end{array}$ & $\begin{array}{l}\text { Low, would occur in } \\
\text { small area relative to } \\
\text { species' range }\end{array}$ & $\begin{array}{l}\text { High, would be } \\
\text { continuous for life } \\
\text { of project }\end{array}$ & $\begin{array}{l}\text { Med, flatfishes in } \\
\text { sand/gravel bottom } \\
\text { habitat where project } \\
\text { located }\end{array}$ & Low & Nelson 2008 \\
\hline \multirow[t]{2}{*}{$\begin{array}{l}\text { Structures on } \\
\text { water's surface }\end{array}$} & Structure & $\begin{array}{l}\text { Possible FAD effect, } \\
\text { changes in predator/prey } \\
\text { interactions }\end{array}$ & Low, see above & $\begin{array}{l}\text { High, would be } \\
\text { continuous for life } \\
\text { of project }\end{array}$ & $\begin{array}{l}\text { Low, flatfishes on } \\
\text { bottom while effects on } \\
\text { surface }\end{array}$ & Low & $\begin{array}{l}\text { Boehlert et } \\
\text { al. } 2008\end{array}$ \\
\hline & Navigation lights & $\begin{array}{l}\text { None, flatfishes not } \\
\text { known to be affected by } \\
\text { lights }\end{array}$ & N/A & N/A & N/A & Low & $\begin{array}{l}\text { Nightingale } \\
\text { et al. } 2006\end{array}$ \\
\hline \multicolumn{8}{|c|}{ Decommissioning } \\
\hline \multirow[t]{2}{*}{ Boat traffic } & $\begin{array}{l}\text { Oil/chemical } \\
\text { release }\end{array}$ & $\begin{array}{l}\text { Toxicity to individuals } \\
\text { and prey }\end{array}$ & $\begin{array}{l}\text { Low for typical small } \\
\text { volume releases, High } \\
\text { for large, catastrophic } \\
\text { spill }\end{array}$ & $\begin{array}{l}\text { Low, occurs over } 2 \\
\text { months }\end{array}$ & $\begin{array}{l}\text { Low, flatfishes on } \\
\text { bottom while oil floats } \\
\text { on surface }\end{array}$ & Low & $\begin{array}{l}\text { Nelson and } \\
\text { Woo } 2008\end{array}$ \\
\hline & $\begin{array}{l}\text { Noise and } \\
\text { vibration }\end{array}$ & $\begin{array}{l}\text { None, flatfishes not very } \\
\text { sensitive to sound }\end{array}$ & N/A & N/A & N/A & Low & $\begin{array}{l}\text { Nedwell et } \\
\text { al. } 2004\end{array}$ \\
\hline \multirow{4}{*}{$\begin{array}{l}\text { Decommissioning } \\
\text { of structures on } \\
\text { water's surface or } \\
\text { seabed }\end{array}$} & $\begin{array}{l}\text { Noise and } \\
\text { vibration }\end{array}$ & $\begin{array}{l}\text { None, flatfishes not very } \\
\text { sensitive to sound }\end{array}$ & N/A & N/A & N/A & Low & $\begin{array}{l}\text { Nedwell et } \\
\text { al. } 2004\end{array}$ \\
\hline & $\begin{array}{l}\text { Oil/chemical } \\
\text { release }\end{array}$ & $\begin{array}{l}\text { Toxicity to individuals } \\
\text { and prey }\end{array}$ & $\begin{array}{l}\text { Low, volume released } \\
\text { would occupy small } \\
\text { area }\end{array}$ & $\begin{array}{l}\text { Low, occurs over } 2 \\
\text { months }\end{array}$ & $\begin{array}{l}\text { Low, flatfishes on } \\
\text { bottom while oil floats } \\
\text { on surface }\end{array}$ & Low & $\begin{array}{l}\text { Nelson and } \\
\text { Woo } 2008\end{array}$ \\
\hline & $\begin{array}{l}\text { Seabed } \\
\text { disturbance }\end{array}$ & $\begin{array}{l}\text { Temporary habitat loss, } \\
\text { changes in prey } \\
\text { resources }\end{array}$ & $\begin{array}{l}\text { Low, would occur in } \\
\text { small area relative to } \\
\text { species’ range }\end{array}$ & $\begin{array}{l}\text { Low, occurs over } 2 \\
\text { months }\end{array}$ & $\begin{array}{l}\text { Low, flatfishes able to } \\
\text { avoid disturbance area }\end{array}$ & Low & Nelson 2008 \\
\hline & $\begin{array}{l}\text { Deconstruction } \\
\text { lights }\end{array}$ & $\begin{array}{l}\text { None, flatfishes not } \\
\text { known to be affected by } \\
\text { lights }\end{array}$ & N/A & N/A & N/A & Low & $\begin{array}{l}\text { Nightingale } \\
\text { et al. } 2006\end{array}$ \\
\hline
\end{tabular}

\begin{tabular}{|c|c|c|}
\hline $\begin{array}{l}\text { Environmental Assessment for Siting Wave \& Tidal } \\
\text { Energy Projects }\end{array}$ & F-30 & $\begin{array}{r}\text { H. T. Harvey \& Associates } \\
24 \text { November } 2009\end{array}$ \\
\hline
\end{tabular}


Appendix F - Effects of Pilot Scale MCT SeaGen Project at the Tacoma Narrows Site

Potential effects on rockfishes, lingcod, and cabezon due to Pilot Scale MCT SeaGen project at Tacoma Narrows

\begin{tabular}{|c|c|c|c|c|c|c|c|}
\hline Project activity & Action & $\begin{array}{l}\text { Description of action's } \\
\text { effect on indicator }\end{array}$ & $\begin{array}{l}\text { Spatial exposure to } \\
\text { indicator (low, med, } \\
\text { high) }\end{array}$ & $\begin{array}{l}\text { Temporal } \\
\text { exposure to } \\
\text { indicator (low, } \\
\text { med, high) }\end{array}$ & $\begin{array}{l}\text { Effect's overlap with } \\
\text { critical life stage, } \\
\text { behavior, habitat or } \\
\text { resource (low, med, } \\
\text { high) }\end{array}$ & $\begin{array}{l}\text { Overall risk } \\
\text { to indicator } \\
\text { (low, med, } \\
\text { high, } \\
\text { unknown) }\end{array}$ & Source(s) \\
\hline \multicolumn{8}{|c|}{ Construction } \\
\hline \multirow[t]{2}{*}{ Boat traffic } & $\begin{array}{l}\text { Oil/chemical } \\
\text { release }\end{array}$ & $\begin{array}{l}\text { Toxicity to individuals } \\
\text { and prey }\end{array}$ & $\begin{array}{l}\text { Low for typical small } \\
\text { volume releases, High } \\
\text { for large, catastrophic } \\
\text { spill }\end{array}$ & $\begin{array}{l}\text { Low, occurs over } 2 \\
\text { months }\end{array}$ & $\begin{array}{l}\text { Low, species in water } \\
\text { column or bottom but } \\
\text { oil floats on surface }\end{array}$ & Low & $\begin{array}{l}\text { Nelson and } \\
\text { Woo } 2008\end{array}$ \\
\hline & $\begin{array}{l}\text { Noise and } \\
\text { vibration }\end{array}$ & $\begin{array}{l}\text { None, species not likely } \\
\text { to be affected }\end{array}$ & N/A & N/A & N/A & Low & N/A \\
\hline \multirow{4}{*}{$\begin{array}{l}\text { Construction of } \\
\text { electrical } \\
\text { collector system, } \\
\text { moorings and } \\
\text { foundations, and } \\
\text { device } \\
\text { installation }\end{array}$} & $\begin{array}{l}\text { Noise and } \\
\text { vibration }\end{array}$ & $\begin{array}{l}\text { None, species not } \\
\text { present in sand/gravel } \\
\text { bottom habitat where } \\
\text { project located }\end{array}$ & N/A & N/A & N/A & Low & N/A \\
\hline & $\begin{array}{l}\text { Oil/chemical } \\
\text { release }\end{array}$ & None (see above) & N/A & N/A & N/A & Low & N/A \\
\hline & $\begin{array}{l}\text { Seabed } \\
\text { disturbance }\end{array}$ & None (see above) & N/A & N/A & N/A & Low & N/A \\
\hline & $\begin{array}{l}\text { Construction } \\
\text { lights }\end{array}$ & None (see above) & N/A & N/A & N/A & Low & N/A \\
\hline \multirow{2}{*}{$\begin{array}{l}\text { Directional } \\
\text { drilling, and } \\
\text { laying cable } \\
\text { under/on seabed }\end{array}$} & $\begin{array}{l}\text { Seabed } \\
\text { disturbance }\end{array}$ & None (see above) & N/A & N/A & N/A & Low & N/A \\
\hline & $\begin{array}{l}\text { Noise and } \\
\text { vibration }\end{array}$ & None (see above) & N/A & N/A & N/A & Low & N/A \\
\hline \multicolumn{8}{|c|}{ Operation and Maintenance } \\
\hline \multirow[t]{2}{*}{ Boat traffic } & $\begin{array}{l}\text { Oil/chemical } \\
\text { release }\end{array}$ & $\begin{array}{l}\text { Toxicity to individuals } \\
\text { and prey }\end{array}$ & $\begin{array}{l}\text { Low for typical small } \\
\text { volume releases, High } \\
\text { for large, catastrophic } \\
\text { spill }\end{array}$ & $\begin{array}{l}\text { Low, wouldn't } \\
\text { persist long-term }\end{array}$ & $\begin{array}{l}\text { Low, species in water } \\
\text { column or bottom but } \\
\text { oil floats on surface }\end{array}$ & Low & $\begin{array}{l}\text { Nelson and } \\
\text { Woo } 2008\end{array}$ \\
\hline & $\begin{array}{l}\text { Noise and } \\
\text { vibration }\end{array}$ & $\begin{array}{l}\text { None, species not likely } \\
\text { to be affected }\end{array}$ & N/A & N/A & N/A & Low & N/A \\
\hline \multirow{2}{*}{$\begin{array}{l}\text { Operation of } \\
\text { turbines or other } \\
\text { moving parts of } \\
\text { devices }\end{array}$} & $\begin{array}{l}\text { Moving device } \\
\text { parts }\end{array}$ & $\begin{array}{l}\text { Collision with turbines } \\
\text { unlikely, rockfishes } \\
\text { occur on bottom }\end{array}$ & N/A & N/A & N/A & Low & \\
\hline & $\begin{array}{l}\text { Noise and } \\
\text { vibration }\end{array}$ & $\begin{array}{l}\text { Possible altered } \\
\text { behavior }\end{array}$ & $\begin{array}{l}\text { Low, would occur in } \\
\text { small area relative to } \\
\text { species' range }\end{array}$ & $\begin{array}{l}\text { High, would be } \\
\text { continuous for life } \\
\text { of project }\end{array}$ & $\begin{array}{l}\text { Med, structures may } \\
\text { attract species to project } \\
\text { area }\end{array}$ & Low & Cada 2008 \\
\hline
\end{tabular}

\begin{tabular}{|c|c|c|}
\hline $\begin{array}{l}\text { Environmental Assessment for Siting Wave \& Tidal } \\
\text { Energy Projects }\end{array}$ & F-31 & $\begin{array}{r}\text { H. T. Harvey \& Associates } \\
24 \text { November } 2009\end{array}$ \\
\hline
\end{tabular}


Appendix F - Effects of Pilot Scale MCT SeaGen Project at the Tacoma Narrows Site

\begin{tabular}{|c|c|c|c|c|c|c|c|}
\hline Project activity & Action & $\begin{array}{l}\text { Description of action's } \\
\text { effect on indicator }\end{array}$ & $\begin{array}{l}\text { Spatial exposure to } \\
\text { indicator (low, med, } \\
\text { high) }\end{array}$ & $\begin{array}{l}\text { Temporal } \\
\text { exposure to } \\
\text { indicator (low, } \\
\text { med, high) }\end{array}$ & $\begin{array}{l}\text { Effect's overlap with } \\
\text { critical life stage, } \\
\text { behavior, habitat or } \\
\text { resource (low, med, } \\
\text { high) }\end{array}$ & $\begin{array}{l}\text { Overall risk } \\
\text { to indicator } \\
\text { (low, med, } \\
\text { high, } \\
\text { unknown) }\end{array}$ & Source(s) \\
\hline \multirow{3}{*}{$\begin{array}{l}\text { Structures in } \\
\text { water column and } \\
\text { on seabed, such } \\
\text { as devices and } \\
\text { moorings and } \\
\text { footings }\end{array}$} & Structure & $\begin{array}{l}\text { Attraction to artificial } \\
\text { reef, changes in fish } \\
\text { community }\end{array}$ & Low, see above & $\begin{array}{l}\text { High, would be } \\
\text { continuous for life } \\
\text { of project }\end{array}$ & $\begin{array}{l}\text { Med, structures may } \\
\text { attract species to project } \\
\text { area }\end{array}$ & Unknown & Nelson 2008 \\
\hline & $\begin{array}{l}\text { Water circulation } \\
\text { changes }\end{array}$ & $\begin{array}{l}\text { None, project not large } \\
\text { enough to affect prey }\end{array}$ & N/A & N/A & N/A & Low & N/A \\
\hline & $\begin{array}{l}\text { Oil/chemical } \\
\text { release }\end{array}$ & $\begin{array}{l}\text { Toxicity to individuals } \\
\text { and prey }\end{array}$ & $\begin{array}{l}\text { Low, would occur in } \\
\text { small area relative to } \\
\text { species' range }\end{array}$ & $\begin{array}{l}\text { Low, wouldn’t } \\
\text { persist long-term }\end{array}$ & $\begin{array}{l}\text { Low, species in water } \\
\text { column or bottom but } \\
\text { oil floats on surface }\end{array}$ & Low & $\begin{array}{l}\text { Nelson and } \\
\text { Woo } 2008\end{array}$ \\
\hline $\begin{array}{l}\text { Electricity } \\
\text { conduction } \\
\text { through cable }\end{array}$ & EMF & $\begin{array}{l}\text { Effects unlikely, species } \\
\text { not known to have } \\
\text { sensitive } \\
\text { electroreceptive sense } \\
\text { organs }\end{array}$ & Low, see above & $\begin{array}{l}\text { High, would be } \\
\text { continuous for life } \\
\text { of project }\end{array}$ & $\begin{array}{l}\text { Med, species may be } \\
\text { present due to attraction } \\
\text { to structure }\end{array}$ & Low & Nelson 2008 \\
\hline \multirow{2}{*}{$\begin{array}{l}\text { Structures on } \\
\text { water's surface }\end{array}$} & Structure & $\begin{array}{l}\text { Possible FAD effect, } \\
\text { changes in predator/prey } \\
\text { interactions }\end{array}$ & Low, see above & $\begin{array}{l}\text { High, would be } \\
\text { continuous for life } \\
\text { of project }\end{array}$ & $\begin{array}{l}\text { Low, species in water } \\
\text { column or bottom }\end{array}$ & Unknown & N/A \\
\hline & Navigation lights & $\begin{array}{l}\text { None, species don’t } \\
\text { occur at surface }\end{array}$ & N/A & N/A & N/A & Low & N/A \\
\hline \multicolumn{8}{|c|}{ Decommissioning } \\
\hline \multirow[t]{2}{*}{ Boat traffic } & $\begin{array}{l}\text { Oil/chemical } \\
\text { release }\end{array}$ & $\begin{array}{l}\text { Toxicity to individuals } \\
\text { and prey }\end{array}$ & $\begin{array}{l}\text { Low for typical small } \\
\text { volume releases, High } \\
\text { for large, catastrophic } \\
\text { spill }\end{array}$ & $\begin{array}{l}\text { Low, occurs over } 2 \\
\text { months }\end{array}$ & $\begin{array}{l}\text { Low, species in water } \\
\text { column or bottom but } \\
\text { oil floats on surface }\end{array}$ & Low & $\begin{array}{l}\text { Nelson and } \\
\text { Woo } 2008\end{array}$ \\
\hline & $\begin{array}{l}\text { Noise and } \\
\text { vibration }\end{array}$ & $\begin{array}{l}\text { None, species not likely } \\
\text { to be affected }\end{array}$ & N/A & N/A & N/A & Low & N/A \\
\hline \multirow{4}{*}{$\begin{array}{l}\text { Decommissioning } \\
\text { of structures on } \\
\text { water's surface or } \\
\text { seabed }\end{array}$} & $\begin{array}{l}\text { Noise and } \\
\text { vibration }\end{array}$ & $\begin{array}{l}\text { Possible altered } \\
\text { behavior }\end{array}$ & $\begin{array}{l}\text { Low, would occur in } \\
\text { small area relative to } \\
\text { species’ range }\end{array}$ & $\begin{array}{l}\text { Low, occurs over } 2 \\
\text { months }\end{array}$ & $\begin{array}{l}\text { Low, species may be } \\
\text { present due to attraction } \\
\text { to structure }\end{array}$ & Low & Cada 2008 \\
\hline & $\begin{array}{l}\text { Oil/chemical } \\
\text { release }\end{array}$ & $\begin{array}{l}\text { Toxicity to individuals } \\
\text { and prey }\end{array}$ & $\begin{array}{l}\text { Low, volume released } \\
\text { would occupy small } \\
\text { area }\end{array}$ & $\begin{array}{l}\text { Low, occurs over } 2 \\
\text { months }\end{array}$ & $\begin{array}{l}\text { Low, species in water } \\
\text { column or bottom but } \\
\text { oil floats on surface }\end{array}$ & Low & $\begin{array}{l}\text { Nelson and } \\
\text { Woo } 2008\end{array}$ \\
\hline & $\begin{array}{l}\text { Seabed } \\
\text { disturbance }\end{array}$ & $\begin{array}{l}\text { Habitat loss, changes in } \\
\text { prey resources }\end{array}$ & $\begin{array}{l}\text { Low, would occur in } \\
\text { small area relative to } \\
\text { species' range }\end{array}$ & $\begin{array}{l}\text { Low, occurs over } 2 \\
\text { months }\end{array}$ & $\begin{array}{l}\text { Low, species may be } \\
\text { present due to attraction } \\
\text { to structure }\end{array}$ & Low & Nelson 2008 \\
\hline & $\begin{array}{l}\text { Deconstruction } \\
\text { lights }\end{array}$ & $\begin{array}{l}\text { None, species don’t } \\
\text { occur at surface }\end{array}$ & N/A & N/A & N/A & Low & N/A \\
\hline
\end{tabular}

\begin{tabular}{|c|c|c|}
\hline $\begin{array}{l}\text { Environmental Assessment for Siting Wave \& Tidal } \\
\text { Energy Projects }\end{array}$ & F-32 & $\begin{array}{r}\text { H. T. Harvey \& Associates } \\
24 \text { November } 2009\end{array}$ \\
\hline
\end{tabular}


Appendix F - Effects of Pilot Scale MCT SeaGen Project at the Tacoma Narrows Site

Potential effects on forage fishes (herring, surf smelt, sand lance, and northern anchovy) due to Pilot Scale MCT SeaGen project at Tacoma Narrows

\begin{tabular}{|c|c|c|c|c|c|c|c|}
\hline Project activity & Action & $\begin{array}{l}\text { Description of action's } \\
\text { effect on indicator }\end{array}$ & $\begin{array}{l}\text { Spatial exposure to } \\
\text { indicator (low, med, } \\
\text { high) }\end{array}$ & $\begin{array}{l}\text { Temporal } \\
\text { exposure to } \\
\text { indicator (low, } \\
\text { med, high) }\end{array}$ & $\begin{array}{l}\text { Effect's overlap with } \\
\text { critical life stage, } \\
\text { behavior, habitat or } \\
\text { resource (low, med, } \\
\text { high) }\end{array}$ & $\begin{array}{l}\text { Overall risk } \\
\text { to indicator } \\
\text { (low, med, } \\
\text { high, } \\
\text { unknown) }\end{array}$ & Source(s) \\
\hline \multicolumn{8}{|c|}{ Construction } \\
\hline \multirow[t]{2}{*}{ Boat traffic } & $\begin{array}{l}\text { Oil/chemical } \\
\text { release }\end{array}$ & $\begin{array}{l}\text { Toxicity to eggs at } \\
\text { spawning grounds }\end{array}$ & $\begin{array}{l}\text { Low for typical small } \\
\text { volume releases, High } \\
\text { for large, catastrophic } \\
\text { spill }\end{array}$ & $\begin{array}{l}\text { Low, occurs over } 2 \\
\text { months }\end{array}$ & $\begin{array}{l}\text { High, spawning occurs } \\
\text { at nearby beaches, } \\
\text { intertidal, and subtidal } \\
\text { zones }\end{array}$ & Low & $\begin{array}{l}\text { Nelson and } \\
\text { Woo 2008, } \\
\text { Penttila 2007 }\end{array}$ \\
\hline & $\begin{array}{l}\text { Noise and } \\
\text { vibration }\end{array}$ & $\begin{array}{l}\text { None, species too motile } \\
\text { to be affected }\end{array}$ & N/A & N/A & N/A & Low & N/A \\
\hline \multirow{4}{*}{$\begin{array}{l}\text { Construction of } \\
\text { electrical } \\
\text { collector system, } \\
\text { moorings and } \\
\text { foundations, and } \\
\text { device } \\
\text { installation }\end{array}$} & $\begin{array}{l}\text { Noise and } \\
\text { vibration }\end{array}$ & $\begin{array}{l}\text { Possible altered } \\
\text { behavior }\end{array}$ & $\begin{array}{l}\text { Low, would occur in } \\
\text { small area relative to } \\
\text { species' range }\end{array}$ & $\begin{array}{l}\text { Low, occurs over } 2 \\
\text { months }\end{array}$ & $\begin{array}{l}\text { Low, fish have patchy } \\
\text { distribution and able to } \\
\text { avoid disturbance area }\end{array}$ & Low & Cada 2008 \\
\hline & $\begin{array}{l}\text { Oil/chemical } \\
\text { release }\end{array}$ & $\begin{array}{l}\text { Toxicity to individuals } \\
\text { and prey }\end{array}$ & $\begin{array}{l}\text { Low, volume released } \\
\text { would occupy small } \\
\text { area }\end{array}$ & $\begin{array}{l}\text { Low, occurs over } 2 \\
\text { months }\end{array}$ & $\begin{array}{l}\text { High, spawning occurs } \\
\text { at nearby beaches, } \\
\text { intertidal, and subtidal } \\
\text { zones }\end{array}$ & Low & $\begin{array}{l}\text { Nelson and } \\
\text { Woo 2008, } \\
\text { Penttila 2007 }\end{array}$ \\
\hline & $\begin{array}{l}\text { Seabed } \\
\text { disturbance }\end{array}$ & $\begin{array}{l}\text { Temporary habitat loss, } \\
\text { changes in prey } \\
\text { resources }\end{array}$ & $\begin{array}{l}\text { Low, would occur in } \\
\text { small area relative to } \\
\text { species' range }\end{array}$ & $\begin{array}{l}\text { Low, occurs over } 2 \\
\text { months }\end{array}$ & $\begin{array}{l}\text { Low, fish have patchy } \\
\text { distribution and able to } \\
\text { avoid disturbance area }\end{array}$ & Low & \\
\hline & $\begin{array}{l}\text { Construction } \\
\text { lights }\end{array}$ & $\begin{array}{l}\text { Aggregation, more } \\
\text { vulnerable to predation }\end{array}$ & $\begin{array}{l}\text { Low, would occur in } \\
\text { small area relative to } \\
\text { species' range }\end{array}$ & $\begin{array}{l}\text { Low, occurs over } 2 \\
\text { months }\end{array}$ & $\begin{array}{l}\text { Low, species } \\
\text { distribution patchy }\end{array}$ & Low & $\begin{array}{l}\text { Nightingale } \\
\text { et al. } 2006\end{array}$ \\
\hline \multirow{2}{*}{$\begin{array}{l}\text { Directional } \\
\text { drilling, and } \\
\text { laying cable } \\
\text { under/on seabed }\end{array}$} & $\begin{array}{l}\text { Seabed } \\
\text { disturbance }\end{array}$ & $\begin{array}{l}\text { Temporary habitat loss, } \\
\text { changes in prey } \\
\text { resources }\end{array}$ & $\begin{array}{l}\text { Low, would occur in } \\
\text { small area relative to } \\
\text { species' range }\end{array}$ & $\begin{array}{l}\text { Low, occurs over } 2 \\
\text { months }\end{array}$ & $\begin{array}{l}\text { Low, fish have patchy } \\
\text { distribution and able to } \\
\text { avoid disturbance area }\end{array}$ & Low & \\
\hline & $\begin{array}{l}\text { Noise and } \\
\text { vibration }\end{array}$ & $\begin{array}{l}\text { Possible altered } \\
\text { behavior }\end{array}$ & $\begin{array}{l}\text { Low, would occur in } \\
\text { small area relative to } \\
\text { species' range }\end{array}$ & $\begin{array}{l}\text { Low, occurs over } 2 \\
\text { months }\end{array}$ & $\begin{array}{l}\text { Low, fish have patchy } \\
\text { distribution and able to } \\
\text { avoid disturbance area }\end{array}$ & Low & Cada 2008 \\
\hline \multicolumn{8}{|c|}{ Operation and Maintenance } \\
\hline \multirow[t]{2}{*}{ Boat traffic } & $\begin{array}{l}\text { Oil/chemical } \\
\text { release }\end{array}$ & $\begin{array}{l}\text { Toxicity to individuals } \\
\text { and prey }\end{array}$ & $\begin{array}{l}\text { Low for typical small } \\
\text { volume releases, High } \\
\text { for large, catastrophic } \\
\text { spill }\end{array}$ & $\begin{array}{l}\text { Low, wouldn't } \\
\text { persist long-term }\end{array}$ & $\begin{array}{l}\text { High, spawning occurs } \\
\text { at nearby beaches, } \\
\text { intertidal, and subtidal } \\
\text { zones }\end{array}$ & Low & $\begin{array}{l}\text { Nelson and } \\
\text { Woo } 2008 \\
\text { Penttila } 2007\end{array}$ \\
\hline & $\begin{array}{l}\text { Noise and } \\
\text { vibration }\end{array}$ & $\begin{array}{l}\text { None, species too motile } \\
\text { to be affected }\end{array}$ & N/A & N/A & N/A & Low & N/A \\
\hline
\end{tabular}

\begin{tabular}{|c|c|c|}
\hline $\begin{array}{l}\text { Environmental Assessment for Siting Wave \& Tidal } \\
\text { Energy Projects }\end{array}$ & F-33 & $\begin{array}{r}\text { H. T. Harvey \& Associates } \\
24 \text { November } 2009\end{array}$ \\
\hline
\end{tabular}


Appendix F - Effects of Pilot Scale MCT SeaGen Project at the Tacoma Narrows Site

\begin{tabular}{|c|c|c|c|c|c|c|c|}
\hline Project activity & Action & $\begin{array}{l}\text { Description of action's } \\
\text { effect on indicator }\end{array}$ & $\begin{array}{l}\text { Spatial exposure to } \\
\text { indicator (low, med, } \\
\text { high) }\end{array}$ & $\begin{array}{l}\text { Temporal } \\
\text { exposure to } \\
\text { indicator (low, } \\
\text { med, high) }\end{array}$ & $\begin{array}{l}\text { Effect's overlap with } \\
\text { critical life stage, } \\
\text { behavior, habitat or } \\
\text { resource (low, med, } \\
\text { high) }\end{array}$ & $\begin{array}{l}\text { Overall risk } \\
\text { to indicator } \\
\text { (low, med, } \\
\text { high, } \\
\text { unknown) } \\
\end{array}$ & Source(s) \\
\hline \multirow{2}{*}{$\begin{array}{l}\text { Operation of } \\
\text { turbines or other } \\
\text { moving parts of } \\
\text { devices }\end{array}$} & $\begin{array}{l}\text { Moving device } \\
\text { parts }\end{array}$ & Collision with turbines & $\begin{array}{l}\text { Low, would occur in } \\
\text { small area relative to } \\
\text { species' range }\end{array}$ & $\begin{array}{l}\text { High, would be } \\
\text { continuous for life } \\
\text { of project }\end{array}$ & $\begin{array}{l}\text { Med, species } \\
\text { distribution patchy but } \\
\text { does occur at } 15 \mathrm{~m} \\
\text { depth of turbines }\end{array}$ & Low & $\begin{array}{l}\text { Cardinale et } \\
\text { al. 2003, } \\
\text { Emmett et al. } \\
2004\end{array}$ \\
\hline & $\begin{array}{l}\text { Noise and } \\
\text { vibration }\end{array}$ & $\begin{array}{l}\text { Possible altered } \\
\text { behavior }\end{array}$ & $\begin{array}{l}\text { Low, would occur in } \\
\text { small area relative to } \\
\text { species' range }\end{array}$ & $\begin{array}{l}\text { High, would be } \\
\text { continuous for life } \\
\text { of project }\end{array}$ & $\begin{array}{l}\text { Low, fish have patchy } \\
\text { distribution and able to } \\
\text { avoid disturbance area }\end{array}$ & Low & Cada 2008 \\
\hline \multirow{3}{*}{$\begin{array}{l}\text { Structures in } \\
\text { water column and } \\
\text { on seabed, such } \\
\text { as devices and } \\
\text { moorings and } \\
\text { footings }\end{array}$} & Structure & $\begin{array}{l}\text { Reef effect, changes in } \\
\text { predator/prey } \\
\text { interactions }\end{array}$ & $\begin{array}{l}\text { Low, would occur in } \\
\text { small area relative to } \\
\text { species' range }\end{array}$ & $\begin{array}{l}\text { High, would be } \\
\text { continuous for life } \\
\text { of project }\end{array}$ & $\begin{array}{l}\text { Low, species } \\
\text { distribution patchy }\end{array}$ & Low & Nelson 2008 \\
\hline & $\begin{array}{l}\text { Water circulation } \\
\text { changes }\end{array}$ & $\begin{array}{l}\text { None, project not large } \\
\text { enough to affect prey }\end{array}$ & N/A & N/A & N/A & Low & N/A \\
\hline & $\begin{array}{l}\text { Oil/chemical } \\
\text { release }\end{array}$ & $\begin{array}{l}\text { Toxicity to individuals } \\
\text { and prey }\end{array}$ & $\begin{array}{l}\text { Low, would occur in } \\
\text { small area relative to } \\
\text { species' range }\end{array}$ & $\begin{array}{l}\text { Low, wouldn't } \\
\text { persist long-term }\end{array}$ & $\begin{array}{l}\text { High, spawning occurs } \\
\text { at nearby beaches, } \\
\text { intertidal, and subtidal } \\
\text { zones }\end{array}$ & Low & $\begin{array}{l}\text { Nelson and } \\
\text { Woo 2008, } \\
\text { Penttila 2007 }\end{array}$ \\
\hline $\begin{array}{l}\text { Electricity } \\
\text { conduction } \\
\text { through cable }\end{array}$ & EMF & $\begin{array}{l}\text { Effects unlikely, species } \\
\text { not known to have } \\
\text { sensitive } \\
\text { electroreceptive sense } \\
\text { organs }\end{array}$ & $\begin{array}{l}\text { Low, would occur in } \\
\text { small area relative to } \\
\text { species' range }\end{array}$ & $\begin{array}{l}\text { High, would be } \\
\text { continuous for life } \\
\text { of project }\end{array}$ & $\begin{array}{l}\text { Low, species } \\
\text { distribution patchy }\end{array}$ & Low & Nelson 2008 \\
\hline \multirow{2}{*}{$\begin{array}{l}\text { Structures on } \\
\text { water's surface }\end{array}$} & Structure & $\begin{array}{l}\text { FAD effect, changes in } \\
\text { predator/prey } \\
\text { interactions }\end{array}$ & $\begin{array}{l}\text { Low, would occur in } \\
\text { small area relative to } \\
\text { species' range }\end{array}$ & $\begin{array}{l}\text { High, would be } \\
\text { continuous for life } \\
\text { of project }\end{array}$ & $\begin{array}{l}\text { Low, species } \\
\text { distribution patchy }\end{array}$ & Low & Nelson 2008 \\
\hline & Navigation lights & $\begin{array}{l}\text { Aggregation, more } \\
\text { vulnerable to predation }\end{array}$ & $\begin{array}{l}\text { Low, would occur in } \\
\text { small area relative to } \\
\text { species' range }\end{array}$ & $\begin{array}{l}\text { High, would be } \\
\text { continuous for life } \\
\text { of project }\end{array}$ & $\begin{array}{l}\text { Low, species } \\
\text { distribution patchy }\end{array}$ & Low & $\begin{array}{l}\text { Nightingale } \\
\text { et al. } 2006\end{array}$ \\
\hline \multicolumn{8}{|c|}{ Decommissioning } \\
\hline \multirow[t]{2}{*}{ Boat traffic } & $\begin{array}{l}\text { Oil/chemical } \\
\text { release }\end{array}$ & $\begin{array}{l}\text { Toxicity to individuals } \\
\text { and prey }\end{array}$ & $\begin{array}{l}\text { Low for typical small } \\
\text { volume releases, High } \\
\text { for large, catastrophic } \\
\text { spill }\end{array}$ & $\begin{array}{l}\text { Low, occurs over } 2 \\
\text { months }\end{array}$ & $\begin{array}{l}\text { High, spawning occurs } \\
\text { at nearby beaches, } \\
\text { intertidal, and subtidal } \\
\text { zones }\end{array}$ & Low & $\begin{array}{l}\text { Nelson and } \\
\text { Woo 2008, } \\
\text { Penttila 2007 }\end{array}$ \\
\hline & $\begin{array}{l}\text { Noise and } \\
\text { vibration }\end{array}$ & $\begin{array}{l}\text { None, species too motile } \\
\text { to be affected }\end{array}$ & N/A & N/A & N/A & Low & N/A \\
\hline $\begin{array}{l}\text { Decommissioning } \\
\text { of structures on } \\
\text { water's surface or }\end{array}$ & $\begin{array}{l}\text { Noise and } \\
\text { vibration }\end{array}$ & $\begin{array}{l}\text { Possible altered } \\
\text { behavior }\end{array}$ & $\begin{array}{l}\text { Low, would occur in } \\
\text { small area relative to } \\
\text { species' range }\end{array}$ & $\begin{array}{l}\text { Low, occurs over } 2 \\
\text { months }\end{array}$ & $\begin{array}{l}\text { Low, fish have patchy } \\
\text { distribution and able to } \\
\text { avoid disturbance area }\end{array}$ & Low & Cada 2008 \\
\hline
\end{tabular}

\begin{tabular}{|c|c|c|}
\hline $\begin{array}{l}\text { Environmental Assessment for Siting Wave \& Tidal } \\
\text { Energy Projects }\end{array}$ & F-34 & $\begin{array}{r}\text { H. T. Harvey \& Associates } \\
24 \text { November } 2009\end{array}$ \\
\hline
\end{tabular}


Appendix F - Effects of Pilot Scale MCT SeaGen Project at the Tacoma Narrows Site

\begin{tabular}{|c|c|c|c|c|c|c|c|}
\hline Project activity & Action & $\begin{array}{l}\text { Description of action's } \\
\text { effect on indicator }\end{array}$ & $\begin{array}{l}\text { Spatial exposure to } \\
\text { indicator (low, med, } \\
\text { high) }\end{array}$ & $\begin{array}{l}\text { Temporal } \\
\text { exposure to } \\
\text { indicator (low, } \\
\text { med, high) }\end{array}$ & $\begin{array}{l}\text { Effect's overlap with } \\
\text { critical life stage, } \\
\text { behavior, habitat or } \\
\text { resource (low, med, } \\
\text { high) }\end{array}$ & $\begin{array}{l}\text { Overall risk } \\
\text { to indicator } \\
\text { (low, med, } \\
\text { high, } \\
\text { unknown) } \\
\end{array}$ & Source(s) \\
\hline \multirow[t]{3}{*}{ seabed } & $\begin{array}{l}\text { Oil/chemical } \\
\text { release }\end{array}$ & $\begin{array}{l}\text { Toxicity to individuals } \\
\text { and prey }\end{array}$ & $\begin{array}{l}\text { Low, volume released } \\
\text { would occupy small } \\
\text { area }\end{array}$ & $\begin{array}{l}\text { Low, occurs over } 2 \\
\text { months }\end{array}$ & $\begin{array}{l}\text { High, spawning occurs } \\
\text { at nearby beaches } \\
\text { intertidal, and subtidal } \\
\text { zones }\end{array}$ & Low & $\begin{array}{l}\text { Nelson and } \\
\text { Woo 2008, } \\
\text { Penttila 2007 }\end{array}$ \\
\hline & $\begin{array}{l}\text { Seabed } \\
\text { disturbance }\end{array}$ & $\begin{array}{l}\text { None, species too motile } \\
\text { and near surface to be } \\
\text { affected }\end{array}$ & N/A & N/A & N/A & Low & N/A \\
\hline & $\begin{array}{l}\text { Deconstruction } \\
\text { lights }\end{array}$ & $\begin{array}{l}\text { Aggregation, more } \\
\text { vulnerable to predation }\end{array}$ & $\begin{array}{l}\text { Low, would occur in } \\
\text { small area relative to } \\
\text { species' range }\end{array}$ & $\begin{array}{l}\text { Low, occurs over } 2 \\
\text { months }\end{array}$ & $\begin{array}{l}\text { Low, species } \\
\text { distribution patchy }\end{array}$ & Low & $\begin{array}{l}\text { Nightingale } \\
\text { et al. } 2006\end{array}$ \\
\hline
\end{tabular}


Appendix F - Effects of Pilot Scale MCT SeaGen Project at the Tacoma Narrows Site

Potential effects on juvenile salmonids due to Pilot Scale MCT SeaGen project at Tacoma Narrows

\begin{tabular}{|c|c|c|c|c|c|c|c|}
\hline Project activity & Action & $\begin{array}{l}\text { Description of action's } \\
\text { effect on indicator }\end{array}$ & $\begin{array}{l}\text { Spatial exposure to } \\
\text { indicator (low, med, } \\
\text { high) }\end{array}$ & $\begin{array}{l}\text { Temporal } \\
\text { exposure to } \\
\text { indicator (low, } \\
\text { med, high) }\end{array}$ & $\begin{array}{l}\text { Effect's overlap with } \\
\text { critical life stage, } \\
\text { behavior, habitat or } \\
\text { resource (low, med, } \\
\text { high) }\end{array}$ & $\begin{array}{l}\text { Overall risk } \\
\text { to indicator } \\
\text { (low, med, } \\
\text { high, } \\
\text { unknown) }\end{array}$ & Source(s) \\
\hline \multicolumn{8}{|c|}{ Construction } \\
\hline \multirow[t]{2}{*}{ Boat traffic } & $\begin{array}{l}\text { Oil/chemical } \\
\text { release }\end{array}$ & $\begin{array}{l}\text { Toxicity to individuals } \\
\text { and prey }\end{array}$ & $\begin{array}{l}\text { Low for typical small } \\
\text { volume releases, High } \\
\text { for large, catastrophic } \\
\text { spill }\end{array}$ & $\begin{array}{l}\text { Low, occurs over } 2 \\
\text { months }\end{array}$ & $\begin{array}{l}\text { Low, species in water } \\
\text { column but oil floats on } \\
\text { surface }\end{array}$ & Low & $\begin{array}{l}\text { Nelson and } \\
\text { Woo } 2008\end{array}$ \\
\hline & $\begin{array}{l}\text { Noise and } \\
\text { vibration }\end{array}$ & $\begin{array}{l}\text { Possible altered } \\
\text { behavior }\end{array}$ & $\begin{array}{l}\text { Low, would occur in } \\
\text { small area relative to } \\
\text { species' range }\end{array}$ & $\begin{array}{l}\text { Low, occurs over } 2 \\
\text { months }\end{array}$ & $\begin{array}{l}\text { Low, species present but } \\
\text { able to avoid } \\
\text { disturbance area }\end{array}$ & Low & $\begin{array}{l}\text { Cada 2008, } \\
\text { Nelson } 2008\end{array}$ \\
\hline \multirow{4}{*}{$\begin{array}{l}\text { Construction of } \\
\text { electrical } \\
\text { collector system, } \\
\text { moorings and } \\
\text { foundations, and } \\
\text { device } \\
\text { installation }\end{array}$} & $\begin{array}{l}\text { Noise and } \\
\text { vibration }\end{array}$ & $\begin{array}{l}\text { Possible altered } \\
\text { behavior }\end{array}$ & $\begin{array}{l}\text { Low, would occur in } \\
\text { small area relative to } \\
\text { species’ range }\end{array}$ & $\begin{array}{l}\text { Low, occurs over } 2 \\
\text { months }\end{array}$ & $\begin{array}{l}\text { Low, species present but } \\
\text { able to avoid } \\
\text { disturbance area }\end{array}$ & Low & $\begin{array}{l}\text { Cada 2008, } \\
\text { Nelson } 2008\end{array}$ \\
\hline & $\begin{array}{l}\text { Oil/chemical } \\
\text { release }\end{array}$ & $\begin{array}{l}\text { Toxicity to individuals } \\
\text { and prey }\end{array}$ & $\begin{array}{l}\text { Low, volume released } \\
\text { would occupy small } \\
\text { area }\end{array}$ & $\begin{array}{l}\text { Low, occurs over } 2 \\
\text { months }\end{array}$ & $\begin{array}{l}\text { Low, species in water } \\
\text { column but oil floats on } \\
\text { surface }\end{array}$ & Low & $\begin{array}{l}\text { Nelson and } \\
\text { Woo } 2008\end{array}$ \\
\hline & $\begin{array}{l}\text { Seabed } \\
\text { disturbance }\end{array}$ & $\begin{array}{l}\text { None, species too motile } \\
\text { and near surface to be } \\
\text { affected }\end{array}$ & N/A & N/A & N/A & Low & N/A \\
\hline & $\begin{array}{l}\text { Construction and } \\
\text { deck lights }\end{array}$ & $\begin{array}{l}\text { Behavioral changes, } \\
\text { more vulnerable to } \\
\text { predation }\end{array}$ & $\begin{array}{l}\text { Low, would occur in } \\
\text { small area relative to } \\
\text { species' range }\end{array}$ & $\begin{array}{l}\text { Low, occurs over } 2 \\
\text { months }\end{array}$ & $\begin{array}{l}\text { Med, species present in } \\
\text { area and could be } \\
\text { attracted to lights }\end{array}$ & Med & $\begin{array}{l}\text { Nelson 2008, } \\
\text { Nightingale } \\
\text { et al. } 2006 \\
\end{array}$ \\
\hline \multirow{2}{*}{$\begin{array}{l}\text { Directional } \\
\text { drilling, and } \\
\text { laying cable } \\
\text { under/on seabed }\end{array}$} & $\begin{array}{l}\text { Seabed } \\
\text { disturbance }\end{array}$ & $\begin{array}{l}\text { None, these species too } \\
\text { motile and near surface } \\
\text { to be affected }\end{array}$ & N/A & N/A & N/A & Low & N/A \\
\hline & $\begin{array}{l}\text { Noise and } \\
\text { vibration }\end{array}$ & $\begin{array}{l}\text { Possible altered } \\
\text { behavior }\end{array}$ & $\begin{array}{l}\text { Low, would occur in } \\
\text { small area relative to } \\
\text { species' range }\end{array}$ & $\begin{array}{l}\text { Low, occurs over } 2 \\
\text { months }\end{array}$ & $\begin{array}{l}\text { Low, species present but } \\
\text { able to avoid } \\
\text { disturbance area }\end{array}$ & Low & $\begin{array}{l}\text { Cada 2008, } \\
\text { Nelson } 2008\end{array}$ \\
\hline \multicolumn{8}{|c|}{ Operation and Maintenance } \\
\hline \multirow[t]{2}{*}{ Boat traffic } & $\begin{array}{l}\text { Oil/chemical } \\
\text { release }\end{array}$ & $\begin{array}{l}\text { Toxicity to individuals } \\
\text { and prey }\end{array}$ & $\begin{array}{l}\text { Low for typical small } \\
\text { volume releases, High } \\
\text { for large, catastrophic } \\
\text { spill }\end{array}$ & $\begin{array}{l}\text { Low, wouldn’t } \\
\text { persist long-term }\end{array}$ & $\begin{array}{l}\text { Low, species in water } \\
\text { column but oil floats on } \\
\text { surface }\end{array}$ & Low & $\begin{array}{l}\text { Nelson and } \\
\text { Woo } 2008\end{array}$ \\
\hline & $\begin{array}{l}\text { Noise and } \\
\text { vibration }\end{array}$ & $\begin{array}{l}\text { Possible altered } \\
\text { behavior }\end{array}$ & $\begin{array}{l}\text { Low, would occur in } \\
\text { small area relative to } \\
\text { species' range }\end{array}$ & $\begin{array}{l}\text { Low, wouldn’t } \\
\text { persist long-term }\end{array}$ & $\begin{array}{l}\text { Low, species present but } \\
\text { able to avoid } \\
\text { disturbance area }\end{array}$ & Low & Nelson 2008 \\
\hline
\end{tabular}

\begin{tabular}{|c|c|c|}
\hline $\begin{array}{l}\text { Environmental Assessment for Siting Wave \& Tidal } \\
\text { Energy Projects }\end{array}$ & F-36 & $\begin{array}{r}\text { H. T. Harvey \& Associates } \\
24 \text { November } 2009\end{array}$ \\
\hline
\end{tabular}


Appendix F - Effects of Pilot Scale MCT SeaGen Project at the Tacoma Narrows Site

\begin{tabular}{|c|c|c|c|c|c|c|c|}
\hline Project activity & Action & $\begin{array}{l}\text { Description of action's } \\
\text { effect on indicator }\end{array}$ & $\begin{array}{l}\text { Spatial exposure to } \\
\text { indicator (low, med, } \\
\text { high) }\end{array}$ & $\begin{array}{l}\text { Temporal } \\
\text { exposure to } \\
\text { indicator (low, } \\
\text { med, high) }\end{array}$ & $\begin{array}{l}\text { Effect's overlap with } \\
\text { critical life stage, } \\
\text { behavior, habitat or } \\
\text { resource (low, med, } \\
\text { high) }\end{array}$ & $\begin{array}{l}\text { Overall risk } \\
\text { to indicator } \\
\text { (low, med, } \\
\text { high, } \\
\text { unknown) } \\
\end{array}$ & Source(s) \\
\hline \multirow{2}{*}{$\begin{array}{l}\text { Operation of } \\
\text { turbines or other } \\
\text { moving parts of } \\
\text { devices }\end{array}$} & $\begin{array}{l}\text { Moving device } \\
\text { parts }\end{array}$ & Collision with turbines & $\begin{array}{l}\text { Low, would occur in } \\
\text { small area relative to } \\
\text { species' range }\end{array}$ & $\begin{array}{l}\text { High, would be } \\
\text { continuous for life } \\
\text { of project }\end{array}$ & $\begin{array}{l}\text { Med, most juvenile } \\
\text { salmon found from } \\
\text { surface to } 12 \mathrm{~m} \text { depth, } \\
\text { turbines are at } 15 \mathrm{~m} \\
\text { depth }\end{array}$ & Unknown & $\begin{array}{l}\text { Emmett et al } \\
2004\end{array}$ \\
\hline & $\begin{array}{l}\text { Noise and } \\
\text { vibration }\end{array}$ & $\begin{array}{l}\text { Possible altered } \\
\text { behavior }\end{array}$ & $\begin{array}{l}\text { Low, would occur in } \\
\text { small area relative to } \\
\text { species' range }\end{array}$ & $\begin{array}{l}\text { High, would be } \\
\text { continuous for life } \\
\text { of project }\end{array}$ & $\begin{array}{l}\text { Low, species present but } \\
\text { able to avoid } \\
\text { disturbance area }\end{array}$ & Low & Cada 2008 \\
\hline \multirow{3}{*}{$\begin{array}{l}\text { Structures in } \\
\text { water column and } \\
\text { on seabed, such } \\
\text { as devices and } \\
\text { moorings and } \\
\text { footings }\end{array}$} & Structure & $\begin{array}{l}\text { Reef effect, attraction of } \\
\text { predators }\end{array}$ & $\begin{array}{l}\text { Low, would occur in } \\
\text { small area relative to } \\
\text { species' range }\end{array}$ & $\begin{array}{l}\text { High, would be } \\
\text { continuous for life } \\
\text { of project }\end{array}$ & $\begin{array}{l}\text { Med, species present in } \\
\text { area in spring, summer } \\
\text { and fall }\end{array}$ & Unknown & Nelson 2008 \\
\hline & $\begin{array}{l}\text { Water circulation } \\
\text { changes }\end{array}$ & $\begin{array}{l}\text { None, project not large } \\
\text { enough to affect prey }\end{array}$ & N/A & N/A & N/A & Low & N/A \\
\hline & $\begin{array}{l}\text { Oil/chemical } \\
\text { release }\end{array}$ & $\begin{array}{l}\text { Toxicity to individuals } \\
\text { and prey }\end{array}$ & $\begin{array}{l}\text { Low, would occur in } \\
\text { small area relative to } \\
\text { species' range }\end{array}$ & $\begin{array}{l}\text { Low, wouldn't } \\
\text { persist long-term }\end{array}$ & $\begin{array}{l}\text { Low, species in water } \\
\text { column but oil floats on } \\
\text { surface }\end{array}$ & Low & $\begin{array}{l}\text { Nelson and } \\
\text { Woo } 2008\end{array}$ \\
\hline $\begin{array}{l}\text { Electricity } \\
\text { conduction } \\
\text { through cable }\end{array}$ & EMF & $\begin{array}{l}\text { Possible behavior, } \\
\text { orientation changes }\end{array}$ & $\begin{array}{l}\text { Low, would occur in } \\
\text { small area relative to } \\
\text { species' range }\end{array}$ & $\begin{array}{l}\text { High, would be } \\
\text { continuous for life } \\
\text { of project }\end{array}$ & $\begin{array}{l}\text { Med, species present in } \\
\text { area in spring, summer } \\
\text { and fall }\end{array}$ & Unknown & Nelson 2008 \\
\hline \multirow{2}{*}{$\begin{array}{l}\text { Structures on } \\
\text { water's surface }\end{array}$} & Structure & $\begin{array}{l}\text { FAD effect, attraction of } \\
\text { predators }\end{array}$ & $\begin{array}{l}\text { Low, would occur in } \\
\text { small area relative to } \\
\text { species' range }\end{array}$ & $\begin{array}{l}\text { High, would be } \\
\text { continuous for life } \\
\text { of project }\end{array}$ & $\begin{array}{l}\text { Med, species present in } \\
\text { area in spring, summer } \\
\text { and fall }\end{array}$ & Unknown & Nelson 2008 \\
\hline & Navigation lights & $\begin{array}{l}\text { Behavioral changes, } \\
\text { more vulnerable to } \\
\text { predation }\end{array}$ & $\begin{array}{l}\text { Low, would occur in } \\
\text { small area relative to } \\
\text { species' range }\end{array}$ & $\begin{array}{l}\text { High, would be } \\
\text { continuous for life } \\
\text { of project }\end{array}$ & $\begin{array}{l}\text { Med, species present in } \\
\text { area in spring, summer } \\
\text { and fall }\end{array}$ & Med & $\begin{array}{l}\text { Nightingale } \\
\text { et al. } 2006\end{array}$ \\
\hline \multicolumn{8}{|c|}{ Decommissioning } \\
\hline \multirow[t]{2}{*}{ Boat traffic } & $\begin{array}{l}\text { Oil/chemical } \\
\text { release }\end{array}$ & $\begin{array}{l}\text { Toxicity to individuals } \\
\text { and prey }\end{array}$ & $\begin{array}{l}\text { Low for typical small } \\
\text { volume releases, High } \\
\text { for large, catastrophic } \\
\text { spill }\end{array}$ & $\begin{array}{l}\text { Low, occurs over } 2 \\
\text { months }\end{array}$ & $\begin{array}{l}\text { Low, species in water } \\
\text { column but oil floats on } \\
\text { surface }\end{array}$ & Low & $\begin{array}{l}\text { Nelson and } \\
\text { Woo } 2008\end{array}$ \\
\hline & $\begin{array}{l}\text { Noise and } \\
\text { vibration }\end{array}$ & $\begin{array}{l}\text { Possible altered } \\
\text { behavior }\end{array}$ & $\begin{array}{l}\text { Low, would occur in } \\
\text { small area relative to } \\
\text { species' range }\end{array}$ & $\begin{array}{l}\text { Low, occurs over } 2 \\
\text { months }\end{array}$ & $\begin{array}{l}\text { Low, species present but } \\
\text { able to avoid } \\
\text { disturbance area }\end{array}$ & Low & $\begin{array}{l}\text { Cada 2008, } \\
\text { Nelson } 2008\end{array}$ \\
\hline $\begin{array}{l}\text { Decommissioning } \\
\text { of structures on } \\
\text { water's surface or }\end{array}$ & $\begin{array}{l}\text { Noise and } \\
\text { vibration }\end{array}$ & $\begin{array}{l}\text { Possible altered } \\
\text { behavior }\end{array}$ & $\begin{array}{l}\text { Low, would occur in } \\
\text { small area relative to } \\
\text { species' range }\end{array}$ & $\begin{array}{l}\text { Low, occurs over } 2 \\
\text { months }\end{array}$ & $\begin{array}{l}\text { Low, species present but } \\
\text { able to avoid } \\
\text { disturbance area }\end{array}$ & Low & $\begin{array}{l}\text { Cada 2008, } \\
\text { Nelson 2008 }\end{array}$ \\
\hline
\end{tabular}

\begin{tabular}{|c|c|c|}
\hline $\begin{array}{l}\text { Environmental Assessment for Siting Wave \& Tidal } \\
\text { Energy Projects }\end{array}$ & F-37 & $\begin{array}{r}\text { H. T. Harvey \& Associates } \\
24 \text { November } 2009\end{array}$ \\
\hline
\end{tabular}


Appendix F - Effects of Pilot Scale MCT SeaGen Project at the Tacoma Narrows Site

\begin{tabular}{|c|c|c|c|c|c|c|c|}
\hline Project activity & Action & $\begin{array}{l}\text { Description of action's } \\
\text { effect on indicator }\end{array}$ & $\begin{array}{l}\text { Spatial exposure to } \\
\text { indicator (low, med, } \\
\text { high) }\end{array}$ & $\begin{array}{l}\text { Temporal } \\
\text { exposure to } \\
\text { indicator (low, } \\
\text { med, high) }\end{array}$ & $\begin{array}{l}\text { Effect's overlap with } \\
\text { critical life stage, } \\
\text { behavior, habitat or } \\
\text { resource (low, med, } \\
\text { high) }\end{array}$ & $\begin{array}{l}\text { Overall risk } \\
\text { to indicator } \\
\text { (low, med, } \\
\text { high, } \\
\text { unknown) } \\
\end{array}$ & Source(s) \\
\hline \multirow[t]{3}{*}{ seabed } & $\begin{array}{l}\text { Oil/chemical } \\
\text { release }\end{array}$ & $\begin{array}{l}\text { Toxicity to individuals } \\
\text { and prey }\end{array}$ & $\begin{array}{l}\text { Low, volume released } \\
\text { would occupy small } \\
\text { area }\end{array}$ & $\begin{array}{l}\text { Low, occurs over } 2 \\
\text { months }\end{array}$ & $\begin{array}{l}\text { Low, species in water } \\
\text { column but oil floats on } \\
\text { surface }\end{array}$ & Low & $\begin{array}{l}\text { Nelson and } \\
\text { Woo } 2008\end{array}$ \\
\hline & $\begin{array}{l}\text { Seabed } \\
\text { disturbance }\end{array}$ & $\begin{array}{l}\text { None, species too motile } \\
\text { and near surface to be } \\
\text { affected }\end{array}$ & N/A & N/A & N/A & Low & N/A \\
\hline & $\begin{array}{l}\text { Deconstruction } \\
\text { and deck lights }\end{array}$ & $\begin{array}{l}\text { Behavioral changes, } \\
\text { more vulnerable to } \\
\text { predation }\end{array}$ & $\begin{array}{l}\text { Low, would occur in } \\
\text { small area relative to } \\
\text { species' range }\end{array}$ & $\begin{array}{l}\text { Low, occurs over } 2 \\
\text { months }\end{array}$ & $\begin{array}{l}\text { Med, species present in } \\
\text { area and could be } \\
\text { attracted to lights }\end{array}$ & Med & $\begin{array}{l}\text { Nightingale } \\
\text { et al. } 2006\end{array}$ \\
\hline
\end{tabular}


Appendix F - Effects of Pilot Scale MCT SeaGen Project at the Tacoma Narrows Site

Potential effects on adult salmonids due to Pilot Scale MCT SeaGen project at Tacoma Narrows

\begin{tabular}{|c|c|c|c|c|c|c|c|}
\hline Project activity & Action & $\begin{array}{l}\text { Description of action's } \\
\text { effect on indicator }\end{array}$ & $\begin{array}{l}\text { Spatial exposure to } \\
\text { indicator (low, med, } \\
\text { high) }\end{array}$ & $\begin{array}{l}\text { Temporal } \\
\text { exposure to } \\
\text { indicator (low, } \\
\text { med, high) }\end{array}$ & $\begin{array}{l}\text { Effect's overlap with } \\
\text { critical life stage, } \\
\text { behavior, habitat or } \\
\text { resource (low, med, } \\
\text { high) }\end{array}$ & $\begin{array}{l}\text { Overall risk } \\
\text { to indicator } \\
\text { (low, med, } \\
\text { high, } \\
\text { unknown) } \\
\end{array}$ & Source(s) \\
\hline \multicolumn{8}{|c|}{ Construction } \\
\hline \multirow[t]{2}{*}{ Boat traffic } & $\begin{array}{l}\text { Oil/chemical } \\
\text { release }\end{array}$ & $\begin{array}{l}\text { Toxicity to individuals } \\
\text { and prey }\end{array}$ & $\begin{array}{l}\text { Low for typical small } \\
\text { volume releases, High } \\
\text { for large, catastrophic } \\
\text { spill }\end{array}$ & $\begin{array}{l}\text { Low, occurs over } 2 \\
\text { months }\end{array}$ & $\begin{array}{l}\text { Low, species in water } \\
\text { column but oil floats on } \\
\text { surface }\end{array}$ & Low & $\begin{array}{l}\text { Nelson and } \\
\text { Woo } 2008\end{array}$ \\
\hline & $\begin{array}{l}\text { Noise and } \\
\text { vibration }\end{array}$ & $\begin{array}{l}\text { Possible altered } \\
\text { behavior }\end{array}$ & $\begin{array}{l}\text { Low, would occur in } \\
\text { small area relative to } \\
\text { species' range }\end{array}$ & $\begin{array}{l}\text { Low, occurs over } 2 \\
\text { months }\end{array}$ & $\begin{array}{l}\text { Low, species present but } \\
\text { able to avoid } \\
\text { disturbance area }\end{array}$ & Low & $\begin{array}{l}\text { Cada 2008, } \\
\text { Nelson } 2008\end{array}$ \\
\hline \multirow{4}{*}{$\begin{array}{l}\text { Construction of } \\
\text { electrical } \\
\text { collector system, } \\
\text { moorings and } \\
\text { foundations, and } \\
\text { device } \\
\text { installation }\end{array}$} & $\begin{array}{l}\text { Noise and } \\
\text { vibration }\end{array}$ & $\begin{array}{l}\text { Possible altered } \\
\text { behavior }\end{array}$ & $\begin{array}{l}\text { Low, would occur in } \\
\text { small area relative to } \\
\text { species' range }\end{array}$ & $\begin{array}{l}\text { Low, occurs over } 2 \\
\text { months }\end{array}$ & $\begin{array}{l}\text { Low, species present but } \\
\text { able to avoid } \\
\text { disturbance area }\end{array}$ & Low & $\begin{array}{l}\text { Cada 2008, } \\
\text { Nelson } 2008\end{array}$ \\
\hline & $\begin{array}{l}\text { Oil/chemical } \\
\text { release }\end{array}$ & $\begin{array}{l}\text { Toxicity to individuals } \\
\text { and prey }\end{array}$ & $\begin{array}{l}\text { Low, volume released } \\
\text { would occupy small } \\
\text { area }\end{array}$ & $\begin{array}{l}\text { Low, occurs over } 2 \\
\text { months }\end{array}$ & $\begin{array}{l}\text { Low, species in water } \\
\text { column but oil floats on } \\
\text { surface }\end{array}$ & Low & $\begin{array}{l}\text { Nelson and } \\
\text { Woo } 2008\end{array}$ \\
\hline & $\begin{array}{l}\text { Seabed } \\
\text { disturbance }\end{array}$ & $\begin{array}{l}\text { None, species too motile } \\
\text { and near surface to be } \\
\text { affected }\end{array}$ & N/A & N/A & N/A & Low & N/A \\
\hline & $\begin{array}{l}\text { Construction } \\
\text { lights }\end{array}$ & $\begin{array}{l}\text { None, not known to be } \\
\text { affected by lights }\end{array}$ & N/A & N/A & N/A & Low & $\begin{array}{l}\text { Nightingale } \\
\text { et al. } 2006\end{array}$ \\
\hline \multirow{2}{*}{$\begin{array}{l}\text { Directional } \\
\text { drilling, and } \\
\text { laying cable } \\
\text { under/on seabed }\end{array}$} & $\begin{array}{l}\text { Seabed } \\
\text { disturbance }\end{array}$ & $\begin{array}{l}\text { None, species too motile } \\
\text { and near surface to be } \\
\text { affected }\end{array}$ & N/A & N/A & N/A & Low & N/A \\
\hline & $\begin{array}{l}\text { Noise and } \\
\text { vibration }\end{array}$ & $\begin{array}{l}\text { Possible altered } \\
\text { behavior }\end{array}$ & $\begin{array}{l}\text { Low, would occur in } \\
\text { small area relative to } \\
\text { species' range }\end{array}$ & $\begin{array}{l}\text { Low, occurs over } 2 \\
\text { months }\end{array}$ & $\begin{array}{l}\text { Low, species present but } \\
\text { able to avoid } \\
\text { disturbance area }\end{array}$ & Low & $\begin{array}{l}\text { Cada 2008, } \\
\text { Nelson } 2008\end{array}$ \\
\hline \multicolumn{8}{|c|}{ Operation and Maintenance } \\
\hline \multirow[t]{2}{*}{ Boat traffic } & $\begin{array}{l}\text { Oil/chemical } \\
\text { release }\end{array}$ & $\begin{array}{l}\text { Toxicity to individuals } \\
\text { and prey }\end{array}$ & $\begin{array}{l}\text { Low for typical small } \\
\text { volume releases, High } \\
\text { for large, catastrophic } \\
\text { spill }\end{array}$ & $\begin{array}{l}\text { Low, wouldn’t } \\
\text { persist long-term }\end{array}$ & $\begin{array}{l}\text { Low, species in water } \\
\text { column but oil floats on } \\
\text { surface }\end{array}$ & Low & $\begin{array}{l}\text { Nelson and } \\
\text { Woo } 2008\end{array}$ \\
\hline & $\begin{array}{l}\text { Noise and } \\
\text { vibration }\end{array}$ & $\begin{array}{l}\text { Possible altered } \\
\text { behavior }\end{array}$ & $\begin{array}{l}\text { Low, would occur in } \\
\text { small area relative to } \\
\text { species' range }\end{array}$ & $\begin{array}{l}\text { Low, wouldn’t } \\
\text { persist long-term }\end{array}$ & $\begin{array}{l}\text { Low, species present but } \\
\text { able to avoid } \\
\text { disturbance area }\end{array}$ & Low & Nelson 2008 \\
\hline
\end{tabular}

\begin{tabular}{|c|c|c|}
\hline $\begin{array}{l}\text { Environmental Assessment for Siting Wave \& Tidal } \\
\text { Energy Projects }\end{array}$ & F-39 & $\begin{array}{r}\text { H. T. Harvey \& Associates } \\
24 \text { November } 2009\end{array}$ \\
\hline
\end{tabular}


Appendix F - Effects of Pilot Scale MCT SeaGen Project at the Tacoma Narrows Site

\begin{tabular}{|c|c|c|c|c|c|c|c|}
\hline Project activity & Action & $\begin{array}{l}\text { Description of action's } \\
\text { effect on indicator }\end{array}$ & $\begin{array}{l}\text { Spatial exposure to } \\
\text { indicator (low, med, } \\
\text { high) }\end{array}$ & $\begin{array}{l}\text { Temporal } \\
\text { exposure to } \\
\text { indicator (low, } \\
\text { med, high) }\end{array}$ & $\begin{array}{l}\text { Effect's overlap with } \\
\text { critical life stage, } \\
\text { behavior, habitat or } \\
\text { resource (low, med, } \\
\text { high) }\end{array}$ & $\begin{array}{l}\text { Overall risk } \\
\text { to indicator } \\
\text { (low, med, } \\
\text { high, } \\
\text { unknown) } \\
\end{array}$ & Source(s) \\
\hline \multirow{2}{*}{$\begin{array}{l}\text { Operation of } \\
\text { turbines or other } \\
\text { moving parts of } \\
\text { devices }\end{array}$} & $\begin{array}{l}\text { Moving device } \\
\text { parts }\end{array}$ & Collision with turbines & $\begin{array}{l}\text { Low, would occur in } \\
\text { small area relative to } \\
\text { species' range }\end{array}$ & $\begin{array}{l}\text { High, would be } \\
\text { continuous for life } \\
\text { of project }\end{array}$ & $\begin{array}{l}\text { Low, most adult salmon } \\
\text { in upper } 10 \text { m of water } \\
\text { column, turbines at } 15 \\
\text { m }\end{array}$ & Low & $\begin{array}{l}\text { Olson and } \\
\text { Quinn 1993, } \\
\text { Ruggerone e } \\
\text { al. 1990 } \\
\end{array}$ \\
\hline & $\begin{array}{l}\text { Noise and } \\
\text { vibration }\end{array}$ & $\begin{array}{l}\text { Possible altered } \\
\text { behavior }\end{array}$ & $\begin{array}{l}\text { Low, would occur in } \\
\text { small area relative to } \\
\text { species' range }\end{array}$ & $\begin{array}{l}\text { High, would be } \\
\text { continuous for life } \\
\text { of project }\end{array}$ & $\begin{array}{l}\text { Low, species present but } \\
\text { able to avoid } \\
\text { disturbance area }\end{array}$ & Low & Cada 2008 \\
\hline \multirow{3}{*}{$\begin{array}{l}\text { Structures in } \\
\text { water column and } \\
\text { on seabed, such } \\
\text { as devices and } \\
\text { moorings and } \\
\text { footings }\end{array}$} & Structure & $\begin{array}{l}\text { Attraction of predators } \\
\text { (reef effect), more } \\
\text { vulnerable to predation }\end{array}$ & $\begin{array}{l}\text { Low, would occur in } \\
\text { small area relative to } \\
\text { species' range }\end{array}$ & $\begin{array}{l}\text { High, would be } \\
\text { continuous for life } \\
\text { of project }\end{array}$ & $\begin{array}{l}\text { Med, species present in } \\
\text { area in spring, summer } \\
\text { and fall }\end{array}$ & Unknown & Nelson 2008 \\
\hline & $\begin{array}{l}\text { Water circulation } \\
\text { changes }\end{array}$ & $\begin{array}{l}\text { None, project not large } \\
\text { enough to affect prey }\end{array}$ & N/A & N/A & N/A & Low & N/A \\
\hline & $\begin{array}{l}\text { Oil/chemical } \\
\text { release }\end{array}$ & $\begin{array}{l}\text { Toxicity to individuals } \\
\text { and prey }\end{array}$ & $\begin{array}{l}\text { Low, would occur in } \\
\text { small area relative to } \\
\text { species' range }\end{array}$ & $\begin{array}{l}\text { Low, wouldn’t } \\
\text { persist long-term }\end{array}$ & $\begin{array}{l}\text { Low, species in water } \\
\text { column but oil floats on } \\
\text { surface }\end{array}$ & Low & $\begin{array}{l}\text { Nelson and } \\
\text { Woo } 2008\end{array}$ \\
\hline $\begin{array}{l}\text { Electricity } \\
\text { conduction } \\
\text { through cable } \\
\end{array}$ & EMF & $\begin{array}{l}\text { Behavior, orientation } \\
\text { changes }\end{array}$ & $\begin{array}{l}\text { Low, would occur in } \\
\text { small area relative to } \\
\text { species' range }\end{array}$ & $\begin{array}{l}\text { High, would be } \\
\text { continuous for life } \\
\text { of project }\end{array}$ & $\begin{array}{l}\text { Med, species present in } \\
\text { area in spring, summer } \\
\text { and fall }\end{array}$ & Unknown & Nelson 2008 \\
\hline \multirow{2}{*}{$\begin{array}{l}\text { Structures on } \\
\text { water's surface }\end{array}$} & Structure & $\begin{array}{l}\text { Attraction of predators } \\
\text { (FAD effect), more } \\
\text { vulnerable to predation }\end{array}$ & $\begin{array}{l}\text { Low, would occur in } \\
\text { small area relative to } \\
\text { species' range }\end{array}$ & $\begin{array}{l}\text { High, would be } \\
\text { continuous for life } \\
\text { of project }\end{array}$ & $\begin{array}{l}\text { Med, species present in } \\
\text { area in spring, summer } \\
\text { and fall }\end{array}$ & Unknown & Nelson 2008 \\
\hline & Navigation lights & $\begin{array}{l}\text { None, not known to be } \\
\text { affected by lights }\end{array}$ & N/A & N/A & N/A & Low & $\begin{array}{l}\text { Nightingale } \\
\text { et al. } 2006\end{array}$ \\
\hline \multicolumn{8}{|c|}{ Decommissioning } \\
\hline \multirow[t]{2}{*}{ Boat traffic } & $\begin{array}{l}\text { Oil/chemical } \\
\text { release }\end{array}$ & $\begin{array}{l}\text { Toxicity to individuals } \\
\text { and prey }\end{array}$ & $\begin{array}{l}\text { Low for typical small } \\
\text { volume releases, High } \\
\text { for large, catastrophic } \\
\text { spill }\end{array}$ & $\begin{array}{l}\text { Low, occurs over } 2 \\
\text { months }\end{array}$ & $\begin{array}{l}\text { Low, species in water } \\
\text { column but oil floats on } \\
\text { surface }\end{array}$ & Low & $\begin{array}{l}\text { Nelson and } \\
\text { Woo } 2008\end{array}$ \\
\hline & $\begin{array}{l}\text { Noise and } \\
\text { vibration }\end{array}$ & $\begin{array}{l}\text { Possible altered } \\
\text { behavior }\end{array}$ & $\begin{array}{l}\text { Low, would occur in } \\
\text { small area relative to } \\
\text { species' range }\end{array}$ & $\begin{array}{l}\text { Low, occurs over } 2 \\
\text { months }\end{array}$ & $\begin{array}{l}\text { Low, species present but } \\
\text { able to avoid } \\
\text { disturbance area }\end{array}$ & Low & $\begin{array}{l}\text { Cada 2008, } \\
\text { Nelson } 2008\end{array}$ \\
\hline $\begin{array}{l}\text { Decommissioning } \\
\text { of structures on } \\
\text { water's surface or }\end{array}$ & $\begin{array}{l}\text { Noise and } \\
\text { vibration }\end{array}$ & $\begin{array}{l}\text { Possible altered } \\
\text { behavior }\end{array}$ & $\begin{array}{l}\text { Low, would occur in } \\
\text { small area relative to } \\
\text { species' range }\end{array}$ & $\begin{array}{l}\text { Low, occurs over } 2 \\
\text { months }\end{array}$ & $\begin{array}{l}\text { Low, species present but } \\
\text { able to avoid } \\
\text { disturbance area }\end{array}$ & Low & $\begin{array}{l}\text { Cada 2008, } \\
\text { Nelson } 2008\end{array}$ \\
\hline
\end{tabular}

\begin{tabular}{|c|c|c|}
\hline $\begin{array}{l}\text { Environmental Assessment for Siting Wave \& Tidal } \\
\text { Energy Projects }\end{array}$ & F-40 & $\begin{array}{r}\text { H. T. Harvey \& Associates } \\
24 \text { November } 2009\end{array}$ \\
\hline
\end{tabular}


Appendix F - Effects of Pilot Scale MCT SeaGen Project at the Tacoma Narrows Site

\begin{tabular}{|c|c|c|c|c|c|c|c|}
\hline Project activity & Action & $\begin{array}{l}\text { Description of action's } \\
\text { effect on indicator }\end{array}$ & $\begin{array}{l}\text { Spatial exposure to } \\
\text { indicator (low, med, } \\
\text { high) }\end{array}$ & $\begin{array}{l}\text { Temporal } \\
\text { exposure to } \\
\text { indicator (low, } \\
\text { med, high) }\end{array}$ & $\begin{array}{l}\text { Effect's overlap with } \\
\text { critical life stage, } \\
\text { behavior, habitat or } \\
\text { resource (low, med, } \\
\text { high) }\end{array}$ & $\begin{array}{l}\text { Overall risk } \\
\text { to indicator } \\
\text { (low, med, } \\
\text { high, } \\
\text { unknown) } \\
\end{array}$ & Source(s) \\
\hline \multirow[t]{3}{*}{ seabed } & $\begin{array}{l}\text { Oil/chemical } \\
\text { release }\end{array}$ & $\begin{array}{l}\text { Toxicity to individuals } \\
\text { and prey }\end{array}$ & $\begin{array}{l}\text { Low, volume released } \\
\text { would occupy small } \\
\text { area }\end{array}$ & $\begin{array}{l}\text { Low, occurs over } 2 \\
\text { months }\end{array}$ & $\begin{array}{l}\text { Low, species in water } \\
\text { column but oil floats on } \\
\text { surface }\end{array}$ & Low & $\begin{array}{l}\text { Nelson and } \\
\text { Woo } 2008\end{array}$ \\
\hline & $\begin{array}{l}\text { Seabed } \\
\text { disturbance }\end{array}$ & $\begin{array}{l}\text { None, species too motile } \\
\text { and near surface to be } \\
\text { affected }\end{array}$ & N/A & N/A & N/A & Low & N/A \\
\hline & $\begin{array}{l}\text { Deconstruction } \\
\text { lights }\end{array}$ & $\begin{array}{l}\text { None, not known to be } \\
\text { affected by lights }\end{array}$ & N/A & N/A & N/A & Low & $\begin{array}{l}\text { Nightingale } \\
\text { et al. } 2006\end{array}$ \\
\hline
\end{tabular}


Appendix F - Effects of Pilot Scale MCT SeaGen Project at the Tacoma Narrows Site

Potential effects on diving ducks, loons, and grebes from Pilot Scale MCT SeaGen project at Tacoma Narrows

\begin{tabular}{|c|c|c|c|c|c|c|c|}
\hline Project activity & Action & $\begin{array}{l}\text { Description of action's } \\
\text { effect on indicator }\end{array}$ & $\begin{array}{l}\text { Spatial exposure to } \\
\text { indicator (low, med, } \\
\text { high) }\end{array}$ & $\begin{array}{l}\text { Temporal } \\
\text { exposure to } \\
\text { indicator (low, } \\
\text { med, high) } \\
\end{array}$ & $\begin{array}{l}\text { Effect's overlap with } \\
\text { critical life stage, } \\
\text { behavior, habitat or } \\
\text { resource (low, med, } \\
\text { high) }\end{array}$ & $\begin{array}{l}\text { Overall risk to } \\
\text { indicator (low, } \\
\text { med, high, } \\
\text { unknown) }\end{array}$ & Source(s) \\
\hline \multicolumn{8}{|c|}{ Construction } \\
\hline \multirow{2}{*}{ Boat traffic } & $\begin{array}{l}\text { Noise and } \\
\text { vibration }\end{array}$ & $\begin{array}{l}\text { Possible movement } \\
\text { away from boat and } \\
\text { disruption of foraging }\end{array}$ & $\begin{array}{l}\text { Low, would occur in } \\
\text { small area relative to } \\
\text { species' range }\end{array}$ & $\begin{array}{l}\text { Low, occurs over } \\
2 \text { months }\end{array}$ & $\begin{array}{l}\text { Med, if done in winter } \\
\text { when birds most } \\
\text { abundant; low if in } \\
\text { summer }\end{array}$ & Low & $\begin{array}{l}\text { LGL Limited } \\
\text { et al. 2009, } \\
\text { SAS [date } \\
\text { unknown] }\end{array}$ \\
\hline & $\begin{array}{l}\text { Oil/chemical } \\
\text { release }\end{array}$ & $\begin{array}{l}\text { Hypothermia, toxicity } \\
\text { to individuals and prey }\end{array}$ & $\begin{array}{l}\text { Low for typical small } \\
\text { volume releases, High } \\
\text { for large, catastrophic } \\
\text { spill }\end{array}$ & $\begin{array}{l}\text { Low, occurs over } \\
2 \text { months }\end{array}$ & Med or low, see above & Low & $\begin{array}{l}\text { Michel et al. } \\
\text { 2007, SAS } \\
\text { [date } \\
\text { unknown] }\end{array}$ \\
\hline \multirow{4}{*}{$\begin{array}{l}\text { Construction of } \\
\text { electrical } \\
\text { collector system, } \\
\text { moorings/ } \\
\text { foundation; } \\
\text { installation of } \\
\text { devices }\end{array}$} & $\begin{array}{l}\text { Noise and } \\
\text { vibration }\end{array}$ & $\begin{array}{l}\text { Possible movement } \\
\text { away from area and } \\
\text { disruption of foraging } \\
\text { or nesting }\end{array}$ & $\begin{array}{l}\text { Low, would occur in } \\
\text { small area relative to } \\
\text { species' range }\end{array}$ & $\begin{array}{l}\text { Low, occurs over } \\
2 \text { months }\end{array}$ & Med or low, see above & Low & $\begin{array}{l}\text { Michel et al. } \\
\text { 2007, SAS } \\
\text { [date } \\
\text { unknown] }\end{array}$ \\
\hline & $\begin{array}{l}\text { Oil/chemical } \\
\text { release }\end{array}$ & $\begin{array}{l}\text { Hypothermia, toxicity } \\
\text { to individuals and prey }\end{array}$ & $\begin{array}{l}\text { Low, volume released } \\
\text { would occupy small area } \\
\text { relative to species' range }\end{array}$ & $\begin{array}{l}\text { Low, occurs over } \\
2 \text { months }\end{array}$ & Med or low, see above & Low & $\begin{array}{l}\text { Michel et al. } \\
\text { 2007, SAS } \\
\text { [date } \\
\text { unknown] }\end{array}$ \\
\hline & Seabed disturbance & $\begin{array}{l}\text { Possible short-term } \\
\text { changes in food } \\
\text { resources }\end{array}$ & $\begin{array}{l}\text { Low, would occur in } \\
\text { small area relative to } \\
\text { species' range }\end{array}$ & $\begin{array}{l}\text { Low, occurs over } \\
2 \text { months }\end{array}$ & Med or low, see above & Low & $\begin{array}{l}\text { Boehlert et al. } \\
\text { 2008, SAS } \\
\text { [date } \\
\text { unknown] }\end{array}$ \\
\hline & Construction lights & $\begin{array}{l}\text { None, these species not } \\
\text { known to be attracted to } \\
\text { lights }\end{array}$ & N/A & N/A & N/A & Low & $\begin{array}{l}\text { Montevecchi } \\
2006\end{array}$ \\
\hline \multirow{2}{*}{$\begin{array}{l}\text { Directional } \\
\text { drilling, and } \\
\text { laying cable } \\
\text { under/on seabed }\end{array}$} & Seabed disturbance & $\begin{array}{l}\text { Possible short-term } \\
\text { changes in food } \\
\text { resources }\end{array}$ & Low, see above & $\begin{array}{l}\text { Low, occurs over } \\
2 \text { months }\end{array}$ & $\begin{array}{l}\text { Med, if done in winter } \\
\text { when birds most } \\
\text { abundant; low if in } \\
\text { summer }\end{array}$ & Low & $\begin{array}{l}\text { Boehlert et al } \\
\text { 2008, SAS } \\
\text { [date } \\
\text { unknown] }\end{array}$ \\
\hline & $\begin{array}{l}\text { Noise and } \\
\text { vibration }\end{array}$ & $\begin{array}{l}\text { Possible movement } \\
\text { away from area and } \\
\text { disruption of foraging } \\
\text { or nesting }\end{array}$ & Low, see above & $\begin{array}{l}\text { Low, occurs over } \\
2 \text { months }\end{array}$ & Med or low, see above & Low & $\begin{array}{l}\text { Michel et al. } \\
\text { 2007, SAS } \\
\text { [date } \\
\text { unknown] }\end{array}$ \\
\hline
\end{tabular}

\begin{tabular}{|c|c|c|}
\hline $\begin{array}{l}\text { Environmental Assessment for Siting Wave \& Tidal } \\
\text { Energy Projects }\end{array}$ & F-42 & $\begin{array}{r}\text { H. T. Harvey \& Associates } \\
24 \text { November } 2009\end{array}$ \\
\hline
\end{tabular}


Appendix F - Effects of Pilot Scale MCT SeaGen Project at the Tacoma Narrows Site

\begin{tabular}{|c|c|c|c|c|c|c|c|}
\hline Project activity & Action & $\begin{array}{l}\text { Description of action's } \\
\text { effect on indicator }\end{array}$ & $\begin{array}{l}\text { Spatial exposure to } \\
\text { indicator (low, med, } \\
\text { high) }\end{array}$ & $\begin{array}{l}\text { Temporal } \\
\text { exposure to } \\
\text { indicator (low, } \\
\text { med, high) }\end{array}$ & $\begin{array}{l}\text { Effect's overlap with } \\
\text { critical life stage, } \\
\text { behavior, habitat or } \\
\text { resource (low, med, } \\
\text { high) }\end{array}$ & $\begin{array}{l}\text { Overall risk to } \\
\text { indicator (low, } \\
\text { med, high, } \\
\text { unknown) }\end{array}$ & Source(s) \\
\hline \multicolumn{8}{|c|}{ Operation and Maintenance } \\
\hline \multirow{2}{*}{ Boat traffic } & $\begin{array}{l}\text { Oil/chemical } \\
\text { release }\end{array}$ & $\begin{array}{l}\text { Hypothermia, toxicity } \\
\text { to individuals and prey }\end{array}$ & $\begin{array}{l}\text { Low for typical small } \\
\text { volume releases, High } \\
\text { for large, catastrophic } \\
\text { spill }\end{array}$ & $\begin{array}{l}\text { Low, only from } \\
\text { periodic trips }\end{array}$ & $\begin{array}{l}\text { Med, boats could pass } \\
\text { through foraging areas } \\
\text { if done in winter }\end{array}$ & Low & $\begin{array}{l}\text { Michel et al. } \\
\text { 2007, SAS } \\
\text { [date } \\
\text { unknown] }\end{array}$ \\
\hline & $\begin{array}{l}\text { Noise and } \\
\text { vibration }\end{array}$ & $\begin{array}{l}\text { Possible movement } \\
\text { away from area and } \\
\text { disruption of foraging } \\
\text { or nesting }\end{array}$ & $\begin{array}{l}\text { Low, would occur in } \\
\text { small area relative to } \\
\text { species' range }\end{array}$ & $\begin{array}{l}\text { Low, only from } \\
\text { periodic trips }\end{array}$ & Med, see above & Low & $\begin{array}{l}\text { LGL Limited } \\
\text { et al. 2009, } \\
\text { SAS [date } \\
\text { unknown] }\end{array}$ \\
\hline \multirow{2}{*}{$\begin{array}{l}\text { Operation of } \\
\text { turbines or other } \\
\text { moving parts of } \\
\text { devices }\end{array}$} & $\begin{array}{l}\text { Moving device } \\
\text { parts }\end{array}$ & $\begin{array}{l}\text { Possible collision while } \\
\text { diving }\end{array}$ & Low, see above & $\begin{array}{l}\text { High, continuous } \\
\text { for life of project }\end{array}$ & $\begin{array}{l}\text { Med, will be present } \\
\text { in winter when birds } \\
\text { most abundant }\end{array}$ & Unknown & $\begin{array}{l}\text { Boehlert et al. } \\
\text { 2008, SAS } \\
\text { [date } \\
\text { unknown] }\end{array}$ \\
\hline & $\begin{array}{l}\text { Noise and } \\
\text { vibration }\end{array}$ & $\begin{array}{l}\text { Possible avoidance of } \\
\text { project area }\end{array}$ & Low, see above & $\begin{array}{l}\text { High, continuous } \\
\text { for life of project }\end{array}$ & Med, see above & Low & $\begin{array}{l}\text { Michel et al. } \\
\text { 2007, SAS } \\
\text { [date } \\
\text { unknown] }\end{array}$ \\
\hline \multirow{3}{*}{$\begin{array}{l}\text { Structures in } \\
\text { water column and } \\
\text { on seabed, such } \\
\text { as devices and } \\
\text { moorings and } \\
\text { footings }\end{array}$} & Structure & $\begin{array}{l}\text { Possible attraction to } \\
\text { increased prey (reef } \\
\text { effect), entanglement } \\
\text { with derelict fishing } \\
\text { gear }\end{array}$ & Low, see above & $\begin{array}{l}\text { High, continuous } \\
\text { for life of project }\end{array}$ & $\begin{array}{l}\text { Med, birds could be } \\
\text { attracted to structure }\end{array}$ & Unknown & $\begin{array}{l}\text { Michel et al. } \\
\text { 2007, SAS } \\
\text { [date } \\
\text { unknown] }\end{array}$ \\
\hline & $\begin{array}{l}\text { Water circulation } \\
\text { changes }\end{array}$ & $\begin{array}{l}\text { None, project not large } \\
\text { enough to affect prey }\end{array}$ & N/A & N/A & N/A & Low & N/A \\
\hline & $\begin{array}{l}\text { Oil/chemical } \\
\text { release }\end{array}$ & $\begin{array}{l}\text { Hypothermia, toxicity } \\
\text { to individuals and prey }\end{array}$ & $\begin{array}{l}\text { Low for typical small } \\
\text { volume releases, High } \\
\text { for catastrophic release } \\
\text { from device failure }\end{array}$ & $\begin{array}{l}\text { Med, continuous } \\
\text { and intermittent } \\
\text { for life of project }\end{array}$ & $\begin{array}{l}\text { Low, could occur in } \\
\text { winter when birds } \\
\text { most abundant }\end{array}$ & Low & $\begin{array}{l}\text { Michel et al. } \\
\text { 2007, SAS } \\
\text { [date } \\
\text { unknown] }\end{array}$ \\
\hline $\begin{array}{l}\text { Structures on } \\
\text { water's surface }\end{array}$ & Navigation lights & $\begin{array}{l}\text { None, species not known } \\
\text { to be attracted to lights }\end{array}$ & N/A & N/A & N/A & Low & $\begin{array}{l}\text { Montevecchi } \\
2006\end{array}$ \\
\hline
\end{tabular}

\begin{tabular}{|c|c|c|}
\hline $\begin{array}{l}\text { Environmental Assessment for Siting Wave \& Tidal } \\
\text { Energy Projects }\end{array}$ & F-43 & $\begin{array}{r}\text { H. T. Harvey \& Associates } \\
24 \text { November } 2009\end{array}$ \\
\hline
\end{tabular}


Appendix F - Effects of Pilot Scale MCT SeaGen Project at the Tacoma Narrows Site

\begin{tabular}{|c|c|c|c|c|c|c|c|}
\hline Project activity & Action & $\begin{array}{l}\text { Description of action's } \\
\text { effect on indicator }\end{array}$ & $\begin{array}{l}\text { Spatial exposure to } \\
\text { indicator (low, med, } \\
\text { high) }\end{array}$ & $\begin{array}{l}\text { Temporal } \\
\text { exposure to } \\
\text { indicator (low, } \\
\text { med, high) }\end{array}$ & $\begin{array}{l}\text { Effect's overlap with } \\
\text { critical life stage, } \\
\text { behavior, habitat or } \\
\text { resource (low, med, } \\
\text { high) }\end{array}$ & $\begin{array}{l}\text { Overall risk to } \\
\text { indicator (low, } \\
\text { med, high, } \\
\text { unknown) }\end{array}$ & Source(s) \\
\hline & Structure & $\begin{array}{l}\text { Possible attraction to } \\
\text { increased prey (FAD } \\
\text { effect), collision while } \\
\text { flying }\end{array}$ & $\begin{array}{l}\text { Low, would occur in } \\
\text { small area relative to } \\
\text { species' range }\end{array}$ & $\begin{array}{l}\text { High, continuous } \\
\text { for life of project }\end{array}$ & $\begin{array}{l}\text { Med, birds could be } \\
\text { attracted to structure }\end{array}$ & Unknown & $\begin{array}{l}\text { Boehlert et al. } \\
\text { 2008, SAS } \\
\text { [date } \\
\text { unknown] }\end{array}$ \\
\hline \multicolumn{8}{|c|}{ Decommissioning } \\
\hline \multirow{2}{*}{ Boat traffic } & $\begin{array}{l}\text { Oil/chemical } \\
\text { release }\end{array}$ & $\begin{array}{l}\text { Hypothermia, toxicity } \\
\text { to individuals and prey }\end{array}$ & $\begin{array}{l}\text { Low for typical small } \\
\text { volume releases, High } \\
\text { for large, catastrophic } \\
\text { spill }\end{array}$ & $\begin{array}{l}\text { Low, occurs over } \\
2 \text { months }\end{array}$ & $\begin{array}{l}\text { Med, if done in winter } \\
\text { when birds most } \\
\text { abundant; low if in } \\
\text { summer }\end{array}$ & Low & $\begin{array}{l}\text { LGL Limited } \\
\text { et al. 2009, } \\
\text { SAS [date } \\
\text { unknown] }\end{array}$ \\
\hline & $\begin{array}{l}\text { Noise and } \\
\text { vibration }\end{array}$ & $\begin{array}{l}\text { Possible movement } \\
\text { away from boat and } \\
\text { disruption of foraging }\end{array}$ & $\begin{array}{l}\text { Low, would occur in } \\
\text { small area relative to } \\
\text { species' range }\end{array}$ & $\begin{array}{l}\text { Low, occurs over } \\
2 \text { months }\end{array}$ & Med or low, see above & Low & $\begin{array}{l}\text { LGL Limited } \\
\text { et al. 2009, } \\
\text { SAS [date } \\
\text { unknown] }\end{array}$ \\
\hline \multirow{4}{*}{$\begin{array}{l}\text { Decommissioning } \\
\text { of structures on } \\
\text { water's surface or } \\
\text { seabed }\end{array}$} & $\begin{array}{l}\text { Noise and } \\
\text { vibration }\end{array}$ & $\begin{array}{l}\text { Possible movement } \\
\text { away from area and } \\
\text { disruption of foraging } \\
\text { or nesting }\end{array}$ & Low, see above & $\begin{array}{l}\text { Low, occurs over } \\
2 \text { months }\end{array}$ & Med or low, see above & Low & $\begin{array}{l}\text { Michel et al. } \\
\text { 2007, SAS } \\
\text { [date } \\
\text { unknown] }\end{array}$ \\
\hline & $\begin{array}{l}\text { Oil/chemical } \\
\text { release }\end{array}$ & $\begin{array}{l}\text { Hypothermia, toxicity } \\
\text { to individuals and prey }\end{array}$ & $\begin{array}{l}\text { Low, volume released } \\
\text { would occupy small area } \\
\text { relative to species' range }\end{array}$ & $\begin{array}{l}\text { Low, occurs over } \\
2 \text { months }\end{array}$ & Med or low, see above & Low & $\begin{array}{l}\text { Michel et al. } \\
\text { 2007, SAS } \\
\text { [date } \\
\text { unknown] }\end{array}$ \\
\hline & Construction lights & $\begin{array}{l}\text { None, these species not } \\
\text { known to be attracted to } \\
\text { lights }\end{array}$ & N/A & N/A & N/A & Low & $\begin{array}{l}\text { Montevecchi } \\
2006\end{array}$ \\
\hline & Seabed disturbance & $\begin{array}{l}\text { Possible short-term } \\
\text { changes in food } \\
\text { resources }\end{array}$ & $\begin{array}{l}\text { Low, would occur in } \\
\text { small area relative to } \\
\text { species' range }\end{array}$ & $\begin{array}{l}\text { Low, occurs over } \\
2 \text { months }\end{array}$ & Med or low, see above & Low & $\begin{array}{l}\text { Boehlert et al. } \\
\text { 2008, SAS } \\
\text { [date } \\
\text { unknown] }\end{array}$ \\
\hline
\end{tabular}

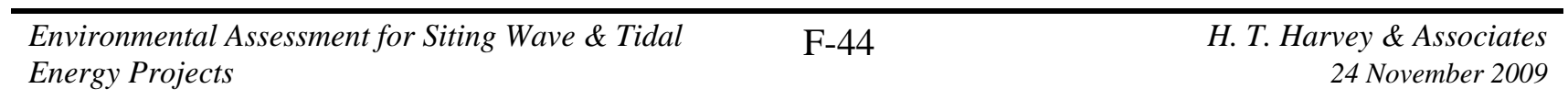


Appendix F - Effects of Pilot Scale MCT SeaGen Project at the Tacoma Narrows Site

Potential effects on cormorants from Pilot Scale MCT SeaGen project at Tacoma Narrows

\begin{tabular}{|c|c|c|c|c|c|c|c|}
\hline Project activity & Action & $\begin{array}{l}\text { Description of action's } \\
\text { effect on indicator }\end{array}$ & $\begin{array}{l}\text { Spatial exposure to } \\
\text { indicator (low, med, } \\
\text { high) }\end{array}$ & $\begin{array}{l}\text { Temporal } \\
\text { exposure to } \\
\text { indicator (low, } \\
\text { med, high) }\end{array}$ & $\begin{array}{l}\text { Effect's overlap } \\
\text { with critical life } \\
\text { stage, behavior, } \\
\text { habitat or resource } \\
\text { (low, med, high) }\end{array}$ & $\begin{array}{l}\text { Overall risk to } \\
\text { indicator (low, } \\
\text { med, high, } \\
\text { unknown) }\end{array}$ & Source(s) \\
\hline \multicolumn{8}{|c|}{ Construction } \\
\hline \multirow{2}{*}{ Boat traffic } & $\begin{array}{l}\text { Noise and } \\
\text { vibration }\end{array}$ & $\begin{array}{l}\text { Possible movement } \\
\text { away from boat and } \\
\text { disruption of foraging }\end{array}$ & $\begin{array}{l}\text { Low, would occur in } \\
\text { small area relative to } \\
\text { species' range }\end{array}$ & $\begin{array}{l}\text { Low, occurs over } 2 \\
\text { months }\end{array}$ & $\begin{array}{l}\text { Med, boats could } \\
\text { pass through foraging } \\
\text { areas }\end{array}$ & Low & $\begin{array}{l}\text { LGL Limited et } \\
\text { al. 2009, SAS } \\
\text { [date unknown] }\end{array}$ \\
\hline & $\begin{array}{l}\text { Oil/chemical } \\
\text { release }\end{array}$ & $\begin{array}{l}\text { Hypothermia, toxicity to } \\
\text { individuals and prey }\end{array}$ & $\begin{array}{l}\text { Low for typical small } \\
\text { volume releases, High } \\
\text { for large, catastrophic } \\
\text { spill }\end{array}$ & $\begin{array}{l}\text { Low, occurs over } 2 \\
\text { months }\end{array}$ & Med, see above & Low & $\begin{array}{l}\text { Michel et al. } \\
\text { 2007, SAS } \\
\text { [date unknown] }\end{array}$ \\
\hline \multirow{4}{*}{$\begin{array}{l}\text { Construction of } \\
\text { electrical collector } \\
\text { system, moorings/ } \\
\text { foundation; } \\
\text { installation of } \\
\text { devices }\end{array}$} & $\begin{array}{l}\text { Noise and } \\
\text { vibration }\end{array}$ & $\begin{array}{l}\text { Possible movement } \\
\text { away from area and } \\
\text { disruption of foraging or } \\
\text { nesting }\end{array}$ & $\begin{array}{l}\text { Low, would occur in } \\
\text { small area relative to } \\
\text { species' range }\end{array}$ & $\begin{array}{l}\text { Low, occurs over } 2 \\
\text { months }\end{array}$ & $\begin{array}{l}\text { Low, project not near } \\
\text { nesting colony or } \\
\text { known foraging area }\end{array}$ & Low & $\begin{array}{l}\text { Michel et al. } \\
\text { 2007, SAS } \\
\text { [date unknown] }\end{array}$ \\
\hline & $\begin{array}{l}\text { Oil/chemical } \\
\text { release }\end{array}$ & $\begin{array}{l}\text { Hypothermia, toxicity to } \\
\text { individuals and prey }\end{array}$ & $\begin{array}{l}\text { Low, volume released } \\
\text { would occupy small } \\
\text { area relative to species' } \\
\text { range }\end{array}$ & $\begin{array}{l}\text { Low, occurs over } 2 \\
\text { months }\end{array}$ & Low, see above & Low & $\begin{array}{l}\text { Michel et al. } \\
\text { 2007, SAS } \\
\text { [date unknown] }\end{array}$ \\
\hline & $\begin{array}{l}\text { Seabed } \\
\text { disturbance }\end{array}$ & $\begin{array}{l}\text { Possible short-term } \\
\text { changes in food } \\
\text { resources }\end{array}$ & $\begin{array}{l}\text { Low, would occur in } \\
\text { small area relative to } \\
\text { species' range }\end{array}$ & $\begin{array}{l}\text { Low, occurs over } 2 \\
\text { months }\end{array}$ & Low, see above & Low & $\begin{array}{l}\text { Boehlert et al. } \\
\text { 2008, SAS } \\
\text { [date unknown] }\end{array}$ \\
\hline & $\begin{array}{l}\text { Construction } \\
\text { lights }\end{array}$ & $\begin{array}{l}\text { None, cormorants not } \\
\text { known to be attracted to } \\
\text { lights }\end{array}$ & N/A & N/A & N/A & Low & $\begin{array}{l}\text { Montevecchi } \\
2006\end{array}$ \\
\hline \multirow{2}{*}{$\begin{array}{l}\text { Directional drilling, } \\
\text { and laying cable } \\
\text { under/on seabed }\end{array}$} & $\begin{array}{l}\text { Seabed } \\
\text { disturbance }\end{array}$ & $\begin{array}{l}\text { Possible short-term } \\
\text { changes in food } \\
\text { resources }\end{array}$ & $\begin{array}{l}\text { Low, would occur in } \\
\text { small area relative to } \\
\text { species' range }\end{array}$ & $\begin{array}{l}\text { Low, occurs over } 2 \\
\text { months }\end{array}$ & $\begin{array}{l}\text { Low, project not near } \\
\text { nesting colony or } \\
\text { known foraging area }\end{array}$ & Low & $\begin{array}{l}\text { Boehlert et al. } \\
\text { 2008, SAS } \\
\text { [date unknown] }\end{array}$ \\
\hline & $\begin{array}{l}\text { Noise and } \\
\text { vibration }\end{array}$ & $\begin{array}{l}\text { Possible movement } \\
\text { away from area and } \\
\text { disruption of foraging or } \\
\text { nesting }\end{array}$ & Low, see above & $\begin{array}{l}\text { Low, occurs over } 2 \\
\text { months }\end{array}$ & Low, see above & Low & $\begin{array}{l}\text { Michel et al. } \\
\text { 2007, SAS } \\
\text { [date unknown] }\end{array}$ \\
\hline \multicolumn{8}{|c|}{ Operation and Maintenance } \\
\hline
\end{tabular}

\begin{tabular}{lrr}
\hline $\begin{array}{l}\text { Environmental Assessment for Siting Wave \& Tidal } \\
\text { Energy Projects }\end{array}$ & F-45 & H. T. Harvey \& Associates \\
24 November 2009
\end{tabular}


Appendix F - Effects of Pilot Scale MCT SeaGen Project at the Tacoma Narrows Site

\begin{tabular}{|c|c|c|c|c|c|c|c|}
\hline Project activity & Action & $\begin{array}{l}\text { Description of action's } \\
\text { effect on indicator }\end{array}$ & $\begin{array}{l}\text { Spatial exposure to } \\
\text { indicator (low, med, } \\
\text { high) }\end{array}$ & $\begin{array}{l}\text { Temporal } \\
\text { exposure to } \\
\text { indicator (low, } \\
\text { med, high) }\end{array}$ & $\begin{array}{l}\text { Effect's overlap } \\
\text { with critical life } \\
\text { stage, behavior, } \\
\text { habitat or resource } \\
\text { (low, med, high) }\end{array}$ & $\begin{array}{l}\text { Overall risk to } \\
\text { indicator (low, } \\
\text { med, high, } \\
\text { unknown) }\end{array}$ & Source(s) \\
\hline \multirow[b]{2}{*}{ Boat traffic } & $\begin{array}{l}\text { Noise and } \\
\text { vibration }\end{array}$ & $\begin{array}{l}\text { Possible movement } \\
\text { away from boat and } \\
\text { disruption of foraging }\end{array}$ & Low, see above & $\begin{array}{l}\text { Low, only from } \\
\text { periodic trips in } \\
\text { summer }\end{array}$ & $\begin{array}{l}\text { Med, boats could } \\
\text { pass through foraging } \\
\text { areas }\end{array}$ & Low & $\begin{array}{l}\text { LGL Limited et } \\
\text { al. 2009, SAS } \\
\text { [date unknown] }\end{array}$ \\
\hline & $\begin{array}{l}\text { Oil/chemical } \\
\text { release }\end{array}$ & $\begin{array}{l}\text { Hypothermia, toxicity to } \\
\text { individuals and prey }\end{array}$ & $\begin{array}{l}\text { Low for typical small } \\
\text { volume releases, High } \\
\text { for large, catastrophic } \\
\text { spill }\end{array}$ & $\begin{array}{l}\text { Low, wouldn’t } \\
\text { persist long-term }\end{array}$ & Med, see above & Low & $\begin{array}{l}\text { Michel et al. } \\
\text { 2007, SAS } \\
\text { [date unknown] }\end{array}$ \\
\hline \multirow{2}{*}{$\begin{array}{l}\text { Operation of } \\
\text { turbines or other } \\
\text { moving parts of } \\
\text { devices }\end{array}$} & $\begin{array}{l}\text { Moving device } \\
\text { parts }\end{array}$ & $\begin{array}{l}\text { Possible collision while } \\
\text { diving }\end{array}$ & $\begin{array}{l}\text { Low, would occur in } \\
\text { small area relative to } \\
\text { species' range }\end{array}$ & $\begin{array}{l}\text { High, continuous } \\
\text { for life of project }\end{array}$ & $\begin{array}{l}\text { Med, cormorants } \\
\text { could be attracted to } \\
\text { structures }\end{array}$ & Unknown & $\begin{array}{l}\text { Michel et al. } \\
\text { 2007, SAS } \\
\text { [date unknown] }\end{array}$ \\
\hline & $\begin{array}{l}\text { Noise and } \\
\text { vibration }\end{array}$ & $\begin{array}{l}\text { Possible avoidance of } \\
\text { project area }\end{array}$ & Low, see above & $\begin{array}{l}\text { High, continuous } \\
\text { for life of project }\end{array}$ & $\begin{array}{l}\text { Low, project not near } \\
\text { nesting colony or } \\
\text { known foraging area }\end{array}$ & Low & $\begin{array}{l}\text { Michel et al. } \\
\text { 2007, SAS } \\
\text { [date unknown] }\end{array}$ \\
\hline \multirow{3}{*}{$\begin{array}{l}\text { Structures in water } \\
\text { column and on } \\
\text { seabed, such as } \\
\text { devices and } \\
\text { moorings and } \\
\text { footings }\end{array}$} & Structure & $\begin{array}{l}\text { Possible attraction to } \\
\text { increased prey (reef } \\
\text { effect), entanglement } \\
\text { with derelict fishing } \\
\text { gear }\end{array}$ & Low, see above & $\begin{array}{l}\text { High, continuous } \\
\text { for life of project }\end{array}$ & $\begin{array}{l}\text { Med, cormorants } \\
\text { could be attracted to } \\
\text { structures }\end{array}$ & Unknown & $\begin{array}{l}\text { Michel et al. } \\
\text { 2007, SAS } \\
\text { [date unknown] }\end{array}$ \\
\hline & $\begin{array}{l}\text { Water } \\
\text { circulation } \\
\text { changes }\end{array}$ & $\begin{array}{l}\text { None, project not large } \\
\text { enough to affect prey }\end{array}$ & N/A & N/A & N/A & Low & N/A \\
\hline & $\begin{array}{l}\text { Oil/chemical } \\
\text { release }\end{array}$ & $\begin{array}{l}\text { Hypothermia, toxicity to } \\
\text { individuals and prey }\end{array}$ & $\begin{array}{l}\text { Low for typical small } \\
\text { volume releases, High } \\
\text { for catastrophic release } \\
\text { from device failure }\end{array}$ & $\begin{array}{l}\text { Med, could be } \\
\text { continuous and } \\
\text { intermittent for life } \\
\text { of project }\end{array}$ & $\begin{array}{l}\text { Med, cormorants } \\
\text { could be attracted to } \\
\text { structures }\end{array}$ & Low & $\begin{array}{l}\text { Michel et al. } \\
\text { 2007, SAS } \\
\text { [date unknown] }\end{array}$ \\
\hline \multirow[b]{2}{*}{$\begin{array}{l}\text { Structures on } \\
\text { water's surface }\end{array}$} & $\begin{array}{l}\text { Navigation } \\
\text { lights }\end{array}$ & $\begin{array}{l}\text { None, cormorants not } \\
\text { known to be attracted to } \\
\text { lights }\end{array}$ & N/A & N/A & N/A & Low & $\begin{array}{l}\text { Montevecchi } \\
2006\end{array}$ \\
\hline & Structure & $\begin{array}{l}\text { Possible roosting } \\
\text { habitat, attraction to } \\
\text { increased prey (FAD } \\
\text { effect), collision while } \\
\text { flying }\end{array}$ & $\begin{array}{l}\text { Low, would occur in } \\
\text { small area relative to } \\
\text { species' range }\end{array}$ & $\begin{array}{l}\text { High, continuous } \\
\text { for life of project }\end{array}$ & $\begin{array}{l}\text { Med, cormorants } \\
\text { present in area year- } \\
\text { round }\end{array}$ & Unknown & $\begin{array}{l}\text { Michel et al. } \\
\text { 2007, SAS } \\
\text { [date unknown] }\end{array}$ \\
\hline \multicolumn{8}{|c|}{ Decommissioning } \\
\hline
\end{tabular}

\begin{tabular}{|c|c|c|}
\hline $\begin{array}{l}\text { Environmental Assessment for Siting Wave \& Tidal } \\
\text { Energy Projects }\end{array}$ & F-46 & $\begin{array}{r}\text { H. T. Harvey \& Associates } \\
24 \text { November } 2009\end{array}$ \\
\hline
\end{tabular}


Appendix F - Effects of Pilot Scale MCT SeaGen Project at the Tacoma Narrows Site

\begin{tabular}{|c|c|c|c|c|c|c|c|}
\hline Project activity & Action & $\begin{array}{l}\text { Description of action's } \\
\text { effect on indicator }\end{array}$ & $\begin{array}{l}\text { Spatial exposure to } \\
\text { indicator (low, med, } \\
\text { high) }\end{array}$ & $\begin{array}{l}\text { Temporal } \\
\text { exposure to } \\
\text { indicator (low, } \\
\text { med, high) }\end{array}$ & $\begin{array}{l}\text { Effect's overlap } \\
\text { with critical life } \\
\text { stage, behavior, } \\
\text { habitat or resource } \\
\text { (low, med, high) }\end{array}$ & $\begin{array}{l}\text { Overall risk to } \\
\text { indicator (low, } \\
\text { med, high, } \\
\text { unknown) }\end{array}$ & Source(s) \\
\hline \multirow{2}{*}{ Boat traffic } & $\begin{array}{l}\text { Oil/chemical } \\
\text { release }\end{array}$ & $\begin{array}{l}\text { Hypothermia, toxicity to } \\
\text { individuals and prey }\end{array}$ & $\begin{array}{l}\text { Low for typical small } \\
\text { volume releases, High } \\
\text { for large, catastrophic } \\
\text { spill }\end{array}$ & $\begin{array}{l}\text { Low, occurs over } 2 \\
\text { months }\end{array}$ & $\begin{array}{l}\text { Med, and boats could } \\
\text { pass through foraging } \\
\text { areas }\end{array}$ & Low & $\begin{array}{l}\text { Michel et al. } \\
\text { 2007, SAS } \\
\text { [date unknown] }\end{array}$ \\
\hline & $\begin{array}{l}\text { Noise and } \\
\text { vibration }\end{array}$ & $\begin{array}{l}\text { Possible movement } \\
\text { away from boat and } \\
\text { disruption of foraging }\end{array}$ & $\begin{array}{l}\text { Low, would occur in } \\
\text { small area relative to } \\
\text { species' range }\end{array}$ & $\begin{array}{l}\text { Low, occurs over } 2 \\
\text { months }\end{array}$ & Med, see above & Low & $\begin{array}{l}\text { LGL Limited et } \\
\text { al. 2009, SAS } \\
\text { [date unknown] }\end{array}$ \\
\hline \multirow{4}{*}{$\begin{array}{l}\text { Decommissioning } \\
\text { of structures on } \\
\text { water's surface or } \\
\text { seabed }\end{array}$} & $\begin{array}{l}\text { Noise and } \\
\text { vibration }\end{array}$ & $\begin{array}{l}\text { Possible movement } \\
\text { away from area and } \\
\text { disruption of foraging or } \\
\text { nesting }\end{array}$ & Low, see above & $\begin{array}{l}\text { Low, occurs over } 2 \\
\text { months }\end{array}$ & $\begin{array}{l}\text { Low, project not near } \\
\text { nesting colony or } \\
\text { known foraging area }\end{array}$ & Low & $\begin{array}{l}\text { Michel et al. } \\
\text { 2007, SAS } \\
\text { [date unknown] }\end{array}$ \\
\hline & $\begin{array}{l}\text { Oil/chemical } \\
\text { release }\end{array}$ & $\begin{array}{l}\text { Hypothermia, toxicity to } \\
\text { individuals and prey }\end{array}$ & $\begin{array}{l}\text { Low, volume released } \\
\text { would occupy small } \\
\text { area relative to species' } \\
\text { range }\end{array}$ & $\begin{array}{l}\text { Low, occurs over } 2 \\
\text { months }\end{array}$ & Low, see above & Low & $\begin{array}{l}\text { Michel et al. } \\
\text { 2007, SAS } \\
\text { [date unknown] }\end{array}$ \\
\hline & $\begin{array}{l}\text { Seabed } \\
\text { disturbance }\end{array}$ & $\begin{array}{l}\text { Possible short-term } \\
\text { changes in food } \\
\text { resources }\end{array}$ & $\begin{array}{l}\text { Low, would occur in } \\
\text { small area relative to } \\
\text { species' range }\end{array}$ & $\begin{array}{l}\text { Low, occurs over } 2 \\
\text { months }\end{array}$ & Low, see above & Low & $\begin{array}{l}\text { Boehlert et al. } \\
\text { 2008, SAS } \\
\text { [date unknown] }\end{array}$ \\
\hline & $\begin{array}{l}\text { Construction } \\
\text { lights }\end{array}$ & $\begin{array}{l}\text { None, cormorants not } \\
\text { known to be attracted to } \\
\text { lights }\end{array}$ & N/A & N/A & N/A & Low & $\begin{array}{l}\text { Montevecchi } \\
2006\end{array}$ \\
\hline
\end{tabular}

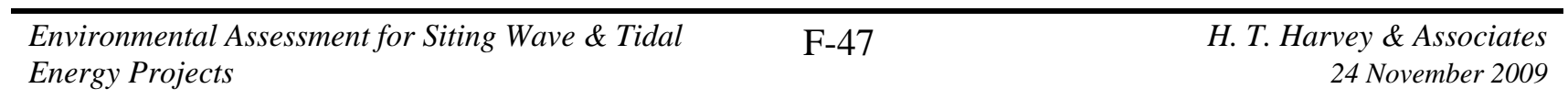


Appendix F - Effects of Pilot Scale MCT SeaGen Project at the Tacoma Narrows Site

Potential effects on gulls from Pilot Scale MCT SeaGen project at Tacoma Narrows

\begin{tabular}{|c|c|c|c|c|c|c|c|}
\hline Project activity & Action & $\begin{array}{l}\text { Description of action's } \\
\text { effect on indicator }\end{array}$ & $\begin{array}{l}\text { Spatial exposure to } \\
\text { indicator (low, med, } \\
\text { high) }\end{array}$ & $\begin{array}{l}\text { Temporal } \\
\text { exposure to } \\
\text { indicator (low, } \\
\text { med, high) } \\
\end{array}$ & $\begin{array}{l}\text { Effect's overlap } \\
\text { with critical life } \\
\text { stage, behavior, } \\
\text { habitat or resource } \\
\text { (low, med, high) } \\
\end{array}$ & $\begin{array}{l}\text { Overall risk to } \\
\text { indicator (low, } \\
\text { med, high, } \\
\text { unknown) }\end{array}$ & Source(s) \\
\hline \multicolumn{8}{|c|}{ Construction } \\
\hline \multirow{2}{*}{ Boat traffic } & $\begin{array}{l}\text { Noise and } \\
\text { vibration }\end{array}$ & $\begin{array}{l}\text { None, gulls follow boats } \\
\text { and do not appear to be } \\
\text { disturbed by boat noise }\end{array}$ & N/A & N/A & N/A & Low & USFWS 2005 \\
\hline & $\begin{array}{l}\text { Oil/chemical } \\
\text { release }\end{array}$ & $\begin{array}{l}\text { Hypothermia, toxicity } \\
\text { to individuals and prey }\end{array}$ & $\begin{array}{l}\text { Low for typical small } \\
\text { volume releases, High } \\
\text { for large, catastrophic } \\
\text { spill }\end{array}$ & $\begin{array}{l}\text { Low, occurs over } \\
2 \text { months }\end{array}$ & $\begin{array}{l}\text { Med, boats could } \\
\text { pass through } \\
\text { foraging areas }\end{array}$ & Low & $\begin{array}{l}\text { Michel et al. } \\
\text { 2007, SAS [date } \\
\text { unknown] }\end{array}$ \\
\hline \multirow{4}{*}{$\begin{array}{l}\text { Construction of } \\
\text { electrical collector } \\
\text { system, } \\
\text { moorings/foundation; } \\
\text { installation of } \\
\text { devices }\end{array}$} & $\begin{array}{l}\text { Noise and } \\
\text { vibration }\end{array}$ & $\begin{array}{l}\text { Possible movement } \\
\text { away from area and } \\
\text { disruption of foraging } \\
\text { or nesting }\end{array}$ & $\begin{array}{l}\text { Low, would occur in } \\
\text { small area relative to } \\
\text { species' range }\end{array}$ & $\begin{array}{l}\text { Low, occurs over } \\
2 \text { months }\end{array}$ & $\begin{array}{l}\text { Low, project not near } \\
\text { nesting colony or } \\
\text { known foraging area }\end{array}$ & Low & $\begin{array}{l}\text { Michel et al. } \\
\text { 2007, SAS [date } \\
\text { unknown] }\end{array}$ \\
\hline & $\begin{array}{l}\text { Oil/chemical } \\
\text { release }\end{array}$ & $\begin{array}{l}\text { Hypothermia, toxicity } \\
\text { to individuals and prey }\end{array}$ & $\begin{array}{l}\text { Low, volume released } \\
\text { would occupy small } \\
\text { area relative to species' } \\
\text { range }\end{array}$ & $\begin{array}{l}\text { Low, occurs over } \\
2 \text { months }\end{array}$ & Low, see above & Low & $\begin{array}{l}\text { Michel et al. } \\
\text { 2007, SAS [date } \\
\text { unknown] }\end{array}$ \\
\hline & $\begin{array}{l}\text { Seabed } \\
\text { disturbance }\end{array}$ & $\begin{array}{l}\text { None, these are surface- } \\
\text { feeders }\end{array}$ & N/A & N/A & N/A & Low & USFWS 2005 \\
\hline & $\begin{array}{l}\text { Construction } \\
\text { lights }\end{array}$ & $\begin{array}{l}\text { None, gulls not known } \\
\text { to be attracted to lights }\end{array}$ & N/A & N/A & N/A & Low & $\begin{array}{l}\text { Montevecchi } \\
2006\end{array}$ \\
\hline \multirow{2}{*}{$\begin{array}{l}\text { Directional drilling, } \\
\text { and laying cable } \\
\text { under/on seabed }\end{array}$} & $\begin{array}{l}\text { Seabed } \\
\text { disturbance }\end{array}$ & $\begin{array}{l}\text { None, these are surface- } \\
\text { feeders }\end{array}$ & N/A & N/A & N/A & Low & USFWS 2005 \\
\hline & $\begin{array}{l}\text { Noise and } \\
\text { vibration }\end{array}$ & $\begin{array}{l}\text { Possible movement } \\
\text { away from area and } \\
\text { disruption of foraging } \\
\text { or nesting }\end{array}$ & $\begin{array}{l}\text { Low, would occur in } \\
\text { small area relative to } \\
\text { species' range }\end{array}$ & $\begin{array}{l}\text { Low, occurs over } \\
2 \text { months }\end{array}$ & $\begin{array}{l}\text { Low, project not near } \\
\text { nesting colony or } \\
\text { known foraging area }\end{array}$ & Low & $\begin{array}{l}\text { Michel et al. } \\
\text { 2007, SAS [date } \\
\text { unknown] }\end{array}$ \\
\hline \multicolumn{8}{|c|}{ Operation and Maintenance } \\
\hline Boat traffic & $\begin{array}{l}\text { Noise and } \\
\text { vibration }\end{array}$ & $\begin{array}{l}\text { None, gulls follow boats } \\
\text { and do not appear to be } \\
\text { disturbed by boat noise }\end{array}$ & N/A & N/A & N/A & Low & USFWS 2005 \\
\hline
\end{tabular}

\begin{tabular}{lrr}
\hline $\begin{array}{l}\text { Environmental Assessment for Siting Wave \& Tidal } \\
\text { Energy Projects }\end{array}$ & F-48 & H. T. Harvey \& Associates \\
24 November 2009
\end{tabular}


Appendix F - Effects of Pilot Scale MCT SeaGen Project at the Tacoma Narrows Site

\begin{tabular}{|c|c|c|c|c|c|c|c|}
\hline Project activity & Action & $\begin{array}{l}\text { Description of action's } \\
\text { effect on indicator }\end{array}$ & $\begin{array}{l}\text { Spatial exposure to } \\
\text { indicator (low, med, } \\
\text { high) }\end{array}$ & $\begin{array}{l}\text { Temporal } \\
\text { exposure to } \\
\text { indicator (low, } \\
\text { med, high) }\end{array}$ & $\begin{array}{l}\text { Effect's overlap } \\
\text { with critical life } \\
\text { stage, behavior, } \\
\text { habitat or resource } \\
\text { (low, med, high) }\end{array}$ & $\begin{array}{l}\text { Overall risk to } \\
\text { indicator (low, } \\
\text { med, high, } \\
\text { unknown) }\end{array}$ & Source(s) \\
\hline & $\begin{array}{l}\text { Oil/chemical } \\
\text { release }\end{array}$ & $\begin{array}{l}\text { Hypothermia, toxicity } \\
\text { to individuals and prey }\end{array}$ & $\begin{array}{l}\text { Low for typical small } \\
\text { volume releases, High } \\
\text { for large, catastrophic } \\
\text { spill }\end{array}$ & $\begin{array}{l}\text { Low, wouldn’t } \\
\text { persist long-term }\end{array}$ & $\begin{array}{l}\text { Med, boats could } \\
\text { pass through } \\
\text { foraging areas }\end{array}$ & Low & $\begin{array}{l}\text { Michel et al. } \\
\text { 2007, SAS [date } \\
\text { unknown] }\end{array}$ \\
\hline \multirow{2}{*}{$\begin{array}{l}\text { Operation of turbines } \\
\text { or other moving parts } \\
\text { of devices }\end{array}$} & $\begin{array}{l}\text { Moving device } \\
\text { parts }\end{array}$ & $\begin{array}{l}\text { None, these are surface- } \\
\text { feeders }\end{array}$ & N/A & N/A & N/A & Low & USFWS 2005 \\
\hline & $\begin{array}{l}\text { Noise and } \\
\text { vibration }\end{array}$ & $\begin{array}{l}\text { Possible avoidance of } \\
\text { project area }\end{array}$ & $\begin{array}{l}\text { Low, would occur in } \\
\text { small area relative to } \\
\text { species' range }\end{array}$ & $\begin{array}{l}\text { High, would be } \\
\text { continuous for life } \\
\text { of project }\end{array}$ & $\begin{array}{l}\text { Low, project not near } \\
\text { nesting colony or } \\
\text { known foraging area }\end{array}$ & Low & $\begin{array}{l}\text { Michel et al. } \\
\text { 2007, SAS [date } \\
\text { unknown] }\end{array}$ \\
\hline \multirow{3}{*}{$\begin{array}{l}\text { Structures in water } \\
\text { column and on } \\
\text { seabed, such as } \\
\text { devices and } \\
\text { moorings and } \\
\text { footings }\end{array}$} & Structure & $\begin{array}{l}\text { None, gulls do not dive } \\
\text { deep enough }\end{array}$ & N/A & N/A & N/A & Low & USFWS 2005 \\
\hline & $\begin{array}{l}\text { Water } \\
\text { circulation } \\
\text { changes }\end{array}$ & $\begin{array}{l}\text { None, project not large } \\
\text { enough to affect prey }\end{array}$ & N/A & N/A & N/A & Low & N/A \\
\hline & $\begin{array}{l}\text { Oil/chemical } \\
\text { release }\end{array}$ & $\begin{array}{l}\text { Hypothermia, toxicity } \\
\text { to individuals and prey }\end{array}$ & $\begin{array}{l}\text { Low for typical small } \\
\text { volume releases, High } \\
\text { for large, catastrophic } \\
\text { release from device } \\
\text { failure }\end{array}$ & $\begin{array}{l}\text { Med, could be } \\
\text { continuous and } \\
\text { intermittent for life } \\
\text { of project }\end{array}$ & $\begin{array}{l}\text { Med, species could } \\
\text { be attracted to } \\
\text { structures }\end{array}$ & Low & $\begin{array}{l}\text { Michel et al. } \\
\text { 2007, SAS [date } \\
\text { unknown] }\end{array}$ \\
\hline \multirow[b]{2}{*}{$\begin{array}{l}\text { Structures on water's } \\
\text { surface }\end{array}$} & $\begin{array}{l}\text { Navigation } \\
\text { lights }\end{array}$ & $\begin{array}{l}\text { None, gulls not known } \\
\text { to be attracted to lights }\end{array}$ & N/A & N/A & N/A & Low & $\begin{array}{l}\text { Montevecchi } \\
2006\end{array}$ \\
\hline & Structure & $\begin{array}{l}\text { Possible roosting on } \\
\text { structures, collision } \\
\text { while flying, attraction } \\
\text { to increased prey (FAD } \\
\text { effect) }\end{array}$ & $\begin{array}{l}\text { Low, would occur in } \\
\text { small area relative to } \\
\text { species' range }\end{array}$ & $\begin{array}{l}\text { High, would be } \\
\text { continuous for life } \\
\text { of project }\end{array}$ & $\begin{array}{l}\text { Med, species could } \\
\text { be attracted to } \\
\text { structures }\end{array}$ & Unknown & $\begin{array}{l}\text { Boehlert et al. } \\
\text { 2008, SAS [date } \\
\text { unknown] }\end{array}$ \\
\hline \multicolumn{8}{|c|}{ Decommissioning } \\
\hline Boat traffic & $\begin{array}{l}\text { Oil/chemical } \\
\text { release }\end{array}$ & $\begin{array}{l}\text { Hypothermia, toxicity } \\
\text { to individuals and prey }\end{array}$ & $\begin{array}{l}\text { Low for typical small } \\
\text { volume releases, High } \\
\text { for large, catastrophic } \\
\text { spill }\end{array}$ & $\begin{array}{l}\text { Low, occurs over } \\
2 \text { months }\end{array}$ & $\begin{array}{l}\text { Med, boats could } \\
\text { pass through } \\
\text { foraging areas }\end{array}$ & Low & $\begin{array}{l}\text { Michel et al. } \\
\text { 2007, SAS [date } \\
\text { unknown] }\end{array}$ \\
\hline
\end{tabular}

\begin{tabular}{|c|c|c|}
\hline $\begin{array}{l}\text { Environmental Assessment for Siting Wave \& Tidal } \\
\text { Energy Projects }\end{array}$ & F-49 & $\begin{array}{r}\text { H. T. Harvey \& Associates } \\
24 \text { November } 2009\end{array}$ \\
\hline
\end{tabular}


Appendix F - Effects of Pilot Scale MCT SeaGen Project at the Tacoma Narrows Site

\begin{tabular}{|c|c|c|c|c|c|c|c|}
\hline Project activity & Action & $\begin{array}{l}\text { Description of action's } \\
\text { effect on indicator }\end{array}$ & $\begin{array}{l}\text { Spatial exposure to } \\
\text { indicator (low, med, } \\
\text { high) }\end{array}$ & $\begin{array}{l}\text { Temporal } \\
\text { exposure to } \\
\text { indicator (low, } \\
\text { med, high) }\end{array}$ & $\begin{array}{l}\text { Effect's overlap } \\
\text { with critical life } \\
\text { stage, behavior, } \\
\text { habitat or resource } \\
\text { (low, med, high) }\end{array}$ & $\begin{array}{l}\text { Overall risk to } \\
\text { indicator (low, } \\
\text { med, high, } \\
\text { unknown) }\end{array}$ & Source(s) \\
\hline & $\begin{array}{l}\text { Noise and } \\
\text { vibration }\end{array}$ & $\begin{array}{l}\text { None, gulls follow boats } \\
\text { and do not appear to be } \\
\text { disturbed by boat noise }\end{array}$ & N/A & N/A & N/A & Low & USFWS 2005 \\
\hline \multirow{4}{*}{$\begin{array}{l}\text { Decommissioning of } \\
\text { structures on water's } \\
\text { surface or seabed }\end{array}$} & $\begin{array}{l}\text { Oil/chemical } \\
\text { release }\end{array}$ & $\begin{array}{l}\text { Hypothermia, toxicity } \\
\text { to individuals and prey }\end{array}$ & $\begin{array}{l}\text { Low, volume released } \\
\text { would occupy small } \\
\text { area }\end{array}$ & $\begin{array}{l}\text { Low, occurs over } \\
2 \text { months }\end{array}$ & $\begin{array}{l}\text { Low, project not near } \\
\text { nesting colony or } \\
\text { known foraging area }\end{array}$ & Low & $\begin{array}{l}\text { Michel et al. } \\
\text { 2007, SAS [date } \\
\text { unknown] }\end{array}$ \\
\hline & $\begin{array}{l}\text { Noise and } \\
\text { vibration }\end{array}$ & $\begin{array}{l}\text { Possible movement } \\
\text { away from area and } \\
\text { disruption of foraging } \\
\text { or nesting }\end{array}$ & $\begin{array}{l}\text { Low, would occur in } \\
\text { small area relative to } \\
\text { species' range }\end{array}$ & $\begin{array}{l}\text { Low, occurs over } \\
2 \text { months }\end{array}$ & Low, see above & Low & USFWS 2005 \\
\hline & $\begin{array}{l}\text { Construction } \\
\text { lights }\end{array}$ & $\begin{array}{l}\text { None, gulls not known } \\
\text { to be attracted to lights }\end{array}$ & N/A & N/A & N/A & Low & $\begin{array}{l}\text { Montevecchi } \\
2006\end{array}$ \\
\hline & $\begin{array}{l}\text { Seabed } \\
\text { disturbance }\end{array}$ & $\begin{array}{l}\text { None, these are surface- } \\
\text { feeders }\end{array}$ & N/A & N/A & N/A & Low & USFWS 2005 \\
\hline
\end{tabular}

\begin{tabular}{lrr}
\hline $\begin{array}{l}\text { Environmental Assessment for Siting Wave \& Tidal } \\
\text { Energy Projects }\end{array}$ & F-50 & H. T. Harvey \& Associates \\
24 November 2009
\end{tabular}


Appendix F - Effects of Pilot Scale MCT SeaGen Project at the Tacoma Narrows Site

Potential effects on alcids and marbled murrelets from Pilot Scale MCT SeaGen project at Tacoma Narrows

\begin{tabular}{|c|c|c|c|c|c|c|c|}
\hline Project activity & Action & $\begin{array}{l}\text { Description of action's } \\
\text { effect on indicator }\end{array}$ & $\begin{array}{l}\text { Spatial exposure to } \\
\text { indicator (low, med, } \\
\text { high) }\end{array}$ & $\begin{array}{l}\text { Temporal } \\
\text { exposure to } \\
\text { indicator (low, } \\
\text { med, high) }\end{array}$ & $\begin{array}{l}\text { Effect's overlap } \\
\text { with critical life } \\
\text { stage, behavior, } \\
\text { habitat or resource } \\
\text { (low, med, high) }\end{array}$ & $\begin{array}{l}\text { Overall risk to } \\
\text { indicator (low, } \\
\text { med, high, } \\
\text { unknown) }\end{array}$ & Source(s) \\
\hline \multicolumn{8}{|c|}{ Construction } \\
\hline \multirow{2}{*}{ Boat traffic } & $\begin{array}{l}\text { Noise and } \\
\text { vibration }\end{array}$ & $\begin{array}{l}\text { Possible movement } \\
\text { away from boat and } \\
\text { disruption of foraging }\end{array}$ & $\begin{array}{l}\text { Low, would occur in } \\
\text { small area relative to } \\
\text { species' range }\end{array}$ & $\begin{array}{l}\text { Low, occurs over } \\
2 \text { months }\end{array}$ & $\begin{array}{l}\text { Med, boats could } \\
\text { pass through } \\
\text { foraging areas }\end{array}$ & Low & $\begin{array}{l}\text { LGL Limited et } \\
\text { al. 2009, SAS } \\
\text { [date unknown] }\end{array}$ \\
\hline & $\begin{array}{l}\text { Oil/chemical } \\
\text { release }\end{array}$ & $\begin{array}{l}\text { Hypothermia, toxicity } \\
\text { to individuals and prey }\end{array}$ & $\begin{array}{l}\text { Low for typical small } \\
\text { volume releases, High } \\
\text { for large spill }\end{array}$ & $\begin{array}{l}\text { Low, occurs over } \\
2 \text { months }\end{array}$ & Med, see above & Low & $\begin{array}{l}\text { Michel et al. } \\
\text { 2007, SAS [date } \\
\text { unknown] }\end{array}$ \\
\hline \multirow{4}{*}{$\begin{array}{l}\text { Construction of } \\
\text { electrical collector } \\
\text { system, } \\
\text { moorings/foundation; } \\
\text { installation of } \\
\text { devices }\end{array}$} & $\begin{array}{l}\text { Noise and } \\
\text { vibration }\end{array}$ & $\begin{array}{l}\text { Possible movement } \\
\text { away from area and } \\
\text { disruption of foraging } \\
\text { or nesting }\end{array}$ & $\begin{array}{l}\text { Low, would occur in } \\
\text { small area relative to } \\
\text { species' range }\end{array}$ & $\begin{array}{l}\text { Low, occurs over } \\
2 \text { months }\end{array}$ & $\begin{array}{l}\text { Low, project not near } \\
\text { nesting colony or } \\
\text { known foraging area }\end{array}$ & Low & $\begin{array}{l}\text { Michel et al. } \\
\text { 2007, SAS [date } \\
\text { unknown] }\end{array}$ \\
\hline & $\begin{array}{l}\text { Oil/chemical } \\
\text { release }\end{array}$ & $\begin{array}{l}\text { Hypothermia, toxicity } \\
\text { to individuals and prey }\end{array}$ & $\begin{array}{l}\text { Low, volume released } \\
\text { would occupy small } \\
\text { area }\end{array}$ & $\begin{array}{l}\text { Low, occurs over } \\
2 \text { months }\end{array}$ & Low, see above & Low & $\begin{array}{l}\text { Michel et al. } \\
\text { 2007, SAS [date } \\
\text { unknown] }\end{array}$ \\
\hline & $\begin{array}{l}\text { Seabed } \\
\text { disturbance }\end{array}$ & $\begin{array}{l}\text { Possible short-term } \\
\text { changes in food } \\
\text { resources }\end{array}$ & $\begin{array}{l}\text { Low, would occur in } \\
\text { small area relative to } \\
\text { species’ range }\end{array}$ & $\begin{array}{l}\text { Low, occurs over } \\
2 \text { months }\end{array}$ & Low, see above & Low & $\begin{array}{l}\text { Boehlert et al. } \\
\text { 2008, SAS [date } \\
\text { unknown] }\end{array}$ \\
\hline & $\begin{array}{l}\text { Construction } \\
\text { lights }\end{array}$ & $\begin{array}{l}\text { Attraction/ } \\
\text { disorientation }\end{array}$ & Low, see above & $\begin{array}{l}\text { Low, occurs over } \\
2 \text { months }\end{array}$ & Low, see above & Low & $\begin{array}{l}\text { Montevecchi } \\
\text { 2006, SAS [date } \\
\text { unknown] }\end{array}$ \\
\hline \multirow{2}{*}{$\begin{array}{l}\text { Directional drilling, } \\
\text { and laying cable } \\
\text { under/on seabed }\end{array}$} & $\begin{array}{l}\text { Seabed } \\
\text { disturbance }\end{array}$ & $\begin{array}{l}\text { Possible short-term } \\
\text { changes in food } \\
\text { resources }\end{array}$ & Low, see above & $\begin{array}{l}\text { Low, occurs over } \\
2 \text { months }\end{array}$ & Low, see above & Low & $\begin{array}{l}\text { Boehlert et al. } \\
\text { 2008, SAS [date } \\
\text { unknown] }\end{array}$ \\
\hline & $\begin{array}{l}\text { Noise and } \\
\text { vibration }\end{array}$ & $\begin{array}{l}\text { Possible movement } \\
\text { from area and } \\
\text { disruption of foraging } \\
\text { or nesting }\end{array}$ & Low, see above & $\begin{array}{l}\text { Low, occurs over } \\
2 \text { months }\end{array}$ & Low, see above & Low & $\begin{array}{l}\text { Michel et al. } \\
\text { 2007, SAS [date } \\
\text { unknown] }\end{array}$ \\
\hline \multicolumn{8}{|c|}{ Operation and Maintenance } \\
\hline \multirow[t]{2}{*}{ Boat traffic } & $\begin{array}{l}\text { Oil/chemical } \\
\text { release }\end{array}$ & $\begin{array}{l}\text { Hypothermia, toxicity } \\
\text { to individuals and prey }\end{array}$ & $\begin{array}{l}\text { Low for typical small } \\
\text { volume releases, High } \\
\text { for large, catastrophic } \\
\text { spill }\end{array}$ & $\begin{array}{l}\text { Low, wouldn't } \\
\text { persist long-term }\end{array}$ & $\begin{array}{l}\text { Med, boats could } \\
\text { pass through } \\
\text { foraging areas }\end{array}$ & Low & $\begin{array}{l}\text { Michel et al. } \\
\text { 2007, SAS [date } \\
\text { unknown] }\end{array}$ \\
\hline & $\begin{array}{l}\text { Noise and } \\
\text { vibration }\end{array}$ & $\begin{array}{l}\text { Possible movement } \\
\text { away from boat and } \\
\text { disruption of foraging }\end{array}$ & $\begin{array}{l}\text { Low, would occur in } \\
\text { small area relative to } \\
\text { species’ range }\end{array}$ & $\begin{array}{l}\text { Low, only from } \\
\text { periodic trips in } \\
\text { summer }\end{array}$ & Med, see above & Low & $\begin{array}{l}\text { LGL Limited et } \\
\text { al. 2009, SAS } \\
\text { [date unknown] }\end{array}$ \\
\hline
\end{tabular}

\begin{tabular}{|c|c|c|}
\hline $\begin{array}{l}\text { Environmental Assessment for Siting Wave \& Tidal } \\
\text { Energy Projects }\end{array}$ & F-51 & $\begin{array}{r}\text { H. T. Harvey \& Associates } \\
24 \text { November } 2009\end{array}$ \\
\hline
\end{tabular}


Appendix F - Effects of Pilot Scale MCT SeaGen Project at the Tacoma Narrows Site

\begin{tabular}{|c|c|c|c|c|c|c|c|}
\hline Project activity & Action & $\begin{array}{l}\text { Description of action's } \\
\text { effect on indicator }\end{array}$ & $\begin{array}{l}\text { Spatial exposure to } \\
\text { indicator (low, med, } \\
\text { high) }\end{array}$ & $\begin{array}{l}\text { Temporal } \\
\text { exposure to } \\
\text { indicator (low, } \\
\text { med, high) }\end{array}$ & $\begin{array}{l}\text { Effect's overlap } \\
\text { with critical life } \\
\text { stage, behavior, } \\
\text { habitat or resource } \\
\text { (low, med, high) } \\
\end{array}$ & $\begin{array}{l}\text { Overall risk to } \\
\text { indicator (low, } \\
\text { med, high, } \\
\text { unknown) }\end{array}$ & Source(s) \\
\hline \multirow{2}{*}{$\begin{array}{l}\text { Operation of turbines } \\
\text { or other moving parts } \\
\text { of devices }\end{array}$} & $\begin{array}{l}\text { Moving device } \\
\text { parts }\end{array}$ & $\begin{array}{l}\text { Possible collision while } \\
\text { diving }\end{array}$ & Low, see above & $\begin{array}{l}\text { High, continuous } \\
\text { for life of project }\end{array}$ & $\begin{array}{l}\text { Med, alcids present } \\
\text { year-round and could } \\
\text { be attracted to } \\
\text { structure }\end{array}$ & Unknown & $\begin{array}{l}\text { Boehlert et al. } \\
\text { 2008, SAS [date } \\
\text { unknown] }\end{array}$ \\
\hline & $\begin{array}{l}\text { Noise and } \\
\text { vibration }\end{array}$ & $\begin{array}{l}\text { Possible avoidance of } \\
\text { project area }\end{array}$ & Low, see above & $\begin{array}{l}\text { High, continuous } \\
\text { for life of project }\end{array}$ & Low, see above & Low & $\begin{array}{l}\text { Michel et al. } \\
\text { 2007, SAS [date } \\
\text { unknown] }\end{array}$ \\
\hline \multirow{3}{*}{$\begin{array}{l}\text { Structures in water } \\
\text { column and on } \\
\text { seabed, such as } \\
\text { devices and } \\
\text { moorings and } \\
\text { footings }\end{array}$} & Structure & $\begin{array}{l}\text { Possible attraction to } \\
\text { increased prey (reef } \\
\text { effect), entanglement } \\
\text { with derelict fishing } \\
\text { gear }\end{array}$ & Low, see above & $\begin{array}{l}\text { High, continuous } \\
\text { for life of project }\end{array}$ & $\begin{array}{l}\text { Med, alcids present } \\
\text { year-round and could } \\
\text { be attracted to } \\
\text { structure }\end{array}$ & Unknown & $\begin{array}{l}\text { Michel et al. } \\
\text { 2007, SAS [date } \\
\text { unknown] }\end{array}$ \\
\hline & $\begin{array}{l}\text { Water } \\
\text { circulation } \\
\text { changes }\end{array}$ & $\begin{array}{l}\text { None, project not large } \\
\text { enough to affect prey }\end{array}$ & N/A & N/A & N/A & Low & N/A \\
\hline & $\begin{array}{l}\text { Oil/chemical } \\
\text { release }\end{array}$ & $\begin{array}{l}\text { Hypothermia, toxicity } \\
\text { to individuals and prey }\end{array}$ & $\begin{array}{l}\text { Low for typical small } \\
\text { volume releases, High } \\
\text { for large, release from } \\
\text { device failure }\end{array}$ & $\begin{array}{l}\text { Med, continuous } \\
\text { and intermittent } \\
\text { for life of project }\end{array}$ & $\begin{array}{l}\text { Low, project not in } \\
\text { known foraging area }\end{array}$ & Low & $\begin{array}{l}\text { Michel et al. } \\
\text { 2007, SAS [date } \\
\text { unknown] }\end{array}$ \\
\hline \multirow{2}{*}{$\begin{array}{l}\text { Structures on water's } \\
\text { surface }\end{array}$} & $\begin{array}{l}\text { Navigation } \\
\text { lights }\end{array}$ & $\begin{array}{l}\text { Attraction/ } \\
\text { disorientation }\end{array}$ & $\begin{array}{l}\text { Low, would occur in } \\
\text { small area relative to } \\
\text { species' range }\end{array}$ & $\begin{array}{l}\text { High, continuous } \\
\text { for life of project }\end{array}$ & $\begin{array}{l}\text { Med, alcids present } \\
\text { year-round and could } \\
\text { be attracted to lights }\end{array}$ & Med & $\begin{array}{l}\text { Montevecchi } \\
\text { 2006, SAS [date } \\
\text { unknown] } \\
\end{array}$ \\
\hline & Structure & $\begin{array}{l}\text { Possible collision while } \\
\text { flying, attraction to } \\
\text { increased prey (FAD } \\
\text { effect) }\end{array}$ & Low, see above & $\begin{array}{l}\text { High, continuous } \\
\text { for life of project }\end{array}$ & $\begin{array}{l}\text { Med, alcids present } \\
\text { year-round and could } \\
\text { be attracted to } \\
\text { structure }\end{array}$ & Unknown & $\begin{array}{l}\text { Boehlert et al. } \\
\text { 2008, SAS [date } \\
\text { unknown] }\end{array}$ \\
\hline \multicolumn{8}{|c|}{ Decommissioning } \\
\hline \multirow[t]{2}{*}{ Boat traffic } & $\begin{array}{l}\text { Oil/chemical } \\
\text { release }\end{array}$ & $\begin{array}{l}\text { Hypothermia, toxicity } \\
\text { to individuals and prey }\end{array}$ & $\begin{array}{l}\text { Low for typical small } \\
\text { volume releases, High } \\
\text { for large, catastrophic } \\
\text { spill }\end{array}$ & $\begin{array}{l}\text { Low, occurs over } \\
2 \text { months }\end{array}$ & $\begin{array}{l}\text { Med, boats could } \\
\text { pass through } \\
\text { foraging areas }\end{array}$ & Low & $\begin{array}{l}\text { Michel et al. } \\
\text { 2007, SAS [date } \\
\text { unknown] }\end{array}$ \\
\hline & $\begin{array}{l}\text { Noise and } \\
\text { vibration }\end{array}$ & $\begin{array}{l}\text { Possible movement } \\
\text { away from boat and } \\
\text { disruption of foraging }\end{array}$ & $\begin{array}{l}\text { Low, would occur in } \\
\text { small area relative to } \\
\text { species' range }\end{array}$ & $\begin{array}{l}\text { Low, occurs over } \\
2 \text { months }\end{array}$ & Med, see above & Low & $\begin{array}{l}\text { LGL Limited et } \\
\text { al. 2009, SAS } \\
\text { [date unknown] }\end{array}$ \\
\hline
\end{tabular}

\begin{tabular}{|c|c|c|}
\hline $\begin{array}{l}\text { Environmental Assessment for Siting Wave \& Tidal } \\
\text { Energy Projects }\end{array}$ & $\mathrm{F}-52$ & $\begin{array}{r}\text { H. T. Harvey \& Associates } \\
24 \text { November } 2009\end{array}$ \\
\hline
\end{tabular}


Appendix F - Effects of Pilot Scale MCT SeaGen Project at the Tacoma Narrows Site

\begin{tabular}{|c|c|c|c|c|c|c|c|}
\hline Project activity & Action & $\begin{array}{l}\text { Description of action's } \\
\text { effect on indicator }\end{array}$ & $\begin{array}{l}\text { Spatial exposure to } \\
\text { indicator (low, med, } \\
\text { high) }\end{array}$ & $\begin{array}{l}\text { Temporal } \\
\text { exposure to } \\
\text { indicator (low, } \\
\text { med, high) } \\
\end{array}$ & $\begin{array}{l}\text { Effect's overlap } \\
\text { with critical life } \\
\text { stage, behavior, } \\
\text { habitat or resource } \\
\text { (low, med, high) }\end{array}$ & $\begin{array}{l}\text { Overall risk to } \\
\text { indicator (low, } \\
\text { med, high, } \\
\text { unknown) }\end{array}$ & Source(s) \\
\hline \multirow{4}{*}{$\begin{array}{l}\text { Decommissioning of } \\
\text { structures on water's } \\
\text { surface or seabed }\end{array}$} & $\begin{array}{l}\text { Noise and } \\
\text { vibration }\end{array}$ & $\begin{array}{l}\text { Possible movement } \\
\text { from area and } \\
\text { disruption of foraging } \\
\text { or nesting }\end{array}$ & Low, see above & $\begin{array}{l}\text { Low, occurs over } \\
2 \text { months }\end{array}$ & $\begin{array}{l}\text { Low, project not near } \\
\text { nesting colony or } \\
\text { known foraging area }\end{array}$ & Low & $\begin{array}{l}\text { Michel et al. } \\
\text { 2007, SAS [date } \\
\text { unknown] }\end{array}$ \\
\hline & $\begin{array}{l}\text { Oil/chemical } \\
\text { release }\end{array}$ & $\begin{array}{l}\text { Hypothermia, toxicity } \\
\text { to individuals and prey }\end{array}$ & $\begin{array}{l}\text { Low, volume released } \\
\text { would occupy small } \\
\text { area relative to species' } \\
\text { range }\end{array}$ & $\begin{array}{l}\text { Low, occurs over } \\
2 \text { months }\end{array}$ & Low, see above & Low & $\begin{array}{l}\text { Michel et al. } \\
\text { 2007, SAS [date } \\
\text { unknown] }\end{array}$ \\
\hline & $\begin{array}{l}\text { Construction } \\
\text { lights }\end{array}$ & $\begin{array}{l}\text { Attraction/ } \\
\text { disorientation }\end{array}$ & $\begin{array}{l}\text { Low, would occur in } \\
\text { small area relative to } \\
\text { species' range }\end{array}$ & $\begin{array}{l}\text { Low, occurs over } \\
2 \text { months }\end{array}$ & Low, see above & Low & $\begin{array}{l}\text { Montevecchi } \\
\text { 2006, SAS [date } \\
\text { unknown] }\end{array}$ \\
\hline & $\begin{array}{l}\text { Seabed } \\
\text { disturbance }\end{array}$ & $\begin{array}{l}\text { Possible short-term } \\
\text { changes in food } \\
\text { resources }\end{array}$ & $\begin{array}{l}\text { Low, would occur in } \\
\text { small area relative to } \\
\text { species' range }\end{array}$ & $\begin{array}{l}\text { Low, occurs over } \\
2 \text { months }\end{array}$ & Low, see above & Low & $\begin{array}{l}\text { Boehlert et al. } \\
\text { 2008, SAS [date } \\
\text { unknown] }\end{array}$ \\
\hline
\end{tabular}

Environmental Assessment for Siting Wave \& Tidal Energy Projects
F-53

H. T. Harvey \& Associates 24 November 2009 
Appendix F - Effects of Pilot Scale MCT SeaGen Project at the Tacoma Narrows Site

\section{References}

Austin M, Delarue J, Johnston HA, Laurinolli M, Leary D, MacGillivray A, O'Neill C, Sneddon H, Warner G. 2009. NaiKun Offshore Wind Energy Project environmental assessment, Volume 4 - Noise and vibration. NaiKun Wind Development Inc. and JASCO Applied Sciences.

Blackwell SB, Lawson JW, Williams MT. 2004. Tolerance by ringed seals (Phoca hispida) to impact pipe-driving and construction sounds at an oil production island. Journal of the Acoustical Society of America. 115(5):2346-2357.

Boehlert GW, McMurray GR, Tortorici CE. 2008. Ecological effects of wave energy development in the Pacific Northwest. National Oceanic and Atmospheric Administration. NMFS-F/SPO-92.

Cada GF. 2008. The potential environmental impacts of marine and hydrokinetic renewable energy technologies. Washington, DC: Wind and Hydropower Technologies Program, U.S. Department of Energy. ER08-1040.

Calambokidis J, Baird RW. 1994. Status of marine mammals in the Strait of Georgia, Puget Sound and the Juan de Fuca Strait and potential human impacts. Canadian Technical Report of Fisheries and Aquatic Sciences No. 1948.

Calambokidis J, Darling JD, Deecke V, Gearin PJ, Gosho ME, Megill W, Tombach CM, Goley D, Toropova C, Gisborne B. 2002. Abundance, range and movements of a feeding aggregation of gray whales (Eschrichtius robustus) from California to southeastern Alaska in 1998. Journal of Cetacean Research and Management. 4(3):267-276.

Cardinale M, Casini M, Arrhenius F, Hakanson N. 2003. Diel variability of spatial distribution of zooplanktivourous fish related to feeding in the Baltic Sea. Aquatic Living Resources. 16:283292.

(CPUC) California Public Utilities Commission. 2009. Sacramento natural gas storage project EIR [Internet]. [cited 14 September 2009]. Available from:

http://www.cpuc.ca.gov/environment/info/dudek/sngs/Appendices/Section\%20D9\%20Noise\%20 and\%20Vibration.pdf.

Dadswell MJ, Rulifson RA. 1994. Macrotidal estuaries: A region of collision between migratory marine animals and tidal power development. Biological Journal of the Linnean Society. 51:93113.

Ebbert JC, Embrey SS, Black RW, Tesoriero AJ, Haggland AL. 2000. Water quality in the Puget Sound Basin, Washington and British Columbia, 1996-98. Tacoma, WA: US Department of the Interior, US Geological Survey. Circular 1216.

Emmett RL, Brodeur R, Orton PM. 2004. The vertical distribution of juvenile salmon (Oncorhynchus spp.) and associated fishes in the Columbia River plume. Fisheries Oceanography. 13(6):392-402.

(EVS) EVS Environmental Consultants. 2003. Status, trends and effects of toxic contaminants in the Puget Sound environment: Recommendations. Olympia, WA: Puget Sound Action Team. 
Hall JE. 2003. Bioconcentration, bioaccumulation, and biomagnification in Puget Sound biota: Assessing the ecological risk of chemical contaminants in Puget Sound. [Internet]. Journal of the Environment. 01 [cited 3 November 2009]. Available from: http://courses.washington.edu/uwtjoe/issues/2003_01/2003_01_02.pdf.

Hatakeyama Y, Soeda H. 1990. Studies on echolocation of porpoises taken in salmon gillnet fisheries. In: Thomas JA, Kastelein RA, editors. Sensory abilities of cetaceans: Laboratory and field evidence. New York, NY: Plenum; p. 269-281.

Herder MJ. 1986. Seasonal movements and hauling site fidelity of harbor seals, Phoca vitulina richardsi, tagged at the Klamath River, California [master's thesis]. [Arcata, CA]: Humboldt State University.

(IALA) International Association of Marine Aids to Navigation and Lighthouse Authorities. 2008. IALA recommendation O-139 on the marking of man-made offshore structures. Saint Germain en Laye, France: IALA-AISM.

Jeffries SJ, Gearin PJ, Huber HH, Saul DL, Pruett DA. 2000. Atlas of seal and sea lion haulout sites in Washington. Olympia, WA: Washington Department of Fish and Wildlife, Wildlife Science Division.

Kastak D, Southall BL, Schusterman RJ, Reichmuth-Kastak CJ. 2005. Underwater temporary threshold shift in pinnipeds: Effects of noise level and duration. Journal of the Acoustical Society of America. 118(5):3154-3163.

Koschinski S, Culik BM, Henriksen OD, Tregenza NC, Ellis GM, Jansen C, Kathe G. 2003. Behavioral reactions of free-ranging porpoises and seals to the noise of a simulated $2 \mathrm{MW}$ windpower generator. Marine Ecology Progess Series. 265:263-273.

Largier J, Behrens D, Robart M. 2008. The potential impact of WEC development on nearshore and shoreline environments through a reduction in nearshore wave energy. In: Nelson PA, editors. Developing wave energy in coastal California: Potential socio-economic and environmental effects. Sacramento, CA: California Energy Commission, PIER Energy-Related Environmental Research Program \& California Ocean Protection Council; p. 57-82.

LGL Limited, KS Biological Services, Pottinger Gaherty Environmental Consultants. 2009. NaiKun Offshore Wind Energy Project environmental assessment, Volume 8 - Marine birds and sea turtles. NaiKun Wind Development Inc.

Michel J, Dunagan H, Boring C, Healy E, Evans W, Dean JM, McGillis A, Hain J. 2007. Worldwide synthesis and analysis of existing information regarding environmental effects of alternative energy uses on the Outer Continental Shelf. Herndon, VA: U.S. Department of the Interior, Minerals Management Service. MMS 2007-038.

(MMS) Minerals Management Service. 2007. Programmatic environmental impact statement for alternative energy development and production and alternate use of facilities on the Outer Continental Shelf. U.S. Department of the Interior. MMS 2007-046. 
Appendix F - Effects of Pilot Scale MCT SeaGen Project at the Tacoma Narrows Site

Montevecchi WA. 2006. Influences of artificial light on marine birds. In: Rich C, Longcore T, editors. Ecological consequences of artificial night lighting. Washington, D.C.: Island Press; p. Chapter 5.

Morton AB, Symonds HK. 2002. Displacement of Orincus orca (L.) by high amplitude sound in British Columbia, Canada. ICES Journal of Marine Science. 59(1):71-80.

Nedwell JR, Edwards B, Turnpenny AWH, Gordon J. 2004. Fish and marine mammal audiograms: A summary of available information. Subacoustech Report ref: 534R0214

Nelson PA. 2008. Ecological effects of wave energy conversion technology on California's marine and anadromous fishes. In: Nelson PA, editors. Developing wave energy in coastal California: Potential socio-economic and environmental effects. Sacramento, CA: California Energy Commission, PIER Energy-Related Environmental Research Program \& California Ocean Protection Council; p. 111-135

Nelson PA, Woo S. 2008. Developing wave energy in coastal California: Potential socioeconomic and environmental effects: Introduction. In: Nelson PA, editors. Developing wave energy in coastal California: Potential socio-economic and environmental effects. Sacramento, CA: California Energy Commission, PIER Energy-Related Environmental Research Program \& California Ocean Protection Council; p. 7-21

Nightingale B, Longcore T, Simenstad CA. 2006. Artificial night lighting and fishes. In: Rich C, Longcore T, editors. Ecological consequences of artificial night lighting. Washington, D.C.: Island Press; p. Chapter 11.

Olson AF, Quinn TP. 1993. Vertical and horizontal movements of adult chinook salmon, Oncorhynchus tshawytscha, in teh Columbia River estuary. Fishery Bulletin. 91:171-178.

Penttila D. 2007. Marine forage fishes in Puget Sound. Seattle, WA: Seattle District, U.S. Army Corps of Engineers. Puget Sound Nearshore Partnership Report No. 2007-03.

Previsic M. 2009. Working draft - Scenario based analysis of environmental and navigation impacts: Wave power scenario descriptions.

Relini G, Relini M, Montanari M. 2000. An offshore buoy as a small artificial island and a fishaggregating device (FAD) in the Mediterranean. Hydrobiologia. 440:65-80.

Richardson WJ, Wursig B. 1997. Influences of man-made noise and other human actions on cetacean behaviour. Marine and Freshwater Behaviour and Physiology. 29:183-209.

Ruggerone GT, Quinn TP, McGregor IA, Wilkinson TD. 1990. Horizontal and vertical movements of adult steelhead trout, Oncorhynchus mykiss, in the Dean and Fisher channels, British Columbia. Canadian Journal of Fisheries and Aquatic Sciences. 47:1963-1969.

(SAS) Seattle Audobon Society. [date unknown]. Puget Trough ecoregion and birding sites [Internet]. Seattle, WA: [cited 3 November 2009]. Available from: http://www.birdweb.org/birdweb/birding_sites_list.aspx?id=3\&emphasis=site. 
Appendix F - Effects of Pilot Scale MCT SeaGen Project at the Tacoma Narrows Site

Schusterman RJ, Balliet RF. 1970. Visual acuity of the harbour seal and the steller sea lion under water. Nature. 226(5245):563-564.

Stroud RK, Roffe TJ. 1979. Causes of death in marine mammals stranded along the Oregon coast. Journal of Wildlife Diseases. 15:91-97.

Snohomish Public Utility District. 2008. Preliminary Application Document: Puget Sound InStream Tidal Power Project. Submitted to FERC January 31, 2008.

Substructure. [date unknown]. How is concrete affected in a marine environment? [Internet]. Portsmouth, NH: [cited 15 September 2009]. Available from: http://www.substructure.com/education/how_is_concrete_affected_in_a_marine_environment.ht $\underline{\mathrm{ml}}$.

Sullivan RM, Stack JD, Houck WJ. 1983. Observations of gray whales (Eschrichtius robustus) along northern California. Journal of Mammalogy. 64(4):689-692.

Todd VLG, Pearse WD, Tregenza NC, Lepper PA, Todd IB. 2009. Diel echolocation activity of harbour porpoises (Phocoena phocoena) around North Sea offshore gas installations. ICES Journal of Marine Science. 66:734-745.

Tougaard J, Henriksen OD, Miller LA. 2009. Underwater noise from three types of offshore wind turbines: Estimation of impact zones for harbor porpoises and harbor seals. Journal of the Acoustical Society of America. 125(6):3766-3773.

(USCG) U. S. Coast Guard. [date unknown]. USCG vessel traffic service - Puget Sound [Internet]. Seattle, WA: U.S. Coast Guard - Thirteenth District; [cited 3 November 2009]. Available from: http://www.uscg.mil/d13/units/factsheets/vts.pdf.

(USFWS) U.S. Fish and Wildlife Service. 2005. Regional Seabird Conservation Plan, Pacific Region. Portland, Oregon: U.S. Fish and Wildlife Service, Migratory Birds and Habitat Programs, Pacific Region.

(USN) U.S. Department of the Navy. 2008. Northwest training range complex draft environmental impact statement / overseas environmental impact statement - Volume 1. Silverdale, WA: U.S. Navy - Commander, U.S. Pacific Fleet.

Van Waerebeek K, Leaper R. 2008. Second report of the IWC vessel strike data standardisation working group. In: editors. IWC 60th Annual Meeting; Santiago, Chile. p. 8.

Wilson B, Batty RS, Daunt F, Carter C. 2007. Collision risks between marine renewable energy devices and mammals, fish and diving birds. Oban, Scotland: The Scottish Executive, Scottish Association for Marine Science.

Yurk H, Trites AW. 2000. Experimental attempts to reduce predation by harbor seals on outmigrating juvenile salmonids. Transactions of the American Fisheries Society. 129:1360-1366. 